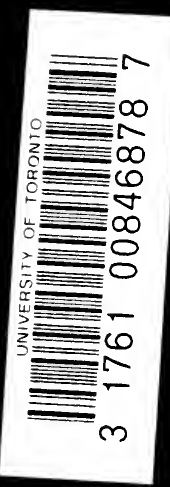




$$
b^{5} T^{3}
$$


JAN1 01997 


\section{Digitized by the Internet Archive in 2007 with funding from Microsoft Corporation}




\section{DISEASES OF THE ARTERIES INCLUDING}

ANGINA PECTORIS 


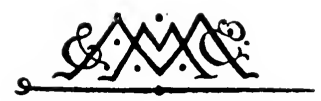

MACMILLAN AND CO., LIMITED

LONDON - BOMBAY · CAICUTTA

MELBOURNE

THE MACMILLAN COMPANY

NEW YORK - BOSTON - CHICAGO

DALLAS - SAN FRANCISCO

THE MACMILLAN CO. OF CANADA, LTd. TORONTO 
$M C$

A

\title{
DISEASES OF THE ARTERIES
}

INCLUDING

\section{ANGINA PECTORIS}

\author{
BY \\ Thomos \\ SIR^CLIFFORD ALLBUTT, K.C.B. \\ M.A., M.D., F.R.C.P., F.R.S., Hon. M.D., LL.D., D.Sc., ETc. \\ REGIUS PROFESSOR OF PHYSIC IN THE UNIVERSITY OF CAMBRIDGE \\ FELLOW OF GONVILLE AND CAIUS COLLEGE \\ HON. FELLOW NEW YORK ACAD. MED., ETC.
}

IN TWO VOLUMES

VOL. I

MACMILLAN AND CO., LIMITED

ST. MARTIN'S STREET, LONDON

I 9 I 5 


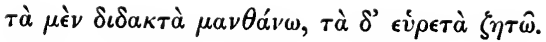




\section{PREFACE}

Those faults of a book which lie in the quality of its author are more apparent to others than to himself, but none better than the author, who has spent many years in the intimacy of his subject, can know its shortcomings. He is beset by the old proverb that so long as more remains to be done nothing seems done. Still a time comes when his book, however far even from relative perfection, must be published, if published at all-the hour when "Tam maun ride." Then it is that to the author the "suicidal impulse" becomes comprehensible; the temptation to make a sudden end of that shed part of himself now drifting on the ebb while the flow of swift thought is streaming far away beyond. No more is there cranny or footnote for new matter or new repentances ; his work is stopped and he swings out of the tide. If this sense of imperfection haunts the composer throughout his work, yet it is keenest when the work is done. Of course we cannot know all, nor anything like all ; much of the detailed structure and of the fuller outlines of every subject is still obscure or out of sight; but can the author reasonably pretend that he possessed interpretation enough to enable him to deal with the facts provisionally? The answer to this question is not for him to give.

But, if I may speak for myself, I have done my best nevertheless to withstand the craving for a show of symmetry; to avoid the attempt, by building up conjectural superstructures, by "letting the fancy outwork nature," to put a semblance of order and 
completion upon data and inference which in the nature of things must as yet be fragmentary and scattered; or to work up into artful categories and systems notions which as yet should be left as guesses or suggestions. As J. F. Payne said of Galen, " the formal completeness of a system may be rather a hindrance than a help to progress." By leaving some data in the background and bringing others into high relief, by painting a little colour here and throwing a little shadow there, it is not difficult to construct an argument far more taking, far more " readable," than pages of outlines still vague, of meanings still tentative, of facts still insecure and unbalanced. Immature "scientific" truth, like the ugly duckling, is plain in feature, in form unattractive, and very cold in colour.

In biology, to ride in search of the truth on logical systems is an error derived from scholastic philosophies. Whatever may be true of them, biology at any rate has not to deal with preexisting potential entities unfolded in time and space. In biology external circumstances, instead of being inessential, enter essentially into the process. The more delicate and complex the balance, the more effectual the tangential influences; hence the potency of external factors in determining the evolution. It has been cleverly said that the living organism is a diagram of incident forces. In a word, the biologist leaves Hegel for Darwin.

No symmetrical scheme of interpretation is possible until a far larger consummation of our sphere of experience is attained; but the principle of a well-known passage of The Ethics (iii. 4) remains true, that "we must understand that this indefiniteness proceeds from our lack of knowledge, and not from any arbitrary nature of the things themselves; for in so far as there were any uncertainty attaching to the things themselves they would not be fit subjects for deliberation at all."

Albeit, we may not flatter ourselves that with vagueness of outline and scrappy thoughts we are putting on the features, or even the vestures, of science; far from it. Better for me that literary suicide, than for this book to increase the volume of 
clinical gossip, in its daily round sorting old phrases to and fro into new combinations. Successfully or not, whether by my own tests or the tests of others, I have tried to adapt my thoughts, not to our language and older concepts, but to such facts as we have; and so to assay the values of current formulas that, poor as I am, I may not pass for rich on false coin.

If in analysing biological co-efficients we lost ourselves, so to speak, in a uniform blend, as for instance of water and treacle, the tracks of observation would be very blind. Happily, functions and rhythms sort themselves in forms, static and dynamic ; as illustrated by tone and mobility, by the members of reflex cycles, by activity and inhibition, by Mendelian factors, and so on : a kind of sorting, as to a sleeper in a railway train or steamship the myriad vibrations blend into a rhythmical burden, or as powders on a drum take form under the sound of a harpstring. Thus, on lines of summation we can track out and chart the processes of organic life in patterns. Man does not fall ill anyhow.

Justly then as we may attribute the unequal and inconclusive characters of our books to states of contemporary knowledge, yet a small voice admonishes us nevertheless of many a fault and negligence. To the works of others, even to some on my own shelves, I have given less than the attention they deserve, and to their authors I must offer a preliminary apology. Notwithstanding, I believe that on the whole the labour put into this work is honest labour, and trust that the winter fruitage of an old tree, if a little withered, may not be unsound; that with the fading it may have some of the mellowness of age.

On another aspect of form I have to offer also some apology. The various chapters of the present volumes have all been published before, some of them, in substance, again and again. Perhaps I ought to have blended these, and recast the work as a whole; in such a remodelling however there were obvious difficulties, not the least being a further loss of time, already 
too great. Moreover the style and construction of the chapters, written at various times and occasions, must have been remodelled, and might in this remodelling have lost life and variety. Thus the book has rather the form of a string of dissertations than of an orderly monograph. Amongst the sections therefore, as first put together, there were many repeated passages; some of these have been pruned away, but some I see yet remain; these may serve for emphasis, and to save cross reference: for redundancies I ask the reader's indulgence. Furthermore, by this freedom of dissertation I have been at liberty to run cursorily over, or even to avoid, many paths of common knowledge, and to write at greater length in ways which are less familiar, or on matters on which I had personal opinions to express.

To a bibliography I make no pretence; amid many public engagements my reading has been unsystematic, and determined by accidents of time and of access to books and journals. I have thought it better therefore to use footnotes at the passages where the articles quoted are applicable. For not a few references I have to thank Dr. Henwood Harvey and Dr. Wolf. To re-verify in proof all the references in my manuscript would have been a heavy undertaking; at hazard I have checked a small percentage of them, and finding these practically correct, I trust that the whole number may not contain much error.

Illustrations I have definitely forgone; effective plates are costly, and I have no features to show of which there are not many and good examples in accessible books.

It may seem that in some earlier sections of this book, as in "Cardiac Physics," mathematical methods were called for. Long ago, with the able assistance of Dr. William Nicolls of Peterhouse, such attempts were made, but they did not carry us far. Some reflections of the late Sir George Darwin therefore, both for their value and for the interest attaching to any words of his, I venture to quote (from a letter to me dated May 31, 1896). Of Dr. Nicolls' work he says, "I think it very praiseworthy." He says 
further that "Larmor (and he) had lookedit over and made certain marginal notes and criticisms"; of these I have taken heed. But to all biologists the important paragraph is as follows: "The author must, and probably does, bear in mind that soluble mathematical problems afford only rough representations of actual biological problems, and that accordingly a careful control by experiment and observation is necessary to ensure that the leading characteristics of the case are included in the mathematical problem." So, as in mathematics I am a child-if even that, I have decided to "stick to my last."

To many kind friends I have to express deep obligations; gladly would I acknowledge my debts particularly were it not that in so doing I might seem to shift some of my own responsibilities to other shoulders. But, in general terms, I would thank the Master of Caius (Dr. Hugh Anderson), Professors Langley, Sherrington, Woodhead, Hopkins, Dixon, and Mines, and Dr. Henry Head and Dr. Walter Fletcher, for particular aids; and these again, and many other colleagues and friends, for the personal instruction and inspiration without which I should have been poor indeed. For hunting up pamphlets and theses not upon the shelves of the University Library, or of our Departmental Library, I have to thank Messrs. Williams \& Norgate, to whom I have been more a troublesome than a profitable customer; likewise I have to thank many authors, especially those unknown to me, for their kindness in sending books and pamphlets. Except in those notes in which I have indicated a secondary source, all the papers quoted have been perused, a practice from which we may learn many things ; for instance, how errors of interpretation arise from the practice of glancing only at the few lines of "Conclusions" at the ends of papers, or at excerpts by the scissors. From summaries many points are omitted; occasionally indeed the "Conclusions" are not quite consistent with the contents of the article itself.

Last, but not least, I have to express my own obligations, to which I may add those of my readers also, to my colleague 
and friend Dr. Aldren Wright for his kindness and skill in compiling the index.

Postscript (March 20, 1915).- The great War burst upon us as these pages, in the early summer of 1914, were finally corrected for press. My publishers then advised me to postpone publication for a while; now however in their opinion the time has come when further delay is more and more difficult. Happily, the materials accumulated during the last nine months seem less than one might have feared. Few, if any, foreign periodicals have come to hand since last July ; probably little work is being done in the laboratories even of neutral countries, most of which are under some military stress. My own materials have indeed grown somewhat; but for these additions no place in the pages can now be made. One case however, with necropsy, seems to me in respect of the alleged series named "Hyperpiesia," "Hypertendus purs," “Die essentielle Hypertonien" (see Vol. I. p. 313) to be of such crucial importance that I venture to drag it in here.

I have repeated the current opinion that cases of "pure" high blood pressure rarely occur in hospital practice. However, when on the outbreak of the War I returned to routine ward duties, I admitted in the first month three such cases. One of them was of a woman beyond middle life, with a systolic pressure of 230 , thick tortuous vessels, big heart, and paroxysmal dyspnea. The urine contained more or less albumin, but, though repeatedly and carefully spun, presented no casts. The specific gravity was good, and lithates were often deposited. She had no nausea, headache, drowsiness, nor neuroretinitis. The second was similar to it, but with the ordinary dyspnea of dilated heart. The third was a very instructive case; with the autopsy it was of almost singular interest. This man was brought into Addenbrooke's Hospital in a grievous condition, but of his previous history little was known. His whole body, except the head and face, was œdematous; the legs were huge, dense and brawny, with lymph stasis and multitudes of clusters of lymph vesicles and nodules on the skin. Extensive excoriations under the thighs and legs, and the orthopnea, caused him great misery. A large curtain of œdema on the lower back showed high pressure 
also in the vena azygos. Deprivation of salt seemed ineffectual; but the withdrawal of a litre of fluid from the right pleura gave some temporary relief. The systolic pressures ranged about 220 , the accessible arteries were thickened and rather tortuous, and the enormous heart, hypertrophied and dilated, displayed at the autopsy dimensions which had been little less conspicuous during life. A mitral regurgitant murmur had been audible now and then, but post-mortem the valves appeared competent. The urine, on the whole scanty, was of good specific gravity and often lateritious. Casts were sought from time to time, but, save an occasional hyaline cylinder, not found. No sign of syphilis.

During these three last months of his life some relief was obtained by medicines. Under diuretics-caffein, digitalis, and so forth, the dropsy gave way a little; but it was not until I administered morphia subcutaneously that any considerable amendment was obtained. A fifth of a grain every evening assuaged all the symptoms, and so far palliated the œdema and cardiac distress that his nights became restful. Although subcutaneous morphia was my own remedy for such states, I had postponed its use because, in such a case in a young man of 29 , I could not but fear the presence of primary contracted kidneys. "Pure" hyperpiesia at such an age must be very rare. However, such it proved to be. The patient died suddenly : after death the heart appeared such as I have described; the aorta in its whole length was conspicuously atheromatous; it was not largely dilated, and presented no mark of syphilis. The larger arteries were universally thickened; a portion of the right radial was excised. Every part of the body was soaked in œdema. The kidneys seemed thus rather large, wet, flabby, and rather pale. The capsule swam off like wet tissue paper, save for a few very small points of adhesion. On section they seemed normal in structure and in the proportions of cortex and medulla. Thin sections of the kidney showed, as in other parts, the general thickening of the arteries, large and small; many of the Malpighian bodies were thus obliterated. This Professor Woodhead reported, in the kidney and elsewhere, as the outstanding and primary disease; the lumina of the tubules were open, and for the most part the epithelium was in situ, but in places cells were breaking down, one or two in a section of a tubule, which in his opinion pointed to a quite recent catarrhal affection belonging only to the later stage of the malady. My personal opinion was that the general œdema had sufficed to produce this tubularchange. The small arteries presented some fibrosis of the media, and the usual patches of atherosclerosis of the intima. The myocardium, on microscopic section, was normal, or betrayed here and there a very early stage of fibrosis. 



\title{
CONTENTS
}

\author{
PART I
}

ARTERIOSCLEROSIS

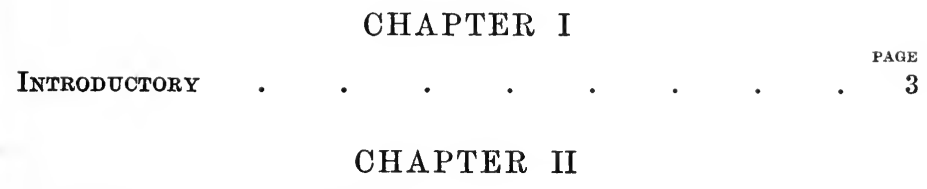

Phystcs of the Circolation . . . . . . .

CHAPTER III

The Vrscosity of the Blood . $\quad$. $\quad . \quad$. 104

CHAPTER IV

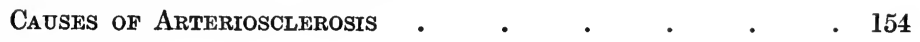

CHAPTER V

Arteriosclerosis AND THe Kidneys . $\quad . \quad$. $\quad . \quad 309$

CHAPTER VI

Symptoms of ARteriosclerosis $\quad$. $\quad . \quad$. $\quad . \quad$. 374

CHAPTER VII

Morbid Anatomy of Arteriosclerosis $\quad . \quad$. $\quad . \quad 455$

xiii 

PART I

ARTERIOSCLEROSIS

VOL. I 



\section{CHAPTER I}

\section{INTRODUCTORY}

HISTORY.- In order to understand the drift of our conceptions of arteriosclerosis we shall do well to look back, if but for a moment, upon the course of our learning in this chapter of medicine. It is remarkable that the ancients, in their interminable scholastic refinements upon the phenomena of the pulse, phenomena first formulated by Herophilus and elaborated later by Galen, in the vessels accessible to touch seem to have paid little attention to the morbid changes of the vascular tunics. In the de Pulsibus, it is true, Galen notes degrees of softness, or hardness of the coat, which may be fleshlike, or dry and hard

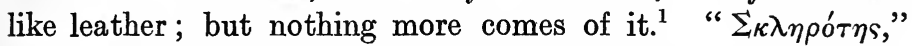
from the context, did not refer to the arterial wall. Asclepiades seems to have ascribed certain hæmorrhages to decay and rupture of the arterial coats. The three tunics of the arteries had been described-probably first by the Alexandrian anatomists ${ }^{2}$ and afterwards by Galen-yet it is not until quite modern times, until, let us say, the illumination of medicine by the torch of Morgagni, that we find more than an occasional allusion to arterial disease. So true is it that we must know that we may see; that in a new field observations do not come about until our thoughts have undergone some correlative preparation. The facts were

1 Pulsus durus and mollis seem to have been indicative of what we now call blood pressure rather than of the state of the coats of the artery. Besides these they had celer, tenuis, tardus, plenus, debilis, languens, etc. etc. Galen wrote sixteen books on the pulse.

${ }^{2}$ See Oribasius, Collect. Med. vol. iii. xxiv. 13 ; pp. 327-328 (of Daremberg's edition). Apollonius of Pergamon (Orib. ii. 64) says the vessels may be stretched too tight, so that the pneuma $(\pi . \phi v \sigma \iota k \delta v)$ can with difficulty pass through the body. 
there all the time, many of them needing no new instruments, no new faculties, for their perception; but the mind's eye was not in focus for them. Thus it was not until Harvey's revolution in physiology that the importance of the arteries as instruments of nutrition was comprehended; before Harvey men were thinking rather of the inward gambols of the pneuma than of the channels in which it expatiated. From the time of Morgagni and Haller onwards however the state of the arterial wall was more commonly, if not very closely or diligently, regarded. Unfortunately, before Laennec and Trousseau, cadaveric staining of the great vessels was taken for an arteritis, as by Peter Frank and Broussais, and even by Bouillaud; thus much observation was wasted by a false pathology. And it was not until the dynamics of the circulation began to receive the attention awakened by the general progress of physics, that by Hales (in 1727) and others blood pressures were studied, or indeed apprehended. ${ }^{1}$ Hales measured the arterial blood pressure in a glass tube; Poiseuille, about one hundred years later, added the mercury column; Ludwig the kymographic pen and drum. As regards the tunics, Clifton Winteringham of York (1689-1748), in his Experimental Enquiry on Some Parts of the Animal Structure, described his tests of the arteries in animals by forcing air into lengths and branches of these vessels ; and he drew up a table of "Comparative Tenacities."

We recognise now, all of us, that in the lapse of man's years one long reckoning of his mortality is, and from all known ages has been, written on the walls of his vessels. We may suppose that in primitive man, by external conditions if not by innate capacity, life was of comparatively brief duration. Domestic animals seem, as a rule, not to live long enough to use up their arteries, or not to live ill enough to abuse them; and amongst these creatures atheroma, though not unknown, has not been

\footnotetext{
1 Puschmann says (Hist. ii. p. 27) that Eustacchi recognised the importance of abnormally high blood pressures; but he does not give chapter and verse. On a cursory search I cannot find such a passage under artery or vein; but there is (Venet. ed. 1563, p. 108) an interesting speculation on arterial faculty of motion, how far cardiac and how far peculiar-but this is the difficulty of the primum movens at which Harvey marvelled, as did Aristotle before him. "Nihilque prohibeat," says Harvey, "quominus eadem facultas illas (arterias) dilatet atque constringat."
} 
commonly observed. ${ }^{1}$ We may reasonably conjecture that as in stable societies human life became protracted the arteries got time to wear out.

Now when we light upon a form of disease which had either escaped the notice of our ancestors, or had been but obscurely perceived by them, we are apt to proclaim the disease as a new one; or at any rate as a return of some vagrant malady after a period of recession. Thus diphtheria, typhoid fever, neurasthenia, and many another malady has been regarded in its turn as a newcomer. But for the most part the new thing is not the morbid series itself but our recognition of it; not the order of nature but the enlargement and sharpening of our clinical wits.

In Egypt as we know, in Chaldea as we may guess, regions protected by desert, marsh, or sea, the circumstances of life were equable and favourable; intervals of domestic peace at home were so far secured that men, of the wealthier classes at any rate, were enabled to carry their bodies to a ripe maturity. In the bodies of the Egyptians, not always of very old persons, Ruffer and Elliott Smith found the aorta commonly presenting atheromatous patches, "ulcers," and calcified plates; and other arteries thickened, beaded, and as brittle as pipes, so as hardly to bear handling. Meneptha, they tell us, ${ }^{2}$ was at his death a baldheaded, decrepit old man with calcareous arteries.

Without pretending, then, to enter upon a history of vascular deterioration, or rather of our perceptions of it, suffice it to say that, long overlooked as it was, arterial disease (atheroma) has been recognised, anatomically if not clinically, for many generations. It was recognised by Riolan the younger (1577-1657) and some of his contemporaries who, like certain eminent pathologists nearer our own day, found satisfaction in final causes; they argued, for instance, that, as in the ripening of years the heart must fail, the arteries were hardened in order that their channels might be kept open-“" in arteriis natura ossicula collo-

1 Such has been the confident opinion of persons conversant with their diseases, from whom I have made enquiries. But it seems likely that in domestic animals also decay is more frequent than is generally supposed, and that it has escaped attention. See Parisot, in more than one paper; e.g. L'Athér. aortique chez les animaux (both domestic and wild), read at the First Congress of Comparative Pathology at Paris, Oct. 1912 (see p. 215).

2 Cairo Scient. Journ., Jan. 1910. 
cavit, ubi opus erat ut aperta manerent." And, in brief summary, arterial degenerations were recognised by Lancisi (1654-1720), ${ }^{1}$ Boerhaave (1668-1738), Winteringham, as I have said, Senac (1693-1770), Haller (in 1757), ${ }^{2}$ Morgagni (in 1764), Bichat (c. 1795), who found arterial disease in seven out of every ten men over the age of 60, Meckel (1781-1833), Scarpa, "steatomatous arteries" (1748-1832), and Hodgson (1810). ${ }^{3}$ Haller's description of arterial disease is very interesting; of the "succus luteolus" or "flavus" "between the muscular and the internal coat," becoming hard at first, but in all degrees softer or pultaceous, or drier; the intima in parts becoming "quasi erosa," or quite ulcerated. 4 It is remarkable that Morgagni says little or nothing about general arteriosclerosis, as, for instance, in the limbs; he describes it in the aorta and large vessels chiefly, but also in the cerebral and in the coronary; of these he said in one case that they were reduced to bony canals. Matthew Baillie mentions coronary atheroma only. Among the recent physicians and histologists who have carried this investigation forward, the names of Rokitansky, Virchow, Guéneau de Mussy, Johnson, Lobstein, Hodgson, Kirkes, ${ }^{5}$ Wilks, Lancereaux, Gull and Sutton, Debove and Letulle, Mahomed, Traube, Ewald, Broadbent, Peter, Potain, Cornil and Ranvier may be mentioned as of pioneers. Corvisart $^{6}$ described arteriosclerosis of the pulmonary artery. The name arteriosclerosis we owe to Lobstein, whose classical memoir may be accepted as the opening of the study of the diffuser lesion, as contrasted with patchy atheroma. ${ }^{7}$ He rightly

1 Paré is occasionally quoted as describing arterial disease, but he speaks of it, so far as I can find, only in connection with, and as a consequence of, aneurysm ( $L i b$. vii. 32 ). Even Lancisi, who fully realised the importance of syphilis as a cause of aneurysm, which Baillie did not (vide iii. 32), failed to attain to the broader view of arterial disease as a forerunner of it.

2 Haller, Opusc. Path. vol. v.

3 For a fuller sketch of the history of this subject see Aitchison, Thesis for M.D., Oxford, Arch. Path. Inst. Lond. Hosp., 1908.

4 Haller, Elem. Phys. xlvi. 4; and Opusc. Path. Obs. lix.

s I include Kirkes' name as some work in this direction is usually credited to him. In his Physiology I find nothing; but I have followed the reference of others to the Med. Times and Gaz. of 1865. Here he supports Bright, who had already associated atheroma with chronic renal disease; but I do not see that he added much of his own. He has other and ampler claims on our gratitude.

- Corvisart, Essai sur les mal. du cœur, ii. 428.

7 Lobstein, Traité d'anat. pathol., Paris, 1833, t. ii. 
considered it as degenerative rather than inflammatory, and thus may be regarded as the forerunner of Gull and Sutton, and Debove and Letulle, whose service it was to enlarge the conception of arterial disease as a general process. Then arose Professor von Basch of Vienna who, on the clinical side, by acute, sagacious, and comparatively precise clinical observations in a large field of experience, checked continually by experiments upon animals, laid the foundations of the systematic knowledge of arteriosclerosis which as physicians we possess today. And if indeed in the progress of knowledge we have found that von Basch's pressure gauge was imperfect, and if in his doctrines we have found something to add to, something to dissent from, and something to correct by a truer analysis, as we suppose, of the correlation of excessive mechanical pressures and arteriosclerosis, nevertheless, in its main features von Basch's work must always hold a classical place in the clinical history of arterial disease.

Meanwhile, on the dynamic side, work of no less distinction and moment was being published by Marey, Ludwig, Ringer, Oliver, Cohnheim, and his brilliant disciple Roy, to whose friendship from the earliest days of my interest in these subjects I owe more than I can tell ; many of Roy's pupils, such as Adami and Rolleston, are happily still working in our midst. Then the highly analytical mind of the physicist was brought to bear on our problems, but the doctrines of these pioneers are now so intimately incorporated with current knowledge, and in the course of this essay must be so frequently cited, that I need not give any further sketch of their services. Marey's early contributions to the measurement of blood pressures are however too little remembered. He made a plethysmographic receptacle for the hand, to which he attached a tambour ; with this instrument, as we shall see, he took the largest oscillations as significant of minimum pressure.

The clinical interpretation of excessive blood pressures and their consequences - "arterial tensions" as they were inadvisedly called-in arterial disease, as opposed to semi-occult and speculative causes such as "blood crases" with their "deposits" and "blastemas," Traube, ${ }^{1}$ Ewald, and Potain

1 Traube, Gesammelte Beiträge, Bd. iii. ss. 164 and 235, etc. 
were the first to unfold; and Riegel ${ }^{1}$ followed in their steps. Sir Lauder Brunton also was investigating blood pressures clinically, in 1871 and previously. But Traube's demonstrations were made rather narrowly upon cases of chronic Bright's disease; and the rise in pressure he explained by the assumption of a vasoconstriction in the renal area, an area too small to make itself felt as a rise of general blood pressure. Ewald and Potain argued for a more general "hypertension," as due to a toxin or to a reflex from renal irritation. Mahomed, ${ }^{2}$ of Guy's Hospital, became impressed more and more with the importance of rising blood pressures; he taught that, obscure as were their causes, as indeed still they are, their significance was profound, and their effects far-reaching. His view of these changes, as I well remember, was larger, and approached more nearly to modern knowledge, than appears literally in the paper to which I now refer. Mahomed urged more and more the significance of these increments of pressure, and the gravity of this factor in clinical interpretation and prognosis $;^{3}$ a salutary warning, whether they were, as he supposed, or were not always significant of renal disease.

My own initiation in this field of research was due to Burdon Sanderson who, under Marey's influence, had begun to work at the sphygmograph; and it was thus that we came into association with the young Guy's physician only too soon to be taken from us. So during the early seventies of last century, Sanderson and Mahomed and I were working together at this subject, chiefly at the form of the pulse; but by means of the sphygmograph we were trying also, as Vierordt was trying, with weights or vernier screws, to get some notion of arterial pressures. For the present, I will say no more than that, to our disappointment, this proved to be impracticable (p. 59). As von Frey observed, a sphygmographic curve is one of which the ordinates are not known. Masing tried to supply them by affixing a R.-R. sphygmometer simultaneously on the upper arm; when he observed the curves good and equal on both sides of an obliterating pressure. The systolic height of the sphygmograph began to fall at $120^{\circ}$,

1 Riegel, Berlin. kl. Wochenschr., 1882, No. 23.

2 Mahomed, Guy's Reports, 1879.

${ }^{3}$ See also Dr. Graham Steell, The Sphygmograph in Medicine. 
and at $150^{\circ}$ disappeared. This is a difficult experiment; and in our early days no external sphygmometer had been devised. Ludwig's kymographic method was applicable only to experiment upon animals. Von Basch's instrument was made known in 1876.

Mahomed at this time introduced a phrase which, with the opinion it signified, has fallen into disuse: namely, "the pre-albuminuric stage of Bright's disease." He formulated an hypothesis that, before Bright's disease manifested itself, there was a period, longer or shorter, of augmented blood pressure; a period during which the encroachment of the disease could be foretold, and perhaps forestalled. Riegel agreed, in 1882.

At this time, and at this stage of the enquiry, we suffered the great loss of our distinguished colleague by a premature death. For, as time went on, I began to find that many of these " prealbuminurics" did not pass into Bright's disease; that on the contrary not a few of them recovered completely: results which rather shook one's notions on the " pre-albuminuric stage of Bright's disease." It was not however until I had watched a certain patient for eighteen years, and many another for shorter periods, that I felt at liberty to publish an opinion upon the subject. That lady, who was the first to come under my own private and continuous observation as a "pre-albuminuric," never fell a victim to Bright's disease, but survived for eighteen years. During this long period she went through a series of morbid changes attended with high blood pressure which I shall describe later ; and ultimately with a large and dilated heart she died of an apoplectic seizure, but without Bright's disease in any customary clinical use of the term. Somewhat later, when I was working alone, I received much encouragement and assistance from my old friend Dr. George Oliver, then of Harrogate, whose contributions, past and present, to the subject are too well known to need my commendation.

Some years later I left Leeds rather suddenly to take up the duties of a Lunacy Commissioner; then, on visiting many asylums, I found a large number of persons suffering from all degrees of arterial disease; yet most of these, as I found somewhat to my surprise, without any apparent aggravations of blood pressure. Thus gradually I became convinced that cases, such as we are 
considering, must be divided, first, into Bright's disease-which I shall consider in another chapter (p. 307); secondly, into the class to which soon afterwards I gave the name Hyperpiesis, a malady in which at or towards middle life blood pressures rise excessively, a malady having a course of its own and deserving the name of a disease ; and thirdly, into at least one other class of arterial degeneration, one not typically associated with rise of blood pressure, a class in which indeed the blood pressure does not exceed, or scarcely exceeds, the rise common to almost all persons in later life; a series of which the course, symptoms, and issues are altogether different. To these I added a convenient if somewhat heterogeneous class of toxic arteriosclerosis.

It is now well known that at this time M. Huchard also was engaged in the same line of research; and in 1893 he published his work on Diseases of the Heart. This work, a reissue and extension, I believe, of certain previous papers which I have never seen, was sent to me for review in the British Medical Journal (Nov. 4, 1893). M. Huchard was then of an age and place in the profession which I hope justified me in criticising his book without reserve, on grounds both physical and clinical. At a later date these dissents would have been expressed, if with no less decision, yet with more deference. The perusal of the book, however, determined me to publish my own observations, and I summed them up in a paper which, at the request of the Hunterian Society, was written in 1894; but it was not read to the Society till its January meeting in 1895. It was in preparing for this paper that I found myself differing in important respects from von Basch also, as I shall explain hereafter. With both these authors however I agreed on the whole, though with certain discriminations, in recognising the disease which then I named Arterial Plethora or Hyperpiesis ; ${ }^{1}$ but I differed from them in distinguishing another mode of arteriosclerosis no less prevalent, a series independent of arterial high pressure, and due to and significant of other causes. And I venture to think that if my distinctions had then been adopted much of the confusion which has obscured, and is still obscuring, the clinical side of arteriosclerosis would have been avoided. Moreover, in my

1 Lately I have preferred the etymology of hyperpiesia for the malady, and hyperpiesis for the hæmodynamic aspect of it. 
review of Huchard's work, I argued that excessive blood pressure in the arteries and high arterial tension are not convertible terms ; that "tension" depends not upon the internal pressure only, as in an inorganic tube, but also upon the inconstant attitudes of tone in the vessels under consideration. Also I urged that if to lateral pressures-pressures, that is, tending to rupture the artery longitudinally, pulsation made no little difference, to longitudinal stretching-tending to rupture the artery transversely, pulsation made all the difference. ${ }^{1}$ If our blood flowed in a uniform stream there would be little tendency to longitudinal stretching, that little only which might come of the fluid friction. In short or twisted vessels we should expect to find the effects of lateral pressures, and in the longer vessels, as in those of the limbs, the effects of longitudinal tension, which probably rips the coils of the spiral fibres (p. 573); an opinion which, with further experience, I held more and more confidently. We shall see hereafter that the lesions of usage in vessels of these two orders of magnitude are accordingly different in relative incidence if not in kind.

Now both von Basch and, after him, Huchard observed, as Mahomed had done, that arteriosclerosis was (might be) preceded by a period of rising pressures; this preliminary period, Mahomed's " pre-albuminuric stage of Bright's disease," was von Basch's "Latent arteriosclerosis," Huchard's "Presclerosis." Thus it will be seen that both von Basch and Huchard had advanced this step, that they recognised, as I had begun to recognise even before Mahomed was taken from us, that rising pressures did not necessarily mean incipient stages of renal disease, in the ordinary sense. In the example of the lady whose case I had watched for eighteen years, guided by our new pre-albuminuric notions I had looked continually for manifestations of chronic renal disease; but years passed on and, as I have said, the dreaded Bright's disease never appeared. Without manifesting any of the clinical symptoms of Bright's disease, such as granular casts in the urine, headache, drowsiness, nausea, anæmia, retinitis, and the rest, to some threescore years and ten, in spite

1 The remarkable development of longitudinal muscular strands in the arterial adventitia, recently emphasised by Drs. Andrewes, J. F. Gaskell, and others, corroborates this principle. 
of heavy arterial pressures, she maintained her ground until the final stage of cardiac defeat; the moment of her death being determined by a cerebral hæmorrhage.

Now while I was observing this case of Mrs. P., at Dr. John Ogle's request I was comparing, for the Medico-Chirurgical Review, the pathological work of George Johnson with that of Gull and Sutton, when I was led to believe that Johnson's allegation of muscular hypertrophy in the arterioles was true, and to suspect that it might be true not for Bright's disease only, but also for certain other modes of arterial high pressure which, whether allied to chronic Bright's disease or not, are yet not identical with it (see pp. 313 and 470). In Mrs. P.'s case no necropsy was obtained; but I may repeat that around her long story, as on a string, I collected the shorter parts of similar stories of other patients, many with necropsies; and that gradually by these experiences my growing opinion was strengthened that, beside chronic renal disease, there were cases bearing some resemblance to it in the eminent feature of rising arterial pressures and hypertrophied heart, and perhaps of hypertrophied arterioles, but in their clinical course and issues differing from it widely, perhaps essentially. At this opinion, in enlargement of that of Traube, von Basch, Potain, Huchard, and others had arrived independently; and it will be discussed in its proper place.

To collect and compare cases with records as long and complete as possible ; to note, to correct, and to interpret the suggestions of the sphygmograph ; at a later date to take up von Basch's sphygmometer and to make allowances for its capricious ways; to follow the experimental work of the physiologists and the pathological events of the deadhouse, and all this in the midst of other professional engagements, was a slow and protracted business; but during the three years 1889-92, when I held the office of Commissioner in Lunacy, I found with new opportunities more leisure. And as in the asylums I was surprised, and for a while disconcerted, to find that, although arteriosclerosis abounded, yet high arterial pressures and hypertrophy of the heart were neither frequent nor conspicuous, the new opinion grew and matured in my mind that, although excessive blood pressure is the antecedent phase of a certain class of cases of arteriosclerosis, and apparently in them a chief cause of the sclerosis, yet that 
an arteriosclerosis might arise, and very commonly does arise, without any, or any considerable, previous excess of pressures; a maxim which was not corroborated till some ten years later, by Grödel, and is even yet imperfectly apprehended. It was thus that I gathered material enough to justify me in publishing the provisional conclusions on arterial disease which I embodied in the paper for the Hunterian Society of London to which I have referred, and two years later (1896) in my Lane Lectures at San Francisco. In my subsequent papers, and in this treatise, I have not had to depart from the principles then laid down.

My own work had been chiefly clinical; but meanwhile on the pathological side of arteriosclerosis, from Virchow onwards, much valuable work was being done. In the first volume of his celebrated Archives this distinguished man placed the whole pathology of arteriosclerosis on its modern basis, ${ }^{1}$ and if it be true that the author dwelt too much on an inflammatory interpretation of the histological phenomena which he demonstrated, comparing them to inflammations of the cornea, or of cartilage, yet, on the other hand, by the cellular pathology were banished the cruder notions of "deposits from the blood in dyscrasias" (the "Auflagerungen" of Rokitansky), and so forth ; thus vindicating the conceptions of Haller, Scarpa, and Lobstein, and later of Cohnheim. Cohnheim accepted Virchow's view of the change as inflammatory, a view of it which has had to submit to much qualification. Langhaas ${ }^{2}$ proposed a distinction between a diffuse and a focal ("knötenformigen") form of arteriosclerosis, and demonstrated the morbid changes in the tunica intima. And thus it was that the work of Marey and Ludwig on cardiac physics, especially the application of graphic methods to the record and elucidation of pressure phenomena in animals and many valuable if less accurate measurements on man, linked together physiological, pathological, and clinical work.

Much misconception is due to the prevalent view of arteriosclerosis as " a disease," a view against which I contended in the Toronto Discussion in 1906 (pp. 374 and 455). Too often it is regarded as a disease, and then subdivided into classes. "The man's

1 Virchow, Über acute Entzündung der Arterien.

${ }^{2}$ Langhaas, Virchow's Arch. vol. xxxvi., a remarkable paper which deserves more attention than it has received. 
real disease was Arteriosclerosis," says one eminent physician. "Arteriosclerosis is the disease, atheroma is but a lesion," says another seer. "Arteriosclerosis," says Huchard, " has its own symptoms, giving the malady son cachet"; he might as well have called dropsy a disease and occupied loose columns with its nature and course, as indeed our ancestors did. A distinguished German friend of mine calls arteriosclerosis " a disease," with its symptoms of hypertrophied heart, especially of the left ventricle, and so on, ${ }^{2}$ and confuses this again with the heart of the obese ; but we shall see that the lesion of arteriosclerosis is found as often without cardiac hypertrophy as with it. Now by a disease we mean, or should mean, a series of symptoms, positive and negative, recurring with fair uniformity; in the antecedents of arteriosclerosis there is no such uniformity, and the formal sub-classes contain processes disparate and alien. To call arteriosclerosis "a disease" is not pathology but necrology, and the error penetrates into and confuses all the therapeutics of the subject. I am glad to have the categorical support of Dr. James Mackenzie in this respect, who says (Diseases of Heart, p. 235 ) that I have " rightly protested against arteriosclerosis being considered a disease." Gull and Sutton, Letulle, and Lancereaux fostered this notion of a similar histological result of many diseases as "a disease," as a general malady which differs case by case only in the points of its attack. Thus chronic Bright's disease is said to be but arteriosclerosis in the renal area; hyperpiesis but arteriosclerosis of the splanchnic area; heart-block but "arteriosclerosis of a cardiobulbar type" (Huchard), and so on: so that without much trouble we have a ready key to differential diagnosis. Local lesions are but episodes. Once admitted on these terms, the use of the titular disease of Arteriosclerosis has no limits. As Dieulafoy ironically says, "Elle résumerait la pathogénie de presque toutes les affections viscérales"; it would govern all "cardiopathies" and most diseases of the nervous system. My old friend von Schrötter summed up such a pathology (in his article in Nothnagel) in the forms of arteriosclerosis : the cerebral form,

1 See Huchard, special report for the Medical Press of June 2, 1909.

2 Bourguignan, one of Huchard's devoted pupils, writes: "Ettre considéré comme in Brightique est . . . un cardio-artériel méconnu" (!). 
the heart form, the renal form, etc.; to which Ortner added the intestinal form. In these vast hypotheses Huchard, the Rupert of medicine, with his dashing physics, his magnificent phrases, and audacious assumptions, has been a leading spirit. $\mathrm{He}$ used to credit some of us with much ignorance because we panted in vain after his systems; I indeed was painfully conscious of a slow mind beside his quick one. But we are learning that phrases such as "cardiopathies artérielles," "cardiosclérose," " dyspnée toxialimentaire," "aortisme héréditaire," "période mitroartérielle," "état méiopragique," etc. etc._" et pondus et ipsa Huc illuc vinclorum immensa volumina trahit "- - either waft us over hollow places or serve for the decoration of platitudes. It is not true that the arterial side of pathogeny and clinics has of late years been neglected, but it is true that less gifted observers have had an unsympathetic distrust of rhetoric. It was said of Michelet that he wrote a style in which the truth could not be told. Ideas are delicate things, and do not always reside in virtuosities of execution. Such terms as "névrosisme," "arthritisme," "Brightisme" -we might as well talk of dermatism or pulmonism-do but create a pretence of knowledge where there is none. It is so much easier to take all for granted than to explore by spade labour. However, as Jack Wilkes "was never a Wilkite," so it was his pupils who carried what von Romberg calls Huchard's "schematisierende Schilderung" into the market-place, and tempted some of us to exclaim with Dieulafoy, in his criticism of Broussais and Bouillaud, "See where the love of system has carried men of such distinction." What Huchard did for us was to stimulate an interest in a side of medicine which had scarcely penetrated into general practice, and to cast a meek discomfiture about the admiring family doctor; this his wondrous neologisms, his French lucidity and logic, his categories and vue d'ensemble achieved delightfully for readers who were not tempted to murmur, "Comment faitesvous donc, Monsieur, pour être si sûr de ces choses-là?" The French School of to-day perceives the frailness of these logical fabrics. Tripier ${ }^{1}$ openly rejoices over the wane of "the arteriosclerosis legend." With justice he complains that by facile diagnosis, vague use of general terms, assumptions for reasons,

1 Tripier, Etudes anat. clin. : Cœur, vaisseaux, etc., Paris, 1909. 
and paper classifications, these rhetoricians were ready to explain anything. As Vaquez says, by such tickets our ignorance is concealed.

At the outset then, as we shall see, these elaborate subdivisions of arteriosclerosis were vitiated by merging things essentially alien in one and the same category ; e.g. the arteriosclerosis of moderate pressures with that of hyperpiesis ; the one primary as regards the arteries, the other secondary. For my part, perhaps, in this essay I have receded into the opposite extreme, into an extreme distrust of categories ; from my own point of view, or indeed under all points of view, perhaps I ought to have divided these several processes the one from the other; but I feel the time has not yet come for radical separations, or much system-building. In manuscript I tried logical schemes more than once, but they led to repetitions, qualifications, and the loss of many a current point of contrast. There is yet too much haze on the horizons; truth is in the making, ideas are in movement; we may envy but we cannot yet enjoy the certainties of the theologian; our facts are still divided and scattered, they cannot yet be packed into their universals ; they lack attraction therefore, their grouping is loose. As Leo X. put it, when compared with doctrines digested into order and formula, and built into a logical tabernacle, "vagi et ancipites videantur." With its Latin tradition, theology can provide completeness for its pupils; the pathologist cannot be pontifical, or not for a long time to come. $\mathrm{He}$ is embarrassed by "l'illogique du vrai." Much then depends upon the precise use of words and names, a matter on which I have often dwelt in other papers; for herein medical authors are really reckless. What are we to think of a clever and eminent colleague who told us the other day that " migraine, epilepsy and asthma are the same disease" ! as though one were to say that Mr. A., his grandfather and his cousin were the same person!

In respect of our present subject I am disposed to adopt the name atherosclerosis, in place of arteriosclerosis, at any rate so far as to indicate the ordinary degenerative lesion of arteries. I admit, lest I should mislead the reader, that $I$ have been rather deliberately inconsistent in this usage; I have used "atherosclerosis" in certain passages where I wished to avail myself of the specific value of the newer name, but otherwise I 
have not cared yet to disturb the common use. ${ }^{1}$ The name of hypertonus for high blood pressure or, more widely, for the malady of arterial plethora, a name introduced by German writers and adopted at home by Dr. Russell, although it draws attention to the mechanism of the malady with which one mode of arteriosclerosis is associated, yet seems to me hardly a clinical word, and to have too wide an application for so special a use. Arterial hypertonicity, in larger or smaller areas, occurs in a multitude of disorders. I have to admit that my name of Hyperpiesis, in its literal sense, applies to more than onefor instance, to both arterial plethora and chronic Bright's disease-so that my name is not without ambiguity; and it has also the fault of emphasising one symptom-a leading one, it is true-above the rest; but so do the names of hydrophobia, measles, whooping-cough, and the like. It is difficult to persuade persons who insist on chaining up a word to its derivation, that this name is to connote, not the high arterial pressure only, but the whole morbid series, not chronic renal disease, of which high arterial pressure is one term. However, I shall use this name, or rather hyperpiesia (p. 313 n.), for lack of a better.

For the other chief series, or process, in which arteriosclerosis is salient, I proposed the name of Involutionary, to which some of my brethren objected. Sir John Broadbent ${ }^{2}$ urged that this mode is often found in persons of not more than 55 years of age; moreover, that it seems to exclude other causes, such as heredity, toxins, strains, etc. Jores' name "senile arteriosclerosis" is open to the same objection. In Haller's works I had observed the Latin verb decrescere, in its several conjugations, used frequently to signify the decline I wanted to indicate $;^{3}$ and, more closely still, Haller used the word decrementum to signify senile involutionary processes. From him therefore I adopted the adjective decrescent to signify this " senile" arteriosclerosis or atherosclerosis, and I think it will serve.

1 The word atheroma has interesting connotations. In the Schol. Oribasius

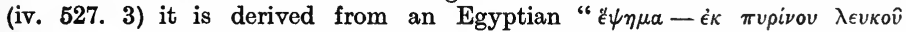
àcúpov," which probably carries us back to the Alexandrian surgery before Christ.

2 Broadbent, John, Brit. Med. Journ., 1906.

3 E.g. "Corpus animale undique decrescebat : elementa solida ad minora intervalla coibant, vasa excaecabantur, terrae portio increscebat...e eae causae in addensando corpore animali valebunt," Lib. xxx. sect. 3.

VOL. I 
Subnormal arterial pressures do not fall within the limits I have fixed for this essay; this study I have yet to begin.

Plebosclerosis also I have omitted, with less excuse. But of this lesion again I know little; it certainly exists, probably abundantly, but may have little clinical significance, unless of course in the portal vein where it is pretty well known. 


\section{CHAPTER II}

\section{PHYSICS OF THE CIRCULATION}

Ir seems desirable at the beginning to touch upon rather than to teach some principles of cardio-arterial physics. This I do apologetically, inexpertly, in the hope of giving some assistance to my clinical brethren. We have seen how earnestly the importance to clinical medicine of some standard of arterial pressures was advocated by von Basch. Keeping before him a mercurial column, by which he continually checked the records of his own imperfect sphygmometer, he endeavoured to formulate the range of pressures of the healthy adult man; and concluded that before middle life and during rest, systolic pressures of 150 and upwards were abnormally high. In elderly persons he observed that for later life systolic pressures about 140-150 were not abnormal ; but that if they steadily exceeded this limit they were to be regarded with anxiety. Some such trespasses arise often enough ; but not infrequently they are due to transient causes, or at any rate, if discovered in good time, may under appropriate treatment be allayed.

Arterial pressures are lowest in infancy and childhood, and, modified only by fluctuations in the growth of the body, rise by slow degrees through youth to age. They are said at birth to be about 35-55. Waking and suckling raise the systolic pressure only about $15 \mathrm{~mm} .^{1}$ This rule applies on the whole to both maxima and minima, but these extremes slowly diverge up to the adult norm of amplitude and onwards.

Of the senile changes the best evidence is given by Wildt, ${ }^{2}$ who,

1 Balard, Gaz. des hôp., May 8, 1913. But at rest pressures are often swinging between points of 15 and $20 \mathrm{~mm}$.

2 Wildt, "Blutdruck in Greisenalter," Zentralbl.f. Herz-u.Gef.-Kr., Feb. 1912. 
after careful consideration of a very complicated subject, recorded observations (1909-10) upon 250 patients of 60 years and upwards in an asylum for old persons at Strassburg. After excluding cases in which failing heart, chronic renal disease, or other malady might vitiate the results, he found the rule to be true that systolic pressures slowly augment with the later years of life. But for diastolic pressures the rule is not quite parallel. Generally speaking, it is true, the diastolic pressure also does rise somewhat, but not to the same amount; pulse amplitude is enlarged, not rarely much enlarged, even so as to resemble the pulse of aortic regurgitation in younger persons. I think we shall accept these observations as in accordance with clinical experience. Now we should naturally connect this kind of pulse with a dilated and probably atheromatous aorta; and in Wildt's experience correctly. He goes so far indeed as to postulate (in such cases) a direct relation between pulse amplitude and sagging of the aorta. That in stiffer tubes, as in degenerate aortas even with but little peripheral arteriosclerosis, pulsatile amplitude enlarges is a well-known physical law. But in peripheral arteriosclerosis, even far advanced, the aorta may be but moderately deteriorated. These figures of Wildt seem discordant with those of Moutier, ${ }^{1}$ but Wildt's results were carefully obtained and seem to accord with general experience. My own comment would be that as the aorta adapts itself best to output at medium diameter, if distended beyond this, in health, more energy is needed, and to get the output taken up systolic pressure rises ; for instance, on making a muscular effort, as the aorta is distended by the rise of pressure, the work of the heart, already called upon for the effort, is multiplied, but as the periphery opens out the aorta recovers its advantage. Such readjustments are far more rapid and effectual in trained persons. On diastole this high stretch of the aorta is no longer called for ; the pressure falls smartly to the rest standard of the individual; the heart need not hypertrophy. Thus the sum of cycle pressure in elderly persons may be no more, if in its phases the curve be more labile. Statically, the senile rise is represented in the artery by a gradual growth of the fibrous strands of the tunica intima. The lower pressures of childhood are probably due to the relative proportions

${ }^{1}$ Moutier, C. rendus de l'Acad. des Sci. cxlii. 10, 1907. 
at that age of heart and arterial tree; the increasing pressures of the later years of life may be due in part to failing resilience of the arteries, and small increments of friction, though probably some katabolic factors, as yet obscurely known to us, are concerned in this alteration. At any rate the rule is so general that a systolic pressure of 120-110 in an elderly man may arouse some suspicion of cardiac fatigue.

We are as yet far from possessing a normal curve of pressures for the daily period; under bodily and mental impulses oscillations are so incessant, and so large, that perhaps no " normal curve" can be formulated. It is a point of no little interest, for example, to determine the arterial pressures during sleep; but the obvious difficulties of taking records upon a person in sleep without lightening or dispelling it have hitherto prevented any conclusive results. It is usually assumed that during sleep arterial pressures rise, and the comparatively passive cerebral arteries may then be distended by afflux of blood during the anabolic activity of the cells in restoring the nervous waste of the day. The not infrequent occurrence of apoplexy and angina pectoris in sleep has been thus explained. But Brooks and Carroll, ${ }^{1}$ on a long series of observations on the phases of blood pressure in 68 normal persons, say that during the first hours of normal sleep pressure fell, but rose as sleep continued, up to waking. Soon after awaking in the morning the readings showed an average fall of $24 \mathrm{~mm}$.; three hours later the average depression was only $12 \mathrm{~mm}$., and from this position pressure slowly rose until the later afternoon, when it gradually fell to its lowest day position. These fluctuations were least in normal or low pressure cases, most in high pressure cases, cases in which the preliminary drop "after sleep" was rapid. In six high pressure cases (average $204 \mathrm{~mm}$.) the night sleep, during first sleep, reduced the pressure by average of $44 \cdot 8$ $\mathrm{mm}$., but the waking pressure was only 22.8 under the day figure. The drop seems to be due to the sleep of night, as in persons not permitted then to sleep no more fall took place than posture and rest would account for. Occasional day sleeps had no effect of reduction of pressures, so that the attempts to turn sleep

1 Brooks and Carroll, Arch. of Int. Med., August 15, 1912; fully quoted Epit. B.M.J. See also Hobhouse's case, p. 658. 
therapeutically to moderation of arterial pressures had little success. Sleep under sedatives did not increase the fall. The influence of meals seems to be too much overridden by other interferences to be reducible to rule. As I believe our sensations of hunger are due to periodical dilatations of the splanchnic vessels under habit, a falling pressure before meals would not surprise me.

In some other directions we have obtained more information. We have decided by experiment, what we had long observed in clinical practice, that irritation of a sensory nerve, such for instance as the sciatic, influences, or may influence, arterial pressures promptly and markedly; and this by determining vasomotor fluctuations, with a mechanism and effects described in the well-known researches of Drs. Gaskell, Langley, Leonard Hill, and many other physiologists. I say "may influence," because the cardiac energy is not a constant; moreover reflex dilatation in other areas may counterbalance the areas of constriction. Although the postulate of a local and immediate muscular or neuro-muscular reflex between arterial muscle and blood pressure within is still in controversy (vide p. 50), yet the arterial system as a whole is directly sensitive to the blood pressure, and to chemical changes in the blood. The "capillary area" reacts, as tested by the injections into a vein of a dilute solution of silver nitrate, or simply of an alkali. ${ }^{1}$ To these enquiries we shall have to return later; at present, in order to keep before us the complexity of these blood pressure reactions, I will emphasise the experiments of Elliott and others on the influence of stimulation of the splanchnic nerves in causing, not only vasoconstriction by direct action on the vessels, but also in causing the adrenals to secrete more adrenin. The action of many drugs, so Professor Mines tells me, is thus dual.

Atmospheric pressures, according to Crile, affect arterial pressures directly. My own experience is to the same effect as regards not absolute barometrical pressures but more or less sudden falls (Vol. II. p. 102). Cold and heat, as we know, affect them directly.

The effect of posture on blood pressures has been empirically

\footnotetext{
${ }^{1}$ See Heger; Ludwig's Festschrift, 1887. A little aneient in date, but I
} bolieve uncontradicted. 
noted of course since the first woman fell down in a faint; and of late years many physiologists have devoted time and experiment to this problem. This is not the place to enter upon a full description of their results as a chapter in physiology, but I may cull a few facts for clinical service. At the same time let me reiterate the admonition, which von Basch so continually urged, that only by submitting again and again to the control of experiment can we divest ourselves of engrained habits of dogma and rough and ready empiricism, and, in respect of our present subject, maintain the general idea of the circulatory mechanism, central and peripheral, as a whole.

Life, like a fountain, consists in a continual ascendancy over gravitation; as life fails heart and vessels, like back and limbs, bow down to their mother earth. It has long been customary with careful physicians at certain moments to test the balance of advantage in the circulation between the surging forces of life and the earthward drag of gravity ; as, for example, by testing the pulse in various postures of body and limb. These methods have been used chiefly for the detection of falling pressures, which may be revealed in abnormal and even alarming degrees. In one case of great physical exhaustion in an otherwise vigorous undergraduate, a patient of Mr. Wingate, gravitation had gained the upper hand so enormously that to raise him towards a sitting posture in bed brought the pulse at the wrist to extinction, and seemed to put his life in instant jeopardy. In another case, of less degree, a pulse of 114 as the patient stood up fell on his recumbency, after few transient vacillations, to 80 . Plesch says that when upright we use about 20 per cent more oxygen than at absolute rest. For the normal relation of gravity to cardio-arterial energy Professor L. Hill has given us some now well-known standards which enable us in disorder to detect, more accurately than we were wont on the older empirical methods, not only the downward fluctuations of low pressures, but also abnormal degrees of resistance to gravitation when arterial pressures are at too high a range. In these last cases the differences between arterial pressures in the standing and recumbent postures may be more and more abated, almost to abolition.

Thus to count the pulse in the upright and in the recumbent position and to compare the numbers, a difference in healthy 
persons of some ten to sixteen beats, is an old clinical device ; and from Marey's time it has been supposed that, caeteris paribus, the blood pressure is inversely as the amount of the difference. When no instrument is at hand this device may be of some service; but we must not assume a very close relation between rates and pressures (p. 399). ${ }^{1}$ Pulse rate of course depends in part on the nervous governance, in part on peripheral resistance; the wall affecting it only as far as its normal balance may be altered. If the blood pressure be very much augmented the rate standing may be even slower than the recumbent rate ; or, as I have said, they may coincide, as when on initial effort the work of the heart is increased, and the left ventricle not emptied. Hesse of Kissingen, at a recent medical Congress, stated that in healthy persons, when recumbent, both the crest and the trough of the curve were lower, but the trough sank more than the crest, so that the wave became ampler, the pressure being unaltered but the output more : in the upright position the order was reversed, the wave became smaller. Quantities of output, other conditions being stable, may thus be guessed at. But, in all pulse recording, the multiplicity and shiftiness of conditions, such, for instance, as the automatic vascular tone of large areas, room temperature, and emotional phases, must never be forgotten; for the reflexes which signal all these fluctuations, constrictions, dilatations, outputs, and so on, are exquisitely ordained.

In this context I take the liberty with $\mathrm{Dr} \cdot{ }^{\cdot} \mathrm{M}^{\circ} \mathrm{Curdy}{ }^{2}$ of adopting the following useful table drawn up for growing children, a table which may be compared with Wildt's records for the elderly.

1 See, e.g., Tigerstedt, Phys. d. Kreislaufes, p. 355, with whom other experimenters agree.

2 M‘Curdy, J. H., School Hygiene, 1912. 


\begin{tabular}{|c|c|c|c|c|c|c|c|c|c|c|}
\hline \multirow{3}{*}{ No. } & \multirow{2}{*}{\multicolumn{2}{|c|}{ Age. }} & \multirow{2}{*}{\multicolumn{2}{|c|}{ Heart Rate. }} & \multicolumn{4}{|c|}{ Blood Pressure. } & \multirow{2}{*}{ 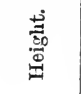 } & \multirow{2}{*}{$\begin{array}{l}30 \\
0 \\
0\end{array}$} \\
\hline & & & & & \multicolumn{2}{|c|}{ Systolic. } & \multicolumn{2}{|c|}{ Diastolic. } & & \\
\hline & Years. & Months. & $\begin{array}{c}\text { Hori- } \\
\text { zontal. }\end{array}$ & $\begin{array}{c}\text { Stand. } \\
\text { ing. }\end{array}$ & Horizontal. & $\begin{array}{c}\text { Stand- } \\
\text { ing. }\end{array}$ & Horizontal. & $\begin{array}{l}\text { Stand. } \\
\text { ing. }\end{array}$ & & \\
\hline 1 & 10 & 8 & 69 & $80 \cdot 2$ & $108 \cdot 2$ & 110 & 61.2 & $63 \cdot 3$ & $146 \cdot 2$ & 40 \\
\hline 2 & 10 & 7 & $77 \cdot 5$ & 84 & $98 \cdot 2$ & 100 & 65 & 65 & $133 \cdot 3$ & $31 \cdot 6$ \\
\hline 3 & 11 & 6 & 73 & 80 & $107 \cdot 7$ & $106 \cdot 7$ & $67 \cdot 7$ & $64 \cdot 2$ & $141 \cdot 6$ & 31 \\
\hline 4 & 11 & 11 & 76 & 79 & $94 \cdot 2$ & $97 \cdot 5$ & $61 \cdot 7$ & $60 \cdot 5$ & 138.7 & $35 \cdot 8$ \\
\hline 5 & 11 & 4 & 93 & 95 & $107 \cdot 7$ & $104 \cdot 5$ & $71 \cdot 7$ & 75 & $150 \cdot 2$ & $39 \cdot 3$ \\
\hline 6 & 12 & 9 & 60 & $64 \cdot 5$ & $102 \cdot 5$ & $103 \cdot 3$ & $64 \cdot 2$ & $68 \cdot 3$ & 136.5 & 30.5 \\
\hline 7 & 12 & 8 & 70 & 67 & $99 \cdot 5$ & $104 \cdot 2$ & $65 \cdot 5$ & 60 & $143 \cdot 6$ & $29 \cdot 6$ \\
\hline 8 & 12 & 10 & 75 & 88 & $108 \cdot 7$ & $106 \cdot 7$ & $68 \cdot 2$ & $72 \cdot 7$ & $145 \cdot 7$ & 38 \\
\hline 9 & 12 & 1 & 90 & $91 \cdot 5$ & $102 \cdot 7$ & 107.5 & $67 \cdot 7$ & $66 \cdot 5$ & $145 \cdot 4$ & $38 \cdot 4$ \\
\hline 10 & 12 & 2 & 66 & 93 & $95 \cdot 2$ & 99 & $67 \cdot 5$ & $61 \cdot 5$ & 152 & 40 \\
\hline 11 & 12 & 10 & 69 & $75 \cdot 5$ & 101 & $107 \cdot 7$ & $66 \cdot 6$ & 80 & $140 \cdot 4$ & $32 \cdot 4$ \\
\hline 12 & 12 & 6 & 92 & 99 & 103 & $97 \cdot 7$ & $66 \cdot 2$ & 70 & $137 \cdot 2$ & $30 \cdot 5$ \\
\hline 13 & 12 & 6 & $78 \cdot 5$ & $87 \cdot 5$ & 104 & $101 \cdot 7$ & 60 & 60 & $153 \cdot 7$ & $43 \cdot 7$ \\
\hline 14 & 12 & 5 & $61 \cdot 5$ & 71 & 95 & 99 & 53 & $67 \cdot 5$ & $146 \cdot 1$ & $28 \cdot 9$ \\
\hline 15 & 13 & 9 & 90 & 94 & $111 \cdot 7$ & 113 & 70 & $69 \cdot 3$ & $147 \cdot 9$ & $34 \cdot 5$ \\
\hline 16 & 13 & 4 & 78 & 80 & $103 \cdot 7$ & 102 & $60 \cdot 7$ & $60 \cdot 7$ & $151 \cdot 2$ & $43 \cdot 3$ \\
\hline 17 & 13 & 8 & 66 & $66 \cdot 5$ & 115 & 115 & $68 \cdot 3$ & $73 \cdot 3$ & 137 & $33 \cdot 2$ \\
\hline 18 & 13 & 4 & 74 & 85 & 90 & 90 & $62 \cdot 5$ & 60 & $135 \cdot 5$ & $27 \cdot 3$ \\
\hline 19 & 13 & 8 & 76 & $86 \cdot 4$ & 103.9 & $100 \cdot 7$ & $64 \cdot 1$ & $65 \cdot 8$ & $149 \cdot 9$ & $36 \cdot 1$ \\
\hline 20 & 13 & 8 & $72 \cdot 4$ & $97 \cdot 2$ & 112 & $103 \cdot 4$ & 64 & 73 & $161 \cdot 2$ & $49 \cdot 6$ \\
\hline 21 & 14 & 11 & 79 & 85 & 120 & $121 \cdot 7$ & $62 \cdot 8$ & $70 \cdot 7$ & $151 \cdot 7$ & $43 \cdot 3$ \\
\hline 22 & 14 & 4 & 80 & $88 \cdot 5$ & $101 \cdot 7$ & $103 \cdot 2$ & $62 \cdot 7$ & 60 & 155 & 40 \\
\hline 23 & 15 & 0 & 95 & 98 & 109 & 110 & 63 & $71 \cdot 7$ & $157 \cdot 2$ & 44 \\
\hline 24 & 14 & 2 & $85 \cdot 8$ & $94 \cdot 8$ & $110 \cdot 9$ & $106 \cdot 2$ & $65 \cdot 4$ & $68 \cdot 4$ & $149 \cdot 8$ & $38 \cdot 1$ \\
\hline 25 & 14 & 4 & $83 \cdot 6$ & $95 \cdot 4$ & $96 \cdot 1$ & $93 \cdot 5$ & $62 \cdot 2$ & 65 & $145 \cdot 6$ & $37 \cdot 5$ \\
\hline 26 & 14 & 10 & 60 & 71 & $100 \cdot 7$ & $105 \cdot 6$ & $65 \cdot 7$ & 70 & $140 \cdot 7$ & $31 \cdot 4$ \\
\hline 27 & 14 & 3 & $74 \cdot 4$ & $87 \cdot 8$ & $96 \cdot 8$ & $107 \cdot 4$ & $63 \cdot 3$ & 68 & $159 \cdot 1$ & $45 \cdot 4$ \\
\hline 28 & 14 & 9 & $81 \cdot 4$ & 90 & 101 & $104 \cdot 4$ & $67 \cdot 7$ & $73 \cdot 8$ & 151 & $35 \cdot 2$ \\
\hline
\end{tabular}

For children Dr. M'Curdy concludes :

1. The heart rate serves as a fair indication of condition.

2. A high heart rate indicates poor condition.

3. A heart rate with wide variations between the horizontal and standing position is indicative of poor vascular adjustment.

4. Low blood pressures (below 100) indicate a weak heart, weak peripheral resistance, or lack of splanchnic adjustment.

5. A fall in pressure on changing from a horizontal to a standing position probably means lack of cardio-vascular adjustment.

To these points I shall return again under the head of Symptoms, p. 399, etc.

The pressures of prepubescents, pubescents, and postpubescents range over the same general field, with a tendency to lowest pressures in the prepubescents, highest in the pubescents, and 
intermediate in the postpubescents. The greatest range of variability is in the prepubescents.

The experiments should be made at periods short enough to determine whether at any time there is a rapid rise; and, if such a rise occur, in what relation to pubescence. It should be related to growth in height, weight, and muscular strength. A series of examinations on the same boys, fall and spring, for a period of years during the change from immaturity to maturity, would seem to fulfil these conditions.

Mental exertion is said (Gibson) to have the same kind of effect as bodily exertion. This coefficient will be considered later (p. 70).

Pressure Gradients and Velocity.-In these functions the efficiency of the circulation may be said to consist; but their coefficients and correlations are so many and complex as almost to defy analysis. In my hands these paragraphs will prove, I fear, to be not only dull but obscure.

For all and every artery to which gauges can be applied the blood pressures per unit of internal surface-systolic and diastolic -are, practically speaking, equal (Dawson); but below these magnitudes the gradient, by increase of friction, falls rapidly; and here lies the resistance, or five-sixths of it. The factors of resistance, the heart being constant, are many and subtle; length of tubes, diameter, ramification, tone and consistence of walls, temperature, viscosity, permeation of leucocytes, tissue work, and so forth. In areas of vasoconstriction, or of structural narrowing, the heart's action being constant, this fall of gradient is of course steeper ; but these areas are so independent of aortic pressures and velocity as to be beyond calculation. Hence one chief difficulty in the discrimination of heart values. Pressure gradients in the arterioles are estimated to fall to about 60-70 $\mathrm{mm}$. ; in medium capillaries to $35-45$; in the more compressible capillary areas to $15-20$; in the finer superficial veins about the same, or in the fine venous plexuses $5 \mathrm{~mm}$. more (p. 55). Dr. Oliver ${ }^{1}$ has endeavoured to turn this law to account by comparing the pressures at the radial or brachial artery with those in the phalangeal vessels. Caeteris paribus, as peripheral resist-

1 Oliver, G., Lancet, July 22, 1905 ; cf. also Dr. Waller's paper, Brit. Med. Journ., 1900, vol. ii. 
ance rises the fall of pressure gradient should be the more rapid; the difficulty is to secure equality of other conditions-to preserve, for instance, a uniform temperature of the fingers, to allow for a small increment of cardiac hypertrophy in those early stages in which such verifying observations are especially required; and so on. Conversely, on administration of vasodilators, as with Professor Dixon's assistance I demonstrated at the Royal Institution in 1905, as unit pressure fell in the brachial artery it rose in the distal areas. In later stages of high pressures, as the heart, however largely developed, is more and more at a disadvantage, the fall of pressure gradient in the peripheral areas should be more evident; but at such stages of disease we have other and plainer clinical means of ascertaining the default of the central circulation.

Speaking generally, the blood pressure signifies the degree of tension not so much of the minor arteries, such as the radial, as of the larger and less muscular arteries ; thus, again speaking generally, a local pulse, among the many things it tells us, does not tell us the sum of blood passing into its district. Stream activity, blood pressure, and resistance are each functions of the other two-within limits that is ; for if resistance gets the upper hand the blood stream must slacken. Still, speaking generally, there is in the carotid no definite relation between pressure and velocity; the conditions are too complex. Erlanger's calculation that the product of pulse pressure-i.e. of the difference between systolic and diastolic pressures-and the pulse rate gives the velocity, is, as no doubt he is aware, a highly abstract maxim, and carries with it, as we shall see, little practical application. With the pressure the calibre may vary, and vasomotor tides are incessant and ubiquitous. In athletic efforts the output of the left ventricle may under normal conditions be amplified to incredible degrees, from four to thirteen times: as stated by considerable authorities ; let us be content with six or eight times. Now if in a case of high pressure, when the aorta is full and tight, a muscular effort be made, its wall cannot expand much more; and in sclerosis this condition will be so much the worse ; this being so, the cardiac output must be restricted, and the pulse amplitude ought to show it. Suppose now we double the arterial pressure, say to 240 , we shall then double the heart's work, or at 
any rate that of the left ventricle; but the radial may nevertheless be so constricted as to be almost empty, and stream pressure imperceptible; although, as recent researches seem to indicate (W. Russell), to instrumental compression the stiffened muscularis may offer a resistance rivalling the central blood pressures. Thus we see that the amount of blood carried to the periphery may be largely independent of the blood pressure; other factors are engaged ; still we may find thus some guide to cardiac energy: on the whole, the better the heart the better the velocity. ${ }^{1}$ Again, in illness we have to deal with larger variables, such as peripheral resistance and heart values; yet possibly, as we may anticipate, with the same pressure readings. In the state supposed, of peripheral constriction with normal arteries, we know that to double the radius of the radial artery would, if the heart were ready for it, multiply its stream activity sixteen times; though of course to widen the bed is, per se, to lower resistance, and plus velocity, even if large additions were made to the quantity of the blood, would mean a falling lateral pressure on the arterial wall. To expand the stream bed is of course so far to slacken the linear velocity - the velocity, for instance, of a swimming corpuscle; but increased area does not necessarily mean retardation (Gaskell, Heidenhain, Bayliss, Volkmann, Dehio, etc.) ; the work of the circulation in the district may be largely enhanced, and the fall of resistance ahead quicken the run behind. As it is difficult to apply abstract physical laws to these biological problems, the term stream activity - "Stromintensität" of German authors-may be used in this context instead of velocity, as giving us for local phases of high activity, if a little loosely, the general conception we want. If the sympathetic be cut in the neck the temperature of the district rises, so that even if velocity be lessened by the dilatation, more blood must be passing through, an increase probably due to the opening of a rich collateral circulation; especially if it be true that in places fine cross vessels connect arterioles and venules. Velocity then in any district depends upon the attitude of its own vessels,

1 On this subject see Hensen, Deutsche Arch. f. klin. Med., 1900, vol. Ixvii. It is to be regretted that in this paper the lamented author did not divide his arteriosclerosis into origins instead of, or as well as, into columns of degrees-" moderate, medium, and extreme." 
and on the cardiac energy ; and by "stream intensity" we shall signify the mechanical energy in relation to the filling of the portions of the vessel under observation-its cardiosystolic local increase of volume in cubic centimetres; ${ }^{\mathbf{1}}$ not the blood pressure. Speaking generally, stream activity in an area makes no demand on the heart; it is provided for by correlative constriction elsewhere. "Stromstärke" and pressure then are in no direct relation; it was found, I think by Hürthle, on the carotid, that as pressure began to fall the "Stromstärke" was more than was calculated. So Hill and Flack also. ${ }^{2}$ This stream activity may be the cause of the damage to the long arteries of the limbs of labourers, and other vessels under similar conditions, such as the precocious thickening of the temporals in migrainous subjects -if this result be verified. ${ }^{3}$ We shall see that it is not upon constricted but upon relaxed arteries that internal pressures tell.

On the other hand, in spite of constricted vessels, within certain narrow limits, the peripheral circulation may be even more abundant; as conversely through dilated vessels the supply may not be increased. Heidenhain's experiments illustrate how in narrowing arterial areas velocity may be augmented, and 0 . Müller (of Tübingen) ${ }^{4}$ found velocity in cutaneous areas increased by cool baths; that is during degrees of vasoconstriction. Dr. Harry Campbell ${ }^{5}$ demurs in some measure to an argument of Sir James Barr that "lateral pressure in contracted arteries is lessened and partly converted into velocity," and enquires "if this proposition be true of a generalised arteriolar contraction?" Well, for this calculation the heart's energy and

1 Christen, loc. cit.; and other authors seem to mean the same.

2 Hill and Flack (Paper at Royal Soc., April 10, 1913) found, on immersing one elbow in warm water and the other in cold, that on the heat-relaxed side, in spite of larger and swifter current, the systolic pressure fell, while on the other side the contracted arteries conducted the systolic wave with almost undiminished force. (But this was a wall wave, and would be better conducted.)

${ }^{3}$ See Allbutt and Rolleston's System of Med. vol. iv. p. 617. Sir William Gowers has published the case of a lady, æt. 50, a life-long sufferer from severe and frequent migraine of the right side of her head, in whom the right temporal (only) was sclerosed. Thoma described a similar condition, in a case of leftsided supra-orbital neuralgia, in which the left temporal (only) was sclerosed. In persons subject to migraine on both sides, both temporals may be affected. This local arterial change stands in no relation to general arteriosclerosis or to the general blood pressures.

4 O. Müller, Kong. innere Med., 1907.

- Campbell, H., Brit. Med. Journ., January 28, 1905. 
terminal resistances would have to be determined. These physiological tides, cardiac and arterial, vasomotor and myogenic, must therefore modify very largely the factors of pressure and velocity, of cardiac propulsion and peripheral resistance; and vascular tone may prove to be the most important of the coefficients of the normal circulation (see Fellner, p. 103).

Plesch puts the mean velocity in the aorta at rest at 424 $\mathrm{mm}$. sec. The rate in smaller vessels is still more difficult to ascertain; in the smallest perhaps $\frac{1}{2}-\frac{1}{3} \mathrm{~mm}$. p. sec. Presumably the vessels in health set themselves at just the widths to serve the momentary and ever-changing needs. The whole circuit of a particle may take 23 sec.

Still, in current conversation there is much confusion between the general arterial pressures and those in particular areas; although the elder Cohnheim, Roy, and others elucidated these problems long ago. To raise pressure experimentally in the carotid and axillary arteries, even to double it, may not react on the heart, nor on the general blood pressure; neither hæmo-dynamically nor by nerve reflex. But if in any area an artery of supply is constricted, a case in which ordinarily there is a corresponding dilatation elsewhere, the blood pressures within the area beyond the constriction must, caeteris paribus, tend not to rise but to fall; any increase of velocity being negligible. In other words, unless of course, as by collaterals and cardiac augmentation, velocity be increased accordingly, a reinforcement which, on account of reciprocal dilatations and of the larger sectional area of the finer vessels, does not ordinarily take place, a peripheral area of constriction, or beyond constriction, is an area not of high but of low pressures (p. 218). Ordinarily, the blood is simply diverted from the constricted to more open areas; and so long as this accommodation obtains the general or central blood pressures are unaffected. But large and powerful constricting areas may force the blood into weaker areas; probably no infrequent event, especially in instable conditions : thus an intense splanchnic constriction may force open cerebral, coronary, or even renal arteries. In health the capacity of the whole tubular system is kept fairly uniform; and although pressures may, and continually do, rise in areas because of constrictions in correlative areas, so that, as we have seen, 
dilatation of vessels in active areas does not necessarily imply retardation of current, yet the heart's work remains ordinarily constant. If however, as we are supposing, areas of constriction are very large, and particularly if the splanchnic or musculocutaneous area be so included that equally large readjustments elsewhere cannot take place, peripheral pressures, in spite even of cardiac hypertrophy and some increments of velocity, tend to fall, and the pressures in the larger trunks to rise; and we see them rise, even enormously; though how far in a given case constriction is extending inwards, is another very interesting and important question (vide p. 298). Clinical experience tells us that in severe cases of arterial spasm the brachial arteries may be constricted to something like half their mean diameter; and, as it is estimated that the amount of cardiac energy expended upon friction is nearly two hundred times that directly available for stream velocity, we see that, notwithstanding velocity is as the square of the impulsive force, to narrow the vessels over areas large enough to raise aortic and intraventricular pressures, and to diminish the output per beat, is with difficulty compensated, even by the heart's wonderful capacity for hypertrophy; thus the lateral pressures upon the walls of the larger vessels must be increased.

These observations I make partly to point out that in respect of blood pressures sclerosis of the larger arteries cannot be any great matter (pp. 185-188), for it is beyond them, in the assumed areas of constriction, that the heart's chief work lies. What does matter, and what may happen, is a fall of stream velocity. Velocity exerts little more than a negligible influence upon pressure levels, as Hensen, on Tigerstedt's calculations, proved against Sahli and Riva Rocci. Nevertheless, as stream velocity, viscosity being constant, is inversely as cross section-total sectional area-of the vessels in a part, and as velocity, peripheral resistance, and blood pressure are each of them a function of the other two, we perceive that changes of pressure have some correlation with changes of velocity, and mean pressure must bear some proportion to mean velocity. The total pressure head is the lateral pressure plus the velocity head. But, during the cardiac cycle, these relations are modified by reflected inertia waves which raise pressure but arrest velocity; so that, as Hürthle 
showed, velocity can only be directly proportionate to pressure when pressures are at high levels. Pressure gradients then give us little help for velocity. Within ordinary limits resistance has more effect on velocity than pressure; for instance, in convalescence velocity rises before pressure, and before other conditions of the pulse attain stability. But as yet we cannot compare velocities in convalescence, or for periods of age; these calculations imply far more accurate methods of measuring systolic and diastolic pressures than (in man) we possess. The total blood should make the circuit in about 30 sec.

It is said that, position and other conditions being equal, velocity is higher in the upper limbs than the lower. We have seen that activity of stream-the "Stromintensität" or "Stromstärke" of German authors-must be largely concerned with velocity in spite of the dilatation of the local blood vessels; as for instance during mastication and salivary activity when the stream velocity in the carotid is multiplied three or four times. In areas of dilatation the velocity rises high during systole, but during diastole falls precipitately. So also after anæmiation of an area, as by compression, the blood returns with an excess of velocity. The biological relations of pressure to velocity then are perplexed; by a vasomotor breeze mechanical calculations may at once be disconcerted; as pressures fall and the peripheral vessels shrink in calibre resistance (friction) must increase. By Hürthle's stromuhr this inconstancy of pressure and velocity relations is demonstrable; but on the whole, viscosity being constant, velocity seems to rise more readily or sooner than pressure. Generally, as we pass away from the heart, velocity falls more quickly than pressure. This is due to arterial elasticity, and might be a measure of it. Velocity may improve with sundry patterns of pulse.

From periphery to centre variations in velocity tell in manifold degrees. Soldiers know that rear ranks are often much disordered by little changes of speed in the van which, there passing almost unnoticed, are propagated down the ranks in ever multiplying oscillations so that the rear ranks are fatigued by the alternations of check and doubling up. A classical instance may be taken from the Peninsular War when, so it is recorded, a few men at the head of a column jumped a ditch, whereby, 
in the successive and multiplying vacillations consequent upon this irregularity, so much time and energy were lost that the detachment did not reach camp till after dark. Now if we carry this illustration into the march of the blood through the vessels we may form some clearer conception of checks to the blood felt in the large arteries, the consequent abnormal stresses upon their walls, and the harassing vacillations of the demands upon the heart. It has often occurred to me that, in such checks to speed, losses of the inertia of the blood stream must be no negligible factor. This principle one used to perceive in the streets when the horses, even if not stopped but only slackened for half a minute, had to pull an omnibus up again to the mean rate. ${ }^{1}$

Arterial Wall and Wave Velocity.-An elastic body offers resistance to two kinds of changes caused by the incidence of forces, namely to change of volume and change of shape. In our aortic system of elastic tubes each cardiac systole loses a fraction of itself as it passes each point in the more elastic kind of artery, most of course in the aorta ; but this is not friction, or not waste ; the energy is paid out again on cardiac diastole, so that in the fine network of the periphery a mean pressure only remains, and the flow is continuous. In rigid tubes on diastole of the pump the flow might almost stop. ${ }^{2}$ The optimum travel of the wave in a healthy artery is about the level of the normal maximum pressure. In higher pressures, if we regard physical laws only, the mobility should become less and less-a hyperbolic curve. Extensions cease to be proportionate to loads. Nicolai, and Herringham and Wills, found the wave rate to rise as elasticity became impaired ; but we shall see that this axiom also is much cut into by contingencies, as by innervation. On the whole, too, the wave would be slower in low pressures, as in a "vagus pulse." If the arteries of the greater magnitudes, either by the hardening of disease or by stretching to the full, become practically nonextensile tubes, the systolic impulse is not thus taken up; so far stream velocity is reduced, but the wall wave accelerated, and

1 For further illustration of researches on nervous influences, calibre, velocity, vascular tone, etc., see G. N. Stewart's many papers, and Cushing, Laboratory Papers, vol. ii., 1912-13.

2 It is generally supposed that a rhythmical passes more blood than a continuous stream; but in doubt of this statement see articles by Gerlach and Hürthle in Pflüger, vol. cxlvii., 1912. Also Hürthle, Berl. klin. Wochenschr., 1912. vOL. I 
the movements approximate to the spasmodic screams of bagpipes without bellows, or to the jarring propulsions of fluid pumped into rigid pipes; so that, unless the heart works harder or the vessels widen, less fluid passes in unit of time. In decrescent sclerosis, as the heart usually does not grow in value, energy is wasted and velocity falls. Or, if the vessels are overfull, the diastolic pressures rise, and the flow is no longer continuous in the periphery but jerky, and less blood is forwarded ; there are more notches on the down-stroke due to the vibration of the whole tree. If then the quantity per minute is to be constant, the heart must work harder, or the bed must enlarge. Increasing length again modifies some of the widening, and everywhere the stiffened walls respond less sensitively, especially in the areas of intermittent friction; so that the large jumpy arteries simulate those of aortic regurgitation. Moreover, in the large vessels, while less potential is stored, inequalities of radius cause a tendency to vortices, which arrest the stream. Such a pulse we see in dilated aortas, as in the syphilitic; the pulse amplitude increases so as to simulate that of aortic regurgitation, though not with the range of a hypertrophied heart; the mean pressure may not be changed. And the aorta can no longer adapt itself to various outputs, though in young persons, if the peripheral vessels be still active, the aortic default is largely compensated by them. Thus here again the readjustive capacities of the animal body are marvellous; if the peripheral vessels retain their quality, or much of it, as often they do even when the aorta is much damaged, yet, as Stadler says, (within limits) the sphygmogram may still present normal features. So with arterial spasm; before any sclerosis the constricted artery becomes so far a rigid tube; and, from what has been said, it follows that within the section friction is increased, energy is wasted, and the tissues are less and less efficiently nourished. It is at medium pressures of course that the resilient properties of the arteries are most effective. ${ }^{1}$

1 Some time after these paragraphs were written, Wells and Hill read an interesting paper to the Royal Society (Proc. Roy. Soc., March 6, 1913), demonstrating these points more fully; but as their results fortify and illustrate these remarks $I$ find no reason for altering my text. Professor Hill's demonstrations are very important, but I cannot agree that the "factor of the wall wave had hitherto not been taken into account." 
The progressive stiffening of the walls of the arteries might then be indicated by the comparative rates of wave propagation-wave velocity as contrasted with stream velocity. As we have seen, this is no very simple consideration. The following table was, I believe, accepted by Tigerstedt, Keyt, and others as approximately correct; the principles, with Professor Dixon's kind assistance, I illustrated in my lecture at the Royal Institution in $1905 .^{1}$

\section{Age Table of Arterial Elasticities}

\begin{tabular}{|c|c|c|c|c|c|c|}
\hline Child æt. $4 \frac{1}{2}$ & . & • & & $\begin{array}{r}W \\
216\end{array}$ & $\begin{array}{l}\text { ve Ve } \\
\mathrm{mm} \text {. }\end{array}$ & $\begin{array}{l}\text { ity. } \\
\text { er sec }\end{array}$ \\
\hline Man æt. 25 & • & - & & 306 & , & , \\
\hline Man æt. 40 & . & . & . & 416 & , & , \\
\hline Man æt. 55 & . & . & . & 510 & , & , \\
\hline $\begin{array}{r}\text { In chronic } \\
\text { Calcifi }\end{array}$ & $\begin{array}{l}\text { Bright's I } \\
\text { or } \\
\text { arteries }\end{array}$ & Disease & \multicolumn{4}{|c|}{ rate still faster. ${ }^{2}$} \\
\hline
\end{tabular}

But Münzer of Prague ${ }^{3}$ also has recorded measurements of pulse-wave propagation. In health he found it to run-aorta to tibial-from 9 to $12 \mathrm{~m}$. per second, and in arteriosclerosis it rose to $15-23 \mathrm{~m}$. per second; in hypotony it fell to $6-7 \mathrm{~m}$. The highest rates (to $20 \mathrm{~m}$. (!) or even more) were attained in hypertonic vessels ("Starrheit"). In Münzer's observations the velocity seemed to depend wholly on the state of the wall, not on the blood pressure; but a comparison, by identical methods, is still to be desired between the waves of a wall in mean, and in constricted, attitudes, and in a wall distended under high pressures. Silbermann has demonstrated in a very interesting way how the pulse-wave rate depends upon the innervation whereby the attitude of the wall is altered from time to time.

Professor L. Hill has pointed out, and Dr. Rolleston ${ }^{4}$ agrees

1 See Nature, Feb. 16, 1905.

2 Compare also M'Curdy's table, p. 25.

3 Münzer, at the Congress of Inner Medicine at Berlin in 1912. See also Pflüger, vol. cxxxvi., 1910; and Münzer and Friberger, quoted by Silbermann in an important contribution in Zentralbl. Herz-u. Gef.-Kranktn., Juli 1913; and Edgren's book; and Münzer again in same journal, Nov. 1, 1913. See also Friberger, and Friberger and Veiel, Deutsche Arch. f. klin. Med. Bd. cvii., 1912. Friberger concludes that the coefficients are elasticity, tone (in the muscular vessels), and degeneration.

4 Rolleston, H. D., Clinical Journal, Aug. 28, 1912. 
with him, the remarkable rule that, whatever the normal difference or no difference (see p. 75), in aortic regurgitation the arterial pressure in the leg in the recumbent patient is much above that in the arm. Professor Hill regards this difference as pathognomonic of aortic regurgitation, and due to a more rapid propagation of wall wave. In aortic regurgitation, besides impaired resiliency, the femoral artery is probably thickened.

In this contrast of wall wave and stream then, if, whether by condensation or distension, the wall be tight, the systolic wall vibration should travel along it so much the faster. Hill demonstrated this by using means of relaxing the artery under observation, when the wave was retarded accordingly. Again, as in the cuff arrangements the veins are obstructed, the stream wave and velocity are for an instant checked as they encounter moments of venous arrest. This is a disadvantage of continuous cuff compression. Because of this and such interferences $I$ have not been able entirely to trust Mackenzie's interesting notes on some palpable differences perceptible between the first few waves as touched by the finger on relaxation of cuff pressure; these in my experience are often due, not necessarily to variations in heart's energy, but to one or other, or to more than one, of these secondary interferences, or to emotion, or respiration, each factor small perhaps; but small interferences are apt to sum up. ${ }^{1}$ The variables, especially in the nervous system, are large and indefinite. Sclerosis presumably would in its degree lessen the power of the wall to respond to the nervous influences, but careful experiments show that a group of pulsewaves gives but a glimpse of the degree of vascular constriction or sclerosis; for instance, deep respirations so modify the wave rate that, if we are to guess at the health or tone of the wall-to discriminate between degeneration of wall and abnormal tension by speed, amplitude, and minimum pressure-we must be careful to measure the wave rate always in the same position of the respiration, which is far from easy. Again, if the wall be indefinitely rigid, the cardiac energy may be, often would be, indefinitely less; and of cardiac energy we have no index. The irritability of pressor and depressor nerves probably varies a good deal with times and states, even in the same individual. Gottlieb

1 See also Mann, Guy's Hosp. Repts., Jan. 25, 1908. 
and Magnus found that digitalis slowed the "pulse-wave" ; but, as they clearly point out, digitalis acts variously on various vascular regions : if it constricts the splanchnic vessels, expansion may occur in other parts, e.g. in skin and muscle, or in the kidneys; atropin also interferes with wave rate, but again vital conditions disturb the physical axiom. We have seen that if a limb be placed in warm water the pulse-wave will be retarded, although, as tested on the other limb, the blood pressure is constant. It seems certain that in spite of rising pressure wave rates may slacken; vascular tension varies, probably under oscillations of nerve centres, of respiration, and so forth; or again with constant blood pressure wave rates may vary under nervous influences. Thus it seems certain that blood pressure is by no means a chief determinant of wave rate. We know, and may easily observe if during the recording of blood pressure we place a small weight in the patient's other hand, or even ask him to close the fist, the apparent pressure may rise even by some $40-50 \mathrm{~mm}$.; but this in my opinion is due as much or more to the immediate effect on the instrument of some sympathetic tension of the muscles of the limb observed (p. 76) than to rise of aortic pressure. However here again many factors are at work; attention for instance.

The friction at the sides, even of atheromatous vessels, if an immobile layer of blood invests the inner walls, is but little, if any. Von Kries thinks the deviation of the axial stream at the forks is negligible; but the incidence of atheroma at the forks, e.g. at the origin of the cervical and intercostal branches, points to the contrary. Eddies probably arise in the relatively large spaces of the heart and great arteries, and possibly at forks, bends, or kinks. It is said that, at blood speed and blood viscosity, eddies do not form in tubes of magnitudes less than the aorta; that in smaller tubes asperities, or even projections, do not cause eddies, but act only as diminishing the radius; an important point in arteriosclerotic physics. However this may be, the friction is mainly within the blood itself. It has always been assumed that the blood wetted the walls of the vessels, so that the outermost cylinder of the blood stream was immobile. Moreover it has been stated that on looking down, on cross section, into the half-full aorta of the rabbit a concave meniscus is to be seen, the blood adhering 
to the sides around. But, not feeling clear about the point, I consulted Dr. John Tait, who most kindly sent me a full reply. ${ }^{1}$ He doubts the adhesion. Starting from some observations by Lister on clotting, he and Hewitt estimated the amount of ether-soluble material-lipoid-in the aortic lining of the ox, and found it to be 8 to 10 per cent. Vascular endothelium is then a lipoid membrane, and adhesion would probably mean clotting. Dr. Tait's letter, and his published papers on these 'subjects, open out many interesting problems on the circulation, some of which I shall mention later (p. 429). Suffice it to say here that Poiseuille's law supposes a wet wall; a point of some importance here, and again afterwards in discussing viscosity, and some of the arguments of Thoma (p. 109). The endothelium, even of atheromatous vessels, living as it does in the blood stream, is remarkably stable.

Resiliency. ${ }^{2}$-Furthermore, Schade, of the Kiel Klinik, has pointed out clearly, and proved by experimental work, that the elastic reactions of the circulation are far more than vascular; that they depend largely upon the cushions about the arteriocapillary bed, which cushions consist for the most part of connective tissue. ${ }^{3}$ Moreover these cushions, or areas, as George Darwin pointed out to me, are "hydrodynamic"; they are infiltrated with incompressible but tidal blood and other juices. Schade, in this interesting paper, describes a gauge with which he has taken records of these elasticities; he finds that they vary much under change of conditions, as in disease. The maintenance of resiliency depends much upon the chemical state of the colloid structures; and he notes that even slight and temporary deviations from the normal, such as slight degrees of œdema, lessen this cushion resiliency remarkably. Thus we shall see how quickly a slight and invisible but widespread aqueous retention may put the heart at a disadvantage. He argues also that unwholesome or relaxed states, not œdema, in so far as they muffle the elasticity of this universal

1 Private letter dated February 6, 1912. If this slip between stream and wall be verified, the validity of Poiseuille's law for the circulation of the blood will be still further impaired. See p. 430 .

2 In a discussion at the Royal Society in 1913 the term "lability" was for good reasons preferred to elasticity or "resiliency."

${ }^{3}$ Schade, H., Zeitschr. f. exp. Med. u. Therap. Bd. xi., 1912. 
cushion, will have a like effect. Thus, unless the heart be equal to a correspondingly increased effort, stream energy will be impaired, the peripheral stream will tend to fall into a pulsatile rhythm, elastic storage in the parts will be reduced, and the peripheral bed will hold more and more residual blood: the stream loses way, as a boat loses way when the oarsmen get less quickly forward. With his gauge Schade thinks he is able to detect incipient degrees of this muffling.

We have regarded the elastic coefficient of the wall as the chief factor of the pulse-wave-not the stream velocity, nor again the blood pressure. The wall wave may be compared to the vibrations of an old or a young tree under a blow upon its stem. No man is so sound, no artery so perennial, as to stand for a lifetime unchanged against the incessant beating of the blood. Professor MacWilliam's researches into the failing elasticity of the aging aorta are too well known to need emphasis. Drs. Herringham and Wills, at a meeting of the Medico-Chirurgical Society on May 31, 1904, demonstrated on 128 aortas in man that this vessel grows in size and strength with age up to 40 years when, so long as it is healthy, the limits of ordinary enlargements, within a small fraction, are reached; up to this age then resiliency should not be seriously reduced, the tube after dilatation should return promptly to its resting diameter. Now even a normal vessel, as it becomes distended, resists deformation more and more; its extensibility diminishes and the wall-wave velocity increases. And so it is with a morbid rigidity. Thus, early in chronic renal disease, as the walls of the vessels are tightened, the wave velocity rises; later, as they stiffen, the effect is permanent. But palpation cannot tell us this ; it must be calculated otherwise. ${ }^{1}$

As age advances, and the inferior connective tissue may be displacing or succeeding elastic fibres, the arterial diameter becomes permanently enlarged, and resilience fails. This enlargement cannot be called compensatory, at any rate not in Thoma's sense. Compensatory, or rather palliative, it may be, in the sense that, as with age distensibility wanes, the vessel is permanently more capacious, and the accumulation of residual blood in the

1 See again Friberger, Deutsche Arch. f. klin. Med. vol. cvii., 1912; also Wells and Hill, paper read at Royal Soc., Feb. 6, 1913, after this text was printed. 
heart, or overwork of the heart, is postponed a little. One effect probably is that cardiac contraction volume and stream velocity both diminish; and as, for a fluid of comparatively low viscosity, enlargement of diameter would favour vortices, to overcome or prevent these more energy must be expendedthat is if the work be done, which however is rarely the case. If a road grows steeper one goes slower. To assume that as the arteries lose elasticity a rise of pressure must follow is to assume that output, function, stream velocity and cardiac capacity are constants. If the heart be still fairly normal, the subjects of arteriosclerosis may retain no little activity, but it lies within a narrower compass. ${ }^{1}$ A slower stream means a slower oxidation. As Dr. Auld pointed out long ago, the elastic fibres themselves deteriorate, a change which has since been more fully demonstrated by Jores and others. But this is not the whole matter; the aorta contains not only elastic fibres sufficient for its physical reactions, but muscular fibres also which endow it with tone and power of self- (reflex ?) adjustment. However, the more or less independent movements of tone in obedience to oscillations of pressure may for the moment be disregarded. A few authors have urged that the stiffer the vessel the more elastic, in a mathematical sense, it becomes ; but elasticity consists not only in resisting deformation but also in quick recovery after compression; in this quality the diseased vessel, stiffer as it may be, deteriorates.

The pulse-wave depends then, mechanically speaking, upon elasticity, gravity, thickness of wall and viscosity; then comes in the nervous system, vasomotor and central, to govern the mechanism.

The Heart and Blood Pressure.-We have glanced at the carriage of the blood as conditioned by arterial and venous pressures, by velocity, by the state of its channels, by tissue activity, by blood viscosity, and so forth; let us turn now to the heart itself, upon which depends the maintenance of all pressures. Can there by way of cardiac inefficiency be a relatively high peripheral resistance without excessive blood pressures, or with inconsiderable excess? Broadbent was perhaps the first physician

1 See Auld, Herringham and Wills (loc. cit.), and Strasburger, Deutsche Arch. f. klin. Med. Bd. xci., 1907. 
to perceive that such a state comes about in the later period of excessive blood pressures when, if I may say so, the heart becomes disheartened; and to this phase he gave the name "virtual tension." Many years ago I ventured to qualify his opinion so far as this, that the so-called "virtual tension" was often, perhaps generally in the cases under contemplation, still positively high. Although, on the principles we have just been discussing, with the yielding heart the circulation is slowing down, and the pressures falling, yet, until the final stage of exhaustion, figures such as 170 to 180 , or even higher, may still be read on the scale. The heart is not doing what it did, the resistance to it is obstinate, yet it is working still at supernormal although no longer compensating pressures. If in some rare instances, although causes of hyperpiesis come into action, the heart yet fails to rise to the emergency, and pressures may thus from the beginning sink, or fail to be maintained, I cannot with certainty say; it is hard to catch such cases at their beginning. Under these conditions the arteries themselves might escape mechanical injury, it is true; but in all other respects the diagnosis and prognosis would be far worse. I can recall one case in which such conditions may have existed :

Mr. D., æt. 51, had a dilated heart and a loud second aortic sound. There was on his visit no mitral or other murmur, but the cardiac rhythm was uneven, and the systolic arterial pressure was registered, at various dates, as 130 to 140 . His arteries to the touch were normal. He was a farmer who had drunk beer freely, and was a large eater. For some years he had been a fat and rather pursy man, and for the last twelve months his breath had been short. The heart's impulse, so far as the fat chest would allow me to appreciate it, was diffuse and rather extended. A latent period of high pressure might, of course, have preceded this neutral phase; but on full consideration of his age, the interval of time, and other facts in his history I conjectured that a flabby heart had never been equal to the readjustment required, and that $a b$ initio the patient's malady was one of "virtual tension,"- - a virtual hyperpiesis. (The unrecorded diastolic pressures may have been raised ?)

Even in health we have to consider if the cardio-arterial becomes ultimately a " self-strained system "; if the reciprocating parts get out of balance with each other; if in time the diligent heart impairs the resilience of the elastic spring upon 
which it operates, and by which it is itself operated. The elastic spring is the spiral arrangement of fibres throughout the cardioarterial system. Were these fibres straight the machine would tend to oscillate periodically in one or two only of its axial planes, and such phases would strain the machine unequally. But a spiral movement round the ideal axis distributes the stresses, almost without such periods. For like reason troops on crossing a slender bridge are ordered to do so out of step, and " at ease." In such a machine balance of impulse is as important as balance of mass. As I shall show under Cardiosclerosis, I think better of the heart than do M. Huchard and his disciples, who involve it and the arterial tree in a common " arterio-cardiopathy." Hearts decay, as all organs decay ; but, in the large majority of the cases we are now considering, the heart shows no little independence of arterial disease and, as I have often shown, may be the soundest limb of a decaying arterial tree. If the change be slow enough, even the very coronaries themselves may grow rigid, or turn to coral, or silt up at their mouths, while the heart, with no incapable muscle nourished from some hidden source, may yet be fighting bravely along, still capable even of driving through a narrow aortic orifice the slenderest of streams at a velocity high enough to maintain an old man or woman at the chimney corner.

Fall of blood pressure of cardiac origin is often attributed to faulty proportions of extrinsic constituents such as the potash or lime salts; or within of choline-like bodies which lower pressures; but falling pressures, except in contrast with increments, do not pertain to my subject. In respect of the cardiac function also, as of the arterial, we have to deal, in both rising and falling phases, with highly complex, indeterminate, and often erratic variables; any one of the five qualities of the heart-excitability, tonicity, conductivity, contractility, rhythm-may vary alone, or in mutable combination with the rest. Contraction and conduction vary independently. Professor Mines, in his interesting papers, ${ }^{1}$ reports certain experiments on the dynamic results of changes in lipoids and lime. To cut off calcium salts reduces contraction more quickly than conduction. Hemmeter, of Johns Hopkins, has illustrated the relation of vagus inhibition to the inorganic salts of

1 E.g. Mines, Journ. of Physiol. vol. xlvi., 1913. 
the heart. Thus, as we see every day, in some cases of cardiac disability the organ is dilated, in others no enlargement is found ; but to every central oscillation of blood pressure the heart must respond, or a sharp drop in velocity would ensue ; and this, as von Frey proved, it does too promptly for any reflex assistance. But temporarily to protect the heart from excessive demands is the function of the vagus, which not only feels instantly every rise of pressure, but probably is always in touch ; on the other hand, if the tissues cry out, the augmentors drive the heart forwards ; and this tissue cry, as calculated by experiment on animals, and by observation of great efforts in man, may for the moment compass even double the normal arterial pressures. Bollinger (quoted by $\mathrm{Krehl}$ ) states that it is not the largest but the swiftest animals which have relatively the biggest hearts, the hearts of birds being relatively the biggest of all. Thus again, as we shall see, when pressures continue persistently in excess, a new and supernormal mean can be established in man for a while, even for many years.

Marey's abstract rule that as pressures rise the rate of the pulse falls, and conversely, is so continually overridden by contingency that we cannot take it as an axiom of practice. It is true that as pressure rises the vagus checks the rate: if the vagi be previously cut or atropined, artificial attempts to raise it will fail; but, for instance, when in early convalescence from infectious fevers, or in under-par persons, the heart and nervous energy are weak, on a slight effort both pressure and rate will rise transiently ; but after exertion, as pressures are disposed to fall, the pulse rate may often fall too, rather than rise. ${ }^{1}$ Lability of pressure gradients may cause them to fluctuate out of concord with the pulse. A slow pulse, caeteris paribus, gives time for a lower diastolic fall of pressure; conversely, excess rate means shorter diastole, less filling of ventricles, less output, and so, with a constant periphery, a disposition to falling pressures. In paroxysmal tachycardia the fall of arterial pressure is due less to heart failure than to diminution, of output by shoriening of the diastolic phase; so that, as seen after a while in dilated right chambers, in swollen jugulars, or even in a distended liver and œdema, the venous pressures rise. The

${ }^{1}$ See Oddo, quoted Lancet, July 9, 1905. 
best outputs in unit time and the best blood pressures are at ordinary pulse rates. On the other hand, if there be sufficient peripheral tone, and the output adequate and equal, a higher pulse rate will, or may, raise systolic arterial pressure. The fall of pressures with accelerated pulse in most of the febrile infections is due in the first instance less to the heart than to dilatation of the peripheral vessels, presumably busy in meeting the excessive tissue and immunity operations.

When with the ordinary sphygmometer we use the finger to ascertain the phases of pressure, we may take either the arrest or the return of the wave as our guide; the opening (return), which is slightly lower, is generally used. It is probably more accurate, but in any case we should observe uniformity of practice. The wave of arrest runs at about $3 \mathrm{~mm}$. higher, but the volume of the current may be not inconsiderable. For we must not assume that arrest of the pulse-wave to the finger on the radial corresponds with closure of the brachial stream; this may require double the pressure. It is said that on arrest of the wave the artery suddenly collapses under this pressure, but we have no evidence of this, and it does not seem probable. After a cuff on the arm has been inflated to the obliteration of the pulse-wave Gärtner's gauge still shows colour in the finger. This is no " überraschende Tatsache," as a member of Koranyi's clinic calls it, 1 but an illustration of a hydrostatic law. The pulsewave is damped before the stream is stopped, or the pressure much reduced; and of course, caeteris paribus, diminution of calibre goes for little. However, I take this point here because the writer had got hold of a useful notion-that as the heart fails in efficiency the difference between the two obliterations may be less and less; and by the use of digitalis, in a responsive case, the difference might increase daily, even up to the normal.

Blood Mass.-It is so well known to physiologists that, within limits which concern the clinical physician, the total mass of the blood has no direct or continuous relation to blood pressures that, were it not that many authors of consideration persist in enumerating blood mass among the causes of pressure variation, I need not have dwelt upon this factor (vide e.g. section on Plethora vera, p. 255). The heart's work, the systoles being uniform in

1 See Deutsche med. Wochenschr., June 2, 1910. 
time, is a function, not of the total blood mass, but of the output -that is, of the mass lifted, and the peripheral resistance. A physical effort, which usually also compresses the abdomen, or a few deep inspirations, may increase the output by 50 per cent without altering the blood pressure. The blood pressure in the horse does not differ very widely from that in the rabbit, yet the heart of the horse, throwing a vastly larger quantity of blood into the circulation, does vastly more work. If, as in certain cases of bulky watery blood, the resistance falls, the output may be doubled or more than doubled, without any rise of pressure ; here again of course the heart is doing more work, but unfortunately, as in these cases the pressure in the coronaries is not rising, it is not fed in due proportion, either by pressure or by quality of blood. It seems probable, from the increased pressures seen temporarily in a few cases of plethora, and of normal pressure in others, that while the heart readily adapts itself to a larger mass of blood the vessels are slower than the heart in doing this. Thus at the beginning of strong exercise the heart seems at once to supply the energy and capacity needed, but two or three minutes, according to the training of the individual, may elapse before the periphery opens out ("second wind"). Again, if fluids of a little higher or a little lower viscosity be injected into an animal, although the heart quickly adapts itself, the systemic pressures temporarily rise, with fall of arteriolar velocity. The capacity of rapid readaptation to variations in blood mass suggests that such fluctuations are common, and that the system is used to them.

Obliteration of Capillary Fields.-Balfour regarded the atherosclerotic obliteration of peripheral vascular areas, with shrinking of the tissues, as a chief or considerable cause of increasing pressures in elderly persons. This hypothesis never commended itself to me, for this senile obliteration of vascular tracts is especially notable, not in the subjects of Hyperpiesia or Granular Kidney, who for the most part are not senile yet in whom pressures rule very high, but in the subjects of "Senile"-my Involutionary or Decrescent-Arteriosclerosis, in whom there is no remarkable rise of pressures. But surely to attribute rise of pressure to obliteration of vascular areas is but the well-worn error, in another guise, of attributing it to increase of blood mass ; 
the argument of both propositions being that the vascular system is too small for its content. In Balfour's hypothesis of restricted area, as in that of increased total volume of blood, it is often forgotten that we are discussing not the vascular system as a whole, but the arterial tree only; now in the whole vascular system there is room enough to accommodate almost any quantity of blood. In old men, and in certain chilly young ones, we may see an inclination of the blood towards the venous side, and in them cardiac contraction volume and stream velocity are probably reduced. Moreover Dr. Mackenzie observes that jugular pulsation is rare in old persons, which supports the opinion that in old age the total volume of the blood is reduced. And in all men we observe that in nature's economy the blood mass is not normally of such bulk as to keep the whole body in universal and simultaneous activity, but is only sufficient to serve alternately now this area and now that, in obedience to partial determinations and periodic activities; moreover, much of the blood, if not the same parcels of it, may lie more or less continually outside the working circulation, as in the abdominal and other reservoirs. Therefore Huchard's opinion that blood mass was a factor in persistently augmented arterial pressure cannot be admitted even for senile arteriosclerosis. Output may be doubled without any rise in pressure. Yet a recent able foreign author, describing rises of pressure, and its cumulative effect of arteriosclerosis, goes on to say that the results of " this capillary atresia and renal atrophy" (not Bright's disease) are an aggravation of the high pressures. But neither of these defaults is related to the high pressure; they both belong to the decrescent series, and neither of them raises arterial pressures perceptibly (vide remarks on Arteriosclerotic Kidney, p. 334).

The Heart and Peripheral Resistance.-It remains for us now to consider the effect of increasing resistance, whether of vascular tonus or of other friction; for, if the heart is thwarted in its efforts to maintain a due velocity, the bodily functions must begin to flag. Senac was perhaps the first to speak of the " arterial heart," and Ludwig the first experimenter to show the importance of the peripheral circulation, of which resistance is but one aspect. As in the history of the Roman Empire-the centre had at times to be sought on the circumference. The higher 
pressure of the aortic system above that of the pulmonary is mainly due to this peripheral resistance. One function of normal resistance is, of course, to increase stability. It is of little use to force up cardiac energy when the periphery is relaxing widely ; on the other hand peripheral constriction in a limb only, say in one leg, would have little effect on the systemic blood pressure ; indeed it is doubtful if constriction of the splanchnic system, without some plus tone elsewhere, would suffice to keep up the normal systemic pressure.

As resistance increases the need for intra-ventricular capacity must increase likewise, and for a more permanent increase than in valvular disease, when the heart undergoes fluctuations in volume comparable with the functional tides of everyday healthy life. Thus, as resistance increases, higher and higher mean quantities of cardiac function are established, grade over grade. The systole lengthens, ${ }^{1}$ as in aortic stenosis ; every individual fibre takes longer to reach its degree of relative abbreviation, and the optimum load is altered. But this stressing of the systole, and increasing contraction volume of the left ventricle, cannot compete with a steady rise of resistance ; very soon output will diminish and residual blood accumulate in the ventricle; and, to meet this extraordinary stress, hypertrophy and dilatation must, and under favourable conditions do, become established. The readjustment may not in all structural dimensions be complete; at each contraction, as perhaps at the undefended space, some residual blood may still lag behind; yet, at equal pulse rates, the quotient of output and velocity grows larger than normal. If the conditions are not favourable; if the blood be infected or impoverished, if the nervous system be fretted, or if other contrary factors be at work, then even approximate readjustment will come short: pressures will fall, ventricular suction (if it have the value commonly assigned to it) will slacken, the venous system will fill, and, as oxygen exchanges are thus reduced in the substance of the heart, the coronary circulation will abate. Such a chain of events is of graver import than some lag in moderate valvular disease, or in stress of bodily over-exertion; temporary accommodations which, within limits, are met out of the ordinary stores of the heart. Contrariwise

1 Vide Luderitz, Zeitschr. f. klin. Med. Bd. xx. S. 374 ; and other authors. 
it is said, on some clinical evidence, that within limits peripheral atony may cause hypertrophy of the heart by its labour to keep the arteries filled. Still we do not know all the conditions; for instance the possible vascular call for adrenin.

Viscosity.-Under this head there was so much to be said, which is not common knowledge, that this section grew into the chapter which follows (vide p. 104).

Validity of Mechanical Laws in Physiology.-From what has gone before we perceive that, however accurate the physical argument, in these problems there is more than a physical quality. We shall see, in the chapter on Viscosity, that in the neurovascular system Poiseuille's law may be subject to interferences so many and so considerable as to lose its abstract values; or for living structures the mathematical laws of elasticity may not have more than a subordinate validity. ${ }^{1}$ Inconstancy of the bed of an area must be a large factor in the regulation of speed; abstractedly, to diminish the channel by one-half is to reduce speed sixteen times; but the critical velocity-the relation of viscosity to diameter-may be so intricated with other conditions as to be very difficult to disentangle and to value. Poiseuille, it is true, did experiment upon living animals, ${ }^{2}$ and concluded that his law held good for the circulation of the blood; that of the four factors-pressure $(p)$, radius $(r)$, length $(l)$, viscosity $(\eta)$, tested by varying any one while the other three were constant-pressure varied directly as the fourth power of the radius, and inversely as length and viscosity. Still, in life, we cannot analyse a compound effect by the method of exclusion. Even physically the law scarcely aids us for so complex a system of tubes of very various diameter; or it holds only for vessels of the calibre where the arteriole passes into the capillary, and where velocity may be directly as pressure. And, so far, all attempts to calculate the dependence of current on $p$ and $\eta$ have failed. In a glass tube to alter $\eta$, the others being constant, has a consistent effect on velocity, but in the artery it is not so; some other factor is at work, in part the inconstancy of cross section. (See also p. 38.) Moreover, the length is not constant; as well as a diametrical, there is a longitudinal tone, the loss of which is plainly to be noted,

1 See Volkmann, Hämodynamik, p. 53, and my Preface.

2 Poiseuille, Recherches sur la force du cœur, Paris, 1828. 
for instance, in the aorta of neurasthenics. Volkmann found moreover that the relation between velocity and pressure varied with the area. We can no longer then assume universally the simple physical formula that velocity is in inverse proportion to the square of the diameter; central nervous controls, secretory and hormonic tensions, the effects of such ingredients as calcium, barium, or potassium salts, or chloroform, may be of as much, or often of more, importance than diameter, and we perceive that living vessels may pass more blood than dead ones. Indeed in any elastic vessels, as Hürthle has shown, ${ }^{1}$ velocity must fall more rapidly as pressures decline, as for instance in a "vagus pulse."

Vasomotor System.-Thus, as regards the elastic tissues, to which physical principles apply with more simplicity, these in the living body are in co-operation with muscular irritability and nervous government. Tension of wall is no longer, as in passively distensible tubes, directly proportionate to a pulsating internal fluid pressure; there is a large, if unmeasured, factor of resistance to tension in variations of tone and pressures in surrounding parts and over large areas, a resistance which is by no means to be taken as uniform. ${ }^{2}$ In some patients the radial arteries are habitually large, in others narrow, in others variable; but the pressure on the content may be the same. And a vasomotor constriction is not a mere throttle valve. A half-turn of a stopcock directly lowers pressures in the area supplied ; but when the pipe is an artery the increase of lateral pressure above the choke sets up, or may set up, another series of events; an active vasodilatation may be aroused in neighbouring or correlated areas, and by anastomosis compass more than a due accommodation for the blood arrested. Such a dilatation is said to take place at the site of insertion of a cannula into an artery of an animal, or even in the brachial artery under variable pressures of the cuff (MacWilliam).

1 Hürthle, “Druck u. Geschwindigkeit," Pflügers Arch., 1912, and Berl. kl. Woch., April 22, 1912. He used optical records of the stromuhr and a very light manometer lever, as described in a previous article in the same number. He gives diagrams of the methods.

${ }^{2}$ Some interesting remarks on reciprocating and independent, or discordant, vascular areas, with their hydrodynamic coefficients, are to be found in an article by Müller, Arch. f. anat. et physiol., 1904; and, as to the brain, by Weber, ibid., 1906.

vOL. I 
Professor Bayliss, ${ }^{1}$ in arguing that muscular arteries, even when cleared of nervous matter, tend to constrict as pressures rise, and to relax as they fall, showed also that such a section of tone may be independent of any nervous arc, or of balance between sympathetic and autonomic systems; it may be simply " myogenic," the sudden stretch of an artery by a rise of pressure being of itself an after-spasm of the unstriped muscle, which seems to possess this excitability; so that by muscle alone the vessels may preserve a normal tone, ${ }^{2}$ and may respond to local anæmias or cachæmias. It seems certain that some local vascular tone remains in or about the walls of arteries after post-ganglionic fibres are divided and cardiac influence removed; for after a temporary drop the tone, in response to heat or cold, or certain irritants, ${ }^{3}$ returns pretty much as before. This is true even of the splanchnic area after severance of all nervous connections. I say " in or about" the walls, as the existence of minute centres in the walls is a very obscure problem. But he showed more than this; if in the rabbit the central end of the depressor nerve be excited of course a fall of blood pressure ensues; but if strychnine be administered to the animal the same experiment causes a rise of pressure. And, conversely, by strychnine the rise of pressure which normally follows the stimulation of a sensory nerve may be converted into a vasodilatation. Chloroform has an opposite action in these respects; so that we see how complex are the conditions with which we have to deal, and how secretly one intercurrent factor or another may drive a system of functions into opposition. And active vasodilatation is of course to be distinguished from passive (atony), especially in the splanchnic area, and in the opening out of vascular areas in corresponding muscles on stimulation of motor nerves (Gaskell and others). We are familiar with such series of events in embolism also; but if the vessels of an area be dilated by section of a vasomotor nerve they return to the normal, or very nearly, in a few days.

1 Bayliss, Physiol. Congress, Wiesbaden, 1907.

2 After these paragraphs were printed I saw MacWilliam and Kesson's important paper on the dilating effect of repeated recompressions of an artery (see p. 75) ; and von Anrep's paper in Journ. Physiol., January 1913, in which he attributes Bayliss's local reaction of the arteries to a gush of adrenin from its glands, and thinks a local reaction has yet to be proved.

${ }^{3}$ See Heger, Ludwig's Festschrift, 1887. 
For such reasons as these a pulsatile may pass more blood than an equable stream; the beat of the wave, independently of mean pressure, may be a coefficient. Other obscure but potent coefficients are the attractions of the working cells for arterial blood, the local oxydative activities ${ }^{1}$-a factor in resistance on which Broadbent was wont to insist, and more recently Sir W. Osler; the chemistry of these metabolites and of the hormones; the heart's energy which may increase velocity even in a dilating vessel, and possibly the quasi-peristaltic endowment of the muscular arteries, suggested by Broadbent, and ably if not convincingly advocated by Hasebroek, and by Grützner. ${ }^{2}$ Hasebroek is of opinion that an artery opens by an automatic rhythm to receive the stream wave and closes after it, so that the blood cannot return from venous to arterial areas. Bayliss's results seem to favour this view, though he does not say so. Energy over a given blood volume in a given area is then an algebraic sum which, however usefully we may guess at it, is, for the present at any rate, beyond particular reckonings. That vessels contract differentially to protect the tissues is

1 Starling and Barcroft think the arterial walls are very sensitive to the metabolites of an organ in activity (p. 236). More than thirty years ago Gaskell (and Chauveau) found that arterioles dilate in very weak solutions of lactic acid, so that thus the muscles in activity are washed.

${ }^{2}$ Hasebroek, Die Blutdrucksteigerung, Wiesbaden, 1910; Grützner, Deutsche Arch.f. klin. Med. Bd. lxxxix. These essays are well worth perusal, whatever our judgment may be upon the facts. To me it seems that the muscular and elastic movements of the arteries depend directly and locally so far upon conditions of pulsation, of branching, and of compressions from without as to overshadow any factor of peristalsis. Hürthle, in an article in Pfügers Arch., 1912, thought that Hasebroek had not yet proved his case; but, while these pages are passing through the press, I find that Hürthle has seen reason to reopen this question. In the Deutsche med. Woch., März 27, 1913, he records a surplus of cardiac propulsive factor of about 16 per cent, apparently not due to fallacy of method: indeed he demonstrates it by more than one method. This surplus be thinks may be due to arterial propulsion; the paper is but a preliminary study. Hasebroek (Pflüger, vol. cxliii., 1912), in returning to the question, urges that in many cases where there is no cardiac compensation the circulation is aided by "the aspiration-component" of the peripheral vessels - an independent peripheral circulation. But is not "aspiration" an awkward noun for a plenum? Professor Langley tells me he is not yet prepared to believe that the contraction of the arteries has a considerable share in keeping up the blood flow. When does the relaxation set in? If during diastole, it would retard the flow nearly as much as the previous contraction helped it; if during systole, the first part of the systole must cause relaxation, and the second part contraction. Barcroft thinks metabolites stronger than dilator nerves. 
improbable, neurovascular reagents may be acceptable to the tissues or may not; the tissues themselves, by their specific affinities, govern the molecular attachments and transits.

How far in arteriosclerosis all these properties and coefficients are abated, or reinforced, is unknown. It is currently said that in this state the carotid-radial interval is prolonged, but is wave or stream velocity intended? We know that in a thickened, or in a tightly distended, artery wave velocity should be increased, but stream velocity might be reduced (p. 33).

In order to ascertain not surface reactions only but also the inward, Weber tried to record the splanchnic waves by inserting inflatable bladders into the rectum or vagina. He found that by this method waves due to diaphragmatic respiration could easily be distinguished. Briefly, Weber found that in normal subjects the abdominal curves varied inversely as those of the arm, so far an interesting verification of theory; thus a normal plethysmographic arm curve might signify a normal splanchnic tonus. Klemperer further proposed to diagnose splanchnic sclerosis, not only by the flatter curve, but also by the deviation from a uniform alternation with the arm.

In chronic Bright's disease, when vascular constriction is perennial, more than the splanchnic area is concerned. In vascular spasm of course we pass from the function of mean tone, the adaptation of continent to mass, away to normal or abnormal redistribution, an influence of other and often remote correlations. Thus, I repeat, we must distinguish between positive and negative vasoconstriction, between an adaptation of the vascular system to imperfectly filled vessels, and an insurrection, of whatever origin, against a good heart and a normal blood distribution. Is it not then better to keep the word tonus accurately for the automatic adaptation, and to discard the German term " hypertonus" altogether; for arterial constriction, due to toxic or other extraordinary causes, is scarcely "tone," but rather a morbid spasm?

There is no evidence, as I have said, that the arterial muscle is readily subject to fatigue; dermatographic lines may be obtainable in articulo mortis. As the vasomotor mechanism has for its function the maintenance of the blood pressures in the large arteries, fatigue in the vessels would be perilous: 
the vasomotor centres may be exhaustible, and their higher cerebral coefficients still more so (see p. 476). But I may perhaps venture upon some application to the unstriped fibre of Professor Sherrington's well-known observations on the tonus of voluntary muscle. The arterial muscle can scarcely be called "a common path," it is true, because there is little competition for it; its stimulus is quite, or almost, single in kind and origin; but nevertheless Sherrington's principle of the "common path" being always held in possession as tonus, the resultant of all peripheral impressions, positive and negative, seems not inapplicable. And I repeat that presumably we may apply with still more assurance to this lowlier agency the farther principle that " the common path does not tire," and that the fatigue, if in our case any, lies in the afferent factor-in a waning of the reflex. The chief seat of fatigue is said to be at synapses where, in extraordinary activity, oxygen fails. But here again in our simple and lowly mechanism the muscle continues to respond directly, ${ }^{1}$ if blindly, to internal pressures, whether the wider and more central reflexes and inhibitions be waning or not. Fatigue of a muscle, or other animal fibre, cannot be compared with "fatigue" of a metal; in this case it means molecular dislocation, in that (within limits, of course) only a temporary functional abeyance. The limits of tone however vary, in unknown degrees, with age, and no doubt in all old tissues molecular dislocation is gradually established. If I may quote myself," " in full vigour of life a stream from the nervous centres maintains a tension curve in steady concert with the other coefficients, a curve lying well within the elastic limits of the particular tissue."

How largely dependent are vasomotor functions upon cerebral causes is illustrated in an interesting article by Bonser of Illinois University. ${ }^{3}$ Bonser made a long series of experiments upon twelve students of his university. Amongst his observations it is pertinent here to say only that intellectual fatigue was accompanied by diminished vasomotor response to emotional changes ;

1 Bayliss, Journ. of Physiol., 1902, and other papers. But see note 2, p. 51.

${ }^{2}$ Allbutt, C., Proc. R. Med. Soc. vol. i. sect. 2, p. 209.

3 Bonser, Psycholog. Rev., March 1903, quoted Lancet, April 13, 1903. 
and recovery from them was slower (as in the dermatography of meningitis). But the psychic influences on blood pressures, and how by disease of the vessels these normal reactions may be modified and prevented, I have yet to consider (p. 68).

The vasomotor system must be re-represented in the cerebral cortex, or be connected with it. In the upper vasomotor centres all districts must be represented, severally and together; so that a general stimulant, or depressant, may issue now upon this area, now upon that - e.g. the splanchnic, or the cutaneous, and so on-before others, and the splanchnic mechanism being, in upright man, of recent organisation, may suffer fatigue, as perhaps is the case in some neurasthenics. I remember observing with Roy that in dogs it was comparatively difficult to produce splanchnic shock; and in them chloroform accidents are very rare ; I cannot recollect any such incident.

The remarkable and, as it appears, effectual capacity of the circulation to counteract the effects of viscosity on blood pressure I shall consider in the section on Viscosity.

Of capillary dynamics we have but little knowledge, yet it is true that here lies the longest circulation time. If, as Broadbent was wont to argue, peripheral resistance lay in this field, it could scarcely be on hydraulic principles ; indeed he himself attributed it to some clogging, or sluggishness of tissue exchanges. Of the immense but still obscure operations of physical coefficients in this peripheral field-coefficients such as osmosis, diffusion, surface tension, absorption, and so forth-I make some tentative appreciations in the section on Viscosity. The enormous pressures which these energies-dialysis, for examplecan bring into play may reduce even gravity to a negligible quantity! We have no exact method of measuring capillary pressure, we can but take the pressure in afferent and efferent vessels and guess at that of the intermediate area. The most systematic estimate of capillary pressures are by Lombard's method. He smears the skin, say the thin skin of the finger, with glycerine or a transparent oil, so that under a lens the fine vessels become visible. The circulation is now arrested intermittently, and fluctuations under pressures recorded by optical means. $\mathrm{He}$ found pressures in the small arteries $60-70 \mathrm{~mm}$. ; in small surface 
veins $15-20 \mathrm{~mm}$., and the capillary mean $40 \mathrm{~mm}$. These figures seem too high. Von Kries estimated the blood pressure in the capillaries at $\frac{1}{3}-\frac{2}{7}$ of the aortic pressure $(20-38 \mathrm{~mm}$. $\mathrm{Hg}$.), and his estimate, though it seems high, has been provisionally accepted. This estimate is probably taken from the pressure of the larger capillaries, and it would rise farther as they enlarged into fine arterioles. Others (on the finger) have made the mean capillary pressure only $7 \mathrm{~mm}$. In the smallest capillaries beyond the arterioles the pressure, peripheral to their tone, can scarcely be more than $10-20 \mathrm{~mm}$; but here again much depends upon this local tone, the hydrodynamic cushion, and the general pressure. Dr. Harry Campbell ${ }^{1}$ reminds us that, in an area remote from arterial pressures, about $10 \mathrm{~mm}$. of pressure seems to suffice for the capillary network of the liver. We may perhaps consider mere capillary resistance as negligible; although many years ago Roy and Brown (loc. cit. p. 57), and Stricker also, showed that the capillaries can contract, probably by the cells of the walls, which clasp the channel and seem to respond to the sympathetic nerves; a demonstration which Bayliss and Langley have verified. The sizes of corpuscles in proportion to capillary diameter are however by no means negligible; they must stem the current, and so reduce mean velocity in the finest channels to $2 \cdot 0$ or $1 \cdot 0$, or even 0.5 . Presumably also some delay must occur during their emigrations.

If then we suppose some such delays in the finer vascular web, and thereupon pressures to rise-for vasodilatation would probably equalise things but for the moment-and the blood stream thereby to be permanently retarded, the heart would work harder to regain velocity; and thus, at the cost of cardio-arterial hypertrophy, and of a heavier stress upon the less muscular vessels, velocity might be regained. Such like, but not the same, was Rosenbach's hypothesis: that by the action of some toxin irritation an excessive metabolism arose in the tissues, and more blood was poured in; that thereby the systolic output was increased, the left ventricle grew in size, and secondarily the right heart also and the lungs (whence an emphysema). This explanation is clearly out of place for decrescent arteriosclerosis, the character of which is not high

1 Campbell, H., Brit. Med. Journ., Jan. 28, 1905. 
central pressures but peripheral atrophy; as an explanation of Hyperpiesia, and so of its consequential arteriosclerosis, it might be urged; but emphysema is generally found, not with the hyperpietic, but with the decrescent disease, and is due in my opinion to decay of the bronchial arteries.

Longitudinally it is true that in detail the systemic capillaries measure only about $5 \mathrm{~mm}$. and, at ordinary velocity, are traversed in about half a second; but if ingravescent causes of retardation be found there, and superactive constriction in middle areas does not intervene, the sum of the capillary area is large enough to make it a considerable factor in the peripheral friction. It is also so roomy that a considerable time may elapse before a change of arterial pressure is felt throughout the area, as for example on the application of a cuff. And it is true that to purge the venous system seems to reduce high arterial pressures. At this point of view Dr. George Oliver's well-known observations on lymph pressures come into consideration; and we cannot forget the marvellous ascent of sap to the tops of the tallest trees, the resistance being overcome not by capillary attraction only, but also by cell dynamics. Leucocytic diapedesis lies nearly altogether in the capillary area, and is favoured by dilatation, with blood afflux and access of oxygen, and by warmth; and rises of pressure in the capillaries dispose to unloading by exudation. Volkmann argued that in secretion and excretion constituents of the blood flowed at various rates through hypothetical stomata, so that in active areas the blood stream might be accelerated by a sort of aspiration, and velocity thus increased behind, while in areas of rest the stream rate would be retarded.

Sir James Barr, Dr. Haig, and others have worked at these capillary problems, and at the effect of lime and other salts upon the current, but Barr does not favour the opinion that high pressures depend upon capillary obstruction. One is tempted indeed to suppose that a reduction of protoplasmic activity might permit the blood to traverse its area with less occupation ; but the rarity of atheroma in the pulmonary vessels, except in mitral stenosis or equivalent states, suggests that, in this cycle at any rate, tissue activity is infinitely less efficacious in forwarding the blood than cardiac propulsion. Again unquestionably the nitrites, as vasodilators, do, by reducing resistance, 
favour the blood current and lower arterial pressure, yet the nitrites do not directly influence the capillary activities. The first effect of vasoconstriction, as we may see in fear, is to blanch the area of it; this holds until in a relatively small field the constriction is wide and persistent enough to cause a venous reflux and plethora, and with this rise of venous pressure we see the capillaries fill readily enough, so long, that is, as the general arterial pressure is constant. If vasoconstriction persist over a large area so that the capacity of the system is diminished, pressures would rise also on the venous side; and in this arteriolar condition all the corresponding capillaries are on the venous side.

As to alleged capillary reflexes, the observations made, save perhaps some of the plethysmographic, seem worthless: the responses must depend also on many other local conditions; on cold and warmth, on phases of vasomotor position, on viscosity of the blood, on metabolic activity in the area, and so forth. Here lie some of the disadvantages of gauges on the principle of Gärtner, and of the time records of blanching after the pressure of a pad or button. If the capillaries are contractile ${ }^{1}$ (p. 55) the movement, so long as the vessels are sound and the blood healthy, is negligible. The flickering in the frog's capillaries is due to waves in the arteriolar tone; the stream being slower, individual corpuscles can be watched and the currents better perceived.

Venous tonus is negligible, even in the portal system ; and collaterals are too abundant to permit of local constrictive anæmias.

Instruments.-Before proceeding to the clinical features of the circulation, I must say something of the mechanical means of registering the phases of arterial pressures. Until his sphygmometer was introduced by von Basch in 1887, my own work-and in respect of man I believe the same is true of that of others-was done entirely with Marey's sphygmograph and cardiograph. Invaluable as were the records of the sphygmograph in revealing the form and rhythm of the pulse, yet, after many attempts to adapt it to even approximate recording of blood pressures, we had to admit that for this purpose another kind of instrument would be required; and about this

1 See Roy and Brown, Journ. Physiol. vol. ii. p. 32. 
time Ludwig's epoch-making kymographic apparatus and researches were opening out new methods and fields of discovery and of record. However, no one who has used the sphygmograph carefully and habitually will have failed to recognise the value of the information and instruction it can give. Some of the earlier workers with the instrument, such as Sanderson, Mahomed, Riegel, Galabin, Roy-I mention a few names as they happen to come into my mind-published excellent curves; then unfortunately books and papers, especially French and English, which had some vogue, were furnished with tracings, some new, many of them hackneyed clichés, not a few of which were unskilful merely as tracings; while many more showed a want of appreciation of the limits and capacities of the sphygmograph itself. Too often the observer had taken pains to read into its records what the instrument was incapable of describing; or had interpreted as physiological, features due only to its mishandling, its defaults, or its inertia. I will venture to repeat with some abbreviation a paragraph from a review written by myself more than twenty years ago ${ }^{1}$ upon many of the published sphygmograms. "The semblance without the reality of precision is a dangerous temptation. Thus we are led to write up to many tracings which are either bad in themselves, or deformed by inertia vibrations or shortcomings in the instrument. There is no such thing as 'the normal pulse tracing'; a good tracing implies not only a sensitive make of instrument, but also a sensitive manipulator. Many an amorphous tracing betrays to the reader that the instrument had not followed the artery; some of its finer waves were beyond the capacity of the instrument used, or of any instrument; or again beyond the user's dexterity. So we are treated to rows of comparatively structureless bumps, especially when under some shift or reflex stimulus the pulse had receded from the pad, or re-engaged with it; so unsteady is the personal equation in these researches. In applying the sphygmograph we meet with four main difficulties : the quantities, properties, and inequalities of the structures of the limb containing the artery; the unequal and unstable application of the pad along the vessel; the lie and pressure of the instrument on the whole underlying wrist; the calibre of the artery. For instance, anacrotic waves are often

1 Brit. Med. Journ., Dec. 31, 1892. 
of instrumental formation, not due to rise of aortic pressure at the end of the outflow. And, as to the interpretation of the tracings, curves significant merely of relaxing arteries have been said to indicate failing heart; or a large output with open periphery has been interpreted as high pressure. Or the bodily temperatures may not be recorded, yet, if febrile, they modify the curves. Obliquities of ascending line and breadth of wave top, important features if of morbid and not of instrumental causation, may, for all that many records contain, have depended only upon the rate of the clockwork; but times and values of abscissæ are often omitted. Again, we are told of 'prolonged or sustained arterial tension ' on curves whose high or low waves had evidently varied with the temporary circumference of the vessel." These critical reflections, it is true, are less called for to-day; but, as many useless curves still find their way into print, they are not without some application, mutatis mutandis, in respect of them, and of the now more popular manometrical records.

The sphygmograph may give some notion of relative pressures if the conditions of application are identical; this however, as we have seen, we cannot ensure; and we are agreed that it cannot give absolute pressures.

Curves taken from widely different pressures often, in their main features, correspond; and such a maxim as that rapid rise and large excursion mean low pressures, and its converse, are always untrustworthy, often erroneous. The position of maximum oscillation lends itself best to the sphygmograph; now, if we take a tracing at this position and then screw down the pad to a higher pressure, we raise its latitude-its horizontal plane upon the paper, but we do not, within limits, alter the curve much in character. Or, if in skilful hands degrees of pressure may be inferred more or less approximately from secondary waves on the declivity of the curve, as by the position of the dicrotic curve for instance, yet again, by manipulation, these secondary notches on this limb can be varied in position. Dr. Pratt of Leicester ${ }^{1}$ has shown that a high or low pressure tracing can be obtained on the same artery, and during the same revolution of the paper, simply by varying the pressure

1 Pratt, R., Lancet, March 18, 1911. 
of the pad on the artery. Thus the dicrotic notch will rise or fall. ${ }^{1}$ The amplitude of the sphygmographic wave (Marey) is in no direct ratio either with the volume of blood in systole, or with the height of pressure in the artery under observation; the only ratio is to the magnitude of local vacillations of pressure, which are inversely as the tension of the arterial walls. Moreover Dr. T. Lewis ${ }^{2}$ has shown how, unless in each case special attention be directed to the conditions of instrumental adaptation, the diastolic or statical phase of the curve is liable, especially under low diastolic pressures, to suffer degrees of defalcation and suppression, or on the other hand of exaggeration, far beyond what was generally suspected. Curves taken before and after venesection, or before and after the administration of $\mathrm{m}$. 2 of amyl nitrite, well illustrate this lability and the delicacy of its conditions. Dr. Lewis finds, by the way, that although the venæ comites affect a tracing only when the venous pressures are excessively high, yet the venous fulness of the limb affects it by interference with the falling limb of the curve. Another of Lewis' many interesting observations is upon the effects on the curves of degrees of compression of the brachial artery, whereby, among other things, he thinks the important factor of blood velocity may be guessed at.

However, in the hands of James Mackenzie, Lewis, Starling, Bayliss, and others at home, and of Wenkebach, Engelmann, and others abroad, all of them expert observers well aware of the limitations as well as of the capacities of the instrument, the sphygmograph has been restored to the position it deserves as a means of research. Any one who will compare the sphygmographic curves of Dr. Mackenzie and of Dr. Lewis, not with those of Galabin, Roy, or Riegel, but with those of most other authors of twenty years ago, will perceive the difference between skilful handling and true comprehension of the capacities, incapacities and accidents of the instrument, and the lack of these accomplishments. The observer must not only himself be well accustomed to the work, but he should, if

1 These conditions were pointed out long ago by Roy in his admirable way; and recently they, or some of them, have been adequately and lucidly discussed again by Dr. Hawthorne, Studies on Clinical Med., 1912.

${ }^{2}$ Practitioner, Feb. 1907, and Journal of Anat. and Phys. vol. xli. 
possible, use always the same instrument; thus the variations in the wrists of the patients, which cannot be neutralised, may be half instinctively allowed for. No little adroitness is required to keep the instrument steady and exactly on the artery. We know how apt is even the sensitive finger to lose perfect adjustment; how the slightest side-slip or rocking alters our sense of the pulse, even, it may be, to the loss of an evasive beat.

But to proceed on the measurement of arterial pressures: I have said that in seeking to represent these pressures by the sphygmograph we were defeated; and as Ludwig's manometric methods were available only for opened arteries in animals, we were driven back upon the first, the readiest, and still least dispensable of pulse gauges, namely the finger, until, with the help of von Basch in 1876, another step forwards was made. By the finger much information of a qualitative kind is to be obtained, and such a work as that of Broadbent on the pulse brings home to us the accumulated values of this ancient and fruitful method. So true is this that a leader writer in the British Medical Journal has rebuked us for attempting to estimate arterial pressures by any other means. Thus, says the writer, "we pauperise our senses and weaken clinical acuity." So eminent a clinical observer as Professor Lindsay also has chidden us for the same vanity1:- "The finger," he has said, "is sufficient for all practical purposes in the estimation of blood pressure." But these and such protests have greeted the invention of every instrument of precision yet introduced into clinical medicine. I am old enough to remember the jokes of old-fashioned physicians at those faddists who thought to make up for lack of clinical acumen and experience by the pretension of the stethoscope; and a little later of the thermometer. Then likewise we were asked, "Why pauperise your senses and impair your own natural endowments by the use of mechanical short cuts to diagnosis?" The truth however is that instruments of precision, so far from pauperising our clinical perceptions, of fever let us say, have on the contrary enriched, enlarged, and, corrected them. Let me rather recall the wise foresight of Leech of Manchester who said that " changes in arterial tension are not often so indicated by clinical phenomena that their extent and direction can be

1 Lindsay, Brit. Med. Journ., July 1, 1905. 
measured without graphic methods." Gibson said that "a long training of his finger beside the sphygmometer had taught him how fallacious the finger must always be." Krehl, whose judgment on such a matter will not be questioned, declares that in palpation of the pulse for pressure the best observers are often deceived. Dr. Byrom Bramwell, while justly vindicating the function of the finger, points out where it fails us. ${ }^{1}$ Dr. Batty Shaw ${ }^{2}$ says : "Continuously maintained high blood pressure has not received the clinical attention it deserves, and simply because our methods of measuring it have been quite inadequate and untrustworthy.... Mere digital examination of the radial pulse will fail again and again to show that the blood pressure is raised." Cook and Briggs, of Johns Hopkins, write that all physicians who have practised sphygmometry admit the deceptiveness of the finger; that "in many conditions of the circulation the finger is the most deceptive and grossly inaccurate of all sphygmometers." In illustration of his argument, Dr. Cook published the following case: A woman, the subject of cancer, was under an anæsthetic for operation when symptoms of "shock" set in, and for this reason the operation was then abandoned. Dr. Cook had been observing the blood pressure throughout, and he noted that as the "shock" came on the blood pressure jumped up to 160 , and the heart dilated; but still the blood pressure rose, to $180 \mathrm{~mm} . \mathrm{Hg}$, and so continued while the cardiac impulse was diffused and widely apparent beyond the nipple. He asked himself indeed, whether the administration of sodium nitrite might not paradoxically have been beneficial, and enabled the operator to proceed? As compared with a pressure gauge, he declared that in such cases the finger on the pulse receives but a tardy and uncertain warning. It was but the other day that a big high-coloured man of 57, a large eater, consulted me "for thick acid urine," "gouty dyspepsia," "oppression round the heart after meals," and so forth. The heart was not obviously enlarged nor to the ear was the aortic second sound much accented, and the radial wall was soft. The pulse seemed too sustained, it is true, but I was astonished to find that I could not with any patience make the

1 Bramwell, B., “Address on Medicine," Lancet, July 29, 1911.

2 Shaw, B., Clin. Journ., March 27, 1907. 
systolic pressure much under 200. Now these cases of hyperpiesia, if caught early, are often quite curable, yet by the finger they are continually overlooked, or misjudged, till the time for cure is past. At the Toronto meeting of the British Medical Association (1906) Dr. M'Phedran said " he frequently found himself in error in his estimate of the pulse, when brought to the test of the sphygmometer" (italics mine). Dr. O.K. Williamson, ${ }^{1}$ and Hopke ${ }^{2}$ also, comparing the two radial pulses in certain cases of thoracic aneurysm, found that the instrument often revealed differences which the finger had failed to detect, and still failed to appreciate. Indeed in more than half his cases of this kind (32 in number) the digital examination in this respect proved useless, if not misleading. Dr. Mackenzie, in some remarks on pulsus alternans, ${ }^{3}$ says of an instance of it, "This variation was imperceptible to the finger," yet on his tracing it became apparent. Hensen reminded us that even in counting the pulse, if we control the record of the finger by that of the watch, we may have to make a large correction. I have met with no physician who has patiently compared his finger impressions with the indications of the sphygmometer, who has not confessed that his finger had much to learn and to unlearn; and that part of the lesson is the fallibility even of the most erudite touch, especially if not systolic pressure only but diastolic also be taken into consideration. For example, in my experience the high blood pressure still maintained in the later stages of a prolonged condition of hyperpiesia, a stage when the heart is relatively failing -Broadbent's "virtual tension"-perceptible as it may be to the "tactus eruditus," is rarely appreciated; yet by the manometer the systolic pressure may be proved to run in these phases as high as 170 or 180 . The same is true of the cases of mitral disease in which, as demonstrated by Dr. H. J. Starling and Dr. H. C. Mann, pressure, even in failing compensation, is often still high. And as a full artery with stiffish walls may give a false impression of high pressure, so in incipient hyperpiesia a substantial rise of pressure, say to 180, may lurk under an artery still soft and a heart still quiet. For further criticism

1 Williamson, O. K., Lancet, Nov. 30, 1907.

2 Hopke, see ref. p. $101 n$.

3 Mackenzie, Jas., Brit. Med. Journ., Oct. 20, 1906, p. 1007. 
of this kind I may refer to Sahli who, in his well-known Lehrbuch, explains clearly where and how the finger as a pressure gauge may fail us.

Now the reason of this fallacy of the finger is not difficult to perceive. The finger gives us no oncographic information, it is apt to take amplitude for pressure, especially in large slow pulses, whereas in high pressure the arterial excursions are less. It perceives the total stroke, not the pressure per square unit; yet a radial may be large and dilated under low pressure, or under very high pressure may be small ("feeble "). In cases of plethora vera (see Vaquez' disease, p. 256) the choke-full radial gives a sense of high pressure, usually false. Let the doubter put a finger upon the abdominal aorta of a thin person, or upon the iliac artery, then upon the radial, then upon the brachial, and he will find it hard to persuade himself that in all these vessels the pressures are practically the same. It has been stated by von Frey, Hensen, and others again and again that at times the pressure in the radial artery may reach the normal even while the finger can scarcely feel the vessel at all; and that in fevers an "atonic" and compressible pulse may simulate low pressures when in fact the pressures are of normal values. Dr. W. Russell remarks, of a case in which the radial artery felt very small, that the systolic record of 170 was "impossible"; the pulse seemed to him "small and feeble," for the finger, as I have said, goes much by the size of a vessel. It is true that the heart was dilated, but over and over again I have found pressures higher than this with dilated heart, as in the later stage of hyperpiesia (without contracted radial); pressures falling, no doubt, but not reduced to that normal abscissa which for so artificial a balance would be perilous. Accustomed as I have been to these comparisons, I find that I cannot be sure of degrees even of systolic pressure between 150 and 180 ; howbeit such differences may be of no little importance in diagnosis. When in ambiguous cases I test my finger by the instrument I consider it a good guess to come by the finger within 20 to $30 \mathrm{~mm}$. either way of the measured systolic pressure; and in any case at the more important diastolic pressure even guesses seem idle. I do my best to guess by keeping the fingers of one hand upon the radial artery, while with the other I manipulate the brachial, 
compressing and releasing it so as to get some notion of the rise, fall, and duration of the waves.

One of our M.D. candidates, having had occasion to note the pulses of five patients in dysentery in whom the radial arteries were very small, "was so struck with their feebleness" that, being a man trained on scientific method, he procured a sphygmometer, when to his amazement he discovered that the systolic pressures, in all five of them, ranged about the normal standard. The finger deceived him by this confusion of total pressures with unit pressures, as if one were to mistake the total steam pressure of a large boiler for its pressure per square inch-a pressure which, of course, may be equal in boilers of very unequal dimensions. To illustrate the opposite fallacy, such as pertains to enlargement of the artery, let us suppose that to a patient in pneumonia a dose of alcohol is administered, and that very soon afterwards the radial artery enlarges and more blood flows through this vessel ; now to the finger the artery thus enlarged may give the impression of a rise of pressure, or at any rate of no fall; although, in the essential and crucial respect of unit estimate, both pressure and velocity may have fallen considerably, as a diastolic reading would probably show. Thus the large relaxed leathery artery of elderly persons is frequently described as one of high pressure, whereas the pressure may be no more than is quasi-normal for the patient's age, and the diastolic pressure low; such a pulse may even be preliminary of asystole, as in an old man's influenza. Under chloroform, as Professor Cushing has demonstrated, fluctuations in arterial diameter may often be misleading to the finger, and fraught with guile for the unwary. $\mathrm{He}$ warns us therefore that during anæsthesia a blood pressure recorder, if not continuously in use, should always be at hand. In July 1902 I saw with Mr. Arthur Cooke in Cambridge a lady of middle age whose blood contained over ten million red corpuscles (p. 260). Her complexion was ruddy almost to a mahogany tint, and her radial artery full as if to bursting, vasodilatation being extreme. To both of us the arterial pressure on a finger estimate felt as if very high, yet it proved to be but 120 . How the state of the arterial wall may interfere with both oncometer measurement and digital estimation is an important point to which I shall vol. I 
return (p. 79). Now the radial wall varies in the same person, often within the limits of a consultation; it feels thicker at one time than another, and fingering the vessel may incite it to dilate (MacWilliam) (p. 75).

Let it be remembered that I am now considering blood pressures only; it is in this estimate only that the sphygmometer pretends to compete with the erudite finger. Even with instrumental aid we are as yet far from being enabled to analyse the factors of the circulation, a highly complex function of many indeterminate variables; of pressures rising, falling, and fluctuating in distribution; of phases varying with the many sources and contingencies of energy in the nervous, secretory, and other storing, directing, and controlling systems of the body. Moreover we have to contend with many defects of our instruments, none of which, unless it be one of the oncometric recording apparatus, is as yet in close and following touch with the vessel, or capable of recording constantly and definitely systolic and diastolic pressures. The finger then can tell us many things which the sphygmometer cannot tell ; so sensitive is it that in my own pulse often, and still better in certain abnormal pulses of the sick, the ordinary respiratory phases may be detected. By the finger again in accessible parts we can appreciate the state and the characters of the arterial walls; we can learn how far the repletion of the vessel is persistent or fluctuating, ${ }^{1}$ precious information not yet so well obtainable by the sphygmometer; the sphygmograph helps us better here: by the finger also we can get a fair notion of kinds and degrees of arrhythmia, although for their ultimate analysis complete graphic curves are necessary. It is to these analyses that the electrocardiograph also is bringing its aids. Besides, as Professor Sahli says, we have little "pressure memory"; we cannot carry a standard in our finger tips, especially not for diastolic pressure. Unfortunately, instruments to record total pressure cycles are too ponderous for ordinary uses; yet without them we can hardly hope to analyse and to solve the many urgent problems of the circulation itself, and of the conditions which modify and control it.

It is remarkable that the champions of "finger only" do not

1 Dr. Leonard Hill thinks that in cases of venous retardation full venæ comites may mislead the finger a little in this observation. 
seem to have " educated" themselves in the use of their natural instrument within its own sphere so fully as the sphygmometrician has generally tried to do. Dr. Ewart, in more than one paper, ${ }^{1}$ has dwelt upon the information which the educated finger can obtain concerning several qualities of the pulse; how the finger can perceive rhythm and rate, and give some idea of form, more of form than of pressures. The radial artery, has for us some disadvantage of inconstancy; as a small and muscular vessel it is more subject to changes of diameter, limb temperatures, tissue demands, flow rate, etc.

How "tone" of the vascular system can be tested without mechanical aid we have considered already, as by comparing the pulse in different positions of the patient ${ }^{2}$ or of the limb. Not in aortic disease only, the radial pulse may be palpated carefully at heart level, then with the arm hanging down, and then again with the arm elevated to the vertical ; and the several pulse rates and qualities compared. When no mechanical gauge is at hand such methods are useful.

The pulse of high systolic pressure, a vehement leaping pulse (pulsus celer) not prolonged, a high pressure chiefly of cardiac origin, differs from the sustained pulse, of lower systolic height, which pertains rather to increased peripheral resistance. Thus, as I shall explain, a trustworthy record of diastolic pressures is of more importance to us than the systolic with which we have usually to be content. I have said that it is a practice with me, while keeping a finger on the radial, with the fingers of the other hand to compress the brachial gradually and repeatedly against the humerus, and thus roughly to guess at the duration and the degree of compression required to arrest and release the stream. But, not to carry this part of the argument too far, let it suffice to add that we need not only to satisfy ourselves as to the data of the pulse for the time being, but also, as in the case of clinical thermometry, to obtain records for comparison, week by week, day by day,

1 Ewart, Wm., Heart Studies and other Essays, 1894.

2 See pp. 23-25. Of the effects of posture, exercise, rest, meals, emotion, fatigue, respiration, etc., upon the normal pressure behaviour, Dr. Oliver, in his books on Blood Pressure, and Potain in La Pression artérielle, have written so well that I do but allude to some of these conditions as occasion requires. For methodical description I refer to those works. 
or even hour by hour, for our own register and for evidence to present to pupils or colleagues, or in published work; for such purposes, if only they can be compassed, records far more definite and permanent than subjective individual perceptions and memories of arterial pressures are required.

In so far as we fail to keep a hand and an eye upon everyday methods of clinical tact, to assert the uses in current practice of empirical qualitative modes of appreciating the subject matter of the medical art, and to uphold such expert impressions against imperfect methods which have more of the seeming than of the reality of scientific precision, the protests and rebukes of which I have spoken have no little justification. For we admit that the instrumental methods at our service are as yet in themselves imperfect and, even so far as they go, imperfectly interpreted. Nevertheless, let us beware lest once more, in the face of history, we set ourselves against a main principle of all scientific progress; that is to say, against methods of quantitative appreciation, in defect of which no substantial foundations of knowledge have ever been permanently laid. The great advances of modern physiology have gone hand in hand with the submission of its operations to measurement. Research has its pedantries no doubt, but so long as clinical medicine is contented to drift, under the empirical wisdom of adepts, without compass, without the information and control of exact measurements, so long will medicine continue to be a hit and miss kind of calling. In the clinical sphere the progress of sphygmometry is slow, and for some time to come must be tentative; but no little has been achieved, and the rate of progress is quickening.

The Influence of Emotion on Instrumental Records.-The influence of emotion, or even of keen attention, on the arterial systolic pressures has been demonstrated by Dr. Martin, by myself, and many others. I insert a curve, one of many such, taken from the arm of one of our laboratory assistants, a cool person well accustomed to us, to our ways, and to our instruments. The cuff was put on before he reclined on the couch, the record began just as he had taken his place. By the timeline it will seem that although his pulse rate had settled down practically to the minimum in ten minutes, yet his blood pressure 
(systolic maxima) was still falling, and did not reach its minimum till after the lapse of about fifty-five minutes. It then remained perfectly level for the following twenty-five minutes, when the experiment ended. Gibson found that, after a clinical demonstration, his own systolic blood pressure, if he recorded it immediately after, had risen some $35-40 \mathrm{~mm}$. above the ordinary level. This interference is often embarrassing in single consultations, when a pressure excess may be either temporary or, as in early granular kidney, persistent. However systolic rises due to nervous excitement can usually with a little pains be discerned. For instance, these emotional augmentations, as in Klemperer's will impulses (p. 238), are commonly systolic only, the diastolic pressure is more steady; by the pulse rate and rhythm

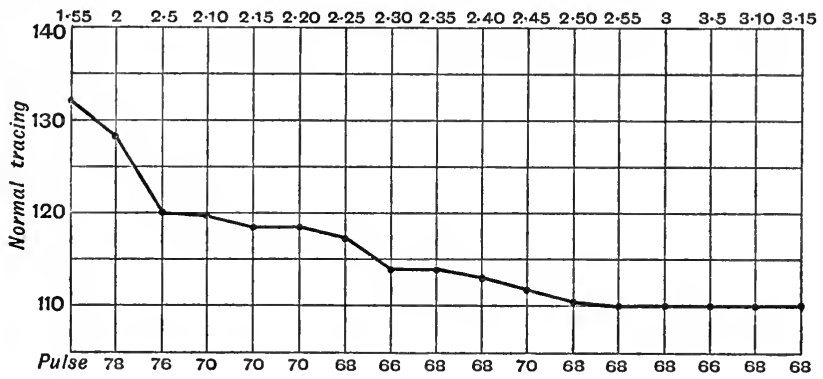

again one may form some opinion as to the degree of nervous excitement or attention. The pressure usually runs higher at the first than on subsequent sittings, and at the beginning than at the end of a consoling visit. In some persons the cardiac and vasomotor centres seem to be very labile. Women are on the whole more susceptible to these fluctuations, but ill-health and apprehension leave none of us unmoved; so that the quicker and simpler methods may be better, even if a little less accurate. Kymographic methods are only for the habituated. Some patients are disturbed by the tightening of the cuff; if so, the finger and the stethoscope must suffice. The pressure gauge should not be brought out till near the conclusion of the interview, after some quiet explanation, when the patient will be told to expect the grip of the cuff ; if then the full pressure be brief, and the colour of the hand watched, generally speaking there will 
be little nervousness or discomfort. The application in winter of a chilly rubber cuff to the arm of a sensitive patient, of either sex, should be avoided; a warm cuff may be applied over thin soft underclothing without mechanical disadvantage. In watching the instrument or the dial the patient is apt to hold his breath, and thus to disturb the record. Tell the patient, both as regards mental and muscular tension, to be as easy as possible, to turn his eyes elsewhere, and to try to regard the matter as indifferent. Still, after all precautions, in an average person calm is not reached for some ten or fifteen minutes of rest. It is better not to repeat the test during the same sitting; the discomposure may be increased, the muscles may tighten, and venous congestion may be more. In abnormal states, say in high pressure cases, rest in bed alone will often reduce pressures by $20-40 \mathrm{~mm}$., so that in such cases the record should, if possible, be deferred.

The "Psycho-physical" effects of emotion upon the vasomotor system, and so upon the blood pressure, offer a curious sphere of study. ${ }^{1}$ These and such researches have been generally on plethysmographic methods, so as to obtain comparative records in areas. This method shows how sensitive are these curves to psychical impressions, so much so that only continually uniform curves can be trusted. Neurasthenics are peculiarly sensitive to these impressions ; in such patients, after all preliminary precautions, even to start the tape may arouse reaction, so that before accepting the record a short run must be given away. Still such a liability may be noted as a subordinate sign of the malady. It is said by house physicians that all pressure records rule higher on days of leaving the hospital. As a voluntary movement of one arm will send up pressure in the other, so any habit spasm, such as a facial twitch, a torticollis, or the like, may vitiate the curve. Although these fluctuations are larger in nervous subjects, in unknown persons it is difficult to tell beforehand which amongst them may prove thus susceptible. On one such patient Schrumpf noted that apprehension of the diagnosis drove up the pressure, which after a quiet and reassuring chat fell on a second and a third observation by

1 See Weber's and Klemperer's well-known studies; Citron, Verein f. inn. Med., Berlin, July 17, 1911 ; Schrumpf of Strassburg, Deutsche med. Wochenschr., Dec. 22, 1910 ; Zabel, Münch. med. Wochenschr., 1910, No. 44. 
33 per cent; then he recalled some of the most vexatious symptoms and worries, such as the sleeplessness, etc., when the pressure rose again by 25 per cent. I have had reason to believe that emotional enhancement of pressures may be greater when already ruling high. The recent paper by Stursberg and Schmidt ${ }^{1}$ on pressure curves in neurasthenia I hope to discuss in an essay on this malady. ${ }^{2}$ For, as I shall show later (Vol. II. p. 61), the diagnostic distinction between hyperpiesia and neurasthenia is often ignored. Fatigue curves likewise may thus be obtained for comparison.

Such interferences as these have been verified in persons only indisposed, as well as in those suffering from heart and arterial disease. Speaking generally, although these psychical factors influence systolic pressure chiefly, yet in arteriosclerosis this rule is not fully available, because in this change the diastolic pressure also may be somewhat augmented (Schrumpf). In the heat and cold tests on the skin it must be remembered that not only the local reflexes but cortical influence also-as pleasure or pain-may enter into the result.

What Klemperer did was to note the effect of concentrated attention, or of any annoyance from the manipulations and the like; he and Citron, O. Müller, and v. Romberg pursued these researches into the sphere of ideas, and accordingly of "suggestion," and they found the effect of cortical excitement upon these vasomotor reactions to be remarkable; by suggestion the pressure curves were reversible, the pressure falling instead of rising ; and conversely. Normal and abnormal reaction curves were thus obtained. The limits of my subject will not allow me to illustrate such mental fluctuations as reflected in the behaviour of the blood vessels, but in passing I may demur to too ready an acceptance of such fugitive phenomena. Psychical stimuli are indefinite in quality and quantity, and too much intermingled with other widespread reactions and influences to be standardised; presumably also the cortical outflow, on its way through the spina-sympathetic system, is liable to absorption resistance, or to shunt; and so likewise for the ingoing sensations.

1 Stursberg and Schmidt, Deutsche Arch. f. kl. Med. Bd. xc.

2 See new edition of my essay on Neurasthenia in Allbutt and Rolleston's (2nd edit.) System of Medicine. 
As regards the patient's attitude during the observation, the conditions should then be as uniform as possible. This in the large majority of instances is easy, as in the hospital or consultingroom the same furniture is at hand, and needs only some change of cushions for tall and short persons. In outside consultations the patient is usually in bed, and this position also is quite convenient. By these precautions, and by keeping as far as possible to the same instrument, some relative uniformity amid one's own results can be maintained. The foregoing considerations warn us that if blood pressures are to be taken with much pretence to scientific accuracy, they must be taken when the patient has been for an hour or two quietly recumbent.

As regards the hour of observation, as I have said (p. 21), we have but little accurate guidance on the normal daily curve of arterial pressures. Gibson, Dr. Oliver, and others have worked at the subject, but our methods are not yet delicate enough for the comparison of small variations. My observations in private practice have been made nearly always between the hours of ten and one o'clock.

In conclusion, we may be content to say that, on the whole, arterial pressure records taken with due precautions in patients at rest are fairly consistent, and uniform enough for practical purposes if not for close comparisons. For my own part for practical purposes I am satisfied to ascertain if a patient's systolic blood pressure be about normal, or about 150 , or about 175 , or about 200, or over; and of his diastolic pressure I get as good an impression as I can. Pressures over 200 usually, but by no means always, mean renal disease.

The Arterial Wall and the Pressure Gauge.-It will be obvious that if the walls of the vessels, widely variable as their states are, count for much in proportion to the dynamic elements of the circulation, our records must lose much or all of the fair uniformity on which we have presumed. The arterial expansion and resilience, which our instrumental curves express, depend in health upon two factors; upon what I may call piston pressure and vascular tone. If the system were inelastic, the curve of pressure would express itself directly as piston velocity ; indeed in an elastic system the curve is essentially dependent on it, but with interferences. Now the vascular walls, 
as we know, are not homogeneous, and therefore in indirect (external) manometry the effect of the wall upon the form of the curve of activity is an algebraic sum of the momentary attitudes of the several tunics. These factors Dr. Harry Campbell discussed lucidly two or three years ago. ${ }^{1}$ He argued that the muscular fibres bear the first stretch of the systole, then the elastic fibres come in. He may have laid too exclusive an emphasis upon this function of the media in resisting lateral pressures, as compared with its function in blood distribution, but my own impression is also that the elastica counts for little in this phase of resistance; in the language of physics, this membrane has little of the elastic property of resisting deformation, but is very resilient. Hence, near the main trunks where the pulsations are most felt and are taken up as potential, the elastica is large; and it thins out-thins out "even to selvage ends" - towards the periphery, where pulsation is almost spent. The adventitia, if in one sense more elastic, is far less distensible and resilient; it is chiefly a protective sheath, and the vehicle of vasa vasorum, lymph channels, and so forth. The tighter the tone of the media the more elastic and the less extensile it is ; the less elastic and the more extensile the looser its tone. Thus the more the relaxation of this coat-to promote, let us say, a rapid afflux of blood inactive work as into the blacksmith's arm-theless is strain of the vessel resisted; the safeguard of tonus is reduced, and ultimately the unresisting vessel becomes impaired. In the passive artery of course the distensibility is most at first; but as the vessel contracts the first yielding to increments of pressure becomes more gradual, and before the adventitia has to carry much of the stress, or the elastica is put fully on the stretch, the central pressure must be very high. Dr. Campbell says that the maximum distensibility lies about $100 \mathrm{~mm}$., which, if so, is at a point a little higher than the maximum oscillation, after which limit it diminishes. How far this stronger contraction of the main opponent to pressure from within is reflected in a corresponding resistance to instrumental compression from without is now to occupy our attention.

What are the effects then upon manometry of the tone, thickness, and condensation of the arterial wall itself? Von Basch

1 Campbell, H., Lancet, Jan. 1, and subseq. 1910. 
satisfied himself that in the readings of the manometer these variable factors of the wall lay within limits so small-about $5 \mathrm{~mm}$. $\mathrm{Hg}$ for a sclerosed artery-as to be negligible. Dr. C. J. Martin came to the same conclusion; namely, that the wall of a thickened artery might interfere by some $7 \mathrm{~mm}$. $\mathrm{Hg}$. Dr. Oliver and Dr. Janeway have held the same opinion, and general experience, by. the fair uniformity of records, has seemed to confirm these conclusions. Professor MacWilliam, with certain reserves to be mentioned presently, still thinks that the factor of the wall is inconsiderable, if not negligible. Ordinary tone, in the brachial artery for instance, seems to me to offer no appreciable resistance to compression. Still I admit that in the face of great eccentricities in pressure records some such interference may well be suspected, and in 1907 Dr. William Russell raised anew the important question of the factor of the arterial wall, and declared it, as demonstrated by a certain scheme which he exhibited, to be not infrequently a copious source of fallacy.

As to the form of the pulse, it has been shown by many observers, by Professor Strauss of Berlin for example, ${ }^{1}$ that pulse form has no direct dependence on blood pressure, that wall elasticity and pulse rate count for more (p. 59). As I have said, by accelerating the clock rate, the tracing from a sclerotic artery may be approximated in form to that from a normal vessel. I may repeat also that Sanderson and Mahomed and I found that plus pressure seemed not of itself to flatten the curve, but sclerosis combined with a certain rate of outflow, and that Dr. Lewis has lately reinforced this principle, and shown the lack of direct relation, in form of curve, between arteriosclerosis and blood pressure. I have alluded also to the question of the compression itself, whether it may set up dilatation, or contraction, of the subject vessel? Herringham and Womack suggested, what MacWilliam has since proved, that on many recompressions the artery may relax, the heart's energy being constant. We are told by Luciani and other physiologists that in internal sphygmometry the insertion of the cannula often causes a relaxation of the artery, and of its principal branches; and furthermore that to compress a large artery in one area may cause

1 See also Roy's paper, Journ. of Physiol., 1879-80, vol. ii. 
a compensatory rise of velocity in a corresponding area; for instance as between the two carotids. It is said that on the ordinary cuff compression of one brachial artery its fellow dilates, and that the afflux may be demonstrated by the plethysmograph. Sahli says that the pressure stimulates to dilatation, and with his sphygmobolometer he reckons in this with other factors; others say this stimulation sets up contraction. And I emphasise MacWilliam's demonstrations of arterial dilatation under repeated recompression ; ${ }^{1}$ but it cannot be said that arteries dilate immediately and regularly under fall of pressure and contract on a rise. For my part I am not convinced that, the aorta excepted, direct local arterial reflexes, or local vascular reactions to pressure variations, such as to affect instrumental records of form or pressure, exist in all or any parts of the arterial tree, unless it be under some vagus influence. ${ }^{2}$ The histologists have not yet told us much concerning nerve end-organs in the arteries. I shall quote some evidence (Angina Pectoris) that they exist in the first part of the aorta, which I have called the " pressure-tambour," and probably elsewhere in the tree $;^{3}$ and it is stated on good authority (Abrams) that by suitable stimulation of the skin of the upper chest contraction of the aorta can be demonstrated.

Comparative Pressures in Arm and Leg.-Dr. O. K. Williamson has recently reminded us of the opinion that under equal conditions - equality of gravitation and otherwise-the systolic blood pressure is higher, or, to speak more strictly, the register of the pressure gauge runs higher, in the leg than in the arm; or that, if in the normal man the pressures in arm and leg are, or may be, identical, yet in high-pressure cases the systolic readings from the leg are, or may be, very considerably higher than those in the arm. ${ }^{4}$ This statement, made first, I think, by Volkmann ${ }^{5}$ and for a while

1 MacWilliam and Kesson, Heart, vol. iv., No. 3, 1913, who tested arteries under all kinds of controls. The article appeared after this book had gone to press. I have inserted what I could.

2 See Kaufmann, Pflügers Arch. Bd. cxlvi. and cxlvii.

3 See my Essay on Angina. Professor Mines has drawn my attention to a clearer affirmative on this matter in the 1912 edition (vol. ii. p. 1) of Schäfer's Quain, on p. 351.

4 Williamson, O. K., discussion, Royal Society of Medicine, April 27, 1909 ; Rolleston, Heart, vol. iv.; Hill and Flack, Heart, vol. i.

5 Volkmann, Hämodynamik, Leipzig, 1850. 
adopted by Hürthle ${ }^{1}$ and others, who compared crural with carotid, seems, as regards the blood pressure itself, to be improbable, as friction does not tell much till the stress reaches the smaller channels. If the observations were correct, their interpretation might depend upon contingent factors ; von Kries attributed the difference to a wave of recoil ; or, as Hürthle said later, ${ }^{2}$ the observations may have been ambiguous or erroneous, as in the use of the same breadth of cuff for arm and leg. Before pronouncing on these difficult physical problems very great caution and many observations are needed. Van Bogaert of Antwerp has made about 800 comparisons of arm and leg, experimenting with various breadths of cuff in each limb; also on persons in various states of health, in all positions, and with due regard to meals and other habitual conditions; he concluded that in arm and leg the pressures, systolic and diastolic, were practically equal. $\mathrm{He}$ used both the R.-R. and the Pachon instruments. In external manometry it must be very difficult to distinguish between blood pressures, degrees of arterial constriction, thick walls-as of the vessels of the legs which have to resist gravitation, and tone of surrounding muscles. My impression is that the tone and volume of the voluntary muscles goes for much; Ludwig Frank, ${ }^{3}$ who tested the brachial pressures in a palsied and flaccid arm against the other (normal) arm, and repeatedly found the reading on the palsied side lower, came to the same conclusion. Herein lies one objection to the use of the forearm for manometry, that the unequal resistance of the two bones, and of the fascia, likewise prevent a quasi-fluid behaviour of the circumambient parts.

Volkmann found in the leg more than a systolic difference; that in various arteries the pulse amplitude varied for the same heart beat, and was much larger ("erheblich grösser") in the crural than in the carotid artery. Dawson, however (loc. cit.) found diastolic pressures equal in all the larger arterial branches. These local fluctuations Hasebroek takes as favourable to his view of a peristaltic forwarding of the blood by the muscular arteries (p. 51). But in these arguments insufficient atten-

1 See Hürthle, Pflügers Arch. vol. xlvii.; but also cxlvii., 1912, p. 593.

2 Hürthle in Pflüger, vol. cx., 1905.

${ }^{3}$ Frank, L., Zeitschr. f. exp. Path. und Ther. Bd. ix. 
tion is paid to the distinction between fluid pressure within the artery and propagation of a wave from the heart's shock along its walls ( $p .33$ ), a propagation the more active as the vascular wall is more rigid, whether because of its denser structure or of a tenser state of it under a high blood pressure. The femoral artery, which has to resist gravity, is a stiff vessel ; so that we should expect it to transmit a quicker and higher wave. But, as I said in a discussion in 1913 at the Royal Society, although a stiffer walled artery would propagate a quicker wave, yet a thinner (elastic) artery, when distended fully by a high blood pressure, might have a similar conductivity. ${ }^{1}$ However, we shall see presently that these differences between one limb and another depend, in part at least, upon degrees of arterial tone, of constriction; and that by frequent (20 to 30 ) recompressions (MacWilliam) the higher record can often, if not always, be brought level with the lower.

If, as occasionally reported, compression of one considerable artery raises the pressure in its fellow of the other side, or in another limb, we must suppose that the heart in the interval has put out more work, or that the circulatory mechanism is out of order.

But let us now come to the examination of Dr. William Russell's reassertion of the inconstant factor of the arterial wall in external measurements of blood pressures. In his first demonstration a schema fitted with indiarubber tubing was exhibited which did not seem to me in pari materia; in any case, its records were not of unit but of total pressures. No scheme with rubber tubes could be regarded as more than tentative and suggestive. Dr. Herringham and I sketched out another testing gauge, on oncometrical principles, in which lengths of arteries might be used. With the help of Mr. Womack, Dr. Herringham constructed a gauge, and made many experiments upon portions

1 It was after this manuscript had gone to press that Wells and Hill read the paper at the Royal Society to which I have alluded. They proved, what I surmised, that by immersing a limb (arm or leg), on Klemperer's method, in hot water and thus relaxing the arteries, marked alterations of pressure records can be produced. Also the interference of vasomotor tides and of output make experiment difficult. Klemperer by the cold and warm water test produced differences of the two sides ranging between a rise of 12 and a fall of $6 \mathrm{~cm}$. of water. See also Hill and Rowland, Heart, vol. iii. No. 2 ; and Rolleston, Heart, vol. iv. p. 82, 1912, who are also unconvinced of the differences. 
of vessels in various states, and obtained amazingly inconsistent results. ${ }^{1}$ In clinical work I had never paid much attention to differences under 10-15 mm., I regarded our methods as too rough for differences so fine; but Dr. Herringham exhibited irregularities of $20-30 \mathrm{~mm}$. Indeed he agreed with Dr. Williamson that in cases of pressures of $200 \mathrm{~mm}$. and upwards the leg pressure (in recumbency) might exceed those of arm by $30-40 \mathrm{~mm}$. In one high pressure case, observed both by himself and Dr. Williamson, there was a difference between the right arm and leg of 72 mm. ; between the left limbs of $36 \mathrm{~mm}$.! After death, portions from the respective arteries were tested in the oncometer, when $60 \mathrm{~mm}$. difference was found between artery from the right arm and the leg, and a considerable but smaller difference between segments from the left limbs. These results were bewildering, so inordinate as to suggest grave fallacies somewhere. In other cases the differences were not so extreme; namely, 8, 14, $5,4,6,12,15,22 \mathrm{~mm}$., vagaries which in my clinical custom I have been used to regard not indeed as negligible, but as, with our instruments, more or less inevitable.

My own observations, on high pressure and decrescent cases respectively, had satisfied me that the opinion of von Basch, Martin, Janeway, and many others, that atherosclerosis of the walls of the arteries altered the pressure records so little (say 5-10 $\mathrm{mm}$.) as to be negligible was justified. Time after time with my mind on this problem I had measured the pressure in such arteries at ordinary systemic pressures, both with the R.-R. and other gauges, and had obtained fairly consistent records. I have, I admit, tested the leg rarely. It is difficult in ordinary practice to allow for the tone of the strong muscles of the limb, a factor which may not have been wholly got rid of by other observers. I anticipate a high or irregular record even from the arm of a strong man, whose muscles do not offer a hydrostatic equality of resistance. Any tendency to contraction of the muscles must be closely watched, as the readings may thus be driven up by $25 \mathrm{~mm}$. and more. It is true, as Hensen says, ${ }^{2}$ and as von Frey had said before, that the mere

1 Herringham and Womack, Proc. Roy. Soc. Med., May 1909.

2 Hensen, "Beiträge z. Physiol. und Path. d. Blutdrucks," Deutsche Arch. f. kl. Med., 1900, p. 436. 
thickness of muscle is no matter, or but little; not so the tone however, which is apt to vary even during an experiment.

Professor L. Hill, at the Royal Society discussion and afterwards, ${ }^{1}$ made some important criticisms upon these erratic results, results which need much sifting. He asked how it was, if the arterial coats were so far concerned, that in arteriosclerosis the measurements in both legs were often identical, for we could scarcely suppose the disease in the two femorals to be equal ? In their oncometrical gauge Herringham and Womack tested other specimens of excised arteries, still with eccentric results, partly I think because the many precautions on which MacWilliam has insisted were not always observed; and the same criticism applies to some at least of Dr. Russell's experiments with dead, half-dead, or surviving, sections of arteries. Again, the diameter of a vessel and the limits of its spasm must not be forgotten ; we cannot reckon, e.g., from the radial to the brachial. However, it is fair to add that Dr. Russell lays no great stress upon his experiments with portions of arteries, and regards them all as fallible. His best evidence is derived from the comparisons of pressures before and after the exhibition of nitrites, when he found ${ }^{2}$ that resistance to the compression of the gauge rose as the wall contracted, and reversely.

I think we must agree with Dr. Russell that, generally speaking, contraction of a muscular artery may raise the resistance to the gauge ; it seems likely that a contracted vessel would oppose external pressures as it opposes the internal. Dr. Williamson, and Drs. Janeway and Park are also of opinion that it is spasm rather than sclerosis which contributes to high resistance $;^{3}$ and that morbid thickenings and stiffenings, or defects of elasticity, go for very little. In this distinction the trained finger should give us much assistance.

However, as these problems cannot be solved without more direct experiment, wealsoin our own laboratories have endeavoured to make some observations on excised arteries ; and we believe with all of MacWilliam's conditions satisfied ; ${ }^{4}$ that is, as to the

1 Hill and Flack, Brit. Med. Journ., Jan. 30, 1909.

2 Russell, Brit. Med. Journ., 1908, ii. p. 1076.

3 Williamson, O. K., Lancet, May 25, p. 1409.

4 As published in many papers, e.g. Royal Society, Nov. 21, 1901 ; and MacWilliam and Mackie, Brit. Med. Journ., Nov. 14, 1908, p. 1477. His 
time after death, the kind of disease of which the owner died, the mode of preparation, and so forth. Of these experiments I will refer to two series for M.D. theses : the first by Dr. Stevenson, in Professor Dixon's laboratory, the second by Dr. Cow, now a Beit student, in the same laboratory. Dr. Stevenson's researches, ${ }^{1}$ by methods similar to those of Herringham and Womack, showed with arteries of the muscular type that von Basch, Janeway, and others were right in saying that in arteriosclerosis the state of the wall was practically negligible; but they agreed with Dr. Russell's in that constriction, on the contrary, had a considerable effect in resisting the compression; and this more and more as pressures rose from $17 \cdot 8$ to 22 per cent. The contractions were excited by running chloroform or barium solution through the sections. Of course many controls were tested, side by side. Stevenson found that for every 10 millimetres of internal pressure the resistance rose on constriction about 1.3. Such were the figures; but he felt unable on the whole to decide more than this-that resistance of the arterial wall counts for more at high than at low pressures. He found, moreover, that this inconstant factor of constriction tells more in arteries like the brachial, to which usually we apply our tests-one in which the wall bears a large proportion to the channel-than, e.g., in the carotid or subclavian, in which the channel is relatively larger. He did not note signs of dilatation of the arteries, excised or in life, under recompressions, but in many an artery noted by the finger as thick and hard the record proved to be low, while others taken by the touch to be normal offered high resistances. In four of Stevenson's sclerotic specimens (brachial) contraction was still good, though three were taken from subjects over 50 years of age. Dr. Harry Campbell pertinently asks why it is assumed that the finger can discern between resistance of coats and resistance of high pressure if the gauge cannot? Does not the

important paper of 1913, quoted in a previous note, appeared too late to inform these pages. Fortunately, he concludes that on the whole, and in the vast majority of cases, the clinical readings are consistent, but liable to err if constriction of the artery under observation sets in. Then recompressions should be used to relax the vessel. Keeping up a continuous pressure may, he thinks, raise the obliteration record.

1 Thesis for M.D., 1910. Paper read again, Pathol. Soc. Great Britain and Ireland, 1910. 
interference of the wall, if it vitiates observation in the one way, vitiate it no less in the other? Dr. Cow ${ }^{1}$ took arteries from animals (sheep and oxen) and from amputations, and, over and above MacWilliam's instructions, he kept them at a uniform temperature, and suspended them in oxydised saline. As in the previous experiments, portions of approximately equal magnitudes but from several areas were compared. The least possible manipulations were used throughout, and these were made as equal as possible for all the specimens. The drum revolved $1 \mathrm{~cm}$. in $42 \mathrm{sec}$. After excluding the cases of known or possible fallacy, 201 experiments remained for comparison. Cow showed that portions excised from peripheral and visceral branches respectively often acted differently, and even contrariwise : constriction in the one might correspond to dilatation in the other. This observation may reconcile many contradictory records of such experiments under pituitin, adrenin, and the like; that constriction in certain peripheral areas is, or may be, accompanied by dilatation in certain visceral areas is clearly known; but some of these contrasts may depend on the degree in which a weaker artery can withstand a rise of systemic pressure?

I think the enormous resistances of wall reckoned by Dr. Russell from experiments with excised arteries, e.g. 70-150 mm., must be erroneous; for what range does it leave for pressures during life? MacWilliam's rules are not mentioned, ${ }^{2}$ and the use of formalin seems, as I have said, to introduce another variable; but experiments on vessels tested without preservatives immediately after death, as by Janeway and Park, ${ }^{3}$ are often vitiated by the inconstancies of survival and quasi-vital reactions. Their careful research indicates however that in youth wall resistance is practically nil; in later life, even in atheroma, it may reach $10 \mathrm{~mm}$., or, if calcified, $15 \mathrm{~mm}$. at most; but this is in the mean position: they admit with Russell, Herringham, and others that by spasm the wall resistance may be raised, even by $30 \mathrm{~mm}$.

1 M.D. Thesis in 1910 .

2 For other possible fallacies of these experiments see L. Hill, Lancet, March 27, 1909.

3 Janeway and Park, Arch. int. Med., Nov. 1910.

VOL. I 
We are told that sclerosis must increase the resistance of the arterial wall; this may or may not be true, so far: but atherosclerosis is unequally distributed, and hydrostatic pressure applied to such an artery would close it not at or about tracts of higher resistance but at the weakest spots, spots of relaxation or decay. It does not seem to be realised that compression of an atherous artery tells, and gives records, not on the strongest but on the weakest cross sections under the cuff.

Whatever the issue of these perplexities, we owe a debt to Dr. Russell for constraining us to revise our data as regards the factor of wall ; for the present my own opinion, and I believe his opinion also, is that where the wall resistance is considerable the resistance is due not to sclerosis but to degrees of constriction; a view of the matter with which Dr. Oliver's coincides. ${ }^{1}$ But constriction, not reinforced by a rise in general pressure, makes for a distal area of low pressure ; yet it may account for the stories of local high pressures (p. 30), or for the most of them. Unfortunately the difficulty of appreciating diastolic pressures, and the scantiness of diastolic records, keep a large part of this argument in obscurity.

The few observations on blood pressure on the central side of amputations, some of them on the thicker vessels of the leg, do not point to considerable differences between external and internal gauging (p. 91). However Uskoff, comparing the records of gauges external and internal to the artery, found the results conflicting, so much so in his opinion as to throw much doubt upon estimates of pressure, whether systolic or diastolic; but the diastolic results were nearer to uniformity.

As in clinical work we all use the brachial artery, and ought to continue to do so in order that the accumulation of data may be so far uniform, and because this vessel is rather less subject than radial to vasomotor fluctuations (see also p. 76), we may leave the resistance of the walls of other vessels to the physiologists. Hensen calculated that the wall of the brachial offers (ccet. par.), only $5 \mathrm{~mm}$. more resistance than that of the radial. ${ }^{2}$ And we must remember that each region of the body has its own build of

1 Oliver, G., Clin. Journ., Sept. 16, 1908.

2 See also Masing (in Dehio's Laboratory), “ Blutdr. d. jungen u. d. bejährten Menschen," Deutsche Arch. f. kl. Med., 1902, vol. lxxiv. p. 254. 
artery; those of the skin are very different from those of-let us say-the kidney, and those of the liver from either. The cerebral arteries contain less contractile tissue; those of the neck, which are exposed to much torsion and pressure, have much thicker coats, a protection which they lose immediately on entering the skull, wherein the vessels are so uniformly supported, as if in a fluid, as not to need it. The wall at the takeoff of the middle cerebral is but $\frac{1}{7}$ th of the vessel in the neck; it consists of a thick intima and inner elastica with but a thin muscular and adventitious coat. The frequent rupture of cerebral vessels, however, indicates that the quasi-hydrostatic support of the encephalic substance is not so serviceable as a strong adventitia with its external elastica. The coronary arteries being, like the iliac, seated upon an organ or organs in incessant motion, are accordingly, like the dorsalis pedis also, protected by thick tunics. But even such arteries as these likewise when they enter into a viscus lose their sheathing; though they very rarely rupture.

Well, the position is then for the present somewhat awkward; are these criticisms justifiable? Have we been heaping up delusive records? That the brachial artery, if more stable than the radial, is liable nevertheless to constriction, every one who has worked with the sphygmometer is aware; for the present we must be content to admit that in states of constriction, even such, for instance, as may arise under the excitement of examination, external measurements may be aberrant. The grip of the cuff is itself, of course, very artificial and not in nature. A compression, or recompressions, may cause a dilatation of the artery, ${ }^{1}$ or again a fall of diastolic pressure in its district, as we see in the distal effects of an embolism. The calibre of an artery is a function of its own muscular contraction and the pressure of blood within; in the aorta and larger vessels the latter coefficient prevails, in the muscular arteries the former. We cannot, I think, measure the calibre; at any rate I never attained any skill with Oliver's gauge; moreover the calibre may vary much-probably it does. The temperature of the room and of the patient must be borne in mind, especially with

${ }^{1}$ Since these words were written MacWilliam, in his last paper, has proved this to be true, if the recompressions be frequent and repeated. 
instruments of the Gärtner kind. And in an early case other evidences of high pressure may be equivocal; the heart may not yet have increased notably in bulk, while audible signs of aortic stress are but impressions, and vary much in individuals-between man and woman for instance, or the nearness of the great vessels to the chest wall. On the undulation of the arterial wall as contrasted with stream pressure I have already insisted-that a stiff vessel wall propagates a wave more quickly than a slack one; and doubtless we often presume we have arrested the current when we have only damped the excursions of the wall (p. 33). As Dr. Janeway says, we must be careful to record, not the obliterating pressure only, which in vasomotor constriction he agrees may be fallacious, but the point of return after obliteration. But all this of course concerns systolic pressure only, a partial record.

The differential arterial and venous pressures in the arm (L. Hill, Moritz, Tabora), an interesting enquiry, have not yet been sufficiently investigated.

Pressure Recorders.-After some hesitation I have determined to discard the bulk of my manuscript on these instruments. I am only too well aware that in so doing I am throwing doubt upon the methods of my own experience, and the sources of my own opinions; still, as my familiarity with these methods has increased, so far from attaining a better assurance as to their capacities I have found but a growing mistrust. For many years past indeed I have discountenanced precise records of pressure in man, as giving a false appearance of accuracy; when in my own notes I enter the precise figures shown on the gauge I regard them but as rough approximations, or short memoranda.

Although not without some bent to mechanics and ready to try all external manometric instruments as they appeared on the market, yet I have never had the leisure carefully to test and compare them, or to improve them. I began my clinical observations with the instruments of von Basch $^{1}$ and Potain; these, before reading Tigerstedt's conclusive demonstration of their incapacities, I had discarded on the production of Hill and

1 The first of these instruments, introduced in 1887. A full account of the literature of the subject will be found by O. Müller, Ergebnisse d. inn. Med., 1908. 
Barnard's arm apparatus, constructed by them in the winter of 1896-97, and exhibited in action about that date at a Cambridge meeting of the Physiological Society. This instrument I used for many years, after altering the cuff to the larger dimension subsequently found necessary. There is no definite rule for the width, the brachial cuff of the man may be used for the thigh of a child; but we must remember, as regards the artery, that velocity depends not on the diameter only but also on the length of a tube, on axial as well as on radial length. I have had little trouble with Hill's larger gauge, none in fact but the need of a comparison and readjustment from time to time with a mercurial column, and sometimes a refitting by the makers. I think I was fortunate in my own instrument, as, generally speaking, aneroids are very liable to fall out of value. And like all external manometers, it is apt to give too high a record for systolic pressures. The dial is convenient, and though the phase of large oscillation is inconveniently extensive, and there is some needle inertia, its suggestions of diastolic pressure do tolerably well for an observer accustomed to it. A little later we were all captured by the vogue of the Riva-Rocci instruments-as in Martin's adaptation and other forms - and for a while I used one of these ; but I soon became dissatisfied with it, especially in regard to what I wanted most, namely the diastolic pressures. For diastolic pressures it is almost absurd. The inertia of a mercurial column is conspicuous, I had nearly said blatant; by the unaided eye one may see its overriding waves, which cannot be eliminated or subtracted $;^{1}$ and this inertia is disturbed by variation in pulse rate. Then into our hands came Oliver's later gauge, the drive of a light short index against a column of air in a glass tube closed at the farther end. This gauge I have used frequently; it is very convenient for the eye, and it seems to me accurate enough for ordinary use; but it will not bear carrying about. Its indications of diastolic pressures, if not so perceptible as in the oscillometer of Pachon (of which I shall speak presently), of von Recklinghausen, or even of Hill and Barnard, are better than by the mercurial column; if they do not give the precise moment of minimum oscillation, which a graphic method only can give, they are more free from inertia inter-

1 Sahli therefore prefers a fine tube with a petroleum column. 
ferences. It is the inertia and backlash, not of columns or needles only but of all the parts of these instruments, which tend to vitiate their indications: so that each instrument has its "personal equation." From a strictly scientific standpoint external methods seem, I fear, all radically inadequate; though we shall learn better and better how to allow for their defects. Without section of an artery no means has been discovered of giving us accurate pressure records all through the curve; the intervening conditions bring in too many sources of fallacy. We must be content with approximations.

The chief difficulty is to get an approximate reckoning of diastolic pressures, for these are the most important. It is the minimum pressure which shows how the blood is getting away. Moreover, resistance being equal, a fall of diastolic pressure is probably the first sign of heart failure. It is said that a fall in both diastolic and systolic pressures is an early sign in tuberculosis; but low pressures are outside my subject. We have seen that systolic pressures, especially in high-pressure cases, vary greatly, obeying many incidents, internal and external; the diastolic are more stable. As the vessels stiffen the diastolic pressures, which, caeteris paribus, are inversely as the elasticity of the system, must fall proportionately. Marey thought that maximum oscillations signified the mean pressure; Sahli takes maximum oscillation as diastolic pressure, but his method must imply some flattening of the vessel ? Howell and Brush, ${ }^{1}$ Masing (loc. cit.), and Dr. Davenport Windle ${ }^{2}$ regard the point of maximum oscillation as the diastolic pressure; the wall is then in elastic equilibrium. Gibson regarded the point of diastolic pressure that at which the large oscillations suddenly begin to diminish. With his oscillometer, von Recklinghausen ${ }^{3}$ (with Erlanger) notes as the approximate maximum pressure the point where the vibrations, after a small augmentation, become suddenly larger; as the minimum or diastolic, where they become

1 Howell and Brush, Boston Med. and Surg. Journ., 1901, p. 146.

2 Windle, D., Lancet, Nov. 18, 1911.

3 Von Recklinghausen's last paper, “Blutdruck b. Menschen," in Beihefte z. med. Klinik, aus dem Lab. f. exp. Pharm. zu Strassburg i. E., 1910, Heft 8, contains a full description of his instrument and much interesting matter besides. His oscillator does not depend exactly upon an aneroid but upon an elastic metal capsule (Bourdon-Röhre), which however, from the point of view of stability, scems to me to come to much the same thing. 
suddenly smaller; the diastolic pressure being signified by the lowest of the large oscillations; though with an unarmed needle this phase is made less definite. The signs then of these periodic oscillations are somewhat arbitrary, and, even with a graphic record before us, are not so easy to determine as the advocates of particular instruments would have us believe.

Dr. Janeway accepts the first diminution of the excursions as the diastolic pressure, and Strasburger and Sahli take the same moment. With Pachon's instrument the beginning of the large oscillating phase is taken as diastolic pressure. To take, with von Recklinghausen, the first enlarging oscillations on the opening side makes a considerable difference, and others (Janowski) take the middle swing. But surely as the excursions diminish the wall must be tightening? As regards tone, we have no guide. Dr. Windle compared the sphygmographical with the sphygmomanometrical curve by affixing Mackenzie's polygraph to the radial artery by means of a tambour, and then from the point of maximum oscillation slowly pumping up the pressure.

In Uskoff's instrument, as in others of this kind, we are directed to begin to read the pressures where the first definite wave appears after arrest-which of course on the tracing is a straight line. But in many cases-not in all-the enlargement at the beginning of the scarcely visible jigging is so gradual that there is a variable play, and with it accordingly a fallacy up to, say, $10 \mathrm{~mm}$.; besides certain constructional defects. Dr. Oliver, in emphasising the importance of diastolic pressures, goes a step farther; he suggests that we may take the maximum oscillation first (which, with Marey, and Roy and Adami, he regards as the mean pressure, and as this measurement does not depend on the capacity of the pad, a small pad will suffice for it); then, if this be normal, he assures us we need not bother ourselves overmuch about the systole, or the rest of the cycle. This alternative would avoid the nervous and other incidental exaltations of systolic pressures. In Graves's disease, for instance, the systolic pressures may rise considerably; but, complications apart, the diastolic are apt rather to fall ; the pulse amplitude is larger.

One of our junior graduates, Dr. Malcolm Donaldson, ${ }^{1}$ carefully

1 Donaldson, M., Thesis for degree M.B., Cantab., July 1912. 
tested some sphygmometers, among them Pachon's and Uskoff's, which latter offered the tempting advantage of parallel tracings of pulse and pressures. Of Pachon's ${ }^{1}$ he gave a fair report; if the absolute figures were not correct, their relations were fairly uniform. To Uskoff's apparatus there is a preliminary objection in the defacing of the smoked paper while on the roll, before it falls under the styles. But the essential defect lies with the indiarubber bulb which protects the tambour from strain. Such a protection is necessary but introduces a capacity for enormous error. The line of expansion or of recoil of the balloon is not a straight line but a curve, so that impulses will tell variously according to the radial phases of the bag. Furthermore, to protect this bag in its turn, it is surrounded by a string net, practically inextensible; what happens when the rubber bulb expands, as it does, to the diameter of the net it is impossible to guess. Of Brugsch's instrument I know no more than is reported by Margulis, ${ }^{2}$ who prefers it, on the whole, to Uskoff's. ${ }^{3}$ For my own use I have thought it better for uniformity of error to stick to the instrument I was accustomed to.

When we are dealing with phases we shall not forget that when we have ascertained maximum and minimum positions we have but a part of the manometrical records we require for clinical use. If it be possible by any external indirect gauging to get kymographic records of the total phases of the undulations of arterial pressures, it may be only by means of such large and costly instruments as those of von Recklinghausen ${ }^{4}$ and of Erlanger ; instruments which have been tested and somewhat simplified by the late Dr. Gibson, whose valuable work in this field is well known. ${ }^{5}$ These machines are, however, for clinical use, still out of reach, unless it be occasionally in the wards of a hospital; and Gibson told me, a few months

1 On Pachon's instrument, see Halls Dally, Brit. Med. Journ., Oct. 11, 1913.

2 Margulis, Zur Sphygmotonographie, Diss. Berlin, Aug. 14, 1911.

s An interesting account of instrumental methods will be found in Dr. Hawthorne's recently published Studies on Clinical Medicine.

"Von Recklinghausen, “Über Blutdruckmessung beim Menschen," Arch. f. exp. Path. u. Pharm. Bd. xlvi. p. 105, contains a useful bibliographical list. Von Recklinghausen's latest instrument is described in the Münch. med. Wochenschr. of 1913, Nos. 15-16. His oscillometer (tonometer) is to be distinguished from his kymographic apparatus.

See, e.g., Gibson, G. A., Quart. Journ. Med., Oct. 1907. 
before his lamented death, that he had not yet brought his instrument up to the standard he desired, and could not yet recommend it unconditionally. Dr. Singer ${ }^{1}$ has made some modifications in Gibson's apparatus which promise to be of service, but he tells me that his instrument likewise is not yet ready for the market.

If then we wish for a fairly satisfactory pulse appreciation we must perceive, infer, and record the pressures as best we can, and then take a sphygmographic tracing (Mackenzie's polygraph); but, whatever pains we may thus be able to take in a few cases for research, we cannot, or we cannot yet, bring all these engines to bear in ordinary work; not to mention frightening our patients into extravagant "tensions."

We must not forget that the first wave is not exactly the first insinuation of the stream under the cuff, but the impact of the wall wave, or wall wave, and stream upon it; this increases as the enclosed parcel of the artery begins to unfold, and depends chiefly, not on blood pressure, but upon pulse amplitude ; in other words, the tapping on the cuff varies in recoil with the depth of diastolic descent. This rule applies to tracings from all cuff instruments. Hensen used to say that he could detect the returning wave by palpation at $3 \mathrm{~mm}$.; can we get a cuff and tambour record as near as this? or must palpation, or auscultation, be added? If we have reason to suppose the pulse amplitude to be large, and that the lever moves much before the entry of the returning stream, we may note the line of returning stream adding itself to that of the cuff impact; thus the descending line may be carried below the abscissa, a dip which, as the stream finds its way through, dwindles out in four or five waves.

It is true that instrumental fallacies touch rather the systolic than the diastolic pressures; could we but know these we should be better able to interpret those. But these diastolic pressures are precisely what we most want, and get least, unless the pulse be very slow; in the slow pulse the instrument has a better chance, the lever has more time to descend: for instance, in the doubled pause after an extrasystole it may fall even below the line. If the height of the pulse wave may be an index of cardiac capacity, in diagnosis and prognosis, as we

1 Singer, Lancet, Feb. 5, 1910, and Quart. Journ. of Med., 1911. 
shall see later, diastolic pressures are more significant. As Broadbent said again and again, 1 the pressure and the fulness of the vessel between the beats (italics mine) were the chief indications. Another reason why we desire to know diastolic rather than systolic pressure is that the mean pressure, a very important condition, lies nearer the minimal phase than the apex of the triangle. The "normal" diastolic pressure seems under middle age to lie about $80-90 \mathrm{~mm}$. to a systolic of 125 ; if over 90 it is abnormal. On the other hand, in health, a slow pulse with large arterial relaxation may carry it down very low, even to $50 \mathrm{~mm}$.; and we are continually observing that while systolic pressure is in excess the diastolic may be normal or subnormal. Let us then give some further consideration to measurements of these diastolic phases so much neglected in our practice.

I have said that if by external methods we can make any sure approximation to these, it is by a well balanced oscillometer; yet here again we have no constants : tone, elasticities, $\mathrm{CO}_{2}$ content, position of the body and of the diaphragm - as to which we cannot always have our own way, mental anxiety, these and other such variables, added to the defects of our instruments, hamper our search after true criterions. Mr. Mummery (in 1908) was sceptical about the records of diastolic pressures, the pressure, that is, immediately preceding ventricular systole, but he used, I think, mercurial gauges only, and perhaps was tempted to ask for more than the instrument was able to give. Indeed Professor MacWilliam thinks oscillation figures untrustworthy, and apt to range too high (p. 96). Moreover, it is in diastolic pressures especially that the peripheral venous congestion and inertia, which I have mentioned as due to the cuff (p. 60), tell against the precision of the record. Still, a long familiarity with Hill and Barnard's instrument gave me some facility in guessing diastolic pressures from it; and oscillometers have since been improved by von Recklinghausen and Pachon of Bordeaux. In the instrument of Pachon, now on its trial, the periods are shorter and more net, and I think there is less instrumental inertia.

Müller and Blauel ${ }^{2}$ and three French physicians, one

1 E.g. Discussion, Med.-chir. Soc., Jan. 1903.

${ }^{2}$ Müller and Blauel, Deutsche Arch.f. klin. Med, vol, xci, 1907. 
of them Heitz, ${ }^{1}$ had opportunities, which they regarded as decisive of taking direct (internal) measurements during amputations on man, ${ }^{2}$ and comparing them with a RivaRocci record. In four cases they found the R.-R. fairly accurate for systolic pressures ("within $10 \mathrm{~mm}$."), but Pachon's oscillometric instrument, which for systolic pressures was fallacious, registering irregularly too high, was more accurate for the diastolic; "indeed it was very accurate." This instrument has been brought before us in England by Dr. Eckenstein and Dr. Halls Dally. Unfortunately it depends upon an aneroid which needs continual testing, but the testing is quite easy. It shows increasing gradients far more sharply and decisively than any other oscillometer, and $I$ also believe it to be the most accurate of our instruments for what we most want, diastolic pressures. It is apt, it is true, to read too high for systolic pressures, aneroids do tend to give excess readings; but, as in any case the tapping of the wave on the armlet interferes with the true reading, $15-20 \mathrm{~mm}$. may be subtracted, and a trifle added for the decreasing phases, which run a little too low. Martinet, ignoring the fallibility of aneroids, relies upon it, for small differences, in my opinion much too confidently. But, as I have said, in the face of these many fallacies I have thought it best for clinical work to stick as far as might be to the same instrument, so that, whatever the errors, they might be uniform. I have therefore not taken the Pachon gauge into use.

Roughly speaking, the diastolic pressure should be to the systolic about $3: 4$, and the "pulse pressure" to the systolic about $1: 4$. The current calculation is that the relation 1 (or 1.5) : 4 indicates normal heart function, but much depends on the pulse rate; with a slow pulse there is more time for the flow through the system. Pulse pressure multiplied by pulse rate is the sum of the work done, and should indicate what the heart is actually doing, but none of these reckonings can tell us its

1 Dehon, Dulne and Heitz, Sem. méd. juin $1^{\mathrm{e}}$, 1912.

2 The first internal measurements of blood pressure in man were, I believe, on the brachial and femoral during amputations, by Faire in 1850; the reference $I$ have mislaid. He found the systolic to vary from 130 to 150 . I have no knowledge of the circumstances, and express no opinion as to the propriety of these experiments. 
capacity (Vol. II. p. 31) ; the conditions of heart's energy and of the periphery are big variables. If we had the product of output and blood pressure, and, still better, if with this we could measure the velocity also, we could make some calculations of the external work done (p. 98). But even then we should have no measure of the heart's internal work, some of it passing into heat no doubt; this internal work may be not only as great as the external but the double or even treble of it.

If, caeteris paribus, the diastolic pressure begins to fall, the heart is slackening, at least in rate. Dr. Janeway says that in arteriosclerosis the diastolic pressure alters less than the systolic, agreeing in this with Israel ; but in which mode of it ? It is in the experience of us all that, as the walls of the great vessels lose their elasticity, the systolic pressure mounts above its proportion to the diastolic, which falls ; the aorta absorbs less energy, most of which goes forward, but the mean pressure may not be exceeded. In aortic regurgitation the difference between the systolic and diastolic figures may be enormous, as much as 105-210 mm. In a certain Adams-Stokes case of my own, at a rate of 32 , the systolic pressure was 180 , the diastolic $90 \mathrm{~mm}$. Thus, with the difference between the systolic and diastolic pressures waxing as the resilience wanes, the blood pressure moves more suddenly and largely, bangs more, and thus racks the machinery. The greater vasomotor lability in youth has a like effect, but the vessels are then much more resilient and energy is stored; moreover these peripheral relaxations are often confined to limited areas, as in blushing and the like. In an early hyperpietic, or nephritic subject, the systolic exorbitancy may be inconsiderable, or variable, while the diastolic is steadily in excess. As age advances the amplitude increases to 80 and upwards; a systolic pressure of 160 may be associated with a diastolic of $80-75$ (Wildt). In unpromising senile pneumonias Wildt found systolic pressures falling, and the diastolic pressures rising - a sign, he says, of heart failure. This may be an important maxim. In those cases the rising diastolic pressures were probably due to the enormous venous inertia - the "hypertension diastolique par obstacle veineuse" of French authors-for Dr. H. C. Mann ${ }^{1}$ says that, as compensa-

1 Mann, Guy's Hospital Gazette, Feb. 22, 1908. 
tion fails, the diastolic pressure begins to fall, and the systolic readings become irregular. When we are observing the blood pressure in diastole the heart, by the aortic valve, is cut off from the arterial system, and the arterial tree may be compared with a distended bag with fine holes in it, so that a rapid fall of pressure would not necessarily mean a failing or weak heart; it might mean, and often does mean, weak stomata - that is, dilated capillary areas; and, if so, the stronger the pressure head the faster the fall. Such a relaxation of the stomata in pneumonia, as in many another malady, may be due to toxic influences; or to simple atony, nervous exhaustion, or defective nutrition. But here again the variables seem infinite.

When we take into consideration all these conditions, static and dynamic; when we remember the volume of the wave, the pressure, the tone and density of the walls, the thready pulse with high pressure, the big pulse with low pressure, meals and the hour of the day as they affect the splanchnic system, the state of training of the individual, the viscosity or velocity of his blood, his lung capacity, his hormones, his mental stabilityto which many inward factors, all modifying or overriding each other, are to be added the tricks of the machine; all these tricks and these sources of fallacy, and the consequent complexity of reckoning, seem to forbid all hope of scientific accuracy, so that some able experimenters doubt the diastolic values of all or any gauge. How then out of this tangle are we to pick out what we want?-systolic, diastolic, and mean pressures, cardiac capacity, splanchnic conditions, tissue activities, and so forth. Well, some of these coefficients are still out of our reach; yet, these confusions notwithstanding, I would plead that on many points observers are fairly agreed. We are agreed that the systolic arterial pressure of a healthy man of the age of 25 is very fairly constant, about 120 ; some, such as young athletes, are under, few over; under morbid conditions the tests must be multiplied, and compared with the clinical data. In a normal adult (male) the diastolic pressure ranges about $80-90$; in children it runs about 75-80. Again, the arithmetical mean pressure (in the aorta) is about equal to the diastolic pressure in the brachial, plus $\frac{1}{3} \mathrm{rd}$ of the pulse pressure there. The geometric mean varies with the form of the curve of descent, which is not a simple linear 
declivity, but a complex of differential pressures and velocities. These approximations indicate fairly well, not indeed what the heart can do, but what, with gravity added, it is doing. ${ }^{1}$ On the whole then, considering all the fallacies personal and instrumental, it is remarkable, and in practice comforting, to find the figures, age for age, in fair agreement.

But appreciations of wave-arrest by touch and sight are yielding place, at any rate for the present, to the auscultatory method. Will this give us better assurance? "Ehret's phenomenon " 2 is this, that at a certain point, while the pressure in the cuff is slowly falling, the finger on the brachial (the radial is too small for the purpose) perceives a sudden throb in this artery. It has been stated that this throb, within a few millimetres, indicates the diastolic pressure, and is so perceptible to the patient that his subjective sensation might be taken as the mark of the recovery ${ }^{3}$ of the wave. However this is a curiosity, and scarcely trustworthy for scientific methods; what is of more importance is that such pulses are audible, as sudden alterations of tension; and it seems probable that their sounds may be taken as marks of the several moments with more accuracy than palpatory signs. This auscultatory method was initiated by Korotkoff, 4 whose methods I tried rather clumsily to verify with a wooden stethoscope. Dr. Oliver, ${ }^{5}$ who did us the service in England of describing this method, improved it by the application of a phonendoscope, a broad flat surface which exerts no concentrated pressure. In 1911 Oliver fitted a sensitive air-tight tambour to the instrument, which has been approved and adopted by MacWilliam, Janeway, and other workers in the field. With this furniture it is said that the manometric cycle can be followed and appreciated in its minimum, mean, and maximum phases, which we are to distinguish, not so much by the quality of tone, as by its relative intensities. Many observers,

1 See able paper by Percy Dawson, Amer. Journ. Physiol. vol. xv., 1905-1906.

2 Ehret, Münch. med. Wochenschr., 1909, No. 12.

a Armstrong, H. G., Brit. Med. Journ., Sept. 21, 1912. This sensation cannot be depended upon in nervous or excitable persons.

4 In a paper read at Petersb. Mil. Med. Acad., Oct. 20, 1905, and again in 1906 , and quoted in many journals. Fellner discovered the same method independently about the same time (Deutsche Arch.f. kl. Med. Bd. 84).

${ }^{6}$ Oliver, G. Two new methods, etc., Roy. Soc. Med., 1910, Clin. Section, pp. 8 and 207, and Quart. Journ. exp. Physiol. vol. iv. No. 1, March 1911. 
both home and foreign, are testifying to the value of this method, ${ }^{1}$ especially, which is of chief importance, for indications of diastolic pressure. Schrumpf ${ }^{2}$ extolls the auscultatory as superior to any other method, and Oliver and MacWilliam are of the same opinion. At the Freiburg discussion, Moritz, on physical grounds which he gave at length, argued that the auscultatory method indicates too high a pressure amplitude ; but Oliver says that, being more delicate, it gives a systolic pressure of 10-15 $\mathrm{mm}$. lower than the palpatory. Lang and Manswetowa made careful comparisons on man and animals between the oscillatory and the auscultatory methods, and, comparing the auditory method with the graphic curves of von Recklinghausen's instrument, found them concordant. It is asserted also that from it we may derive some information as to the capacity of the heart; this I cannot deny, as I have not myself made any careful comparisons of the method, but the arguments alleged for it are far from conclusive. Between contrary opinions time must decide. On the whole, Ettinger thinks that minus tone and plus "murmur" mean a failing heart; this opinion at first sight does not seem probable, for, if the murmur signifies an eddy at the critical strait of the vessel, its loudness must depend somewhat upon the heart's energy. With stiffening of the walls all these phenomena are intensified. On the intricate, and often erratic, variables in these estimates I have said much already; among them Ettinger emphasises the temporary diameter of the vessel, the degree of elasticity, the size of the wave, the rate and viscosity of the blood, and the heart's energy : to these I may add the degree of venous congestion of the part.

The method is thus described; as, from arrest, the air is slowly let out of the armlet, and the wave begins to traverse the brachial artery, a throb breaks the silence. The transition, at

1 See Goodman and Howell, American Journ. Med. Sci., Sept. 1911, who find in this method an approximation to dynamic values; Hoffman, Petersburg med. Wochenschr., 1910 (quoted in other journals); Bickel (of the Bonn Clinic), Zeitschr.f. exp. Path. u. Ther. Bd. v. Hft. 3, Jan. 1909; Fischer, Deutsche med. Wochenschr., 1909, an important article; Lang and Manswetowa, Deutsche Arch.f.klin. Med. Bd. xciv. Hfte. 5-6 (controls by animal experiment); Schrumpf, Moritz, and others, Protokoll, Naturf. Gesellsch. Freiburg i. Br., Juni 9, 1909, see German journals of the time ; and Ettinger, Wiener kl. Wochenschr., 1907, a valuable contribution; MacWilliam and pupils, many important articles.

2 Schrumpf and Zabel, "Auscult. Blutdruck.," Münch. med. Wochenschr., No. 14, 1909. 
about $60-90 \mathrm{~mm}$., from silence to throb is quite sharp and fairly uniform in all persons, rising or falling with the arterial pressures. On increasing the cuff pressure the throb is intensified, and this phase is maintained for some $20 \mathrm{~mm}$. ; then it gradually wanes, but ceases sharply. Thus a plus and a minus limit are laid down; both sharp, so it is said, to $1 \mathrm{~mm}$., the lower signifying the diastolic pressure. Ettinger and Oliver formulate four phases: sharpness (click or faint pistol shot), murmurishness (not " murmur" in its full sense), loudness, and dulness ; then (5th) silence. The murmurish phase is inconstant, often absent. The third is the feature said to be directly as the vigour of the heart. Sterzing's ${ }^{1}$ description of the quality of the sounds agrees with that of Oliver, and he warns us likewise that the phenomena may be much altered by the contact of a stethoscope; he also uses a phonendoscope. Dehio ${ }^{2}$ says the loud tone indicates the complete collapse of the artery in diastole, first obtained, and the minimum pressure overcome, by external cuff pressure. This he regards as the note of the diastolic pressure; as the moment of the waning of the sound betokens the systolic. Thus he also regards the auscultatory method as the best for diastolic record (beginning of phase 4), and Professor MacWilliam ${ }^{3}$ is of the same opinion. In disease by differences, especially diastolic, as yet unexplained, the auditory and the oscillatory signs are discordant. Dr. Windle ${ }^{4}$ puts the sounds phonetically as swish to dup; dup rises to a maximum and dies out as the air-escapes. On releasing external pressure the sounds take the inverse order. On the precise interpretation of these sounds observers are not yet agreed; probably they differ somewhat with the magnitude of the vessel under the ear. The beginning of the fourth phase seems to be the diastolic moment. Von Recklinghausen is less sure; he compares the auditory with the palpatory method, to the disadvantage of the auditory, which, he says, is at best not more accurate. The precision of its data he has

1 Sterzing, in Reinhold's clinic, Deutsche med. Wochenschr., Oct. 28, 1909.

2 Dehio, quoted Zentralbl. f. Herz- u. Gefasskr. 1913, No. 9 (May).

- Melvin and Murray, Q. J. Exp. Physiol., July 1914; also Warfield, Interstate Med. Journ. xix. 1912, Nov. 10, and Arch. Int. Med. x., Sept. 1912.

- Windle, D., Lancet, Dec. 9, 1911. 
tested beside the kymographic record, when he found it often quite fallacious ("versagt nicht selten ganz"); ${ }^{1}$ he regards the auditory characters as rather unsteady and capricious ("labil und launisch"), and says that a little change in the congestion of the arm may modify them. To avoid this, for diastolic pressures, MacWilliam raises the pressure slowly from zero till the diastolic note appears. Some other experts, among whom was Gibson, agree with these criticisms ; for my part, I think more depends on phases of vascular tone than is fully realised, but $I$ hope that in this method we have the most useful index, by indirect measurement, of pressures systolic and diastolic, yet available.

Electro-cardiography, invaluable in the interpretation of cardiac function, especially of rhythm, gives us as yet little assistance in the study of pressures or of sclerosis.

Of small and handy machines for ordinary, purposes many skilful observers, such as 0 . K. Williamson and Gossage, speak well of Professor Hill's little gauge. I carry it in my bag, and find it decidedly helpful, in a "subjective" sense. Its index is unstable under manipulation, so that it is difficult to exhibit a record to a bystander not used to its little ways; as a handy rough test however for one's own eye it is quite worth using. Herz of Vienna has produced an instrument, "simple, handy, and cheap," which I have not seen ; unfortunately, Neimark, ${ }^{2}$ who has tested it, found it wanting: when carefully compared with the R.-R. instrument, or with von Recklinghausen's graphic machine, it proved to be inconstant. Like an apparatus recently suggested by Dr. Oliver, in order to avoid the discomforts of the ordinary cuff, it consists in part of an armlet which is only partly filled, so that its higher pressures are effected by the hand of the observer; as the armlet is squeezed the index moves from zero to the point of pulse arrest or return. For this purpose Oliver has provided a small sack bandage which can be folded round the arm, which seems better than the narrow and less adaptable cuff figured in the Herz instrument. Neimark however does not take to the hand-pressure method; he says that the degrees slip up and down with every vacillation of the hand, as indeed they do in the small Hill instrument.

1 Von Recklinghausen, loc. cit. (Beihefte, p. 226).

${ }^{2}$ Neimark, Diss., Berlin, Dec. 8, 1911.

VOL. I 
Gärtner's gauge has fallen out of use ; its fallacies were many and glaring. In cold weather it is difficult to obtain any pulsation from the finger without careful adjustment of a warm-water bath ; and, if this initial difficulty be got over, its records are still very inconstant, the errors ranging to 20 per cent and upwards; especially as regards those diastolic pressures which we need most. The gradient of fall from the forearm to the finger ends is very rapid. ${ }^{1}$ Fleischer of Berlin, in Strauss' Poliklinik, devised a gauge in which he relied, not on fleeting colour tests, but on plethysmographic reactions in the whole finger. ${ }^{2}$ The finger, after any necessary warming, is passed into a stall of rubber around which flows a regulated volume of mercury; as the pulses of the finger are propagated to the mercury, they repeat themselves on a graduated column springing from the upper surface of the vessel. I procured the instrument, which seemed to have certain merits, but I could not agree that the inertia of the metal was indifferent, and the large mass of metal chilled the finger. As moreover the workmanship of the instrument was in certain respects defective, I put it aside.

Measurements of the Work done.-If we can obtain a fairly approximate notion of maximum and minimum pressures we may obtain also, as I have said, a notion of the work which the heart, whatsoever its capacity, is actually performing (p.92). The amplitude-frequency product is said to be equal to volume per second divided by the arterial diameter. Or by Haldane and Smith's, or Plesch's, method we may learn the relations of quantity per beat and per minute. It is said that during the pulse cycle the peripheral resistance may be taken as constant; within ordinary limits of diastolic phase this may be true. Yet the estimates of work are widely apart; those of Volkmann, Hürthle, von Frey, Magnus, Hensen, so vary, from $\frac{1}{5}$ th to $\frac{1}{3}$ rd of the minimum figure, that it is not worth while to quote them textually. Hensen's attempts to measure output by the ordinary methods in man, careful as they were, issued in very divergent, if not contradictory results. Gumprecht, working with Mosso, calculated the pulse amplitude at $30-60 \mathrm{~mm}$., presumably under different conditions. In the rare instances of direct measurement of the output on the open

1 Seo Müller u. Breuer, Deutsche Arch. f. kl. Med. Bd. xci., 1907.

2 Fleischer, Berl. klin. Wochenschr., 1908, No. 4. 
artery of man, the pulse amplitude has been recorded at $20 \mathrm{~mm}$. Masing calculated the mean output $=30$. The rate of oscillation is of course independent of amplitude, which is proportionate to the length of the swinging column (libration) and the elastic coefficient of the wall, together with, unfortunately an obscurer factor, the optimum load of the cardiac muscle. Were the tube rigid and the column one of mercury, the rate and the amplitude would be mutually dependent. However, the total work going on is the output multiplied by the mean pressure ; and the output, other things being equal, is correlated with the amplitude, but, as I have said before, not with the pressure-an axiom too often forgotten. In dilated and hypertrophied hearts the contraction volume, whether put out or not, must always be plus. The height of the rising wave is very often taken as a measure of output or amplitude, but, as we have seen, it cannot be so reckoned. Strasburger's researches on "pulse pressure" (amplitude) are well known, and Fellner and Rudinger, ${ }^{1}$ by applying a manometer to one femoral artery in a dog, and to the other Hïrthle's tonograph, showed that, although the pressures of internal measurement are of course lower than those apparent externally, and some conditions are variable, yet for practical purposes the relation of arterial pressures and heart-work may thus be guessed at. But no one, to my knowledge, has considered how far diminutions of extensibility, due to excess of connective tissue in the wall, may prevent the ampler excursion which is assumed in arteriosclerosis, at any rate in the largest vessels (p. 86). Extensibility is not elasticity, and in so far as the vessel may have dilated its excursions will be less. So far then from becoming a pulse of the celer type, the swing of it might be within narrower compass. In any case a large pulsus celer of this kind is not inconsistent with a heart positively enfeebled. As more than once I have been told that, as by the compression of the cuff the peripheral resistance is put out of existence, the systolic pressure should give a measure of the heart's contraction, it may be well to point out that the arm area, whether by inclusion or exclusion, is far too small to affect the systemic pressures.

However certain instruments have been designed to give us,

1 Fellner and Rudinger, Zeitschr. f. kl. Med., 1905. 
what as clinical observers we anxiously desire, a direct measurement of the heart's work in the circulation, or at any rate of the output per beat. It is to Professor Sahli that we owe the most assiduous attempts of this kind. The instruments we have already discussed give us no more than the resultant of the many forces engaged in the cycle; but if we were able to analyse these in such a way as to give us the several values of the factors concerned, we should be enabled to provide ourselves with data of great importance. Hopke (see p. 101 n.) and others think these instruments (Hopke used Christen's) give us not only such details, but a value of the whole function (output?). They may do so, if repeated on the same individual at the same hour. In previous papers ${ }^{1}$ I have pointed out how little our methods of investigation do for the enquirer who wants to know in a particular case what quality of heart muscle he has to deal with (Vol. II. p. 25), and I a m not persuaded that by any instrument yet designed we are enabled to make such analysis. Hopke however, following Christen, gives results in gram-centimetres ; and typical normal curves (1) adult, (2) child : the child's like an adult's with a weaker heart (weaker periphery?). He says that hypertony gives a characteristic curve, the energy crossing the resistance curve. He points out truly that when in some cachectic states the pressure seems to the finger not bad, by this energy curve the poverty of the pulse is betrayed. He worked out energy curves also with the forearm warmed and cooled, and under adrenalin, digitalis, etc.; but I cannot pursue this part of the subject any farther. In 1907 Sahli 2 introduced, under the name of "Sphygmobolometer," an instrument which was to register the "vital force" ("lebendige Kraft") of the pulsation, and thereby a measure of the work, and indirectly of the volume, of the systole of the heart ${ }^{3}$ - say 60 gram-metres per stroke of left ventricle. It is to record the energy effectively given out, the cardiac potential it cannot give; it may give the "Leistung," but not the " Leistungsfähigkeit." The method seems inadequate, for what we want as clinicians is this potential, the cardiac capacity, which could be tested only by tasks of work done under the operation of the

1 E.g. Brit. Med. Journ., March 23, 1912.

2 Sahli, Deutsche med. Wochenschr., 1907.

3 Sahli, Klin. Methoden, 5te Aufl. 1908. 
instrument. But Sahli ${ }^{1}$ is quite aware of this limitation. In private conversation he assures us that with his instrument he does not pretend to test the reserve energy of the heart. I should have thought that for his purpose plethysmographic methods would have been better? A pad exerts only a partial pressure upon the artery and can hardly be kept plumb upon it without slipping. Sahli suggests that a weight lifted by the unoccupied arm might be a cardiac test (p. 61); but such an effort would, I fear, be difficult to arrange without vitiating the observation. In a truly scientific spirit Sahli has listened to many criticisms on his instrument, urged especially by Christen ${ }^{2}$ who brought to bear upon it a battery of physics and mathematics; and has reconstructed his apparatus four or five times. For the principles and applications of a measure of the mass and energy of the blood effectually thrown into the system, the reader must be referred to the scheme and description of Sahli's instrument, which he showed at the London Congress of 1913.

Christen has himself designed an instrument for this purpose, and kindly demonstrated it to me in Bern. In no carping spirit I trust, I may confess to an abiding prejudice that, as I have repeated again and again in this work, the hæmodynamic conditions are so subtle, and so many, and the diameter of the radial artery so inconstant, as still to offer almost insuperable difficulties to analysis. The very large variations of output under normal conditions are not the least of the difficulties. Mathematics, freely used by Sahli and Christen, may serve now and then to test principles (Preface); but experience of mathematics in biology shows that its methods, admirable as checks, are far too abstract for interpretative or constructive applications; definite results can be obtained only by eliminating many of the intimate factors which go to make up the sum of the physiological process. And in this

${ }^{1}$ Sahli, loc. cit. vol. i. p. 187; also Correspondenzbl. Schw. Ärzte, No. 16, 1911.

2 Christen, “Die neuen Methoden d. dyn. Pulsdiagnostik," Zeitschr. f. kl. Med. Bd. lxxiii. Hfte. 1 and 2; and Zeitschr. f. exp. Path. und Ther., 1909 and 1910, Bd. vi. and vii. Also Ed. Med. Journ., Oct. 1912. Christen, in the first paper, considers the state of the arterial wall. Hopke (of von Bergmann's Klinik), Exper. u. klin. Untersuchungen über Kreislaufdiagnostik mit dem Energometer, 1913. (On my reprint no name of journal.) Christen has since (1914) published his researches in an interesting book with ingenious charts. 
opinion I have been reinforced by Cambridge mathematicians. I have submitted Christen's design and argument to two of my friends here, the one a physicist and physiologist, the other a mathematician. They were both of opinion that there is no proof that these curves ("sphygmobolometrical" (Sahli) or "energometrical" (Christen)) bear any constant relation to the energy-output of the heart, the conditions being, as I have said, too complex. For instance, in the apparatus the compression of the air of the cuff is assumed to be isothermal; but this is not so: the compression is so rapid that it is adiabatic, a condition which upsets the whole of the mathematical reasoning. The instrument is not proved therefore, as a measure of cardiac energy, to be physically accurate, though there may be margin enough for clinical approximations. The only way of ascertaining under what conditions such an instrument would work would be, instead of the heart, to attach it to an artificial pump, whose energy could be so measured that the curve of vasomotor and pressure conditions, etc., should bear a constant relation to the phases of that energy. Münzer of Prague, ${ }^{1}$ who has published observations with Sahli's instrument, says that in arteriosclerosis this instrument indicates that the throw ("Wurfkraft") of the pulse-wave is considerably raised; this may be true, but we must remember that, on account of the loss of resilience, far less of the heart's energy is taken up en route by the large elastic vessels, a quantity which is quite indefinite, and in each individual an unknown variable. Admiring as I do the work of these experimenters, for the reasons I have mentioned I fear as yet we can place their records in no more effective light than those of other and simpler manometers. Still I hope that the method is one of promise for the future.

It must be understood that the volume which Sahli and Christen seek to measure is not the " pulse pressure," with which I have dealt already, but the exerted mechanical energy related to the filling of the portion of the vessel observed; the systolic volume (according to them) increases in cubic centimetres measurable by the volume of air displaced in the instrument. This energy I suspect in the same individual and in health is very inconstant. For instance, in fever filling may be fair, but at low energy; in a

1 Münzer, Wiener kl. Wochenschr., 1910, No. 38, and other papers. 
cachexia both the filling and the energy manifested may be low ; in aortic disease both may be plus; in the "wiry" pulse of renal disease, while the filling is small, the energy put out may be enormous. Christen, ${ }^{1}$ who provides a logarithmic differential scale giving to each beat the percentage of mean pressure, claims for his instrument a direct record of energy independent of elasticity and of the volume of displaced air.

Warfield (p. 96) is of opinion that a shortening of his 3rd auscultatory phase signifies a feeble cardiac effort; a lengthening of it a powerful stroke. I have alluded to the energy value of the arrest of the palpable wave.

In respect of these energy computations, Fellner ${ }^{2}$ affirms that from the three components, pulse pressure, stream rate, and volume (plethysmographic), he can separate velocity. He compresses the whole arm so as to emptyit of blood arterial and venous-as Hürthle did, by the way-with an Esmarch's bandage ; then he notes the time when, on gradual release of the compression, colour returns (on the Gärtner method) to the finger tips. He has controlled his method by the stream-clock on animals. He calculates, on 1000 observations, that the variations of velocity in the brachial artery lie between 2-400 $\mathrm{mm}$. per second. By dividing the output by the velocity he reckons the calibre of the vessels and, according to von Recklinghausen, their elasticity is equal to the quotient of pulse pressure into pulse rate (see p. 31).

In the sum of all this discussion I reiterate that, when we consider the fallacies and difficulties of external or indirect manometry, its frictions, its inertias, its mechanical faults, the fat, lean, muscular, and bony arms, the changing arterial walls, the interferences of stream energy and wall wave, and the subtle and various influences of hormones, we may be surprised to find so near a clinical agreement. The outcome of it all is that if we wish to have uniform data we must keep to the same instrument, the same adjustment, the same limb and part of limb, the same observer, and, I had almost run on to say, the same patient!

\footnotetext{
1 I am sorry Christen's book reached me too late for any modification of these paragraphs.

2 Fellner, B., Deutsche med. Wochenschr., Feb. 4, 1909.
} 


\section{CHAPTER III}

\section{THE VISCOSITY OF THE BLOOD ${ }^{1}$}

From the time of Vesalius modern investigators have been occupied in discovery of the statical conditions of the living organism, conditions which lend themselves to description and to demonstration: it is the task of our own day upon this foundation to discover the dynamic conditions; but these, being in continual flux, elude our diagrams, and vanish before they are seen. Nevertheless, as I have said, the analytical methods of the modern physicist are penetrating in chemistry and medicine. Without the laws of motion an accurate and comprehensive knowledge of mechanical principles cannot be had, nor the properties of living solids and fluids understood. The mechanics of the human body are being calculated by such methods as those of Sherrington on the muscular confederacies; of Hales, Marey, Ludwig, von Basch, and their followers on the arterial pressures and so forth; of Pfeffer, Sanderson, Waller, Arrhenius, Einthoven on the molecular physics-to mention but a few of these pioneers; again, on the chemical physics by work such as that on "internal secretions," and of Starling on the hormones; and on the subtlest biological processes by the researches of Ehrlich and others in respect of such problems as those of immunity. When we realise the swiftness, the counterplay, and the evanescence of these dynamic activities, we admire the patience and assiduity which in several departments have analysed and recorded many of their fleeting phenomena, and calculated their motions. On other lines perseverance has found

1 This section, now re-edited, was published in the Quart. Journ. of Med., April 1911, vol. iv. No. 15. 
less reward. For instance, in the physics of the circulation, if much has been done, yet for the most part, as we have seen already in the pages of this book, these dynamical currents are still slipping through our snares and escaping our reckonings. Considerable, indeed signally successful, as the investigations of a host of observers into the tides of the blood have been, yet we have seen that even in the delineations of its massive mechanics there is still much to be desired; while on the chemical and electrical sides of hæmodynamics we lack even definite outlines of the molecular activities of the bodily juices and surfaces, and have attained as yet to few principles applicable to practice. However, it is one of the conditions of advance that from time to time we should survey our position; and in the following article I have essayed in respect of the viscosity of the blood some such humble but necessary task.

Viscosity, or the degree of cohesion of the particles of a fluid, as a factor in the efficiency of the circulation of the blood and lymph, although not without recognition in Germany ${ }^{1}$ and France, has received little attention among English observers. In America, by the work of Russell Burton-Opitz ${ }^{2}$ and others, this factor is well recognised; yet nevertheless I read last year, in a useful little treatise on arteriosclerosis by an American physician, this astonishing sentence: "The viscosity of the blood, as such, probably has very little effect on the resistance to the flow." In this sentence what does "on" mean? and what " as such"? Can it be that the author believes that the blood runs as freely along its channels as water would do, or alcohol ? And a British critic of my studies on the arteries lately bantered me for that I " still cling to viscosity." 3 Well, if a man of science may have any prepossessions, I will admit that I " cling to "gravitation, to the conservation of energy also, and, whether applicable to our present subject or not, likewise to the law or laws of Poiseuille; and to many another theory which has survived by

\footnotetext{
${ }^{1}$ See, e.g., Senator's article on the treatment 'of arteriosclerosis, Ther. $d$. Gegenwart, März 1907.

${ }_{2}^{2}$ Burton-Opitz, Journ. of Amer. Med. Assoc., July 29, 1911, and many other papers.

${ }^{3}$ Sir Lauder Brunton gives the weight of his authority to the need of a due regard to this factor, and gives some interesting illustrations in the Lancet of May 10, 1913.
} 
universal consent. And when we know that friction in a fluid must multiply with every increase in viscosity, with every increase, that is, of the cohesion of the particles of the fluid, and when we remember also that nearly 200 times more of the heart's energy is expended in overcoming friction than in direct transference of velocity to the stream, we shall scrutinise most carefully any degrees, however small, in the stickiness of the circulating fluid; for these, calibres being constant, will be multiplied many times in frictional resistance. And in the degree of internal friction (viscosity) the velocity of the stream is reduced, although in living blood vessels, contrasted with straight inorganic pipes of uniform bore, the pressure may remain unchanged. It is true notwithstanding that in the circulating fluids viscosity may be so balanced as to be virtually a constant; and, if so, may be accepted as on both sides of our equations and currently disregarded; and if it be said that in comparison with a widespread area of vasomotor constriction viscosity would become a vanishing factor, we may be ready to agree. It is true that this other chief factor in resistance, the dimensions of the tubes, is far from constancy; under the influence of the nervous system and otherwise, it is continually shifting within large limits. That the arterial is a pulsating stream, Hürthle says, is no great matter.

On the whole, relative degrees of viscosity are most difficult to measure, and, even if measurable, our application of physical laws to vital fluids and their continents is still beset with fallacies ; however, the standard of it, as a vital condition, is so incessantly readjusted by the protective harmonies of the organism as to maintain it, so it is said, not only in relative stability, as is the case with the normal arterial pressures, but also in more or less abiding equilibrium. Still, as Thoma, in an able study of the subject, said, ${ }^{1}$ this compensation may come about in different ways; the heart work may be excessive, or other divergences may accumulate. The heart may or may not maintain the same output; or if viscosity fall and pressures are constant, and vessel diameters constant, more blood would be sent through, and the heart's work increased. If blood pressure be normal the velocity through the capillary system may nevertheless be abnormal. It is most difficult to ascertain at once both the 1 Thoma, Deutsche Arch. f. kl. Med. vol. xcix., 1910. 
arterial and venous pressures and the output per beat and per second. But Thoma (p. 98) will not allow viscosity, because somehow it may be readily compensated, to be put aside as indifferent. In some abnormal states the fluctuations of viscosity may be extreme; yet not only in health, but under the ordinary evolutions of disease, they may be either so confined, or so rapidly rejusted, as to elude our relatively clumsy methods of measurement. Even in the laboratory the physiologist has made little progress in this research; his results are as yet far from consistency, and his methods out of the reach of the clinical physician. For these reasons then, as in 1898 (Lane Lectures) I had dwelt upon the essential importance of viscosity, I have since refrained from discussing it; I have attended rather to the fluctuations of vasomotor tone and of alternative irrigation areas which, as they oscillate within larger limits and under simpler conditions, are predominant, are therefore clinically more perceptible, and to our still imperfect instruments afford more consistent records.

Far then from concentrating my attention upon viscosity, I have not ventured beyond occasional allusions to it, which however, as against a customary neglect of this factor, may have appeared to my readers as comparative emphasis. And indeed this factor can never be far out of our thoughts, for if it should appear that in states of disorder viscosity may vary considerably the consequences cannot but be heavy; the handicap upon the heart might possibly be enormous. So, while giving my own time to those clinical phenomena for whose appreciation I am better equipped, I have also collected for some years what I could find in the researches of observers skilful in the measurement of physical coefficients, and have tried to piece together such approximations to knowledge as from time to time they have offered to us. Determann, whose researches in this field are well known, has published a tract ${ }^{1}$ of larger scope, the first treatise, I think, devoted to the subject; to it I am greatly indebted for the revision of my own notes, and moreover for the example of a lucid and fairly adequate comprehension of the whole matter so far as it has been carried.

1 Determann, Die Viskosität des menschlichen Blutes, Wiesbaden, 1910. Its usefulness is impaired by the lack of an index. 
Of methods it would not become me to seem as of my own knowledge to speak; my own experiments have been of no importance, and those which I have witnessed have been comparatively few. What the difficulties and the fallacies of the research are we shall see presently: difficulties with venesection (in man), with the coagulation of the blood, with its gas content, with changing temperatures, and indeed with the very application of physical-laws to living conditions. As we shall see, there are two kinds of viscometers; one on the principle of comparing the flow of the sample with that of water, the other of the rate of transit of a body moving in the fluid. Hürthle's method, valuable as are his results, is applicable only to animals. Hirsch and Beck's viscometer, which is frequently used in Europe, requires not inconsiderable quantities of blood, and takes it in the venous state, a state in which, as we shall see, it is of higher viscosity; whereas we prefer to know the blood as the left ventricle lifts it. The earlier viscometers did not provide for constancy of temperature; but in his last model Determann, by jacketing the tubes, aims at this constancy, and moreover to provide blood enough for the estimations by a simple puncture of ear or finger; in his instrument $0.2 \mathrm{grm}$. is sufficient. Walter Hess ${ }^{1}$ in his instrument, by fixing two glass capillaries parallel and near together, enables the observer to compare the rate of the blood under examination with that of a flow of water. As a ready and quick way (in a minute or two) of ascertaining the viscosity at the bedside this instrument seems hitherto the best; it can be cleansed quickly, and if not closely accurate, will probably sin less on the whole than he who uses it.

The comparison of glass capillaries with the fine blood vessels, and the safety of the inference from the one to the other, have of course been thoroughly discussed by all experimenters in this field. It is said that the technique may be trusted, for certain observers $^{2}$ have stated that the blood column in the aorta of

1 Hess (Walter, of Zürich), Deutsche Arch. f. klin. Med., 1903-4, lxxix. 128; and (on instruments) Münch. med. Wochenschr., 1907, liv. 2, 1590; in collaboration with Determann, “Die Viskosität des Blutes bei Gesunden," Deutsche Arch.f. klin. Med., 1908, xciv.; sce also Traupp, "Etudes sur la viscométrie," quoted Jahresb. $f$. Kinderheilk. vol. xxxiii. suppls. (See also p. $116 n$.)

${ }^{2}$ E.g. Hirsch in 1906 and Beck, loc. cit. But to the contrary see Wolfgang Heubner, Arch. f. exp. Path. u. Pharm. Bd. lii. p. 149. 
freshly killed animals, by adhesion of the blood around the wall, forms a concave meniscus, that the blood does wet the wall of an artery, and that it does not slip; or if it does that the slip is occasional and negligible. ${ }^{1}$ And in the peripheral vessels pulsation may be neglected, as in them oscillation is merging into a continuous flow. The term "capillary tube" is not physiological ; it does not signify identity of diameter with the capillaries of the circulation, but tubes of small if not constant diameter in which fluids behave otherwise than in large or middle-sized channels. Of course in full tubes there is no "capillary attraction," as this attraction lies between contiguous surfaces of fluid and air, or of two fluids of different qualities. However, to eliminate any fallacy of tube diameters, Determann in his estimations has frequently used in the same experiment tubes of three calibres; namely, tubes traversed by water in $7 \cdot 6$ sec., $12 \cdot 3$ sec., and 58 sec. respectively. He found however that within these limits the calibre of the tubes was unimportant. Tigerstedt ${ }^{2}$ agrees with Hürthle and Hirsch and Beck that Poiseuille's law may be said, with very slight margin of error, to express the hydrodynamic processes in the circulation (but comp. p. 38), but other experimenters do not hesitate to bring these methods into serious doubt; they are far from sure that Poiseuille's law, which is true for solutions, holds also for suspensions, and especially for suspensions in which the floating particles are, relatively speaking, large, and also various in size. May not such corpuscles transform the mode of motion and of friction from cylinder within cylinder into that of one cylinder of the same rate in all parts of the transverse section? This question is asked also by Rothmann. ${ }^{3}$ If so, the result cannot be accepted until the tube diameters in relation to the size and number of the corpuscles are known, a complex function not to be settled by simple application of Poiseuille's law. This problem I must leave to those competent to deal

1 As such is still the current opinion, I must now leave this as I wrote it in 1911, for the Quarterly Journal of Medicine, and previously in the Lancet, Feb. 14, 1903; but this opinion is now doubted. See Dr. John Tait's observations on page 38 ; the question is for the present in suspense.

2 Tigerstedt, quoted by Krone, Deutsche med. Wochenschr., 1910, xxxvi. 1438.

3 Rothmann, "Ist das Poiseuille'sche Gesetz für Suspensionen gültig ?" Rothmann thinks at any rate the wall is wetted. Berl. $k l$. Wochenschr., No. 22, Juni 2, 1913. Rothmann is Assistant in the Breslau Physiological Institute. 
with it; but if it be true, as in Tait's experiments, that the blood does not wet the wall of a blood vessel, Poiseuille's law will not hold for these channels-an awkward dilemma!

Coagulability and Viscosity.-Our graduate Dr. W. L. Johnson, in his thesis for M.B. Cambridge, 1912, described what is really not exactly a viscometer, but a very simple coagulometer; it seems to act well, and may be worthy of mention here. Glass tubing drawn to between 1.23 and $1.37 \mathrm{~mm}$. calibre, is cut into lengths of about $2 \mathrm{~cm}$., and one end of each piece is partially closed in flame. Very small shot, picked out as about $10 \mathrm{mgr}$., are boiled in spirit and dried. One shot is placed in each tube. After the blood is introduced the ends are placed between the jaws of a dressing forceps, on each of which a little plasticine has been placed, and thus the tube is dipped in water kept at $35-8^{\circ} \mathrm{C}$. by an electric lamp plunged in it, which serves also to illuminate the tube. The tube is now so rotated that the shot rolls to and fro, and the time of its passage is noted. In each case three or four readings are made. Johnson claims constancy for his method. ${ }^{1}$ Dr. Welsh ${ }^{2}$ however, in nine various patients, found no direct relations between coagulation time (Addis method) and viscosity; but Mayesima, like Dr. Turner (p. 150), is of opinion that the shorter the coagulation time the higher the viscosity. In a preliminary essay Holmgren ${ }^{3}$ discusses the relations of viscosity to coagulation time. Having shown the considerable influence of the polymorphs upon viscosity, and of viscosity upon coagulation, he proposed to try whether or no a like relation exists between leucocyte form ("Leucocytenbild") and viscosity (p. 125). Up to the present his result is that viscosity depends largely upon the polymorph : lymphocyte quotient. If this be verified, many scattered facts may be pulled together; either as reagents, such as glycerine, or as baths (p.147), acting respectively as excitants of "polynucleosis," or as determinants of the areas of polymorphic distribution. Holmgren's rule may find support in the low viscosity of typhoid blood. The effect of gases in the blood, especially of $\mathrm{CO}_{2}$, may be by some

\footnotetext{
1 See also on "Coagulometry," Dale and Laidlaw, Journ. Path. and Bact. vol. xvi., 1912.

Welsh, Heart, vol. iii. No. 1, 1911.

3olmgren, I., Deutsche med. Wochenschr., Jan. 30, 1913.
} 
such mediation: and so likewise with blood pressures. But coagulation must surely depend also specifically on calcium content, on the presence not of any amount of calcium salts, but of an optimum amount. To add these salts by the mouth when calcium is not in defect probably would have no effect at all. In arterial experiment a neutral salt, if concentrated, prevents coagulation.

As regards the interference by the coagulation of the blood observed, it seems that during the first moments of venesection the blood does not undergo substantial alteration; still under this condition the observation has to be made too rapidly for careful work. Defibrinising agents-such as oxalic acid-may alter its qualities considerably. The instruments of Determann and Hess are not identical in their physical conditions ; for instance, Determann's tubes are wider; their records are therefore not directly comparable: those of each however are consistent within themselves and give relative values. Determann uses hirudin in minute quantity, but it is suspected that even this agent may interfere with the normal properties of the blood; certainly it must facilitate the precipitation of the corpuscles. Dr. Welsh, in a case of hæmophilia in which coagulation time was very slow, found that hirudin did not modify the viscosity; the viscosity with and without the hirudin was $6 \cdot 108$. In the case of animals an anæsthetic, morphia especially, seems to have some modifying effect; in chloroform narcosis the viscosity of the blood is said to rise. Scarpa ${ }^{1}$ and Luziani ${ }^{2}$ have analysed viscosity problems, and discussed forms of viscometers with solutions of pyrocatechine, hydrochinone, etc., with the aid of mathematical methods with which I am not conversant. That these papers did not seem to me to come to much was probably my fault.

Professor Wenkebach told me, in 1911, that two of his pupils, a physicist and a chemist, claimed to have made an "ideal" viscometer, avoiding all known fallacies, and constant for temperature. It was to be, and presumably now is, described in Dutch, a language out of my reach. Cheinisse ${ }^{3}$ thinks that

1 Scarpa, "Lo Sperimentale sulla viscosimetria e tensione superficiale del sangue," Arch. de la biol. norm. et path., Nov. 1911.

${ }^{2}$ Luziani, ibid. fasc. May and June 1910.

3 Cheinisse, L., Sem. méd., Déc. 21, 1910. 
with the improved instruments studies on viscosity are issuing from an academic into practical life. He notes how largely observers are agreed in comparisons of age, sex, diurnal curves, etc. It is true, as we shall see presently, that these kindred qualities offer to us in electrolysis, in refraction, in osmotic pressures, and in chemistry, alternative and controlling methods of measurement.

However, experimenters are as yet far from satisfied with any one of the various viscometers hitherto invented; and Adam, ${ }^{1}$ in the laboratory of W. His of Göttingen, whose work in this field gives weight to his opinion, thinks that instead of advancing to greater simplification, as is usual in instrumental progress, the viscometer, if it is to meet the many various contingencies inherent in the nature of the research, must become more elaborate; although on some points our imperfect instruments may be even too delicate. For we are baffled not only by the delicacy of the mechanics of the matter, but also, and yet more, by the infinite complexity of this and the kindred problems in their physical, chemical, and vital potencies.

For with the mind's eye let us endeavour to obtain a vision, or a glimpse, of these myriad and infinitely various modes of energy, streaming forth in all dimensions of space throughout the animal body; forces so fleeting and yet so perennial, so intricate and yet so harmonious, so universal and yet so nicely specific, so subtle and elusive yet so mighty. We live but in the balance of them; in their spring so swift, so quickening, yet ever implicit with the cadences in which they are to vanish when the symphony is over. With a pertinacity which indeed is born of them we, with our microscopes, our manometers, our viscometers, our electrometers, our polarimeters, year after year are scheming diagrams of their mazes, plotting out their centres, their rings, and their chains, threading them now and then, and aiming here and there at some exact measures of their features; streamlines tensive, diffusive, osmotic, ionic, chemic, and, what we are fain still to call, vital; the dynamic salts permeating and spinning the more static colloids, and passing them by evanescent phases from suspension to solution and

${ }^{1}$ Adam, Zeitschr. f. klin. Med., Berlin, 1909, lxviii. 177. 
oxidation. ${ }^{1}$ Nay, how vain it is to try with words to paint these influences; every molecule a world, every cell a solar system, every organism a prismatic fountain of strength, animation, and immeasurable purpose !- " the great Chime and Symphony of nature." Vain indeed are such words, and yet without them, without some endeavour to express this vast and multitudinous rhythm, this mazy and symphonic dance, we might isolate one by one diffusion, osmosis, ionic stresses, gaseous tensions, and so forth, as we might pin out upon a board the several items of the body-its bones, its tendons, its nerves, and yet form no vivid conception of its functions as a whole. And if we do attain to any vision of this unity in complexity, of the poise of these bewildering rhythms, in our first marvel at this infinite concert of harmonious energies may we not recoil, as Darwin in his amaze at the beauty of the human eye recoiled, in momentary incredulity from his own theory?

A certain effect, or expression rather, of this concord of infinite velocities and exchanges is that we can seize no moments of arrest during which by our machinery static measurements can be made. Not having the cosmic eye, nor infinite mind, we halt between arbitrary classification and functional jumble. If from phase to phase fluctuations must be, or may be, large, they are consentaneously held up again; still unless, on account of the fragmentary outlook of the human mind and of other limits of its faculties, we can after some arbitrary manner catch them flying, and get some kinetographic records of exposure, we must give up the hope of attaining to a scientific knowledge of the functions of the circulation. If the viscometer cannot become a handy clinical instrument, yet the clinician must demand from the physiologist some approximate calculations of the potent if not dominant factor of viscosity. If, like the hydrogen ions, in health always, and commonly in disease, it be so incessantly equilibrated as to be virtually a constant, so much the easier for us; but we shall not be satisfied to assume this constancy-at any rate not in states of disorder-until laboratory experiments convince us either of uniformity or of approximation to it.

1 For an interesting survey of these intimate physico-chemical laws see Schryver, S. B., Proc. Roy. Soc., Aug. 12, 1910.

VOL. I 
Certain well-known observations of Dr. Oliver have suggested that under high arterial pressure the space relations of blood and lymph are altered, and therewith the density of the circulating fluid. The younger Erb, ${ }^{1}$ as we shall see (p. 118), came also to the conclusion that with the rise of arterial pressures the blood concentrates-contains more solids; and that as pressures fall it becomes more dilute; though this reaction is slower than the previous concentration. From this point of view two papers read to the Royal Society by Mr. Mallock (Nov. 24,1910 ) are of interest; in these, by inorganic apparatus, he demonstrated that in "viscous flow the character of the stream differs according to whether the flow is towards decreasing or increasing pressure." However subsequent observers think that in the animal body such changes are not due, or are not notably due, to mere exudation and return of lymph from the blood vessels, and that the ebbs and flows of blood distribution in areas predominate over those of lymph filtration. Besides, such and so free are the molecular reciprocations amid all the bodily fluids, that in these respects the lymph, which is actually the medium of the cells, may be taken as the equivalent of the blood; indeed Botazzi ${ }^{2}$ and his pupils seem to have proved this upon animals; so that little or nothing is to be gained by separating blood from lymph as one of our necessary if arbitrary divisions of the subject. To Botazzi's work I shall make some reference later (p. 118).

What analytic divisions then can we conveniently make? In the first place our experiments are restricted to capillary, or virtually capillary, tubes, concerning the physics of which, by the labours of Poiseuille and others, we are instructed already; and within such tubes in the body it is that the circulatory frictions are most tenacious. The currents in larger vessels, and within the viscera, are affected by other conditions also. As we approach the peripheral areas we find the resistance rising rapidly. And in the blood it is of importance to watch not only its behaviour as a whole, but also, as we shall see, dividing the corpuscular elements from the plasma, to calculate the viscosity of the plasma and the blood severally. By the corpuscles them-

1 Erb, Deutsche Arch. f. klin. Med., 1907.

2 Botazzi, Biochem. Zeitschr., 1908, vii., quoted by Determann 
selves, merely as foreign bodies, the viscosity of the blood is largely increased; moreover we shall see that this effect of them is more complex than that of indifferent foreign bodies. Another chief division in our analysis speaks for itself, namely, the distinction of the colloid from the saline constituents, the classes and phases of each of which behave in ways of their own.

On such lines we have to try to reach some vision of the infinite reciprocal play of the modes of energy in which the living web of life is ever weaving; but in this vision we perceive that the influence of contingency must be incessant, and in our experiments difficult to neutralise. Let us glance at some of them. We shall anticipate rightly that the molecular dances will be different at different ages, and may be different in the sexes. Again, that animals and plants will differ from each other and from man; and in our experiments we can keep animals far better in equilibrium in respect of external conditions than is possible with man. In youth it is fairly certain that the capillary wall, and other membranes concerned, are more permeable than in age ; and herein may lie more or less of the lagging of function in the elderly, and perhaps some of the stiffening of blood pressures in them. By experiment upon 122 persons, divided in age by decades, W. Hess ${ }^{1}$ found that the viscosity of the whole blood rises from childhood to adult life, and perhaps towards middle life; but after that maturity the variations show no consistent tendency, progressive or retrogressive. The mean of adult normal males was 4.74 (water is the unit and the viscosity coefficient is usually designated by $\eta$ ), the extremes being 4.0 to 5.5 ; the plasma had a mean of 1.86 : of adult females the mean was $4 \cdot 40$; the extremes 4.0 to $5 \cdot 4$. The viscosity of men seems thus to rule higher than that of women, at all ages, a rule verified by Austrian, ${ }^{2}$ and by Welsh; it is probably due to a lower red content-cells and hæmoglobin. A viscosity in a man below 4.3 or above $5 \cdot 3$, or in a woman below 3.9 or above $4 \cdot 9$, Hess would regard as abnormal. Welsh thinks these and such figures, being taken too much from hospital patients, are too low, and that in vigorous, active persons viscosity may rise over 6.0. Sweating (p. 119) may be concerned in this result;

1 Hess, W., Deutsche Arch. f. klin. Med., 1908, xciv. 404.

2 Austrian, Johns Hopk. Hosp. Bull. vol. xxii. No. 238, 1911. 
Welsh has indeed found the red count high in athletes $(5,800,000$ to $6,000,000$ ). Probably the irregular variations lie in varying percentages of red corpuscles and hæmoglobin. Besides, the corpuscles must themselves, as requiring shoves in narrowest vessels, exercise a mechanically retarding influence, so that in such channels maximum velocity may fall to 1.0 or even to 0.5 . Nevertheless, even if $\eta$ be within limits of the possible normal, and indeed if the hæmoglobin figure also be not positively abnormal, yet, according to Hess, if the quotient viscosity hæmoglobin be outside the limits of 17-21, the condition is abnormal. To the hæmoglobin factor we shall return.

There are other difficulties also in defining the limits of the normal. In woman, for instance, is pregnancy, is menstruation, within the normal? In woman viscosity seems to rise before the climacteric, and to fall after it. Ribaudi, ${ }^{1}$ using Hess's instrument, ${ }^{2}$ found in pregnancy a fall of viscosity of the whole blood, especially in the latter time of it, until just before parturition, when it rose-especially if the birth were a hard one, or in eclampsia-to fall immediately after delivery. The viscosity of the blood is higher in the fœtus than in the mother. During suckling the viscosity of the maternal blood does not rise. Similarly there is, he says, a premenstrual rise of viscosity, a fall during menstruation, and a rise to normal immediately after it. Again, idiosyncrasy plays its part, perhaps a considerable one; between various species of animals Hürthle found such differences : dog's blood he found about 4.7 ; cat's blood about $4 \cdot 2$; rabbit's blood about $3 \cdot 3 .^{3}$ Then again there are certain fluctuations during the day; the effects of meal-hours, exercise, diet, nervous and temperamental influences (especially vasomotor), and so forth. Climate and altitude likewise have, as we shall see, their effects on the viscosity. I shall not quote many

1 Ribaudi, quoted in Arch. des mal. du cour, 1909, 256.

2 Kagan and others have shown that this instrument is liable to some fallacies. See Kagan, G., Deutsche Arch. f. klin. Med. Bd. 102, Hefte 1 u. 2, 1911. It is too simple for the complexity of the requirements (p.112), and sedimentation of the red corpuscles also vitiates the readings. Determann and Hess have since made a combined instrument, avoiding the faults of either. See also Münzer and Bloch, Zeitschr. f. exp. Path. u. Therap. ii. 2, 1912. Hess, in the Berlin. klin. Wochenschr. of 1912 , discusses instrumental capacity and the influence of pressures on the coefficients of blood viscosity.

${ }^{3}$ Hürthle, Pflügers Arch. f. ges. Physiol., Bonn, 1900, lxxxii. 438. 
figures in these enquiries, for as yet, such are the discordances of various observers, we have little firm ground for generalisation from them. Concerning diet and blood viscosity, if some definite conclusions could be formulated they would be very helpful to the physician; but the reports are discordant. Hürthle, and Burton-Opitz, upon animals, inferred that changes of diet were reflected largely in the viscosity curve, even in variations so large as 20 per cent. Determann, on the contrary (in man), comparing vegetarian and animal diets, strictly observed for some days, failed to convince himself that these extremes became perceptible in terms of viscosity; Julius Bence ${ }^{1}$ likewise failed to find any loss of viscosity on a vegetarian dietary. It seems to me that the blood, in gathering and selecting its own materials out of the food-stuffs, may well neutralise irregularities of supplies, at any rate for some days; proteins are to be got out of either diet, and extremer variations in electrolytes, e.g. salts, are probably more effectual. In our everyday work much must depend on such adaptations; though over much longer periods - a year or a decade of yearsmore permanent alterations might become established. We shall see that in plethorics with high blood pressures Determann himself has noted a higher viscosity, and it is said that in animal experiments large feeds of flesh raise the viscosity. However, comparisons between carnivora and man in this respect may be invalid. It seems likely that, in dogs for instance, the viscosity of the blood would fluctuate more violently on conversion to a vegetable diet than in omnivorous man. I fear the pig would present a nearer parallel with ourselves. In the careful and repeated experiments of Burton-Opitz on dogs the most definite results seemed to be that pining (presumably water was given to them) reduced viscosity, and full flesh-diet increased it; in rabbits, that viscosity varied more with the watery constituent of the food. It would seem that feeding and fasting should offer more crucial differences, though it is not easy to determine the optimum moment of cell satiety. It seems certain that the meals determine some fluctuations of viscosity; these seem to be larger in the morning than in the evening; the daily excursions reaching even 12 per cent. In experimental estimates

1 Bence, Julius, Deutsche med. Wochenschr., 1905 
these normal daily waves must not be forgotten. Hitherto the conflicting results of good observers is rather baffling.

I have referred to Erb's opinion that viscosity varies as blood pressure more or less inversely; but the evidence in this respect seems to me as yet too slender to be trusted. It may be that high pressure expels the watery part of the blood and so raises the apparent percentage of corpuscles, and so the viscosity. As pressure rises, so rise, it is said, cell content, hæmoglobin value, and specific gravity; and conversely as pressure falls. But these phases are subject to great interference and many internal reciprocities (p. 144). And overriding all is the governance of the relations between the running blood and the reservoirs of lymph and formed elements, which, if in considerable part physical, must also in no small part be subject to the nervous system. It is stated, for instance, that if viscosity be raised with gelatin, the cord divided below the bulb, and the vagi resected so that nervous readjustment cannot take place, osmosis and surface tension have free play, and pressure and viscosity rise together. ${ }^{1}$ Japelli verifies the statement that the injection of gelatin (within limits, of course), although in arterioles and capillaries it raises pressures for a moment (probably an arrest of velocity before a peripheral dilatation?), does not raise aortic pressure. On the degrees of independence of pressure and viscosity, Rubino gives two examples of febrile conditions: in the first case, with a viscosity of $5 \cdot 23$ the blood pressure was 130 ; in the second, with a viscosity of 4.08 the pressure was 150 . But this is familiar ground to us all, as seen eminently in polyglobuly.

Pressure may vary as viscosity at a critical phase; fluctuations of pressure may be rapidly compensated by an interchange between vessels and tissues. Erb, junior, ${ }^{2}$ as also Hess at Marburg, worked at this question, but concluded that although it is of great importance by methods of exclusion to get at the underlying relations between viscosity and pressure, yet, if the phase be at all prolonged, in the living and complete animal the nervous machinery dominates the balances, perhaps by the pulmonary coefficient. Erb tested the concentration and

1 Japelli (in Botazzi's laboratory, Arch. d. fisiol. vol. iv., 1907; quoted Rubino (loc. cit. p. 119 n.). 1905.

${ }^{2}$ Erb, “Blutdruck u. Konzentration d. Blutes," Münch. med. Wochenschr., 
pressure relation in rabbits by injecting adrenalin, and found the concentration of the blood to vary as the pressure; but this agent may act merely mechanically, or also as a chemical substance. The truth is, to repeat what I have already said, to gauge the effect of viscosity on pressure we must know all the simultaneous conditions, and these, if not infinite, are yet manifold and complex. ${ }^{1}$ (See Martinet and others, p. 143 et seq.)

Concerning work and exercise the evidence is less conflicting. Sweating, it is agreed, raises viscosity by about 1.25. Apart from this factor, Blunschy ${ }^{2}$ found from his own and from other experiments that prolonged exertion, such as ski-running, reduces viscosity considerably, while short forced efforts raise it. Notwithstanding, it was found that by derivation from the tissues a reduction towards the normal was soon brought about (Lommel, ${ }^{3}$ Hess, etc.). Böhme (Kiel), speaking in a physiological discussion, ${ }^{4}$ said that brief severe exertion (on the ergostat) concentrated the serum, the albuminous content rising $7 \cdot 8-8 \cdot 5$ per cent : it returned to normal in about five minutes. This he explained by the higher osmotic pressure in the muscle during work, the water passing into the muscle. An important feature of these, and of some other careful experiments, is that relatively large figures are registered, so that there is room for some experimental error. For instance, on long marches as much as 13.46 per cent of difference has been recorded; an estimate which ought to leave a secure result of, say, 7 or 8 per cent. And Hasebroek, in an interesting discussion at one of the German Medical Associations, ${ }^{5}$ remarked that in estimating blood pressures during

1 See Rubino, C., "Il Coefficiente di viscosità sui valori di pressione," Gaz. d. ospedali e di clinica, Feb. 2, 1908, an interesting article based on competent knowledge and personal experience. Rubino enters into far more detail than I can find room for; see also Gouget, "Quelques travaux récents sur la viscosité," Presse méd., juillet $1^{\mathrm{er}}, 1911$.

2 Blunschy's paper was a Zürich dissertation of 1908 of which I have seen only extracts in year-books, and other quotations. His curves of normal daily fluctuations, etc., are republished in Determann's book, p. 66. For many of his observations he seems to have used hospital patients more or less indifferently, many of them probably out of health ? In any case, Vaquez accepts his conclusions with doubt and reserve, especially in respect of alcohol.

${ }^{3}$ Lommel, Deutsche Arch. f. klin. Med., 1904, lxxx. 308 (quoted by Krone, vide No. 2); and Münch. med. Wochenschr., 1908, No. 6 (quoted by Determann, loc. cit.).

4 Reported in Deutsche med. Wochenschr., May 5, 1910.

5 Hasebroek, reported in Deutsche med. Wochenschr., 1909, xxxv. 1414. 
exertion, to avoid fallacy he had to estimate viscosity also-a correction in which Deneke agreed. Blunschy also, on this occasion, referred to his own experiments (mentioned above), and added that during bodily exertion he had noted movements of pressure and viscosity to be parallel; and for us clinical physicians the important factors, with blood of constant value, are its speed and the heart work expended on it. Hasebroek indeed expressed the opinion that Oertel's therapeutical methods owe more to reduction of viscosity than to reinforcement of the heart itself. He found also, as we have found in England, that in the long run, in trained men, by exercise the arterial pressures are reduced.

Climate is too large and vague a condition to detain us long at present. Mayesima (p. 149) finds as a rule the viscosity lower in the Japanese than in the European; the number of his red corpuscles is fewer; and Matsuo, ${ }^{1}$ another Japanese physiologist, makes the same comparison with like results, but suggests that the lower figure may be due to the vegetarian diet. In altitude we have a more definite factor, and one for which we have some definite data. Determann and others have found that at high altitudes viscosity increases substantially; this is probably due, at any rate in part, to the well-known increase of red corpuscles; and we find the same rule in cyanosis, in which these corpuscles more abound. It is well ascertained that the blood of normal residents in high altitudes, such as St. Moritz, is richer in red corpuscles; they have probably a more capacious blood-making apparatus and lung capacity. It is in these persons that Determann finds a corresponding excess of viscosity (by $17 \cdot 4$ per cent) ; and von Koranyi has found that this excess can be reduced by the inhalation of oxygen. It is not always remembered however that as coefficients of viscosity we have to estimate, not the corpuscles only, but also the hæmoglobin.

The density and volume of the several secretions and excretions of the body, difficult as they are to follow, are yet much concerned in the problem before us ; for presumably by their fluctuations the equilibrium of the corporeal fluids is largely provided. When, for example under a sweat, the viscosity of the blood rises, and

${ }^{1}$ Matsuo, Deutsche Arch. f. Klin. Med. Bd. cvi. Hefte 5 and 6, 1912. 
is compensated by derivation from the tissues, the canals and reservoirs of the system will give up some of their aqueous element; and in some measure the electrolytic elements also will be readjusted. It has been my own experience, and I believe that of mountaineers generally, that during long climbs one does not desire much food; nor indeed after coming home is it wise to eat largely or too soon, for the secretions of the parts of digestion have rendered up their juices to prevent concentration of the arterial blood, and therewith increased labour of the heart. The scantiness of the urine and the dryness of the mouth in prolonged exercise are familiar to every one.

So far I have sketched out the sort of influence exerted by outward conditions upon the viscosity of the blood; I may now ask the reader to follow me in pursuing this train of thought into the motions, qualities, and exchanges within the blood itself. This part of the inquiry is traversed by certain important but indeterminable factors, indeterminable at any rate by handy methods. Two of these factors, not easily to be appreciated currently during experimental work, are the velocity of the blood stream and the output of the left ventricle. Until these moments can be reckoned in, our observations must still have something conventional about them. Meanwhile however we may make a little progress with otherconditions on which they have somedependence.

And this seems to be the place to point out that viscosity is not specific gravity, an assumption not infrequently made, even by eminent writers on the circulation ; there is not, indeed, any direct relation between these two coefficients. A normal or even a high specific gravity is not inconsistent with a low viscosity. The "strong salts" and the "weak salts" differ widely in molecular weight, and accordingly in the effect of equal doses. Of the solid constituents of the blood some, such as sodium and calcium chloride, have as concerns viscosity a positive effect; others, such as sodium iodide, or the acetates and organic salts, a negative. Sodium formate, as we should expect, lies on the limits between the two classes. Again, as we shall see, 'the gas content of the blood largely affects its viscosity; by carbonic acid this is increased, by oxygen it is reduced. Furthermore, Grawitz ${ }^{1}$ showed

${ }^{1}$ Grawitz, Zeitschr. f. klin. Med., 1893 ; quoted Saundby, Brit. Med. Journ., May 18, 1907. 
that stimulation of the vasomotor nerves increases the specific gravity of the blood. May I digress for a moment to point out that the specific gravity is by no means a negligible factor? A long series of observations by Roy, Lloyd Jones, and myself proved that in the normal man the specific gravity is maintained fairly constant, being 1058-60; but after the age of 40 tends to fall, ${ }^{1}$ especially in males. If in the elderly it should rise, the rise is often coincident with a rise of arterial pressure also, in which case cerebral hæmorrhage is to be feared, and may be preventable. In hyperpiesia I have never found this variable to be subnormal, and if pressures abate the specific gravity falls likewise. Conversely, if in a case of suspicion of cerebral hæmorrhage the specific gravity be low, the diagnosis-as in respect of embolic or thrombotic alternatives-should be revised (Lloyd Jones and myself). In this case the arteriosclerosis will probably be of the kind which I have called involutionary or decrescent, as opposed to that ensuing upon prolonged high pressures. At the same time, although in a case of arteriosclerosis, or high arterial pressure, the specific gravity be high, or rising, and hæmorrhage be feared, yet a falling specific gravity may not be propitious; for if not consistent with cerebral hæmorrhage it may signify failing reactions. For instance, to return to viscosity, Hirsch and Beck (loc. cit.) have shown, contrary to the presumption of Ewald, that if in the earliest stage of granular kidney-the disease above all in which peripheral resistance is high-viscosity may rise, it may soon fall, the fall: being| probably due to the hydræmia; or in part to anæmia, as it is often said that the specific gravity of the blood runs parallel with the hæmoglobin content of its red cells. But the whole question of molecular concentration in the sundry kinds of renal disease has yet to be dealt with. Generally speaking, in later life falling arterial pressures are attended with falling specific gravity. Specific gravity then is no guide to viscosity ; indeed the relation of the two factors often moves inversely, ${ }^{2}$ so that each factor must be measured separately.

1 For the percentages of water in the blood in infants and in eertain disorders see Lust, F., Jahrbuch f. Kinderheilk. Hefte 1 and 2, 1911.

2 Burton-Opitz, Stud. Rockefeller Inst. Med. Research, vi. 190; and Hirsch and Beck, Deutsche Arch. f. klin. Med., 1900-1, lxix. 503. 
Now, having cleared our path by making these distinctions, let us concentrate our attention again upon viscosity itself which, signifying internal friction, must be a dominant factor in the circulation of the blood; indeed were the small vessels of constant calibre, area and ramification, it would be a tyrannical factor. The law of flow in capillary tubes-known as Poiseuille's law-is this:- That, at a constant temperature, the quantity of flow of water in unit of time is directly as the pressure and as the fourth power of the radius, but inversely as the length. With the cone of outflow and the vena contracta we have not to deal, except to remember that this cone is the expression of the section of the flow ; for the flow is regarded as consisting of cylinders of increasing velocities, the one within the other. The outermost cylinder, that in contact with the wall, if it wets the wall, as in the blood vessel we have presumed that it does, ${ }^{1}$ is stationary, it changes by diffusion only; but from this cylinder inwards the successive layers, or cylinders, of the fluid travel each by the other with increasing velocities as they lie nearer to the central or axial cylinder, and farther and farther from the more and more sluggish peripheral cylinders. Thus we see why the outflow is conical. Now as each of these cylinders is continually engaged in shearing stresses in relation to the pair without and within it, the stickier the fluid the more the internal friction, and the harder-caeteris paribus - the heart must work, if the velocity is to be kept up ; for much of this friction is turned to heat or other modes of energy and, so far as velocity is concerned, is lost. Thus to inject gelatine into the living animal raises the blood viscosity in about parallel measure. Now as in man the viscosity of the blood is four to five times that of water, we may apprehend how enormously more is the energy required to circulate blood than water; and how severely, caeteris paribus, the heart will be handicapped by even small increases in this viscosity, in tubes which are not straight, in beds of friction so extensive and so ramified as the vessels of upright man, and in a current which is not continuous: caeteris paribus, I repeat; for, according to the accepted law, the fourth power

1 This wetting is now called into question; see Dr. Tait's observations, p. 38. See also von Recklinghausen (Arch. f. exp. Path. u. Pharm., 1906, vol. lvi.) on the validity of Poiseuille's law as concerns the blood vessels. 
of the radii of the tubes and their lengths, variables of wide limits, have to be reckoned with also. But it seems quite clear that as friction takes up an enormous percentage of the heart's energy, and as again friction varies as the fourth power of the radius, a very slight increase of radius will make a very large fall in resistance. Again, we are assuming the motion to be rectilinear, which probably, at any rate in the middle and small vessels, it is ; but there is a certain "critical velocity," depending on the ratio of the kinematic viscosity of the fluid to the diameter of a pipe, at or about which the transition takes place from linear to turbulent motion, as shown by Osborne Reynolds by means of coloured filaments in the stream. If turbulence arises then Poiseuille's law is interfered with, and paradoxically the thinner the fluid the more readily will turbulence set in on rise of pressure-head, and the flow be retarded. Determann thinks the system is endowed with an adaptive capacity so that viscosity value rises as pressure falls, and conversely (vide pp. 118 and 143). To meet this variable, Determann has constructed a new viscometer ${ }^{1}$ designed to prevent high or inconstant pressures setting up turbulence, an interference/ with the Poiseuille law which, he says, may happen in the Hess and Münzer-Bloch instruments. However, I suggest that turbulence is a factor of tubes much larger than those of viscometers. But another obscure factor we must not forget, namely, that it is in the capillaries that the work is done; there the physiological exchanges take place between the nutritive juices and the cells ('Gewebsgefühl'), which can scarcely be without some effect in speeding or delaying the blood. This effect Broadbent believed, in some morbid conditions, to be a considerable factor in delay of the blood in the capillaries. And we shall see that the gas content also is a large factor of the velocity of the stream, or at any rate of its viscosity. Whatsoever these coefficients, Müller, Brodie, and du Bois Reymond ${ }^{2}$ carefully studied that of the viscosity in the stream through the capillary system (on Brodie's method) in cats. Quantitative

\footnotetext{
1 Determann, Deutsche med. Wochenschr., Report of Freiburger Med. Gesellsch. Sitzung 30 . Mai, 1911; and also Zeitschr. f. klin. Med. Bd. 73, Hefte
3 and 4. 2 Müller, T. G. Brodie, and du Bois Reymond, Disc. Physik. Gesellsch. Berlin,
Dec. 6, 1907.
} 
differences apart, Poiseuille's theory on the whole held good for the systemic capillaries. Within the viscera the vasomotor fluctuations were too variable for accurate appreciations.

The Blood not a Homogeneous Fluid.-Thus far the blood has been taken as if it were a homogeneous fluid, as it were water thickened more or less with glycerin. But we well know that such is not its condition; that in the plasma colloids and saline matters are mingled, and that in the plasma are suspended a large number of corpuscles, red and white. Now if we suppose these bodies to be equally distributed throughout the fluid, and to be of constant diameter, would such a compound stream behave as if homogeneous? In making the comparison the clotting embarrasses us; in defibrinised blood the corpuscles sink before the observation can be completed, and the addition of chemical agents to delay clotting presumably interferes with the reaction of the blood thus treated; even Determann's parsimonious use of hirudin is said to be open to this demur (p. 111). Professor Mott ${ }^{1}$ has suggested that nucleo-protein, which has so rapid a coagulative action on the blood, "may have a viscosing effect on the blood "; and Dr. Arthur Bodington, in his Cambridge M.D. thesis for 1903, expressed a similar opinion. In this place a description of the several methods by which these difficulties have been surmounted, or reduced, would be superfluous; suffice it to say that expert observers, such as Beck and Hirsch, ${ }^{2}$ Hürthle, and Tigerstedt, ${ }^{3}$ are of opinion that in respect of Poiseuille's law the blood may be considered to behave as a homogeneous fluid within small limits of error. Notwithstanding this approximation, it is of some importance in our analyses, as we shall see, to reckon severally the part played by the plasma and by the whole blood. Burton-Opitz says, as we should suppose, that in the normal condition the viscosity of the serum is without influence on the blood pressure; but if in health the serum viscosity may be called a constant the effect of a fluctuation would be felt the more. Of laking the blood we will speak presently.

The plasma consists of colloids suspended, and merging by

1 Mott, Phil. Trans., London, 1899, Ser. B, exci. 211.

2 Beck and Hirsch, Arch. f. exper. Path. und Pharmakol., 1906, liv. 54.

3 Tigerstedt, quoted by Krone, Deutsche med. Wochenschr., 1910, xxxvi. 1438. 
degrees towards solution, in the serum; this consists of a solution of salines and proteins, on which its properties depend. The plasma without the corpuscles is of course of much lower viscosity than the whole blood, it is generally said as $2: 3 ;^{1}$ the viscosity of the blood depends not on the corpuscles only, but upon the plasma content also; more upon the colloids than upon the salts, which have very different effects upon viscosity. Calcium chloride may in some solutions be osmotically much "stronger" than sodium chloride, and both of course far "stronger" than, say, the acetates (see pp. 121 and 132). The viscosity of the whole blood is higher than that of a mixture of the corpuscles in the same proportion with salt and water. It is not proved that free calcium ions affect the viscosity, but rather the fixed lime salts, for whose estimation we have no ready method (Sir James Barr ${ }^{2}$ and others). Barr is of opinion that although the fixed lime (albuminates of calcium) is concerned in the viscosity of the blood, free calcium ions may cause contraction of the arterioles. The ions drive the osmotic pressure; and although $\mathrm{Ca}$ is as to viscosity negative, yet its presence is essential to such other co-efficients as rhythmical contraction. There is no evidence of a higher viscosity in the blood of residents on limestone soils. By excess of hydrogen ions viscosity is presumably increased. Again, if the corpuscles are not uniformly diffused, viscosity will vary in areas, rising in those in which the corpuscular content is denser, and falling in those in which it is scantier. Thus in asphyxia the rise of viscosity may be due to the more numerous corpuscles, or to variations in their size; but here, as we shall see, the effect of carbon dioxide comes in. That in leucocytosis or erythrocytosis viscosity must rise seems an obvious inference; as a matter of observation it may rise over 6.0 ; and although total blood-volume may be increased, this increase, in a profusion of reds, is in less relative proportion. In two cases published by Dr. Parkes Weber ${ }^{3}$ in which the red cells attained $8-8 \frac{1}{2}$ and over 10 millions respectively, and the hæmoglobin 200 per cent, although the blood pressure (full systolic ?)

1 See Lisbourne et Margarot, quoted Arch. des mal. du cœur, etc., mai 1913.

2 Barr, Sir Jas., Brit. Med. Journ., Sept. 24, 1910.

3 Weber, Parkes, the first case, Lancet, London, 1905, i. 1254 ; the second case, Parkes Weber and Dorner, Lancet, 1911. 
was only 160 [a rough mean between Haldane's Riva-Rocci (157 mm. on two observations) and Weber's Hill and Barnard (165 mm.)], the viscosity was such that the heart, in spite of this moderate systemic pressure, was failing, at the age of 29 , under the aggravated friction thus engendered, and the considerable (in the second case $75 \mathrm{~cm}$. per $100 \mathrm{grm}$. body weight) excess of total blood-volume to be driven. Hutchinson and Miller, ${ }^{1}$ in a similar case, recorded a high degree of fibrosis of the vessels, which they reasonably attributed to the high frictional resistance of $7 \frac{1}{2}-8$ million reds ; an observation which favours the assumption that viscosity is at least a vera causa of arteriosclerosis ; Dr. Craig's Cambridge M.D. thesis on excessive arterial pressures in a certain kind of melancholia is well known; now Bruce and Alexander, ${ }^{2}$ on the high pressures in such cases of insanity, say that these pressures (of 140-180 mm.) are associated with a leucocytosis. It occurred to me that in the high viscosities of polycythæmia the heart should be enlarged on both sides; but, in such notes of the few necropsies I can lay my hands upon, the heart is spoken of in general terms only, as hypertrophied or dilated. When a rise of arterial pressure is due preponderantly to large areas of vasoconstriction the stress, as in chronic renal disease, falls upon the left ventricle in corresponding degree; if however the resistance be due to increased viscosity the stress should be felt on both sides of the circulation alike. It is to be desired that in future the state of the several chambers of the heart should be carefully noted on Müller's method. It is also to be desired that when in such cases the corpuscles are reduced in number, as for instance under therapeutical methods, that the effect on blood pressure, and, if possible, on velocity, should be accurately noted. We have seen that in granular kidney, unless in the earliest stage of it, the viscosity of the blood is not much increased, if at all, ${ }^{3}$ a notable point when we consider the polyuria of the disease; now it is in granular kidney that hypertrophy is developed often wholly, always very preponderantly, in the left ventricle. It may seem surprising at first sight, if the corpuscles be so large a factor in viscosity, that in the cases of heavy poly-

1 Hutchinson and Miller, Lancet, London, 1906, i. 744.

2 Bruce and Alexander, Journ. Mental Sci., London, 1890, xlvi. 725.

3 Beck and Hirsch, Arch. f. exper. Path. und Pharmakol., 1906, liv. 54. 
cythæmia to which I have referred the arterial pressures do not rise enormously; certain compensatory adaptations seem to meet the stress, and among them vasomotor dilatation must take a principal part. I have pointed out how large must be the effect produced by quite a small increase of arterial radius, if over wide areas; yet even if such a dilatation do yield more than a cubic increase of space, the extra burden of the heart may not be annulled thereby. ${ }^{1}$ How perilous, how mortal, enormous increases of the corpuscles, or relative increases, may be, is illustrated in phases of certain colliquative diseases, such as cholera; conditions in which the marvellous effects of intravenous injections of saline water in reducing the viscosity are likewise conspicuous; now these cases are probably but eminent examples of an injurious process which, in many diseases, in certain fevers for example, may constitute a considerable if occult cause of danger to life.

I have already hinted that in the viscosity of the corpuscles there is more than the mere mutual friction of multitudinous bodies. We are apt to forget in the discussion, not only of this mechanical question, but also of associated physical questions, that the surfaces of the corpuscles being bathed in the plasma, and wet with the colloid fluid in which they are suspended, partake so far of its qualities. But there is a simple method of ascertaining by elimination the mere mechanical conditions of these solids, this is by laking the blood; in laked blood we have an opportunity of observing what effects, other than by mechanical suspension, the corpuscular elements may have on the physical reactions of the blood; by their stroma, for instance, their hæmoglobin, their gaseous content, and so forth. Determann, ${ }^{2}$ Adam, ${ }^{3}$ Hess, Blunschy, ${ }^{4}$ have given especial attention to these functions, which seem to be after this manner. Laked blood proves to be not less, but more, viscous than normal blood; for this reason, that the corpuscles contain within them a substance more viscous than the plasma, which substance, while within, is prevented from exercising this property to the

\footnotetext{
1 Hess, "Viskosität d. Blutes und Herzarbeit," Vierteljahrsschr. d. Naturf.Ges. in Zürich, 1906.

2 Determann, Zeitschr. f. klin. Med., Berlin, 1909, lxviii. 177, and 73 (3-4).

3 Adam, loc. cit., Berlin, 1909, lxviii. 177.

- Blunschy, quoted by Krone and by Determann.
} 
full. Thus it falls out that when laked the molecular friction in the relatively homogeneous fluid is higher than between the corpuscles and the serum; the stroma, segregating into granules (Adam), sets up more friction than do the bodies of the intact corpuscles themselves. On removing these suspended granules by the centrifuge Adam found the friction reduced.

But this is not all; by another series of experiments these industrious observers proceeded to demonstrate that, even after the removal of the stroma, there was still a notable excess of viscosity ; and this they have traced to the compound coefficient of hæmoglobin and gas content. Oxygen or carbon dioxide, gases which produce no effect of viscosity upon plasma deprived of corpuscles, affect its viscosity instantly when to it are added crystals of pure hæmoglobin. We see then that in laked blood this compound factor comes into full activity, and how important it is in these comparisons to take heed, when testing viscosity, that the blood be taken from a standard area; venous, capillary, or arterial.

Here comes into view a most important principle in disease, especially of disease of the lungs or heart ; namely, that excess of carbon dioxide ${ }^{1}$ means excess of viscosity. This may be quickly demonstrated on the same person by comparing the blood from a simple finger puncture with another specimen taken after producing local cyanosis ; but observers of cyanotic blood do not, or often do not, remember to compare the specimens in respect of cell content: now we know that $\mathrm{CO}_{2}$ alters the number, the form, and the surface of the red cells. However, this rise of viscosity is demonstrable in vitro where the number of corpuscles is constant. It seems fairly certain that in defective oxidation, in mitral insufficiency for instance, at the moment when the velocity of the blood should receive a compensatory acceleration, its viscosity is rising; thus the burden of the heart increases in a vicious circle. $^{2}$ In cyanosis it is a familiar experience with me, as I doubt not with my brethren,

1 Determann, Die Viskosität des menschlichen Blutes, Wiesbaden, 1910; and Hess, Münch. med. Wochenschr., 1907. (The gas content of various bloods was estimated on Barcroft's method.) Burton-Opitz, "Visk. d. Blutes," Pflügers Arch. Bd. cxix. Hefte 6-8.

${ }^{2}$ See Bence, Julius, Deutsche med. Wochenschr., 1905 ; a careful series of experiments.

voL. I 
that although on the mere mechanics of pulmonary respiration it may not always be easy to explain the beneficial action of oxygen inhalation, yet in many instances the patient clings to his oxygen-pipe with affection, or even with desperation. Now we know that in suffocation, as in narcosis, tracheal stenosis, and the like, the blood viscosity is extremely high ; if, therefore, access of oxygen favours an attenuation of the viscidity of the blood, it should thereby ease the heart and forward the bloodstream; if accumulation of carbon dioxide makes for an increase of viscidity and a sluggish current, the comforting effect of oxygen inhalation becomes intelligible. The principle can be demonstrated readily as follows:- Some blood is drawn from a vein and, to prevent coagulation, a very little hirudin is added, and the viscosity tested. It is then shaken in air, to oxidise it, and the viscosity again measured, when it will be found to have fallen considerably. And, besides this relief by oxidation, we may presume in the body some further fall in friction by a diminution in the number of red corpuscles; for these in defect of oxygen multiply, in abundance of it are reduced in number. In heart disease von Korànyi has shown by blood-counts that inhalations of oxygen and a rise of tension of the gas in the blood are followed by a fall in the number of reds. Ströbell's ${ }^{1}$ experiments indicate that in chronic tracheal stenosis the heart becomes hypertrophied.

It would seem indeed that of all factors of viscosity carbon dioxide is the chief, for the changes in viscosity by these gases are less of the plasma than of the corpuscles; so that in diseases of the heart and lungs, as Determann says, a vicious circle is established of plus viscosity, plus resistance, plus $\mathrm{CO}_{2}$, and so round to plus viscosity again-a gyration which the heart may be unable, by increasing the pressure head, to break through. ${ }^{2}$ Furthermore, as tissues are declining to hydration and acidification, at their surfacesalbumin is ionised and friction thus increased. To this $\mathrm{CO}_{2}$, a weak acid, contributes; and as in the presence of other acids its tension rises there is less alkali to spare for its removal. This factor must tell heavily in fevers in which the alkalinity of the blood is reduced; still more in "acidosis";

1 Ströbell, Zeitschr.f.d.ges. exp. Med. vol. i., 1913 (quoted Zentralbl.H.u.G.).

${ }^{2}$ On this aspect of the matter, see also Bence, "Visk. d. Blutes," Zeitschr. f. klin. Med., 1906, vol. Iviii. 
and not a little in heavy muscular exertion with lactic acidification. ${ }^{1}$ Thus, as in life there is no direct correspondence between arterial pressures and viscosity, we have seen indeed that they may move contrariwise, we have assumed some large compensatory factors of another kind, probably vasomotor, of which as yet we have no accurate knowledge. The problem then is far from solution, and will be until we have some clinical means of ascertaining cardiac output, velocity of current, total mass of the blood, and breadth of peripheral bed. ${ }^{2} \mathrm{We}$ may suppose however that cases of high pressure plus viscosity would favour cerebral hæmorrhage; of low pressure, thrombosis.

Now although, as we have seen, under these conditions the corpuscular changes are a main factor in viscosity, yet the behaviour of oxygen and carbon dioxide are not quite reciprocal. And viscosity depends so far on the state of the plasma that it cannot be measured simply by the number and sizes of the corpuscles; though presumably the blood as a whole grows more and more viscous as it approaches the lungs. Oxygen combines with the hæmoglobin to form oxyhæmoglobin, but is not very stably fixed; while carbon dioxide attaches itself not only to the reds but also to constituents of the serum, as to mineral alkali and protein bodies. Hydræmia, again, must often be an interfering factor, and one difficult to estimate (see Martinet, p. 143). An excess of $\mathrm{CO}_{2}$ favours the penetration of chlor-ions and water into the corpuscles, with a corresponding movement outwards of albuminoids, and consequent rise of viscosity dependent in part on increase of colloid in the plasma, in part on swelling of the corpuscles; at any rate such is the reaction in vitro, and it is consistent with certain experiments in vivo. And, if we may have ascertained the values of these factors for the blood in general, we must still remember that the states of the blood, and the interchanges between blood and tissues, may vary widely in the several areas. Again, although any two or more qualities of the blood taken separately may

1 See Bechold, Die Kolloide in Biologie, 1912.

2 Haldane and Lorrain Smith's carbon monoxide method is not yet for clinical work; and that of von Plesch, if more readily applicable, seems to be open to some adverse criticism; vide Müller, Deutsche Arch. f. klin. Med., 1909, vol. xcrii. p. 559. 
lie within normal limits, yet their quotient may be abnormal ; as illustrated by Hess's statement, already quoted, that even if hæmoglobin and viscosity taken separately may lie within normal extremes, yet if these figures divided give a figure which does not fall within 17-21, the blood is of abnormal composition. Bachmann, ${ }^{1}$ working with Hess and Eichhorst at Zürich, gives

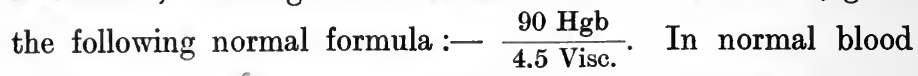
observers concur at present in stating that viscosity and hæmoglobin ought to run almost parallel; but that in disease this relation is often disturbed.

In the next place we may discuss the saline constituents of the blood, which leads to another set of conditions, conditions affecting both corpuscles and plasma ; namely diffusion, osmosis, electrolysis, and the calculation of the crystalloids of the bloodto certain laws, that is, of chemical physics. ${ }^{2}$ It is by the disaggregating energy of salts in solution on the proteins that the activity of life is maintained; and the inorganic salt solutions, being less viscous than the organic-their diffusion rate from a limiting surface being higher, have more disaggregating capacity; their action is a function of this viscosity and of the surface tensions. The higher the surface tension and the viscosity the weaker, of course, the disaggregating action. Salts have, of course, other disaggregating capacities; some of them have, for instance, varying measures of solvent action on globulins. ${ }^{3}$ And the well-known rise of blood-pressure obtained by injection of normal salt solution is said to be accompanied by an increase of corpuscles (20-30 per cent), apparent or real.

Again, a very important condition of life must be that of adsorption of salts, as for example of chlorides, the greater as the surface tension of the solution is lower, on surfaces of colloid molecules; which property by "sterical inhibition" makes for storage of energy. It prevents the chemical interactions of the large colloid molecules, and prevents, or moderates, condensation and precipitation. The reactions of toxins and antitoxins may

1 Bachmann, Deutsche Arch. f. klin. Med., Oct. 1908; a careful paper with observations on 400 cases.

2 See Roger, "Introd. à l'étude de la viscosité du sang," Arch. de méd. expér., Sept. 1908.

${ }^{3}$ See various papers by Hardy and Mellanby. 
depend more or less on adsorption phases. ${ }^{1}$ Thus the ordinary interchanges of chemical mass action are inhibited, and the time rates more or less retarded ; these rates are now closely occupying the attention of chemists, every reaction probably having its own time rate, depending on concentration, temperature, cataclysts, etc. It is probable that these time rates are of the inner secrets of biology ; for instance, ordinary mass action may depend upon the optimum times and quantities of fresh supplies, and on several specific reaction velocities, as of ferments. Thus by subtle measurements we mightobtain a modulus for carbohydrate, protein, and fat changes respectively, and in the presence of each, as of protein change in the presence of carbohydrate; and so obtain some sort of rules for practical service. The primary factor of osmosis is that, of a number of different substances in solutions of equal percentage strength, the solution of substances of small molecular weight contain more molecules than those which contain substances of heavy molecular weight. In the normal body the salts, in their quantity relation to the other ingredients, preserve a remarkable equilibrium. Besides Ringer's proof that calcium was necessary for rhythmical motion, we know also that it is necessary for the building up of protein complexes in cell-nuclei ; substances, therefore, which precipitate calcium from these cells are poisons. ${ }^{2}$ An excess of calcium ions which, by their electric charges and mobilities, fortifies systole, pushes up tone, even to the arrest of the heart in systole. Sir James Barr sent to me, on February 6, 1911, the two following notes :-in J. B., after the calcium content of the blood had been raised, the visc. was 6.45 ; cap. velocity $4 \mathrm{~mm}$. in $\frac{3}{5} \mathrm{sec}$. ; calcium index 0.5 ; urine normal, slight excess of lime: in P. H., visc. 7.06 ; cap. velocity $4 \mathrm{~mm}$. in $1 \frac{2}{5}$ sec.; calcium index 0.8 ; urine 1010 acid, slight excess of lime. When in vain we try to realise the vast extent and diversity of surfaces throughout the infinite channels and recesses of the body, we shall not be surprised to hear that surface or osmotic tension has been regarded as the

1 I may note here in passing the very curious but as yet incomplete observations by Grünbaum and Coplans at Leeds on filtration through sterile asbestos.

2 As bearing in an interesting way on these chemical laws I may refer to Sir James Barr's lecture on Rheumatoid Arthritis in the Brit. Med. Journ. for April 12, 1913. 
overruling mode of energy in the organism, plant, or animal. For instance, the opinion is prevalent that muscular activity is not comparable with an internal combustion engine, but consists rather in differential surface or osmotic reactions between the colloid contractile elements and the more saline sarcoplasm. If, by bathing in isotonic sugar solution, the salts of the fluid parts be extracted the muscle is reduced to inactivity; by returning its salts its activity can as readily be restored. ${ }^{1}$ Thus, while the vast stream or system of energy is working between the inward and the outward, the molecular forces just indicated are striving within, and with confederate intensities almost as universal.

What part filtration may play in these animated processes is uncertain, for the data of relative local mechanical pressures, and of the distribution of gases, are out of our reach, at any rate at present. We recognise filtration in certain areas, as in the renal glomeruli, though the glomerular function is not one of mere filtration; and it may be no inconsiderable factor elsewhere; but diffusion and osmosis are universal, and govern the balances of viscosity. The enormous pressures osmosis brings into play may almost reduce even gravity to a negligible quantity. If evaporation from the leaves of a tree keeps up a negative (ascending) pressure, it does but aid osmosis in the ascent of the sap; and such is the potency of osmosis in the simpler animals. Thus the nutrition of the cells and the mechanical ease of the heart and circulation are favoured by isoviscosity and isotony, as these in their turn are regulated by the bywash of the secretions and excretions, and are probably under some influences of innervation. And these reactions with the tissues are by no means confined to blood and lymph, but also in the blood itself are incessantly at work between its plasma and its corpuscular elements. Such here and there are the peeps we may get into the intense and immeasurable reciprocations of these biological coefficients in the healthy body.

Chemical analysis on the ordinary method, although it gives us indispensable information as to the ultimate and mediate components of the blood and lymph, throws no independent light upon their physical attractions and repulsions, and gives

"I may remark here that "normal saline" is usually a little too dilute; the physiological balance at which the red corpuscles remain unaltered is $\mathbf{0 . 9}$. 
little insight into the physical and electric vibrations which constitute the dynamics of these fluids. For instance, many enzymes, which may be colloidal, elude measurement and even separation. We shall see however that we are not without some way of measuring these activities, for in electrolysis we have a means of ascertaining approximately the rate of osmosis, and indirectly of molecular friction. Hampered as we are by the imperfection, or alleged imperfection, of viscometers, it is well to attack these problems also by way of the electric resistance of the blood. Dr. Dawson Turner, of Edinburgh, ${ }^{1}$ made a number of experiments to ascertain the time occupied by sodium chloride to reach the blood, the mean normal resistance at $15 \cdot 6^{\circ} \mathrm{C}$. being $550 \mathrm{ohms}$ (Turner), the specific resistance 93.5. In five cases the blood-resistance was measured before taking 30 grains of the salt, and at five minutes' intervals afterwards. The average time taken for the first lowering of the resistance of the blood was $\mathbf{1 5 . 4}$ minutes, and for the maximum effect 21.4 minutes. Dr. J. Edmunds, of Ann Arbor, also told me (in September, 1907) that by interposing a length of the femoral artery between two hooks connected with positive and negative poles, and recording the variations of conductivity of the blood, he was enabled to observe the intervals, which were very brief, between his injecting a saline solution into the jugular vein and the consequent rise in the conductivity of the blood.

The tests of freezing and boiling points, as we shall see (p. 141), have proved disappointing in practical medicine. For instance, the milk and the serum of the cow have the same freezing-points, but the electric conductivity of the milk, because it contains relatively more organic molecules, is lower. Besides, not even all the inorganic particles in these fluids are in full electric activity, for some, in combination with proteid substances (Loeb's ionproteids), exert less electromotive and osmotic energy or even none. It is not the number of ions but their rapidity, their driving power against the indifferent colloids, which is of working importance. And here we observe again the irrelevance in these measurements of the specific gravity (p. 121). Speaking generally, colloids are of low electric conductivity, salts of high conductivity; but salts, as we have seen, are far from being of equal effect on

1 Turner, Dawson, Nature, London, 1902, lxvi. 127. 
viscosity, or conductivity, or freezing-point. We have seen that the specific ionic effects of the various salts are far from identical ; some raise viscosity, others, such as the iodides, reduce it. So that the concentrations in blood and urine must be taken of each constituent. ${ }^{1}$ The salts incessantly bombarding and penetrating the colloids thereby promote the various dynamic activities in the fluid, from diffusion onwards. Thus viscosity falls; and at temperatures higher than the atmosphere, but under that of incipient coagulation, these operations are more and more active. Globulin, insoluble in water, becomes soluble in weak saline; and Pauli, ${ }^{2}$ obtaining by careful dialysis a neutral albuminous solution, found on adding to it gradually small increments of salt (sodium chloride) that the viscosity did not rise, but fell. He explains this paradoxical reduction of inner friction on the addition of solids by supposing that particles of salt, attaching themselves to the albuminous molecule, form a more slippery albumin-salt-ion combination. Yet, as we shall see, in practice the viscometer fails to register any definite fall in the blood of patients under treatment with the ordinary doses of iodide of potassium. We cannot, it is true, regard the flow in glass-tubes as identical with that in the finer arteries, but the approximation is supposed to be fairly representative; it may be that in the body the exchanges between the fluids and the cells, and the vasomotor readaptations, are so rapidly compensatory that we fail to catch the oscillations. And if under iodide of potassium no fall of arterial pressure has yet been proved, the belief of clinical observers, for what it may be worth, that by the administration of this salt the circulation is eased, is notwithstanding very prevalent.

Diffusion, the passage through intermolecular fluid spaces, as contrasted with osmosis, which is selective passage through the interstitial spaces of a porous solid, is, under the circumstances of which we are speaking, a universal factor; and in colloid fluids is particularly efficient, for thick fluids of differential densities lend

1 A. V. Hill, in Barcroft's recent work.

2 Pauli, Kolloidchemische Studien an Eiweiss, Dresden, 1908; and Kolloidchemische Zeitschr. Bd. iii. (I have not seen either of these papers, but they are freely quoted in other essays and handbooks; e.g. Philip, Physical Chemistry, 
themselves less readily to mere mingling. Other things equal, the diffusion depends on viscosity; the more viscous the slower the reaction. It is by diffusion only that the stationary outermost cylinder of blood which wets the wall of the vessel is continually being changed. It is true that colloids are of low diffusibility, but by the disaggregating salts their diffusion is promoted, and the physical chemist finds that, in respect of diffusibility, substances in solution behave as gases. Thus by variations in kind and quantity of saline content, some salts being positive in respect of viscosity, others negative, continual activity in the blood is provoked; the proteid or colloid particlesare continually sliding along lines of least frictional resistance and, as it were at their thin edges, passing fractionally from suspension into solution. In a particular area of the circulation diffusion depends also of course on the velocity of the stream in that area.

To attempt to pursue the several lines of these researches -the mechanical, the electrical, the chemical-would carry us beyond our present purposes; it must be accepted that in the process of biological analysis the separation of the modes of energy by which the tissues of the body are woven, repaired, and demolished, and the concentration of our attention upon each severally, is an arbitrary convention by which we endeavour, and indeed are enabled more or less, to apprehend and discriminate the lines and degrees of energy; yet in these partial reckonings we are not to forget the whole, not to lose comprehension of the blend of these various aspects, the merging of these functions the one into the other, the unity in complexity. Thus, in passing on from the mode of energy which we have called diffusion, the steps by which we advance to osmosis are imperceptible; and onward again in the discussion of osmosis-i.e. the selective absorption by membranes from fluids, as contrasted with filtration through hypothetical pores-we soon find ourselves involved in certain other functions, or aspects of function, such as the electrolytic or refractive coefficients which under analysis must receive their several expression and recognition; so again under certain other conditions, such as resistance pressure head velocity, and with certain other properties also, such as volume molecular and atomic weight, and so forth, we are dealing in 
many forms of ubiquitous and protean but harmonious modes of energy, incessantly transforming themselves the one into the other. Thus it is that we often interpret one by the other, as osmosis by refraction, or by electro-conductivity; and thus, as I have hinted already, by electrolysis we obtain one more sorely needed method of measurement, electro-conductivity and osmosis being terms either mutually convertible, or of similar significance; the electro-conductivity of a fluid being an index of inner friction. Although some years ago Professor Starling, and other observers later, showed that colloids have, or may have, some slight electro-conductivity, yet, generally speaking, they are nonconductors, and their inner friction is high; without the impulses of saline ingredients they have no osmotic pressure, or nearly none; and by this virtue of its colloids it is that the blood is protected against the continual fluctuations in the densities of its saline constituents. We have seen moreover that while some electrolytes lower the viscosity of colloids, others raise it.

Now, to pass from the blood itself, we come to consider the relations of this complex fluid to the multiform cells of the body; for between these and the fluids, as Professors Waymouth Reid, Loeb, and others have shown, there is an incessant play of specific alternating phases, according to the differences in specific endowment of the cells, differences which, for lack of a better word, we may call for the present vital; differences at any rate of internal structure whereby they exercise selective functions so nice that only certain kinds of reciprocation, or of physiological permeability, ${ }^{1}$ can take place in each area; thus the kidneys select some ingredients, the pancreas others, the gastro-intestinal canal others, and so forth. A remarkable example of these "vital" peculiarities is the acquired osmotic impermeability, for obvious purposes, of the healthy epithelium of the bladder in situ; an adiabatic state which fails when the coat is injured or deteriorated. ${ }^{2}$ When salts are held against the apparent disposition of osmosis, this attitude may be maintained by surface tension. To remove the living epithelium from

${ }^{1}$ Vide Boruttau, loc. cit., 1908, p. 106, "Die einzelnen Zellarten specifischer Vermittlung," etc.

${ }^{2}$ Upon the function of lipoids as investments of, or as clews amid, the molecules I have not touched ; experts are not as yet in substantial agreement on these points. 
the intestine may make absorption not easier but more difficult. Again, in like manner it is not easy on physico-chemical grounds to understand the transfer by the renal membranes of the urea from the blood, in which the urea is in very low dilution, to the urine in which its proportion is high; or again, their refusal of sugar, which is more abundant in the blood than urea. Another physiological idiosyncrasy we shall note presently in the red blood-cells. The specific build and reaction of the sundry tissue cells, the peculiar and complementary function of each, and the consequent various, but in its concert complete and harmonious, play with the aggregate of the many foods, and the mutual phases of the nutritive juices, give us a notion, however inadequate, of the enormous power which these physico-chemical, and apparently also peculiarly physiological, processes incessantly at work in the body must exert, even under small changes of salt content; a notion likewise of an equilibrium so perfect in its evolution that, whatsoever the variety of cell-structure and operation, of changing blood content, of the strife of opposing tensions, of the calls of the hormones, of the interventions of the nervous system, of the affairs of the whole body engaged in its outer world, the oscillations in health are so small, and balanced so perpetually, as to maintain not only stability but a practical equilibrium; such an equilibrium, indeed, as to baffle the investigator in his endeavour to analyse it. Throughout this vast area of surface, and field of reciprocal function, there is practically isosmosis and isoviscosity; although on the balance we must presume a slight proclivity of osmotic pressure from tissues to blood in its mean between arterial and venous; and that the lymph on the balance must be slightly less viscous-less colloid-than the blood.

And as with these solids and fluids as wholes, so with their parts; as between the corporeal cells and fluids, so between the blood corpuscles and the plasma, a continual osmotic reciprocation is in play. Among their other endowments the corpuscles play a considerable part in the balances and reserves of the whole blood. Permeable to some inorganic ions and not to others, under such influences they change in form-swell or shrinkaccording to the swings of plasmatic constituents or their own lability; so that on account of its corpuscles the viscosity of 
the whole blood varies more than that of the plasma. We have seen that much of the viscosity depends on hæmoglobin and gas content; ${ }^{1}$ on the addition of $\mathrm{CO}_{2}$ the chlor-ions penetrate from the plasma into the corpuscles, so that their water content increases, and the molecular concentration of the plasma increases. Sodium chloride is one of the most active ionizing and osmotic agents in the plasma. The plasma is more electro-conductive, the corpuscles are more colloid; and, as already stated, are permeable to some salts, not permeable to others. At present it would seem that by some physical or biological endowment of the corpuscular edge, or "membrane," the inorganic constituents of the cells and the plasma stand various and unequal ; the corpuscle being rich in potassium and phosphate, the plasma in sodium and chloride. ${ }^{2}$ It is at this boundary, in its origin almost ideal, that we note the initiation of inhibition, that fundamental character of organised life, of survival by an economic faculty preventing dissipation of energy, and storing it for further and larger ends. For this auto-limitation, viscosity and friction and, as we have seen, adsorption, putting the brake upon physico-chemical processes, give the conditions. ${ }^{3}$ And it is here that for a moment we may glance again at one of the most practical problems of the clinical physician, at the resources of the body, its reserves or potential. In advancing years we suspect that the tissues become less permeable; osmosis is less active, less free : for to equalise the rises and falls of viscosity, or osmotic tension, the cells must be readily pervious to water. For example, in laboratory experiments with yeast the cells must be fresh, " hatched" within an hour or two ; cells of the day before are far less sensitive.

It has been said that for reserve or discharge the secretions and excretions by their fluidity or viscidity play a large part in equalising viscosity, balances in which the nervous system must play a large but incalculable part; of these agencies the

1 Adam, Zeitschr. f. klin. Med., Berlin, 1909, Ixviii. 177.

2 Boruttau, Med. Physik, 1908; Philip, Physical Chemistry, 1910, and other authors on this subject. The observations of Barcroft and Orbeli (Journ. of Physiol., Jan. 1911) on the effect of lactic acid also in sweeping out low-tension oxygen from the tissues, are very interesting.

'For an able study of these phases see Garrison, "Physiology and the Second Law of Thermo-dynamics," N. York Med. Journ., Sept. 25, 1900; and papers by Barcroft and by Hopkins, published after my text was printed. 
function of the urine must be very important, sharing indeed with the respiration the chief part; to the urine therefore no little attention has been given, and much help was anticipated, by von Korànyi and others, from cryoscopy in estimating these exchanges. Unfortunately, Sir John Bradford, ${ }^{1}$ Sir James Barr (in private letter), Sahli, ${ }^{2}$ Winternitz, $^{3}$ and other careful observers, have found its indications, which of course fluctuate largely in health, unavailable, at any rate in this respect; as likewise those of electro-conductivity (p. 321). It tells us no more than the concentration at the moment of the dissolved substances, we get no more out of it than out of specific gravity. To weigh the ashes from a locomotive does not go far in a computation of its efficiency. For instance, if the kidneys fail to carry off the waste products the molecular concentration of the blood, and therefore its freezing-point, should rise, and its electro-conductivity fall ; but other variables are such that even in renal default this is not regularly the case; and cryoscopy of the urine, and conversely of the blood, fail to tell whether after operation on one kidney the other is healthy or not. Now to test the concentration of the blood, and so indirectly the renal efficiency, by cryoscopy needs a venesection of $20 \mathrm{~cm}$.; and even then it seems to me impossible to exclude falls of concentration due to variations of cardiac energy, or other causes of rising venous pressures. If electroconductivity should fall independently of the freezing-point it would suggest a retention of organic molecules. A little proteid, however toxic, would scarcely alter the freezing-point. The freezing-points of the whole blood and of the plasma are practically identical; suspended particles make no difference ; quartz dust, for instance, suspended in a similar solution makes no difference ; but the conductivity, on the contrary, of the whole blood is but half that of the serum from which the corpuscles, which obstruct conduction, have been removed.

Of the effect of glands and hormones upon viscosity we really know nothing. On the sum of such data as these which

1 Allbutt and Rolleston, System of Medicine, edit. 2, iv. pt. i. 535.

2 Sahli, Klin. Methoden, edit. 5, 1909, ii. 748. Sahli criticises von Korànyi's methods as fallacious.

3 Winternitz, in Krause's Lehrbuch, 1909, 259-60 
we have been considering $\mathrm{Du}$ Pré Denning and Watson ${ }^{1}$ formed the provisional opinion that viscosity seemed to be not an independent but a dependent variable; one not dominant in the circulation, but by its adaptive changes contributory to the maintenance of its consistency and efficiency. At present indeed it is not clear from what has gone before that in pathological states its fluctuations are always beneficial; but with so much in the evidence that is obscure, or even contradictory, we must for the present hold our judgment in suspense. From these inconclusive data and still speculative notions, vitally important as the facts may be, and enlarging as these concepts are to our physiological vision, it must be admitted that few principles can as yet be formulated for application to practical medicine. Although, as Hess says, ${ }^{2}$ (other things being equal) a stickier blood must increase the burden of the heart on both sides, yet it is true that this variable quality may be met by so many readjustments as to be virtually a constant. If a thicker blood flow more slowly water will soon gather in the body; if a thinner and less valuable blood flow more rapidly the urinary effluent will soon be increased, and the normal viscosity restored. Yet a thinner blood, if, as is probable, it be in larger mass, and if, as less nutritious per unit, it be run at greater speed, and with equal aortic pressure this would be the case, the heart would be no less burdened; though not necessarily under any rise of blood-pressure. I am still disposed to think that a superviscous blood may be concerned in some modes of arterial stress.

Upon the influence of the nervous system on viscosity it is difficult to say more than that it must be considerable. In another chapter I have referred to experiments showing that certain sections of the spinal cord have a remarkable effect in determining the apparent number of cells in the blood. To stimulate the cord " high up" may gorge the capillaries with red corpuscles; and to stimulate the vasomotor system directly increases the viscosity, probably by the same mechanism.

However, further research on this subject will surely lead to

${ }^{1}$ Du Pré Denning and Watson, Proc. Royal Soc., Oct. 24, 1900 ; and No. 78,1906 .

${ }_{2}^{2}$ Hess, Deutsche Arch. f. klin. Med., 1908, xciv. 404. 
some clinical and therapeutical results. For there is evidence that in abnormal states these readjustments, in health so ready, in disorder may lag; it is alleged, for instance, that in the state of excessive blood-pressure described independently by Huchard and by myself in 1893-94 as precursory of arteriosclerosis, and named by me Hyperpiesis, by Huchard Presclerosis, the viscosity of the blood is, or may be, raised ; the observations on this point are however as yet too few, and too lacking in authority, to establish a rule (p. 118). But since this chapter first appeared Martinet, ${ }^{1}$ in an able treatise, by the French love of symmetry and logical completeness made a little too plausible and trim, has published some further researches and many clever diagrams, on the relations between viscosity and blood-pressure. He used the instrument of Hess. He concluded that the physiological variation is within very narrow limits-say, 0.3 to 0.4 , at most $(3 \cdot 8$ to $4 \cdot 5)$, but that the pathological limits are wider by far : in one individual, and within a few days, he recorded a rise from 5 to $7 \cdot 8$. In his experience the pathological limits were 1.9 and 7.8. Martinet lays much stress upon watery dilution of the blood, and accordingly on the efficiency of renal filtration and pulmonary exhalation-the degrees of dilution of the proteids being determined by refractometric methods. In chronic renal disease therefore some cases present a plus, others a minus, viscosity-points already brought out by Hirsch and Beck, ${ }^{2}$ Lommel, ${ }^{3}$ and Determann (loc. cit.), but which are all the clearer for this verification, as they bear closely upon our clinical problems. For instance, of 22 cases of excessive blood-pressure, whether renal or hyperpietic, four had a normal viscosity and seven a plus viscosity (an addition to the burden of the overlaboured heart which cannot be overlooked). Ambard's researches on the urates in the urine and the blood respectively bear closely on these postulates (p. 321). In "plethoriques simples" again the viscosity and the pressure were both in excess, and these cases offer a favourable prognosis; if the viscosity be

1 Martinet, Pressions artérielles et viscosité sanguine, 1912.

2 Hirsch and Beck, "Studien d. Viskosität," etc., Deutsche Arch. f. klin. Med. Bd. Ixix., 1901; and Arch. f. exp. Path. u. Ther. Bd. liv., 1905.

3 Lommel, Deutsche Arch. f. klin. Med., 1904, lxxx. 308 (quoted by Krone, in Deutsche med. Wochenschr., 1910, xxxvi. 1438); and Münch. med. Wochenschr., 1908, No. 6. 
low we have to do with renal default. In seven the viscosity was reduced; chlorides and water were retained; the blood was diluted, and the tissues were wet. In valvular, say in mitral, disease, as systole is in default and venous pressures rise, $\mathrm{CO}_{2}$ would raise viscosity, while hydræmia ${ }^{1}$ would lower it. Yet we admit that the curves of arterial pressure and viscosity do not run parallel; the distribution of the cell elements in the blood must come also into the reckoning. As pressure falls the refractometric signs and viscosity rise together as $\mathrm{CO}_{2}$ accumulates, until-say under digitalis-diuresis may set in, when arterial pressures will rise, and viscosity fall in respect of respiratory exhalation of $\mathrm{CO}_{2}$, but rise in respect of the escape of water by the kidneysa complicated sum. Briefly, he says, minus pressure and plus viscosity mean pulmonary insufficiency and venosity. Hess, for some time past, has laid great stress upon the coefficient of pulmonary exhalation; in pulmonary disease of course, this balance must be more or less gravely disturbed, and more work thrown on renal filtration. All observers agree that hydrogogue purgatives raise viscosity, while, caeteris paribus, both maximum and minimum pressure fall (unless tissues water-logged). In one of Martinet's patients, in a mitral asystolic phase, under digitalis a three days' diuresis set in, and the cedema disappeared; the urine fell then to the normal quantity and the viscosity of the blood ran up, for three days, to 5.8 and even to $6 \cdot 0$. Martinet thus obtains three formulas:

+ PR. and + Viscosity = plethora sanguinis (hyperglobuly or glykæmia).

-PR. and - Viscosity = hypoglobuly (anæmia).

+ PR. and - Viscosity = hydræmia (renal insufficiency).

The iodides, he says, are useful when pressures are high and viscosity also, but bad if pressures be high but viscosity low. In the former cases free dilution is required, but not in the latter; good points, if duly verified, for therapeutics. Martinet opines that in cases commonly called plethoric (my hyperpiesia) the plus viscosity is due to plus red corpuscles-say 6 to $8,000,000$ (p. 260); but of any special relation of polymorphs to viscosity or coagulation time, or of Austrian's observation (loc. cit.) that although

1 Hydræmia must not, of course, be confused with hydration, which has, probably, but a slight influence on viscosity, if any. 
in chronic nephritis the viscosity of the blood may fall (hydræmia), yet in uræmia that of the plasma rises (due to retention products? -Holmgren, p. 110), a clinical point of much interest, he says nothing (see remarks on Giesböck's cases (pp. 256-60). Austrian found the viscosity coefficient at least normal in thirteen renal cases out of twenty-two; and in some high-pressure cases he had seen it even minus. For Martinet then the chief determinants of viscosity are pulmonary and renal efficiency. He gives an interesting sketch of the cyanotic habit, of persons with weak hearts and blue or livid extremities; torpid, shivery, and venous: in these he says viscosity rules high.

The difficulty of attaining to exact knowledge in this subject is illustrated by the uncertainty which in these respects still surrounds the effects of iodide of potassium upon the arterial circulation. We have noted how tenaciously, in spite of the lack of definite evidence of this virtue, medical practitioners cling to the prescription of this salt in "arteriosclerosis" ; and this in justifiable disregard of pharmacological opinions-justifiable, that is, if it appears to them that the clinical effects are decisively in its favour. Yet, so far as observation of normal men and animals has gone, little evidence of the effect of moderate doses of iodide of potassium in reducing viscosity of the blood has been obtained. Ottfried Müller and Inada, ${ }^{\mathbf{1}}$ whose researches were carried out under Romberg's supervision (Hirsch and Beck's instrument), found that the iodide had a "favourable effect upon arteriosclerosis," 2 not by any effect upon the vessels, but upon the blood viscosity, which in 9 out of 11 men was reduced in degrees from $1 \cdot 7$ to $8 \cdot 3$ per cent. They stated, moreover, that they had demonstrated a gain of four seconds in stream velocity. But on the other hand Adam, ${ }^{3}$ who administered potassium iodide in full doses-i.e. 45 grains a dayfound a reduction of viscosity in only 6 of 30 , and that doses of 20-30 grains made no difference at all. Determann likewise has been unable to detect any reduction of viscosity under

1 Müller (Ottfried) and Inada, Deutsche med. Wochenschr., 1904, xxx. 1731 ; and Ther. $d$. Gegenwart, 1906, 360-361; see also the reports of the Kongr. f. inn. Med., Wiesbaden, 1909.

2 Romberg, Lehrbuch d. Herzkrankheiten; who upon this ground recommends iodide of potassium in coronary sclerosis.

${ }^{3}$ Adam, Zeitschr. f. klin. Med., Berlin, 1909, lxviii. 177.

VOL. I 
the salt. ${ }^{1}$ He says that, if the iodides be useful in high blood pressure, the explanation must be sought elsewhere. In vitro it is well known that iodide of potassium reduces viscosity in watery solutions of serum-albumin, and in blood plasma; and we have recognised the large effect which a relatively small reduction would have on the whole blood. Müller, in response to those criticisms, returned to his experiments ${ }^{2}$ and reaffirmed his conclusions. He gave about 4-6 gr. of P. I., three to five times a day, with an equal quantity of bicarbonate of soda, for months-say a year-but with eight to ten days' pause every month. He warns us that near the iodide ingestion food must be avoided lest iodine be set free and upset the stomach. The observations of Martinet I have just quoted (p. 144). This conflict of evidence as to the effect of the iodides may be due in part to a time difference; or possibly to an alleged effect of potassium salts, when injected into a vein, of constricting the arterioles. This property, if it exist, appears not to have been taken into consideration. In the discussion at the Wiesbaden Congress (1909) to which I have referred, Umber of Altona said that in his wards Jorns, in a long series of cases, had noted reductions of viscosity on administration of the iodides, not in very large doses but over long periods of time. Boveri ${ }^{3}$ administered iodine compounds to twelve persons with " hypertension," some with and some without arteriosclerosis, and tested the whole blood, and the serum, at intervals of 10 , $20,30,40$ days. In eight of the cases there was a fall of viscosity from 10 per cent downwards. The differences are not large, and (in my source at any rate) the hours of the day on each examination are not mentioned. On the whole Boveri says there seems to be a parallelism between arteriosclerosis-presumably the kind consequent upon the "hypertension"-and the viscosity of the blood, and (contrariwise) iodine ingestion. But it is to be observed that these falls were in the whole blood,

1 Determann, "Blutviskosität bei Ioddarreichung," Deutsche med. Wochenschr., 14. Mai, 1908, p. 871 ; Lommel, Deutsche Arch. f. klin. Med., 1904, Ixxx. 308 (quoted by Krone, vide No. 2); and Münch. med. Wochenschr., 1908, No. 6 (quoted by Determann, loc. cit.).

2 Müller, Fortsch. d. deutsch. Klinik, 1910, p. 358.

3 Boveri, "Viscosité du sang et iode," La Presse méd., 1908 ; extracted in Arch. des mal. du cour, 1909, 255. 
the serum in all the cases being recorded as stationary; the effect therefore-if the effect were substantial-was in a reduction of the number of corpuscles, or the quantity of hæmoglobin. It has been suggested that a comparison of the blood of Graves's disease and of myxœdema, and again of the blood before and after thyroid operation, might throw some light on the effects of iodine. A few experiments upon animals by Burton-Opitz and others seem to indicate pathological fluctuations of this kind.

Mineral waters, as affecting blood viscosity, have received some attention, and are credited with no little power of reducing it. This effect seems to have been noted chiefly in those big feeders and plethorics who frequent such resorts, and whose blood, as we have seen, is alleged to be overviscous (see purgatives, p. 144). If this prove to be true, both as to the hyperviscosity and the relief of it, we shall have gained a very useful indication for scientific treatment. But we shall beware of increasing the volume of blood in a system perhaps already overloaded.

In the study of the effects of baths a few investigations have been made by Determann and others. Opitz says ${ }^{1}$ that viscosity depends in part on outer conditions; cold baths raise viscosity, warm baths attenuate it. The most of the effect is during the first 10-15 minutes. In these external applications of air, water, light, etc., the action of the vasomotor system and the partial distributions of the corpuscles must go for much; so that differential observations upon the whole blood and the plasma are required. It is generally agreed that in hot-air baths the viscosity rises; in hot-water baths no such rise is observed, respiration and perspiration being no doubt the variables. So far as the physics are concerned we know that the coefficient of internal friction varies with the temperature. In the pack Determann has recorded, in the cold stage, a rise of viscosity; in the hot reaction a fall. After short cold baths he observed a consistent rise. Kündig ${ }^{2}$ also has reported on these methods, but the results obtained were variable and inconclusive.

Venesection reduces viscosity, but not in corresponding proportion, and, as to the whole blood, it may soon be made up

1 Burton-Opitz, Journ. of Amer. Med. Assoc., July 29, 1911.

${ }^{2}$ Kündig, Diss., Jena, 1903, quoted by Krone (loc. cit.). 
again; that of the plasma however usually remains lower for some weeks (Vol. II. p. 118).

Of viscosity in disease we know little. Hunger is said to increase viscosity in dogs and rabbits. ${ }^{1}$ In the Wiesbaden discussion Umber stated that a very high viscosity (8.27) had been found in the diabetic coma of a patient in whom six months previously, in spite of acidosis, the blood viscosity had been found nearly normal $(5 \cdot 61)$. But is this "nearly normal"? Most authors agree (Austrian, Martinet, etc.) that in glycæmia the viscosity is high (let us say 5.61 ?) ; 8.27 is enormous. In diabetes mellitus the electrical resistance of the blood is high, as is that of the urine also, as, from the low conductivity of sugar, we should expect. Blood containing uric acid is said to present no alteration of viscosity. In anæmias, as we have seen in respect of polycythæmias, the viscosity, caeteris paribus, will vary as the number of corpuscles in the area from which the blood is drawn. ${ }^{2}$ In simple anæmias it falls a little. Matsuo (loc. cit.) makes the interesting remark that in anæmia viscosity readings run proportional to bruit de diable, which begins at and under $2 \cdot 8$. In menorrhagia, in Grave's disease, in "acidosis," which need not be a fulminating malady, and no doubt in other affections, the calcium loss may be excessive, and viscosity reduced. Albumin may then appear in the urine (Barr). In leukæmias, in spite of a low hæmoglobin content in some of them, increased viscosity is often noted; and this pretty surely depends on total increase of corpuscles-for it may be noted here that the white corpuscles count for viscosity at least as much as the red, some think for more. In splenic leukæmia Determann found that the white corpuscles conduced in their proportion more to the viscosity than did the reds. ${ }^{3}$ Usually in these cases to inhale oxygen does reduce the viscosity; but much depends on the number of the reds, and on the hæmoglobin values. In a case of hæmoglobinuria studied by Determann the liberation, during the attacks, of viscous substances from the reds enhanced the blood viscosity, which, as these bodies were restored, regularly fell again. Here at any rate clinical

1 Krotkow, "Russk. Wratch," No. 42, 1912, extr. in Deutsche med. Wochenschr., 1912.

3 The viscosity value of the platelets is unknown. 
observation seems to correspond with the experiments with laked blood (p. 128).

In pernicious anæmia Dr. Dawson Turner ${ }^{1}$ found the electrical resistance of the blood fallen to $300 \mathrm{ohms}$ (about one-half), while the resistance of the urine was doubled; "hence the striking fact that while the urine contained too few salts the blood contained an abnormal amount," as if "the kidneys were at fault." In another case of pernicious anæmia he found the electrical resistance of the blood down to 300 ohms, and the urine raised to 88 (instead of the normal 45) ohms. In beriberi Mayesima ${ }^{2}$ says that viscosity does not fall. But in all diseases we shall have to reckon with variations of hæmoglobin and gas content-the most definite of the results so far obtained - of protein content, of salt content, of plasma, of whole blood, of number and size of corpuscles. The exosmotic drive which may take place in dropsy on elimination of salt from the dietary is astonishing, and must be associated with wide viscous, osmotic, and electrolytic changes. Mayesima (loc. cit.) found that after all operations which irritate the peritoneum, or affect the spinal marrow (p. 142), as after fractures, acute purulent inflammations, venous stasis, and extirpation of the spleen, viscosity rises. Zuntz and Cohnheim ${ }^{3}$ also showed the remarkable effect of sections of the spinal cord in determining local afflux of red cells and so local rise of viscosity; the capillaries being gorged with blood cells. Viscosity falls in extensive operations on soft parts, in anæmia and leukæmia, in chronic nephritis (Cheinisse, but see Martinet, p. 145), in liver cirrhosis, and in malignant growths. It rises on injection of gelatine or diuretin, but falls on infusion of common salt and iodide (but not bromide) of potassium.

Some authors say that a rise of hydrogen ions in the blood raises viscosity ; as in gout, uræmia, acetonæmia, fatigue, and so on : and that in these states under the administration of alkalies

1 Turner, Dawson, Proc. Royal Soc. Edin., 1891-92, vol. xix. p. 20, quoted by himself, Nature, 1902, lxvi. 127.

${ }^{2}$ Mayesima, J., "Viskosität d. Blutes," Mitt. a. d. Grenzgeb. d. Med. u. Chir. Bd. xxiv. H. 3.

3 Zuntz and Cohnheim, Berl. klin. Wochenschr., 1886, quoted Saundby Brit. Med. Journ., May 18, 1907. 
viscosity falls. The mutual adhesion of the reds may be variously modified; but as yet our data are too scanty to reason upon. The viscosity coefficient in heart disease depends chiefly upon the cyanosis, if any.

In epilepsy Dr. John Turner, ${ }^{1}$ who considers that coagulation time is a measure of viscosity (p. 110), says that coagulation time is much shortened, and this the more the more frequent the seizures. He thinks the bromides retard the coagulation rate. Between coagulation and blood pressure he found no relation. The blood pressures in epileptics were often low, this he attributes to small ill-developed heart. He quotes Besta ${ }^{2}$ as having arrived at the same conclusions.

In fevers the mere rise of temperature is attended with fall of viscidity (see p. 147) ${ }^{3}$ but if the fever be prolonged the evaporation of water will act contrariwise; this may explain Mayesima's denial that viscosity falls in fevers, and the divergent results of Riegel, who registered a rise of viscosity in fevers. Venesection, as already remarked, by reduction of the corpuscles in number reduces viscosity in some, but not in close, proportion to its amount, possibly up to 12 per cent, and accelerates the stream velocity; an effect which, as regards the plasma, continues for a considerable time, the corpuscles - under ordinary circumstances-being soon made up again. Welsh observed the result in seventeen various cases; mitral disease, renal disease, eclampsia, etc. The effect seems to abide for some considerable period.

In typhoid fever Bachmann ${ }^{4}$ found a fall of viscosity, in spite of some excess of $\mathrm{CO}_{2}$; but in pneumonia a rise, not due, in his opinion, either to blood-pressure or to leucocytosis, and apparently not to excess of $\mathrm{CO}_{2}$, as the rise was noted also in mild cases. He considers that this difference may be regarded as a diagnostic point. Also that viscosity rises in epidemic meningitis and very much in cerebral hæmorrhage. There is some consent of evidence that in diphtheria the viscosity of the plasma is increased,

1 Turner, J., Journ. Mental Sci., Oct. 1907, a paper in many ways interesting.

${ }^{2}$ Besta, Rev. sperim. de freniat. vol. xxxii., abstr. J. M. Sc., April 1907.

3 Burton-Opitz, Stud. Rockefeller Inst. Med. Res., 1907, vi. 190, and Journ. Amer. Med. Assoc., July 29, 1911.

4 Bachmann, Deutsche Arch. f. klin. Med., 1908, xciv. 409. 
though outside the body we know that the peptonising influence of bacteria, as of trypsin, is to make albuminous fluids less viscid, and, by releasing free ions (e.g. amino-acids), to increase conductivity also. Would not further investigations of bacterial actions, and comparison with venoms, lead on these lines to a better knowledge of catalytic poisoning?

Czépai and von Torday, ${ }^{1}$ in a paper which deserves more attention than it has received, conclude, on methods carefully specified, and in 118 cases - 61 of tubercle and 57 of various kinds of disease - that a value of viscosity quotient over 4.0 in a mixture of tuberculinised serum with absolute alcohol is in their experiences "absolutely a specific" sign of tuberculosis; in a few cases of syphilis an alcoholic extract of the fœtal liver gave a much higher quotient still. The admixture is 0.2 ccm. old tuberculin $+0.2 \mathrm{ccm}$. absolute alcohol $+0.1 \mathrm{ccm}$. of 0.9 per cent $\mathrm{NaCl}$ solution. But, if we may rely upon Eisler and Laub, ${ }^{2}$ the serum in mild cases of tuberculosis is much as in health, i.e. from 1·8-2.5 (method of Czépai and von Torday). But in severe cases it runs higher-from 3.0 upwards. A fall of serum viscosity is of favourable omen. On the whole in tuberculous guinea-pigs the reaction proved to be much the same.

In respect of drugs other than the iodides (p. 145), the accurate method of the pharmacologists should give us definite results, but as yet there is not much to build upon. As to alcohol, $\mathrm{M}^{\mathrm{C}}$ Caskey, ${ }^{3}$ and Determann, found that it increased the viscosity, but Bachmann that alcohol reduced it. Burton-Opitz, ${ }^{4}$ injecting alcohol, in varying degrees of dilution, into animals- $(a)$ into the facial vein, $(b)$ into the stomach or duodenum-found the blood in all cases more viscous ; but especially under method $(b)$; viz. from 4.95 to $5 \cdot 71$ (at $87^{\circ}$ C.). The maximal effect arrived in 5-10 minutes, and persisted for 30-35 minutes (see p. 247). Digitalis is said to have no effect upon the viscosity ; strophanthus, adrenalin, and caffein slightly and transiently to raise it,

1 Czépai and von Torday, Deutsche med. Wochenschr., Aug. 10, 1911.

2 Eisler and Laub, "Viskositätsbestimmung bei Tuberculose," Wien. klin. Wochenschr., 1912, No. 20.

3 M'Caskey, Journ. Amer. Med. Assoc., Chicago, 1908, li. 1653 (quoted by Krone).

4 Burton-Opitz, Journ. Physiol., Cambridge, U.S.A., 1905, xxxii. 8. 
camphor to reduce it. The interesting statement is made that the nitrites temporarily reduce viscosity. ${ }^{1}$

The conclusion of this review, from the point of practical medicine, seems to be that if the estimation of viscosity, unless in respect of calcium content, be as yet of little clinical service, it is desirable notwithstanding that academic observations on the subject should still be pursued, seeing that it is a study of those dynamical problems of molecular constitution, osmotic pressures, electro-conductivity, and chemical physics which are the principles of life. Moreover, while patiently we continue to penetrate to the arcana-to the intimate and ultimate nature and genesis of the materials we have to study and to handle, we may obtain meanwhile some useful tools for rough and immediate reckonings. And if in the course of evolution the periods and fluctuations of these forces have become so rapidly adjustable as to elude us, our methods of research must be refined to a corresponding nicety. That these secret influences have an enormous effect upon the stresses and forces of the circulation, upon its pressure heads, its maintenance, its distributions and its issues is no matter of speculation but of verified principles and definite data. The difficulties in the present problems lie not so much in the principles as in the complexity of their common sphere of operation. So far as research enables us to pry into the future it seems as if, without postulating any "vital" agency, these physico-chemical principles, with their time relations, will compass much of the interpretation of life.

This review was made in order to learn, if possible, the degrees of dependence of cardio-arterial integrity upon the qualities of the fluid these vessels contain and drive. Subtle changes in the content, acting over long periods of time, would induce corresponding modifications in the continent; whatsoever the causes, the arteries do suffer modifications which are large and conspicuous. And if it be true that to accommodate a more viscous fluid, as in polycythæmia for instance, adjustments of vascular capacity in the whole, or in very large areas, do take place, yet in the long run no secondary adjustments can be

${ }^{1}$ I refrain from giving the many references to these and many other experiments; they are far from conclusive, and need extensive verifications. 
equivalent to the normal balance. If the vessels be so dilated that the blood-pressure is little altered, yet the frictional pressures must be driving the systole and diastole to act within much narrower limits of vascular elasticity, and in time to strain the arteries. Since these words were first published Dr. Welsh has said (loc. cit.): "There can be no question but that blood of high viscosity requires more driving power to force it through the capillaries than does blood of lower viscosity, and that the dilating or contracting of the arterioles does not alter this fact (postulate ?), although it may partly compensate it." We know that in man, as years go on, blood pressures rise a little, and the arterial coats thicken and degrade; while simultaneously the left ventricle may take on small degrees of hypertrophy. Osmotic changes become less active; the shins may present a slight œdema, and the urine may exhibit poverty of solids and traces of albumin, so that the watchful physician may prescribe some reduction of salt intake. If by sclerosis in some areas the radius of the finer vessels be diminished the blood would so far be relatively more viscous; if also the endothelium were unhealthy thrombosis would be favoured. And alongside these senile changes the peripheral vascular areas may be shrinking, but therewith, and perhaps in indirect proportion, the total volume of the blood falling (p. 46). Such is the intricacy of the mechanical system which we have to interpret.

Finally; as of the conclusions of these studies on viscosity which seem better established, one is the increase of viscosity on increments of carbon dioxide, so in many diseases is the vicious circle-plus viscosity, minus velocity, plus $\mathrm{CO}_{2}$, and round to increasing viscosity. Hence one advantage set up, of inhalation of oxygen in cases of inadequate aeration. Now, as the fine vessels of the lungs are comparatively of constant radius, excessive internal friction must tell sooner on them. This effect we are little able to measure directly, but in practice we meet daily with cases of gradually encroaching cyanosis, due for instance to bronchial, emphysematous, or more general causes, which lead to or are associated with enlargement of the right side of the heart. It seems probable that in these cases, as in other multiples of cyanosis, increments of viscosity may play no inconsiderable part in the adversity. 


\section{CHAPTER IV}

\section{CAUSES OF ARTERIOSCLEROSIS}

Ir is when we come to study the causes of diseases that we are most disconcerted by the breadth and depth of our ignorance; it is here that our path is most cumbered with guesses, presumptions, and conjectures, the untimely and sterile fruitage of minds which cannot bear to wait for the facts, and are ready to forget that the use of hypothesis lies not in the display of ingenuity but in the labour of verification. Again and again the data required are not before us, or not fully before us; indeed, even so far as we have them, it may be impossible to track them severally through the perplexities of individual lives. Moreover, we have seen that arteriosclerosis is not "a disease," but an anatomical result of several morbid processes; and again how far the name "arteriosclerosis" signifies not a unity within itself, not a uniform concept, but is an omnibus name including two or more main divisions; such as the "hyperpietic" and the "involutionary," or " decrescent," kinds. In discussing the causes of "arteriosclerosis" then we shall have to keep an eye to these separate processes, or, if the processes or some of them should prove at bottom to be akin, at any rate to the several directions and various features of the paths which lead ultimately to the one and to the other. Again, in respect of the areas of the body, we shall see that arteriosclerosis is not a universal process, it prevails now in this area, now in that; determinations which may throw some light upon causation.

The Correlation of Arteriosclerosis and Blood Pressure.Many years elapsed before my main, and I venture to think 
crucial, distinction published in 1894-5, and again in 1896, between arterial lesion with high blood pressure, and without it, was admitted, or indeed taken into consideration. And at the present day, to the confusion of our clinical and pathological teaching, this distinction is commonly disregarded. Senator was one of the first to apprehend it, and, so far as I know, independently. He writes: "Among the early signs of arteriosclerosis great importance is generally attached to an increase in arterial tension. ... According to my experience this sign in arteriosclerosis is a very inconstant one." Yet he did not comprehend that the arterial lesion of the high pressure type and of the decrescent type respectively, belonged to distinct clinical series -to different " diseases." Like many eminent teachers after him, he attributed the difference in pressures simply to differences in cardiac energy; that is, to incidental fluctuations in the same case, not to processes different in nature. Von Basch, when confronted with cases of arteriosclerosis with moderate pressures, took refuge in the same conjecture. He likewise did not realise that the arteriosclerotics with moderate pressures often attain great length of years, and as a class live longer than the high pressure patients, renal or hyperpietic. Ewald, in a paper written in $1877,{ }^{1}$ was perhaps the first to observe that in not a few cases of atheroma, including the smaller vessels, the heart is not hypertrophied. Fränkel (in the 1894 edition of Eulenburg's Real-Encyklopädie) remarked incidentally that arteriosclerosis is found in many cases in which no previous excess of pressures could be inferred from the state of the heart ; and in these cases he attributed the lesion to "plethora." But he did not follow up the distinction. And here I may refer to Dr. M'Crorie's series of necropsies in the Glasgow Med. Journ., 1892. Councilman in about half of his autopsies on cases of arteriosclerosis found the heart hypertrophied, and made the important observation that cardiac hypertrophy was conspicuous not in senile cases but in those of " big middle-aged men." Then Dr. Janeway ${ }^{2}$ admitted that although in many cases of arteriosclerosis the blood pressures range high, yet in others they are not inordinate; but this difference he too

1 Ewald, Virchow's Arch., 1877.

2 Janeway, Clin. Study of Blood Pressure, 1904, p. 143. 
explained by the supposition of an enfeebled heart, and likewise regarded these contrasted states as but phases of one process. So again Dr. Coley of Philadelphia, while confirming the difference, explained it by cardiac insufficiency ; and this, I believe, is still Dr. Herringham's interpretation. Thus for all these authors the character of pressure is one of accident and degree, and lies in no radical difference of process. Janeway however did proffer the alternative explanation of Hasenfeld and Hirsch, that pressures rise in proportion to the degree of arteriosclerosis in the splanchnic or supradiaphragmatic areas. This hypothesis, considered elsewhere (p. 188), is now abandoned: ${ }^{1}$ as von Romberg ${ }^{2}$ says, extensive splanchnic arteriosclerosis is rare ("sehr selten "); it is now ascertained indeed that even high degrees of splanchnic and thoracic arteriosclerosis do not themselves give rise to any clinically recognisable hypertrophy of the left ventricle. If, he truly added, it should increase the work of the heart, this would not necessarily imply any rise of pressures. That the attacks of high pressure which arise occasionally in elderly persons are transient and curable (p. 452) indicates that the cause of these cannot lie in any static arterial alteration. In these cases it supervenes upon a senile arteriosclerosis, it may be of conspicuous degree and ubiquity, which however, neither before the intervention nor after it, had any notable effect upon the arterial pressures.

The next observation I will quote upon this part of the subject is by Dr. Cautley, ${ }^{3}$ who emphasised the absence of high blood pressure, and of cardiac hypertrophy, in syphilitic arterial disease; he brought forward a case of this disease in a man, aged 40 (primary infection fifteen years before), in whom, although the arteries were extensively diseased, there had been "no arterial tension and no cardiac hypertrophy." The abdominal aorta was transformed into a thick rigid tube. Although the patient was scarcely middle aged, the case reads like one of original syphilitic arterial disease merging into "senile" arteriosclerosis (vide p. 296). Such cases abound, yet nevertheless this emphatic demonstration was much needed.

1 Sec, e.g., Marchand, Verhandl. Kong. f. innere Med., 1904.

2 Discussion on Arteriosclerosis at Budapest Congress, in 1909.

${ }^{3}$ Cautley, Lancet, April 6, 1901. 
But it was not until 1904 (ten years after my opinion on the point was first published) that clinical observers were compelled to listen to the distinction between these clinical characters of arteriosclerosis by the appearance of no less than three important papers, especially directed to this fundamental distinction, by Groedel of Nauheim, ${ }^{1}$ by Dunin, ${ }^{2}$ and by Sawada. ${ }^{3}$ Groedel's announcement at the Congress attracted more attention than the other two papers, and is now well known. But Dunin made 1000 observations on 440 arteriosclerotic cases, and found no constant or approximate proportion between arteriosclerosis and blood pressure. With the advance of years a moderate increase of pressure is normal, but in his younger arteriosclerotic patients the pressures were often rather low than high. Of 380 patients, he found the blood pressures in 80 normal, or somewhat less ; in the remaining 300 it was raised : a proportion of high pressure cases, by the way, much larger than later observations have established; probably the sum contained many renal cases. Dunin, with proper caution, made no deductions for the gradual quasi-normal rise with age-say to 140 or 150 . Sawada, from this point of view, examined 206 cases of arteriosclerosis; in " 122 uncomplicated cases" only 12.3 per cent proved to be associated with high pressures. Of the rest 75 were complicated with cardiac or with renal disease, or both; and upon the whole number the high pressure cases were $30^{\circ} 6$ per cent, a far lower proportion than Dunin's; but of course there is much chance in the collection of such lists. Krehl ${ }^{4}$ then threw the weight of his opinion into the same scale, and quoted a series of necropsies in which extensive disease of the aorta and visceral arteries was found, yet in which there had been no high blood pressure during life; but his article was written for a somewhat different purpose.

From the date of these papers medical opinion began to veer. At the British Medical Association in 1905 Dreschfeld, " divided

1 Groedel, Verhandl. Kongr. f. innere Med., 1904.

2 Dunin, Zeitschr. f. klin. Med., 1904.

3 Sawada, Deutsche med. Wochenschr., Nov. 12, 1904.

- In the new edition of his book (1912) Krehl is not lucid. He says in one sentence (p. 31) that in high pressures atherosclerosis accounts for much; in anotber that the cause lies in " hypertonie."

5 Dreschfeld, Brit. Med. Journ., August 1905. 
cases of arteriosclerosis into those with high tension and those with low"; but at that meeting Dr. Herringham, in accepting the difference, attributed it, as I have said, to degrees of myocardial degeneration; and assumed that among elderly people those live longer in whom, in response to an alleged increase of peripheral resistance due to arteriosclerosis, the heart is hypertrophied. We are all indebted to Dr. Herringham for his important researches on these subjects, but here I cannot follow him. We shall see presently that arterial decay does not itself set up cardiac hypertrophy; furthermore that the cases in which the heart is hypertrophied are cases of hyperpiesia or of renal disease, the arteriosclerosis being not the cause of the high blood pressures but their effect. These persons die not later but sooner-on the whole much sooner; ordinarily of cardiac defeat or of apoplexy. About this time Dr. Henry Campbell accepted my statement of the matter, and argued that "this should not surprise us, seeing that thickening of vessels need not necessarily increase the resistance which they oppose to the blood flowso long as a generalised arteriolar hypotonus (or a tone not higher than normal ?) prevails" To assume that by the stiffening of the tubes and loss of their elasticity the heart is hypertrophied or injured ${ }^{1}$ is not justifiable unless we assume also that the circulation is kept as efficient as before, of which there may be no evidence, or much to the contrary. The first effect is probably a retarded blood stream; the curve is round topped, and the pulse amplitude increased (vide p. 31). Dr. Rolleston agreed with me that "very often, as a matter of fact, the general blood pressure is not raised, even when obstructive arteriosclerosis seems extensive and severe, and the heart's energy unabated." Dr. Chalmers Watson ${ }^{2}$ kindly informed me, in 1907, "I have been examining the blood pressure of every patient with thick arteries, but not more than half the number had distinctly raised blood pressure. . . . I fully share your view as to the necessity of recognising a class of cases of arteriosclerosis in which there is no obvious rise of pressure." Again, in my Address at Toronto, in 1906, I set forth

\footnotetext{
${ }^{1}$ As by many authors, see, e.g., Grossmann, Wien. med. Wochenschr., 1910, Nos. 39-40.

2 Private letter, January 1907.
} 
these differences, and discussed them with Dr. Rudolf and some of his colleagues, and in 1908 Dr. Rudolf stated ${ }^{1}$ that he now agreed that in many cases of well-marked arteriosclerosis the blood pressure is not augmented; but he still seemed disposed, with Senator, Janeway, and Herringham, to attribute this stability to cardiac defect, which was not to realise my view of the pressor and the non-pressor arteriosclerosis as of different series. I was much interested therefore to read in a yet later article by Dr. Rudolf ${ }^{2}$ that he had adopted my original classification in full. He had found high pressures in not more than half of his arterial cases, and in most of the rest that the difference was not due to heart failure. At the British Medical Association Meeting in 1908, Dr. Newton Pitt admitted that " half his cases (of arteriosclerosis) were not associated with hypertrophy of the heart," ${ }^{3}$ and in the same year Dr. George Oliver, in one of the not least interesting of his many able contributions to this subject," wrote that in " a large percentage of his cases of arterial thickening the blood pressure is not only not at all raised but may be somewhat sub-normal . . . very extensive atheroma is consistent with low blood pressures," and refers these cases to my Involutionary (or Decrescent) class, as set forth by me at the Hunterian Society in 1894 (Trans. 1895) and in the Lane Lectures of 1896. But Dr. Oliver proposed yet another class, in which arteriosclerosis for some time precedes a rise in pressure which nevertheless may ensue quite definitely. In these cases he alleges a " general fibroid change all over the body and all vessels, arteries, capillaries and veins, "including the kidneys." He identifies it with the angio-sclerosis of Thoma, and distinguishes it by the insusceptibility of such vessels to the action of the nitritesthe increase of friction being of mechanical rather than vasomotor origin; static rather than dynamic. These cases I have not been able to discriminate. The arteriosclerotic kidney of Ziegler, Lorrain Smith and myself, and J. F. Gaskell (p. 334) is not associated with exorbitantly high pressures; genuine "Granular kidney" is so associated, but from the very outset. Dr. Oliver's cases may have been of the kind which I have described

1 Rudolf, Amer. Journ. Med. Sci., Sept. 1908.

2 Rudolf, Brit. Med. Journ., Nov. 26, 1910.

3 Pitt, N., Brit. Med. Journ. and Lancet, Aug. 8, 1908.

- Oliver, G., Clinical Journ., Sept. 16, 1908. 
as episodes of high pressure in the course of decrescent (involutionary) arteriosclerosis (p. 452); but these episodes, on such treatment as Dr. Oliver would employ, would be transitory, lasting for a few weeks only. But to proceed : in Osler and $\mathrm{M}^{\top} \mathrm{Crae}$ 's System Sir William Osler accepts my original three classes of arteriosclerosis; namely, High pressure arteriosclerosis (Hyperpiesis and chronic renal disease); Involutionary or Decrescent ("Senile"); and Infectious or Toxic (typhoid, syphilis, diabetes, lead, etc.). I must now content myself with one more reference, to Israel (loc. cit.), who submitted 16 cases of "senile" (my "decrescent") arteriosclerosis to a minute analysis; in these there was no augmentation of blood pressure ("ohne Druckerhöhung "), and in none was the heart enlarged (" kein einziges Mal eine Dilatation verzeichnet"). He added that of course in these cases, as in many others of various kinds, if the heart yields by intrinsic default dilatation might ensue. For such default he uses the name "cardiosclerosis," to be discussed in another chapter (vol. ii. p. 1). Israel found enhanced blood pressures in 64 per cent of arteriosclerotics, but he adds that many of these proved to be renal. He and the other authors whom I have quoted have avoided the vicious circle of argument that high pressures prove arteriosclerosis and arteriosclerosis proves high pressures; a maze in which many medical writers have gone and still go astray. Their testimony disposes also of the remnant hypothesis that all these decrescent cases began as augmentations of pressure.

In illustration of the distinction, I would quote the following case, on this point of interpretation, from Dr. Guthrie Rankin. ${ }^{1}$

Male, æt. 37. No syphilis, but gouty, and a free liver. Keen in body and mind. Urine scanty and lithatic. No albumin. Mental depression, furred tongue, sleeplessness, headache, lassitude, irritability of temper, dizziness, etc. Pulse of exceedingly high tension. No murmur. "Gradual development of moderate, but unmistakable tortuosity of the arteries." Then slight albuminuria, but no casts nor renal epithelium.

I quote this case as a typical case of hyperpiesia, and would venture, in view of the distinction I am making, with great respect to question Dr. Rankin's opinion that this case was one

\footnotetext{
1 Rankin, G., Practitioner, May 1904.
} 
of " bad material used for the tubing" (i.e. my decrescent kind). In these cases the cardiac hypertrophy does not " ensue upon the loss of arterial elasticity" - the high pressures come first and the arterial changes are consequential.

Vascular Spasm in Arteriosclerosis.-We have dwelt upon the principle that an artery is not an inorganic tube, but one endowed with a nervous mechanism, an endowment which profoundly modifies its reactions to the blood pressures, variable as these are. Willis, who first described these nerves, ${ }^{1}$ perhaps with Lower's assistance, regarded them as "bridles and reins by which the sanguineous vessels, either by straining or pulling themselves together, sometimes retard, sometimes incite, the course of the blood according to the needs of the viscera." Now by some observers it is asserted that in disease the arteries are not less but more susceptible to the nervous influence. There is indeed an impression abroad that in arteriosclerosis the vessels, or territories of them, if lamed by the disease, are even more liable to spasm than in health, and less under control (pp. 216 and 433). ${ }^{2}$ We hear more in this respect of vasoconstriction than of vasodilatation. Sir W. Osler admits that we have very little direct evidence of these incidental visceral angiospasms, but the formula is very handy for an explanation of all sorts of problems. Hence come Nothnagel's audacious phrase of "vascular colic," and the "vascular crises," by which formulas abdominal pains, vomitings, cerebral seizures (pp. 416 and 445), and many another periodical disorder, are explainable offhand, as by the "strictum and laxum" of the Methodists. That, in so far as the muscular coat is concerned, the more it is diseased the more readily or touchily it responds to such stimulation is, on the face of it, a more anomalous postulate than its advocates seem to realise. It might be argued that if the muscular coat be damaged in parcels, intermediate parcels may be brought into degrees of morbid irritability, but if so. the frailer parcels more centrally situated would be imperilled. I admit that the muscular elements of the peripheral arteries are more persistent than is generally believed ; and the nerve-endings on these vessels, being placed externally,

1 Willis, Anatomy of Brain, ch. xxvii. p. 138, quoted Ferrier, Harv. Or., 1902.

2 See, e.g., Osler, Luml. Lect., Lancet, March 26, 1910, pp. 842-843.

VOL. I 
may for some while escape the sclerosis beginning from within. Until demolition is far gone, both nerve and muscle may be capable of these capricious phenomena, and the balance of tone, whether for constriction or expansion, may be more unstable than in health. However we have no direct evidence to this effect; indeed such evidence as we have is to the contrary. Plethysmographic experiments upon the arm in arteriosclerosis, experiments carried out by more than one skilful investigator (von Romberg, ${ }^{1}$ O. Müller, ${ }^{2}$ Thayer, and others), indicate so far that the response of the vasomotor mechanism-nerve and muscle-to stimulation, as, for example, by the touch of a piece of ice, which in the normal person may bring down the scale by $4 \mathrm{~cm}$., or, on the contrary, by a warm application, is in arteriosclerosis not enhanced but diminished; although the reflex path is presumably open, there is in sclerosis so progressive a diminution in response of the volume of the arm that declining degrees of response might be taken as proportionate to advancing degrees of sclerosis, and as a test for them. So in cases of severe arteriosclerosis the limb may not respond at all. As Curschmann contested these results, they were carefully repeated and verified; psychical conditions being excluded, which must be difficult. On the application of the ice, in proportion to the degree of the arteriosclerosis (gewöhnlich und gradatim) the curve flattened until in extreme sclerosis it became almost a straight line. This maxim, that thus arteriosclerosis of a limb and its degrees may be appreciated, Klemperer felt able to formulate (at the Kongr. cit.). ${ }^{3}$ Dr. $\mathrm{M}^{6}$ Phedran ${ }^{4}$ used nitrites as a test of arterial mobility, watching the pressure curves under their use, and found that when the coats are healthy the blood pressure falls promptly and definitely ; but when sclerosed the impression of these salts is slow and indefinite. H. W. Cook likewise (loc. cit.) found in arteriosclerosis a decided loss of ability on the part of the artery to respond to vasodilator drugs.

1 Von Romberg, 21ter Kongr. f. inner. Med., 1904, and Deutsche med. Wochenschr., Oct. 28, 1909.

${ }_{2}^{2}$ Müller, O., Deutsche med. Wochenschr., 1906, Nos. 38 and 39 ; also Deutsche Klinik, 1910, and Zeitschr. f. klin. Med. vol. lxxv.

${ }^{3}$ See also observations of Klemperer, Citron, Simon, and others at the same session, July 17, 1911.

4 Reported at the Toronto Meeting, Brit. Med. 'Ass., 1906. 
It is not improbable that another incalculable factor may be concerned in these lapses; namely, an impairment of the system in one or more of the vasomotor centres. We have besides to learn how far in age the vessels of the splanchnic area may slacken in their peculiar function as regulators of the circulation in the upright body of man ; or how far in spasm and relaxation they may waver. Perhaps careful measurements of the capacity of normal and arteriosclerotic individuals in upright and recumbent positions might give us a criterion of diagnosis. As regards morbid anatomy we have seen (p. 457) that, in decrescent arteriosclerosis at any rate, the arteriolar areas of the splanchnic area are less prone to decay than those of the pancreas and spleen. Hess and Bermann ${ }^{1}$ again have recently verified these observations in all substantial points concerning arteriosclerosis, comparing also in normal states the reflected influences upon the other limb, and the dilatation of vessels under painful stimulation. In sclerosis the responses were often dissociated, e.g. normal to warmth and defective to cold. A test for arteriosclerosis may be introduced here, which has been mentioned by Hertzell. ${ }^{2}$ The patient lies down and a pneumatic band is applied to each leg and to one arm; these three bands are blown up to points of arrest. If the vessels be sound, the blood pressure should rise very little in the other arm-say $5 \mathrm{~mm}$.; but in case of sclerosis it rises far more, even to $60 \mathrm{~mm}$. or more. The readaptive capacity is reduced. Not having tried this experiment I have no right to any opinion about it, but one may presume all the other variables to be constant. Still all this, if helpful, is not quite conclusive. The limbs we can watch, more or less well, directly; what we want to know is the states and capacities of internal areas. Moreover we shall see that, especially in sclerosis of the vessels of the limbs, the media is said to be the primary seat of decay; if so, the mobility of the arteries may be more affected than in definite atherosclerosis; the one process may arise and progress out of step with the other. In renal disease, probably for that considerable period during which the media may be hypertrophied, the vessels are not less but more active. I suspect in hyperpiesia likewise this is the

1 Hess and Bermann, Wien. med. Wochenschr., Aug. 7, 1913.

2 Hertzell, Berl. klin. Wochenschr., 1913, No. 12. 
case, and that the vessels which fail actively to respond are the seat of Mönckeberg's mode of sclerosis (p. 178). It is generally admitted that in later life there is an increasing lack of quick peripheral adaptation, that on exertion the central blood pressures rise too extremely; thus elderly persons chill more easily and tire more quickly. And I have remarked that the "dead fingers" of old persons are the effect not of arterial spasm but of tardy stream and atrophied arterioles. In their legs and feet " dermatographic" phenomena are not easily produced, but if, by the tip or length of the finger, pressure be made upon the skin for a second or two, the tint returns to the blanched skin very slowly. However, for the armchair speculator, peripheral vascular cramps and expansions are so convenient that it is heartless to throw doubt on this ready resource; and as yet he is safe in its inaccessibility to verification. I am of course as far from denying that such vascular phases occur in arteriosclerosis as we ought to be from assuming them; we are without sufficient data either way.

One demur there is, moreover, which seems inevitable : we cannot well hold, as some writers do, both to the irritable spasms of the muscularis and the initial medial sagging of Thoma (pp. 486 et seq.).

Heredity.-At the very outset then of a discussion of causes we were met by the necessity of distinguishing the several kinds of arteriosclerosis. In respect of hereditary influences for instance, the family histories have never been divided into those of high blood pressure and those of premature arterial decay; and yet without this primary discrimination these histories are worthless. Surely there is all the distinction in the world between a family disposed to arterial pressures so excessive that by it the vessels are damaged consequentially, and a family disposed to primary and essential arterial decay, without such high pressures? 1 And in our experience we see this difference every day. We are acquainted, if we will but think of it, with the families in which, generation after generation, individual lives are ended by cerebral hcemorrhage without the ordinary symptoms of renal disease; and with those

1 In this respect I think Dr. Stengel's interesting article in Amer. Journ. Med. Sci., 1905, is indecisive. 
on the contrary in which, if death come by stroke, it is not by the hæmorrhage of high pressures but by the occlusion of arterial twigs not subjected to excessive blood pressure but already in decay. Dr. Mott, extending his data in the second edition of Allbutt and Rolleston's System, says still more confidently (p. 595), "The relative infrequency of intracerebral hæmorrhage in the insane with atheromatous arteries is probably due to the fact that they have not been the subjects of prolonged high arterial pressure, as shown by the absence of cardiac hypertrophy." The inmates of a pauper asylum or workhouse are not usually full-blooded persons; a rise of pressures in them would suggest chronic renal disease. It is scarcely necessary to illustrate the prevalence of sanguineous apoplexy in certain "plethoric" families; this hereditary proneness to apoplexy has long been known and recognised, even by the public (vide p.412). Broadbent explicitly insisted on heredity in high blood pressure, and on "hereditary hypertension" as the precursor of apoplexy. In a certain family, some of whom came under my clinical observation, all of them lifelong friends of mine, the men, generation after generation, were carried off about the same age (65-70) by this kind of stroke. They were big florid muscular countrymen, keen sportsmen, and not averse from the pleasures of the table and the cellar; the two whom I examined had large leathery arteries under very high and sustained pressures. Among themselves this mode of death, and about such an age, was recognised, and calmly anticipated. Renal disease, as ordinarily understood, did not appear among them. With such examples however I am appealing to an experience so familiar that I need not labour the argument. Some while ago I remember that Dr. James Mackenzie published in one of the journals a note of high pressures in two sisters, members of a "ruddy-faced" family; the patients' father and uncle died of apoplexy. Professor Raymond ${ }^{1}$ again was convinced of this disposition to high pressures and apoplexy as a family fate, some individuals ruddy but some spare and sallow, and narrated the following calamitous story: The patient, a man æt. 43, had " high arterial tension" and an aortic systolic murmur; his grand1 Raymond, Prog. méd., mars 30, 1907. 
father died early of cerebral hæmorrhage; his father's sister died of the same stroke at æt. 57 ; his father's brother suffered a cerebral hæmorrhage at æt. 52, and a second (fatal) at æt. 59 ; his own sister (living) had had an attack; of five cousins three had died of the same, and the other two had cerebral hæmorrhages at the ages of 52 and 57 respectively. A gentleman, æt. 60, who called on me about a skin affection, proved to have a very high blood pressure ; in answer to some cautious remark of mine he frankly said that his family had a tendency to "blood to the head"; for three generations his immediate ancestors on the paternal side had, to his own knowledge, died of cerebral hæmorrhage (see also p. 412). I have assumed that every practitioner of experience is well aware of this common hereditary peril ; but the point on which I now desire to insist is that in these families the proclivity is not in the first place to arterial disease - to arteriosclerosis - but to the prevalence of high arterial pressures-as hyperpiesia or as renal disease-damaging these vessels, and ultimately rupturing one or more of them. Nay, under such pressures rupture may take place without degeneration apparent to the microscope. Dr. Savill gave me a section, now in our pathological collection, in which the media, healthy so far as histological methods could detect, was giving way at one point in its circumference where the coat, suddenly thinning, pouted with the adventitia outwards; but complete rupture had not taken place. I see that von Recklinghausen has expressed the same opinion. ${ }^{1}$

But with the involutionary or decrescent kind of arteriosclerosis it is otherwise ; this proclivity also seems to be hereditary, but not with the bent towards excessive blood pressures; I have just taken the maximum pressure in a man æt. 80 with extremely tortuous and calcified vessels; it ran 125-30. The arteries of certain families may be made of less durable stuff. In the Jews it is said that the decrescent arteriosclerosis is frequent and rather precocious. Or again a disposition to certain katabolic perversities may run in families. Yet, as we shall see, these people, liable as indeed they are to obsolescence of the decaying vessels, in the brain as elsewhere, and to a general atrophy resulting from the increasing inability of the degraded vessels and slacker stream

1 Von Recklinghausen, F. Handb. allgem. Pathol. des Kreislaufs, 1883, p. 84. 
to convey a sufficiency of blood and lymph, are not very liable to cerebral hæmorrhage. Now it is in these subjects, even with vessels "exasperated by bony scales," that, as I urged again and again in 1894 and in 1896, the arterial pressures are not exorbitant; but until ten years later, when Groedel repeated the observation, I could get no hearing. Dr. H. D. Rolleston ${ }^{1}$ expresses a recent change of common opinion when he says the belief that arteriosclerosis is the cause of high arterial pressures is contradicted by the innumerable cases in which, with general atheroma and widespread arterial degeneration yet without heart failure, the blood pressures are but little raised, or even do not reach the normal; though, as he justly adds (a suspicion to be discussed later), some toxins may act both as stimulants to rise of pressure and as corrosives of the arterial tunics. Dr. Mott's opinion, founded on an unusually large experience, I have already quoted, and shall have to quote again.

In respect of arteriosclerosis then there are two modes of hereditary transmission, the direct and the indirect ; the direct, that form which I have named the Decrescent, or primary, which consists apparently either in an original frailty or toxic susceptibility of the arterial structure, a frailty or susceptibility which, like other such peculiarities, may run in families; the indirect, that form which I have named the Hyperpietic, a secondary event, which apparently depends not upon an original arterial frailty but upon a proclivity to some ill-understood poisoning or perversion making for high blood pressures ; and to these high pressures it is that the soundest arterial structures, thus subjected to præternatural stresses, will succumb.

Age.-Marvellous as in the human frame are the symmetry of parts and the harmony of function, yet it cannot be supposed that the stealthy hours carry away no qualities of tissue, no quantities of energy. Although in many old men the accessible arteries are soft and straight enough, and in a few cases even necropsy, to the naked eye, betrays no detriment, yet on the whole arterial resilience is inversely as the years of its duration. A few days before these lines were written, a brisk old gentleman, aged 78, called upon me for slight Menière's symptoms, suggestive, at least, of aural sclerosis; yet all his

1 Rolleston, H. D., Clin. Journ., June 21, 1905. 
accessible arteries were perfectly soft, even in the legs; and the radial pulse in a thin wrist in full daylight was invisible. The heart seemed perfectly normal, even on some test of effort. Again, I remember an instance of necropsy in a man who died, of an intercurrent malady, in his 83rd year, whose aorta and arteries and cardiac valve cusps to the naked eye seemed intact; to the microscope probably some fibrosis would have been revealed; and doubtless, as von Recklinghausen says (loc. cit.), molecular fatigue, or I would add the waning of autolytic capacities in the cells, may go far without coming within microscopic ken; for, accidents apart, it is by cellular and molecular fatigue that we number our days. The late Dr. Hudson of Mildenhall once took me to see a man aged 100 years, who was receiving the congratulations of his friends on that birthday. He allowed us to note that his arteries were barely perceptible to the touch, and that his heart was to all physical examination normal. The blood pressure to the finger seemed rather under than over the normal. Dr. Hudson, who had brought him through an attack of pneumonia eighteen months before, said that his health and faculties, including his memory for recent events, were good. He ate well, and slept well.

He had as white a head and fresh a cheek

As ever were produced by youth and age

Engendering in the blood of hale fourscore.

There are many cases on record of persons of 90 years and upwards in whose bodies on necropsy the larger vessels presented no signs of arteriosclerosis, at any rate none to the eye. Morgagni, ${ }^{1}$ in a woman probably about æt. 90 , was surprised to find little sign of arterial disease, except slight white spots in the descending aorta and in the iliacs, sites which, as we know, are among the first to exhibit those signs of decay. Charcot, of a woman in the Salpêtrière, aged 103, reported complete integrity of the structures of the heart and arteries. Sir George Stokes's accessible arteries at the age of 83 were soft and straight; his maximum pressure 135, his minimum about 100 .

Nevertheless during advancing years a gradual systolic rise of $20-30 \mathrm{~mm}$. is the rule. Schell, ${ }^{2}$ who measured the

${ }^{1}$ De S. et C. M., Ep. Ixvii. 11.

2 Schell, Virchow's Arch. Bd. cxci. H. i. 
larger arteries in many bodies at various ages, found that with age resiliency had so decreased that these vessels had grown longer and broader. This he attributed to the prolonged stresses of the circulating blood. It is not quite true then that, in the common acceptance of the words, " a man is as old as his arteries." It is true, as Roy used to say, that old age is most common in advanced life; yet many a man grows old while his arteries keep a fair aspect; and conversely many a man, labourer or not, with rough and grotesquely distorted arteries, at any rate in the limbs, lives actively and cheerfully to a ripe antiquity. Jores has pointed out that in arteriosclerosis many of the finervascular webs may, and commonly do, remain unaltered; even for instance in a myocardium whose coronary stems and branches may be decayed. Broadly, but not universally, it is true that arterial degeneration is a senile change; yet I cannot follow Jores and others in a formal adoption of "Senile Arteriosclerosis" as a name. It predominates or arises in the period of senectus, not of senium; if it has not appeared in the earlier it will not come to much in the later. Lancereaux declared emphatically that arteriosclerosis is not a disease of extremer age, but usually appears, if it is to appear at all, between 60 and 65 . Here again however $I$ have to distinguish between high pressure arteriosclerosis and the decrescent form; the form due to vascular strain, in hyperpiesia or kidney disease, occurs on the average at an age somewhat younger than the "senile," distinctively so called. If the pathological effect in both may be the same, the kind due to Hyperpiesis or to "Granular Kidney" is not infrequent in much younger persons; though it is true that in these younger and less vulnerable subjects the strain of high pressures takes a much longer time to make its mark on the arteries. Although the "senile" form is naturally an affection of more advanced years, yet it is often antedated by the inroads of infectious disease.

High pressure arteriosclerosis then is certainly not "senile"; it occurs at an earlier period of life, we might say roughly a decade earlier ; though, as resistance to strain may then delay it by perhaps five years, the age period of high pressure arteriosclerosis, and that of the decrescent form, if they do not coincide, may overlap. And the longer survival of the decrescentarteriosclerotics 
separates the age incidence still farther. Probably, if we leave out on both sides the exceptionally young cases, to be discussed severally, the age period of my Hyperpiesia (Huchard's "Presclerosis" ; von Basch's "Latent Sclerosis") would lie over an age period of, say, 45-60, with an average duration of ten to fifteen years; that of my Decrescent Arteriosclerosis-the "Senile" form-over a period beginning about the age of 55, commonly perhaps with the ossification of the costal cartilages, and with an indefinite duration. For while Hyperpiesia, if uncontrolled by medicine and unbroken by accident, is a mortal disease, one of which the unimpeded progress brings life to an end by failure of the heart to keep up the fight, one in which however life is often cut short incidentally by sanguineous apoplexy, decrescent arteriosclerosis on the other hand, whether in inward or in outward trunks, is not incompatible with even extreme old age.

In this age-comparison chronic renal diseases are omitted; these maladies have their own cycle. Moreover, in respect of its bearings on our present subject, I shall consider renal disease in another chapter (see Renal chapter, p. 309). If here again we disregard the young cases, which however are not a few, the age incidence of Granular Kidney would be about that of Hyperpiesia.

In a later section I shall deal fully with the arteriosclerosis of infectious origin, the chief of which is syphilis. Before me are the notes of a case of syphilis in which the primary infection was incurred at the age of 17 , and after this an arterial affection appeared within the year. For such kinds of arteriosclerosissyphilitic, influenzal, typhoidal, etc. (see p. 281) - there can be no age averages. Lancereaux's remark that if in any individual arteriosclerosis has not appeared by the age of 60-65, it will not appear at all, is a reflection which must be taken to apply to mere senile arteriosclerosis. If we do not take painful note of slighter degrees of arterial thickening in the limbs, which may be no great matter, it is so far fairly true. Hyperpiesia, as I shall show afterwards, may appear at any age; it is no infrequent contingent disorder in elderly people, when, if detected in good time, it is usually readily curable; if not, however transient, it is a serious menace to decadent structures. I am disposed to think that if an individual have a proclivity to hyperpiesia so strong as to defy treatment, he will have it comparatively early in life, 
say by the age of 50 ; when it appears late in life it is not so ingrained, it is often a curable incident. The oldest instance I happen to remember among my primary hyperpietics was in a man, at the time of the diagnosis, aged 76 ; but the disorder was then evidently of long standing, medical aid had not been sought till the heart was in defeat; no doubt the malady had been going on for ten or fifteen years at least. Still, even with this allowance, it was a belated example. About a year afterwards an attack of aphasia occurred, with recovery; and a little later a right-sided hemiplegia; but the patient survived about two years longer. The sanguineous apoplexy which waits upon hyperpiesis occurs, as a rule, somewhat earlier in life.

Observing the same divisions between the arteriosclerosis of decrescence, of hyperpiesis, and of infection, let us turn now to the young cases, and first to early hyperpiesis. Bourguignon says that " arteriosclerosis, formerly regarded as "a normal malady" of old persons, is becoming every day more and more precocious." The arteriosclerotic of 40 years of age is no longer a rarity. Strictly speaking, perhaps, in the young arteriosclerosis proper does not often, if ever, follow high pressures ; yet to high pressures they are subject not infrequently, and to thickened arterial walls, as I pointed out many years ago; but this consequent thickening, if any, in my experience passes away with its causes, and, infections apart, may amount to nothing worse than a hypertrophy of the muscular coat of the media. The vessel wall, though too palpable, feels softer than in senile arteriosclerosis. ${ }^{1}$ The vessel is not closely contracted. I drew von Schrötter's attention to one of these cases, and he promised to investigate them, if possible histologically; but they were hard to find on the post-mortem table. It is not easy to picture atherosclerosis in the artery of a child, which, like a rabbit's aorta, is little more than an epithelial layer upon the undulating elastica interna. Like the rabbit's vessel, it seems scarcely capable of atherosclerosis. Von Romberg, who also has recognised these juvenile cases, says we have no information as to the ultimate issue

1 See also Russell, W., Lancet, June 1, 1901. Dr. Russell says that in these cases the thickening of the vessel disappears under nitrites. It may be so ; but I think his sphygmographic tracings are insufficient to prove this. $\mathrm{He}$ will probably agree that a small difference in height in tracings of the same character means little (vide p. 60). 
of them, nor any knowledge of their anatomical nature. His youngest case was at the age of 12 . Since then however some information has come in. Fahr ${ }^{1}$ examined a number of persons after intervals of some years from dates when the arteries had been before noted as thickened, and the heart as hypertrophied, and who since those notes had suffered from no illness. He found that in the large majority of cases the abnormal conditions had wholly vanished; but not in all. In some, besides more or less enlargement of the heart and thickening of the arteries, the heart had failed in rhythm, or a systolic murmur had appeared at the apex. Fahr infers that in these cases the vascular material was of inferior quality. Is it not at least as probable that in these cases some later causes had been at work? Other authors have reported juvenile cases of this kind (Fränkel, Bäumler, Thayer and Councilman, Marchand, O. Müller, Oppenheim) and have generally attributed them to infections. 0 . Müller ${ }^{2}$ quotes cases from Seitz (at ages of 12, 13 and 14), Fischl Martin (age 9), Filatou and Halm (age 12), Sonné and Chiari (age 13). In one of his own cases the aorta of a child, aged 5, was atheromatous. Among older writers, I have read, in Hodgson's Diseases of Arteries, of a case in an infant aged 15 months, communicated to him by his friend George Young; and Scarpa ${ }^{3}$ and Portal ${ }^{4}$ each records a similar case. Hirschfelder ${ }^{5}$ records the case of a boy æt. 6 , ill of an acute nephritis and pneumonia following a B. coli abscess of the buttocks and a B. coli cystitis, in whom the arterial pressures were continuously low throughout, yet the accessible arteries became tortuous and thick. The boy made a complete recovery, and the arterial affection passed clean away.

But there is more than this to be said: these last were probably infectious cases in which the blood pressures were as usual in children, yet with these not infrequent cases it is my present purpose to compare others in which there is no known history of infection but the blood pressure is temporarily raised and the radial thick. In this observation 0 . Müller agrees : he has noted

1 Fahr, Deutsche Arch. f. klin. Med., 1911.

2 Müller, O., Deutsche Klinik, 1910.

3 In his work on Aneurysm.

4 Portal, Cours d'an. méd. vol. iii. p. 133.

5 Hirschfelder, loc. cit., p. 345, 2nd ed. 
palpable arteries in young persons, under puberty, and again about the age of 25 ; and in some of them a heaving heart-beat, definite hypertrophy of the left ventricle, and a reinforced second aortic sound. These patients are " bilious"; the tongue is heavily coated, the abdomen tumid, the bowels irregular, the stools offensive ; and the child or youth is languid and pasty-faced. Under gentle mercurial and other appropriate treatment the whole trouble clears up, and the arteries after some while recover their natural quality (p. 618). In these cases I think the vessels are merely temporarily hypertrophied. Broadbent, in the discussion on my paper at the Medical Chirurgical Society, in January 1903, agreed with me concerning these cases; he said: "The condition ... high systolic pressure and fulness between the beats (italics mine) is sometimes recognisable in the child, and the schoolboy; and often in men under 30." As these cases will be considered again under the head of Symptoms they may be held over for the present. The arteriosclerosis of certain infections however, as we shall see presently, if not very infrequent in children is perhaps more prone to appear in adolescents. Dr. Mott says that "in asylums atheroma of the aorta is common on the post-mortem table, even in comparatively young people." It is not usually attended with high pressures, and "is more fibrous than atheromatous in its nature." These are probably of infectious origin; processes which will be considered in a later part of this chapter (see p. 281).

As I was collecting notes for these paragraphs on age, a gentleman, aged 30, presented himself for insurance with an excellent personal and family history. With one exception, all tests which I could bring to bear on him were favourable. Certainly his heart and kidneys were healthy, yet his accessible arteries were definitely thickened, and the emptied vessels felt like tape. But the pulse was not sustained, and the systolic blood pressure was about 120. Lancereaux ${ }^{1}$ recorded his cases of arteriosclerosis in decades between 30-40, 40-50, and 50-60; but he did not, of course, distinguish between the decrescent and the hyperpietic forms. He added however the interesting remark, which is true I think of the decrescent form, and worth remembering, that the younger the patient the more active the process. Of such are the two cases which follow:

1 Lancereaux, Paris Med. Soc., 1908. 
Mr. J., seen with Mr. Wingate, May 1904. Æt. 43. Weakly and dyspeptic ; often pains of " neuralgic" or of uncertain nature. No probability of syphilis. Habits quiet and temperate. No anxieties ; business easy and sufficient. Ârteries, so far as traceable, very degenerate; those of the feet and lower leg thick, non-pulsating cords. Pressures "always extremely low" (Wingate). No history of infectious disease. The pulse at my visit extremely weak and compressible.

Young man, æt. 26, seen by many of us in hospital. No history of typhoid or other infection. A weakly person with little energy. Arteries thick, wherever accessible. Blood pressures, at various dates, never above normal ; usually rather below. About the same time I had under my care an undergraduate subject to occasional fits of epilepsy, which seemed to be amenable to treatment. Habits, on account of his malady, very careful. No syphilis or other infection. Both radials were conspicuous, and rolled thickly under the finger. Systolic pressure never above 120. No history of any infection. (See also Longworth's case, Female æt. 29, p. 187.)

Yet in some elderly persons also the degeneration is rapid. The arterial thickening which we find not infrequently in early middle life is generally a mark of some past infection, as explained pp. 281 et seq.

We come now to certain extremer manifestations of arteriosclerosis in youths and children, and even in infants, not high pressure cases, and often with calcification. Brault, in 1897, contested the too positive axiom that arteriosclerosis was unknown in youth. Andral published cases at the ages of 15 months, 7,18 , and 24 years. H. Martin, at the age of 9 ; Durante, in an infant born prematurely at the seventh month (Soc. Anat., 1899); Gee, at the age of 7 ; Marchand, at 22 and 27 ; and many other such instances are on record by Filatou, Chiari, Simnitzki, etc., the majority probably being syphilitic. Chiari's infant was syphilitic and died aged 15 months. The same congenital infection was present in three cases, aged 10,11, and 12, published in his M.D. thesis (Camb., 1901) by Dr. Graham Forbes; in these the cerebral arteries and brain were much diseased. ${ }^{1}$ If not a few of these very precocious cases are syphilitic, this is not true of all; indeed some cases attributed to syphilis arise from more respectable causes; but with these "congenital or infantile" cases I

1 See also Bury, Brain, April 1883. 
have really little concern. To pass onwards to youth and adolescence, I reported some years ago, and showed to my pupils and friends, that in cases of diabetes in girls and boys in their teens the accessible arteries may be considerably thickened (p. 279); now in these cases the pressure is usually far from high. The artery is uniformly thickened, not nodular. In an undergraduate who consulted me for "heart strain" at football, I found the heart, as also the stomach, dilated, and wanting in tone. His pulse in recumbency was 80 ; on standing up 120 . Under treatment these symptoms abated, and in fourteen weeks he was well; but I had noted that his radial tunics were decidedly thick, and the vessel " tuby"; though the blood pressure (systolic) was well under 120. In him again there was no definite story of any infection, but these overdone hearts are most commonly due to some passing infection. Dr. Ainslie Hollis, to whose instructive papers on arteriosclerosis we are all much indebted, found such elevated patches in the aorta of a boy æt. 11, who died under his care from appendicitis. ${ }^{1} \mathrm{He}$ found an atheromatous aorta in a girl aged $2 \frac{1}{2} ;$ a similar condition of the aorta in a girl, æt. 17, who died of pneumonia; another again in a girl, æt. 18, who died ten days after a burn : 12 out of a series of 52 cases of arteriosclerosis were, at death, under æt. 23. Such instances, if looked for by observers intent upon the point, would prove to be not infrequent. As we shall see under Infections, attention has been fixed too exclusively, if still insufficiently, upon the heart. Certain cases of thick arteries in the young, reported by Dr. West, ${ }^{2}$ which with me he is disposed to attribute to a transient medial hypertrophy, were associated, or at least some of them, with raised blood pressures; but probably the examples I have just been considering as due to some infection, noted or unnoted, were not associated with high pressures. Simnitzky's ${ }^{3}$ paper on this subject is well known. This author, who unfortunately included no clinical notes in his article, rested his argument upon necropsies only, and his

1 The reprint in my hands bears no date nor reference.

2 West, S., Lancet, Feb. 11, 1899.

3 Simnitzky (Prague Institute, a pupil of Chiari), Zeitschr. f. Heilkunde, April 1903. "Häufigkeit von A.s.schen Veränderungen d. jugend. Individuen." 
researches were chiefly directed to the aorta. He reported 37 cases of altered arteries, at ages ranging from 2 to 25 ; that is 27.5 per cent of his series of autopsies on children. For the most part there were no direct clinical symptoms of the arterial disease. In all of them the changes were of the atherosclerotic kind, and in 18 the history of infection was definite, and belonged to one or other of the following kinds: rheumatic fever, diphtheria, scarlet fever, measles, smallpox, typhoid, pneumococcus, tuberculosis; in all but seven an infection had occurred. Syphilis was excluded from his series. Simnitzky calculates that in 27 per cent of necropsies in persons under æt. 25 some signs of arterial affection-at least of the aorta-are to be found, and that these for the most part are due to infections. Fraentzel writes that in the Franco-German war he observed not a few young men with thickened arteries; and further that these men succumbed to fatigue while others of no more apparent strength, but with normal vessels, got through fairly well.

In two only of Simnitzky's cases were the vasa vasorum affected in such a degree and manner as to suggest that the sclerosis might have depended primarily upon them. In cases of recovery from infection, he also thinks that probably for the most part the arterial lesions clear up entirely. Josué, in his book on Arteriosclerosis (1903), and Oppenheimer, ${ }^{1}$ testified to the same or similar effect, except that Oppenheimer regarded the change as more of the kind of primary medial degeneration with atrophy of elastic fibre. Certain authors, Saltykow for instance, ${ }^{2}$ push these observations so far as to declare that arteriosclerosis, in its germination, is not a disease of age ; that its beginnings, as some opine concerning tuberculosis, are to be sought in adolescence, and even in childhood, though it may not manifest itself till years likewise begin to tell. Saltykow, whose views may be taken rather from chemical than from clinical experience, slights mechanical causes, repudiates "wear and tear," and relies too much on poisons, such as alcohol.

It can scarcely be said then that " these young cases attest the hereditary nature of arteriosclerosis, because in them none of the recognised etiological factors can be found"; none of the

1 Oppenheimer, Virchow's Arch., 1905.

2 Saltykow, Korr.-Bl. f. schw. Ärzte, 1911. 
conventional causes may be found, it is true, but we shall have to grant a much larger field to infectious causes in arteriosclerosis than we have been wont to do (p. 281). In the well-known case of Bryant and Hale White ${ }^{1}$ (an infant of 6 months), the child had suffered from congenital phimosis with retention of urine, dilatation of the ureters, double hydronephrosis, and atrophy of the kidneys. Probably there was no syphilis in the case. All the arteries of the body were affected, and many of the smaller ones obliterated. In a collection of cases in his graduation thesis (Camb. 1901) on cirrhosis of the liver in children, Dr. Bull of Ashbourne reported of a child (Case XIX.) aged 5, with cirrhosis and ascites and anasarca, that the arteries were hard; although the heart and kidneys were reported (at St. Bartholomew's) to be normal. The parents had given brandy to the child "for a long time." Perhaps the most important paper on this part of the subject is by Wiesel of Vienna, ${ }^{2}$ who, from this point of view, investigated 300 necropsies, and reported minutely on 80 of young, many of them very young, children who had succumbed to diphtheria, scarlet fever, and other infections; the clinical kind of the infection is of less pathological moment, for the characters of the morbid histology are much the same in all (p. 287). Such difference in form as there may be depends chiefly upon the stage of the disease. About the end of the first week changes become visible, in the aorta, carotids, and coronaries, etc., as minute yellowish patches, some presenting calcareous points. Under the microscope these changes are found widespread throughout the arterial tree; indeed they predominate in the peripheral vessels. The first change is an infiltration of the media and vacuolation of the muscular layer; then the elastic fibres break up, and the muscular tunic undergoes necrosis. The endothelium survives longer, and the intima is involved only in a small minority of the cases.

As regards later stages of this lesion, as revealed by a few necropsies after death from other causes, the result of healing is some increase of fibrous tissue, probably not very harmful. Even if high-pressure thickening in comparatively young persons be not mere muscular hypertrophy but some lesion, in early life

1 At the Meeting of the Royal Med.-Ch. Soc., Jan. 12, 1903.

2 Wiesel, Zeitschr. f. Heilkunde, 1906, Bd. xlvii.

VOL. I 
the arteries probably have considerable powers of regeneration. Elastic and muscular elements can be restored, as experiments and surgical operations on animals have demonstrated; if experimentally an artery be laid bare from its external supports, and consequently thickens, on the restoration of its normal conditions it returns to its proper measure in three or four weeks.

Wiesel's investigations are then corroborative of those of Flexner and Thayer in typhoid. In its acute form the process suggests, clinically speaking, "Mönckeberg's" rather than Jores' arteriosclerosis. Ortner, von Romberg, Marchand, Bäumler testify to the same or similar effect, and I need not multiply instances; but I may refer to a good paper by Levy-Frankel, ${ }^{1}$ who writes fully, on 11 of his own cases and 34 collected, of arterial disease in children. In the majority of his cases syphilis was the bane; but rheumatic, scarlet, and typhoid fevers also had their victims. In cases of infection a fibrous aorta may be overlooked and the vessel regarded as not abnormal; yet this hyperplasia is the sequel of small cell infiltration, and of blocked or congested vasa vasorum.

Von Romberg calculated that of necropsies of children under the age of 15 arteriosclerosis was found in 5.88 per cent.

The calcareous plates which form about spots of imperfect vascular development in fœtal life are curiosities on which I need not dwell; and for many other instances and references I may refer to Fremont-Smith's full and accessible paper. One of his examples however I am tempted to quote: "In 1890," he writes, "I attended a . . . man aged thirty-two, ... with sclerotic and beaded arteries. He gave a history of luetic infection in his eighteenth year . . . my attention was drawn to his son, a boy aged twelve years, who by his pinched and haggard appearance, by his complexion, figure and carriage, suggested the withering of age rather than the freshness of youth. Examination revealed sclerosis in all palpable arteries, with calcification as advanced as in his parent; the beading could not have been further exaggerated at any stage of life." There was no kidney disease, and the author's diagnosis was congenital syphilis. Whether syphilitic or not, coronary and cerebral arteriosclerosis are not rarely found in young persons ; coronary atheroma is even frequent in young

${ }^{1}$ Levy-Frankel, Arch. des mal. du cour, oct. 1, 1912. 
subjects of chronic renal disease. I remember hearing Dr. Mott say that he had found atheromatous arteries very frequent in juvenile forms of General Paralysis of the Insane.

Sex.-In hospital records the number of male to female arteriosclerotics is as 3 to 1 ; but this by no means represents the true proportion. Medical statistics based upon hospital statistics are misleading. It is scarcely necessary to point out that selection is at work in many directions. My experience of private practice is still what I have often stated of it before; namely, that the difference between the sexes in this liability, at any rate to the decrescent kind, is inconsiderable; and for this opinion we have also the authority of Dr. M. Bruce. However, here again an indiscriminate gathering of all kinds of thickened arteries under one title accentuates the error of regarding arteriosclerosis as " a Disease," instead of, what it really is, an effect of diseases many and various. For example, syphilitic arterial disease is, no doubt, far less common in women than in men; and, if tobacco has anything to do with sclerosis, I hope we may still assume that Englishwomen do not smoke. Women again are on the whole less exposed to current infections than men, and less exposed moreover to whatever arterial attrition may result from business cares or muscular stresses. What these may be we shall consider presently. The arterial thickening due to hyperpiesis is, I think, as hyperpiesia itself, less frequent in women, though not uncommon with them. Ordinary decrescent atherosclerosis is indeed so frequent in both sexes as to show little proportional difference; we find it in all conditions of life, in castle, vicarage, and cottage. Dr. Newton Pitt agrees that atheroma is about equal in the two sexes. There is no conclusive evidence that the menopause, save as a signal of the watch, nor that repeated pregnancies, with their reputed toxic influences, have much to do with its provocation. Dr. Mott says after the climacteric both sexes are alike obnoxious to this decay; but that before the age of 50, often that is in the hyperpietic kind, it is more frequent in men. On this side of the comparison we must not forget that the arteriosclerosis of bodily labour may be confined, or chiefly confined, to the limbs. Some of my most instructive cases-whether hyperpietic or decrescent- 
have been in women; because in the circumstances of women we enjoy more continuity of observation, and the contingencies are fewer.

Of Race, Country, and Climate in respect of arteriosclerosis we know little, and this little suggests that these factors are at most of subordinate importance. Estimates of frequency in this region of the earth or of that depend much on the diligence, opportunities, and bias of the observer. It is said to be more frequent in the United States, and to appear there at an earlier age; but for some time past the Americans have been closely watching their arteries. It is said to be frequent in England "because the English are large meat-eaters"; they may be, but surely the traveller who at cosmopolitan hotel tables observes the customs of his continental neighbours will not be convinced that the Englishman is the most voracious of the guests; nor do I think that the case histories of spas, of Marienbad for example, would give this testimony. It seems that in all countries the plethoric form of arteriosclerosis prevails rather among the high feeders, that is, among the wealthier classes, especially in the colder regions where a more plentiful and richer feeding is required, and that the decrescent form prevails rather among the poor. My own impression of hospital practice is to this effect. The impression one received from Abercrombie and Watson was that sanguineous apoplexy, a frequent result of high pressures, was more common among the gentry than among the working classes. Occupation cannot be forgotten in this connection. In hot countries, where the foods are simpler and lighter, the decrescent form of arteriosclerosis will be relatively more frequent, and this seems to have been the case with the ancient Egyptians, with whom neither a rich meat diet, nor alcohol, nor tobacco, nor syphilis, nor gout, nor modern "worry" had much place; yet we have seen (p. 5) that in their bodies arteriosclerosis is common, often extensive, ${ }^{1}$ and not very late in life. Professor Pick says that in Bohemia, where the hospital patients come of a poor class, the arteriosclerosis, unless it be a part of chronic Bright's disease, is, generally speaking, not attended with high blood pressure. Of the liability of the Jews to decrescent sclerosis

${ }^{1}$ See Journ. of Path. and Bact. vol. xv., 1911. 
I have spoken. Medical men who have practised in hot countries report that, although in these climates blood pressures rule low, arteriosclerosis is frequent enough; and as senility comes earlier in life so does senile arteriosclerosis. Physicians practising in India, such as Professor $\mathrm{M}^{\mathrm{C}} \mathrm{Cay}$ of Calcutta, tell us that arterial systolic pressures rule lower among the natives than in Western Europe (90-105), yet that their arteriosclerosis is precocious ; and it is of the senile variety. Their hæmoglobin is also 25 per cent less. Warfield ${ }^{1}$ and Camac ${ }^{2}$ say that arteriosclerosis is more common, and appears earlier in the black races than in the Caucasian; but Warfield adds that, beside their physical labour, syphilis and aneurysm are very prevalent among them. The Reports of the Massachusetts General Hospital (Oct. 1908) do not bear out the greater proclivity of the negro in the States. I gather from these reports, though the reporters do not observe my distinction between the two main kinds of the arterial disease, that it is the decrescent form with which they are mostly concerned. Dr. Oliver made comparative estimates of blood pressures in hot and cold weather respectively, and satisfied himself that, on the whole, in continuously hot weather blood pressure in the brachial artery fell, while in the phalangeal vessels there was a rise. Dr. John Lambert ${ }^{3}$ observed that on bright days, with a high barometer, blood pressures rose, with a sense of well-being, especially in persons with whom pressures ran naturally rather low. But the data for these factors of causation are as yet defective. Dr. Stengel, basing his conclusion on certain statistics, attributes an apparently rapid increase of arteriosclerosis to a city life; he does not distinguish between the two chief clinical kinds, and forgets, I think, that in recent city practice diagnosis has become far more searching; moreover, of late years the subject has attracted far more attention.

On Pike's Peak (14,109 ft.) Dr. Haldane and his companions found pulse and blood pressures little affected. Kronecker, after ascending to the Jungfraujoch by rail, found no change in blood pressure, though a little bother at the station below had caised it

1 Warfield, Arteriosclerosis, St. Louis, 1908.

2 Camac, Amer. Journ. Med. Sci., 1905.

${ }^{3}$ Lambert, Thesis for M.D., Cambridge, 1908. 
to 160 . Sir Lauder Brunton has come to the same conclusion. This may be true for healthy men, but I am sure a level of 3000 to 4000 feet disturbs persons already the subjects of high pressure (Vol. II., p. 102).

It has been said, by Rumpf for instance, that calcareous water, or large milk consumption, promotes calcification of the arteries. But, as Dr. Percy Lewis remarks, ${ }^{1}$ "If the degenerative process wants lime there is plenty to be had, both in the body and in the food, without calling on chalk in the water, and so forth." In my district of Cambridge the drinking water is hard, but I have not found any difference in the prevalence or severity of arteriosclerosis between this district and the manufacturing districts of West Riding, where the moorland water is of the softest. Gouget, who knows well a certain calcareous district in Champagne, around the Aube, where the water is hard even to milkiness, says arteriosclerosis is not more prevalent there than elsewhere. The fact is, calcification is a secondary and ultimate event, the coffin, not the cause of decay. Indeed, as we shall see later (p. 505), calcification may be rather a good than an evil; it may give some substance to a perishing vessel.

Mechanical Causes.--When, among the momenta aetiologica, we undertake to discuss the mechanics of arteriosclerosis, we approach the centre, or one centre, of our subject. In dealing with the causes we cannot exclude from consideration certain alleged mechanical consequences of this affection, and may discover some reciprocations of cause and effect. Not only so, but on a discrimination of the mechanical conditions, whether as causes or effects, depends the truth of my version of arteriosclerosis. This subsection therefore must run to some length.

For instance, are certain manifestations of excessive arterial pressure dependent on a pre-existing arteriosclerosis? Or do these rises of pressure precede the affection, and possibly produce it? In the beginning of this chapter I argued that arteriosclerosis, with high pressures and without them, were not incidental variations of one general process but were two several kinds. In the following paragraphs I shall proceed, with as little repetition as convenient, to find out what may be the mechanical conditions

1 Lewis, P., Brit. Med. Journ., July 22, 1911. 
and correlations of arterial disease and blood pressures. We have seen that von Basch and the majority of authors down to the present day have opined that the arteriosclerosis comes first, and when established so impedes the blood stream that resistance increases, and therewith the pressures in the larger trunks and onwards. Potain ${ }^{1}$ likewise, following von Basch, always would have it that high arterial pressure was the result of sclerosis; though he admitted that, sclerosis once established, these stresses would exert a further deformative reaction upon the weakened tube ; so far at any rate as the peripheral areas, in which normally the pressure gradients fall quickly. Then to the contrary Huchard in 1893, I myself independently in 1894, Edgren ${ }^{2}$ in 1898, and afterwards Thoma and many other authors, urged that the high pressures, however brought about, were not the result but a cause of arteriosclerosis. H. W. Cook (loc. cit.) says that in 1904 Broadbent and I were still alone in opposing the view of "hypertension" as the effect of arteriosclerosis. For Huchard to the end of his life, arteriosclerosis signified always a previous phase, observed or unobserved, of excessive pressures, a phase perhaps of the past, but always implied and essential. So likewise for Edgren, and in France for Hayem, Lancereaux, Chantemesse, all arteriosclerosis meant exorbitant blood pressures ; though Edgren admitted toxic and nervous causes as subordinate and indirect.

But with this single category in my own first paper (1894), as in all my later papers, I was not content. From the first I have reiterated, and must try to drive it home (see p. 155) that arteriosclerosis may arise, and very often does arise, without any preliminary phase of high pressures; that in time, under ordinary pressures, and in almost all persons, the arteries give way, the exceptions in old age being rare; so that from middle life onwards arteriosclerosis becomes as frequent under ordinary as under excessive pressures, present or past. For past phases of morbidly high and persistent pressures leave plain and indelible records upon the heart. How is it then that although Traube saw that high pressures occur without sclerosis, and sclerosis without high pressures, and although Hope in $1832^{3}$

1 Potain, La Pression artérielle, Paris, 1902.

2 Edgren, Die Arteriosklerose, 1898 ; a valuable treatise.

3 Hope, James, Treatment of the Diseases of the Heart, 1832. 
asked, "What is it that calls this depraved condition of the blood vessels into activity?" and replied, "It appears to me that overdistension of the arteries by the force of the circulation is what, principally at least, produces this effect," yet to this day authors, both on the clinical and pathological sides, fail to clear their minds of this confusion, and continually speak of arteriosclerosis as the cause rather than as the effect of blood pressures? In essay after essay, even the most recent, we still read of " alterations in the vessels leading to high pressure," and meet the assumption that arteriosclerosis and high pressures are but the static and dynamic aspects of one and the same "malady." For example, at the Toronto discussion, in 1906, an eminent British Professor of Clinical Medicine roundly declared that "arterial tension might be the result of arteriosclerosis but was never the cause of it "; ${ }^{1}$ and thus did but express roundly what is still the opinion in vogue. It is but the other day that in a leading article of a medical journal I read that " the existence of generalised arteriosclerosis may be inferred with a fair degree of certainty from blood pressure measurements." Indeed the great authority of Dr. Mott seems to incline to these opinions; he speaks of arteriosclerosis coming first, then a rise of pressures, and, thirdly, " a multiple effect of both ; so that the arterial lesion may be both cause and effect of high pressures." "In most cases," he says, " of arteriosclerosis we find a combination of thickened arteries, a high-pressure pulse, and a hypertrophied heart"; but in his sum of cases not a few are included in which the kidneys were under suspicion.

How do these prepossessions arise? Well, in the old story that because the arteries lose elasticity the heart has more work to do; therefore it hypertrophies, and pressures rise. But why? if the added heart work does no more than make up the loss. Thus an eminent English author is now arguing that as with advancing years the arteries lose elasticity, they offer an increased resistance to the blood stream, and consequently the heart hypertrophies. But it is not in "senile arteriosclerosis" that the heart hypertrophies; and under high pressures the heart waxes some time, for some years indeed, before arteriosclerosis sets in. It is not probable that loss of elasticity is concerned in the moderate

${ }_{1}$ Lancet, Sept. 15, 1906, p. 729. 
quasi-normal rise of pressures which is the rule in middle and later life, but by dissipation of energy it may slow the stream. Even Gouget, ${ }^{1}$ who has had to admit at last that high pressure could no longer be regarded as the sole cause of arteriosclerosis, still declares that this degeneration, if spread over a large number of vessels, must augment arterial tension (" a pour conséquence obligée une exagération de la tension artérielle "), at first by itself ("par ellemême"), then by hypertrophy of the heart. This view prevailed at an important discussion in $1905,{ }^{2}$ when however the late Dr. Foxwell brought forward good arguments to the contrary. ${ }^{3}$ As von Romberg says, ${ }^{4}$ sclerosis, even if it should increase heart work, cannot raise blood pressure; loss of elasticity does not make more heart work; but makes it less effectual and the stream jerky; ${ }^{5}$ as we see it in the relaxed carotids of healthy persons under emotion, or in Graves' disease. In mechanics, to substitute rigid for elastic tubing of the same calibre reduces pressure. I shall argue that in a decayed aorta the sensibility of the arch as a blood-pressure tambour may be impaired, and pressures so far be less well governed.

In 1910 the Paris correspondent of the Lancet, $^{6}$ after stating untruly that " before Huchard's discoveries the attention of medical men had been directed almost entirely to lesions of the cardiac orifices" (in this respect ignoring all von Basch's pioneer and far more accurate researches, the work of Potain, of Sanderson, of Oliver, of Broadbent, of Brunton, and many others), said that " to him (Huchard) belongs the merit of showing that many cases of heart disease are due to a functional disorder of the circulation, which he called arterial hypertension, and to arteriosclerosis of which this hypertension is only the first stage" (italics mine). My own observations (published in 1894, showed that at best Huchard's view was but a partial one, and that the cases of arteriosclerosis without substantial increase of pressure were as many; to this class I then gave the name

1 Gouget, Artériosclérose, 1907.

2 See Brit. Med. Journ., Oct. 21, 1905.

3 Same journal, p. 1028.

4 I think in his article in Deutsche med. Wochenschr., Oct. 28, 1909.

5 As bearing on this subject see Josué, Arch. des mal. du cœur, avril, 1909.

6 Lancet, Dec. 24, 1910. 
"Involutionary," but afterwards, in response to some criticisms, "Decrescent." Teissier shows some disposition to accept my views of the several kinds of arteriosclerosis, and divides them into "Arteriosclerosis" - by which hyperpiesia seems to be intended, and "Atheroma," to correspond with my decrescent form in which he admits that pressures do not rise. He well contrasts the therapeutics of each. But we shall see that we cannot thus distinguish the processes as "arteriosclerosis" and " atheroma" (p. 510). Ortner" has now to admit that uncomplicated arteriosclerosis may produce neither cardiac hypertrophy nor high pressures; but in another place he still calls ${ }^{2}$ high blood pressure " the leading symptom of established arteriosclerosis." Jores ${ }^{3}$ says categorically "even in far advanced arteriosclerosis hypertrophy of the heart may be absent or very slight." I repeat then that for the assertion that arteriosclerosis is itself a cause of pressures abnormally high for the patient's age we have no evidence whatever. When time after time cases are pointed out of high arterial pressures without disease at any rate of any accessible arteries, and furthermore, of the cure of this condition, which von Basch himself candidly admitted-indeed he narrated many such cases, but dismissed them as "pseudo-arteriosclerosis" - it was, and still is, argued nevertheless that the rise is due to arteriosclerosis, but of vessels inaccessible to sight and touch, as, for example, in the mesenteric and aortic areas (Hasenfeld and Hirsch), or in wide ramifications in peripheral areas (Balfour). Broadbent, ${ }^{4}$ in discussing my view eight years after its publication, testified to cases of arteriosclerosis " in which the blood pressure was not high, and never had been." Masing ${ }^{5}$ also supported my view decisively, saying that the blood pressures bear no definite relation to arteriosclerosis "das Mass der Entwickelung des sclerotischen Prozesses ist keineswegs entscheidend für die Höhe des Blutdruckes." He gave an example of a man, aged 68, with well-marked (" ausgesprocbenem ") rigid and snaky arteries, whose blood pressure did not exceed 120. One may see dozens

1 Ortner, Fortschr. d. D. Klinik, Bd. i. 1910.

2 Id., Jahreskr. Feb. 1911.

3 In his art. in Deutsche Arch. f. klin. Med., Sept. 1908.

- Broadbent, W. H., Lancet, Feb. 7, 1903.

5 I think in his paper in Deutsche Arch.f. klin. Med., vol. lxxiv., 1902. 
of such in any large workhouse. Any quantity of evidence can be produced in proof of this postulate ; for the sake of illustration I insert the following examples.

From a list of cases recorded by Dr. Stephen Longworth, of the Melton (Suffolk) Asylum, who has kindly taken many observations for me, I select a few to illustrate the compatibility of arteriosclerosis with ordinary or low pressures. His work was in the female blocks. In all of these the urine was carefully and repeatedly examined for albumin, casts, etc., and found normal. The estimations, save one, were made in the sitting position with the usual precautions. More than one instrument was used, but the readings were compared with each other and with a mercury column.

(F., æt. 66. Quiet secondary dementia; physical state good. Heart normal, not enlarged. Arteries hard and tortuous. Systolic pressure $95-100$; pulse rate 68.

(F., æt. 66. Recurrent mania, subacute ; physical state fairly good. Arteries hard and tortuous. Heart normal ; not enlarged. Systolic pressure 115 ; pulse rate 80 .

(F., æt. 36. Stupor after mania; reduced health and anæmia. Radials distinctly hard and rolling under the finger; heart normal. Systolic pressure $65 \mathrm{~mm}$.; rate 64 . After one month's treatment (with nourishment and tonics) and general improvement, systolic pressure 105. After a further two months, when the general report was much the same, systolic pressure still 105 . In another month, on complete recovery and discharge, pressure had risen to 115.

(F., æt. 54. Subacute mania; physical state good. Arteries distinctly sclerosed. Systolic pressure 115 ; rate 68 . Heart normal. The pressure remained the same during her cure and discharge. During the illness traces of albumin appeared, but without casts.

(F., æt. 48. Chronic melancholia, with constant acute mental depression, delusions and hallucinations of sight, etc. Heart normal. Arteries markedly sclerosed. Systolic pressure 130 ; rate 60.

(F., æt. 29. Stupor following mania during pregnancy. Two months confined (at seven months). Physical state poor and illnourished. Confined to bed. Arteries tortuous and prominent. Heart normal. Systolic pressure (recumbent) 140 ; rate 52.

Dr. Longworth finds in many of such cases a low specific gravity of the urine (about 1014), but without casts or other sign of renal disease; and this often in patients who recover. 
Hasenfeld and Hirsch, in their well-known paper, ${ }^{1}$ alleged that if arteriosclerosis in peripheral arterial branches might not be attended with a substantial rise of pressure, with ensuing hypertrophy of the heart, yet that this consequence did follow when the disease occupied the aortic and mesenteric areas. They urged, moreover, that, in cases of high arterial pressure, even if in some cases to the eye the mesenteric trunks might have a fair seeming, yet, if microscopic search were pursued to their finer network, extensive sclerosis of these ramifications would be revealed. When, on its publication, I read Hasenfeld's paper, I thought his own cases by no means bore out his conclusions. However, it is sufficient to say that, although more than fifteen years have elapsed since Hasenfeld and Hirsch's paper was published, years during which their conclusions have been reasserted and built upon again and again, yet they have never been verified in the author's sense; namely, that high pressures and cardiac hypertrophy depend upon arteriosclerosis in the mesenteric area, and in this area, or the thoracic aortic, only or mainly.

No careful comparison of degrees of arteriosclerosis of the several areas of the body with the coincident ranges of blood pressure during life has been published. Hasenfeld and Hirsch admit that sclerosis in vessels other than the splanchnic and supradiaphragmatic "have no influence on blood pressure"; and contrariwise there is ample store of necroptic observation to prove that in many cases of arteriosclerosis without notable rise of pressure the aortic and mesenteric arteries have been, at any rate in their main trunks, extensively sclerosed; and we shall see that, while in these sclerosis is not frequent, in their finer ramifications it is rare. We learn from many pathologists, including Jores, that the finer ramifications of the mesenteric branches are generally intact. Again, if disease began in this area, what about the explanation of high pressures by spasm of the splanchnic system? Mesenteric arteriosclerosis, in so far as it may impair the supporting mechanism, would tend to a fall

1 Hasenfeld and Hirsch, Deutsche Arch. f. klin. Med. Bd. lix., 1897. Hasenfeld has since admitted (1913) that his explanation does not solve the problem. It is now too late to alter my text. 
of arterial pressures; on the other hand, if an arterial irritant were to set up an extensive constriction, the constriction would be the effective factor in rise of pressure; sclerosis being in this respect superfluous if not contrary. The aorta comes early in the order of liability to disease, and generally takes a large place in all extents of arteriosclerosis, whether with excessive pressures or without.

Thus later observers are compelled to admit a lack of parallelism between the courses of arterial pressure and the degrees of mesenteric arteriosclerosis; also that pressures may reach high levels before sclerosis in this area has progressed very far; and conversely. The late Dr. Savill, with whom I had frequent communications in this matter, whose field of experience in workhouse practice and necropsy was very large, whose diligence was indefatigable, and who compared arterial ramifications, magnitude by magnitude, in many areas, was unable to discover any such parallel, or any such guide to pressure levels. Thayer and Fabian (loc. cit.) concluded that on the whole radial sclerosis runs fairly parallel to the visceral and aortic. Von Romberg, who has fully admitted that high pressure may precede all sclerotic change, has himself produced postmortem evidence that in such cases the abdominal vessels ("Unterleibs Arterien") often show no correlative disease. Marchand ${ }^{1}$ is quite clear that rise of pressure is not due to mesenteric and cœliac arteriosclerosis, and proceeds to say that arteriosclerosis without cardiac hypertrophy is common; indeed that, as in some cases the heart is primarily degenerate, he doubts the alleged high-pressure effects of arteriosclerosis (" sehr fraglich ist, wieviel davon Rechnung der Arteriosklerose zu setzen ist"). It is true that, as the mesenteric postulate became less and less tenable, von Romberg and Hirsch began to lay more emphasis on the aortic side of the explanation; but everyday experience tells us that even in enormous aortic deformation, as in the Hodgson's aorta now known to be syphilitic, cardiac hypertrophy, unless by some complication, does not arise. When atheroma of the aorta is associated with hypertrophy of the heart the story is the other way: high pressures first, cardiac increase second, aortic injury third. It is surely common experience, whatever the

1 Marchand, art. "Arteriosclerosis" in Eulenburg, 4th ed. 
state of the mesentery, that the aorta, the orifices of the carotids, the subclavians, etc., may all be extensively diseased without any cardiac hypertrophy, or history of high blood pressures. Marchand (loc. cit.) agrees that in such cases the hypertrophy is "by no means constant," and cannot be attributed to the sclerosis; although in some of them a concomitant emphysema with hypertrophy of the right ventricle proves that, had there been any call for it, the heart was capable of hypertrophy. He says also, and quite truly, that cardiac hypertrophy cannot depend upon areas of arteriosclerosis. At most, sclerosis of the large vessels could but affect the systolic maxima; it could not raise the minima, which are of more importance. In the recent clinical descriptions of colicky symptoms attributed to abdominal arteriosclerosis (p. 445) ${ }^{1}$ it does not appear that any conspicuously high range of blood pressure is registered.

In my view, as time and experience have increased, the arteriosclerosis due to high pressures has taken a less rather than a larger compass. More than 50 per cent of Groedel's cases showed no signs of excessive blood pressures. Vessels which are incessantly subjected to hydrostatic stresses cannot but betray their effects ; and these the more as by one co-operating frailty or another their coats may be less fit to withstand them; but by arteriosclerosis of high pressure, properly so called, we mean surely lesions primarily and mainly thus produced in vessels previously sound. If, as frequently we find in highpressure cases, the arterial integrity is not otherwise impaired, it takes a longer and severer persistence of excessive pressures to damage them than is generally supposed.

In a young adult person-say, under 45-suffering from Bright's disease with inordinate arterial pressures, the aorta and vessels may, for a couple of years or more, as proved again and again by post-mortem examination, show no visible injury, and the heart itself no more change than a sound hypertrophy; so large are the cardiac capacities. Now if the rise of pressure does not follow but precedes the vascular strain, all the busy speculation as to the way in which arteriosclerosis causes high pressures is thrown away. 1903.

${ }^{1}$ E.g. Ortner, Klin. Vorträge, N.F., Nr. 347; Innere Med. Nr. 102, Jan., 
In a shopman, æt. 39 , with no renal disease, but a very high range of blood pressures during life, the heart after death weighed $42 \mathrm{oz}$., but the aorta was fairly normal. He was working till he came under my observation in the Addenbrooke's Hospital, only three weeks before his death.

Another case, also from the mortuary of the Addenbrooke's Hospital, may serve as a converse illustration. The autopsy was made by Dr. Aldren Wright. Male æt. about 55. Lead colic twelve years before. Albuminuria and granular casts. Blood pressures unknown. Kidneys small, scarcely any cortex. The collapsed heart weighed $19 \mathrm{oz}$., but was very degenerate; the finger could be thrust easily through the ventricular wall. Aortic and mitral cusps normal and fairly translucent. Aorta somewhat, not much, dilated, and inner surface a darker yellow, but no atheromatous patches. Here a long-standing early proneness of the heart to degeneration presumably had spared the aorta. There was some degree of peripheral arterial thickening (toxic ?).

In incipient hyperpiesia, so far as clinical observation goes, these parts and their functions may, on cure of the malady, return to something like integrity; but much of course depends upon the age, the constitution, and the habits of the patient. On the other hand, we may observe again and again well-marked or extreme sclerosis of peripheral arteries with aortic stenosis ; that is, with scanty output, low aortic pressures, and low systolic wave.

The corollary however that we have at least two clinical modes of arterial lesion-the high pressure and the decrescent - whether histologically identical or different - that the corresponding clinical series are therefore different, and that, as I reiterated at Toronto in 1906, " arteriosclerosis," be it single or multiple in form, is not "a disease but a pathological consequence of several diseases," is not yet realised. Although doubts are expressed by Mönckeberg, Adami, and others as to its pathological unity, a problem which we will consider hereafter (p. 483), in current medical writing the unity of the process, as a clinical series, is still assumed. It is needless to say that in the causes of the decrescent form mechanical stresses must of course play a large part, stresses which may be taken into the reckoning much in the same way as in the hyperpietic form; the difference in the decrescent form being not so much 
in the incidence of the strains as in the lower quality of the vascular structure, and in the presumptive co-operation of other coefficients of decay, such as metabolic perversions and incidental poisons, of which I shall speak hereafter. In this place I am not dealing with the causes of high blood pressurethese, so far as they are known, will be considered as they arise,-but with the causes of arteriosclerosis. So far then I do but maintain my dissent from the prevalent doctrine that, in this way or in that, arteriosclerosis itself is a cause of any considerable rise of blood pressure, of rises, let us say, above 140-150 mm. (See also p. 462.)

That high pressures and friction are competent to set up atherosclerosis was clearly shown by Roy and Adami; and I have adduced familiar proof of it in the alteration of the arterial wall at critical points, as at bifurcations, at narrows normal or morbid, and again at dilatations with change of wave leading to distortion and elongation. In its earliest manifestations the points and lines of atheroma are points and lines of stress, whether in valves or arteries. In congenital stenosis at the site of the ductus botalli the aorta, on the hither side, and the carotids and cerebral arteries, are extremely diseased (atherosclerosis), while on the farther side the tree is normal. Here no toxin is concerned. How far along the tree central pressures tell peripherally, that is in areas beyond the critical fall of pressure gradient, is unknown. This knowledge we need. Much must depend upon the habitual attitudes of tone in the several districts of the body.

If then, besides ordinary wear and tear, there are many other causes of atherosclerosis, we must not forget that in none can the mechanical coefficient be absent, or even negligible. If so be that in this way or that, by toxic agencies, as Krehl insists, or by originally defective quality, the consistence and resistance of the arterial coats are reduced, it is nevertheless by mechanical stresses that the undoing is directly effected; every increment of stress, whether of mean or of systolic pressure, must co-operate with whatsoever toxic or morbid proclivity, and multiply its effects; impellit labantem. But we are still faced by the enquiry, if atherosclerosis does much itself to aggravate blood pressures or stresses, and so accelerate its own career? 
Now, in estimating the mechanical stresses in the cardio-arterial system, we shall not forget that its motions, if they contain the elements of dissolution, are nevertheless themselves the form and condition of life. If the term of their own duration is implicit within them, yet meanwhile they are coefficients of work and development. The pulsations, on which, like the weaver's shuttle, the molecules of the arterial coats travel to and fro, promote the systole and diastole of the vasa vasorum by which the coats are nourished. By a reciprocal action, while doing work they also, as it were, open and close the valves of their own feed. And this illustrative instance is but one of the many mechanical coefficients of a perfect circulation. Within certain limits, the larger the oscillations the more the nutritive exchanges; but the normal limits are narrow; protracted distension may expel all juices, both afferent and efferent, and extreme oscillations lead to strain (p. 209). It is for the vasomotor mechanism, by increase of tone, to moderate these excursions; as well as to determine the irrigations of the several areas of the system. As we see in aortic regurgitation, and in the muscular efforts of elderly men whose vessels are less elastic, high maximum pressures-wider differences between systole and diastole-are apt to strain the vessel; and usually this means a larger output and a larger mean arterial diameter. Such a high upstroke however must be distinguished from high pressure, as resistance may be low and output large. Such a state we find during active muscular exercise ; on sitting down to rest the musculo-cutaneous arteries contract and, while the maximum falls, mean pressure may actually rise. That condition of maximum oscillation and large afflux of blood it may be, rather than a high mean range of pressures, which damages the limb arteries of labourers (vide p. 205). Marchand lays great stress not only on high pressures as a cause of arterial decay but especially on large oscillations of pressure (" häufige starke Schwankung"). Agreed, but let us be clear; this rule scarcely stand alone. We have seen that oscillation is a coefficient of nutrition, that high pressures through all phases of the cycle militate against nutrition, and this tension, though narrow as oscillation, is for the arteries an adverse condition of the circulation. What Marchand, and we all, mean is that the artery is protected by tone, and that if over long periods tone is in

vOL. I 
abeyance the arteries get battered. Experimentally, it is true that to excise one kidney in an animal so that the other grows in size and activity may not produce sclerosis of the correspondingly voluminous renal artery; but is not the time too short? If concurrently adrenin be injected this renal artery soon becomes impaired. ${ }^{1}$ Within limits then, mechanical stresses are a coefficient of life, and in their default lies death. Within limits; but what in these limits is excess? Well, we may regard the excess in two directions : in the height of the systolic wave, as in aortic regurgitation, or in resistance to arterial systole; that is, in an excessive amplitude, or in a high mean tension; or of course in a co-operation of both factors, as in certain phases of chronic Bright's disease. In the first of these conditions the molecule is driven outwards to the verge of its attraction to its neighbours; all the molecules may be driven on the outward curve dangerously out of touch of each other, and dangerously near, if not upon, the extreme limits of the cohesion of the material. The limit of elasticity of all materials is far within the breaking limit; and by this separation the molecules are liable, in this place and in that, to fail to return precisely to their own seats, when the group may take a new set and altered sizes of particles would create new stresses. ${ }^{2}$ We may presume that herein, in molecular groups which have failed to come back, may lie some explanation of focal detriments. At the spots where normal molecular cohesion is strained there is still, of course, some adhesion between the gliding surfaces; hence the new set, and consequent bias of function. But, if the stress be mitigated, perhaps some recovery of pattern may take place, or resolution in a mean direction. In this way perhaps, by dissipation of the energy which should have restored each molecule to its own place, a lower if more static kind of fibre may result; or the lower fibre surviving may increase at the expense of the higher.

1 See Frugoni, Sem. méd. No. 9, 1913.

2 We cannot of course suppose that even normally the molecules of functioning parts all return absolutely to their place before the action; were it so life would be not a development and involution, but a stationary oscillation. But we eonceive of these movements as having their critical phases, whether of development or of strain. Tissue memory tends to carry the molecules back towards their own places, incident forces to deflect them. 
It seems then that mechanical stresses accumulating upon sound arteries in comparatively young persons, as for instance in chronic Bright's disease, may be regarded severally as follows: (1) The normal cardiac systolic, relieved normally by diastole. It is frequently averred that under lateral pressures an artery does not expand, nor under axial pressure lengthen; and it is true that no calipers seem delicate enough to indicate such expansions. But with a plethysmographic apparatus and an optical record, lateral expansion on cardiac systole can be demonstrated; and even to a close scrutiny of the naked eye a serpentine shift of position, that is, an extension lengthwise, may be perceptible (p. 461). Rationally, all arteries of elastic quality must widen, or how could energy be stored to equalise, as is equalised, the flow in the capillaries through diastole? (2) A cardiac effort moderately increased, but with no great disturbance in the proportions of arterial diastole and systole ; an enhancement of work consistent with fibrotic or even muscular hypertrophy of the component parts of the vessel, especially of the media and intima; for, as we know, hypertrophy is due to increase, not of persistent but of intermittent stresses. (3) A largely reinforced cardiac systole, but still with corresponding fall and duration of diastole, as in aortic regurgitation, leading to hyperplasia, fibrotic or fibro-muscular. The effects of such an alteration are seen in the aorta, or in the gradual sclerosis of the tricuspid valve in mitral stenosis, or of the mitral cusps as in chronic renal disease (p. 519). In 192 cases of chronic renal high pressure, Sir James Goodhart found these curtains thickened, even to contraction, in 25 per cent. Thus in time, the interval depending on the age and nutritive capacity of the patient, lesion accumulates; the excessive expansions trespass upon the elastic limits of the materials; their molecules, as we have supposed, forced too far asunder continually fail to return to their original seats ; tensile and shearing strains are thus established (p. 513), and the structure takes a new set: yet, so long as diastole (arterial systole) is not inadequate in duration, rest, nutrition and time for recoil are not prevented ; though, as in the well-known yellow specks or patches on the inner aspect of the overstretched aorta, significant alterations begin to appear. (4) A rise of (cardiac) diastolic pressures, as in chronic Bright's disease and in Hyper- 
piesia, so that the wall of the vessel now loses its vaso-systolic release, while, worse still perhaps, the vasa vasorum are prevented from expanding to deliver their tale of blood to the tunics which they have to feed. Thus high levels of diastolic pressures are more mischievous to the arteries than increments of cardio-systolic pressures with normal diastolic (arterial systolic) rest and repletion. As then the mean blood pressures become exorbitant, so molecular strain- " fatigue," as in many materials it is called-tensile and shearing strains, and probably a ripping action on the spiral thread of the muscular fibres, conspire with the squeezing out of vasa vasorum to damage and impoverish the coats, especially the media and intima. Moreover, it may be that, when high parietal tension occludes the vasa vasorum, these vessels themselves also undergo an occlusive process of degeneration, such as we see in experimental researches on the ligature of vessels (see also pp. 460-2).

So then it seems to be with high pressures in arteries otherwise sound and well nourished. Dr. John Cowan, in an interesting essay on the history and evidences of arteriosclerosis, ${ }^{1}$ says that excessive blood pressure, whether with or without renal disorder, always damages the vessels; sooner or later. And these reflections, for arteries less sound, and for persons less well nourished, are still more apt; in such constitutions, in so far as the vessels may themselves be frail, the evil consequences of stress will arise the more readily, and strain be the quicker. And this is, in fact, what we see in senile or decrescent arteriosclerosis, a mode in which, as I have urged again and again from 1894 and 1896 onwards, high pressures are not implied. In the arteriosclerosis of elderly persons, as in them the elastic limits have become much narrower, the vessels fall into a cognate mode of decay under stresses not extraordinary. "Time that gave doth now his gift confound."

The play of external contingent forces upon the arteries, especially upon those of the limbs, may not be inconsiderable; but this factor will be considered later.

Up to this point then the argument seems clear; we are agreed that under persistent rises of pressure, as in the "Prealbuminuric Stage" of Mahomed, the "Latent Sclerosis" of

1 Cowan, John, Practitioner, August 1905. 
von Basch, the "Presclerosis" of Huchard, my Hyperpiesia, and again in chronic Bright's disease, especially, that is, when the diastolic pressures are lifted up, yet also in very high systolic expansions with ampler diastole, as in aortic regurgitation, arteriosclerosis results sooner or later, and takes after the ordinary, the intimal and subintimal, pattern. Young and sound vessels may resist this degeneration for years, but surely it comes about even in them. Speaking generally then, a thickened artery means plus stress or minus elastic value, or an algebraic sum of both in various proportions.

In a later paragraph, under the easy phrase of "wear and tear," I shall discuss the infinite accessory factors which, besides the directly mechanical, impair the arteries; but here I must tarry a little to interpose that we are not by any means sure that we are dealing always with anatomical constants. We may have to recognise four histological modes of arterial alteration : atherosclerosis, as described by Langhaas and Jores; the strain of the media in the vessels of the limbs, called by Adami " Mönckeberg's sclerosis," a name I accept for present convenience (p. 482); the simple intimal connective hyperplasia found in healthy elders, which is liable, however, in spots to slow fatty degeneration, and the syphilitic. Connective tissue hyperplasia does not always lie within the terms of disease ; it is a process which begins even in childhood (p.489). One or other of these anatomical forms more than the rest, connective hyperplasia for instance, may be significant of mere mechanical stress; to engender atherosclerosis some auxiliary causes, toxic perhaps in nature, may, as urged by Dr. Mitchell Bruce, be in cooperation. It is generally to this complexity of causation that our German colleagues apply the term "Abnutzung." But these ambiguities we shall endeavour to appreciate in the later paragraphs.

As regards the vascular conditions of mechanical stress: the intima, in its inclusive sense, is the blood-vessel proper, the media and the adventitia being its investing supports; and in this respect the media does the chief work, for so long as the media is efficient but little of the tensile stress reaches the adventitia, a tunic which serves rather as a carrier of the subordinate channels of blood and lymph, and as a bond with 
surrounding parts. When however the media yields, these channels in a squeezed adventitia may, as we have seen, be straitened or stopped. And, while upon these outward relations of the vessels, we may remark that these vary with the distributions of the branches: some vessels, like the descending aorta, are closely attached to surrounding structures; others, if not closely attached to the surrounding parts, are so embedded in them-in muscle or fat for example, as to be none the less well supported or packed; others again, like the cerebral, temporal and coronary branches, are attached but loosely, or stand free from packing; so loose or so free as to be almost unsupported. It is easy to perceive in the early thickening and tortuosity of unsupported branches, even in persons wholly free from general arteriosclerosis, how important for the integrity of the vessels these external relations must be. ${ }^{1}$ Thusthe basal cerebral arteries, which are not well embedded, and are moreover possessed but of feeble muscular tone, suffer comparatively early; and their twigs, when impaired in quality and unprotected by constriction of their affluents, are peculiarly liable under rising pressures to give way. As an artery yields lengthwise it must be thrown into secondary curves between any two of its points of closer attachment; on each oscillation it rises out of its bed and, as friction is thus increased and vortices possibly formed, we should suppose that the defect, like all defects, would multiply itself ; and such is Albrecht's ${ }^{2}$ conclusion. Oberndorfer however, who has also made a careful comparison of arteries in supported, unsupported (e.g. temporal and coronary), and immobile positions, disagrees with Albrecht in supposing that the vessels, or parts of vessels, most liable to arteriosclerosis are the unsupported. If it does seem true for some, such as the temporal, he reminds us of the negative instances, of which he gives many; e.g. the trunk of the iliac, which is often rigid and calcareous while its limb branches are wholly, or comparatively, unaffected. The coronaries are not supported and, although muscular, are feebly innervated in order that they may be supple to the paces of the heart and generally passive to distension, in obedience to the rate of oxidation in cardiac excitement (Barcroft, loc.

1 See again Schade (p. 38).

2 Albrecht, Deutsche Arch. f. klin. Med., 1911. 
cit.), and on any access of high blood pressures may respond instantly to the heart's need; a response which, if opposed by the constrictions so often assumed, ${ }^{1}$ might be impeded. The dependent arteries, those of the legs for example, have to withstand another stress, that of gravitation; and, so long as this does not exceed the normal, we find no more than a relative thickness of these branches, but if-as in varicose veins of the leg-pressures rise in the dorsalis pedis and posterior tibial this thickening palpably increases, if still perhaps within limits of healthy hypertrophy. If in a comparatively young subject the varicose veins are cured by operation the arterial thickening will subside ${ }^{2}$ but if the venous disease continues the co-operating arteries fall into an irremediable sclerosis.

I may repeat that we have no reason to suppose, or none at any rate to assume, that any one diameter in tone is more apt to produce medial hypertrophy than another (p. 48 et seq.); but, as in the heart, plus or minus tone may make a large difference in exposure of the vessel to lateral pressures and tensile strain. In high-pressure cases, with obvious constriction of the palpable arteries, the diameters to which constriction may confine them, and the extent of the constriction inwards towards the larger magnitudes, are very variable; in many cases the radials are tightly constricted, but the brachials apparently not so; in others both brachials and carotids feel narrow and tight. The capacity of the carotids for inordinate changes of diameter is conspicuous in their expansion in Graves' disease; and that of the aorta itself in pseudo-aneurysmal throbbing of its abdominal portion. In other patients again, whatever constriction there may be, if any, in the outward periphery, or in visceral areas, the radials themselves may be of ordinary diameter, or even stretched and leathery. When the radials are constricted the protection against strain of their own coats must be enormous (p. 218); and as a matter of fact in these cases, for a long part of their course at any rate, the constricted walls present an even texture to the touch. Arteries of low tone, such as the cerebral and the coronary, which work

1 See Essay on Angina Pectoris.

${ }^{2}$ Sce Vignolo, Società Editrice Libr. Milano, 1900. 
nearer to their elastic limits, are the more liable, as experience proves, to suffer injury, even in time at ordinary pressures.

The obscure part which may be played by cellular and capillary function in arteriosclerosis I shall consider again presently; Broadbent and Thoma were disposed to find in these areas a chief part in peripheral augmentations of blood pressure. Briefly to anticipate such considerations, I may say here that Thoma, fortifying his position by quantitative reckonings, postulated a constant relation between the parenchymatous weight of an organ and the mean blood current through its capillaries; that the weight of the kidney, for example, is in constant relation to the mean bed of its vessels. Thus the capillary provinces may be said to determine, or to express, the mean quantity of blood passing in time-unit through the terminal arterioles; though probably, in such coefficients as rate and viscosity, there are individual differences. Now upon this, says Thoma, if velocity rises then tonus falls, whereupon the artery enlarges; and conversely. Tonus really, in his view of the matter, depends on velocities. Other things being equal, of course a rise of piston pressure, even in a somewhat wider area, must increase velocity; but vasomotor tides, muscular movements, and even posture are also concerned in it (vide p. 27). Sir James Barr ${ }^{1}$ says that by lowering a limb capillary velocity may be doubled. He adds that the extra-vascular pressure in capillary areas amounts to about a quarter of the capillary pressure from which it is derived. Broadbent's attention was drawn more to tissue needs: "The arterioles contract or relax in obedience to the demands of the tissues; ultimately, therefore, the causes of arteriosclerosis are in the relation of blood and tissues." This he illustrated by the behaviour of the arterioles, with fall of pressure, in pyrexia, an activity due to the cellular insurrection against the toxin. Chauveau demonstrated that, into muscular areas, the flow on demand may be increased fivefold. We have to reckon also with hormonic influences, which are hard to read, but are engaged in the sum of events. By calculations of lime and other salts something may be done to register and appreciate these tissue activities, as Blair Bell and

\footnotetext{
1 Barr, Jas., Brit. Med. Journ., August 25, 1906 ; Broadbent, W. H., Lancet, Feb. 7, 1903.
} 
Barr are wont to do; as also by other tests of metabolic processes.

At present however, having indicated the part of the vasomotor innervation and tissue activity in preventing or modifying mechanical stress on the arterial coats, enormously increased inwards as the pressure may be, I may return to the proofs of the effects of the mechanical factor by alteration of the texture of the arteries. To one of these proofs, that of the effects of varicose veins upon the posterior tibial artery, I have already alluded (p. 199); but, before travelling into the sphere of disease, we may find some of the evidence we want in healthy arteries, or at any rate in arteries barely entered into disease. Of Thoma's observations, also, on the fibroid increase of the intima in areas of growing stress-e.g. that consequent upon the closure of the ductus botalli -I have spoken already, observations which have been verified by critical observers, such as Jores who indeed traces it in adolescence into arteries such as the carotids. This fibrous growth Fuchs attributes to longitudinal stresses. Taking these incidents as known, I may return to another character upon the confines of health, namely, the buttressing of the channels at points of stress, especially at points of bifurcation. At these junctions the mouths of the divergent channels are fortified by a small angular pad of additional tissue, a firmer welding developed in response to heavier impact, and perhaps, in larger vessels, to vortices. This provision is best seen at the bifurcations from the aorta ; especially where, as at the intercostal openings, the branches diverge at large angles. And further, it is at such points as these that the earliest signs of strain begin to appear. By infinite degrees normal and conservative fibrous buttressing passes over into hyperplasia and degenerative accumulation; the elastic limits grow smaller, and are sooner exceeded. And in advancing life (after the fortieth year) a retrograde character becomes manifest in the falling proportion of elastic to connective fibre, and in the penetration of this fibre between the elastic and muscular elements. ${ }^{1}$

1 The mutual development and retrogression of this group of constituents is more fully described, and in a very interesting way, by Aschoff (loc. cit.), but I cannot give more space to them here. 
Pulmonary Arteriosclerosis.-Here let us turn to another manifestation of the effects of mechanical strain, one which is almost crucial. Such is the capacity of the reservoirs on the right side of the heart, and indeed of the lungs also, that vast quantities of blood may be heaped up there without stemming the current so far as to raise the systemic pressure, harmful as loss of velocity may be. Thus, under stresses which ordinarily may be great, and indeed at times exorbitant, the pulmonary artery suffers little. Still, in extremer cases of pressure on the right side with hypertrophy of the right ventricle, as for instance in advanced degrees of mitral stenosis, emphysema, chronic bronchitis, or congenital malformations, the pulmonary artery suffers strain and injury more commonly than is usually supposed, for Torhorst, ${ }^{1}$ by closer research, demonstrated internal proliferation in many cases in which, at first sight, it was not apparent. Good specimens of this arteriosclerosis may be seen in any large pathological museum. ${ }^{2}$ Again, while the main trunk may seem normal, and even its two branches, patches of sclerosis may be obvious beyond them. For an illustrative instance I may quote Langdon Brown and Durno, ${ }^{3}$ who published a case of patent ductus arteriosus in which " the pulmonary artery and its two main branches were dilated uniformly, the walls showing large plaques of atheroma." The right ventricle was very largely hypertrophied $-\frac{7}{8}$ ths inch thick as against $\frac{1}{2}$ inch of the left. The patient was 33 years of age. Dr. Brown calculated that in his case the pulmonary artery was submitted to a pressure more than double the normal. Dr. David Lees ${ }^{4}$ has published a case of atheroma of the pulmonary artery in a child, aged 9 , with mitral stenosis ; the aorta was unaffected. Kitamura ${ }^{5}$ describes it in a "beer heart" much enlarged on both right and left sides. There was no

1 Torhorst, Ziegler's Beiträge, Bd. Ixxvii. p. 380, 1904.

2 A good drawing of atherosclerosis of this vessel is given by Letulle, p. 165. Syphilis of this vessel (sec Rogers, L., Quart. Journ. Med., vol. ii., 1908) is of course excluded in this place.

3 Brown, Langdon, and Durno, Lancet, June 13, 1908.

- Lees, D., Clinical Journal, Oct. 9, 1901; Mott, F.; Allbutt and Rollcston's System of Med. vol. iv. p. 587, who quote two cases from Romberg ; Foxwell, Brit. Med. Journ., Oct. 21, 1905, p. 1028. See also Avrilaga, Arch. des mal. du cœur, aô̂t 1913, and Frugoni, Arterioskl. pulm., Milano, 1912.

s Kitamura, Zeitschr. f. klin. Med. vol. lxv., 1908. 
mitral disease. The myocardium seemed normal. He attributed the pulmonary sclerosis to plethora, perhaps with hyperglobuly. Professor Marchand, who has kindly shown me his own specimens, has drawn attention to these cases as crucial instances of atheroma due to high pressures, and, in cases of congenital stenosis at this spot, has compared them with similar cases of atheroma of the aorta in its portion anterior to the spot of the ductus botalli. Professor Mönckeberg ${ }^{1}$ has commented upon two remarkable cases of the kind; but, as the principle is the same (there was large hypertrophy of the right ventricle in both), I need not pursue them into detail. In the same series of events ensuing upon mitral stenosis we see sclerosis establishing itself likewise in the tricuspid valve. Posselt, in an article on the Clinical Diagnosis of "Pulmonalarteriensklerose," in Volkmann's Beiträge for 1908, brought together much valuable matter on this subject, but more as a problem in clinical diagnosis than in respect of the pathology. One of our graduates, Dr. D. G. Pearson, for his thesis (January 1910), examined the parts of ten cases of pulmonary atherosclerosis, nine of which were due to uncomplicated mitral stenosis in young subjects in whom the right ventricle only (not the left) was much hypertrophied. In these cases he calculated that the pulmonary pressure had risen nearly, or quite, to the aortic standard. In all the pulmonary arterial system was sclerosed. In two cases the branches "gaped" all through the lungs. In one case, of a boy aged 12, the date of the first sense of distress was recorded, and by the date of death it appeared that these sclerotic changes had been produced in a few months. The tenth case, one of aortic regurgitation with a "Flint murmur," was very interesting, for in this case there was no mitral obstruction whatever, but an insufficiency; and the pulmonary sclerosis seemed to prove that the aortic reflux on residual blood had sufficed to maintain the high pressure in the pulmonary area. As we have argued in respect of systemic arteries, if the vessel can recover itself during diastole it may escape injury, Dr. Pearson did not find the lesion in any case of mere mitral regurgitation, however high the venous pressure; he regarded

1 Mönckeberg, Deutsche med. Wochenschr. No. 31, 1907. 
the lesion as pertaining to mitral stenosis. ${ }^{1}$ The illustration can be made still more crucial, and Torhorst (loc. cit.), Steell, Ehlers, ${ }^{2}$ have very carefully described cases in which closure of one lung threw an excess of work upon the branch of the pulmonary artery of the other side. ${ }^{3}$ In such cases the strained branch only became atheromatous. The following may be given as an example :-

F. æt. 39. Pleuropulmonary fibrosis of right lung and hypertrophy of right ventricle. Right pulmonary branch normal and without signs of strain ; left branch atherosclerotic-" "both nodular and diffuse," all through its ramifications. Syphilis was excluded. The histological characters were identical with those of an atheromatous aorta. (By the way, on Thoma's hypothesis the slowing of the current in the right branch should have caused compensatory increase of the intima of this nevertheless normal branch.)

A parallel mechanical damage can be seen in the portal vein in many cases of cirrhosis of the liver with dropsy. ${ }^{4}$ The intima is thickened and the media deteriorated. Simmonds thinks bacterial toxic as well as mechanical causes are herein concerned, and declines to accept the change as atherosclerosis, "for it is not inflammatory"; but neither is atherosclerosis inflammatory.

The rate of the pulse, that is the mere number of blows upon the walls of the arteries in unit of time, apart from their energy, is probably, within the limits of life, negligible. Generally speaking, the quicker the pulse the lighter the several blows. There is less time, it is true, when the pulse is rapid, for the molecules to return to their former positions, so that in this respect a slow pulse would seem to give them a better chance; and they do not get that comparatively long rest in which, as we familiarly observe in the edge of a razor, materials tend to regain their elasticity, or at any rate their original positions.

Again, as Dr. Rolleston has shown, the mere mechanical effect of stress on vascular structures may be witnessed not

1 In certain cases supposed to be syphilitic, Dr. Leonard Rogers regarded the hypertrophy of the right ventricle as consequential. Rogers, Cases in Bengal Quart. Journ. Med., Oct. 1908.

2 Ehlers, Virchow's Arch., 1904, Bd. 178, H. 3.

3 A good account of the arteriosclerosis of the pulmonary artery, with bibliography, will be found in the Arch. des mal. du cour, 1910, p. 218.

" Pylophlebosclerosis. See Simmonds of Hamburg, Virchow's Arch. vol. ccvii., 1912 (seven cases), and other papers. 
in the valves and arteries only, but also in the effect of mitral stenosis upon the lining of the left auricle.

In thus considering fatigue of the arteries by the incessant beat of the blood upon their walls, we naturally turn to observe the effect of muscular effort, in persons exposed to more than ordinary stresses of this kind. Artificial stimulation of the cerebral motor area causes a general rise of blood pressure; at the same time the plethysmograph exhibits an increase of volume of all four limbs, while the splanchnic vessels are correspondingly reduced. ${ }^{1}$ Moreover the same effect in kind is produced by the mere suggestion of the effort. One of the first pathologists to publish definite observations on this side of the problem, on the effects of prolonged muscular exertion on the arteries, was Tschigajew, in a paper entitled "The importance of muscular work in the production of arteriosclerosis." 2 It is to this effect: that in Russian peasants, during the heavy labours of the summer and harvest seasons, the vascular walls thicken; but this thickening-“" a pseudosclerosis due only to thickening of the media"-dwindles in the winter, when the vessels return to their ordinary consistency; in certain other labourers however, such as iron-workers, men who are occupied in hard work more continuously, a genuine and permanent arteriosclerosis is prematurely established. Yet, interesting as this is, we cannot forget that iron-workers are probably exposed to many other injurious conditions of life; unwholesome workshops, habits of drink, syphilis, and so on; and we need facts for a comparison of outdoor labour with indoor, as in warehousemen and inside porters, and again with wage-earners of the same class not so heavily laboured. Also it is imperfectly known how far this labourers' arteriosclerosis extends into the various regions of the body; whether the strain of muscular effort falls simply or mainly upon the arteries of the limbs, a comparatively small matter, or sets up likewise a general arteriosclerosis extending to the inward parts? Even then the still deeper problem remains, whether this degenera-

1 Weber, Internat. Physiol. Congr., Heidelberg, 1907.

2 Tschigajew, Wratsch, 1894 , p. 353 . I must not pretend to have consulted this paper, but Dr. Mott kindly sent to me certain extracts from it; I find a notice of it also in Virchow's Jahresbericht for 1895. 
tion be due directly to mechanical stress-strain, or to the ampler derivation of blood to parts in extraordinary activity, or again to the accumulation of more metabolites in tissues and blood? I wonder if Hawkeye had thick radials? Contusive friction cannot perhaps be left out of reckoning. These questions come not of mere curiosity; for in so far as the sclerosis may be due to increased blood flow in response to predominant tissue calls in any part, so far we may anticipate concerning the heart, or the brain likewise, in persons whose occupations make large and incessant demands upon these respective energies, that such local tides may imperil the integrity of the respective vessels, and so of the more precious organs which they supply.

Again, at the Duisseldorf Congress in 1912, ${ }^{1}$ Dr. Remy of Paris drew attention to the common occurrence of arteriosclerosis in labourers at and about the age of 35, "especially if they were addicted to alcohol"; he mentioned syphilis also as an important factor. And when many years ago in Leeds I was first interested in the effects of muscular exertion upon the heart I was surprised in hospital and dispensary practice to find a high frequency of arteriosclerosis among labouring men; arteriosclerosis without any other signs of ailment, unless it were in some aspect of premature senility, or perhaps in some disability of tardiness and lack of endurance. This evidence however impressed me less in a large city, where other noxious influences, such as drink and syphilis, are at work, than since it has done in Cambridgeshire, where the country labourer is less exposed to these ill influences, and works in the fresh air. In the Eastern Counties I found the frequency of arteriosclerosis in the labouring population was no less; perhaps in these half-fed labourers it is even higher. Moreover, I soon learnt that I was not alone in this impression. Mr. Wilkin of Wickhambrook practises in a district of which a large part of the soil is a damp clay, the other part is a light dry upland (around Newmarket). The contrast of these different areas has often been a subject of discussion between us, and is not without some bearing on the present subject. In the damp clay part of his district Mr. Wilkin finds "rheumatism" in large proportion, both rheumatic fever and "chronic rheumatism." Rheumatic fever marks its way by

${ }^{1}$ See report in the Lancet, August 1912. 
the signposts of heart disease; among the chronic rheumatic patients he seldom finds valvular lesions, but in their accessible arteries of these he does find a large proportion of arteriosclerosis, without definite signs or symptoms of cardiac disease (p. 289). Romberg, who at Hesse practises among a hard-labouring people in a hilly country, found between ages 30-49 that one-seventh of his list showed arteriosclerosis, and between 40-49 one-third. In the women it was less prevalent, and in them appeared usually in the legs before the arms. But he also found a very large proportion of defective hearts, and much neurosis and neurasthenia, and believes that in his folk other factors besides toil are concerned. Putting aside renal disease, he also was disposed to say that among his patients thickened arteries were associated with chronic "rheumatism"; one main difference being in that these cases the blood pressures are not excessive, whereas in renal disease of course they rule high. The chronic rheumatism and arteriosclerosis he is disposed to put down in part to malnutrition (poor feeding-too much carbohydrate material, too little nitrogenous), but chiefly to "constant exposure to damp"; as with the Irish labourer whose cottage "was damp enough to give rheumatism to a wild duck." In Hertz's lists (loc. cit.) labour was returned in the first place as a cause of arteriosclerosis, and the large majority of cases observed were no doubt of the labouring class; but extremes of temperature and external frictions and contusions cannot be left out of the reckoning. Professor Leonard Hill ${ }^{1}$ thinks indeed that toil is not of itself a sufficient cause, but that the vascular wall must be "predisposed by some toxic agency to degenerate." Thayer and Brush ${ }^{2}$ (after a study of the toxic causes of arteriosclerosis) conclude that palpable radial arteries are very frequent in persons occupied with heavy physical labour; but, they add, such persons are commonly given to drink. So perhaps neither in city nor country may the causation of arteriosclerosis "be attributable to manual labour merely; the problem seems to be more complicated. ' On the other hand, as a member of a Committee of the Home Office, I have lately visited certain large works in the cities of the north, where

1 Hill, L., Allbutt and Rolleston, System, Art. “ Path. Cereb. Circ.” p. 263.

2 Thayer and Brush, Journ. Amer. Med. Ass., 1904. 
again I was much impressed by the prevalence of arteriosclerosis in the heavier labourers. In Sheffield we called up about twenty men, between 40 and 50 years of age, occupied in the heavier departments of a forge, and my guide selected for us healthy men of sober habits; yet in these there was not one without some thickening of the radial arteries; and a considerable proportion of the men were also emphysematous. Dr. Mitchell Bruce ${ }^{1}$ likewise, from his hospital experience, reports arteriosclerosis as prevalent among manual labourers; and adds that in some of these who worked mainly with the right hand the right radial was thicker than the left. Marchand mentions a remarkable case in a woman, aged 35 , who (from infantile palsy) was lame of the right leg; the left leg had to do all the work. In this leg the femoral artery showed extreme medial calcification, the rest of the arteries of the body, including the other leg, being healthy. Other observers have testified to untimely arteriosclerosis in the legs of postmen. Boveri ${ }^{2}$ also, in an argument to the same effect, stated that arteriosclerosis was prone to occupy the limbs of heaviest labour, and thus was conspicuous on one side, or limb, more than on another, according to the relative incidence of the muscular exertion. Dr. Harry Campbell ${ }^{3}$ has observed the same arterial thickening in the arteries of the limbs of peasants, and attributes it to a continual hypotonus of these vessels during the afflux of blood to the active limbs, an explanation to be considered presently. Friedrich, in 1384 labourers under the age of 40 , found arteriosclerosis in the limbs of $1000 .^{4}$ Warfield (loc. cit.) states that arteriosclerosis appears at early ages in stevedores, whose muscular labour is very heavy. Bäumler, ${ }^{5}$ summing up his investigation of this subject, says that in labourers he has found the arteries of the arms, and especially those of the arm chiefly used, to be widened, tortuous, and calcified; the more used arm, in cases where the work is thus distributed, being strikingly more affected

1 Bruce, M., Lancet, July 15, 1911.

${ }^{2}$ Boveri, Riform. med., No. 30-31, 190?, quoted Deutsche med. Wochenschr., where the year was unfortunately omitted.

${ }^{3}$ Campbell, H., Brit. Med. Journ., Feb. 3, 1906, and Lancet, Feb. 19 and April 2, 1910.

4 Friedrich, Münch. med. Wochenschr., 1909, p. 2080.

5 Bäumler, Berl. klin. Wochenschr., 1905. 
than the other. He collected a number of left-handed workmen, and found in them that this side was decidedly more affected than the other. But in heavy weight lifters he found the aorta also to be gravely damaged. He supports the opinion that in sclerosis of this origin the patches arise at points of giving way ("Nachgeben") of the media. At the same time he argues that excessive activity in some other area, in the brain for instance by work or worry, in the stomach, in the kidney, may also be a factor in the deterioration of the limb vessels. If so, katabolites or other such baneful ingredients present themselves again as possibly concerned in it; for at first sight excessive activity in any area would seem to imply vasodilatation, and so far a fall of pressure. Klotz, ${ }^{1}$ on the sclerosis of the arteries of a blacksmith's arm, says they "suffer under the increased blood pressure"; but he does not explain how. Does he mean systemic or local pressure? We have discussed (p. 28) the "Strom-Intensität" of German authors, by which they mean abundant afflux with probably some increase of velocity. Claude Bernard, as we know, demonstrated that vasodilatation in a small area raises blood pressure in the vessels peripheral to the dilatation, but that (caeteris paribus) the aortic pressure would fall. To effect this rapid afflux some other areas must close, as no doubt they do; and it is not the constricted but the lax vessel which suffers from " high tension." It is said that " periodic and intermittent work has more effect in injuring the arteries than more continued stresses." It will need more evidence than has yet been produced to prove such a postulate. My own opinion is, as I have said, that in the working limbs the arteries suffer from a large swift output telling upon them while in a state of relaxation, and that this in the long arteries of the limb may produce "Mönckeberg's" form of sclerosis (p. 482) which, while deforming these vessels, need not signify disease in the vessels of other parts, where indeed pressures may be proportionately relieved. I look back upon two or three sportsmen of temperate habits, of great energy and muscular activity, riding hard, rowing hard, and so forth, whose arteries became very thick (in the limbs) before middle life. And I repeat that in these cases of arteriosclerosis of labourers, as in a like class of senile cases, the 1 Klotz, O., Science, N.S. vol. xxxiii., June 1911.

VOL. I 
arterial disease may be of the limbs only, as Orth, Mönckeberg, Marchand, and others say; not exactly or mainly atherosclerosis but, at any rate in part, a primary medial decay.

Dr. Elliott Dickson and his brother, of Lochgelly, Fife, made a long series of very careful observations on the state of the accessible arteries and the blood pressures in coal miners; and in December 1907 were good enough to forward their manuscript for my perusal. At that time they had examined, from this point of view, 500 miners ; 250 completed cases were observed consecutively. A few control cases also, other than miners, were added. The brothers Dickson enclosed to me also the full notes of 19 of the cases, "chosen pretty much at random," as specimens of the material of their tables, and of their method of recording the points of special observation. Besides the state of the radials, pulse rate and blood pressures, were noted the size of the heart and the character of its sounds; also personal habits as to alcohol, meat, tobacco, and conditions of work. In 200 of these 250 miners there was no sign nor symptom indicative of variation from the normal save the condition of the radials; in the minority were various cases, such as of a plumber who had worked with lead-piping for three years, in whom the systolic pressure was 155; and so on. In the 200 ordinary cases the pressures were "always well within the normal." The radials were entered as $\mathrm{A}, \mathrm{B}$, and $\mathrm{C}$; that is, A, a healthy artery (impalpable); B, easily flattened, but then feeling like thick tape; C, thick, rough, and tortuous. To these letters a plus or minus sign was frequently added. Only 18 cases were entered plainly as A. As to age, 191 were over æt. 20 , and of these 12 only had normal radials. They found no relation between the indications of the instrument (broad cuff R.-R.) and the state of the radial wall. For instance, in case 3 of the full notes I see the systolic pressure was 150 with arteries A (æt. 35); while in case 2 (æt. 65) with arteries C, this pressure was 112 . Case 74, with a pressure of 155, was queried, but no other abnormal sign was found, and this was the only case (in the short complete list) with so high a pressure; the average run was from 110-135; the mean about 120 . Some cases of "Allbutt's Hyperpiesis" were observed, but were excluded from this category. Of the 19 full reports I see 5 men 
took no alcohol ; the others averaged an expenditure on alcohol of about $6 \mathrm{~s}$. a week, a sum about equally divided between whisky and beer. The Dicksons were unable to discover any relation between arteriosclerosis and tobacco. Although these observations could be made on the limbs only, yet in a very few cases where, after accidental death, a piece of the radial artery could be secured, the lesion proved to be typical atherosclerosis, and this only. This I have seen in the excellent sections shown to me by Dr. E. Dickson. The media may be somewhat hypertrophied, but it does not present the degenerative features described by Mönckeberg (p. 482). As many of the observations were made in cases of persons employed in the mines as overlookers, or others not engaged in manual labour, the Drs. Dickson are disposed to attribute the arterial lesion not to labour but to some (gaseous ?) poison in the mines of their district. If this be so, their cases would belong to the toxic class (p. 232).

In big beer drinkers and gluttons the arteries, whether by the bulk of intake or by toxic influences upon vessels or kidneys, suffer sooner and more severely. And this deterioration, even on small increments of age, tends, like other defects, to multiply itself ; for as the arteries stiffen-in ordinary persons after the age of 60 -a muscular effort may send up the systolic pressure twice as high as in men of 20-30 years whose vessels are supple. Thus Hazebroek may be right in making some distinction between "Mehrarbeit" (overwork) and "Uंberspannung" (over-distension) ; between, for instance, the pulses of muscular exertion and those of aortic regurgitation. This may be due to excessive systolic stretching, that to afflux of large volumes of blood ("Strom-Intensität," p. 28). Continual large dilatation of the vessels of the limbs is quite consistent with ordinary and, in the case of vigorous heart, with rising blood pressures. And, as I have said elsewhere, protracted and continuous stresses, even if well within elastic limits, engender strain.

But, in thus roughly discussing our notions of predominant causes, we must remember that we are dealing with dynamical problems of infinite complexity; even when we omit toxic and other external conditions, we are calculating compound effects of maximal and minimal pressures, pulse amplitudes, resistances, and other variables; a complexity which baffles our 
attempts to ascertain from graphic data the functional capacity of the particular heart. ${ }^{1}$ Enormous muscular development again must increase waste over a vast area, and thus throw more cleansing work on the circulation, not to mention the delivery of a fouler blood to the myocardium itself.

The so-called "strong men," worked up to huge muscular development, are generally supposed to be unhealthy; the muscular system becoming as it were a parasite upon the frame : they stand disease badly, so it is said, and become senile early. In this context some of us may recall a well-known passage in The Republic (403 E): “I am afraid, I said, that a habit of body such as our ordinary athletes have is but a sleepy sort of thing, and rather perilous to health. Do you not observe that these athletes sleep away their lives, and if they depart in ever so slight a degree from their customary regimen are subject to most dangerous illnesses?" This is remarkable as direct testimony from an observer early in the fourth century B.c., when these athletes were held in high esteem for their prowess, and when our suspicions of metabolic perversions were unknown.

On the other hand, not a few coefficients are, as Zuntz and his pupils have proved, working wonderfully for conservative ends, especially of course in periods before the advent of old age. If an active effort of short duration causes rises of pulse rate and of maximal and minimal pressures, whereby the heart is urged, in a continuance of exercise the vessels open out, and the systolic and diastolic pressures sink towards the normal; in the intervals of rest probably below it. But these points $I$ have discussed elsewhere, I will not dwell upon them here.

Lactic Acid.-Oswald Loeb ${ }^{2}$ has recorded certain experiments which, if verified, seem, in this matter of causation, to be of importance. After trying the effects of various substances as vessel poisons, he found, generally speaking, bacterial toxins to be far more frequently destructive to these tissues than other bodies such as alcohol, nicotin, etc. With cholesterin over long periods of time he obtained sclerotic effects (see p. 252), yet, as he remarks, it is impossible to say that in such periods the

1 See Fantus and Staehelin (Berlin), "Blutdruck während Muskelarbeit," Ztschr.f. klin. Med. Bd. Ixx. He. 5 und 6; Otis, Amer.Journ. Med. Sci. vol. cxliii., 1912 ; and my article on "Heart Strain " in A. and R.'s System of Medicine.

${ }^{2}$ Loeb, O., Deutsche med. Wochenschr., Sept. 18, 1913. 
hutch rabbit may not fall into this disease of itself. But in experimenting with bodies of these kinds, and with alcohols, etc., he discovered that the aldehyds-of the aliphatic class only-were followed by this degeneration with a high degree of certainty. It began to appear that in so far as ordinary alcohol had any effect on the arteries it was by its aldehyd. He suggests that in diabetes, by oxydation of sugar, glycerin-aldehyd, and acetoaldehyd and glycol-aldehyd may be of the offending agents. And aldehyds arise from fatty acids, especially the amido-acids from leucin, etc. Now of these series lactic acid, arising out of aceto- and glycol-aldehyd, is in his opinion one of the most injurious. By the mouth, lactic acid, at the rate of $1 \mathrm{~g}$. per kilo of animal, constantly produced multiple degenerative patches in the aorta, while other acids, on controls, had no such effect; although these controls often became cachectic while the lactic animals continued in fair condition. Now Loeb points out with reason that in the limbs of labourers lactic acid must abound; indeed he finds that, the normal content being about $15-20 \mathrm{mg}$. to $100 \mathrm{~g}$. of blood, after hard exertion the content may rise to $150 \mathrm{mg}$. and more. The same increase follows defective oxydation, and certain liver affections (Hoppe Seyler and his school). It may accumulate also in districts of the body, as in the muscular system of labourers. Whatsoever the coefficient of overwork of the vessels, a surplus of lactic acid in indolence, or large affluxes of lactic-laden blood in heavy labour, may be concerned in the result.

To turn to other areas of effort; that the cerebral arteries are more diseased in brain workers I have no evidence. Von Romberg and Klemperer ${ }^{1}$ urge that bodily work is more apt to injure the limb arteries (and it is these of which we are now speaking) if the labour be of a vexatious kind-a "psychogenic arteriosclerosis." In the labourer's life there is more beer than worry; besides, to interpret the early stages of hyperpiesis as neurasthenia is no very infrequent error of diagnosis. Judges, who as a rule live long, seem to have no more tendency to arteriosclerosis in the cerebral area than other people. Cynics may say that with years they become even too ingenious. Apoplexy and sclerotic occlusion in the cerebral vessels are at least as frequent

1 Romberg and Klemperer, Verhandl. Kongr. inn. Med., 1904. 
in thoughtless persons, idle or laborious, as in professional men, sedentary as their habits often are. Dr. Mott is disposed to think that a sedentary life is more conducive to cerebral arteriosclerosis than physical labour, but such impressions depend very much upon the spheres of observation of individual physicians. During active intellectual work the blood pressure is said to rise a little, by way of cardiac reinforcement and vasomotor constriction in other large areas. For instance, Bickel of Bonn ${ }^{1}$ reports, on plethysmographic methods, that pressures rise in intellectual as they do in emotional work. An augmentation of heart work is partly concerned in the rise, as well as arterial constriction. But in pathological states the curve is other than in the normal; in mental work the volume of the outward districts increases; whereas in health volume lessens in the arm and ear, while in brain and bowels it rises, the blood being thus diverted to the brain. While engaged in brain work I am always conscious of a tendency to cool skin and to cold legs and feet. So far as we know, these cerebral affluxes oscillate within small limits, limits much narrower than those correlated with muscular efforts. The scholar, accidents apart, seems to live long.

From observation of animals we get very little help in the interpretation of arteriosclerosis. As Haller pointed out in this respect, the lives of wild animals are extended scarcely beyond the period of fullest activity ; and in confinement their lives are shortened by unnatural conditions. Fordomestic animals reared for food life is brief; and even draught animals, oxen and horses, rarely live more than twenty years. Elephants and parrots he excepted. Birds are said to have the longest lives of all animals, but our data are scanty. ${ }^{2}$ Haller, who seemed to think that longevity was inversely as sexual expenditure, stated that doves " cum in justo matrimonio vivunt" live long. He noted, however, an induration of the arteries in old horses ("petrifactio scutellata aortæ"). Hodgson says this lesion is rare in the brutes, even in those so long lived as the elephant and the eagle; but he gives no grounds for his assertion. On enquiry at the Zoological Gardens of London, and at veterinary institutions, I

1 Bickel, “Kreislauf u. psychische Vorgänge," Neurol. Zentralbl., Nov. 2, 1914.

2 On "Longevity in Mammals and Birds," C. Mitchell, Proc. Zool. Soc., London, 1911. 
have found that very little information concerning arterial disease is to be had; it is reported as rare or insignificant, and little has been published on the subject. We do not see what we do not look for. Dr. Hans Lyding, ${ }^{1}$ to whose paper I have been referred, says on the contrary that sclerous changes in the arteries of cattle are much more frequent than is generally supposed, ${ }^{2}$ especially in stall-fed cattle; he suggests a toxic origin. Lyding had no opportunities of investigating the matter in draught-oxen. An old bear which died at the age of $23 \mathrm{had}$ a single patch in his aorta, and no more. Of 100 cases of atheroma in cattle Lyding found it to be considerable in 35 ; but in all the disease was confined to the setting on of the aortic valve, the concavity of the arch-especially about the scar of the ductus botalli, the aorta descendens, and the bifurcations of the intercostal and lumbar arteries. Parisot of Nancy ${ }^{3}$ says that atheromatous arteries are by no means uncommon in animals, both wild and domestic; rather more in herbivora, such as old rabbits and hares, among which the percentage is considerable. The spontaneous disease in rabbits is, he says, after the kind of that produced by compression, or by adrenin. The probability is then that observation has been at fault. Zuiserling ${ }^{4}$ finds that, omitting parasitic disease, which, he says, Lyding failed to do, horses are subject to necrosis and calcification of the media of the arteries with vasomotor decay, after the manner of the Mönckeberg variety in the limbs of man. Faber notes this kind of arterial disease in horses, but Zuiserling says that in them the precise atherosclerosis of man does occur, though more rarely. Again, in old horses Kitt ${ }^{5}$ has described sclerosis in the valves, sinuses, and arch of the aorta, patches presenting to the naked eye much the same appearances as in man; that Charcot demonstrated in them atheroma of the abdominal aorta and of the vessels of the legs is an old story. Thoma in 17 dogs found atheroma once; Lyding in a much larger number only twice. Hutch rabbits

1 Lyding, H., Zeitschr. f. Tiermedicin, Bd. xi. He. 4-5, 1907.

2 I find Faber, who has examined many animals, is of the same opinion. He says it is histologically the same as the human form.

3 Premier Congr. Internat. de Pathol. Comp., Paris, Oct. 1912.

4 Zuiserling, W. D., Virchow's Arch., Bd. cexiii., Juli 16, 1913.

5 Kitt, Lehrbuch path. Anat. d. Hausthiere, Bd. ii. 
are said in laboratories to present about 3 per cent of aortic atheroma, so that experiments on atheroma in these animals must be made on larger numbers. But Steinbiss says, as we find in Cambridge, that, if kept under natural conditions, atheroma is rare in them.

Thus it would seem, so far as animals go, that a life of indolence and high feeding may be as effectual to promote cardio-aortic atheroma (we have no observations on general arteriosclerosis in them) as hard muscular work; but here the distinction of the kinds of change, if real, is of much interest. In cows Ball says that the vascular decay is of the so-called Mönckeberg's variety (vide p. 482), that it is much disposed to calcification, and consists primarily in a yielding of the media, the vasa vasorum being fairly normal; but that in the draught-horse it is of the sub-intimal hyperplastic kind, as elucidated by Jores. In respect of the cerebral arteries, we have some evidence that in the horse these may fall into sclerosis. In one horse Ball found the left sylvian and posterior cerebral arteries almost occluded by the disease.

Vasoconstriction in a large territory, such as the mesenteric, may perhaps, without the musculo-cutaneous, be extensive enough by itself to raise systemic arterial pressures (vide p. 218). Professor Hill translates the splanchnic capacity into terms of pressure as about $50 \mathrm{~mm}$. $\mathrm{Hg}$.; and the vascular network of the skin is said to have a capacity of from one-half to twothirds of the blood mass. Thus to drive the coach with the breaks on must rack the machinery. Splanchnic, musculocutaneous, and cerebral tone should mutually balance each other ; so that constriction in the splanchnic or musculo-cutaneous area alone ought not to drive up pressures very high, nor maintain them perpetually. These observations, with their bearings on physiological balances and on pathological high pressures, were made familiar to us all long ago by Cohnheim and Roy. Whether sclerosed vessels anywhere have a morbid proclivity to constriction is a notion which I have discussed on another page (161); this notion has rested hitherto chiefly on speculative grounds, and probably is over-rated. It is popular because of its convenience as a resource in guesswork. Hypertrophy of the left ventricle alone, without rise of peripheral 
resistance, will not keep up a high pressure; and the evidence at present is that persistent elevations of systolic and diastolic pressures, with narrowing amplitude, are due to extensive regions of vasoconstriction in arterioles presumably healthy which cannot be, or are not, compensated by dilatation elsewhere, and must therefore, by raising left intraventricular stresses, establish a corresponding growth of this part of the heart. Krehl has been quoted to the effect that so persistent a spasm of vascular districts is a priori unlikely; but if we turn to his context ${ }^{1}$ we shall see that he is referring not to systemic arterial pressures but to alleged rises of pressure in areas, or local anæmias. To me it is remarkable that the only too able digestions of big eaters should be compatible with widespread splanchnic constriction. Professor Langley suggests that the depressor mechanism may become enfeebled or rusty; and Mr. Mummery ${ }^{2}$ has pointed out that in old persons slight falls of pressure, e.g. to $90-100$, produce shock.

Paroxysmal and capricious as vasomotor waves may be, healthy vessels do seem capable, in some districts at any rate, of permanent, even perennial spasm. Two remarkable observations on man, as convincing as if experiments on animals, were published by Gibson. $^{3}$ In these cases, both in men, dislodged kidney had set up a permanent vasoconstriction simulating Raynaud's disease; by fixation of the kidney in both cases a permanent release was obtained. Cushing, in his brainpressure experiments, showed vividly how on compression of the brain the arterial pressure rose, while the splanchnic vessels visibly contracted, to dilate again as the compression was relaxed and the pressures fell. Roy and I carried out some similar experiments with similar results. Von Basch vividly describes, on irritation of the splanchnic nerve, the crimping up of the small and smallest arteries, and the collapse of the small veins passing from emptied capillaries; the part behaving, as he says, "like a squeezed sponge." Thus all the viscera, except the kidneys, are blanched. The extruded blood accumulates in the heart and large vessels and in the lungs and extravisceral

1 Krehl, Path. Physiol. p. 3, 4th ed.

2 Mummery, L., Lancet, March 25, 1905.

${ }^{3}$ Gibson, Geo. A., West Canada Med. Journ., April 19. 
areas, augmenting arterial pressure. Von Basch, assuming as he did arteriosclerosis, visible or invisible, to be at the bottom of every case of persistent high pressure, by no means forgot the potency of vasoconstrictions, though he was apt to overlook its practical importance. In his own experiments he calculated that vasoconstriction in the muscular area alone, or in the cutaneous alone, were sufficient to cause an enduring rise of aortic pressure. But this rise is by no means so prompt and effective as when the splanchnic area is the seat of the constriction; and if to the splanchnic some further area of constriction be added, it is, of course, the more stubborn. But these experiments on animals do not prove that such a state can be permanent; in animals perhaps it could not be, for in man the upright position demands from the splanchnic mechanism a capacity of permanence, and such permanent constrictions would save its arterioles from the stress of high systemic blood pressures, as indeed Jores has demonstrated to be the case.

Dr. William Russell ${ }^{1}$ (who adopts from the German the term hypertonus) argues as if it were the constricted vessels which undergo sclerosis, but, so far as our evidence goes, vasoconstriction does not provoke arterial disease in the districts so affected as ligature does, and within the district supplied pressures of course must not rise but fall (p. 30). I have long urged that a constricted artery is protected from strain, and Dr. Harry Campbell has agreed with me (p. 199). Whether or no the trunks serving it become strained depends upon the extent of the constriction; if the trunk or trunks of supply are included in the sweep of the constriction they are so far protected; if outside its limits, and the constriction be wide enough to raise systemic pressure, they will suffer with the rest of the high-pressure areas; and vessels of low contractile capacity, such as the coronary and the cerebral, will suffer, as these do, disproportionately (p. 477). As yet we have no means of estimating directly the blood pressure in the aorta and arteries of the greater magnitudes; the records which our instruments give us are taken rather towards the sphere of peripheral pressures.

We have seen (p. 44) that increase of the total volume of the blood, regarded by Huchard as a cause of increase of blood

1 Russell, W., Arterial Hypertonus and Sclerosis, 1907. 
pressure, has within clinical limits no such abiding effect (see Plethora); and the gradual obliteration of capillary areas, supposed by Balfour and Dr. Mackenzie ${ }^{1}$ to raise pressure by crowding up the blood into smaller compass, is but the blood mass hypothesis in another aspect. Mackenzie's words are: "Consequently the ventricle has to contract more strongly to raise the arterial pressure, and so embarrass the degenerated heart." One answer is that the work is not done, ${ }^{2}$ the whole machine runs slow. The truth is the relations of the blood mass to its continents do not come into this problem. If we ligature the aorta below the exits of the visceral branches, and by severance of the cord paralyse the splanchnic vessels, there is no rise of pressure. Beneke's statements on the subject ${ }^{3}$ seem to me to be fallible. In any case we are told, by pathologists of the eminence of Cohnheim ${ }^{4}$ and by many later authors, that in old age the total volume of the blood decreases; the surplus blood being "consumed" and removed and a correspondingly less bulk produced.

Concerning viscosity I have written in another chapter (p. 104).

At this point of the argument I must set a problem which hitherto, I think, has not been considered; namely, if the effect of extensive vasoconstrictions be to raise pressures in the larger arteries and the left ventricle, and if sclerosis in these vessels be thus accounted for, how far and in what directions does or may this secondary lesion extend? If histologically it seem identical with decrescent arteriosclerosis, is it so in its distribution? If with considerable rise of pressure there be, as no doubt there are, some peripheral areas not under constriction, does the stress effect travel so far in these open areas, and overcome friction so far, as to strain the finer arteries and the arterioles? Or is this consequential strain limited to those central vessels which sustain the first brunt of the high pressure? In "granular kidney" we know that in the kidney, spleen, pancreas, etc., sclerosis of the finer vessels is an early if not an incipient phenomenon, and becomes apparent long before strain may arise in the aorta,

1 Mackenzie, Jas., Diseases of Heart, p. 237.

2 See p. 217, Mummery on shock in elderly persons.

${ }^{3}$ Quoted M. Bruce, Lett. Lect., 1901.

4 Cohnheim, Syd. Soc. Trans., 1889, vol. i. pp. 131-132. 
notwithstanding hypertrophy of the left ventricle. This peripheral disease in these finer vessels can scarcely then be due to the high pressures, unless we suppose them to be far more vulnerable than the larger vessels; moreover, it is not easy to see why systemic high pressures should pick and choose among the vessels of the several viscera, unless it be that in the liver the finer vessels may be saved by the portal network. If then mechanical or strain sclerosis certainly does, as we all agree, in time affect the aorta and large vessels, are there then in chronic Bright's disease two kinds of arteriosclerosis at work? or, if one kind, then two categories of causes; mechanical lesion in some areas and toxic lesion in others? And if this duplicity be supposed on the findings of morbid anatomy, can we assume that in hyperpiesia, without renal symptoms, mechanical causes, so far as sclerosis is concerned, are at work alone? Some experimental data bearing on this side of the problem will be mentioned a little farther on. These considerations make one hesitate whether in renal or other high pressure cases to attribute arteriosclerosis anywhere wholly to mechanical strain. This problem however I must be content to state, I cannot solve it.

It is obviously very difficult to test in animals the mechanical conditions which we are discussing, either in combination or singly, but the experiments of Lewin and Larkin ${ }^{1}$ have attracted some attention. These observers made an arterio-venous anastomosis between the external carotid and the external jugular vessels, to see if the higher carotid pressure would set up hypertrophic or sclerotic changes in the vein. In some of the animals (dogs) the arterial pressure was aggravated by injections of adrenalin. The dogs lived for periods of 60-100 days-one lived for 120 days-but the results were negative. This, I think, we might have anticipated. Probably the local rise of pressure -of which no measures are given-was very transient; and in any case an operation so considerable, conditions so unnatural, and the differences between arterial and venous structure offer abundant room for fallacy.

Adrenalin and Arteriosclerosis. - A large number of experiments have been made with drugs and other agents to test the vulnerability of the arteries, and with none more frequently

1 Lewin and Larkin, Journ. Exp. Med., 1911. 
than with adrenalin (Josué, and numerous later verifications), which, as an active pressor substance, appears to be especially apt for this purpose. Josué ${ }^{1}$ and Gilbert and Lion ${ }^{2}$ were, I think, the first to publish definite results. Their researches were made by the intravenous injection of adrenalin into animals, at intervals, for longer or shorter periods of time. By this means conspicuous changes were produced in the aorta, of which I shall speak presently. Pearce and Stanton ${ }^{3}$ verified their results in respect of rabbits, and indeed it is on these animals that nearly all such researches have been made. The effects seem in them to have been confined to the aorta; though Erb (junior) reported a like effect in the renal arteries. We have seen that the percentage of spontaneous aortic disease in hutch rabbits is taken at about 3 per cent, but probably this is a low estimate, and the liability runs higher than this. ${ }^{4}$ But I will not discuss these figures, for, as Handelsmann says, much must depend upon the feeding and housing. And, as Manouélian of the Pasteur Institute found his positive experimental results (loc. cit.) to be 84.9 per cent, and in his most decisive experiments still higher, there is a large margin for any spontaneous sources of fallacy. The first effects are in the form of minute necrotic areas of granular degeneration in the muscular coat. The intima is not much affected, but opposite the foci in the media some little proliferation may be seen, and occasionally some tendency to form fibre. In more advanced stages the areas of degeneration are larger, or continuous, but are still practically confined to the media. The adventitia and vasa vasorum are unaffected. A very remarkable part of the changes in these aortas is the rapid advance to calcification. If, without defeating the experimental design by too sudden an injury, the effects of the injections can be carried to a certain degree rather rapidly, the media may be found degenerated over considerable lengths without calcification; but this does not usually happen; usually within these brief experimental periods calcification sets in, not as a tardy and incidental but as an

1 Josué, La Presse médicale, 1903.

2 Gilbert and Lion, "Athérome expérimentale," Arch. de méd. expér., 1904.

3 Pearce and Stanton, Laboratory Studies, Albany, 1905.

"Weinberg, "Athérome spont. chez le lapin," C. rendus de la Soc. de Biologie, déc. 3, 1908. 
early and essential effect. Moreover not a few observers have discovered in such tracts true bone with osteoblasts, Haversian canals, and even rudimentary marrow. The elastica breaks up and becomes disintegrated, even into granular relics. There is no doubt then that laboratory animals, chiefly rabbits ${ }^{1}$ it is true, after all due deductions for fallacy, under longer or shorter courses of adrenalin, have with fair uniformity exhibited very definite degrees of arterial disease; though almost exclusively of the aorta. But here again my problem, or a part of it, reappears. For a while it remained in doubt, indeed it is in some doubt still, whether these changes were due simply to the pressor effects of the agent or, more or less, to a toxic influence. C. Otto of Warsaw, ${ }^{2}$ after experimenting with many drugs on this method, concluded that they acted, if at all, in the same way-as pressor substances, producing their lesions just mechanically. He agreed with other experimenters that the media suffers first, then the intima ; finally the adventitia may show signs of irritation. In the media the muscular fibres suffer first, then the elastic fibres break up, and ultimately the intima thickens and degenerates. Ludwig Braun ${ }^{3}$ concluded that the effects of adrenalin on the arteries were not mechanical but toxic, because they were not prevented by a simultaneous administration of amyl nitrite. Dr. Rickett however demonstrated that amyl nitrite does not prevent an enormous forcing of pressure by the adrenalin injections, a rise which in spite of nitrites will endure for five minutes. It has been argued that thickening of the arteries in Addison's disease, even in young subjects, occurs too frequently for mere coincidence; but the lesions provoked by experimental adrenism are attended with plus pressures; in Addison's disease adrenin is lacking and pressures are low. But I have been unable to verify the clinical facts alleged. Mr. Markham, in a thesis for our M.B. degree, reported the arterial pressures in ten cases of Addison's disease as ranging between 74-85 mm. In one of his cases an injection of adrenalin forced up the pressure only from 84 to 112 .

1 Pearce and Stanton (Lab. Stud., Albany, 1905) failed to produce these results in dogs; F. Fischer of Bonn likewise, Deut. med. Woch., 1905. Leersum u. Rasser support Harvey (p. 223) (Z. f. exp. Ph. u. Th., June 1914).

${ }^{2}$ Otto, C., Virchow's Arch. Bd. cciii. H. 3.

3 Braun, L., "Zur Frage d. Art.-scler.," Münch. med. Wochenschr., 1905. 
This doubt between mechanical and toxic causes seemed to be set at rest by further experiments which, by bringing to bear stresses merely mechanical, still produced lesions of the aorta identical in character with those caused by adrenalin. I may especially refer to the long and assiduous research of Dr. Harvey in our laboratories, ${ }^{1}$ who, extending the method of aortic compression of Roy and Adami, by periodical compression of the abdominal aorta (in rabbits) set up the lesions in the upper portion of the vessel. The vasa vasorum and the adventitia were unaffected. (See also Adami, p. 229.)

A graver difficulty is that in none of these cases (my own observations have not been made entirely on Harvey's preparations) have the lesions, similar as they are among themselves, resembled the arteriosclerosis (atherosclerosis) of man If in certain respects they show some analogy, or even some deeper resemblance, to medial degeneration ("Mönckeberg's") with rapid and extensive calcification, the media being demolished by sudden and sharp strains such indeed as in life do not occur, in no instance to my knowledge have the intimal and sub-intimal changes of arteriosclerosis proper (atherosclerosis) been produced mechanically, unless it be in Manouélian's monkeys. Dr. Rickett, ${ }^{2}$ in our laboratories, produced these aortic lesions, that is a deterioration of the muscular fibres of the aorta, followed quickly by calcification, by nicotine, squill, barium chloride, and adrenalin; but no reactive signs appeared, no connective tissue proliferation, not even the fatty stain reaction of the kind demonstrated by Jores; nor any manifolding of the elastic layer, which merely loses its wrinkles and breaks up. In this layer the earliest changes (I quote from Adami, Rickett, Harvey, and Drummond) are stretching and interruption of elastic fibres, then fibrillation of them with granular disintegration. The muscular fibres likewise degenerate, and calcareous matter is deposited outside the elastica, entering into the media. The literature of this subject is very large ; I am

1 Harvey, Virchow's Arch. Bd. cxcvi. H. 2. See'also similar and confirmatory results and inferences of Biedl and Braun, "Zur Path. d. exp. Arteriosklerose," Wien. klin. Wochenschr. No. 20, 1909. Dr. Andrewes regards Harvey's results as cogent. Lubarsch, on the other hand, has said that he failed to verify them.

${ }^{2}$ Rickett, Journ. Pathol. and Bact., Oct. 1907 ; and Thesis for M.D., Camb., 1907. 
indebted to Dr. Harvey for assistance in references, and find a general agreement that experimental arterial disease is of the "medial type," the process being apparently too brusque for a gradual thickening of the intima. But some observers, ${ }^{1}$ in cases in which the strain was made as gradual as possible, record a preliminary hypertrophy of the media. Dr. Mott also compares these experimental effects to the "medial type of human arteriosclerosis," as described in the extremities (vide p. 482). There is however some evidence, obtained by retarding the blood current in one limb only, that by a slower method an increase of connective tissue, as compared with the vessels on the other side, may be obtained (the " regenerative form" of Jores); but these records need confirmation. Dr. Rickett, comparing the adrenalin experiments with others, regarded the lesions as purely mechanical; as did likewise Pearce, ${ }^{2}$ Stanton and Baldauf. Experimental work seems, as I have said, to be too brusque to produce ordinary arteriosclerosis; but Councilman, Sajous, ${ }^{3}$ and others, have suggested that if adrenalin in some excess were insensibly diffused through the arterial tree over long periods of time, it might set up arterial lesions, and perhaps arteriosclerosis of the typical intimal (atherous) kind. This may or may not be the case; as yet the evidence is not for it. Lion and Gilbert's results also bore wholly upon the media, but were regarded by them as toxic; while the recorded tendency to aneurysm in the experimental disease indicates the media rather than the intima as the seat of the mischief. It must be remembered however that the rabbit's aorta has not the complete intima of man, and is little more than a layer of epithelium; it is lacking in the elastic and musculo-connective layer which plays its part in human arteriosclerosis. Thus the aorta of the laboratory animal is differently constructed from that of man; the time conditions are different, and certain animals, the rabbit especially, seem

1 Victor Ball, Thèse de Lyon, 1907 ; Papadia, Arch. gén. de méd., août 1906.

2 Pearce and others, “Exper. Art.-scler.," Journ. of Exp. Med., 1906, continued in Proc. Rockefeller Instit. vol. vi., 1907. See also to same or similar effect Nowicki u. Hornowski, Virchow's Arch., May 7, 1908, and Drummond, Journ. of Physiol., 1904, vol. xxxi. Drummond thinks that the elastica is torn, and the media thus exposed first to the brunt of the stress.

${ }^{3}$ Sajous, Med. Assoc. of New York, 1906. 
peculiarly susceptible to these strains. Ball seems to have failed to produce such lesions in the horse or dog, though in experiments on the horse with injection of adrenalin the blood pressure would rise to double the normal; there it would remain for a minute and a half, and then gradually (in five minutes) fall back to normal. During the experiment the animal was distressed; the heart and respiration were much disturbed, and sweat broke out over the body.

As I have hinted in passing, there are no signs to suggest that the vasa vasorum, or a leucocytic invasion, have any part in the initiation of these changes.

As to the extent of the experimental arterial lesions, whether they are confined to the aorta or are more generally disposed throughout the arterial tree, I have said that observers differ. Handelsman ${ }^{1}$ reported the smaller vessels sound ; in them were no necrosis of the media, no calcification. He made a great point of keeping all his rabbits free and well nourished, for cachectic rabbits are certainly prone to exhibit necrotic foci in the aortic media. Erb (junior) at first thought the changes were more widely distributed, but he found reason later to change his mind, and reported their seat as almost exclusively aortic, or at most aortic and renal. All observers however agree that the left ventricle of the heart becomes much enlarged and dilated, and that venous hyperæmia and hæmorrhages may be found in the lungs and kidneys. Histologically, as Stewart has shown, this cardiac enlargement is not, or is in part only, a genuine muscular hypertrophy.

In my chapter on Arteriosclerosis and the Kidneys (p. 309) I shall discuss the hypothesis which maintains that in Bright's disease the high arterial pressures are due to irritation of the adrenal bodies, with profusion of adrenalin; and shall express the opinion that as yet we have little evidence that such is the nexus, and not a little to the contrary. Josué, in $1903,{ }^{2}$ was, I think, the first to attribute arteriosclerosis to some change, presumably of an irritative kind, in the adrenal bodies (p. 221). From my point of view, much of this argument on both sides

1 Handelsman, Inaug. Diss., Berlin, Dec. 4, 1906-a useful essay with bibliography. Also Elliott, T. R., Q.J. Med., Oct. 1914 ; appeared too late for me. ${ }^{2}$ Josué, Soc. d. Biologie, Nov. 14, 1903.

VOL. I 
is confused by neglect of the distinction between the arteriosclerosis due to high pressures and that of decrescence. However, this matters the less if the adrenal surmise be baseless. Taking arteriosclerosis in the lump, it will appear that no constant or characteristic alteration of the adrenals is associated with it; whatsoever alterations there may be in them are but such as are prone to occur indifferently in other maladies likewise; most of them are of the nature of degradation rather than of stimulus or of enlarged capacity, and moreover have often occurred in the cortex with a normal medulla. Increase of size (adenoma) need not of course mean any abiding excess of function. Dr. Hodge of Woodford Green, in his thesis for M.D. at Cambridge, brought together no inconsiderable number of cases of adrenal adenoma in which the blood pressures, or at least the state of the arteries, had been noted; in none was found any evidence of correlation between the adenoma and the state of the vessels. Frank ${ }^{1}$ found that in many cases of high blood pressure, the adrenal bodies were not concerned, nor probably any part of the chromaffin system; and Münzer is decisively of the same opinion. ${ }^{2}$ Münzer says that a middleaged friend of his took adrenalin, subcutaneously, for asthma, continually for eighteen months, but that neither in heart nor vessels did any alteration appear. In some infections, as in diphtheria, the toxin injures the adrenal bodies, and blood pressure falls, making one of the perils of convalescence. Dr. Mott, in some high-pressure cases, carefully weighed the adrenals and found no reason to attribute the perversion to them. Indeed these bodies often proved to be wasted. Handelsman also (loc. cit.), in a series of cases of atheroma, weighed the adrenal bodies and, comparing the weights with those of control bodies taken from non-atheromatous cases, failed to find any correspondences; and he urged further objections which are substantially those I have stated above. In many cases of arteriosclerosis the vessels of the adrenals are themselves the seat of intimal sclerosis, a change which can scarcely make for profusion of their specific product. Nowicki and

1 Frank, "Chromaffinsystem v. chron. Hypertonie des Menschen," Deutsche Arch. f. klin. Med. Bd. ciii. p. 194 (1911).

2 Münzer, Zentralbl. f. Herz-u. Gefässkr., Nov. 1, 1913. 
Hornowski ${ }^{1}$ of Lemberg, again, made a very full examination of this question, and scrutinised the adrenals in 36 cases of arteriosclerosis (ages 44-77), controlling them by 122 cases in which this change was wholly absent. They concluded that in form and weight there was no difference. In old persons, with arteriosclerosis, or without it, or with but little of it, they often found the adrenal cortex to be the seat of an increase of the capsular and trabecular connective tissue with encroachment upon the parenchyma; but in none did the medulla show any change, excepting of course sclerosis of its vessels; nor were the associated nervous structures altered. They consider, apparently with justice, that they carried this research far beyond that of Josué, and even beyond that of Handelsman (who examined these bodies in 15 cases of arteriosclerosis). Bittorf and others, at the Wiesbaden Congress for Internal Medicine, supported the same conclusion. In an anatomical sense then there is at any rate no apparent connection between arteriosclerosis and adrenal disease, medullary or cortical. Biedl's views of the function of the cortex need not concern us here. Parkinson, ${ }^{2}$ who did find some association of high pressure with high adrenal or with general chromaffin activity, also concluded notwithstanding that the negative instances were at least as frequent, and that this aspect of the problem was, as yet at any rate, anything but illuminating.

Still, the chromaffin system may have some obscure relation to irregular arterial pressures. Dr. Langdon Brown ${ }^{3}$ suspects some excess of adrenal secretion in cases of men of middle life, with high pressure and glycosuria.

Hugh Stewart ${ }^{4}$ has called into question the nature of the vasoconstrictory substance of defibrinated blood, asserting that it is not adrenin..$^{5}$ It acts directly on muscle, and throws the bowel into spasm; whereas adrenin inhibits peristalsis. In

1 Nowicki and Hornowski, "Nebennieren bei Arteriensklerose," Virchow's Arch. Bd. cxcii. H. 2, May 1908. See also to same effect Bröking u. Trendelenburg, Deutsche Arch. f. klin. Med. vol. xcvi.

2 Parkinson, Trans. Pathol. Soc., 1907, p. 187. ' See also Frank, L., Deutsche Arch. f. klin. Med. vol. ciii., 1911.

3 Brown, L., Clin. Journ., Oct. 1, 1913.

4 Stewart, Hugh, Journ. Exp. Med. vol. xiv., 1911.

5 On methodical tests for adrenin see Joseph, Studies Rockefeller Inst. vol. xvi., 1913 ; and on the chemical constitution of these bodies see Dakin, Proc. Roy. Soc., 1905. 
this opinion Janeway and Park agree; and Janeway ${ }^{1}$ agrees further with Hodges and $M^{\prime}$ Clure that the adrenal bodies secrete too little of their peculiar product, and secrete it too slowly to maintain the tone of the whole vascular system, or to serve as more than a reserve for emergencies. Some recent observers are asserting that very minute doses of adrenin lower arterial pressure by vasodilatation. Still, when we think of Addison's disease, and of the ill effects (in the guinea pig) of removing even one of them we cannot underrate the values of these organs. And the big heart of hyperpiesa, on the other hand, is made of good stuff. If however in these cases the adrenal bodies are in any way concerned, we may recall the opinion of Sajous ${ }^{2}$ that their secretion is a means of oxygen exchange, and is partly identified with the albuminous molecule of the red corpuscle; if so, it may no doubt play a direct part in all tissue metabolism. I shall touch on this point again in connection with the specific infections. But, once more, none of these observers has taken into consideration my original postulate, now abundantly verified, that manyprobably most - cases of arteriosclerosis are not attended at any period with arterial pressures excessive for the age of the patient.

It is worth while however to note the order in which, under the stresses of injected adrenalin and cognate substances, certain arterial areas undergo constriction, while others not so held up are strained and damaged, a question asked already on the clinical side. Gaskell, Cushny, Dixon, Harvey and others have described such series for us. Under adrenalin the vessels are constricted in the skin, stomach and bowels, mesentery, and uterus; not in the brain, heart, lung, nor liver, areas in which vasomotor tone is weaker: these areas therefore are liable to become loaded with blood; and in some of them, if the heart's energy be augmented, which, subject to uncertain vagus restraints, it usually is, the vessels, or at any rate the aorta, become strained. But the efferent outflows - cranial, thoracico-lumbar, sacral-

1 Janeway, Amer. Journ. Med. Sci., May 1913-a full discussion of these problems; and for a similar survey see Rolleston, H. D., Lancet, Sept. 28, 1907 ; Langendorf, Zentralbl. f. Physiol., 1907, and Möller, S., "Beiträge z. Wirkung d. Nebennieren-Extractes," Therap. Monatsheft, Dec. 1905, and Jan.-Feb. 1906. Möller's papers I have not seen.

${ }^{2}$ Sajous, Journ. Med. Assoc., New York, 1906. 
are not uniform; the activity varies in areas (Gaskell, Cushny, Dixon), and is best marked in the thoracico-lumbar. This is curious, if adrenalin acts directly on the muscular coat. However we must not forget that in mechanical experiment distribution of lesion is dependent, at least to some extent, on the position of the animal. In Professor Adami's laboratory rabbits were suspended head down for three minutes a day for 130 days, when the thinning of the media of the aorta with calcareous deposit, after the manner described by Dr. Harvey (p. 223), was observed; in them the cervical and cranial vessels also were notably affected.

In any case then extensive constriction is only the proximate cause, and the antecedents have to be sought beyond. Why and how is it that these widespread constrictions come about? The conjectural answer is that the constriction is set up by some poison in the system, autogenous or heterogenous. If so it be, we have a remarkable instance of the irony of things, in the abundant generation for evil of an agent of high pressure of which, for good in a therapeutical sense, we are often in sore need. Our remedies for falling peripheral pressures, as in collapse, are few, difficult, and fallible; strychnine and the fugitive adrenalin and pituitin being perhaps our only available pharmaceutical vasoconstrictors.

Concerning the pituitary body and other hormones, so interestingly discussed on speculative grounds by the late Dr. Gibson in the address on Medicine at Liverpool in 1912, I have no more to say than concerning the adrenals; but in chronic disease of the pituitary body cardiac hyperplasia (let me repeat not a genuine hypertrophy) and atherosclerosis may be enormous. ${ }^{1}$ Secretin seems to cause a fall of pressure ; for although it constricts many areas it dilates other large ones, such as, it is said, the splanchnic. As Gouget says, ${ }^{2}$ we cannot arrange our glands into pressor and depressor ranks. I have argued that these inward glands are not to be regarded as quite separate

1 Vide L. Humphry's case, etc. (p. 271). For a study of Hypophysitic Extracts see Lewis, Miller, and Matthews, Arch. of Intern. Med., June 15, 1911 (quoted Epit. B. Med. Journal). Dr. Blair Bell thinks this extract more active in pathological than in normal states.

${ }^{2}$ Gouget, Journ. méd. Fr. ii. 52, 1912, quoted Zentralblatt f. Herz-u. Gefässkr., March 1912. 
and peculiar. Whether a secretion shall be discharged outwards or inwards, whether its waste product can be utilised or not, is an accident. To manufacture some product, useful, useless, or harmful, is the property of all cells. Parkes Weber, Lorand of Carlsbad, Ewald, H. D. Rolleston, Leonard Williams and others have suggested that senile arteriosclerosis - especially when precocious-may be due to thyroid deficiency. We have as yet no evidence of this, indeed Sir Victor Horsley and other competent observers deny any such relation. They have reminded us however that in elderly persons the thyroid often undergoes some involution, and that these senile changes may be comparable in a measure with degrees of myxœdema; or, again, as Dr. Rolleston puts it, ${ }^{1}$ the suprarenals may get too big a pull upon a thyroid injured by some infection. In my own elderly body five grains a day of thyroid extract taken experimentally for about five days in one week, instead of a perennial spring, set up tachycardia, fine tremor, and petulance, when I was fain to stop the experiment, and therewith my venture for carnal immortality. I have tried the same drug on a few arteriosclerotics of the decrescent kind, whom I supposed to be rather more torpid than myself, but with no signs of advantage. Atropine of course palsies the autonomic system, but choline stimulates it. The chromaffin substances influence the sympathetic but not the autonomic system. ${ }^{2}$

Ovarian extract is said to lower blood pressure, and rises of pressure at the climacteric have been attributed to poverty of it in the system; at any rate it seems probable that at this period the balance of these hormonic glands is deranged. ${ }^{3}$ But in elderly people the rule is, not that arterial pressures, as in myxœdema, decline, ${ }^{4}$ but rise rather.

It is often suggested that the normal balance of arterial health and pressure ${ }^{5}$ is maintained by the antagonistic action of several internal secretions; so that arteriosclerotic states may arise, for instance, from defect of one or other of the inward

1 Rolleston, H. D., Clin. Med. Journ., June 21, 1905.

2 See Kinneir Wilson, Brit. Med. Journ., June 14, 1913, p. $1261 . \quad 3$ Ibid.

4 According to some authors, in myxœdema the blood pressure is raised, according to others it is depressed or normal. The myxœdema is probably not the determining factor in the blood pressure.

${ }^{5}$ See Fearnsides, E. G., Lancet, July 4, 1914. 
glands of the thyroid, of the adrenals and chromaffin bodies, such as the carotid body, the pituitary, the ovaries after the menopause, and so on. In Dr. Fearnsides' case of "dyspituitarism" under thyroid extract pressures of 180 fell to 120 . Some slow cases of arteriosclerosis with chronic glycosuria, attributed to atrophy of the pancreas with connective hyperplasia, may originate in a preceding sclerosis of its finer vessels.

Barium chloride acts simply as a mechanical cause of high pressure, by means of a widespread vasoconstriction. It seems to act directly on the arterial muscle, and with a vigour proportionate to the amount of muscle in the several branches. When injected intravenously into rabbits it thus raises pressure, and provokes changes in the aorta rather more like atheroma than the effects of adrenalin; namely, intimal disease with splitting of the elastica, a result which so far makes for the opinion that these changes are not toxic but merely mechanical. The virtues of certain other and better known agents which may force up arterial pressures, such as squill and digitalis, are too complex for experimental work in this particular direction, and in any case they are less energetic than adrenalin; caffeine is less active still.

Excessive ingestion or retention of common salt is said to raise arterial pressures, most readily in subjects already arteriosclerotic $;^{1}$ a rise which quickly subsides on free excretion of it. The rise of arterial pressure after meals has been credited to the salt. The part of salt in the economy is too large and various to be discussed here incidentally, and needs far more research. The œdema correlated with salt retention is chiefly in the musculo-cutaneous system, but each organ has yet to be tested for normal and abnormal retentions of it. Widal and Javal estimate it in the blood serum by electro-conductivity. The normal content is $0.562 \mathrm{grm}$. Chloride metabolism is a far more complex matter than we usually suppose, but we shall touch upon it again in some later chapters (vide Rickett, loc. cit.). I have found that to cut out salt from the diet of healthy persons for a few days produces no changes of pressure.

There are certain synthetic substances which are credited with

1 Bayer, Journ. Exp. Path. and Pharm., July 1907. Ambard and Beaujard, Arch. gén. de méd., 1904. 
pressor virtue, certain amines, ketones, and the corresponding secondary alcohols. Some of these have been used in experiment; pyrocatechin, which, by the way, is not a pressor substance, is said to have affected the aorta and other vessels, after the manner of adrenalin, but so far the results are not sufficiently convincing to enter into the present argument. ${ }^{1}$ Of the effects of lactic acid, as it may be generated during a suboxidation in the muscular field, I have spoken already (p. 212). Here again the arterial lesions attributed to it in experiment on animals do not constitute the arteriosclerosis of human disease, but the medial necrosis with calcification (p. 482); a kind of change which, as we have seen, some " hypotensive" substances seem capable of producing, at any rate in the elementary aorta of the rabbit (vide p. 234).

Toxic Causes.-We shall now proceed to discuss the multifarious agents which, under the general conception of animal poisons and "toxins," entering the body from without or engendered within it, impair and disintegrate, so it is supposed, the coats of the vessels. And to these we advance with uncertain steps across the marches of "wear and tear," marches haunted by unnumbered ills of obscure origin, some of them engendered even by psychic causes, which may issue at length in arteriosclerosis, and may be directly related to it as cause and effect. But we shall remember that in many cases " apparently toxic," in persons under middle age, especially in renal disease, the strain of the aorta shows itself as a sequel at a comparatively late date (see p. 498) after a long continuance of high pressures.

But when we come to enquire into the influence of poisons, known or suspected, upon the coats of the arteries, we are arrested at the outset by many difficulties. For instance, arteries do not all behave in the same way to the same agent, and animals differ much in their several reactions to drugs and secretions. Thus under adrenalin, while all the other arteries contract, the weaker coronary and cerebral arteries are dilated. The pulmonary artery, before its entry into the lungs, is constricted by it, but the intrapulmonary twigs remain unaffected, or dilate. ${ }^{2}$ Again,

1 For these experiments I may make a general reference to the well-known papers of Cushny, Dixon, Dale, Barger, Laidlaw, Widal, Vaquez, Achard, Strauss and others. Also to Scholz and Hinkel, Deutsche Arch. f. klin. Med. Bd. cxii. He. 3 and 4.

2 Cow, Douglas, Thesis for M.D., Cambridge, Dec. 23, 1910. 
in observing the behaviour of the arteries under such agents we have to discover whether the response, if any, be directly from the muscular or nervo-muscular mechanism, or if it be a local reaction to an irritant in the blood (p. 51 n.), or again a direct reaction to lateral pressures. And I have insisted upon another difficulty, concerning mixed kinds and origins of arteriosclerosis, that the arterial disease known by this name may in nature be more than one? If, after our wont, we suppose one kind of poison to act directly upon the walls of the vessels, corroding or inflaming them, as seems to be the case with some of the "infections," and another to act upon the peripheral mechanism, setting up a wide field of constriction such as to force up the main blood pressures to the point of strain, it would seem that in these cases we should have to do with two kinds at least, or at any rate with two processes, of arterial lesion and reaction : these cases, poison or no poison, would fall into the class of strained wall, those into the class of deterioration; and at first sight we should anticipate that the histological phenomena would in the two cases differ also. Carbon dioxide is regarded by some authors as the poison associated with high pressures; asphyxia of the medullary centres with consequent intense vasoconstriction, therefore less and less cardiac output, and so on. I do but mention this hypothesis, which however clinical experience has done little to recommend. Edgren admitted two kinds of cause only, (1) the toxic, and (2) the nervous; the toxic being autogenous or heterogenous, but often acting through the nervous mechanisms. Lubarsch emphasises the toxic side in causation almost exclusively.

Thus when we come to consider the part of toxic substances in the causation of arteriosclerosis we find ourselves if poor in knowledge yet rich in conjecture. We regard more strictly as poisons substances, such as lead or strychnine, which are measurable by dose, constant in virulence, and theoretically recoverable from the excretions and the tissues of their selection. We regard as toxins those whose properties partake rather of the protean qualities of living matter in respect of zymotic energy, of incubation, of multiplication, of transformation, and of relative imponderability. To restrict the term "toxin" to bacterial products may be too narrow; we are 
not sure that decomposition of food itself, simply in the course of oxydation, may not pass through toxic phases. These two classes of poison are dealt with each by its appropriate analytic method; or, in the case of substances which move on the borders between these classes, such as certain katabolites -or kakobolites as for the moment we may call them-of the amine or xanthine order, by a combination of chemical and biological methods.

While then such are the differences of agents, we have also to bear in mind the differences of the several arteriosclerotic series: the high pressure mode, the decrescent mode, and thirdly the convenient but largely overlapping class of toxic modes. When we have fathomed the depths of these reactions we shall probably be enabled in great part to redistribute our provisional class of toxic arteriosclerosis, and to remit the poisons of some of them, such perhaps as certain recognised substances of the adrenic, ergotic, or amine series, to the high pressure class, and others of them, some of a more gradual and stealthy nature, to the decrescent class. For it is a common surmise, strengthened on the one hand by the occasional precocity of decrescent arteriosclerosis, and on the other by its absence in a few old men who have lived no sheltered life, that something more than mere wear and tear-more than mere detrition-may be concerned in its establishment (p. 236). The recent experiments with adrenalin, whether this body be on the aorta simply a pressor, or a pressor and also a toxic agent, have at any rate perpetuated discussion of toxic agency, and led to the investigation of toxic bodies, such as those of the amino-N. order (Dale, Barger, Dixon, Laidlaw), and of other perverters of metabolism, pressor and depressor. ${ }^{1}$ Yet, if this advance be made, it seems probable that specific kinds of arterial disease, kinds such as the syphilitic, for instance, or the plumbic, will remain, for which a toxic class must be maintained. Furthermore, as regards arteriosclerosis of the first and second classes, and the various contributory toxins, we shall perceive a further difference; namely, between those which produce arterial lesion directly, and those which, as causes of

1 See, e.g., Hirsch und Thorspecken, Deutsche med. Wochenschr., 1912, He. 5-6; Bain's papers (loc. cit.), and so on. 
excessive blood pressure, compass it but indirectly, or, as in the just cited case of adrenalin, a pressor substance may injure the vessels directly by both toxic and pressor qualities. For there may be, probably there are, bodies which thus damage the vessels both directly as toxic to them, and also mechanically as pressor substances. Von Leersum ${ }^{1}$ urges the validity of the results he obtained, namely very great rises of blood pressure, by feeding rabbits upon liver presumed to contain intestinal toxic substances. He produced no arterial lesion, it is true, nor did he obtain hypertrophy of the heart; but the time was perhaps too short even for cardiac hypertrophy. Only one other observer, I think, has as yet published corroborations of von Leersum's results. So far then these suggestions are little better than guesswork; still, by a continuance of careful experiments with a few poisonous and toxic substances whose properties are known or suspected, each in its turn, we shall gradually clear some way before us. To take a simple case, that of carbon dioxide; in the first stage of asphyxia a great rise of arterial pressure occurs, due apparently to excitation of the vasomotor centre in the medulla with contraction of the systemic muscular arteries, but perhaps to some direct action on their muscle, perhaps also to a rise of viscosity by the $\mathrm{CO}_{2}$. But the effect is transient. Lead, which is said to be a vasoconstrictor, may have a compound effect of excessive pressures due to extensive vasoconstriction, to renal impairment, and perhaps again to some circulating poison, lead itself, or dependent on lead, which corrodes the vessels.

Furthermore, the physician, while gratefully taking information from the physiologist, must not forget that drugs act very differently upon different kinds of animals and on man. In the dog nitrite of amyl causes but little fall of pressure, because an acceleration of the heart prevents it; and in many respects the horizontal dogs, rabbits, guinea-pigs, and the erect human being, manifest different reactions to the same reagent. In some cases a like effect may arise from different caiuses; as in one animal a fall in pressure may be due to vascular relaxation, in another to cardiac enfeeblement under the same conditions.

We know enough of exercise, of muscular metabolism, of the

1 Von Leersum, Zeitschr. f. exp. Path. u. Ther., Aug. 31, 1912. 
relations of oxygen and lactic acid, and so forth, to be alive to the many ways in which by fatigue metabolism may be perverted ; how noxious metabolites, circulating in the blood in this way or that, carried perhaps by way of the vasa vasorum, may corrode the arterial tunics. Dr. Gaskell, in his well-known paper in the Journal of Physiology, suggested that acid metabolites, due to the activity of an organ, might play a part in relaxing the muscles of the small arteries supplying the organ by altering the composition of the lymph bathing such arteries (p. 51 n.). This agency, so far as it would affect blood pressure at all, would tend towards its relaxation. It is remarkable however that hitherto experiment with poisons, even such as lead, seem, so far as I know, to have failed to produce definite arterial disease.

If wear and tear ("Abnutzung ") is to mean strictly fatigue of the coats by frictional and oscillatory stresses, it should have been contained wholly in the previous section; it is in this sense that in former papers I have used the phrase. But Dr. Mitchell Bruce $^{1}$ proposes to extend the term, from the mechanical causes already considered, into a more comprehensive sense, a sense to cover not only psychical pressor (p. 70) as well as obvious physical strain, but also an incalculable " series of small, insidious, mostly unperceived actions upon the circulation, of an unfavourable character," influences to which, with their alleged perversions of wholesome katabolism, he would chiefly attribute my "decrescent or involutionary form" (of arteriosclerosis) (p. 532). From this point of view in another passage Dr. Bruce discusses more fully "worry and anxiety" as a cause of arteriosclerosis, being herein, I observe, a disciple of

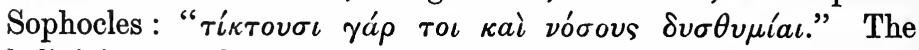
belief is prevalent that mental anxiety, worry, care, distress, acting over long periods of time will cause this vascular deterioration; Lancereaux was as eloquent about neuroses as Huchard about toxins; but this impression is aggravated more or less by an error in diagnosis, that is by the mistaking of some cases of hyperpiesia in its earlier stages for neurasthenia. It is also asserted that vascular instability under psychic storms is very trying to the coats of the vessels, but I am not yet prepared to

1 Bruce, M., "Lumleian Lect.," July 8, 1911 ; Lancet, p. 71. 
admit that excitable, emotional persons are more subject to arteriosclerosis than persons of equable temper. I am not yet convinced that causes of this kind take a leading place in the production of my "Decrescent" mode of arteriosclerosis; I am inclined rather to expect from them some effect of hyperpiesis, and if so perhaps ultimately of the sclerosis consequent upon this. The life of a wealthy inhabitant of ancient Egypt does not at first sight suggest to us the excitements, the haste, and the mental harass of the Stock Exchange; yet in the Egyptians arteriosclerosis, apparently of my "Decrescent" kind, was prevalent, even in Rameses II. himself, who however may have been worried by Hittite "unrest." We are too apt to look wistfully back for a Golden Age in which passions were calm and occupations tranquil. But the tyrannous passions and internecine raids of savage peoples can scarcely have made for emotional peace, or for circulatory and secretory equilibrium ; and we do not know that the conditions of life among such peoples were or are more wholesome than our own. And I repeat that the age, comparatively golden, of Egypt, ${ }^{1}$ and probably of Chaldæa, before Edom and Assyria " woke up," appears to have made rather conspicuously for decrescent arteriosclerosis, if it were only by the prolongation of life; as does the quiet boudoir of the rector's wife, or the cloistered life and peaceful occupations of the philosopher. Thirty-seven years ago ${ }^{2}$ I published certain evidence in favour of emotional harass as a cause of "Granular Kidney," an opinion which met then with much acceptance, and since again at the hands of Sir James Goodhart ${ }^{3}$; now it may be that Granular Kidney is set up by some toxin, as yet unknown, upon which pressor effects would immediately follow; or possibly by emotional harass metabolism may be so perverted as to engender either corrosive metabolites of endogenous formation which attack the coats of the vessels directly, or a pressor toxin damaging them indirectly by strain. We know how in incipient diabetes worry or a sleepless night may throw sugar into the urine. Speculative as this argument is, yet in its context some very interesting statements,

1 See Ruffer, Journ. Path. and Bact. vol. xv.

2 Brit. Med. Journ., Feb. 10, 1877.

3 Goodhart, Sir J., Harveian Oration, 1912. 
made at the Wiesbaden Congress of Inner Medicine in April 1907, may be mentioned. It was stated by Weber of Berlin, and the statement was corroborated by Klemperer, that, even in hypnotic trance, to excite the idea, "the psychical factors, the mental motor impression of an effort" ("einer Arbeit"), without any part of the effort itself, raises arterial pressure (vide p. 69). Thus it may be that incessantly agitating ideas, with oscillations of the blood pressure no less incessant, may in time tell upon the coats of the vessels, or affect them indirectly by excesses of imperfectly eliminated waste. It is said by House physicians that the blood pressure rises in convalescents on the day of leaving the hospital. But Dr. Bruce himself remembers, with some perplexity, that death from cerebral hæmorrhage is greater per million in the country than in London; though we must not forget that nowadays many citizens and publicmen reside in the country. Moreover, apoplexy connotes not decrescent but pressor sclerosis. I agree however with Dr. Bruce that "hard intellectual work does not of itself damage the organs of circulation" (vide p. 213). He is of opinion that "worry, suspense, anxious anticipation, disappointment, consciousness of failure or of failing health, the 'hunted' feeling that comes of overwork and arrears, regret, sorrow, despair" ... "wear out not only the nervous but also the cardiovascular system." Age without years. It may be so ; Fräntzel, it is true, noticed a large proportion of palpable arterial coats in soldiers after long war and exposure, but hereunto no doubt many evil factors conspire. Yet when I remember the large number of cases of arteriosclerosis, both decrescent and hyperpietic, in which no such history was apparent, and the large number of cases without cardioarterial consequences in which such misery had abounded, one is disposed to demur that Dr. Bruce's experience is drawn more especially from certain classes of society.

Before weighing the evidence, clinical and other, of autogenous toxins which may poison the arteries, let us set out from what we know of certain more obvious agencies.

Diet.-That diet, in the ordinary acceptation of this word as a scheme or selection of food, has any part in the generation of decrescent arteriosclerosis I have no knowledge. It is 
frequent both in the wealthy and in the poor; in the Hospital and in the Hall. But that one of the kinds of arteriosclerosis is due to excessive, or relatively excessive, feeding with meat and wine, and that this kind is caused by a persistence of high arterial pressures, as opposed to von Basch's opinion that the high pressures were the consequence of the arteriosclerosis, has been my opinion from the first, an opinion which Huchard and I stated independently in 1893-94; my difference from Huchard being, as I have said, that he gave this explanation of high pressures to all arteriosclerosis, whereas I applied it to one mode only, and this a secondary mode; the event seen ultimately in the malady I have called Hyperpiesia, in kidney diseases, and perhaps in other morbid processes with excessive arterial pressures. Cohnheim (loc. cit.), who recognised high blood pressure and big heart in gluttons, said then we had no data for its interpretation. Traube was one of the first physicians, in recent times, to marshal clinical evidence on luxus consumption, and to argue the question on scientific lines. He argued, and in this Fräntzel followed him, that with gluttony arose an overcharge of the veins and a fall of centrifugal velocity, which propagated a rise of pressure backwards to the capillaries and arteries, a condition which he treated with laxatives:

... When we have stuffed

These pipes and these conveyances of our blood

With wine and feeding.

And he and his followers then pressed the familiar argument that as the renal excretory superficies was inadequate to the increased demand, the efforts of the circulation to pass blood through it were multiplied. On this followed George Johnson's arteriolar hypertrophy, and so on. The arteries of the alimentary tract are among the most muscular in the body, and have a very complex nervous endowment-a mingled vasoconstrictor, vasodilator and vagus supply; and in no arteries is medial hypertrophy, without calcification, so well marked, and even so palpable. This change, when absent elsewhere in the system, ${ }^{1}$ may be

1 Vide Rössle, "Über Hypertrophie u. Organkorrelation," Münch. med. Wochenschr., 1908, No. 8. 
found here. ${ }^{1}$ Dr. Stengel ${ }^{2}$ emphasises as causes overfeeding and hereditary predisposition, and says that regulation of diet and habits, if they cannot cure, may alleviate the malady, even in its more advanced stages. Sir Lauder Brunton, Dr. W. Russell, and many others are of the same opinion. "Man ist, was er isst." Still I think we must hold even this common opinion with some qualification. We cannot say that all hyperpietics are, or have been, gluttons, though a great many of them, both men and women, are, or have been; and Vaquez ${ }^{3}$ is, in my opinion, too sceptical of overfeeding as a cause of arterial disease. Overfeeding is a relative question: what is overfeeding for one person may not be injurious overfeeding, though it may be superfluity, for another. Human engines vary much in consumption of fuel ; some men can do long and active work on very little food ; others, especially big men, need a somewhat liberal table, and give way without it. I can work well on half the food which other active men seem reasonably and by experience to require. In some notable persons, rich or even enormous overfeeding, even with somewhat inactive habits in a muscular sense, has been consistent with long and energetic life. And not all such persons are obese; some are, others might " find a mansion in the case of a treble hautboy." Many heavy feeders, friends and patients, have I known, who, with arteries soft and blood pressures moderate, have lived, or happily still are living, to a good old age. We may be pretty sure then that mere surplusage of nutriment is not always a direct cause of arteriosclerosis of any kind; there are other and intercessory coefficients. Physiologists are of opinion that in normal persons all the food is absorbed, and has therefore to be used or destroyed. What then is the factor of evil ?

1 In the British Medical Journal for Feb. 8, 1913, is a remarkable collection of proverbs of diet. The crowd of them which warn against gluttony, against digging one's grave with one's teeth, is in all times and all peoples innumerable, so that the ills of overfeeding have been manifest to all shrewd observers. From Theognis and Hippocrates, through Latin, medieval, and modern Europe, these saws, such as " Non plures gladio quam cecidere gula," and so on, have abounded. Madame du Deffand, from her large experience of hospitality, wrote of feeding: “C'est un des quatre fins de l'homme; j'ai oublié les trois autres."

2 Stengel, Amer. Med., Jan. 2, 1904.

3 Vaquez, Address to the Physicotherapeutic Congress at Berlin in 1913. 
Is it a true plethora, mere overwork in katabolism, or the generation of toxic by-products? I have said that we, as other engines, differ widely amongst ourselves not only in the quantities of food we need for given work, but also in our capacities for dealing with it economically. As men in their capacity for disposing of sugar in the system vary widely, so no doubt they vary widely in their capacities for other metabolisms. A small eater may yet be a bad metaboliser; and I have come across not a few moderate or small eaters who, notwithstanding, became subjects of hyperpiesia; and not a few who, in spite of ample outdoor exercise, could not indulge their appetites with impunity. To this side of the question we shall return under the head of Gout (p. 273). Meanwhile I would urge that, if one man can be gluttonous with impunity, and another on a moderate diet becomes "plethoric," nevertheless the average man who indulges his appetite for food and drink, especially if his habits be sedentary, runs no little risk of high arterial pressures, and of imperilling the integrity of his arteries. As Fanny Burney neatly put it, Thrale's miscalculation of his digestive powers ended in an apoplexy. Let us remember then the well-known passage in The Republic: "And do you not hold it disgraceful to require medical aid, unless it be for a wound or an attack of illness incidental to the time of the year - to require it, I mean, owing to our laziness and the life we lead, and to get ourselves so stuffed with humours and wind, like quagmires, as to compel the clever sons of Asclepius to call diseases by new-fangled names?" 1 A good cook, as some one observed, is more to be feared when one is in perfect health than a bad doctor when one is ill! Concerning the part of butcher's meat in particular in making for high pressures, I shall speak presently under the head of autogenous toxins.

To the experiments of Professor Chittenden I need do no more than refer, so well known are they; and Dr. William Russell and others have emphasised their importance in clinical medicine. Dr. Russell "was amazed 'at the statement that excessive protein feeding led in the individual to no bad results." 2 Professor Saundby says that the diet of the Trappist monk, who is by no means an idler, is as follows :

1 Plato, Rep. $405 \mathrm{D}$.

${ }^{2}$ Russell, W., Brit. Med. Journ., Sept. 3, 1911.

VOL. I 


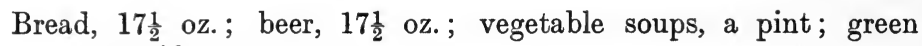
vegetables, $6 \mathrm{oz}$.

That is :

$\begin{array}{lrrr}\text { Protein } & . & 62 \text { grms. } & 254 \text { heat units. } \\ \text { Fat } & . & 10 \text { grms. } & 93 \quad " \\ \text { Carbohydrates } & . & 450 \text { grms. } & \frac{1845}{2192} \quad "\end{array}$

Mr. Fletcher's diet in our laboratories $=1606$ heat units. Professor Chittenden's (he is a light spare man) $=1700$ heat units. $\mathrm{Mr}$. Fletcher's diet was 40 grms. of protein a day, for 6 days only, which is no test of endurance. Professor Chittenden's athletes, while under his eye, consumed 47-55 grms. of protein ; university athletes usually consume it at least up to the Voit standard of 120 grms. Professor Saundby says that 2000 cal., containing 50 grms. of protein, is a sufficient diet for a 140-lb. man in business or professional life; and this he illustrates thus :

Breakfast : 1 egg $=75$ cal.; toast, 3 oz. $=207$ cal. ; butter, $\frac{1}{2}$ oz. $=120 \mathrm{cal}$. ; milk, $\frac{1}{4}$ pint $=90 \mathrm{cal}$. ; tea $=30 \mathrm{cal}$.

Lunch: Rice pudding, 6 oz. $=500$ cal. ; cheese, $1 \frac{1}{2}$ oz. $=168$ cal. ; bread, 2 oz. $=138$ cal. ; fruit $=40$ cal.

Dinner: Clear soup, $\frac{1}{2}$ pint (say 30 cal.) ; fish, 2 oz. $=56$ cal. ; meat, 2 oz. $=56$ cal.; potato, 4 oz. $=90$ cal.; green vegetable $=20$ cal. ; pudding, 4 oz. $=320$ cal. ; savoury, $1 \mathrm{oz} .=70 \mathrm{cal}$. ; halfpint light wine $=50 \mathrm{cal}$.

The whole three meals to equal a little over 2000 cals.; or in grammes carbohydr. 286, protein 50, fat 26 .

Chittenden's athletes in full exercise consumed, in all, under 3000 cals. per diem. I fear this looks awkward for some of us, but in my experience those tight reckonings, if they may serve for a few months or a year, do not perpetually suffice under all kinds of stresses. Moreover, in restricted diets there is a danger of omitting or reducing some subtle element, as in the case of the skin of the rice, which is essential to health.

Asa Gray ${ }^{1}$ said of plants that " with high feeding and culture comes vastly increased liability to disease." It is stated that in the healthy man on a milk and vegetable diet arterial pressures fall, but I find no note of any accurate work on this point. In discussing the effects of overfeeding upon arteriosclerosis ${ }^{1}$ Gray, Asa, Darwiniana, pp. 338, etc. 
then it must be understood that, so far as my argument is concerned, this habit bears only upon the hyperpietic arterial affection; upon the causation of decrescent arteriosclerosis it has little or no bearing. Huchard attributes a spread of arteriosclerosis (he did not distinguish the modes) to the increased use of meat during the nineteenth century. But we do not know that the lesion, of whatever origin, is more prevalent than in ages past ; and again, while it is true that the consumption of meat is more widespread, we do not know that individual consumption is more : it is probably less, as the culture of vegetables in Western Europe has extended enormously. Mougeot, at Royat, ${ }^{1}$ sees much of gout and atheroma attributable to excess of high feeding, especially on meat, for 20-30 years. He narrates the parallel cases of a man and wife who for years had thus fed themselves up. Such a couple I also well remember. In many earlier papers, I have argued that although gluttony, especially on a diet rich in meat and wine, seems potent, however we may explain it, to force up arterial pressures in some persons ; yet it does not so in all. It is said that in sanatorium treatment high feeding, with a gain say of $14 \mathrm{lb}$. in five weeks, raises the blood pressure by $20-25$ degrees (see p. 245). But on this statement also we need more observations. Furthermore, as I have said, under heredity the disposition to this consequence is a character not only of certain individuals, but also of certain families (p. 164). At my time of life, I repeat that I look back upon many a family in which fathers, sons, and brothers, often hearty rubicund men, men of large appetites and of great mental and muscular vigour, were obnoxious to high arterial pressures, and fell by apoplexies, or by defeat of overtaxed hearts. This habit of body, especially as due to "intemperantia in victu, et vita sedentaria," was well known to our forefathers. ${ }^{2}$ We may find it in middle-aged sedentary men, gouty, bibulous, and addicted to overfeeding; but I repeat that this series of morbid events is apt to occur also in persons of other build, aspect, and habits, in whom, if we may speak of hyperalimentation or "luxus consumption," it is only in a relative sense of these words; such sufferers may

1 Mougeot, Zentralbl. f. Herz- und Gefässkr., Oct. 1, 1913.

2 E.g. Selle, De curandis hominum morbis, Berolini, 1798. 
be, often are, quite moderate eaters and temperate in drink, as contrasted, in Wiseman's phrase, with " men of gross bodies and disorderly habits." In them the defect is qualitative rather than quantitative; in them, whether in the liver or elsewhere, there must be some flaw whereby the food, and especially its nitrogenous elements, in some way go wrong. The error may be one of katabolism, or of glandular incapacity, and so innate or self-engendered; or it may be the work of some microbe to whose machinations individuals may be peculiarly susceptible; or again, it may be due to some element of our common diet which disagrees with individuals. It seems probable then that in a person of sound health and vigorous metabolism some overfeeding may do no great harm, especially if not continuous ; but that in persons of lower visceral quality or capacity, whether by fatiguing certain departments of digestion, such as the liver, or by defect of some antidotal or antilytic substances, or by imperfect oxydation of katabolites, or again by co-operation with other causes of imperfect combustion, some of them possibly emotional, or finally by accumulation and putrefaction of waste in the bowel with resorption of toxic products-whatever the explanation may prove to be-overfeeding, or liberal feeding, especially on meat and alcohol, or indeed feeding not, as custom goes, excessive, but relatively excessive for particular individuals, sets up and maintains high arterial pressures, and this the more if the muscular exercise be insufficient. This may come about by exciting vasoconstriction in large areas, which pressures, if long continued, inflict injuries upon the coats of other vessels, and especially on the intima.

I make no attempt now to fasten exclusive attention upon any particular group of katabolites - such as the xanthine group; for our knowledge of these bodies is scarcely begun. In my opinion then, as in that of Huchard and his school, the arteriosclerosis thus produced is the consequence not the cause of high pressures, and is probably of purely mechanical origin, and owes little, or not much, to a corrosion by toxins ; but I did not, and I do not, agree with Huchard that this view covers the whole field of arteriosclerosis; there is, I repeat, a kind or kinds of arteriosclerosis, at least as numerous, in which high pressures-pressures of any considerable height-are not 
concerned, whether as cause or consequence. It is in the production of this kind of arteriosclerosis that overfeeding plays no obvious part; on the contrary, although consistent with a self-indulgent life, it is not inconsistent even with privation, being indeed itself an atrophy. That, as stated by Woods Hutchinson and others, ${ }^{1}$ arteriosclerosis, apart from kidney disease, is caused by adversity and privation, if true, is true particularly of the decrescent, not of the hyperpietic mode. Either mode may occur in abstinent, or even ascetic persons, as in the clergy, in maiden ladies of slender means, in teetotallers and vegetarians. Privation is no guarantee against hyperpiesia, yet in such persons the decrescent malady is far and away more frequent, the hyperpietic is rare. Thus hyperpietic cardioarterial disease, frequent enough in private practice, is sparsely found in hospital practice, in which field decrescent arteriosclerosis abounds.

Whether mere overfeeding, apart from a toxæmia, can raise the blood pressure, as if by a parenchymatous surfeit, is a question which, as we have seen, is as yet unanswered; but Dr. H. T. Starling of Norwich has contributed something towards an answer. ${ }^{2}$ In a sanatorium Dr. Starling made manometrical observations upon eighteen patients submitted to that gross stuffing system which for a few years had so extravagant a vogue ; simultaneous control observations were made upon nurses living under the same climatic and other general conditions. The mean age of the patients was 30 , of the nurses 29 . The results indicated that in the patients on the stuffing system the systolic pressures rose notably, averaging about $140 \mathrm{~mm}$., and ranging on the whole 10 degrees above those of the nurses. And we shall not forget that commonly in phthisis the arterial pressures range rather under than over the average. It was also remarked that this heightening of arterial pressures occurred, wholly or chiefly, during the periods of regaining weight, while presumably the nutritive system was ill-balanced; when such patients had been under treatment for some time, had done well, and had brought their weights up to the normal level, the pressures receded also to the normal.

1 Hutchinson, Woods, Brit. Med. Journ., Sept. 23, 1911.

2 Starling, H. T., Lancet, Sept. 29, 1906. 
It is convenient now, in order to set our dietetic data as free as possible from incidental interferences, to discuss certain substances which lie on the confines of diet, and mingle with it, such as alcohol and tobacco.

Alcohol is glibly assumed by many writers to be a definite cause of arteriosclerosis ; but really for some of us everything and anything seems good enough, or bad enough, to be a cause of this lesion. Now, if such a cause it be, we shall ask if it acts as a depraving influence, slowly sapping the arterial walls directly -i.e. decrescent arteriosclerosis; or if, acting as a pressor substance, it damages them consequentially by high tensions. What then is the influence, if any, of alcohol upon blood pressure? To this question it is not easy to give a plain answer. Much depends upon the dose, much again upon the mode of administration and the concurrence of other factors. Moreover in animal experiment on this question it is almost impracticable to imitate the conditions of sottish habits in man. Here again however we are met continually by the fallacy of accepting systolic pressures only. Experiments on man, or most of them, fail to record diastolic pressures. Now Raff, ${ }^{1}$ by von Recklinghausen's machine, measured the effect of doses of alcohol on man, after two hours' rest, and carefully assured himself that the circulation was little disturbed. And when drunkards on admission to hospital were deprived of alcohol, the systolic pressure rose for the first few days, and then slowly returned to its former position, the diastolic pressure being unchanged throughout. Aubertin reported that by keeping certain animals under the continuous influence of alcohol over long periods, such as two years, arterial degeneration ensued; but Fahr, who repeated the experiments, failed to verify this sequence. However, experiment has thrown some light upon the immediate effects of alcohol on the circulation; and a comparison of the testimony of expert observers indicates that, if doses of alcohol raise blood pressure at all, the rise is transient; that in the main the effect is towards lower pressures. The initial rise seems to depend upon the dose and the mode of administration. If alcohol be given by the mouth a rise of systolic pressures usually occurs at once, especially in persons not accustomed to its use. The rise

\footnotetext{
1 Raff, Deutsche Arch. f. Klin. Med. Bd. cxii. He. 3 and 4.
} 
is usually $5-15 \mathrm{~mm}$. but may reach $30 \mathrm{~mm}$. Its duration is variable-the fall may set in after intervals of 5-30 minutes; for instance, a succession of small doses may keep up the pressures for twenty or thirty minutes, while a large dose (say $50 \mathrm{~cm}$. of a 50 per cent alcoholic solution, Kochmann) may send down the arterial pressure $(10 \mathrm{~mm}$.) at once, and stimulation of a sensory nerve does not raise it. The rule seems to be that from the moment when the alcohol reaches the blood current pressures fall; thus intravenous injections of alcohol are followed promptly by fall of pressure, without preliminary rise (see p. 151). The transitory rise of pressure then may be but a momentary reflex vasoconstriction in the splanchnic area through the afferent fibres of the gastro-intestinal surface. The heart at first is retarded by the usual vagus protection against high pressure; but as by the afferent stimulation the heart seems also to be stimulated this retardation is transient, and a phase of acceleration quickly succeeds it. In like manner the vasoconstriction is transient, and soon gives way to vasodilatation, at any rate in peripheral areas, and to such an extent as to mask and override any areas of persisting constriction; so that low systemic pressures prevail. On the whole then resistance falls: although pressures and velocities are in inconstant relations yet, the widening of channels being compensated more or less by an accelerated heart beat, blood velocity is promoted $;^{1}$ and this is the rule during the first stage of the "cordial," till by the drug the heart is enfeebled and pressures sink. Thus, although perfusion of a heart with fractional parts of alcohol accelerates the flow, if the doses are increased the heart is soon poisoned. It would seem therefore that if alcohol is a cause of arteriosclerosis this is not by way of excessive arterial tension. Lancereaux's careful experiments also were negative.

And if we turn to the clinical side of the enquiry we shall find no stronger evidence to connect alcohol with arteriosclerosis. Mitchell Bruce (loc. cit.), James Barr, ${ }^{2}$ and Cabot ${ }^{3}$ agree with

1 For experimental facts see, among other papers, Dennig and others, “Alc. u. Blutdk.," Deutsche Arch.f. klin. Med. Bd. xcvi. He. 1 and 2; Kochmann, Deutsche med. Wochenschr., 1905, No. 24 ; Brooks, "Alcohol and Blood Pressure," Journ. Amer. Assoc., July 30, 1910 ; Wood and Hoyt, Univ. Penn. Bull. xviii. 70.

${ }^{2}$ Barr, Sir J., Brit. Med. Journ., July 1, 1905.

3 Cabot, Amer. Journ. Med. Sci., 1904, vol. xliii. p. 774. 
Crile and other clinical observers in a negative opinion. Cabot, from his valuable statistical tables, reports that, if we select drunkards under 50 years of age, arterial deterioration will be found only in about 6 per cent; taking all drunkards the prevalence was 50 per cent: and conversely, in well-marked cases of arteriosclerosis he found no notable prevalence of abuse of alcohol. Fahr (loc. cit.), in 309 necropsies on drunkards in the harbour Hospital at Hamburg, found the incidence of arteriosclerosis to be no more than normal. Brault pointed out that the incidence of arteriosclerosis in cases of cirrhosis of the liver was not conspicuous. In topers of all ages Dr. Bruce ${ }^{1}$ found thickening of the radial artery only in one-third. Dr. Mott's opinion is that arteriosclerosis is not a frequent feature in the alcoholic cases of general hospitals, and Dr. Andrewes agrees with him (loc. cit.). Max Herz of Vienna sent out a circular to Austro-Hungarian physicians inviting their opinion as to certain alleged causes of arteriosclerosis : ${ }^{2}$ he received 822 useful replies. In such a crowd of witnesses great allowance has to be made for the various spheres of practice, and for personal prejudices and impressions; but some points seem to come out saliently. Alcohol ranked in the third column, not very high. The vineyard folk, who drink heavily, and the peasantry, who consume much spirit, for even the children drink brandy, proved not to be especially liable to arteriosclerosis. Gluttony too came out low; but the mass of the people cannot attain to gluttony, and the returns did indicate a prevalence of arteriosclerosis in the trading classes. For many years I have pointed out to students how commonly one finds in cases of alcoholic cirrhosis-even "potatores (as Wepfer says) qui tanquam gurgites et voragines de poculorum magnitudine certent"- that the accessible arteries are quite soft, and the blood pressures by no means high. And after death, as many other observers also testify, little is to be found of lesion in the main arteries but trifling spots and superficial patches of fatty degeneration. Therefore Aubertin's explanation that alcohol causes arterial disease indirectly by exciting the

1 Bruce, M., Lancet, March 23, 1901.

2 Herz, Max, “ ̈̈tiol. d. Arteriensklerose," Wiener klin. Wochenschr. No. $44,1911$. 
adrenals is not wanted. Conversely, as I have remarked before, the ancient Egyptians and Orientals, as we learn from investigators in these lands (Elliott Smith, Ruffer, and others), peoples whom we suppose to have been for ages sparing both in the use of alcohol and of animal food, were, as Orientals still are, very liable to extensive and even precocious decrescent arteriosclerosis. During a Mussulman pilgrimage, Dr. Ruffer made more than 800 necropsies on "persons who certainly had never touched alcohol in their lives." $\mathrm{He}$ found in them arterial disease as frequent and as early as in persons who observe no such abstinence. We cannot then follow Huchard in his confident assertion that alcohol causes arterial disease by setting up constriction of the smaller arteries ; nor, in respect of copious bibbers, can we agree with him and Traube that increase of blood mass, however injurious it may be, raises arterial pressures (see p. 44). In alcoholism the plethora is rather on the venous side; and, if drunkenness be associated with sedentary habits, the veins lose their main motor, the voluntary muscular machine.

Alcohol then is not "a cause in eminent domain"; but it is a potent ally of any other poison which may be in co-operation with it. Vaquez thinks that alcohol conspires with other causes to injure the heart and vessels of heavy beer drinkers. The beer drinker, again, suffers from doses of arsenical poisoning which through the healthy man would pass nearly or altogether harmlessly; the whisky drinker suffers from traces of lead in the tap water which he adds to his glass, but which leave other consumers of the same water unscathed. So likewise Thayer and Brush ${ }^{1}$ observed that under alcoholic habits the effects on the limb arteries of physical labour, and again, more widely, of typhoid fever, were greatly multiplied. This is decrescent arteriosclerosis, or it may be chiefly medial and of the limbs. And, on the facts of experience, I think that greedy feeders, if to a rich meat diet they add an abundance of wine or other alcoholic drink, are more liable to " high tension" than gormandising teetotallers; indeed in recent years many gross feeders have turned to moderation in alcohol, some are virtually or even strict abstainers.

1 Thayer and Brush, Amer. Journ. Med. Sci., 1904. 
Tobacco, as glibly as alcohol, is declared to be a cause of arteriosclerosis, especially by Huchard and his school, who have erected "Tabagism" into a serious and polyphasic malady; though on a slender foundation. Cohnheim disbelieved in the alleged sclerosing effects of either tobacco or alcohol. ${ }^{1}$ In Germany Traube, who seems to have been the first witness against tobacco, and after him Erb, von Schrötter, and Klemperer have looked upon tobacco as a cause of arterial degeneration. Ortner thinks it injures preferentially the abdominal vessels. No doubt we meet with many smokers profoundly affected with arteriosclerosis; smokers are many, especially in German circles; and, as arteriosclerotics also are many, the normal coincidence must be a large one. But the frequency of arteriosclerosis in elderly women is sufficient to make us pause in so facile a connection of cause and effect. Moreover tobacco, if it be much of a poison, is a very slow poison, at any rate to most persons ; so that its effects, being mingled with the other conditions of senility, are almost impossible of discrimination. The late Dr. Dixon Mann, ${ }^{2}$ a careful and thoughtful observer whose death we are now lamenting, collected many opinions to the effect that the use of tobacco resulted in arteriosclerosis; but he was not less ready to admit that proof was very difficult. Edgren was of opinion that tobacco had no permanent effect on the vessels. Dr. Mitchell Bruce ${ }^{3}$ says that, apt as is tobacco to disorder the heart, even gravely, "it does not, in my experience, produce either enlargement of the heart (i.e. no abiding high pressures-C. A.) or thickening of the arteries." Rénon holds the same opinion, but less decisively. Dr. George Oliver ${ }^{4}$ is unable to say that arteriosclerosis is "more frequent in habitual smokers than in non-smokers; and in some great smokers (he) had failed to discover any evidence of this disease." Lancereaux $^{5}$ (p. 247) excluded from the list of causes both alcohol and tobacco.

The researches on nicotine by Professor Langley and his pupils indicate that the drug produces vasoconstriction and rises of

1 Cohnheim, Syd. Soc. Edn., vol. i. p. 67.

2 Mann, D., Brit. Med. Journ., Dec. 5, 1908.

${ }^{3}$ Bruce, M., loc. cit. Luml. Lect.

4 Oliver, Clin. Journ., Sept. 16, 1908.

s Lancereaux, Méd. Soc., Paris, June 1908. 
pressure; John ${ }^{1}$ makes the important statement that it does raise diastolic pressures. It is said to act as adrenin, but the experis mental doses are far larger than a smoker's dose. Adler and Hensel ${ }^{2}$ found that the effects of injections of nicotine (taking this, as is now supposed by Professor Dixon and most observers, to be the injurious constituent of tobacco smoke) in rabbits varied widely in different individuals. In some the wall of the aorta was attenuated, and calcareous deposit abundant; even so much that the vessel would become pouched on its posterior or lateral aspect. The chalk deposits began as minute points in the media, and the authors detected the first lesion in the muscle cells of this coat, the intima being difficult to see and describe. We can scarcely expect, as I have said, that experiments on animals can help us much in questions of inveterate habit. But, if tobacco be a cause of arteriosclerosis at all, it is, as clinical observation of many copious smokers will tell us, not in the consequential mode due to hyperpiesis but in the decrescent form. Conversely, in many oldish inveterate smokers I have found the arteries accessible to palpation to be normal. Smoke as a man may, it is not alleged that he will fall into an arteriosclerosis before his later life, yet, if tobacco is to damage the vessels, surely a quarter of a century's use-say at æt. 45-should suffice; but do we in smokers find arteriosclerosis thus antedated? Professor Dixon has proved that in the habitual smoker an antibody is generated, and some users of tobacco may be less capable of engendering it; but in this case surely they would be disposed to smoke less, or not at all.

Workers in tobacco have, I believe, been examined from this point of view, but I think without definice results. Hensen did indeed relate an important case in a tobacco worker aged 25, which was attributed to his occupation. He presented well-marked arteriosclerosis and a dilated aorta. The pulse was irregular and the heart's apex a little out to the left. No other cause was known, but the evidence of his previous condition, for instance as to the possibility of syphilis, was inadequate. In Herz's returns (loc. cit.) those from the tobacco trades

1 John, M., “ Beeinfl. d. Blutdrucks durch Tabakrauchen," Zeitschr. f. exp. Path. u. Ther. xiv, 1913.

2 Adler and Hensel, New York, Journ. Med. Res., Sept. 1906. 
manifested no extraordinary prevalence of arteriosclerosis. Dr. Parkes Weber, ${ }^{1}$ it is true, speaks of " obliterative arteritis," not syphilitic, as prone to occur in poor Russian Jews, many of them engaged in tobacco factories, who smoke cigarettes ad libitum. The ancient Egyptians (p. 249) presumably did not smoke.

Obesity and high pressure have little in common, although in diabetes a low pressure arteriosclerosis is frequent, even in the young. As both hyperpiesia and obesity may have their origin in gluttony, so they are apt to meet in the same person; on the other hand, as von Basch observed at Marienbad, many fat old persons have quite soft arteries and pulses; their hearts, like the rest of their muscles, tend rather to want of tone, to flaccidity and deterioration, than to hypertrophy. There is some inconstant and unknown variable. Fat people are of two main kinds: the hereditary, often flabby and rather anæmic, soon tired; and the big "malmsey-nosed" feeders, who have abundant blood and often, if not hampered by fat, great energy ; the blood pressures of these persons do often run above normal.

Cholestercemia.-The presence of doubly refracting (anisotropic) fats in the products of atheroma has long been well known; but recently it has been suggested that cholesterin is no accidental element in the process. It is suggested that cholesterin is essentially concerned with the fatty streaks and spots in the vessels after certain infections, after typhoid fever for instance; and it is asked if these are strictly atheromatous? It is said that they have not the connective hyperplasia of atheroma; and again, that in young persons they present swollen but not degenerated cells of the intima. Some suppose that the relations of cholesterin to the vessel walls is altered so that this substance is deposited passively in the arterial tissue, while arteriosclerosis is a more chronic disorder of cholesterin metabolism. Others again take the broader view that the fatty spots of the infections are atheromatous, and that these and ordinary atheroma are due not to wear and tear and strain, but to the cholesteræmia. ${ }^{2}$ Wacker and Hueck gave pure cholesterin to rabbits--15 grms.

1 Weber, Parkes, Lancet, Jan. 18, 1908.

${ }^{2}$ See, for instance, Wacker u. Hueck, of the Munich Path. Inst., in Münch. med. Wochenschr., Sept. 23, 1913 ; also Zinserling, Zeitschr. f. Pathol. Bd. xxiv. H. 14, quoted Deutsche med. Wochenschr., 1913, pp. 1695-6. 
cholesterin in 3000 grms. of oatmeal-and say that thus they obtained true atherosclerosis in these animals; also that two Russian physicians, whom they quote, obtained it independently in the same experimental way. They also administered certain toxins whereby they obtained cholesteræmia, and in five months atheroma. Besides free cholesterin in the blood they found much more as esters in the serum, and as a cholesteatosis of the liver. The adrenals are said, in the cortex, to be always rich in cholesterin; moreover that injections of adrenin produce excess of cholesterin in the blood, and excision of these organs a deficiency; and that injury of the sympathetic (chromaffin system ?) produces a local cholesteatosis. They think that muscular activity influences cholesterin metabolism, and, as to connective hyperplasia, that its degree is a matter of reaction in durations of time and stress. They go farther still, to say that dyspnea is not without association with cholesteræmia.

However Cantieri ${ }^{1}$ did not find any constant relation between cholesteræmia and arterial disease; that in arteriosclerosis, whether with excess of pressures or not, cholesterin might be present in the blood or absent, and that in the serum there are great fluctuations from one case to another, or even in the same person; the quantities, in the whole or the sick, depending on diet. In nephritis however he found cholesteræmia usual, even if without high pressures; and otherwise that there was no relation between pressures and cholesterin. On feeding man with cholesterin no rise of pressure appeared.

Saltykow, ${ }^{2}$ still later in date, published an elaborate paper based on experiment on rabbits. He began by stating that the rabbit's aorta is capable of true atheroma, by way of a very tenuous layer of connective tissue under the endothelium, which may undergo hyperplasia, and show sudanophile granules and larger fatty particles. Saltykow found that to obtain definite results fat must be fed to the rabbits with proteid; he used milk, which contains cholesterin; this he thinks is carried ("fats and doubly refracting lipoids") by the vasa vasorum to the aorta. The cholesterin is then deposited, and about the deposit a secondary reactive process takes place. In remarkable consent

1 Cantieri, Wien. klin. Wochenschr. vol. xxvi. p. 42, 1913.

2 Saltykow, Ziegler's Beiträge, Bd. lvii. H. 3, Jan. 20, 1914. 
with other observers Anitschkow ${ }^{1}$ also reports identical results in the aorta of rabbits fed with yolk of egg and brain substance, and affirms that the aortic lesion thus produced is not "of the adrenalin type," but is morphologically and microchemically identical with human atherosclerosis. Saltykow however admits that many co-operative factors are concerned in arteriosclerosis, and that in man a diet rich in cholesterin would not suffice to produce it. Anitschkow says the doses given in experiment are enormous, but that small doses suffice if the blood pressure be also forced up. The Russian observers, and Steinbiss, regard arteriosclerosis simply as a disturbance of metabolism, and Faber is not convinced of any reactive phase ; for my part, I think in arteriosclerosis it is impossible to overlook a variable but often decisive connective hyperplasia. In Saltykow's experiments the media always participated more or less.

The atheromatous artery contains much more cholesterine than the normal vessel-up to $16-17$ per cent more; the normal aorta should contain only about two parts per thousand. But it seems that neither in arteriosclerosis nor in hyperpiesis is there any proved excess of cholesterin in the blood, unless the kidneys are failing, when large accumulations of it may occur (with, I suppose, an excess of hæmolysis ?). Cholesterin has not the escape into the tissues which urea and salt have. Lemoine ${ }^{2}$ thinks that if cholesterin, being slightly soluble, is deposited as "plâques d'athérome," and causes arteriosclerosis, we should try to dissolve it away: as some say of calcification (p. 505). In the blood the normal cholesterin content is very small; in the several tissues the content is various. In old persons the blood contains not more cholesterin but less. ${ }^{3}$ There is no direct relation between rise of blood pressure and the amount of cholesterin in the blood, save, as I have said, in renal disease, when the relation seems to be not of high pressure but of defective excretion. ${ }^{4}$ The liver, not the suprarenals, regulates cholesterin metabolism (Aschoff, reported to me by Dr. M'Nee).

1 Anitschkow, N., Ziegler's Beiträge, Bd. lvi., 1913; and Deutsche med. Wochenschr., Jun. 11, 1914.

2 Lemoine, Gaz. des prat., Sept. 1, 1913, quoted in other journals. Also Bull. de la Soc. Méd. des Hôp., 1912, No. 28.

${ }^{3}$ See Linossier, Arch. des maladies de lapparat digestif, etc., mai 1912. Quoted Arch. des maladies du cœur, oct. 1913.

${ }^{4}$ Cantieri, C., loc. cit. The following papers also, among many others, may 
Plethora.-But, before we can appreciate perversions of metabolism and consequent toxic issues, we must enquire if there be such a condition as a true plethora, a considerable and an abiding excess of blood mass (p. 44); and, if so, whether or no mere repletion can mechanically strain its vascular continent. In my first papers on the subject ${ }^{1}$ I assumed, a little carelessly, that such a condition might grow, and by distention might strain the cardio-arterial tree. But afterwards I remembered that Cohnheim and Roy had said that a " plethora vera," save as a transient modulation, cannot be, or cannot persist ; that any such excess, as, for instance, in experimental hydræmia, is promptly shed off by the urine. Krehl however contested both this opinion and the validity of the experiments, good up to a certain point only, on which it was based. There is some evidence that when with a bulky hydræmic blood all the tissues are saturated, and the kidneys are torpid, so that albumin may appear in the urine, blood pressures, even if already high, may be forced up. Anæmia and cyanosis alike irritate the vasomotor centre, but in anæmia vascular friction is less. This point is considered also in my chapter on Viscosity (p. 148). And we must remember that the capacity of the whole vascular system is very great, perhaps 25 per cent larger than its mean volume. As the bulk of the blood increases the veins fill; and conversely. On injection, say of defibrinated blood, the rise of arterial pressure is transient; but the venous pressure rises so long as the injection is continued; so that if the heart keep up, the velocity must increase. As the vessels expand to receive the excess of fluid the peripheral resistance falls. Bollinger and his disciples urged that fluid surfeit was not only possible, but, as in beer drinkers, not rarely reached so high a mass as to tax the heart and vessels beyond their capacity. In these patients however, by lowering the tone and quality of the myocardium, the alcohol co-operates. Vaquez, in his description of Polycythæmia rubra, emphasised the impression of the clinical observers (e.g. of Bollinger and others) that a "plethora vera" may become established. The

be indicated : Dorée, Ellis, Fraser, Gardner, Proc. Roy. Soc., various papers, 1908-12; M'Nee, J. W., good summary, Quart. Journ. Med., April 1914, and letters to me, July 1914 ; Elliott, T. R., Adr. Cortex (ibid., Oct. 1914, pp. 72 and 83). Grigant, many papers, chiefly chemical, C. rendus Soc. biol., 1913.

1 Hunterian Soc., 1894, and Lane Lectures, 1896. 
late Professor Senator, ${ }^{1}$ who opened a discussion on this subject at the Buda-Pest Congress in 1909, convinced himself, by the plethysmographic and the Haldane-Smith methods, ${ }^{2}$ that a plethora vera does happen; and not only in Polycythæmia rubra and in "Geisböck's disease "-which I may briefly indicate here as P. rubra without enlargement of the spleen-but also with enhanced blood pressures and hypertrophy of the left ventricle. Lymphoid metaplasia of the marrow, as it does not concern my argument, I omit. Geisböck's paper ${ }^{3}$ contains a very interesting table of 18 cases, in which the red corpuscles ranged from six to eight millions or more. He found no regular parallel between the number of corpuscles and the blood pressures; indeed, we know that in the Vaquez-Osler (splenic) cases the red cells may run over ten millions, still with a normal blood pressure and heart volume; yet in many of his own cases Geisböck did, in fact, find the arterial pressures excessive, and the pulse, though usually regular, prone to extra-systoles. Lucas ${ }^{4}$ says the blood pressure is raised in about one-third of the cases of erythræmia. In 50 per cent (of all the cases) traces of albumin, more or less slight, were noted. It is scarcely out of place to digress for a moment to point out how like to my class of Hyperpiesia are some of the cases on Geisböck's list. Take this, for instance :

Male aet. 54. Red faced. Blood pressure $=200-210$. Dizziness and headache. On venesection blood pressure fell to 156 , and two days later it was 150. Nitrites reduced it still farther, ultimately to 120. Red cells however, ten days after bleeding, still ten million; whites 9680. Urine normal. The symptoms, including rise of pressures, returned; but a second bleeding, and this from the radial artery, relieved the condition greatly, and for ten days the blood pressures ranged about 120 . But again they rose, and the patient grew worse for three months, when diarrhœa set in, on which pressures moderated to 120 ; much improvement then followed and continued, so that the man returned to work. When the radial artery was opened its coats seemed normal.

\footnotetext{
1 Senator, Polyzythämie und Plethora, Berlin, 1911.

2 Dreyer, Ray and Walker (Dept. Path., Oxford, 1913) are not sure that the $\mathrm{CO}_{2}$ method is trustworthy, as the results are inconsistent; and they give other reasons. They find that blood volume is normally proportionate not to body weight but to body surface.

${ }_{3}^{3}$ Geisböck, Deutsche Arch. f. klin. Med. vol. Ixxxiii. (1905) p. 396.

4 Lucas, Arch. of int. Med. vol. x. p. 567, 1912. Quoted Janeway.
} 
In Dr. Hale White's cases 1 " the blood pressures were normal," which was remarkable considering the volume of the blood (said to be three times the normal, the red cells double the normal, and the whites also in excess. Stähelin, ${ }^{2}$ under the title of Polycythæmia hypertonica records seven cases in which the blood pressures were over 200. In none of these was the spleen enlarged. Geisböck's variety of the disease seems more prone to high pressures; unfortunately in Hyperpiesia I have made no corpuscular counts. (See however p. 308 note).

In the next place, let me report certain evidence which $\mathrm{Hart}^{3}$ produces from the side of morbid anatomy. The author says that in the corpses of certain persons, at an age of full vigour, he finds an obese condition, but powerful muscles therewith, and a strong bony frame. There is œdema, but the body is charged throughout, even the arteries also, with an overflowing dark violet blood. Both right and left ventricles are enlarged in muscle and in capacity, a condition for which no ordinary cause may be found. The kidneys likewise, though structurally normal, are hypertrophied. The spleen is full of blood and somewhat tough, but not otherwise altered. The systemic arteries, beyond an increased capacity, present little or no change; but the pulmonary artery up to its finest ramifications is affected with atherosclerosis. In one case the pulmonary artery and its branches, though much dilated, were not atheromatous. The marrow of the long bones presents a lymphoid hyperplasia. Hart pertinently compares the great capacity of the systemic arterial system, whereby it can adapt itself to variations of blood mass, with the pulmonary arterial system, which is not endowed with the same vasomotor capacity, so compensation of excessive friction can be met only by the capacity of the lungs. He also emphasises, what other observers have recently shown, the wide limits of distensibility possessed by the normal aorta. But, says Hart, on the aortic side the increased diameter is not obtained at the expense of resiliency, it seems to be a true development; the vessel is not only enlarged in lumen, but still, age for age,

1 White, W. Hale, Lancet, Jan. 6, 1912. The blood volumes were measured by Dr. Gordon Douglas on Haldane and Smith's method.

2 Stähelin, Berl. klin. Wochenschr., Jan. 16, 1911.

3 Hart, Deutsche med. Wochenschr. No. 17, April 25, 1912. I am much indebted to Hart's paper.

vOL. I 
possesses no less than its ordinary elasticity; and from the aorta a guess may be made as to a cognate development in the whole tree, or at any rate in the vessels of greater magnitudes. These independent observations of a morbid anatomist are of great interest in explaining how in the presence of high blood pressure and cardiac hypertrophy the aorta may be free from disease, an apparent anomaly which, in the younger subjects of chronic Bright's disease has often arrested my attention on the post-mortem table; and to this I think all observant pathologists can bear witness. Strasburger ${ }^{1}$ also has recently described this quasi-normal hypertrophy of the aorta under such and similar conditions. In one of Strasburger's cases, a huge man weighing $26 \frac{1}{2}$ stones, the aorta, when submitted to $40-240 \mathrm{~mm}$. of $\mathrm{Hg}$ pressure, showed a distensibility of $86.0 \mathrm{c.cm}$. At a pressure of $80-160$ its diameter was 28.5 c.cm., that is, its tensile limits were 74-78 per cent wider than in the average man! In like manner, Strasburger demonstrated that, age for age, the aorta of woman has narrower tensile limits than that of man; and in woman accordingly the mean blood pressure averages ten $\mathrm{mm}$. lower than in man, the minute-volume of cardiac output being so much the less. Hart adds that there is no direct relation between the size of the aorta and the weight or height of the body; but Dreyer and Ainley Walker ${ }^{2}$ have shown since that its sectional area varies as the bodily surface. In the cases of our text Hart suggests that the blood vascular increase may be correlated with the excessive activity of the bone marrow; and that, accidents such as thrombosis apart, life is imperilled not so much by the state of the blood itself as by the ultimate failure of the circulatory system which it entails. In advanced life, of course, this limit of cardiorenal capacity is reached the sooner. Like the physiologists however he is indisposed for several reasons to assume that a mere aqueous increase of blood volume can thus persist; one of his reasons is that in diabetes insipidus large volumes of water are drunk and got rid of; but here, as in diabetes mellitus, the diabetes probably precedes the thirst and imbibition.

1 Quoted by Hart without a reference, but probably with all the conclusive paragraphs.

2 Dreyer and Walker, Journ. of Physiol., June 12, 1912. 
Much of this balance must depend on the function of the kidneys (p. 144).

Hess ${ }^{1}$ by careful management maintained an experimental plethora, with some increase of viscosity, for more than two months, ${ }^{2}$ and found the arterial pressures still unaltered, and the heart not enlarged. Presumably in this experiment the work of the heart was not increased, for the duration of the blood circuit may be considerably lengthened, and in the splanchnic vessels amount almost to stasis. The vast potations of some beer drinkers seem more to the point, and Hess speculates on the possibility that alcohol may stimulate the bone marrow to over-production. As to this I can only say that in the two, perhaps three, cases of $\mathrm{P}$. rubra which I have seen, alcoholic excess was certainly out of the question, and indulgence at table not probable. It may be that between serum gravity and corpuscular richness there is some mutual balance, or satisfaction. But Hart himself frankly says that having searched the long bone marrow of drunkards for abnormal features, he found those changes only which Bleichröder pointed out as pertaining to cirrhosis of the liver. The only cause which commends itself to him is gluttony, an antecedent which, in some at least of the cases he has collected, certainly existed ; and he supposes that Bollinger's beer heart-which, with Senator and others, he accepts as accurate observation-is the product rather of excess of the alcohol and other feeding substances in solution than of the aqueous elements. Unfortunately Bollinger in his cases made no blood counts. That meals rich in nitrogenous constituents do produce a physiological polycythæmia, a true, if a transient, plethora, is known ; and possibly this, at any rate in persons of a certain, perhaps hereditary, habit, may, under industrious feeding and excessive demand for oxygen, issue in an abiding "plethora vera." Dr. Haldane in a private letter to me (dated July 21, 1907) says that although in health blood mass does not vary more than 10-15 per cent in either direction, in many morbid conditions it may be permanently increased or diminished. Dr. H. D. MacCulloch (also in a private letter) suggests that blood platelets are

1 Hess, Deutsche Arch. f. klin. Med. vol. xcv., 1909.

${ }^{2}$ Vide chapter on Viscosity, p. 142, etc. 
"amputations" by vasoconstriction, and if so should be more abundant in pressor conditions: and that an accumulation of these might aggravate the arterial pressure.

I have seen it stated that in polycythæmia the dicrotic wave is wholly suppressed; if this prove to be true it will be very interesting, as we all know how this wave is exaggerated after hæmorrhage. There are on record, if I remember right, some experiments by yon Frey which showed that waves on the descending limb of the sphygmogram vary inversely as the specific gravity of the circulating fluid. Guinea-pigs are said to show no dicrotic wave.

I have not hesitated to dwell at length upon this part of the subject, because I think by this way some important light may come. Hart regrets the lack of clinical data, or at any rate of clinical data in coordination with the pathological; and here I confess a fault of my own. The cases which I have called Hyperpiesia may be of the same kind as those, or some of those, described by Geisböck, cases, that is, of "plethora vera" with polycythæmia, hypertrophied heart, and high arterial pressure, but without the splenic enlargement of the Vaquez cases; and I have to confess to the omission of blood counts. If hereafter we find in them an excess of red cells, my hyperpiesia cases may rank with those of Geisböck (but see note, p. 308). Polycythæmia rubra in which the spleen is enlarged, but in which the heart is not enlarged, is a rarer disease, and falls into another category. As I write (in July 1912), a woman of about 50 with an enlarged spleen and eleven million reds is under the care of Mr. Arthur Cooke of Cambridge; although her skin is deeply and universally reddened, and her very large, slightly thickened, curved radial (and other accessible) arteries stand out visibly, chokeful of blood, yet I cannot satisfy myself that her heart is hypertrophied, and her blood pressure runs at about 120. Here the finger estimate was very deceptive, for to the touch the blood pressure seemed very high. Monro and Teacher ${ }^{1}$ have published an interesting paper based on three cases. In one, a mixed case with enlargement of the spleen, the pressures ranged from 180-200 mm., and the radials were thick. The kidneys were smooth on the surface;

${ }^{1}$ Monro and Teacher, Lancet, April 12, 1913. 
the cortex and glomeruli were diminished, but there was much healthy kidney intact, and the reduction was attributed by the authors to vascular occlusion. No syphilis: Wa. R. negative.

Autogenetic Toxins.-Let us now proceed to consider certain toxic substances supposed to be generated within the body by some perversion or defect of its own metabolism. ${ }^{1}$ In one sense we have heard, especially in France, a little too much of them, for their very existence is as yet largely hypothetical; we are not as yet justified in making use of them, in explanation and in therapeutical doctrine, as if the processes were definite, and the substances familiar. Yet we meet with page after page of such guesswork as "due to a faulty metabolism giving rise to auto-intoxication" - a sentence which in the present state of our knowledge is mere verbiage, or a kind of talk which bears no scrutiny. Moreover, all these speculations are confused, if not vitiated, by the lack of discrimination between the two modes of arteriosclerosis - the mode with high pressures, and consequential upon them, and the primary mode, without high pressures. We shall do well to start from principles such as these, and then tentatively to seek for some firm ground beyond. One eminent author, beginning with the notion that all arteriosclerosis means high pressures, attributes these primarily to the action of a toxin or toxins which, irritating the tissues, provoke a wasteful metabolism, and thereupon a call for larger supplies of blood, so that the capacity of the heart is increased; and so on. Others argue about bacterial decomposition in the bowels with production or disengagement of poisonous or toxic substances hence, continually or fitfully, absorbed into the body. Others again believe that the fault lies with some organ, such as the liver, which fails to purify the products, or prevent the perversion, of normal metabolism.

In respect of overfeeding, it is said that the chemical affinities of the tissue cells are strongest for proteins, less for carbohydrates, least for fats; and it is suggested accordingly that order in the issues of proteins, whether as concerns excess or perversion, is more important to the body than in the issues of carbohydrates and fat. It is therefore in the field of protein

1 See Dr. Cowan's careful article in the Practitioner for Aug. 1905, with historical notes; and Simnitzky, Zeitschr. f. Heilk. vol. xxiv. 
physiology chiefly that observers have engaged in the search for bodies, hypertensive or poisonous, apt to be injurious to arterial tissues. The great difficulty of the research is that the bodily tissues and fluids on the one hand, as for instance the serum, and on the other the excretions, such as the urine and fæces, are, as wholes, too complex for experiment; and of their constituents some at any rate, and these perhaps the more virulent, are so unstable as to elude precise separation and tests. The blood serum, at first sight a promising field for search, is so subtly constituted as to be almost beyond the reach of physical chemistry. ${ }^{1}$ No pressor substances have been found as yet in the cerebrospinal fluids of apoplectic and other high pressure cases. Metchnikoff has stated that the mucosa of the intestine is capable of absorbing the toxins produced by three chief putrefactive anaerobes in the human intestine; but agglutination and other tests for the influence of $\mathrm{B}$. coli in the blood have so far proved negative. ${ }^{2}$ The emphasis which Metchnikoff has laid upon the colon as the probable seat of diffusible toxins is well known, but we are still on very uncertain ground. Probably the lower end of the ileum is more to be suspected. Roger and Garnier, ${ }^{3}$ in experiments which seem to have been carefully planned, made extracts of the intestinal contents at several levels; these extracts they injected intravenously into dogs and rabbits. Extracts from the large intestine, unless it were those from the cæcum, proved much less injurious; those from higher levels more virulent. A milk diet reduced their toxicity, but did not neutralise it; to pass them through the liver or lung also moderated their activity, so much so that the lethal dose of the extracts injected into the portal was three times that of the extracts injected into a peripheral vein. The toxins were apparently albuminoid, and very unstable. But in these mainly biochemical experiments the factor of arterial pressures was not dealt with. Lubarsch reported that feeding rabbits for some time on liver produced the well-known aortic degeneration of these animals, as under high arterial pressure. Some later observers, as von Leersum, have failed to verify these vascular

1 Vide Manwaring, Rockefeller Inst. Rpts., 1907.

2 Goodall, Lancet, Sept. 30, 1911.

3 Roger and Garnier, Rev. de méd., Aug. 10, 1906. 
changes, but von Leersum agrees that a very prolonged course of this food (e.g. dried and powdered liver) raises the blood pressure (p. 235), ${ }^{1}$ and he found the heart in these rabbits much hypertrophied. Stuckey, ${ }^{2}$ by a rich animal diet-flesh juice, milk, eggs-says he produced in rabbits an intimal atheroma of the aorta with but slight changes in the media, a form identical with the human. Fahr of Mannheim (an authority on this subject has reported ${ }^{3}$ likewise that he had fed rabbits on animal diet, and found consequential degenerative changes in the intima; and subsequently a degenerative enlargement, not a hypertrophy, of the adrenal bodies, both arteries and adrenals deteriorating together. $\mathrm{He}$ attributed the degeneration in the aorta to causes both toxic and mechanical. Steinbiss of Düsseldorf, reports ${ }^{4}$ that in such rabbits as can be kept alive, say for three months, on albuminous food, with as little fat as possible ("Eiweissnahrung") this diet causes glycosuria and disease of the aorta, and peripheral arteries, identical with the effects of adrenin injection; and this the more surely the longer the survival. Many died, but some survived; one to 121 days. The finer peripheral vessels also were sclerosed, especially the renal (see p. 351), but not so those of intermediate magnitudes. A very small addition of vegetable food prolongs life indefinitely, and the aortic changes come on gradually, as yellow spots and streaks, resembling more closely the intimal type in man, as contrasted with the acute adrenin type of the rabbit. However such a diet is so wholly alien to the rabbit's constitution that these experiments can scarcely be taken as crucial.

Still, that the daily use of animal flesh favours the growth of bacteria in the large bowel is generally admitted. Repeated bacteriological analyses of the fæces, as carried out at the Battle Creek Sanatorium, show that on a change from a flesh to a nonflesh diet the number of intestinal bacteria are very markedly diminished, and the benefits of this change are speedily reflected

1 See von Leersum, “Alimentare Blutdrucksteigerung," Zeitschr. f. exp. Path. und Ther. Bd. xi. H. 3, p. 409, 1912.

2 Stuckey, article quoted in Centralbl. f. Herz- u. Gefässkrankheiten, Dec. 1912, p. 399.

3 Fahr, Deutsche path. Gesellsch. zu Strassburg, April 1912.

4 Steinbiss, Virchow's Arch. Bd. cexii. Heft 1, 1913. 
in the feelings and progress of the patient. Spa physicians of experience will probably admit that a large part of their success in dealing with gouty and rheumatic disorders is due, not only to the baths, massage, electrical and other methods, but in great measure also to the substantial diminution in the daily quantities of animal food.

Now animal extracts as a class (unless, of course, taken from peculiar parts, such as certain of the inward working glands) are hypotensive (cf. Popielski's vaso-dilatin in the mucosa of the alimentary canal. Stewart ${ }^{1}$ however, who made many experiments with fæces, taken both from normal men and from men suffering under high blood pressures, which he injected into dogs, concluded that in some of them pressor substances occurred; but, as I have said, fæces are much too complex for more than rough appreciations. Moreover such pressor constituents as may thus have betrayed themselves were apparently not very potent, were not in any degree comparable with adrenalin for instance. On the other hand, it is true that an amine of intestinal putrefaction separated by Barger and Dale ${ }^{2}$ causes intense bronchial spasm, and strong contractions of the uterus. These authors aptly reflect that such effects of this amine, a poison rather than a toxin, may depend not on side chain action, but on physical or physico-chemical reactions; on solubility, on quality of cell membrane, on degree of access to responsive cell constituents, on adsorption, and so forth; but it dilates the arterioles and so reduces blood pressure. Barger thinks this is Popielski's vasodilatin, This differential action on the histidine base is very curious. Dosage is of course a factor of importance; for certain agents, in small doses stimulant, are in larger doses depressive. Possibly in minute doses all protoplasmic poisons may be primarily stimulant.

Bacteriological observations seem as yet no less in the haze. ${ }^{3}$ Some states of disorder, such as butyric fermentation, states marked locally by gas in the bowels, and constitutionally by hæmolysis, are associated with $\mathrm{B}$. äerogenes capsulatus rather

1 Stewart, G. N., Journ. Exp. Med., 1911.

2 Barger and Dale, Journ. of Physiol., 1910.

3 Vide Harter, Journ. Biol. Chem., Aug. 1906. 
than with B. coli ; in other cases streptococci are the prevailing organisms, while a third kind of disorder is indicated by its products, as of the indol series. In these three kinds of intestinal disorder there seems to be no great likelihood of extensive vasoconstrictive action, and therewith of high pressures; though they may, some of them, engender toxins of a kind to prey upon organised tissues, such as the arterial tunics. This evil attribute, as we shall see presently, not a few specific infections possess. But the less specific kinds, if they do interfere with the arteries, are more likely insidiously to cooperate in the sum of effects leading to the decrescent kind of arteriosclerosis; for this reason it is that $I$ have not called it " senile," for thus it may occur, and often does occur, before old age. Work upon the intestinal anaerobes is in progress, but as yet no definite conclusions have been published. At the Swiss Medical Association Meeting at St. Gall in 1911, "Senile arteriosclerosis" was attributed to an obscure and perennial corrosion by many and various toxins, often initiated, especially if of specific origin, in much earlier life. ${ }^{1}$

Such are the insidious effects we presume the more specific infective toxins to possess; but we do not know whether ordinary mechanical stresses acting, in Sir William Osler's phrase, upon originally bad tubing, or upon tubing of abated tone, are or are not competent alone to compass the same ends. Alienist physicians are becoming more and more disposed to suspect that certain kinds of mental disease, if remotely determined by a peculiar susceptibility of the nervous elements of the individual, depend immediately upon the influence of toxic bodies, for the most part autogenous; and that when such diseases become permanent this result may be due in part to damage done to the blood vessels distributed to the parts concerned. ${ }^{2}$ Thus in these cases the prognosis grows worse with the age of the patient. However all, or nearly all, of these speculations are as yet little more than guesswork.

Hitherto then we find ourselves carried back rather to arteriosclerosis of the decrescent form, a form not ordinarily attended with abnormally high pressures; but, to return to

1 Vide, e.g., Saltykow, Deutsche med. Wochenschr., 1911.

2 Vide, e.g., Robertson, Brit. Med. Journ., Oct. 26, 1906, and later papers. 
pressor bodies, are we justified in saying that there is no evidence in overfeeding of any generation of pressor toxins apt to damage the arteries indirectly, not by corrosion but by tensile strain $;^{1}$ or perhaps by exerting a twofold influence, both directly baneful to the vascular tissue and provocative of high pressure?-a twofold mischief with which adrenalin has been credited.

Many observers, as we have seen, ${ }^{2}$ attribute a considerable measure of this kind of neutralising capacity to the liver. For instance, rises in blood pressure have been vaguely attributed to falling alkalinity of the blood, and certainly the liver seems able to destroy sarcolactic acid; on the other hand, Bayliss and Starling extracted a substance from the intestinal wall which, on intravenous injection, lowered the blood pressure, and this substance also the liver neutralised. I remember Roger and Josué, I forget where reported, injected intestinal extract into a branch of the portal vein with no effect on the arterial pressures, and concluded that the liver protects the system. In obstinate constipation and in dilated colon the arterial pressures do not rise. The patient is usually meagre and sallow, the eyeballs yellow, and the orbits darkly ringed; some hyperpietics are of spare and sallow habit, but the majority are plump and ruddy complexioned. Choline does not raise pressure but lowers it; partly by reducing cardiac energy, partly by dilating the peripheral vessels; if atropin be previously administered, by plus output per minute and splanchnic constriction, the fall is converted to an augmentation. We may assume then that the liver, and active muscle likewise, can destroy pressor substances, adrenalin for instance; and, by destroying them or sparing them, make for regulation of pressures. Adrenalin indeed is used up rapidly in the act of vasoconstriction. ${ }^{3}$ And, as it seems probable that the liver is susceptible to nervous influences, if it be true that emotional causes may promote arteriosclerosis, we may guess that in part, at any rate, the function of the liver may be the seat of some disorder allowing the passage of destructors of vascular integrity. Thus, whether

1 Abelous, and Abelous and Bardier, Journ. de physiol. et path. vol. x., 1908, and various papers between 1906 and 1909.

2 See also Delaunay, Gaz. hebd. des sci. méd., July 21, 1907.

3 Elliott, Journ. of Physiol. 1904-5-6. 
by secreting compensatory substances, or by a kind of two-way potential such as we observe in certain ferments, the liver may balance arterial pressures, or rather the bodies influencing them, in both directions, pressor and depressor.

Let us in the next place advert to any evidence we may discover by way of the kidneys ; though here again we find ourselves still in obscurity, the data being vague and few. The "uræmic poison," like the bile, seems to retard the pulse. Little definite knowledge has been added, as regards verification, to Abelous' announcement ${ }^{1}$ of two pressor substances (amines) normally excreted in the urine but, according to Dr. Bain, ${ }^{2}$ in cases of gout and excessive arterial pressure abnormally retained; the one (" urohypertensine ") derivable from leucin, the other from tyrosin. These two bodies, according to Abelous and Bardier, Rosenheim and Walpole, ${ }^{3}$ and Dale and Dixon, ${ }^{4}$ are to be obtained from urine and fæces, and from decomposition of albumin ; some such decomposition in the intestine is suggested. One of them seems to be identical with one principle of ergot (Dixon and Harvey). It is alleged that some such products of nitrogenous waste, and with such it is that the kidneys are occupied, acting also, like adrenalin, ${ }^{5}$ on the plain muscle of the vessels, stimulate the vasomotor centre, and compass a cardiac hypertrophy. Nicotine, ergotoxin, indolethylamine, etc., have degrees of selective action on vasomotor centres (spinal cord) and plain muscle respectively, with consequently various modifications of kindred functional coefficients; though, if peripheral vessels be stimulated only up to a certain degree or extent, this measure may fall short of resistance to a normal or heightened cardiac energy. Sir James Barr, ${ }^{6}$ in the course of some cryoscopic experiments on the blood in a series of cases of arteriosclerosis, was convinced that, of the toxins which raise pressure, some raise it by direct action on the arterial muscle, some by stimulating the corresponding vasomotor centres, as strychnine

${ }^{1}$ Vide Laidlaw, Biochem. Journ., 1911; and Dale and Laidlaw, Journ. of Physiol., 1911, vol. xliii. No. 2.

2 Bäin, Lancet, 1909 and 1910.

3 Barger and Walpole, Journ. of Physiol., 1909, vol. xxxviii.

4 Dale and Dixon, Journ. of Physiol., 1909; Barger and Dale, ibid.

5 For evidence as to ultimate effect on the structure of the vessels, the animals of experiment do not survive long enough; but see Etienne, Journ. d. physiol. et pathol. gén. tome xv. No. 1, Jan. 15, 1913.

6 Barr, Sir J., Brit. Med. Journ., Jan. 14. 1905. 
is supposed to do; while others-e.g. iodothyrin-have a depressor action. Viperine poisons paralyse the vasomotor centre, so that the splanchnic vessels are seen vividly to dilate; a fall of pressure which may be arrested by dividing the cervical cord, ${ }^{1}$ when however the heart can still respond to adrenalin. Colubrine poisons affect the respiratory centre, but much depends on the dose. Cobra venom reinforces vasoconstriction and cardiac tone, but has also a cardio-inhibitory action, factors which obscure each other; large doses are apt to carry over into relaxation. Asphyxia, as is well known, raises blood pressure till the end, or until the heart fails (vide chap. Viscosity, p. 129).

Eichler ${ }^{2}$ (see p. 359) found in the serum of nephritics, and of nephrotomised animals, a vasoconstrictor substance like adrenalin. Similar observations have been published by Schlayer $;^{\mathbf{3}}$ and Esh, ${ }^{4}$ working with v. Behring, found a poison in the serum of eclamptic women which he identified with that of the urine.

Tyrosin, when inoculated with fæces, is converted into tyramine, a pressor substance nearly akin to adrenalin, a conversion which may prove to be an important link in the causation of high arterial pressures due to intestinal poisons. Harvey, by injections into animals (rabbits), has with this toxin produced various characteristic degrees of renal disease, from thickened arterioles to general parenchymatous degeneration. In 15 of them definite aortic damage was also found. Some of these rabbits were kept alive for periods from eighty days to over a year. Only one of the rabbits was immune. Out of 33,20 betrayed definite renal disease ; 25 aortic lesions ; 10 cardiac hypertrophy; 13 were negative. The chronic renal deterioration seemed due to arteriosclerosis of the renal vessels, which may accord with the observations of Jores, Aschoff, and Gaskell. ${ }^{5}$ But the order of events differed from Granular kidney: first, injured aorta, secondly, sclerosis of the renal arteries; thirdly, epithelial reaction in the kidney, and, fourthly, more general arteriosclerosis with further renal change, such as distended glomeruli, thickened Bowman's capsule, etc., and

1 Rogers, L., Proc. Roy. Soc., 1903.

2 Eichler, Berl. klin. Wochenschr., Nov. 11, 1907.

3 Schlayer, Deutsche med. Wochenschr., Nov. 14, 1907.

4 Esh, Münch. med. Wochenschr., Feb. 19, 1912.

5 See my chapter on the renal factor, pp. 339 and 351. 
consequent detritus, round cell infiltration, and new connective tissue supplanting the glandular parts destroyed.

But we have not yet quite done with the alternative of toxi-albumins. Handowsky and Pick, ${ }^{1}$ of the Pharmacological Laboratory of Vienna, found ox serum, as others have done, strongly vasoconstrictive, and proceeded to test horse serum. This serum, after a while (5-6 days) became also strongly vasoconstrictive. All bacterial influence was excluded. The constrictive substance, which they compared to adrenalin, did not pass a parchment filter. It was not globulin. The authors regarded it not as crystalloid but due to a disaggregation of a colloid complex, a change of physical state. A Petrograd physiologist ${ }^{2}$ found the same (?) substance in serum which, after coagulation which sets it free, applied to the rabbit's ear constricted the vessels. The adrenals had been previously extirpated. The fresh plasma had no effect. As it was not destroyed by boiling, this observer took the substance to be crystalloid, perhaps derived from the blood cells.

The publications of Metchnikoff and his pupils seem then to have done little, if with great respect I may say so, to reinforce the elaborate but slender fabric of toxi-alimentary hypothesis, constructed chiefly by the French clinicians. Huchard's dogmatic assertion that, in cardiac dyspnea for example, to forbid all meat and meat extracts, and to substitute a milk diet, brings sure relief, and that to return, however sparingly, to such foods recalls the distress, even if his rule be verified by further experience, admits of too many interpretations to allow us to accept the "toxi-alimentary" doctrine so confidently and so extensively built upon it. For this "toxi-alimentary" dogma in particular no experimental proof was produced; not even analyses of the urine before and after these prescriptions; and analyses of bowel discharges before and after colectomies in England and elsewhere have hitherto been perfunctory, and practically worthless. Vaquez, for instance, declares that the factor concerned is but the quantity of ingested salt. Many of us looked with interest to a discussion, now past, at the Royal Society of Medicine on "alimentary toxins"; but, ably as

1 Handowsky u. Pick, Arch. f. exp. Path. u. Pharm. Bd. Ixxi., Dec. 24, 1912.

2 Extract, Zentralbl. f. Physiol., 1913, No. 11. 
the subject was introduced by Dr. Hale White, and profuse as were the speeches, so that the publication of the "symposium" cost the Society $£ 235$, yet the gleaning was scanty. We looked for experiments on the behaviour of various comparatively simple and even crystallisable substances, not beyond chemical separation, derived from the several areas of the canal ; for experiments on their absorption, physical or selective (of colon absorption we know nothing); for comparisons of the content of the portal vein with that of the vena cava, whereby the function of the liver and the passage of such substances from stage to stage in the body might be elucidated; and so on. But our slender array of facts received little increment; of proofs we had few, of clinical gossip a surfeit. Dr. E. Mellanby, it is true, gave us some substantial help. The indoxyl group, the treacherous auxiliaries of many and laborious speculations, he "threw overboard," as relatively innocuous or insignificant, and directed attention to the amines produced by certain species of intestinal bacteria by splitting off $\mathrm{CO}_{2}$ from the proteolytic amino-acids. With the jettison of the indoxyl series the ordinary coliform-organisms, which have so long held our attention, fade into comparative neglect: the old vague suggestions lose their footing, save that tryptophane, the indol amino-acid of Hopkins and Cole produced by B. coli, has a somewhat remarkable, if slight, toxicity.

The products of ordinary septic organisms being then, so far as common experience goes, all depressor, even in nonfebrile phases, let us return to the hypertensive amine, or amines. Now these amines are near, perilously near, the amino-acids of nutrition, and bring us back to the difficult problem of normal and abnormal intestinal absorption and selection, which seems to be the key of the position, ${ }^{1}$ but of which we know really nothing. Those bodies, some pressor, more depressor, are normal ingredients of the intestinal processes, and under some unknown conditions may pass into the body. Vasoconstriction in the intestinal area would seem opposed to absorption. Dogs' urine normally contains a de-

1 Since this was written, Dr. Mellanby has made the same comment : Lancet, May 3, 1913. He suggests that specific intestinal secretions may be the determinants. See also Mutch, Quart. Journ. Med., July 1914. 
pressor substance, precipitable by alcohol, which in artificial nephritis cannot escape. ${ }^{1}$ It is from the point of view of hyperpiesis that pressor substances are of chief importance; decrescent arteriosclerosis may be in part a result of slow and long absorption from the bowel of depraving substances, neither pressor nor depressor; but of such processes we have much conjecture and no evidence. The supposed lactic acid control of certain morbid bacterial conditions of the intestine has made no way.

Those basic substances then which are actively depressor have, as yet, no recognition as arterial poisons. For the present we know only one or two intestinal substances capable of affecting the blood pressure, e.g. the ergot-like body already mentioned. This substance must be studied with the chromaffin secretion and the pituitary, which may be apt to break loose from proper control and perturb the balance of the circulation. In disease of the hypophysis (posterior lobe) a pressor substance, which as yet I think has not been analysed, is found abundantly in the urine; and in these cases the heightened arterial pressure and enlarged heart simulate Hyperpiesia. In Dr. Humphry's two cases ${ }^{2}$ Professor Dixon found that $10 \mathrm{cc}$. of the urines injected into a cat drove up the blood pressure 70-80 mm. Now a similar injection of normal urine drops the pressure. It is true that the big heart of pituitary disease, as of adrenalin, is not hypertrophied in proportion to its often enormous increase of bulk, which consists in large part of degrading tissues; but some of the increase must be genuine, for the systemic blood pressure in these cases is usually increased, if not greatly. In Dr. Humphry's first case, though at death the heart weighed $2 \mathrm{lb} .13 \mathrm{oz}$., the systolic blood pressure was only 160 (Male æt. 39). There was general thickening of the arteries. But of the effects of pituitin I have spoken elsewhere (p. 225).

Professor Hopkins and Mr. Sidney Cole have kindly drawn my attention to papers by van Slyke and Meyer, ${ }^{3}$ in which it is stated that, in protein digestion, free amino-acids, not any incompletely broken down complexes, are absorbed into the blood (see p. 322). Their method consists in an estimate

1 Pearce, Journ. Exp. Med., 1910, vol. xii. p. 128.

2 Humphry, L., Brit. Med. Journ. vol. ii., 1910, p. 1047.

3 Van Slyke and Meyer, Journ. Biol. Chem. vols. xii. and xvi. 
(by a special apparatus and accurate technique) of amino-nitrogen in the blood and tissues. Such nitrogen is present in the blood to the extent of 3-5 mgms. per 100 cc., and rises to $10-11 \mathrm{mgms}$. after a meal of meat. This is not urea nor uric acid nitrogen, but amino-acid nitrogen. In the liver the amount rises from 40-50 mgms. to $120 \mathrm{mgms}$. or thereabouts, the high figure subsequently falling as the amino-acids are metabolised in the liver. Van Slyke offers proof, from quantitative considerations, that we absorb all of the total non-proteid $\mathrm{N}$; but part of this will be in acids; not therefore representing waste products. Thus his estimation giving us this amino-acid $\mathrm{N}$ can be carried out. Deducting this from the total, the rest will be $\mathrm{N}$ in the form of urea, ammonia and uric acid, which together would vary as renal efficiency. Then, if by Folin's new colorimetric method we estimate, thirdly, uric acid, the remainder will be urea and ammonia. Thus when in high protein feeding $\mathrm{N}$ is retained in the tissues it is not as stored digestion products but as body protein. I have given this sketch of van Slyke's research, not because his method is practicable in clinical work, but because it opens our horizon and indicates a line of work. If we succeed in inventing a clinical method of detecting retention products in the blood, and of watching the descent of these simplifying bodies towards the kidneys and outwards, we shall have something like a key to renal efficiency and its correlations. (See "Ambard's Constant," p. 322.)

The sum of this part of the argument then is, so far as arteriosclerosis is concerned, that certain observations which we owe to Abelous and others offer evidence with some clearness to show that one, or two, definite and separable crystalline products produced from protein by bacteria, especially by a specific bacillus of the coli group, can affect the blood pressure. Certain amino-acids of protein (e.g. histidin) may be converted, by the organisms which deprive them of carbonic acid, into basic substances with a pressor action. One of them-that derived from the substance tyrosin-is chemically related to adrenalin and, if less powerful, yet produces potent pressor effects. Another, that derived from histidin, turns out to be one of the active constituents of ergot (Dixon and others), and exercises an ecbolic as well as a pressor action. How far these 
bodies are absorbed, and under what conditions, and how far their effects, if any, are of quantitative importance yet remains to be determined. It is true ${ }^{1}$ that these bases are present in small quantity in the intestinal wall ; but of their absorption, or of the permeability of the gut, in health, in disease, in advancing age, or again of some lesion necessary for their passage, we know nothing; nor again do we know how far, if absorbed, the normal liver modifies them, or the abnormal lets them pass. If they pass unmodified, the tyrosin derivative especially must aggravate blood pressures by acting locally on the vessels, and herein may lie the secret of the malady of Hyperpiesia. Such poisons, moreover, may not only crimp up the arterioles, but also do some direct injury to their walls.

Gout.-All these hypotheses Lancereaux ${ }^{2}$ rather scornfully put away, and denied that any modification of diet would reduce the evil : but his scepticism carried him too far; he disbelieved no less in the influence of infections, even of syphilis, in arterial disease, and referred the solution of the whole problem in vague terms to trophic nerve disorder. I think before his lamented death our distinguished colleague had fallen a little out of touch with recent work on the subject, work which, incomplete as it is, points at present in other directions. It is fair however to add that Lancereaux regarded gout as a cause of trophic nerve changes, and herein we may find some common ground. The belief is prevalent that gout is intimately concerned with arteriosclerosis; moreover Huchard, in his rhetorical way, propounded that "Gout is to the arteries what rheumatism is to the heart." There are no statistics on which to base any such an axiom; and here again we are baffled by the confusion which consists in the lack of discrimination between hyperpietic and decrescent arteriosclerosis, and in the obscurity of the nature of gout itself. Professor Elliott Smith, in all his experience of ancient anatomy in Egypt, met with one case only of gout, and this was in the early Christian period; though arteriosclerosis, as we have seen, was present abundantly, now and in all periods. From the

1 Mellanby and Twort, Journ. of Physiol. vol. xlv. p. 53. What is true of histidin is probably true of others.

2 Lancereaux, Paris, Acad. Méd., June 2, 1908.

VOL. I 
days of Sydenham to the present time we have learned but one new principle about gout; namely, from Alfred Garrod, the occurrence in this disease of relatively large quantities of uric acid in the blood. Between gout and arteriosclerosis there is this common ground; as in the kind of arteriosclerosis consequent upon persistent high blood pressure we have seen reason to accept overfeeding as one among its causes, so is this habit in the individual, or in successive generations, confidently and almost universally, presumed to engender gout. And, broadly speaking, both hyperpiesis and gout are wont to manifest themselves at or about middle life, when the energies of digestion and assimilation, and of muscular exercise, become less and less efficient. But what and whence, on this urinary side or elsewhere, may be the peccant bias or poison no one has any notion. By Garrod's discovery suspicions of uric acid were deepened almost into conviction, but this relict is now generally abandoned to the quack; although it may be that as a by-product of gout uric acid is so far significant as to be some measure of it. The notion current is that the specific cause of gout is of the kind of xanthine bodies, and other purins ; that is, some antecedent or associate of uric acid. Both for exogenous and endogenous purins and for uric acid it is true that the capacity of dealing with them (" urikolytische Kraft") varies much in different individuals, and probably at different ages of life; and presumably is connected somehow or other with the oxygenating activity in certain areas: and the same, or something like it, may be true for hyperpiesia. Injections of xanthine bodies have not so far been found to raise arterial pressures, or they do so but transiently at the moment of operation; however more experiments in this direction are wanted. At present we have no direct evidence of harm done by, or of clinical effects due to, purin retention. Uric acid, notwithstanding its frequent insolubility, seems to be excreted from the body as readily as other substances of the same order, and is indeed itself, if toxic at all, one of the less toxic purins ; as is manifest enough in leucocythæmia. ${ }^{1}$ Urates then, if they may be a sign, are no considerable cause of artnrial disease, and are not found in the coats. We may perhaps go so far as this, that, if purin

${ }^{1}$ See many papers by Hopkins, Walker Hall, Langdon Brown, etc. 
excretion be high but yet the intake not excessive, we may reasonably suspect hepatic insufficiency.

On empirical grounds the physician is strongly disposed to connect gout with arteriosclerosis, and certain not inconsiderable authors go so far as bravely to declare - although, so far as I am aware, no such evidence is before us-that "the poison of gout is a vasoconstrictor toxin." If so, gout and high arterial pressures ought to be inseparable, or at least in frequent association. Now many years ago I pointed out that, whether true or not of "irregular gout," of frank gout this is certainly not true, not even in an attack; and Dr. Mitchell Bruce and other physicians of large experience have supported my opinion. Dr. Luff ${ }^{1}$ also agrees that, if the kidneys are normal, gout is not a high pressure disease. Dr. Lambert, who took the subject of gout and blood pressures for his M.D. thesis, ${ }^{2}$ concluded that before middle life in gouty persons, otherwise normal and free from renal disease, the blood pressures are unaltered; and that after middle life the alteration is no more than usual in all persons. Lambert thinks however that in chronic tophaceous gout arterial pressures, on the whole of persons examined, do rule somewhat higher; but many of these patients are elderly and their cases complicated. In one uncomplicated case, of a man æt. 68 who had suffered from frank arthritic gout for twenty-eight years, the systolic pressure was 130 ; in another, æt. 55, who had suffered thus for seventeen years, it was only 115 . Geisböck ${ }^{3}$ found on careful comparisons that in the majority of his patients suffering from " uratic gout" the arterial pressures were normal; and this, he points out, was the more notable as in not a few of them a trace of albumin appeared in the urine. Dr. Edgecombe, ${ }^{4}$ at Harrogate, finds that in articular and tophaceous gout, when allowance is made for the age of the patient, the blood pressure is usually normal or subnormal. On the other hand, Dr. George Oliver thinks, with many other bath physicians, that in gout there is some cloying of the finer vessels, and that the attack of acute gout

1 Luff, Brit. Med. Journ., May 10, 1913.

2 Lambert, Thesis for M.D., Cambridge, 1908. Also published in Bull. Stud. Specific Diseases; Camb. ed. Strangeways, vol. ii., Oct. 1908.

3 Geisböck, Deutsche Arch. f. klin. Med., 1905.

4 Edgecombe, Baln. Soc., Dec. 12, 1907. 
which is apt to follow bath treatment is due to a setting free of blood and lymph exchanges. But such cloying, unless of very wide extent, would not affect systemic pressures. I may allude here to two cases now under my observation :

Mr. B., a temperate and active man, æt. 48 , of gouty parentage. Has had already ten attacks of podagra; yet his arterial pressure is perfectly normal, and his accessible arteries quite soft.

A butler in Addenbrooke's Hospital; æt. 51; free liver. Gouty for many years. Hands, feet, olecranon, ears, etc., full of uratic deposits. Radials very slightly thickened, not more than quite common at his age; not tortuous nor even visible. To the finger the pulse is not tense nor sustained ; to the gauge the systolic pressures at various times and at most have never exceeded 140 .

It must be conceded then that regular gout and high pressures are not more than incidentally associated. However it is commonly asserted that, whatever the pressures, gout and arteriosclerosis are somehow akin. Yet even this vague apprehension, as regards regular gout, is doubtful. Orth, in the early editions of his Lehrbuch at any rate (my copy is dated 1887), concluded that in spite of a prolonged and suspicious scrutiny he could not perceive that gout played any part in arteriosclerosis. The writer on gout in Eulenburg (4th ed.) says the same thing; and Ebstein ${ }^{1}$ on the whole is disposed to agree. For my part I think that arteriosclerosis, whether with high pressures or not, is a frequent, though by no means an obligatory, concomitant of gout, especially of irregular gout. In my first paper on the subject of high pressure arteriosclerosis (in 1894), I noted this association of irregular or suspected gout with high arterial pressures, but was unable to connect the two processes. I thought that in this respect podagrous gout differed from the "suppressed" modes. Dr. Mitchell Bruce, whose careful notes and digests of private practice put one to shame, has found "the radial pulse more tense in irregular than in regular gout"; in his irregular cases the arterial pressures were raised in more than 50 per cent, and in the majority the arteries were thickened. These patients (men) suffered from irregular fluttering intermittent hearts, and some of them from anginal and precordial pain ; the women suffered also from "faintnesses." It would

1 Ebstein, Gicht, Wiesbaden, 1906. 
seem then as if the vulgar notion were true, that podagra is, not so much gout as a relief of gout.

In respect of arterial disease Dr. Bruce pointed out ${ }^{1}$ the frequency in gouty subjects of degeneration in the aortic area; an aortic systolic murmur was audible in 12 of 29 of them. Aortic regurgitation of course is rarely a consequence of atheroma.

Under the head of " chronic rheumatism " Guéneau de Mussy noted a frequency of arteriosclerosis, and Rosenbach and others have followed him. The name is, of course, a vague one, and the condition itself no less equivocal ; but many of such cases may be, or doubtless are, of gouty nature.

On the whole then, it appears as yet that if in its regular forms gout may not be very conducive to arteriosclerosis, yet that the kind or kinds of perversion which we must for the present be content to call irregular, or suppressed, gout, are apt to promote arterial disease, sometimes by setting up high blood pressures with consequential strains, sometimes perhaps by endogenous toxins, decrescent modes of decay; the two processes, the "regular and the irregular" gout, if we may judge by their several issues, not being identical. In irregular gout the perversion may drive up blood pressure, with or without independent detriment of the arterial tunics; in the regular form the poison or deranged metabolism may attack these tunics directly, so that, independently of any pressure, they may yield; for we have noted that Mitchell Bruce found the " radial pulse more often tense in the subjects of irregular than of regular gout." The pressor facts point to some vice of metabolism rather than to a mere defect of antilysins (Hort), or of "stimulins" to growth and repair ; conditions which might however account for a decrescent arteriosclerosis without the mediation of excessive blood pressure.

In many cases then, gout and high arterial pressure seem rather in correlation than to be cause and effect, as probably in the following case :

Mr. A., æt. 68, a patient of Dr. Hayward of Wimbledon, a fine big man, full of energy and vivacity. He was incessantly engaged in very large affairs, and saw much company. He did not hesitate

1 Bruce, M., "L. Lect," Lancet, March 23, 1901. Murchison, Sir Dyce Duckworth, and Dr. Norman Moore have given like testimony. 
to admit that as regards food, wine, and tobacco he lived generously. For some few years Dr. Hayward had found his blood pressures ranging (systolic) about 200, and for some shorter time past more or less of a cloud of albumin in the urine. Never any sugar. He had never shown any sign of uræmia ; no œdema, no headaches, no nausea or vomiting, no retinal affection, no drowsiness. He inherited gout definitely. His own first attack (podagra) was at the age of 35 ; and attacks in hands and feet had recurred from time to time since. I found the systolic blood pressures above $200 \mathrm{~mm}$., diastolic near 180. Heart large, and beginning to dilate, but myocardium probably healthy. Arteries rather thick. Second aortic sound ringing at base and apex. A few fine crepitations at left pulmonary base. There was a ring of albumin in the urine, but a long search failed to discover any casts or renal epithelium. Some uric acid crystals, one or two cells (leucocytes?), and a shred or two of vesicle epithelium constituted all the deposit in drops on many slides. This urine had stood in a conical glass for an hour or two, and the drops were taken by pipette from the bottom.

If now we turn to lead poisoning, the symptoms of which have some kinship with gout, or at least a remarkable resemblance to it, we find a more definite story; we find the rule to be one of both arterial high pressures and of arterial thickening. Lancereaux $^{1}$ says that Desplanches first drew attention to this result, as based upon four necropsies, in which, with renal disease, extensive atheroma was found. Bureau (loc. cit.) gives a case of exposure to lead from æt. 15. Death æt. 34: the whole arterial system affected. There was no gout. The thickening is so constant and so early a phenomenon that one must assume it to be not merely the result of strain; some toxic influence, plumbic directly or indirectly, must also conspire to the result. It is said that in cases caught early and cured the thickening disappears as if, at any rate at first, it were due only to medial hypermyotrophy, a condition in marked contrast with the high brief systole and low diastole of alcohol. Gibson, who had opportunities of watching cases of lead poisoning from beginning to end, says that whether renal disease be apparent or not, the arterial pressures are high throughout. These observations are the less equivocal as lead poisoning with these symptoms often occurs, like syphilis, early in life, un-

1 Lancereaux, Dict. Encycl. art. "Artérite." 
complicated by senile degradations. I have recent notes of plumbism in a man, æt. 41 , in whom the blood pressures were excessive and the radial arteries tortuous and thick far beyond mere hypertrophy. The arterial pressure will fall of course if the heart becomes weary. In a recent degree examination a painter, æt. 51, presented extremely diseased arteries, not only thickened but also beset with granules of calcification. We, as examiners, had to decide whether the candidates should describe his blood pressure by the finger as high or moderate, not a very easy task; however we all agreed that by the finger alone it would be difficult to say that the pressure was augmented; yet he had granular and epithelial casts in his urine. Now on examining the heart it was found to be largely dilated, but poor in impulse; his legs were puffy, and crepitations were audible at the bases of his lungs. No record of the blood pressure was found in the notes. This was what Broadbent used to call "virtual tension," the lapsing of long periods of high pressure by the defeat of the heart. Aubertin, quoting also Bernard and Bigart, states that lead injures the vessels indirectly by exciting the adrenals.

It has been suggested that minute traces of lead may be concerned in many of the cases of premature arteriosclerosis the origin of which is obscure; and we are reminded of the Reports on Foods, which reveal traces of lead in wine, beer, cider, syphons, etc., in groceries, and even in bread. Dr. Putnam therefore examined the urines of 100 persons in reputed health, and detected lead in 17 per cent of them. These persons must have lived in a very adulterated market.

A few years ago a notion was abroad that diabetes was a disease of high arterial pressures. The notion seems to have originated with Potain, but, if so, this master of clinical medicine soon altered his opinion. His own gauge was not a trustworthy one, and probably at first he happened to try it on some cases of glycosuria with granular kidney. Diabetes is not a disease of high pressure, though glycosuria is no countercheck to such rises if under some concomitant conditions they may be generated; and some authors attribute arteriosclerosis in diabetes to the meat diet. In so far as diabetes may sap the power of the heart it is a disease of low pressures ; the occasional 
records of hypertrophy of the heart are of cases complicated with renal disease. When therefore in simple diabetes we find the arteries thickened we may be pretty sure that the sclerosis is due not to high tension, but to some toxic causes, not of a pressor kind (p. 450). Some recent observers have declared that arteriosclerosis does not occur in young diabetics, but, as my pupils of many years past can testify, this statement is wide of the truth. Many years ago I pointed out the occurrence of thick arteries in young diabetics, one of the youngest being a girl in the Addenbrooke's hospital, aged 14. I have notes of many instances of such arteries in children and in young and comparatively young adults suffering from the malady. I think that in diabetic patients some thickening is the rule; their consequent liability to gangrene is familiar knowledge. I may select this case from my notes:

A man aged 25, a diabetic. No sign or probability of syphilis. Sugar on ordinary diet abundant, and not wholly removable by the strictest diet. The man did badly; his knee jerks disappeared, and his foot extension weakened. But there was no rise of measured arterial pressure at any time; often, indeed, it was well under normal, especially as the patient became emaciated and cyanotic, and the heart suffered more in its nutrition. The pulse was never sustained, and was always very compressible. Notwithstanding, over the few years he had to live the arteries slowly thickened, becoming more and more tapy to the touch. In him the vessels never calcified; presumably they would have done had he lived longer, for in diabetics gritty vessels are common enough.

In elderly persons of course the incidence of arteriosclerosis and of glycosuria respectively is high enough to make their coincidence so frequent that neither could be regarded as the cause of the other. Such glycosuria is often of the milder senile type; the sugar slight and intermittent, and often accompanied with a little albumin. A more plausible suggestion is that the glycosuria may be a result of arteriosclerosis in particular areas, as, for example, in the pancreas (Naunyn), a suggestion which occurred no doubt to many of us in past times before we had had the opportunity of witnessing the gradual advance of the vascular changes as a sequel of diabetes in young persons. That such may be the causation of some cases of mild glycosuria in elderly persons is possible enough. 
These views of the relations of diabetes to arteriosclerosis have been verified by more recent observers. Elliott agrees that diabetes has no effect in raising blood pressures. ${ }^{1}$ Janeway is of the same opinion. Severie, ${ }^{2}$ after examination of 29 diabetics, found among them many ranges of arterial pressure, even up to 240 , a figure surely indicative of the renal disease which frequently appears in the later course of diabetes. ${ }^{3}$ Thus many old gouty glycosurics present a high blood pressure-cases which often end in apoplexy or angina pectoris ; it is the young diabetics who, as a rule, are disposed rather to low pressures, especially if prone to phthisis; yet in many of them the arteries obviously deteriorate. What may be the direct agent in this detriment to the vessels we cannot tell at present; perhaps an under-alkaline blood. In those too frequent cases of rapid diabetes which occur in young subjects arterial degeneration may fail, only because in them survival is too brief to include it. Nevertheless, I have seen not a few cases of longer duration in which it never appeared.

We are hearing something now, from Neubauer, Weiland, and others, of "blood sugar" and high arterial pressures. Port, of Göttingen, has recently reviewed these hypotheses ${ }^{4}$ and shows that the association is due to some common cause. Thus injection of adrenin will bring them together. He quotes several cases of a very high blood pressure (non-renal) in which there was no hyperglycæmia, except in apoplexy: we know that in brain diseases, e.g. tumours-sugar may appear in the urine. The argument that arteriosclerosis in diabetes is due to large meat consumption will not do. Hospital physicians see too many cases among the poor to accept this notion.

Infections.-As of late years our views of natural processes have enlarged, we have been enabled to trace chains of causes farther and farther back, and to discover in his bygone history many of the sources of defects or perversions of the adult individual. As in some cases adult tuberculosis may be the shoots of a seed sown in infancy or childhood, so it is surmised

1 Elliott, Journ. Amer. Med. Assoc., July 1907.

2 Severie, Sem. méd., juillet 31, 1907.

3 See discussion Aron, Israel, and others, Berlin. Med. Gesellsch., Feb. 19, 1913, reported in the German journals about that date.

4 Port, Deutsche med. Wochenschr., Jan. 9, 1913. 
that in adults arteriosclerosis may likewise creep on under and after some toxic influence which in early life impaired the arterial tissues; the slow accumulation of stresses_-" wear and tear"-telling on vessels whose tone and elasticity had been sapped in infancy or childhood by some infection. I have said that for this reason I have eschewed Jores' name of "Senile arteriosclerosis," because it seems to ignore these more precocious, latent, and often specific coefficients, and to throw the effect entirely upon simple and inveterate stresses. Until our knowledge is riper we shall do well to keep the door open for certain co-operative detriments which may not become obvious till after the lapse of many a decade.

One by one the common infections of children have fallen under suspicion, and the harm they do, both acute and chronic, has been demonstrated, especially by the French School. That the infectious fevers attack the arteries is confirmed by Dr. Andrewes, who writes (Rpt. L.G.B. 1913) that it is rare in necropsy of such young patients to miss local spots of fatty change in the intima of the aorta and larger branches. Occasionally in infections the lesion may be tracked along the vasa vasorum. These changes he illustrates by excellent plates. Lesions, both focal and diffuse, such as necrotic spots in the media and lesion of the intima, have been brought home to typhoid fever, rheumatic fever, influenza, small-pox, malaria, scarlet fever, diphtheria; and to such ubiquitous mischief-makers as streptococci, staphylococci, and the colon bacillus (p. 528). The diphtheria toxin especially seems, in experimental tests, to attack the vessels with some constancy. The poisonous substances secreted or excreted by these bacteria are so subtle as hitherto to have eluded all attempts to isolate them; but they are not alien from plant and animal venoms, and are probably perversions or developments of normal secretions, each one with its own specific affinities in the cellular world. However, it is not my task to discuss these bodies except in so far as they jeopardise the arterial tunics.

Of the acuterfocal inroads upon the larger trunks, and especially upon the aorta, I shall report the observations of the French and other pathologists in my chapter on Aortitis (Vol. II. p. 189); so that here I will deal rather with the more diffuse or universal 
lesions. I have however alluded (p. 221) to the experiments of Gilbert and Lion-who were first in this field of experiment ${ }^{1}$ on the production of atherosclerosis by the injection of bacterial emulsions, of Josué, of Klotz, and of Saltykow ; ${ }^{2}$ results which have obtained substantial verification. ${ }^{3}$ Setting syphilis aside for the moment, the infection whose toxic effect upon the arterial system is best known is that of typhoid fever, on which we have learned much from the labours of Professor Thayer, ${ }^{4}$ and of Landouzy and Sireday, ${ }^{5}$ who noted slow arterial changes as due to infections. Josué in animals produced atheroma by injections of typhoid bacilli and toxin. Thayer investigated 183 cases of recovery in cases of typhoid fever which had been under treatment in the Johns Hopkins Hospital during the fourteen previous years. The ages ran from 3 to 69 years, and the periods from discharge from one month to thirteen years. Of these individuals 48 per cent showed palpable radial arteries; the same number of control cases showing but 17 per cent. In 25 per cent of these individuals whose ages lay between 10-20, these arteries were thickened, and in 50 per cent of those whose ages lay between 20-50; in all the cases between 50-60 the vessels were very palpable and "fibroid," a percentage far above the normal incidence, twice or three times more. Of the cases cited the following may be extracted as an example:

Boy, æt. 13. Typhoid fever. Discharged November 1896. Recalled for examination January 4, 1903. Radial wall much thickened, can be rolled under the finger. The temporals tortuous, prominent and thick. The heart was hypertrophied, and the arterial pressure raised.

In all these sclerotic cases the blood pressures ranged higher than in a control series of normal persons under like conditions and of the same ages. In a few of them the pressures were considerably augmented, and the heart hypertrophied; but these cases I think must have been complicated with renal or other disorders.

1 Gilbert et Lion, Comptes rendus de la Soc. de Biologie, Oct. 12, 1889 ; and later papers, e.g. Arch. de méd. expér., Jan. 1904.

2 Saltykow (infections by streptococci), Beitr. f. path. Anat. u. Pathol., 1908.

3 E.g. Manouélian of the Pasteur Inst., Ann. de l'Inst. Past., Jan. 25, 1913.

4 Thayer, Amer. Journ. Med. Sci., March 1904-a paper read to the N.Y. Acad. Med., 1903; and Thayer and Brush, Journ. Amer. Med. Assoc., 1904.

5 Landouzy et Sireday, "Contrib. à l'hist. de l'artériosclérose typhoidique," Rev. de méd., 1885. 
In many others, although the arteries were thicker than normal, the arterial pressures were not at all, or but little increased; and such has been my experience. Since Dr. Thayer's paper was published I have come across not a few cases of unduly thick arteries in which a history of typhoid was obtained, but in these chance cases the blood pressure was not augmented. I saw recently, with Dr. Aldren Wright, Mr. M. æt. 20, in the second week of definite typhoid fever. We noticed, independently, that even already the radial arteries were tapelike when empty, and rolled roundly under the finger when full; but the pulse was dicrotic, and systolic blood pressure rather under than over 95. In the active undergraduate normal arterial pressures run low. ${ }^{1}$ In some of Thayer's cases were valvular lesions, on the doubtful interpretation of which I need not dwell. During the fever of course the arterial pressures run on the lower side-say 90-95; whatever the temperature and the pulse rates, the pulse is usually no doubt relatively slow. During convalescence the pressures return to normal. I may take occasion to say, in respect of blood pressure in most of the acute infections, that pressures rule low rather than high; partly because of some reduction in cardiac values, partly because of peripheral relaxation. The rises of pressure recorded in the earlier or later history of sufferers from typhoid fever need further study and discrimination. The opinion of Thayer and Brush that the thickening of the vessels in or after typhoid fever is due to "overstrain of the vascular walls from high tension, however caused," is not on the face of it very probable. I suspect that in typhoid fever, as in the class of infections generally, the injury to these tissues is due more directly to the poison itself. The mental symptoms occasionally seen in typhoid convalescents, such as loss of memory, incoherence, and even slight dementia, may be due to effects of the poison mediately on the vessels, and not directly upon the cerebral tissue itself.

For these infective vascular changes the terms arteritis (and aortitis) are often used, especially by French writers; but we should hesitate to use these terms: Dr. Andrewes ${ }^{2}$ thinks

1 See art. on Heart Stress, Allbutt and Rolleston's System, 2nd ed.

${ }^{2}$ Andrewes, F. W., loc. cit. 
that the arterial disease is in part at least inflammatory, but for the most part the process is degenerative, the reparative reaction, if any, being in typhoid of the slightest (p. 522).

On turning from the diffuse to local lesions, I find in Brouardel's System that typhoid aortitis was demonstrated by MM. Landouzy and Sireday, but no reference is given; I have made some brief search for this source about the probable dates-say 1885-87but without success, and I have not pursued it because our debt herein to the French School is of earlier date. Infectious aortitis will be discussed in my Cavendish Lecture (Vol. II. p. 148). Laennec and Louis recognised the evil influence of typhoid fever upon the heart; and our closer knowledge of cardio-arterial disease in the infections dates from Hayem's well-known paper. ${ }^{1}$ Barié has recently verified these observations. Clinically the liability of arteries, such as the femoral or tibial, to acute obliterative lesion in typhoid is freely admitted in France and England, though it seems to be doubted in Germany (e.g. by Romberg and the late Professor Curschmann). The thrombosis of typhoid fever-as of other infections-may be due to focal patches of arteritis. In a previous paper I have attributed the sites of circulatory disorders of the arms and legs, at any rate in part, to a mechanical cause, and thus explained the relative date of their appearance. As the patient emerges from his prostration, and regains some power of movement, he is apt to throw one leg over the other, or turning a little sideways to lie upon a limb.

Still, it may be asked, before we go farther, of what kind is the arterial lesion caused by these infections ; is it of the subintimal kind, or is it an attack upon the media (necrotic foci), to which some allusion has been made? In syphilis of course the characteristic lesions are medial, or medial and adventitial (pp. 296 and 478) ; and Dr. Cowan is disposed to think the other infections act similarly. It seems agreed that the infections enter by the vasa vasorum, along the tracks of which leucocytic exudations may be observed; and that preferentially they attack the arch of the aorta, a thick wall richly supplied by these minor vessels. On the other hand, he notes how frequently after acute infections fresh, often translucent, patches of " atheroma" arise, in which sometimes bacteria may be detected (and thence might set up

1 Hayem, Arch. de physiol. norm. et path., 1870. 
infective endocarditis). But probably in more recent study of the subject he will admit that this mode of arterial disease is often in site and kind subintimal (atherosclerosis). I think it is clear that we cannot draw the definite distinction between endartérite scléreuse and mésartérite calcifiante so as to attribute that to toxins and cognate substances, and this to lead, gout, etc.; though it is true that the effects of these agents have more of the nature and reactions of inflammation, and tend to connective hyperplasia. Klotz ${ }^{1}$ says that diphtheria, typhoid and the like toxins do not attack the media but the intima, and Saltykow ${ }^{2}$ agrees with him; Adami however assents with doubt, remarking that, if so, the change would soon stop the imbibition of nourishment to the inner media and implicate it also in a vicious circle. In animal experiments, or rather in rabbit experiments, the media seems to be the primary seat of the reaction and lesion, but, again, the rabbit's aorta differs too much from that of man to permit us readily to apply conclusions from the one to the other. In monkey experiments, at the Pasteur Institute, with streptococcal and staphylococcal emulsions, Manouélian (loc. cit.) and Metchnikoff ${ }^{3}$ produced lesions far more closely like the aortic atheroma of man, especially by repeated injections of less virulence; but in the rabbit (86 of them were infected) the effects in 73 resembled those of the mechanical strains; as if its rudimentary aorta had but this one mode of response. The elastic fibres seem first to break up, and are the seat of the first calcification, and the muscular fibres soon follow. In the monkey -5 out of 6 - the change was more internal, and calcification was absent or relatively slight, hyaline change being more evident. But the morbid effects were not uniform. No microbes were detected, I believe, in the parts examined. Duval ${ }^{4}$ states that in rabbits infected with glanders proliferation of the endothelium of the small vessels occurs, but Adami comments on this, as one may on all experiments of the kind, as I have commented upon experimental arterial lesions in rabbits, that no direct comparison can be made between such arterial lesions in these animals and arteriosclerosis in man, and that the results

1 Klotz, quoted Adami, Amer. Journ. Med. Sci., Oct. 1909.

2 Saltykow, Ziegler's Beitr., 1908, vol. xlii. p. 187.

3 Metchnikoff, Ann. de l'Inst. Pasteur, Oct. 1910.

4 Duval, Journ. Exp. Med., 1907, ix. p. 241. 
in apes are more homologous. Whether or no there is any radical distinction between diffuse arteriosclerosis and focal, or whether we should call these infections arteriosclerosis at all, is a question which as yet we are scarcely prepared to answer. Infectious arteritis around septic foci is the prevalent conception of the process. When in infections the aorta has been attacked, acute or chronic foci of atheroma have been found in tubercle, pneumococcic, streptococcic and staphylococcic affections (Manouélian), anthrax, erysipelas, rheumatic fever, smallpox, scarlet fever, typhoid fever. In this vessel at any rate the lesions are focal, and we may perhaps surmise for the present that such is the mode of distribution of the lesions in all the arteries. Künne ${ }^{1}$ reports a necropsy on scarlet fever in a girl aged 19; extensive lesions were found in the media, but the adventitia also was affected. The media had yielded in many places, but there were no aneurysms. The aortitis of rheumatic fever will be described later (Vol. II. p. 150).

But to return to typhoid fever; among the most exact researches on this subject which have fallen under my notice are those of Wiesel ${ }^{2}$ of Vienna, who made minute postmortem examinations of the blood vessels in a large number (about 300) of mortal cases of typhoid fever, scarlet fever, diphtheria, and other infections in cases under 24 years of age. To take typhoid first, and omitting the cardiac pathology, in carefully selected young cases he found well-marked lesions in the aorta and pulmonary artery, and in the coronary, radial, iliac, femoral, tibial, hepatic and superior mesenteric vessels, especially in the intestinal branches, the pulmonary, and the anterior coronary (as also in their veins); the cerebral vessels were least affected. Sometimes the lesion had advanced so far in smaller arteries, but not in the coronary, as to close the channel. Wiesel thinks indeed that this arterial effect of typhoid is universal and constant, being thus of higher frequency than the wellknown cardiac changes; though ordinarily there is nevertheless a quick and complete restitutio ad integrum; so that ill consequences are rare. Ultimately in some instances he obtained

1 Künne, Frankf. Zeitschr. f. Pathol. Bd. v. H. 1, extr. Zentralblatt f. Herzund Gefässkr., Jan. 1911.

${ }^{2}$ Wiesel, Zeitschr. f. Heilkunde, Feb. 1905 and July 1906. (See also p. 477.) 
necropsies on cases of recovery from typhoid in which death had occurred "one or more years later," from other causes ; in these he found the vessels to all seeming sound again; although on close investigation here and there spots could be detected where connective tissue had supplanted the muscular; and in some spots, where the intima had been attacked, signs were observed of Jores' " regenerative" (proliferative) change (vide p. 522-3).

Now to these changes Wiesel also thinks that the name of arteriosclerosis - or at any rate of atherosclerosis-ought not to be applied. They are not primarily affections of the intima, but of media and intima. Moreover, I remember that Simnitzky, ${ }^{1}$ in his large collection of such cases, found in some calcification, said to be medial; but Wiesel did not find calcification to be a feature of these specific cases.

The minuter morbid histology is thus described by Wiesel on iliac, femoral, and radial arteries. He found the elastica broken up by a degenerative change, so that in places the fibres, imbibing little stain, or none, vanished altogether, or were reduced to a granular state. Thus far, especially when seated in the medial portion of the aorta, the changes, which are in agreement with the description of Manouélian of the Pasteur Institute (loc. cit.), differ from the manifolding of the elastica in atherosclerosis. The muscularis wastes, but, as it is largely supplanted by connective hyperplasia (" eine stärkere Ausbildung des intramuskularen Fasernetzes"), he did not hesitate to use the suffix "-itis," and to speak of a "mesarteritis" which seemed to run parallel in time and degree with the myocarditis. I have said that Dr. Andrewes also is disposed to regard the process as inflammatory. Curschmann, on the other hand, denied that this change was an endarteritis and, from the nature of the patches on the aorta, was disposed to accept the process as atherosclerotic. But if so, the rapid and complete restitutio ad integrum is the more remarkable.

Thayer thinks that in the typhoid and rheumatic fevers arterial lesions are most frequent; but in many at least of his patients hard labour, or irregular habits, or both, were conspicuous. As concerns rheumatic fever, while I have gathered no little evidence of arteritic and atherosclerotic lesion of the thoracic aorta in this

1 Simnitzky, loc. cit. 
disease, I have lighted upon little of the diffuser forms of arteriosclerosis as a sequel of it. For the aortic lesions I would refer to Aortitis (Vol. II., p. 152), and Angina Pectoris (Vol. II., p. 422); and for interstitial arteritis of the myocardium to Dr. Carey Coombs' preparations. Concerning general arteriosclerosis as a sequel of this infection, I have only this case to offer :

A patient in the Addenbrooke's Hospital, aged 35, with mitral disease and a dilated heart, attributable to severe attack of rheumatic fever at the age of 14, and unfit ever since for hard work, presented also thick and leathery arteries (radials, brachials, and other accessible vessels). There were no signs of aortic valvular disease, nor of renal disease, and the blood pressures were moderate.

We are agreed that "chronic rheumatism" is too vague a term for scientific use, and that some of the cases so called are gouty. Still the chronic malady to which I have already referred as dependent upon cold and damp, whatsoever its nature, is probably in some cases attended with arteriosclerosis, as first pointed out by Guéneau de Mussy, and verified by Rosenbach and others. On another page (206) I have spoken of the experience of Mr. Wilkin of Wickhambrook, whose practice is divided between two districts, one a heavy clay, the other-the Newmarket sidea dry upland. On the clay side, where he has to deal with large numbers of cases of " chronic rheumatism," he finds that in these cases thickened arteries are frequent, but without rise of blood pressure. Syphilis is very rare in his practice, and lead poisoning practically unknown. Renal disease was excluded from these comparisons. This so-called rheumatism is not followed by heart disease. Professor Stockman found in an excised portion of a joint thus affected, "that the small blood vessels showed very distinct peri- and endarteritis, with marked connective tissue proliferation."

Diphtheria.-Concerning diphtheria, Dr. Aikman of Guernsey ${ }^{1}$ has published the following curious observations. In cases in which he had injected doses of anti-serum insufficient to control the attack in a few days a perceptible thickening of the accessible arteries set in. In the elderly of course the change was masked by senile decay, and under æt. 3 the artery in a fat arm was not easy to appreciate, but in youths and persons

1 Aikman, Brit. Med. Journ., April 21, 1906 ; an interesting letter, VOL. I 
under middle age the change was evident enough. No corresponding renal disorder could be discovered in these persons. This thickening usually disappeared in a few weeks. In those cases, on the other hand, in which he had injected anti-serum sufficient to control the attack the arterial thickening never set in. He had observed a similar change in the arteries after influenza and typhoid fever, but usually in them also it disappeared " in nine or ten weeks." The thickening appears rather suddenly, but disappears very slowly. Many cases of diphtheritic aortitis are on record (vide Aortitis, Vol. II., p. 164).

Here again, Wiesel's laborious researches, which have not been confined to typhoid fever, come to our assistance. He examined 200 cases of acute infections other than typhoid, again selecting young and otherwise normal subjects, and following up his examination through arteries of all magnitudes to the capillaries. Of these 80 were of diphtheria, and 80 were of scarlet fever. In diphtheria, says Wiesel, small opaque grey spots may become visible on the aorta even by the sixth or seventh day (but at this date rarely beyond the aorta) ; indeed, so far as the aorta is concerned, this observation was applicable to all the infections. Moreover, in diphtheria, by the microscope, a degenerative process was almost always revealed in the coronaries (this " never wholly failed"). At the very beginning, before either intima or media is disintegrated, the media is soaked in serum ("Durchtränkung"), and by it the elastic lamels and muscular fibres are forced apart, or squeezed together. This juice is a homogeneous badly staining substance, with a leucocyte here and there in it. In aspect, though not in nature, these changes vary of course somewhat with the magnitude or structural variety of the particular vessel examined (i.e. with the relations of the tunics). Then the elastic fibres begin to refuse the stain, and by the eighteenth day these and the muscular fibres break up, and fall into necrosis.

These patches of damage in the media, patches of almost homogeneous matter in which a few casual nuclei and some scraps of elastic fibre may be seen, run in size about $5 \mathrm{~mm}$. In the severer foci the intima does not escape, but also begins to swell towards the lumen; the adventitia is usually intact. Such patches were found in about 40 per cent of the diphtheria cases, and were frequent in scarlet fever, influenza, septic diseases, 
and erysipelas ; in pneumonia they were rarer, and in measles and chicken-pox rarer still. In none of them were any calcareous nodules to be detected, as seen in adrenalin experiments. As regards restitutio ad integrum, later autopsies on survival cases were difficult to obtain, but such material as the author was able to obtain indicated that this took place by new vascular twigs from the adventitia, nuclear proliferation, and substitutive connective tissue. The author reminds us that to crush a healthy artery does not thus lead to dissolution of the elastic fibres, or at any rate not until active inflammation has continued for a while. I think Jores demonstrated that elastic fibre, or the elastic layer, is not insusceptible to exudative and proliferative changes (in influenza?). I may remark by the way that in practically all these infections the blood pressure is rather low than high.

If it be true then, as in Wiesel's judgment, that these toxic effects of the infections are not exactly atherosclerosis, but medial disease with but occasional and consequential intimal lesion, in speaking clinically of the thickened arteries in young persons which may follow such maladies, we shall have to revise our common language, if not our concepts. For these observers, and Marchand likewise in similar observations, have been careful to exclude renal complications. Thus we find a disposition to separate what Wiesel rather barbarously calls "Arteritis chronica postinfectiosa" from atherosclerosis. It may be noted that, save in respect of the adventitia, these infections in their incidence on the media fall into line with the syphilitic and rheumatic. However, I think Professor Marchand is of opinion that syphilis starts rather in the outer elastica than in the adventitia.

In influenza the arterial thickening is likened by Fort ${ }^{1}$ to that of typhoid. Of its effects on the aorta I shall treat in the chapter on Aortitis. Fort says truly that in influenza the arterial change is most commonly seen in persons over the age of 30 , and perhaps rather predisposed to degeneration. It is most frequent, and appears first, in the vessels of the lower limbs and, at the beginning at any rate, seems often to be unilateral. It may have a sudden onset and, if in a considerable vessel, with active pain, even severe enough to need morphia. On

1 Fort, Thèse de Paris, 1901. 
this may follow the usual loss of distal pulse with dangers of gangrene. Of a certain patient Dr. Mitchell Bruce ${ }^{1}$ says that after an attack of influenza a systolic apex murmur appeared, and physical signs of dilatation of the aortic arch, which progressed until, with associated causes, death ensued.

Tubercle, as we shall see, occurs in the aorta rather as a rarity. Its toxin is credited occasionally with the effect of general arteriosclerosis. Tubercle is but too common an experience in the practice of all of us, and for my own part I have never observed clinically any such changes in the arteries of the tuberculous which could be regarded as more than contingent in nature; although of course an arterial district is often involved in a tuberculous inflammation (a true arteritis). There is no definite evidence that tubercle is apt to produce a general arteriosclerosis. $^{2}$

Of the effects of syphilis on the arterial structures we know so much, and on this subject, in the section on Morbid Anatomy and on Aortitis, I shall have so much to say, that in this context I may be content with a brief summary. Hasenfeld and Szili of Budapest used the Wasserman test in 188 old ("hochbetagten") men with general arteriosclerosis, not clinically distinguishable from the ordinary form, and found it positive in $20 .^{3}$ The results of such examinations depend a good deal upon the kind of population investigated; still such reports are significant of that co-operation of alien toxins with " wear and tear " which so often presses itself upon our attention (p. 236). Dr. Mott ${ }^{4}$ is " convinced that syphilis is an underlying factor in a large proportion of cases of cerebral arteriosclerosis in persons of both sexes between the ages of 45 and 55 who have acquired syphilis in earlier life." If in some such vessels a recent syphilitic arteritis may be distinctive, ultimately the changes may be indistinguishable from ordinary arteriosclerosis (p. 480). We are not surprised to read again and again that in General Paralysis of the Insane, even in its juvenile

1 Bruce, M., Luml. L., Lect. III.

2 Faber, whose book (Die Arteriosklerose, 1912) did not reach me until this manuscript was made up for press, states, somewhat to my surprise, that arteriosclerosis is quite common in tuberculosis, and comes early. I can now do no more than refer to this able essay.

3 Hasenfeld and Szili, Deutsche med. Wochenschr., Oct. 28, 1909, p. 1903.

4 Mott, F., Allbutt and Rolleston's System of Med. vol. iv. p. 613. 
form (as in one patient æt. 18), " atheroma of the aorta" is very frequent (Vol. II., p. 181). Dr. Mitchell Bruce (Lettsom. Lect., 1901) stated that in 28 cases of syphilis with cardiac symptoms 9 presented aortic disease, and all the others had ringing second sounds over the aorta (p. 389). In half the cases pain of an anginal character had occurred (vide Essay on "A. Pectoris," Vol. II., p. 424). In two-thirds the radial artery was sclerosed (p. 295). He agrees with Dr. Mott, and, if I may say so, with myself, that these processes are still imperfectly understood.

So far as my experience goes, syphilitic arteriosclerosis usually appears in two to three years from the primary inoculation; sometimes it seems precocious, in other cases it is delayed, or apparently delayed. Marchand quotes from Darier and Nonne well-authenticated instances of syphilitic arterial lesion eighteen months, nine months, eight months, and five months respectively after primary infection. Benda, quoted in Aschoff's Lehrbuch, reports such a case a year and a half after infection. But we must not confuse delay of obvious arterial changes with delay of clinical consequences; even within the arteries themselves these changes often progress silently and latently. Moreover the history of this specific infection is often very slippery: one who confesses to the infection may still have good reason to conceal its date and occasion; and middle-aged sinners are tempted to push back the date of the scourge to the reckless days of youth. Not only so, but other poisons, as of influenza or of typhoid, or again the deteriorations of alcohol, may have intervened. Still I suppose it is true-I have some evidence to suggest it - that signs of general syphilitic arteriosclerosis may be delayed even for twenty years. In hereditary syphilis, on the other hand, as we have seen, the invasion of general arteriosclerosis may be very precocious. (See under "Age," p. 174.)

With syphilitic aortitis- "schwielige Aortensklerose," the "Mesoaortitis productiva" of Chiari, the "miliary gumma" of Létulle-I shall deal under the head of Aortitis (Vol. II., p. 167); here I am considering the specific lesion only as it occurs in the vessels of smaller magnitudes. Syphilis of the large vessels, as we shall see, was recognised even by Paré, and by Bianchi (quoted by Morgagni) ; to Morgagni it was of course well known. This great pathologist seems also to have perceived syphilitic disease 
of the smaller vessels, ${ }^{1}$ but it was not until Virchow's paper appeared (in the first volume of his Archives) that small-vessel syphilis was clearly recognised. ${ }^{2}$ Then followed Wilks's descriptions, in 1863, of visceral and vascular syphilis, stubbornly contested by his contemporaries. Wilks wrote, in his Lectures on "Pathology" (1859):- "The arteries are in all probability susceptible of the same influence; that is, a deposit, atheromatous or of an analogous kind, forms within the coats, leading to various consequences; thus, in some cases of aneurysm and of softening of the brain from diseased vessels, syphilis has been the most probable cause." And in his Guy's Lectures on "Syphilis " (Lancet, Feb. 9, 1867), after speaking of syphilitic meningitis, etc., he points out how otherwise also "the blood vessels by a fibroid thickening of their walls, as in other structures of the body (bring about a) ramollissement of the cerebral substance." Two years later (St. George's Reports, 1869) I was enabled to give the histological description, from the brain of a soldier who presented ample evidences of lues and died of specific cerebral disease. Heubner's fuller account of syphilis in this area appeared in $1874 .^{3}$

The question which meets us at the outset is whether in nature the lesion be single or various? As syphilis may be a disease not of this tunic or that but of the whole vessel, and as its kinds, or modes of incidence, bear directly upon clinical diagnosis, it is desirable, in order to answer this question, to anticipate some considerable part of the histology of vascular lues. In the chapter on Morbid Anatomy (p. 478) the division of the subject into lesions of the several coats respectively is less applicable to syphilis, which is prone to attack them all. In the earlier years of my observation of syphilitic vascular disease it so happened that I found, or thought I found, universally, even in the younger cases, a morbid change, apparently fibrous (p. 480), more or less throughout the accessible arteries ; for it so fell out that amongst a long series of advanced syphilitic cases which came under my notice a sclerosis of these arteries was so frequent as to

1 Comp. Proksch, Arch. f. Derm., 1878.

2 See Sternberg's retrospect, which I know only from Canstatt's Jahrbericht, 1861, Bd. iv. S. 328.

${ }^{3}$ Die luetischen Erkr. in d. Hirnarterien, Leipzig, 1874. 
tempt me to suppose it to be a general feature. This however proved not to be the case; in the turn of experience another run of cases occurred in which, although for example the aorta might be manifestly diseased, yet the accessible arteries were to the touch unaffected. Dr. Bruce, in his notes of syphilis in persons under the age of 40 or 45 , says that in two-thirds of all the cases, besides the more specific features, the arteries of the limbs were thickened evenly to the touch, not presenting palpable calcification. I saw a man, æt. 43, at Osborne with Col. Kilkelly, in whom all accessible arteries were thick, and the characteristic clanging second sound of syphilitic aorta audible. The infection dated from a few years before. The pressures were moderate. But in many cases of aortic syphilis the peripheral vessels are soft enough. I am speaking here of palpable arteries only; but of course, while these are exempt, other arteries of the periphery - the cerebral for instance - may be affected. I recall many such cases; one in a medical man, infected in the course of his profession, whose symptoms pointed unmistakably to cerebral syphilis, and apparently to an infection of the basilar artery; yet in him every palpable artery was to the touch normal. Conversely, about the same time another patient, near the same age, presented similar cerebral symptoms, but in this case every accessible artery was much thickened. Professor Osler describes three cases of the "diffuse sclerotic type with no limitation of area." 1 My first patient, the medical man, made a complete recovery under mercurial inunction, and after many years was still living and well ; in the second case, although by the same means the earliest symptoms were wholly and permanently dissipated, dulness appeared under the manubrium sterni and extended to the right; the dilated anfractuous aorta of Hodgson was diagnosed, and at death some years later this diagnosis was fully verified by necropsy. I may state by the way that as, in the last few months of life, two or three years after the first definite signs of the aortic disease, this distinctive process, according to its wont, extended visibly to the innominate artery, an enterprising surgeon of no little note distressed the patient by the opinion that this vessel ought long before to have been operated upon for aneurysm!

1 Osler, Sir W., Luml. Lect., April 9, 1910. 
Why in some cases syphilitic arterial disease is thus general or universal, why in others it is limited, and by no apparent rule, to this, that, or the other district of the arterial tree, as, for instance, to the ascending aorta, and why again it appears in at least two shapes-as a focal lesion, as a more diffuse fibrosis, or in places with intimal lesion not unlike atherosclerosis -are questions as yet beyond our comprehension; but as regards the generalised cases, or at any rate as regards cases of syphilitic sclerosis of the vessels of the limbs and of the aorta, I think that this distribution may be determined by functional stress. Such is the impression I have received from cases under my own observation, for this has happened in men engaged by business or sport in hard muscular exercise.

And now to return to the enquiry concerning the nature and forms of this syphilitic arterial disease in areas, if in kind or in shape it be one or several. This question we ask as, in its more diffused distribution, such as I have alluded to, in the limbs, the change is supposed by some pathologists to be a diffuse fibrosis rather than extended lesions of the types recognised in the aorta, or in the cerebral arteries, and other particular districts. Let us then try to learn the essential nature of the well-known and often-described syphilitic arterial disease, whether it be single or multiform, whether distinct in nature from atherosclerosis, or but a variety of this. In some cases, in later stages, and in elderly persons in whom atherosclerosis may, and usually does, supervene upon the specific lesion of the vessels, it may be difficult in a particular specimen, even with a fat stain, to draw clear distinctions between syphilitic and atherosclerotic lesions, especially in disease of old standing; so if we are to form a judgment upon real or alleged histological distinctions we must examine the lesions in their earlier and clearer stages. If we turn to a recent author of mark on the subject, such as Dr. Mott ${ }^{1}$ or Professor Adami, and compare our own observations with theirs, we shall find that, whether there be also a diffuse fibrosis of the long arteries or not, the inward arteries are commonly attacked in a way which, if not in nature, may be different in form from the invasion of the aorta and larger vessels.

1 Mott, F. W., Allbutt and Rolleston's System of Med., art. "Arterial Disease," p. 567. 
It is generally true that the cerebral, the coronary, the hepatic, the renal arteries and so on, and furthermore the veins also, are invaded primarily from the outer side, and, in contrast with atherosclerosis, by a process which is not from the beginning virtually degenerative but is inflammatory or sub-inflammatory, the result of an irritant; so that, logically speaking, syphilitic arteritis is not atherosclerosis, and has no proper place in this category. Atherosclerosis, being degenerative, never propagates itself to contiguous tissues. In syphilis the adventitia is attacked, as it seems, by some irritant passing in the periarterial lymph channels, channels especially notable of course in the brain where we see an arachnitis involving the vessel, and thence a periarteritis with the inflammatory features of proliferation, infiltration, diapedesis and so forth; the vasa vasorum being manifestly in active participation in the process. It is true nevertheless that, although this process is inflammatory, the inflammation from the beginning verges upon degeneration, and may soon be merged in it, and that then the intima becomes involved also. Yet if this first inflammatory stage be promptly met by specific treatment, it would seem from clinical evidence that the drift to degeneration may be prevented, or at any rate arrested, and cure effected; a cure which in the earliest phases may be practically complete, possibly even without a scar (local fibrosis). On the other hand, if the mischief be not arrested, it may advance in a manner not closely like atherosclerosis, but so destructively as to reduce the arterial branch to a thin and hard, fibrous, not calcified, cord (arteritis obliterans). A typical and carefully described case was read by Buzzard and Sankey before the Pathological Society so long ago as 1877. ${ }^{1}$ They exhibited the adventitia as the seat of an active small-cell infiltration with increased activity of the vasa vasorum, new or newly visible twigs of which penetrated into the intima; the media presented a similar proliferation, and the muscular fibres had passed into granular degeneration. . In the intima were no plasma cells but some fibrous hyperplasia. In a girl æt. 19, subject of congenital syphilis, Biermann ${ }^{2}$ reported arteritis (apparently) universal. The posterior tibials on both legs were

1 Pathol. Soc., Report of Meeting in Lancet, Feb. 10, 1877.

2 Biermann, Deutsche med. Wochenschr., 1911, No. 25. 
thus obliterated, and the left radial was much reduced in calibre. Yet even then, if the anatomy of the circulation permits, as the parcel of the vessel above the block dilates, a more or less efficient collateral circulation, according to the vascular supplies of the part, may thence be established.

Now, if we suppose a cerebral vessel to be caught quite in the early stage, the changes in the intima described by Heubner and myself will not yet have appeared; otherwise they soon do appear; and they may depend primarily, as Baumgarten argued, upon processes in the adventitia and vasa vasorum, whether by a propagation of the specific irritation or merely by interference with nutrition. However this may be, in the form we described, the media, instead of falling before the first inroads of poison, as it does in the aorta, may be practically intact. The adventitia, or even the intima, succumbs first, the intima swelling even to direct occlusion of the lumen ; yet, white and cord-like as such a basal cerebral artery may be, it contains no lime. In these cases the intima may be greatly thickened, and breaking through the marvellously enduring endothelium combine with thrombosis to stop up the channel ; or a patch may present itself in an "ulcerative" form very like an atheromatous ulcer." ${ }^{1}$ Spirochætes have been found in such lesions in early cases.

In the larger vessels the occurrence of independent luetic foci in the intima, to which I have alluded, is rare. However, I repeat, when such intimal changes are established, it may become difficult or impossible, especially in elderly persons, and in some arteries, to discriminate between syphilitic and atherosclerotic disease. The rule, originally my own, ${ }^{2}$ that, unless mingled with atheroma in elderly persons, the specific process is much less subject, in itself indeed is not subject, to fatty and calcareous deposit, but is more disposed to fibrous and thrombotic occlusion, has been generally accepted. A scrutiny of the perivascular tracts also may aid in the distinction; a perivascular fibrosis, or an extension of gummous material, may have penetrated far into these, and have obliterated

1 A coloured illustration of syphilitic cerebral artery, from a chimpanzee, is given in Grünbaum's Morbid Histology, p. 106.

2 See Fagge's Textbook, 3rd ed., vol. i. p. 551. 
considerable batches of small vessels. In $1869 \mathrm{I}$ demonstrated in certain syphilised brains nodular masses, as large as a filbert or more, in which, by diffuse degradation of the web of and about the vessels, parcels of cerebral matter were reduced to a coarse necrotic lump, blending at the circumference with the more normal substance around them. ${ }^{1}$

The syphilitic poison is as ready to attack veins and lymphatics as arteries, perhaps more ready.

Whether in association with General Paralysis, with the palsies of consecutive atrophies, or again with partial convulsions, distinctive characters of luetic vascular disease in the brain, spinal cord and retina have been carefully described by many writers ; but similar changes, again not always easy to discriminate from atherosclerosis, are found not rarely in other arterial districts. If, wherever they be found, they are associated with characteristic vascular and perivascular gumma, for instance in the coronary or hepatic districts, the recognition of the specific nature becomes plain. Still it is impossible to offer for common use, as a canon of distinction, this rule - that in syphilis the disease begins from without the vessel and proceeds inwards, whereas in atherosclerosis the process is from within outwards. As a generalisation this statement has much truth in it, but in practice the histological characters may be indecisive; indeed the several coats are often so confused, especially in the elastic vessels, that the order of change is no longer apparent; the luetic nature of small deposits often eludes demonstration, and we have to make our diagnosis on the associated conditions : these, however, if minute search is made, are usually decisive.

Notwithstanding, disease of the media is in syphilis both characteristic and independent, and is, moreover, if only as a source of aneurysm, of great practical importance. The media is prone to suffer before the intima, and in a more specific form. Not in the elastic arteries only, indeed in vessels of the muscular type, granulomatous foci, isolated in the media, may readily be demonstrated. And not in the aorta only, but also in the arteries of secondary magnitudes, little constellations of punctate lesion, rather presumed than known to be "gummous," are in luetic

1 A similar lesion is described and figured by Niesl in his Beiträge, Bd. i. H. 1, Berlin, 1913. 
disease frequent, and to the microscope conspicuous, in the media; granulomatous patches quite independent of adventitia or intima. But around them, and in other places, plasma cells may be seen in groups and layers, and a fibrosis pushing inwards and outwards from adventitia and intima; the outer media being usually involved more or less from the beginning in the morbid activities of the adventitia. ${ }^{1}$ I have said that in these respects the process resembles the changes produced in Mott's and in Sumikawa's experiments of painting the outside of a vessel with some irritant. On this primary luetic disease of the outer elastica and media, injuring and enfeebling this coat, ensue those warpings and bulgings of the vessel which give rise to more or less sacculated extrusions of the wall, to "dissecting" hæmorrhages, or, in the finer twigs, to thrombosis. When patches of fat or lime are seen, they suggest, or even announce, a blend of atherosclerotic with syphilitic disease, as also would a wellmarked splitting of the elastica (p. 520), a layer which in syphilis is more prone to decay than to multiplication.

Is this medial disease gumma? Well, as Dr. Andrewes once said to me, "gumma" is rather a clinical and macroscopical than a pathological term. In histology the ordinary minute cellnests in the arterial coats are in nature homologous with more conspicuous gummatous swellings; that is, in vascularity of young granulation tissue, abundant plasma cells, early necrosis, and (in the artery) obliterative inflammatory processes. Moreover there is a larger proportion, than in atherosclerosis, of fibrotic (productive) to degenerative products. Histologically there is more activity in the stellate and spindle cells, more exudate. The lumen may be uniformly or crescentically narrowed, or if the intima be deeply engaged, even closed; and often it is actively vascularised, though at the same time, e.g. in the brain, it blends with the media and this with the adventitia which may be adherent to the pia mater, and this again to the cerebral substance. Probably the lymph sheath is always an active area of the infection. Thus Jores also compares luetic arterial disease with his "regenerative" ("Bindegewebswucherung") form, comparing it with the "endarteritis" of ligature, of pulmonary cavities, and of meningitis. My own impression

1 A coloured illustration is given in Létulle; for reference see p. $202 n$. 
is that the distinction in the smaller vessels between atherosclerosis and diffuse syphilitic sclerosis is a true one, but is apt to be obscured by coincident atherosclerosis or, except in its fresher stages, to lose its peculiar characters. To quote Dr. Andrewes again (loc. cit. p. 226)-although he thinks that the specific thickening is more fibrous all through the wall, and the lumen more narrowed, he regards this as a matter of degree rather than of histological kind. Moreover, such a change is not confined to syphilis, but may be seen in cerebral meningitis, in tuberculosis, and so on. These, we repeat, are arteritis, not atherous degeneration; they are inflammatory, and often begin from the adventitia as such. However Stein 1 has reported a case of syphilis in which disease of the common iliac artery presented the features of ordinary atherosclerosis and not of aortic medial disease. The elastic fibres were multiplied and in the intima was the ordinary swelling of fatty decay. The media was fibrosed and the adventitia thickened, but about the artery there was no specific criterion. Josue also admits that the histological discrimination may at times be very difficult. Still, for minor vessels, hard as it may be to identify particular cases, we may perhaps venture to make the broad contrast, that diffuse syphilitic sclerosis consists more of fibre; that the adventitia is involved, and the vasa vasorum also; that the proneness to fatty necrosis and calcification, if it exist at all, is far less ; and that in fresh cases small-cell infiltration and other evidences of inflammation may be detected, and often foci of syphilitic deposit in the media. It is true that some of these changes are due rather to particular ways of interference with nutrition than to specific tissue change; still, however engendered, they enter into the sum of our interpretative data.

Summarily then, whether in syphilis, besides the now wellknown irritative process of a granulomatous character, attacking most frequently and commonly in the first place the adventitia, or also frequently, early, and independently, the outer media, but rarely, in a primary and independent sense, the intima, seats in which, in the aorta, the spirochæta has often been detected, whether, besides this now well-known process, there may be another in which the sclerosis is general and diffuse, possibly

1 Stein, Virchow's Arch. Bd. ccxi., 1913, H. 1. 
"parasyphilitic," of a fibrous rather than an atheromatous nature, and falling especially upon the vessels of the limbs, and whether furthermore there may be an endarterial form in which the process takes something of the order and semblance of atherosclerosis, starting in the intima only or chiefly-a mode which certainly has been detected (Teacher, p. 479); whether syphilitic arteritis be thus multiform or, more broadly speaking, twofold, after the first two modes, the third being no more than the common blend in later life of ordinary senile fibrotic or atherosclerotic changes with a foregoing syphilitic lesion, we are not yet in a position to decide. Yet after all, disease certainly syphilitic, and syphilitic only, is, in its more advanced stages when the whole vessel is involved - say in the coronary or cerebral area-often hard to distinguish from atherosclerosis; indeed we must admit that histologically the distinction may occasionally be impossible.

In respect of my main clinical division of Arteriosclerosis into that associated with high pressures and that not so associated, I wish here to impress upon the reader that grievous and extensive as syphilitic arterial disease may be, salient, in many cases, as a great deformity of the thoracic aorta, with or without a general peripheral thickening, yet, unless complicated by renal or by other incidents, in no one of these cases, and we see scores of them, have I chanced to find the blood pressure ranging above normal. And in not a few of my own cases observations to this effect have been confirmed by expert colleagues.

Upon the remarkable cases of universal arterial degradation in congenital syphilis, cases of which no inconsiderable number are now recorded, I do not return; histologically these arterial ruins are too far gone for interpretative data, and their interest is other than histological (p. 174). On the systematic morbid anatomy of syphilitic aortitis and arteriosclerosis I offer some further remarks in later chapter (p. 478). ${ }^{1}$

Of the poisonous effect of some other microbes upon the blood vessels, the following case, quoted from Dr. Andrewes' essay in the Report of the Medical Officer for 1913 (Infectious Origin), is a remarkable example:

1 For a fuller discussion of this subject I would refer to Dr. Mott's article on Arterial Disease in Allbutt and Rolleston's System of Medicine. 
A girl, aged 17, died after an illness of four months' duration -a pyelo-nephritis due to $B$. coli. There was no suggestion of syphilis. Apart from a few superficial fatty streaks along the descending aorta, the lesions were limited to the transverse arch and to the origins of the great vessels of the neck. Here were large projecting intimal plâques up to half an inch in diameter, firm in consistency, and covered by an intact endothelium. They resembled the ordinary plâques of nodular arteriosclerosis, except that they were purely fibrous and contained no elastic elements. Fatty and even calcareous changes were present in the deeper parts of the thickenings. No bacteria were demonstrable in the intimal plâques ; but in the adventitia, and extending a little way along the vasa vasorum into the media, were scanty areas of inflammatory infiltration, and in these situations I found a few groups of bacilli, negative to Gram's stain. Cultures could not be carried out, as the specimens had been put into formalin. (Illustrations given in original paper.) See Vol. II., p. 160.

Filtered staphylococcan toxins are said to constrict the arteries (of cats). Foreign serums-e.g. of sheep, ox, swineare said, on cats, to have a like effect. ${ }^{1}$

The association of cancer with arteriosclerosis, noted by Edgren, is probably fortuitous; both maladies pertain on the whole to advanced life. Still now and then one is much impressed by this association in very marked degrees, and we may be led to speculate upon cytolytic toxins, or upon degenerative proclivities common to both; but such speculations are too vague for present consideration.

This seems a fitting place to consider certain cases of arterial disease attributed to neuritis, itself probably the consequence of some bane, as in gout. In Tabes, General Paralysis, and other infections, arterial degenerations and neuritis may be attributable to the common cause of both. But the part which diseased nervous strands may play in determining a sclerosis of the associated arteries has been much discussed; for instance, by Virchow, Vulpian, Lobstein, Nothnagel, A. Fränkel, Martin, Bervoet, Bechterew, Marchand, Chantemesse, Eichhorst, one might add by Bernard and Brown-Séquard, both from the clinical and the experimental point of view; but without much more result than the collection of many curious

${ }^{1}$ Läwen u. Dittler in Hering's (Leipzig) laboratory, Zeitschr. f. exp. Med., Bd. i. H. 1, 1913. 
cases ; such, for instance, as that of Chantemesse ${ }^{1}$ in which an injury to the brachial plexus was followed by well-marked arteriosclerosis of the wounded arm only, the other arm, both as to nerves and vessels, remaining quite normal. Similar results have been described as dependent upon injuries of the sciatic, the peroneal, and the tibial nerves, and of others no doubt; but it is often difficult to say in what relation the nervous and the vascular lesions stood towards each other. Oguro, working under Hansemann, ${ }^{2}$ detected in preparations of arteriosclerosis from 6 cases a concomitant affection of the local nerves, and leaned to the belief that the nervous change might be the primary lesion; but he was frank enough to add that in 19 similar cases his results were negative. Israel thinks that the vessels thus suffer when under nervous influences pressures rise; or, I might add, when the vessels dilate and undergo atonic stretching. Aschoff thinks Israel's explanation very dubious, and so do I; however I have considered the point elsewhere. Fränkel's alleged alteration of arteries in areas in which he had cut such a nerve as the sciatic is not very convincing; but in all such experiments it is very difficult to bring temporary lesions into line with the slower tooth of time. Most writers follow Bechterew. ${ }^{3}$ Fränkel reported that after section or irritation of the sciatic, followed by lively vasomotor effects, morbid changes eventually, but after a long time, appeared in the smaller arteries. They began in the adventitia, then the unstriped fibres became opaque and granular, and as new vessels appeared connective tissue increased, both in media and intima. The same changes are said to follow pathological neuritis. Similar changes occur in the veins and lymphatics. Dr. Todd, ${ }^{4}$ in a case of cervical rib with trophic palsy of the sympathetic fibres, found disease of the intima of the arteries leading to thrombosis. He points out however from Schäfer's Physiology (Langley, and others), that the sympathetic does not pass down the main vessel but-in this instance-

1 Read at the Paris Medical Association in February 1908.

2 Hansemann, Virchow's Arch. Bd. excviii. H. 3.

3 Bechterew, Die Funkt. d. Nervencentra, trans. Weinburg, H. 1, 1908, pp. 544 et seq.

4 Todd, W., Journ. Anat. and Physiol. vol. xxvii. Photograph by Hirsch, Lancet, May 17, 1913. See also Bruce, A. N., Arch. Exp. Path. and Pharm. No. 63, 1910 ; and Fränkel (quoted Todd), Wien. klin. Wochenschr. vol, ix., 1896 ; see also Todd, Journ. Nerv. and Mental Dis, vol. xl., July 1913. 
by the sciatic and anterior crural nerves. More definite are certain experimental results, obtained by Manouélian, of the Pasteur Institute. ${ }^{1}$ He sought to erase a nerve supplying an artery directly and, after other endeavours, stripped off the sympathetic nerve passing to the abdominal aorta, near the origin of the left renal artery, and to the pulmonary artery respectively, in dogs. The dissections were most carefully made, so as to avoid any other disturbance of the parts. The effects in "sclerotic foci," which seemed constant, were degeneration of the elastic and muscular fibres and atherosclerosis, but calcification scarcely occurred; the parts took on a hyaline form of decay. The author promised to pursue these researches. Foerster of Breslau ${ }^{2}$ describes a painful neuritis (sciatic, brachial, etc., and more rarely trigeminal) due to sclerosis of the vasa nervorum, which he distinguishes from senile neuritis, in which the nerve is more tender to pressure and the arteriosclerosis perhaps not so obvious. His point of view is, conversely, of disease of nerve following upon arteriosclerosis. The article does not admit of abridgment. There were no losses of reflex, nor palsies, until muscular atrophy also set in. Such inferences Klotz (loc. cit.) contradicts, saying that the sclerosis due to the nerve lesions is septic, a gradual infectious arteritis, going on to chronic fibrosis. And many microbes take neural routes. In the larger vessels there may be a mesarteritis with conspicuous hypervascularity. Or under the intimal changes there may be a medial degeneration, due to prevention of nutrition from the lumen. Sir William Gowers speaks of an "atheromatous neuritis" -an atrophic inflammation, or fibroid reaction.

Of "thrombo-angitis" (Raynaud's disease, certain obliterative arterial diseases, chronic and acute, and so forth), I say little. These are not arteriosclerosis, nor thrombosis of merely sclerosed or constricted vessels. Some other factors, probably including epithelial destruction, are concerned in these obscure and often grievous and painful lesions. ${ }^{3}$ Syphilis may be concerned in some few of them; and other infections in some others. To

1 Manouélian, Ann. de l'Institut Pasteur, tome xxvii., 1913.

2 Report of discussion in Deutsche med. Wochenschr., 1912, and Wien. med. Wochenschr., Jan. 25, 1913.

3 See Buerger, Amer. Journ. Med. Sci. vol. cxxxvi., 1908.

VOL. I 
witness the active and agonizing progress of acute obliterative arteritis, as one sees it occasionally,-I am thinking of one of my few cases, an attack in the arteries of the left arm of a middle-aged woman, - is to be compelled to guess at some virulent infection as the cause of such an outbreak.

Some remarkable cases of arteriosclerosis are on record which stand on the confines between general and local nervous influences ; such as an early and concurrent invasion of this malady after shocks or accidents affecting the nervous system more or less as a whole, as in "Traumatic Neurasthenia." To ascertain the true values of evidence of this kind, we need more data than we usually get. Rivalta has published a striking suggestion of such a sequence. ${ }^{1}$ The patient, a young man æt. 29, who had never suffered from syphilis, and was not addicted to alcohol or tobacco, fell from his saddle on his head. He vomited, and was insensible for ten days, and remained in bed for twenty-five days. A month after he got about it was noticed that his temporal arteries were increased to the dimensions of a goose quill. The walls were thickened and very tortuous. The radials and other accessible arteries were also much thickened and "tense." His lay friends noticed the change in the temporal arteries. What was even stranger is that six months later all sign of the arterial lesion had vanished!

But I will not dwell on points of no great importance to my subject, as in many of the cases uniformity of data and testimony are still wanting. In " nervous" subjects, of whom we all see a good many, I have carefully looked for signs of precocious arterial . decay, but have not been impressed hitherto by their undue frequency; I am not yet prepared therefore to agree with Mitchell Bruce and Israel, if they lay much emphasis on causes of this kind. "Neurotics," it seems to me, often nurse themselves into old age without presenting undue manifestations of arterial disease. Aschoff thinks Israel's postulates dubious, and indeed Dr. Bruce, in asking if disease of the cerebral arteries in women may be attributed to "their precordial oppressions, flushings and false angina," says judiciously that "many other

1 Rivalta, Il Policlinico, March 1907; Epitome, Brit. Med. Journ., 1907. I can only refer to the article by Rumpf, "Arterioskl. u. Unfall," Deutsche med. Wochenschr., 21. Mai 1914, which appeared too late for this book. 
explanations may reasonably be offered." 1 Moreover, apoplexy (sanguineous) and "hemiplegia" are too often lumped together, a confusion I deprecate and have tried on another page to clear up. The nerves of the sympathetic system may, as has been suggested, exercise a "trophic influence" upon the heart and blood vessels, but this is a mere guess; and the probability that the accelerator, if unchecked, is quite capable of wearing the heart out, is some evidence to the contrary.

Cachexia, as a vague term for failing and perverted nutrition, or cytolysis, may affect the arteries as in the following case :

Male æt. 30. Seen with Dr. Huntly at Sudbury. Tall, sallow, dark complexion. Profound cachexia. Rapid cancer of liver. No syphilis. Vessels ill filled and pressures very low, but all accessible arteries thick. Aorta probably altered, as it was visible and palpable in jugulum. Heart's sounds short and weak, but second aortic clacking as if vessel atheromatous.

Finally, I must allude to pregnancy as an alleged cause of high pressures and consequent arterial disease, with or without chronic renal disease. That pressures may rise in the later weeks of pregnancy, and if at or above $150 \mathrm{~mm}$. cause in the mind of the obstetrician even more anxiety than the appearance of albumin, is true; especially if, on delivery, the pressure does not fall. Setting aside the many cases of hyperpiesia in childless or unmarried women, there is no convincing evidence that a normal pregnancy raises pressures notably, except of course during the act of parturition. Dr. Galabin first drew attention to the importance of observing arterial pressures in pregnancy, and since that time many observers, such as Oliphant Nicholson, Durosiez, Gerhardt, have investigated the matter with conflicting results. Stengel and Stanton made careful records in 70 cases, ${ }^{2}$ and their conclusion, which seemed convincing, was that, whatever displacements of the heart may occur, there is, notwithstanding, no abnormal increase of pressure nor cardiac hypertrophy. Vogeler of New York, Dr. O. Nicholson, and Dr. H. Starling, ${ }^{3}$ while emphasising the duty of watching for any persistent excess above 130-50, and of keeping any parturient whose

1 Bruce, M., Luml. L., Lect. III.

2 Stengel and Stanton, Penn. Univ. Med. Bull., Sept. 1904.

3 Starling, H., Lancet, Sept. 10, 1910. 
pressure is over 130 under close supervision, have corroborated this opinion on the whole. My own impression is that in so far as pregnancy may cause renal disease it may promote a consequential arteriosclerosis, but no further; and that a slow pulse and labouring heart are the chief diagnostic signs. But for an adequate discussion of this subject I must refer the reader to the papers of the authors whose names I have mentioned.

In revising this chapter on the Causes of Arteriosclerosis I sympathise with a reviewer who, after reading a certain dissertation on another disease, exclaimed, "We would ask what the conditions are which do not bring on this malady!" And every failing element is both cause and effect.

Note to p. 260, par 3, l. 12.-In conversation with Professor Hochhaus he informs me that in the course of hospital routine blood counts have been made in many cases of my hyperpiesia (i.e. persistent high blood pressure and cardiac hypertrophy without clinical evidence of renal disease), and that had these counts presented any abnormality his attention would surely have been drawn to it. On the other hand, I found in Addenbrooke's Hospital, on taking charge of wards in August 1914, two cases of hyperpiesia, one in a man of about 48, the other in a woman over 50. Both had pressures of 190-200. Though albumin appeared variably, most sedulous tests of the urine in both cases failed to reveal any casts whatever. The specific gravity ranged in both at 1020 and over. Now in the man the red corpuscle count was but little under six millions, in the woman five millions, a range which for an elderly, worn and anæmic-looking woman was unexpectedly high. The heart in both cases was enormous. The man had large dropsy of the legs and one pleura, but no orbital nor subconjunctival œdema. 


\section{CHAPTER V}

\section{ARTERIOSCLEROSIS AND THE KIDNEYS 1}

INTRODUCTION.-The problem which we have now to consider is one of the most alluring and the most baffling in pathologyalluring, perhaps, by its very subtlety. Whether we think to approach it by the way of clinical observation, or of the exacter methods of clinical research-the methods of chemistry and physics, or, again, by way of experiments in animals or of empirical tests in man, we find ourselves on every side disconcerted. These ways are strewed with conjectures and speculations withering untimely even before the early spring of a working hypothesis. At the bedside the physician, who indeed has made more of the task than his comrades of the laboratory, justly believing himself to have delineated certain broad lines of difference between kinds of kidney disease, when he has followed his cases into the dead-house, is disconcerted to hear that his "tubular" cases, being also "interstitial," and his interstitial cases being likewise tubular, the pathologist will have none of his divisions. Moreover, he is informed that, although a certain group of these diseases may be more obviously and extensively correlated with cardio-arterial disease, yet few cases of chronic Bright's disease fail to reveal to the pathologist-at least within the relics

1 This essay is an extension of an address delivered before the Kensington Division of the Metropolitan Counties Branch in 1911. See British Medical Journal, April 15 and 22, 1911; and in this journal for Aug. 10, 1912 is an abstract of a paper to the same effect read at the Brit. Med. Assoc. Ann. Meeting in 1912. In re-editing the chapter for this book I find many paragraphs in repetition of matters similarly dealt with already under Arteriosclerosis ; but after some hesitation I have decided for the most part to leave them in place. If true, reiteration is the way of conviction; in any case a reader of this chapter will find the perusal less tedious if, for parts of the argument, his labours are not increased by frequent cross reference, 
of the kidney-some measure of arterial disease. The physicist and the chemist, studying in Bright's disease the freezing-points and the electro-conductivity of the urine or of the blood, told us but yesterday that evidence of retained katabolic products of a non-conductive quality-and therefore of organic nature-was secured; to-day we are told, on authority as good, that had these investigators thought of applying the same tests to the normal man they. would have received much the same response; and that, in either case, the variables are too many to issue in consistent results.

Again, but yesterday we were told that in renal disease the urine indicated a lack of nitrogenous waste-waste remaining in the body to poison it; to-day we hear that, other things, especially food values, being equal, the total urine in renal disease is, save in extremis, often as well loaded as in health. Once more, we were to have found some standard of renal efficiency in the rates of discharge of conspicuous bodies-such as methylene blue or potassium iodide,-now these tests likewise are said to have failed in constancy ; the kidney, it seems, may work hard on one set of bodies, and either neglect others or, more probably, refuse to take notice of them until properly presented to it. Moreover chloride and iodide excretion depend upon obscure movements of the circulation, and upon variable tissue affinities. Finally, the physiologists were surmising that some of the occult influences of renal function might be due to an internal secretion, perhaps of a pressor quality; and there were rumours of a hypothetical "renin"; later investigators turn us back with the announcement that if renal extract, like almost any other organic extract, may raise blood pressure momentarily, yet the main effect is depressor or neutral. Well may Professor Adami say, in his Principles of Pathology, that arterio-renal problems are among the most complicated and obscure in the whole of pathology.

But from these diversions we must turn to the part of the subject which is to be the matter of the present discourse; namely, the relations of arteriosclerosis to the kidney, sick or sound.

Historical.-A generation ago, when " high arterial tension" 
began to awaken the interest of physicians, this condition was regarded as belonging essentially to disease of the kidneys. Such was Traube's view of it; such were George Johnson's, ${ }^{1}$ Burdon-Sanderson's, Mahomed's likewise. We have seen (p. 8) that the first shifting of this attitude was, in part, due to Mahomed, who proved that persistently excessive arterial pressures were not rarely to be found in persons in whom symptoms of renal disease had not become manifest; still Mahomed supposed, none the less, that this increase of tension essentially pertained to renal disease in one kind or another. In his brief but fruitful life Mahomed did not get beyond the doctrine that these high arterial pressures constituted an initiatory, latent foreboding of renal disease, ${ }^{2}$ a stage which he denoted as the "prealbuminuric stage"; not very happily as it proved, for, as we now see much more clearly, albuminuria is by no means a peculiar or decisive symptom of Bright's disease. Not only so, but as Huchard and I argued fifteen years later, albumen and high-blood pressures, even when taken together, are not by any means decisive of a diagnosis of chronic renal disease in the ordinary clinical sense of the term.

Soon afterwards arose another dislocation of opinion, when Lancereaux, Gull and Sutton, and Debove and Letulle ${ }^{3}$ contested Johnson's alleged arterial hypertrophy, and in so doing lifted the whole problem of cardio-arterial disease, and especially of the granular kidney proper, from the narrower notion of a kidney disease into the larger conception of a general constitutional distemper, of which the kidney disease and the vascular disease were but coefficients. On the other hand, in their larger view they lost sight of such distinctions as the "Granular" kidney, the Secondary Contracted (" senile white") and the Senile. Moreover, unfortunately, they named arteriosclerosis an arterio-capillary fibrosis, which it is not, or only in some part. Besides, the exaggerated notions of the effects on blood pressure of vasoconstriction in the kidneys, as interpreted especially by Traube, were shaken' by Cohnheim's proofs that arterial constriction confined to the renal areas is quite

1 Johnson's original papers were in the Brit. Med. Journ., 1867, and Trans. R. Med. Chir. Soc., 1872 and 1877.

2 Researches in the period 1875-1881, vide Trans. Intern. Med. Cong., 1881.

3 Med.-Chir. Trans., 1872. 
inadequate to produce even a temporary rise of general arterial pressures. Chauffard, in a survey of the variable and somewhat inconstant correlation of renal diseases with cardio-vascular changes, perceived that the relation was not thus merely mechanical.

Gull and Sutton were misled by the imperfections of their histological methods, especially by their use of glycerine. Johnson, who in Chauffard's words, was the opener of the histological period of renal disease, was too positive in his assertions and interpretation (p. 470). While preparing for a now obsolete article in the British and Foreign Medico-Chirurgical Review (p. 12), certain suspicions that Mahomed's prealbuminuric stage (p. 11) did not always issue in what, clinically speaking, we classify as one or other kind of Bright's disease-suspicions I had discussed with our lamented friend shortly before his death-grew and took definite form in my mind. By the nature of this enquiry, however, a long period of time had necessarily to elapse before it was possible by experience to be assured that excessive blood pressure may enter into more than one clinical series; and a still longer interval before I could hope to carry this persuasion to others. To compare morbid series so protracted as these was, generally speaking, possible only in private practice; yet in private practice at the end of such a clinical series to obtain a necropsy was a very infrequent opportunity. On the other hand, in hospital practice such patients came and went too irregularly for long and continuous observations. I have explained that sphygmographic curves, however suggestive to a practised operator, give no record of pressures. We had no pressure gauges; and even yet the graphic record of any continuous course of blood pressures depends too much on the occasional zeal of a house-physician. At any rate no trustworthy and continuous series of such records, such as they are for instance in the courses of pyrexia, were then, if they be now, open to the enquirer. Still in time, by observing a few private cases throughout, by watching, as I have said, one case in particular of hyperpiesis without Bright's disease-a thread upon which much of my material was strung-through its period of eighteen years, by selecting and patching longer and shorter terms of other cases seen piecemeal, and, latterly, on the inven- 
tion of pressure gauges, I was enabled, by approximate verifications, to apply some standards of comparison, and to satisfy myself that a certain process marked by excessive arterial pressures may, and often does, arise without the initiation, intercurrence, or sequel of the events known to the clinical physician as chronic renal disease in any of its forms ; but is significant of another and a different clinical series, though one ultimately damaging the heart and arteries in like manner, and no less irreparably. This alternative clinical series I named at first "Senile Plethora," 1 but, the malady being not one of senility, I suggested the alternative name of "Hyperpiesis." 2 Unfortunately, many too literal authors persist in detaching this name from the malady, or total morbid series, thereby indicated, to confine it to the mere phenomenon of high blood pressure under whatsoever circumstances. German authors now distinguish between "die essentielle Hypertonien" as contrasted with " die nephritischen Hypertonien." But, whatever our terms, I think it is now admitted that, although in Bright's disease, or in most kinds or modes of it, persistent excess of arterial pressures is an obligate condition, a like persistent excess may nevertheless, in obedience to some causes as yet unknown, arise independently, and indeed be engaged in another morbid series.

During the years of which I have spoken Huchard was occupied in similar observations, and also in discriminating this alternative clinical series, not Bright's disease, though correlated with high pressures. He attached to it the name of "Presclerosis," a name even less happy than mine, as it seemed to signify, and, indeed, was intended by him to signify, that the disease, when fully manifest, would be that of Arteriosclerosis. To this issue I shall return presently.

Now, to digress for a moment from renal disease and to return to arteriosclerosis, Professor Osler, so far back as the

1 The plethora of the ancients, with its characteristic pulse, headache, vertigo, epistaxis, etc., and relief by venesection was no doubt in great part "Granular kidney"; but the class would contain also Hyperpiesia.

2 In my first publication on this subject, an address to the Hunterian Society, written in 1894 from materials collected between 1870 and 1889 in which year I passed, for three years, from private to asylum practice- the term " hyperpiesis" was introduced as an alternative name. In 1896, in my Lane Lectures I preferred this name. It has occurred to me since that while hyperpiesis is the process, the correct form for the malady is hyperpiesia. 
third edition of his text-book, New York, 1898 (p. 771), when speaking of arteriosclerosis, concluded :

There are certainly two groups of cases, one in which the arteriosclerosis is the first change, and the other in which it appears to be secondary to a primary affection of the kidneys. The former occurs, I believe, with much greater frequency than has been supposed.

Dr. Samuel West, in his Lettsomian Lectures for 1899, spoke on the problem under discussion no less definitely; although, as I shall say presently, I cannot quite accept his views on the whole. However, he separated from Bright's disease, as I had done, the deteriorated kidneys connected with advanced atheromatous disease, or the-

"So-called senile or gouty kidneys" ... because, although "often markedly interstitial ... they need not necessarily be granular, and are often large." ... "Moreover, the cases need not run the clinical course of granular kidney."

I italicise this sentence as it coincided very nearly with my previous discrimination. To judge by the context, Dr. West clearly intended by the name "red granular kidney" to signify, not a mere aspect of the kidney in the literal meaning of the adjectives, but something more specific ; namely, a particular series of clinical events-a certain morbid process or " disease." That Dr. West had not, in 1899 at any rate, accepted my further distinction between arterial degeneration secondary to excessive pressures and the decrescent form-indeed, he did not then record any arterial pressures-made his testimony peculiarly valuable by its clear separation of the group of renal patients from other arteriosclerotics, and of the "Granular" proper from other rough or deformed kidneys. I must assume that Dr. West's contrasted group of non-nephritic arteriosclerotics was a mixture of the cases I had distinguished as decrescent (or involutionary) and hyperpietic respectively. At that time indeed he may have regarded all high-pressure cases as renal.

Again, whatever pathologists may decide concerning the late Dr. Savill's testimony to Johnson's arterial hypermyotrophy, ${ }^{1}$ I may at least quote his seventh proposition : ${ }^{2}$ That arterio-

1 Jores is disposed to admit a stage of medial hypermyotrophy (vide p. 471).

2 Paper read before the Royal Medical and Chirurgical Society, January $15,1897$. 
sclerosis may be independent of renal disease, as "in 22 of the 31 cases (forming the basis of his paper), the urine was healthy during life, and the kidneys were found normal after death, excepting for the slight degree of fibrosis commonly found in all the organs of aged people." If Dr. Savill had thought fit to accept my distinctions between infective, involutionary, and hyperpietic arterial disease, the proportion of the highpressure cases would have been considerably lower; but the argument would not have lost its importance.

For further weighty testimony to the same effect I must pass over some intermediate years and come to the

Opinions of the Present Day.-Professor Krehl, whose authority in such matters is unquestionable, says ${ }^{1}$ that we are disposed to attribute all persistently high blood pressure to nephritis, which assuredly is an error (" es ist gewiss nicht richtig, alle starken Erhöhungen des arteriellen Drucks auf eine chronische Nephritis oder Arteriosklerose zurückführen "); to the sense of the words "oder Arteriosklerose" I shall refer again. He relied upon a "series of cases with high arterial pressure in which during life there was no reason ("Anhaltspunkt") to assume the presence of chronic nephritis ; and in one of these, in which a necropsy was obtained, neither macroscopically nor microscopically were there any signs of renal disease."

Dr. John Cowan of Glasgow, with whose researches in this respect I became acquainted when he was preparing for the M.D. thesis which he read at Cambridge in 1902, ${ }^{2}$ expressed the following opinions :

Arteriosclerosis is very frequently associated with chronic renal disease, especially the granular variety; but it may occur without any renal lesion at all, or associated with one which is evidently secondary to itself (arteriosclerotic kidney). The one common factor of all (?) these cases is the presence of continued high arterial tension, which is now accepted as its immediate cause.

That in this broad division Dr. Cowan did not discriminate as I had done, and do still, between arteriosclerosis, the result

1 Krehl, Deutsche med. Wochenschr., 1905, p. 1872.

2 Cowan, J., Thesis for M.D. Camb., 1902 ; and again Practr., March 1906. 
of high pressure, and those of toxic and of involutionary origin, need not concern us now.

Dr. Graham Steell ${ }^{1}$ writes of cases of sustained pulse of high tension, with hypertrophy of the left ventricle and loud second aortic sound, leading to dyspnœa and mitral regurgitation, which, notwithstanding, are not Bright's disease.

And, further, at Toronto, in 1906, Dr. Stengel reiterated what Huchard and I had pointed out many years before, that albuminuria in cases of arteriosclerosis must not be taken alone as evidence of Bright's disease, nor necessarily as a foreboding of it. Dr. Stengel argues that the appearance of albumin in the urine in arteriosclerosis may be independent of renal disease, and that in such cases renal disease may be and often is absent. If, now and then in terminal stages, convulsions occur, they are the result simply of failing circulation. ${ }^{2}$ Senator makes the same admission, pointing out that albumin is a late symptom, and even then transitory ; it is not, as in "Granular kidney," brought out by meals or exercise. Dr. Tyson of Folkstone ${ }^{3}$ to the same effect relates his own experience of cases in later middle and elderly life of albuminuria without casts or other symptoms of renal disease. One such patient he accepted for insurance at a heavily loaded premium, and it turned out a safe transaction.

Dr. Heitz" says that, "as things stand it does not seem to me any longer possible always to ascribe persistent hypertension to a renal lesion." $\mathrm{He}$ falls back upon the chromaffin system (see p. 220). And in $1907^{5}$ Sir John Rose Bradford, while giving the same warning against the fallibility of a diagnosis of renal disease merely on the result of one examination of the urine, said that no mistake is more common than to diagnose acute or chronic nephritis merely on the presence of albumen. He argues, in a phrase which I would emphasise, that "diagnosis must depend on clinical examination."

1 Steell, G., Med. Chron., Dec. 1902.

${ }^{2}$ Stengel, Amer. Journ. Med. Sci. vol. cxxxv. p. 196, 1908 ; an interesting article on arteriosclerosis and the nervous system.

3 Tyson, Clin. Journ., July 11, 1906.

4 Heitz, Jean, Journ. méd. français, Feb. 15, 1912 ; quoted Arch. de mal. du cœur, mai 1912.

- Bradford, Sir J. R., British Medical Journal, March 31, 1907. 
Dr. Janeway (loc. cit.) says, and Dr. Tyson of Philadelphia agrees with him: "Excluding nephritis, however, there are fairly numerous cases with high arterial pressure and hypertrophied hearts," and "in the beginning without arteriosclerosis." Among many others in the United States, Dr. Riesman ${ }^{1}$ is one of the most recent witnesses to the same effect. Sir W. Osler's testimony I have quoted already (p. 314). Dr. Leonard Williams ${ }^{2}$ says that high arterial tension is, in the minds of far too many, so indissolubly connected with renal disease that if, by examination of the urine and otherwise, they persuade themselves that the kidneys are not affected, they are unable to realise that the excessive pressure can be doing any harm.

Fränkel is of Krehl's opinion; he says :

That general arteriosclerosis can be the result of renal disease is seen in the young "Nephritikern," but that high-pressure cases exist independently of it (of renal disease) is seen in many cases in which the urine, if it may contain a little albumen, is of full colour and specific gravity, with but few casts of any kind, and practically no granular casts.

Geigel, in some reflections which seem not to distinguish between the arteriosclerosis due in the main to exorbitant blood pressures and that due to other causes, says that the relation of high arterial pressure to renal disease is not understood, for the arteriosclerosis may be an independent event. Fiessinger ${ }^{3}$ reported that out of 160 high-pressure cases 84 were renal ; 44 were " arteriosclerosis without renal disease." Of such cases he emphasised the curability (in early stages); 30 of these patients were more or less obese. Faber of Copenhagen ${ }^{4}$ writes: "Unquestionably, however, there are cases of chronic high-blood pressure without renal lesion" ("chronischer Blutdrucksteigerung ohne Nierenleiden "). See also Loeb, p. 357.

The great assertor that high blood pressures always signify renal disease is Strauss. But, as Vaquez remarks, surely when from first to last no clinical sign of renal affection appears, analyse on the latest methods as you may, such a postulate is

1 Riesman, Amer. Journ. Med. Sci., April 1913.

2 Williams, L., Clin. Journ., 1907.

3 Fiessinger, Bull. de l'Acad. de Méd., April 18, 1912.

- Faber, A., Die Arteriensklerose, Jena, 1912, p. 13. 
at least premature, and from the point of view of clinical medicine baseless (see "Ambard's Constant," pp. 272 and 322). Von Romberg, who, with Strauss, had regarded all high-pressure cases as latent Bright's disease, collected notes of cases of plus pressures without evidences of renal disease. In one of these he obtained a necropsy, and found the kidneys practically normal, both to the eye and to the microscope ; but he cynically objected to these cases being regarded as the arteriosclerosis of high pressure, lest by this view of the matter physicians should be induced to treat this symptom! Surely if we are to discover causes and to neutralise or prevent them, we must discriminate between the several series of phenomena as well as we can. In the pathological laboratory reports of Berlin City Hospital I see that cases of high arterial pressure have recently been divided into the two classes of "Arteriosclerosis" and "Chronic Nephritis," which betrays the still prevalent assumption that arteriosclerosis is the cause of persistently excessive pressures rather than-in one of its kinds-the effect of it; yet still the division does serve provisionally to segregate the renal from other cases of similar, and often no less exorbitant, arterial pressures.

But it may be said, where is your post-mortem evidence? Well, necropsies on cases of hyperpiesia are hard to get. In hospital these patients are few, they do not appear till the final breakdown, and then are entered as " heart disease," or apoplexy. The previous history is unknown, a consequential arteriosclerosis is found in many districts of the body, including the renal, the kidneys are not scrutinised from our present point of view, and we are put off with common phrases, such as "kidneys slightly granular," and so forth. Hyperpiesia as a clinical series belongs chiefly to private practice, and in private practice necropsies are rarely to be had; by consultants almost never. But not long ago I did obtain a necropsy in a man æt. 37. History imperfect. A drinker. High blood pressures. Died of an apoplexy. Heart hypertrophied. Kidneys to naked eye normal, except renal artery stiff and open. Microscopically, the renal vessels were thickened, but the parenchyma of the organs was unaffected. The arterial disease was most severe and extensive in the brain. Dr. Herringham (loc. cit.) reported that in 8 high-pressure 
cases out of a series of 26 he found cardiac hypertrophy without any disease of the renal arteries. Such cases are comparatively brief, or in early stages before the inroads of consequential sclerosis. In later stages Fischer of Nauheim ${ }^{1}$ reported on 42 necropsies of persistent high blood pressure : of these, 28 were clinically renal disease, and such was the post-mortem confirmation; in 14, in which there was no clinical evidence of renal disease, 7 proved to be renal and 7 "showed a few spots of lesion." But the writer does not compare these last cases with control necropsies, taken at random, nor does he carefully discriminate between the kinds of the renal disease. It seems clear that some of these were but "cardiac" or "mitral" kidney ("Stauungsniere").

Clinical Criterions.-On my own testimony and on data obtained from other authors I have stated that the presence of albumen in the urine is of itself no criterion. About $1878 \mathrm{I}$ was consulted by a medical friend, who believed himself to be the subject of chronic renal disease. He had a large heart, a sustained pulse of high resistance, some slight œdema on the shins, and a little albumen in the urine, which was however of good colour and specific gravity, and often lateritious. On repeated examinations but a few casts were found, and these hyaline. As my experience then stood, I was unable to give a definite diagnosis; but, in the absence of the ordinary clinical symptoms of renal disease, in the absence, for instance, of characteristic headache, of nausea, of anæmia, of retinal lesion, and so forth, I ventured to speak encouragingly. My patient was a man of means and had lived well-too well. On a restricted dietary, some rest, and courses of alterative and laxative drugs, his life was spared for many years; no symptoms of renal disease appeared, and he reached old age before at last his labouring heart was defeated. This case was one of the early signals on my path; and, as I discovered more and more cases of a similar kind, I ceased to regard albuminuria, even with high arterial pressure, as necessarily indicative of Bright's disease' in the clinical sense of the term. A friend of mine, a well-known student of heart disease, published a while ago the following case of "very chronic Bright's disease," essentially a wrong diagnosis :

\footnotetext{
1 Fischer, Deutsche Arch. f. llin Med. vol. cix., 1913.
} 
Male, æt. 45. A trace of albumen had been observed in his urine from time to time without any sense of ailment. The urine was otherwise normal. The blood pressure was high. This went on without any inconvenience for ten years, when he was seized with a severe attack of angina.

Such cases are frequent enough, and end either cataclysmically in apoplexy or angina, or decline by the more gradual defeat of the heart. In the urinary deposit may be found a few leucocytic or epithelial cells and hyaline casts, but few or no granular casts, which, if found repeatedly and in some numbers, are of grave omen, and probably significant of chronic Bright's disease. I speak here of ordinary slide examinations of deposit in a conical glass, after our old-fashioned way. Hyaline casts we know occur in many fairly normal conditions, e.g. hard exercise, oxaluria, etc. But Dr. Lestock Thornton of Exmouth urges, probably with truth, that with modern spinners we shall discover more granular casts in cases not definitely renal, especially in old people, than we are wont to expect. ${ }^{1}$

The mean age of the subjects of Granular kidney again is somewhat lower, not than that of early gathering pressures in hyperpiesia, but than that of the later and graver consequences of their long-continued stress-the last stage, which too often is the first to be submitted to the physician, or to be detected by him. One of my M.B. candidates found the mean age of death in 261 cases of "Granular kidney" (definite cases selected from hospital and post-mortem records) to be 56. This age however is too high; the cases probably include, besides the true primary contracted kidney, many "arteriosclerotic kidneys." "Contracted white forms" have of course a still lower mean age incidence. In hyperpiesia the ultimate defeat of the heart is accomplished, so far as I can reckon or guess, about a mean age of some five to ten years higher. The arteriosclerotic (senile) kidney runs a decade more higher in age than the primary contracted kidney ("Granular" proper).

As regards the more refined methods of appreciating renal values we meet with some difficulty. The researches of Sir John Rose Bradford, of Pässler, of Heineke, ${ }^{2}$ of von Noorden, of Ritter,

1 Thornton, L., Lancet, June 7, 1913.

2 Heineke, Virchow's Arch. vol. cxevi. 
and others have shown that although in damaged kidneys nitrogenous secretion may, as we have said, be up to the normal in amount for short periods, yet it is prone to drop suddenly, or to shade off gradually, into periods of inefficiency. Such periods may be due to the variable relations of cystalloids and colloids in the blood. It is remarkable in nephrotomies that urine discharged by the wound from a grievously mutilated kidney, may be of quite good chemical value. Dr. Thomson Walker, ${ }^{1}$ after examining urines by the methods of cryoscopy, toxicity to animals, electric resistance, and so forth, discussed this source of fallacy. He decided that the phloridzin and methylene blue tests were, on the whole, the most trustworthy ; but, as the phloridzin method is somewhat delicate, he regarded that by methylene blue as better for ordinary use. Moreover it is perhaps safer than phloridzin. Dr. Walker published some interesting charts of the behaviour of these substances. To the methylene blue test I have already alluded, and I have said also that cryoscopy of the blood or urine, as I am informed by friends who have practised it, has proved an untrustworthy test, at any rate as a measure of renal affections. Dr. Lydiard Wilson, in his M.D. thesis, Camb., 1910, in testing cryoscopic methods for blood and urine obtained inconsistent results. Sir James Barr wrote to me: "I have had to be careful so far not to draw any decisive inferences from it." 2 The electro-conductivity of the urine also for our present purpose is discarded, but I think that on the whole a good sodium chloride excretion is favourable. Krause ${ }^{3}$ looks sceptically upon all methods of testing renal values. He says, if we desire to form an opinion of the efficiency of the kidneys by urinary analysis, we may take into consideration together specific gravity, a fall of molecular concentration (by cryoscopy), a fall in nitrogenous excretion, a fall in sugar on phloridzin injection, and a fainter colour discharge on indigo-carmine injection; and, when all is done, we may pay too much heed to small differences.

A more profitable line of investigation seems to be in the direction of blood content. Ambard compared the uric elimination of the urine for a definite period, and compared it with the

1 Walker, Thomson, Lancet, March 16, 1907.

2 See chapter on Viscosity, p. 141, where these questions are discussed more at length.

3 Krause, Lehrbuch, ed. 1909.

vOL. I 
same content of the blood for the same time. The constant, the quotient of the urea nitrogen of the blood divided by the square root of the urinary output, was stated as 0.07 , or lying between 0.063 and 0.08 ; in renal insufficiency it might reach 1.20 or 2.0 , possibly more. Opinions concerning " $A m b a r d$ 's urea constant" have varied, but at the London International Congress ${ }^{1}$ Professor Folin stated that determinations of urea, and total non-coagulable nitrogen in the blood, can now be made with $5 \mathrm{c.cm}$. of blood; and that by diet these products can be reduced to a level at which the kidneys can adequately deal with them, and kept at such levels. Higher concentrations than 25 per litre of urine are abnormal. Carrion ${ }^{2}$ also has given similar testimony. Aubertin and $\mathrm{Parvu}^{3}$ declare that this urea curve reveals the slightest disturbance of renal function, and this notably in renal cases $(0 \cdot 12-0 \cdot 14)$. Widal indeed finds a parallel between a rising blood content and uraemic symptoms - nausea, torpor, anæmia, etc. In simple high pressure without albuminuria, or with traces only, it stands at 0.076-0.090 (i.e. normal), which indicates that in "hypertendus purs," even over $200 \mathrm{~mm}$., the renal function is not reduced. With this conclusion Ambard ${ }^{4}$ and Vaquez agree. Nitesco ${ }^{5}$ contributes 8 cases in which, without any renal symptoms, the arterial pressures ruled high, with "bruit de galop" and a little albumin, but no casts, yet with excessive urea content. If so, hyperpiesia might seem after all to be a kind of renal disorder. But I would point out that, at the stage reported, cases of hyperpiesia are cases of fatigued heart, and the kidneys "cardiac" (Stauungsniere). If in "cardiac kidney," as perhaps in all declining cases, the urea content of the blood be indeed in excess, we do not count this phase among the kidney diseases proper.

Maragliano's contradiction of Ambard is well known. ${ }^{6} \mathrm{He}$

1 See also Folin and Denis, Journ. Biol. Chem. vol. xiv., 1913.

2 Quoted from the Spanish in various journals.

3 Aubertin et Parvu, Soc. de Biologie, Dec. 21, 1912, and Tribune méd., January 1913 ; quoted Zentralbl. für Herz- u. Gefässkrankheiten, June 15, 1913; and, a later verification, Berlin Intern. Congr. Physiotherapie, March 1913, and Arch. des mal. du cour, mai 1913. Vaquez took part in this discussion.

4 Ambard, Soc. méd. des hôp., déc. 5, 1913.

5 Nitesco, C. rendus de la Soc. de Biol., juillet 4, 1913.

- Maragliano, Gazz. dei osped., Oct. 7, 1909, reported in Epit. Brit. Med. Journ., about same date. See also Virchow's Jahresbericht for 1902 (published 1903) and contemporary journals ; and Lewis and others, Heart, vol. v. i. 1913. 
denies the dependence of symptoms, even of chronic nephritis, on retention and auto-intoxication by renal inefficiency. $\mathrm{He}$ thinks there is no constant; that any excess of extractives in blood over the urine is only what may be observed in the normal, if the number of observations be large enough. He goes so far as to say that, if diminished intake be not forgotten, and a sufficient period taken, as much of the nitrogenous products are excreted by a diseased as by a normal kidney (p. 321). The proportion of inorganic salts in the urine he ascribes - here I think rightly - to conditions of heart and circulation. He supposes some cytolytic poison, due to a false cellular metabolism; a perversion of the whole system, setting up a nephrolysis, or a necrobiosis of which the kidney is but one exponent.

Unfortunately the urea method is not yet available for clinical work, though T. Lewis, Widal, and others are making academic use of it.

As regards uric acid, Folin and Marshall, and Brugsch and Kristeller claim for their methods some advantages over that of Hopkins and Wörner, so that fluctuations of uric acid in the blood varying between 0.1 and $2 \mathrm{mg}$. per 100 grms. can now be accurately estimated.

However another test is coming into favour, Rowntree's ${ }^{1}$ phthalein test, which is said to give results parallel to those of the methods of Ambard and Folin. The reagent should be excreted over 10 per cent in three hours; in health its elimination varies from 20 to 10 per cent. If in three hours the excretion has fallen below 10 per cent-to 5 per cent, or traces-the uræmic outlook is proportionately grave.

At the last moment I have seen Professor Walker Hall's review of Uricæmia and Gout in the Quarterly Journal of Medicine, vol. vii. No. 25, Oct. 1913. Professor Hall accepts the estimation of "uricæmia" as a working hypothesis, but points out truly that our information concerning uricæmia in gout, etc., is as yet very slender. He discusses the operation of ectophan as an accelerator of uric excretion.

Into the elaborate postural and drink volume tests of renal values under experiment by the French school (Vaquez and Cottet and others), their normal standards, and comparisons between

1 Rowntree, L. G., Trans. Cong. Amer. Phys. and Surgeons, vol. ix., 1913. 
the hydraulic capacity and excretion of the kidneys, and the states and potentials of kidney and heart respectively, I cannot now enter. The experiments are complicated and the results as yet indecisive. The quantities and values of urine are said to vary with the "pulse-pressure." Cæteris paribus, this may be; but the coefficients are far too complex and inconstant for any such clinical maxim.

Morbid Anatomy.-In respect of the kidneys, what, in the confused heap of cases huddled under the title of Arteriosclerosis, do we find after death? We find in some of them kidneys which are either irreproachable, or so little deteriorated, as to be acceptable as "fairly healthy" ; in many we find "cardiac kidney"; in not a few genuine "Granular kidney"; and in another large class an atrophic fibrosis, from slight or moderate degrees to extensive layers, streaks or bands with more or less conspicuous deformation.

But, as we enter into the histology of renal disease, there are certain conditions of a preliminary kind which we are to bear in mind. We must remember that the parts of organisms so highly differentiated as the mammalia have themselves become so specialised in structure as to stamp a peculiar character not only upon their normal functions but also upon their defects. As the more peculiar the structure of an organ, the more peculiar its business, and the wider its differentiation from the forms and the functions of co-operating parts, so with its faults and impairments; these likewise, especially in initial stages, will have their special features and their limits. Thus, in the kidney, we should expect the features of derangements of function and of structure to have much of their own; as the liver, the heart again, the nervous centres, and the rest go right and wrong each after its own fashion. The kidney consists, it is true, of vessels, secretory cells, and connective fibreelements which all over the body obey certain general laws of growth and decay; yet notwithstanding, because in the kidney they have special dispositions, relations and functions, so their modes of growth and decay in the kidney proceed on lines congruous among themselves but differential in respect of other organs. And within the structure itself the reciprocal changes of 
the tissues will prove to vary more quantitatively than in quality. Renal disease has its own histological features, features not those of disease of heart substance, of the nervous structures, of the gastro-intestinal, and so on ; to the observation of renal disease then we must bring certain views, views obtained from a consent of experts, views not precisely those deriving from observation of disease in other organs. For instance, if the arteries distributed in the kidney are terminal arteries, an interference with one of them will partake of the form of an isolated infarction. Also, as by their tufts in the glomeruli and in their relation to capsular and connective tissues they are peculiar, so their affections will have features concordant and differential, and play within a narrower range. Furthermore, the canaliculi, as they take origin from the tufts, depend in a peculiar manner upon the functions and the disorders of these sources. Thus it is that diseases of the kidney, because it is a kidney, have characters peculiar and narrow; are not those of diseases of any other part.

Now, if this be so, the several diseases of the kidney, in so far as they present features which mark them off from diseases of other parts, will among themselves and amid their particulars present certain and considerable resemblances, resemblances in the features which separate them from diseases elsewhere. In all of them we shall expect to observe common characters, and this especially in the earlier stages of shortcoming or injury ; for, in so far as failure or injury may have proceeded, these structures will have descended from peculiar to more general characters: returning into the common paths of dissolution of all living tissues, they will be melting into the common forms of tissue decay. Thus it is that in the earlier stages of impairment we shall expect to discern the characters specific of the renal diseases but alien from the diseases of other parts ; in the later stages we shall expect to find these characters drifting into a universal kind of wreck. But as it is chancy to catch an internal viscus in an initial stage of defect, but easy to lay hands upon it in the ultimate issue, so we are apt to make too much of the ruins in which the metamorphoses characteristic of the organ have vanished.

To press these considerations a little closer. In the early stages - the stages of initiation-we find in the kidney, according 
to its several arterial units, a lobular arrangement of its affections, yet within each area a mutual dependence of disordered constituents ; we shall scarcely anticipate that disease will express itself in single tissues, such as the connective or the parenchymatous or the vascular, because these elements in the terminal arterial unit live a common life-the disorder of one is the disorder of all. For instance, the blood on leaving a tuft has lost much of its water, but is not yet relieved of the corresponding quantity of waste ; $\mathbf{1}$ if then this waste happen to be abnormal, or, still worse, if it contain some toxic ingredient, the efferent vessels and the tubes, however considerable their capacity of repair, must undergo at least temporary disturbance. Probably the tufts themselves and their capsules must suffer also; no ideal limits can be set to the implication of the whole functional unit. That nevertheless in the kidney substance the visible effects are generally scattered and focal is curious; but this inconsistency, as it is not a peculiarity of the kidney, nor of terminal arterioles, I do not stay now to consider; it is notable in the affections of most, if not of all, organs-of myocardial lesions for instance, and may depend on vascular areas. For some obscure reason, over all dimensions of an organ there is not equivalence; some sections seem to be more vulnerable than others (p. 528). Especially as regards the kidney then, we are not to look now for disease of vessels, now for hyperplasia of fibre, now for degeneration of the secreting cells, but, if in some irregular proportions of incidence, for a group lesion. So investigation tells us that, strictly speaking, there is no such process as an acute interstitial nephritis, as exclusively contrasted with a parenchymatous or with an arterial nephritis; ${ }^{2}$ or if at the outset there be any such difference, this will soon become blurred, and blurred more and more until the special features of renal disease are confounded in general dissolution. Lecorché and Talamon, in their well-known treatise on Albuminuria and Bright's disease in the 1888 edition of Brouardel's System,

1 It is true that the tufts are supposed to be a little more than filtersto have some excretory virtue, but not so much as to invalidate these reflections, which, I find, are made in principle by Adami and Nichols also.

2 See especially Löhlein, who endeavoured to combine clinical with pathological research ("Über die entzündlichen Veränderungen d. Glomeruli d. mensch. Nieren," Arb. aus d. Path. Inst. Leipzig Heft 4, 1906). 
insisted on this principle. Yet it is with these ultimate stages, when specific features are waning or obliterated, that pathologists have for the most part to be content. For such reasons the name Chronic Interstitial Nephritis is misleading and indeed wrong; the original perversion is, to say the least, as much in the blood-vessels.

It is understood then that although disease of sundry kinds, of different nature and clinical course, may affect the kidneys, yet when we come to the ultimate states, with which we have usually to be content, we are examining documents in which the inscriptions of whatsoever disease have become blurred, if not erased; moreover that, if indeed we obtain access to specimens in early stages, we must expect, even from the outset, that the peculiar structure of the kidney will by its very peculiarity be limited in its range of expression; its manifestations can only be in its own vernacular.

Now, as we have to study such kidneys as we can get, and as their morbid anatomy in its early stages is peculiar in mode, and in its later, that is to say in most of our specimens, is falling back into chaos, we shall be the more persuaded of the importance of controlling necropsies by clinical records. We shall deprecate the seclusion of pathology from clinical medicine, each in its solitary office; we shall regret that laborious papers should be written on the morbid anatomy by pathologists who are often ignorant of the clinical history of the cases from which their materials were detached, or inexpert in co-ordinating the two lines of study. In terminal states for instance, the two shrunken kidneys, both correlated with high pressures and hypertrophied heart, which in this country we have been accustomed fairly to distinguish as the small white and the granular, may, alien as they are in nature, be so far reduced by decay to a common denominator that microscopic analysis no longer marks any signal differences. Let us grant then that a classification of renal diseases cannot be founded on a mere anatomical basis, or at any rate not in obsolete stages. The diagnosis must include the clinical history.

It is in the earlier stages that we shall seek what I may call the provincial features of its tissue changes, the stages of kidney diseases on which the specific conditions inherent in the organ 
itself stamp each kind, however different, with its own seal; in them all, while each retains its own quality, the range of expression will be limited by the renal style of organic reaction.

If these thoughts seem tedious, I must plead again that they are too often forgotten. Thus, in Germany rather than in our own country it is true, we peruse diligent researches into obsolete Schrumpfniere (shrunken kidneys), painful endeavours still to decipher in these relics the characters of the several modes of Bright's disease. If in shrivelled kidneys remnants of differential character may still be detected, yet the seals are broken; at this stage - a stage in which the peculiar renal devices are obsolescent-we can hardly expect to decipher the notes of foregone specific series. If in these blurred characters we are to read anything, I reiterate that we must have with us continually the whole morbid series-the clinical story. When at length the old amyloid kidneys, small whites, terminal whites, granulars, are merged in a common degradation, what scrutiny then can discern the original relations of time, place, or quality between parenchymatous, arterial and fibrotic perversions? A skilled pathologist may guess, or indeed may pronounce with some confidence, upon the kind, but he can no longer read in the organ the series of its morbid processes in the past. The mutual interdependence and the integration of function and structure in the kidney being such as we have seen, we shall not be surprised if, ultimately, no clear divisions betwixt parenchymatous, interstitial, or glomerular disease can be made. In the proclivities of this vitiated tissue or that we may find variations in the relative rates or quantities of change, but upon them all we shall find the seal of renal idiosyncrasy; we shall, I repeat with emphasis, be more and more convinced that it is only by a close combination of clinical with pathological investigations that we can frame any fruitful classification of renal diseases-a classification based not upon superficial but upon profounder affinities.

Jores, Prym, and Roth, in their minute histological descriptions of the shrunken kidney, admit-or, rather, in his latest and maturest essay, ${ }^{1}$ Jores admits-that, invaluable as dissections may be, yet the anatomist, without the clinical histories of the

1 Jores, Virchow's Archiv, 1909. 
cases, cannot go very far to interpret the various diseases by which the kidneys had been damaged. When an author on the subject so eminent as Professor Saundby hesitates to follow the English 1 "dualistic" conception of chronic parenchymatous and interstitial nephritis (I use these equivocal names for convenience), because on the post-mortem table intermediate forms can be shown between the large white and the small kidney, the validity of his argument is not convincing. For instance, even setting aside other clinical considerations, upon what notes of anatomical aspect or of tissue change in ultimate shrunken kidneys can we explain the correlation from the outset of high pressures and cardiac hypertrophy in some of them, and their uncertainty and irregularity in others (e.g. p. 367)? All classifications are of course arbitrary, but the separation of the large congested or white (inflammatory?) kidney, primarily epithelial and often healed, from the primary shrinking kidney, primarily arterial and never healing, seems to me on grounds both clinical and anatomical to be fully justified. Gull and Sutton were dualists ; in their broadest view of renal disease they did not overlook this distinction : and about the same time Lancereaux distinguished the "néphrite d'origine artérielle."; all these observers, with Lecorché and Talamon in 1888, insisted that the granular kidney could not be the end of the large white kidney.

With these reflections let us turn to consider so much of renal disease as may bear on arteriosclerosis ; for such are the limits of my subject. What, with the indispensable assistance of Professor Lorrain Smith, I argued before the Association of Physicians at Liverpool in the summer of 1910 , and by myself, as well as I could, before the Royal Medico-Chirurgical Society on January 13,1903 , I would now, for the sake of the study of arteriosclerosis, consider a little more fully.

In coming to close quarters with the pathological anatomy of the kidney, I would note that one broad result of the researches of Jores and his pupils is, that in shrunken kidney, whether small white or "granular," the arteriosclerosis, even if restricted to the renal arterioles alone, is arteriosclerosis proper-that is, sub-

1 I speak of it as English because we owe the phrase to Johnson and to Grainger Stewart, whose divisions and subdivisions have held their ground; though it is true that Virchow also was a "dualist," and from the two "Brightic" forms separated the amyloid. 
intimal hyperplasia with splitting of the internal elastic layer. ${ }^{1}$ This renal arterial disease then does not differ, except in a peculiar severity in the Granular kidney, from atherosclerosis elsewhere, as generally recognised. If renal disease without arterial disease is rare, yet there is no arterial disease peculiar in kind to the kidney; and generally in renal disease the state of the arteries is atherosclerosis. There are however at least two other kinds of change in them which need discrimination-namely, endarteritis obliterans, and the hypertrophy of the media alleged by Johnson, Ewald, Savill, and later writers.

The former, endarteritis obliterans, is incidental to a twig caught in a focus of inflammation, which thus becomes itself inflamed but, in unmixed cases, does not turn fatty nor calcify. The distinctions to the microscope are that the elastica is intact; no fatty granules are seen as in atherosclerosis, all the coats are involved, there is more cell proliferation, and more persistent fibrosis; so that the vessel tends directly to occlusion of lumen, with consequent atrophy of the area of its supply (canaliculus). Excellent illustrations of this process are to be seen in the fourth edition of Woodhead's Practical Pathology, pp. 347-49. Endarteritis obliterans is then a local incident, with secondary consequences. Forasmuch as arteriosclerosis, on the contrary, thrombosis apart, does not tend so directly to closure, it is less of a menace to the nutrition of its unit.

The other kind, hypertrophy of the media, that is to say, pure hypertrophy, not fibrosis, is prima facie no improbable event (p. 470). One is tempted indeed to presume that, under the lateral pressures of high tension, it would affirm itself. Jores is not indisposed to grant that the assertions to this effect of certain English observers, accepted by Ewald, may have some truth in them; although (as I and others have pointed out before, and as I ventured to point out to Johnson himself upon his own specimens), some of them were of the intimal sclerotic type (p. 470). But, as I have said elsewhere, for these dimensions

1 In the discussion on my paper at Kensington some speakers seemed not to be clear as to the precise meaning of the name "arteriosclerosis." Pathologists are now quite precise in applying it to the definite process mentioned in the text, a process in nature identical with that which in large vessels is called atheroma ; so that Marchand's and Aschoff's name "atherosclerosis," now coming into use, will probably be adopted generally. 
we have no standard of calculation. How are we to determine the precise taper of the section from its bifurcation; the true proportion of the coats to the lumen; and, above all, the degree of contraction of the muscle on artificial fixation? (MacWilliam and others.) Before we can decide on any limits between a physiological and a pathological increase we must find such standards; and we must ascertain if this simple hypertrophy occurs in states of high pressures other than of renal disease. In many cases recorded as hypertrophy of the media, Jores also suspects that the thickening was not primarily of the media, but was ordinary subintimal arteriosclerosis; and he reminds us that, when it occurs, unequivocal arteriosclerosis will probably be found in other vessels of the body. In any case a media thus altered may be unstable, more prone than it should be to degeneration, fibrous or necrotic.

The affections of the arteries in the kidney are, then :

1. Atherosclerosis, as already designated ; this is the frequent alteration.

2. Endarteritis obliterans, an incidental and secondary condition.

3. Thick media, perhaps at first a hypermyotrophy.

4. Infective changes, such as due to scarlatina, syphilis, etc., which may attack the vessels, but usually appear first in the tubular epithelium. And to these classes may be added the accidental events of infarction and of embolism.

The next notable result of the work of Jores and his school is that, although in primary contracted kidney atherosclerosis is found not in the kidney only but also in the splenic arteries, the pancreatic, the cerebral, etc., yet, whatever the state of their larger trunks, the intimate vascular network of the liver, of the myocardium, and of the skeletal muscles is usually unaffected. The coronary trunks, for instance, may be conspicuously atheromatous; yet on tracking them into their intramuscular network these vessels will generally prove free from disease. If I may judge from current opinion, this very important discovery is not generally known; yet, among other postulates, it helps us to account for that no less remarkable and very salutary maintenance of the nutrition of the walls of the left ventricle in many cases of damaged coronary stems which was demonstrated, but not 
explained, by Kanthack and myself. We have discarded (p. 188) the notion of Hasenfeld ${ }^{1}$ that increase of arterial pressures depended upon implication of the mesenteric vessels, an opinion still surviving here and there; arteriosclerosis arises first not in the vessels of the alimentary canal, but in those of the spleen and pancreas; and this is so uniformly the case that, in order to avoid the toil of searching through the body for evidences of arteriosclerosis, for cursory purposes it is sufficient to record the state of the vessels of the spleen; next in order after them (p. 456) atherosclerosis usually attacks not those of the bowels or liver but those of the heart and of the brain.

In the renal vessels then atherosclerosis is the characteristic change; but is systemic atherosclerosis an essential part of all the renal affections of which we are speaking? Apparently not, though it is rarely quite absent; if assiduously sought for it is commonly to be found. Roth, a disciple of Jores, has described ${ }^{2}$ six cases of diseased kidney, apparently not all of one kind, without general arterial disease $;^{3}$ Löhlein ${ }^{4} 3$ cases; and Jores himself 1 case at least, as follows (Case III. in his 1904 paper) :-

Female, æt. 45. Uræmic coma. Heart not much enlarged and wholly of left ventricle. A few yellow opaque spots and streaks in thoracic aorta, coronaries, and iliacs. All other vessels (including splenic and cerebral) normal. Renal arteries mostly free from disease, but twigs running between the cortex and medulla showed a good deal of hyperplastic intimal change.

Indeed Jores says plainly that sclerosis of the smaller arteries is not found in all cases (e.g. scarlet fever nephritis), even although the heart may be hypertrophied. Still these cases are few, and most of them in young persons. Heineke ${ }^{5}$ regrets, as I have done, that for lack of the clinical records such observations as these should have lost much of their value. Prym ${ }^{6}$

1 Hasenfeld, Deutsche Arch. f. klin. Med. vol. lix.

2 Roth, Virchow's Archiv, 1907, vol. clxxxviii.

3 In only one was the blood pressure noted as being very high; in only one are retinal changes mentioned. None was mortal by apoplexy, all by uræmia. Some of them were probably "small whites."

${ }^{4}$ In a local laboratory report which I have not seen, but which has been fully quoted in various periodicals.

5 Heineke, Virchow's Archiv, Bd. cxcvi.

6 Prym, Virchow's Archiv, 1909. 
reports, on the other hand, that in sixty-six out of seventy persons after 40 years of age the intima of the fine and medium arteries of the kidneys is thickened, often without arteriosclerosis elsewhere in the body. This change, which even in the kidney may not amount to arteriosclerosis, or even trespass beyond physiological limits, he considers as normal for middle-aged persons; a point which needs verification. Aschoff and Gaskell would, I think, demur to this statement. Except in the occasional cases of primary contracting kidney in young persons, the main renal artery, and indeed in this form of disease the aorta also, pretty surely likewise present evidences of degeneration. Crossing these limits, the arteriosclerosis so commonly present in diseased kidneys may be obvious as a part of a general arteriosclerosis, the main renal arteries stanaing open and stiff; or, on the other hand, may be so slight and intimate as to be overlooked by the pathologist who does not minutely examine the intravisceral twigs, and especially the vasa afferentia.

However, unable as we may be in the absence of clinical data to sort the heap of obsolete kidneys examined, and to attach the kinds each to the specific morbid clinical series to which it belonged, yet by this character or that we may try to distribute the kidneys of some of the records without serious error.

For instance, we may suspect one or two of them to be amyloid, though in some researches these are expressly excluded. More confidently we may assume many, if not all, of the exceptional specimens referred to as without arteriosclerosis to have been "small whites." Our English dualists are pretty surely right in assuming that the genuine "granulars" all present arterial lesions - at least in the interlobular and afferent arteries of affected units. Jores states that in all his specimens, even in those secured in early stages, arteriosclerosis, if nowhere else in the organ or outside it, was found in the vasa afferentia of the lobules affected; also pretty surely in the spleen and pancreas, and often in the brain. The presumption is that it begins in these viscera; but the distinction between small white and granular kidney, whether resting on necropsy, on clinical history, or on both sets of data, was not made. In one specimen the arteriosclerosis was of the vas afferens only, "sharp at its offshoot" from the interlobulare. 
Now, although this kidney was apparently not, or not yet, an ordinary "granular"-for it was pale yellow, smooth, and undiminished in cortex-yet, as it by no means stands alone, and as there is no arterial disease peculiar to the granular kidney, the site of the initiation of the arterial affection is significant.

We may take it for the present that of the granular kidney arteriosclerosis of a peculiar severity is a primary and invariable feature, and begins in the intimate vascular ramifications; although, as an epiphenomenon, due to prolonged high tension, an arteriosclerosis of the larger vessels may appear, but later. I would add that in many a necropsy, especially of subacute disease of no long duration in comparatively young persons, no arteriosclerosis, or but a nominal degree of it, is to be found in the vessels of the larger magnitudes.

But the chief question for us is whether the "Granular kidney" is, or is not, an essential part of general arteriosclerosis? In other words, Is it, as we commonly read, especially in French writings, a general arteriosclerosis invading the renal artery which makes the proper Granular kidney? In "Granular kidney" the patches of nephritic fibrous proliferation are generally to be found in close connection with the arterioles. This mischief, under the influence of toxins, may creep along the lymphatics also, as it does in tubular nephritis, when it is associated with nuclear and lymphocytic infiltration, and assumes larger proportions (Woodhead, Mott). On this dilemma I will begin by citing again, from our joint paper at Liverpool, ${ }^{1}$ the conclusions of Dr. Lorrain Smith. Passing over the fibrosis of the parenchymatous and of the acute interstitial forms of nephritis, he says: "The fibrosis of so-called chronic interstitial nephritis occurs in limited areas, alternative areas being free from fibrosis. In the fibrous patch the glomerulus is converted gradually into a mass of hyaline fibrous tissue; the tubules are to a large extent destroyed, atrophied rudiments alone remaining. So far, the secreting mechanism disappears; but the unit next to it-artery tube and interstitial tissue-may be normal, unless indeed it be hypertrophied. Now from this state, he adds, the 'arterio-

1 Our paper was read and specimens exhibited at the Liverpool Meeting of the Physicians' Association in 1910. 
sclerotic kidney'-the kidney, that is, which partakes in a general arteriosclerosis-differs considerably. In this disease a unit deprived by the sclerosis of its free supply of blood is replaced by a small amount of fibrous tissue, and resembles an infarcted area." This, he concludes, is not nephritis in any form. In unaffected areas-in areas, that is, in which vessels may be fairly normal, or, if affected, may still be pervious-the glomeruli, tubules, and interstitium are in the same degree normal. Although both forms present the focal distribution to which I have already alluded as not inconsistent with the effects of toxins here and elsewhere, we may call the Arteriosclerotic a withering kidney, as contrasted with the Granular kind, which seems to consist in a slow irritative process. These arguments are the same as those which I urged in 1903, but without the same valuable pathological corroboration.

Now I venture to think that, even in the reports of necropsies, such discernment as I have indicated is not always manifest; a triple confusion, often under the head of "latent nephritis," between this senile kidney, hyperpiesia, and chronic primary renal disease if not universal, is far too common. For instance, from no inconsiderable an author I take this case.

Male, æt. 73. "Senile arteriosclerosis; hypertension (no record given); latent nephritis." Traces of albumin. Sp. gr. 1025-31. Constituents and concentration capacity undisturbed. A granular cast found rarely. (Simply a senile kidney, and the term nephritis unjustifiable. The blood pressures probably ranged but a little over the normal.)

Huchard never distinguished between the senile and the "Granular" kidney. Dr. Stengel recognised the senile form on clinical grounds, and pointed out that in this form a little albumin in the urine is negligible (p. 316). With these problems in view I have run my eye over a wilderness of postmortem reports which for the most part put us off with such loose phrases as "kidneys granular," "kidneys slightly granular," and so on ; much or little "granularity" being reported not with a specific or even a precise meaning, but on superficial appearances, and according to the general notions and personal vocabulary of the reporter. If in superficial aspect a kidney may be described as "granulated," the adjective may well 
convey only a subordinate fact; and " early interstitial degeneration " is meaningless. Post-mortem clerks often, and, I venture to think, professed morbid anatomists sometimes, forget that as a technical term "granular kidney" does not mean merely an organ rough and deformed, shrunken, fibrous, or senile, but a certain disease, a fairly uniform series of clinical phenomena. So, as a name in nosology, "Granular kidney" is to be attached, not to any kidney whose capsule is rough, shrivelled, or wrinkled, and the substance fibrous, but is to signify a certain, usually long, series of morbid events, positive and negative, recurring in clinical experience with fairly uniform symptoms, and in morbid anatomy with fairly uniform alterations of character; especially in the kidneys, the arteries, and the heart. Even Ortner ${ }^{1}$ in his diligent study seems not to perceive this difference between the primary contracting kidney and the decrescent or arteriosclerotic kidney; or that in hyperpiesia without renal symptoms the kidney at death may be the terminal kidney, the "cardiac kidney," of failing circulation. He admits that the inconstancy of these phenomena puzzle him, and that he finds the causes of big heart and high pressures to be hard to trace, a sentiment which I humbly share; yet I think Ortner would find some points become clearer were he to realise that decrescent arteriosclerosis and senile kidney do not raise pressures, nor labour the heart, as we observe in "Granular Kidney." Lancereaux likewise confused the arteriosclerotic and the granular kidney together, including both as "an actual nephritis of arthritic origin"; and confusion of arteriosclerotic renal atrophy with the "nephritis" of "Granular Kidney" is still too common. Indeed the term nephritis is not appropriate to either of them. To propose explanatory divisions of my own is to give hostages to fortune; were I to do so it would be a threefold division, thus : (1) cytolytic cases ("tubular nephritis"); (2) arteriolytic cases ("granular kidney"); (3) atrophic cases (senile kidney).

Fibrosis may appear specifically as an active strangling invasion, as in "Dupuytren's contraction," or as a cheaper substitute for a "nobler" tissue; for instance, as a phase of atrophy, when the blood supply has been very gradually curtailed by obsolescences in the vascular tree. As specific tissue dis-

1 Ortner, Fortschr. d. deutschen Klinik, Bd. i., 1910. 
appears, a nuclear and fibrillar proliferation appears more or less secretly in the connective elements. For increase of connective tissue, as contrasted with necrosis, there must be some chronicity ; it wants time.

The decrescent arteriosclerotic process, in such patients, Ziegler demonstrated also in the other parts of the body, characteristically in the pancreas. Ewald, in 1881, virtually agreed with Ziegler, and pointed out moreover that in arteriosclerosis the vascular change and its fibrotic consequences may be found in many organs, while the kidneys are but little affected, or even unaffected. This interpretation it was that Dr. West, in the Lettsomian Lectures already quoted, rightly accepted and pursued. He " excluded from the category of Granular kidney certain ... degenerated kidneys . . . in advanced atheromatous disease, and in the chronic gout of elderly persons ... kidneys often marked by interstitial change, and often not small, but large ; and not running the clinical course of Granular kidney." Thus Dr. West emphasised the axiom that in the name "Granular Kidney" the adjective does not mean the superficial glance of a morbid anatomist, but a certain disease regarded in its processes and conditions, clinical and pathological, as a whole. Contrariwise, of the fibrous kidneys thus reckoned at random as " granular," Dr. Rolleston says : ${ }^{1}$

They are in the great majority of cases the result of atrophy from diminished blood supply, depending upon arteriosclerosis of the renal arteries... where this is most marked there the tubules and glomeruli are most atrophied and the hardier fibrous tissue takes its place.

Recently, again, Sir John Rose Bradford writes ${ }^{2}$ emphatically that in spite of occasional transition forms, and of some obscurities of pathogeny, the true Granular kidney is to be distinguished from the arteriosclerotic kidney; he also relies on the clinical course. Senator, to whose excellent book there is unfortunately no subject index, admits, on pp. 117 and 291 et seq., that the contracted kidney of irritative origin is to be

1 In a discussion of a paper of mine at the Medico-Chirurgical Society, January 1903 (vide Med.-Chir. Trans. vol. Ixxxvi.).

2 Bradford, Sir J. R., Allbutt and Rolleston's System of Medicine, second edition.

VOL. I 
discriminated from the arteriosclerotic ("Nierensklerose"). Adami and Nichols ${ }^{1}$ likewise consider the arteriosclerotic kidney as etiologically and clinically different from the "Granular"; although on the post-mortem table the difference may not always, so far as the kidneys go, be easy to exhibit. Of shrunken kidneys, they distinguish four kinds-the two forms of small white, the granular form, and the arteriosclerotic. In the arteriosclerotic they agree that an atrophic, scarcely toxic, process is concerned. Eichhorst, in the sixth edition of his text-book, is rather confused among the many shrunken kidneys, but agrees that the distinction of the Granular and the arteriosclerotic forms rests upon the clinical history. Although Senator ${ }^{2}$ felt obliged to admit the distinction, he urged that transition cases were common, which is contrary to Lorrain Smith's experience and mine. Moreover I cannot admit that the arteriosclerotic and the Granular kidney proper, if they may blend, are but degrees of one process, or on the other hand that the "Primary contracting kidney" is an incident, a local manifestation, of general senile arteriosclerosis. In the simple sclerotic kidney the heart is not enlarged, or but very slightly, and the blood pressures for the time of life are not excessive; in Granular kidney it is always very much enlarged, and the blood pressures range very high. Wildt ${ }^{3}$ has written on this contrast of pressures. He works in a hospital for old persons, and there he examined the kidneys after death of 250 old folk among whom renal atrophy, kidneys often scarred and very rough, was common; yet arterial pressures above the normal were very unusual. The contrast of these with his cases of primary contracted kidney is very definite. I have quoted Dr. Mott to the same effect. Wildt's testimony is the more remarkable as he seems disposed to interpret all rises of pressure in terms of renal disease. Ribbert emphasises this "deep difference under a superficial resemblance." Ebstein says, in his Handbuch, that this distinction was drawn by Wagner, and he quotes to this effect from Wagner's article in Ziemmsen, vol. xii. The Leipzig school agrees. Dr. Stengel's emphasis on the difference, and his disregard of a little albumin in the urine, I have quoted.

1 Adami and Nichols, Systemic Pathology, 1910, p. 721, etc.

2 Senator, Erkr. d. Nieren, 2nd ed., 1902, p. 306.

3 Wildt, Zentralblatt f. Herz- und Gefässkrankheiten, Feb. 1912. 
The decrescent kidney is, generally speaking, smaller than the granular, and softer to the touch. The extent of change in the small renal arteries is not directly proportional to that in the aorta; the aorta may be much diseased while the renal arteriosclerosis is but slight. The interstitial hyperplasia is less in the decrescent than in the Granular kidney (Mott). ${ }^{1}$ Indeed, the arteriosclerotic kidney (decrescent, senile) is of little clinical importance save as part of a general closing in of capacity and reserve. In the Granular kidney proper the change is found early in the smallest arteries of the kidney, especially in the afferents, but also in the interlobular; the thickening is enormous, even to closure of the channel, and the fat reaction salient, as bright red rings; changes far greater than in other contracted kidneys (J. F. Gaskell). Aschoff writes to the same effect; ${ }^{2}$ he gives an excellent figure of the arteriosclerotic form, pointing out that it arises with atherosclerosis of extrarenal origin; he traces its origin in the advancing alterations of age, in the presence of atherosclerosis in other areas, and so on. In "Granular kidney" he describes the primary arteriosclerosis of its fine inner vessels, and the later appearance of the systemic atherosclerosis (p. 351). Fahr of Hamburg, ${ }^{3}$ working upon a large quantity of material, says that in the kidney, as in many other parts of the body, arteriosclerosis is very common, and frequently reaches high degrees, even causing much shrinkage of the organ, with comparatively little harm to (" ohne erheblich zu schaden ") its parenchyma, and often without previous high pressures. I have ventured to say that Jores and his school lack the clinical clues - they cannot get clear of anatomical ruins, and betray again and again some unfamiliarity with the several morbid series which may merge ultimately in a common dissolution; as for instance, when he, and some other authors, say that, as general arteriosclerosis penetrates to the finer vessels of the kidney, the case becomes specifically one of renal disease. We shall note in another paragraph a like confusion of kind, when nephritis is inferred from simple arterio-

1 Mott, F., Allbutt and Rolleston's System, vol. vi. p. 595 ; an article to which I am much indebted.

${ }^{2}$ Aschoff, Pathol. Anat., 1909.

3 Fahr, Virchow's Archiv, Bd. cxcv. Heft 2. 
sclerosis of the retinal vessels; the erroneous assumption here also being that when arteriosclerosis of any order, "senile" for example, extends to the retinal arteries the result is "albuminuric retinitis" (see p. 434). And in the lists of cases published by himself, Prym, and Roth, the reader perceives that from a clinical point of view a more discriminating collection might have been made. Roth admits that all his 6 cases of "Schrumpfniere" without arteriosclerosis were what we call " small whites." The kidneys of Case II. had a smooth surface, and of some others parts were smooth and parts warty. The same may be said of the cases cited in Dr. Herringham's able paper (9 out of 126), in which there was disease of the renal arteries without hypertrophied hearts ; that is, senile kidney, not primary renal disease at all. Jores' Case IV. (in his 1904 paper) was evidently one of senile kidney only, as follows :

Male, æt. 78. Capsule easily detached (" löslich "), some (" ziemlich ") increase of interstitial tissue. Very little change in epithelium. Renal, splenic, pancreatic, and cerebral arteries sclerosed.

I trust that I have made our gratitude to Jores for his accomplished work on arteriosclerosis too manifest to be misunderstood if I enter a caveat on a side of the subject on which he does not, I believe, profess to be an expert. The difficulty of disentangling these states on necropsy only is admitted; especially in Granular kidney proper arising in persons of middle life in whom general arteriosclerosis had made some independent progress. Here the cyanotic ("cardiac") kidney lends us some interpretation. Prolonged congestion causes hyperplasia, first round the glomerules, then between the tubules; then appears a slight contraction and roughness of the surface : but with all this the destruction of the parenchyma may be relatively little.

Furthermore, as I have said, the age incidence of "Granular"-primary contracting-kidney is at least ten years younger than that of the arteriosclerotic (decrescent) kidney (J. F. Gaskell, and Lorrain Smith and myself). Many " senile" kidneys are taken from aged persons, nearly all from patients over 50 years of age.

When therefore morbid anatomists have to speak of these rough fibrosed kidneys they must use an adjective less equivocal 
than "granular," one not already earmarked, or current in the sphere of the physician, and even by him much misused. The name "Granular Kidney"-not "Granular Kidneys" in the concrete plural - an unfortunate invention signifying only a surface resemblance of many kidneys diverse in nature, is now a bee in the pathological bonnet. It has at any rate the advantage of not committing us to any frail hypothesis concerning the nature of the disease. ${ }^{1}$ Still some less equivocal name is much to be desired; "Primary contracted kidney" may serve for the present, but here again the name is cumbrous, and the adjective "primary" may beg a question.

Nevertheless, it may be reasonably argued that, if many units are obsolete in function, it matters little whether this destruction be due to occlusion or to a chronic inflammation. Yet of this demur I think that certain qualifications are necessary ; we have seen that endarteritis obliterans, an irritative process, makes more surely for complete vascular occlusion than does arteriosclerosis. It seems to me that in the Granular kidney the arteries, renal as well as others, are far thicker than in the senile or arteriosclerotic kidney; so that the parenchyma may suffer more. This difference is well seen in the cerebral arteries. Besides, it is here that we find ourselves faced by the farther question of the nature and incidence of a hypothetical toxin behind Granular kidney, and whether such toxin confines itself to raiding the vessels, or attacks the connective tissue and the parenchyma also? I have always suspected that in the foci of the primary granular organ the parenchyma suffers directly, and by some process more corrosive than mere withering; and Dr. Tyson expresses the same opinion (loc. cit. p. 342). Thus the plumbous kidney, which smoulders into the Granular kidney, if caught in an early stage presents-so Sir Thomas Oliver informs us-a universal parenchymatous affection, seen first in cloudy swelling of the epithelium, then in granular degeneration and increase of connective tissue with invasion from the capsule; and the whole organ shirinks. It seems to me then that, although in a state of ultimate wreck such original differences of process may be erased, yet that the Granular

1 For differentiation of syphilitic renal fibrosis, see J. Rose Bradford, Croonian Lecture, 1904 ; and Loomis, New York Path. Soc. Trans. 1880. 
kidney is the result of some process more virulent than an atrophy. Notwithstanding, as fractional parts of healthy kidney suffice for life, the focal distribution of its virulence does enable the kidneys to keep at work for long periods without serious breakdown; thus it is that for years the disease may lie latent. It is alleged indeed that in Granular and arteriosclerotic kidney the intact areas may grow to make up for the defective (p. 334). ${ }^{1}$ In cases where the whole organ is presumably at some disadvantage, this is very doubtful; I have never seen any such hypertrophy. We must be careful not to mistake extension for growth. Were not age against it, it would be more likely to happen in the arteriosclerotic (senile) kidney where the atrophy is a kind of ablation.

Bright's Disease and High Pressures.-Of the amyloid disease high arterial pressure is not a feature ; but in acute parenchymatous nephritis and in acute interstitial nephritis, although in these maladies, and even in cases ending in recovery, the arterial pressures may rise suddenly to no inconsiderable heights and burden the heart severely, yet in them high pressures are somewhat irregular and inconstant in their occurrence. I have spoken of the "dualists" in England and in America, the physicians who from the time of Johnson and Wilks have divided chronic Bright's disease into Chronic Parenchymatous Nephritis and Granular Kidney; a distinction still recognised by Tyson ${ }^{2}$ in Philadelphia and Rose Bradford ${ }^{3}$ in London. All biological classifications are of course arbitrary, and divided by no hard and fast lines ; but, broadly speaking, this "dualism" corresponds with clinical experience. In most cases the contrast of symptoms suffices to determine a diagnosis between the two kinds; and even in cases of doubt the hesitation of differential diagnosis probably lies more often in the data, or opportunities of exact observation, than in a blend of the main morbid processes. Whether in a few cases the differential features be conspicuous or not, Granular Kidney and Chronic Parenchymatous Nephritis are, each of them, a fairly consistent clinical series throughout.

1 E.g. see Kawashima, Zeitschr. f. exp. Path. u. Ther. Bd. viii. H. 3, 1911.

2 In his valuable work on Bright's Disease and Diabetes.

${ }^{3}$ Bradford, Sir J. R., loc. cit. 
In the "Chronic Parenchymatous" clinical series high pressures, if common, are not invariable. In this disease, and under its correlative high pressures, the heart, whether by dilatation or hypertrophy, is usually enlarged on both sides, if not to the same degrees; in some necropsies no very great enlargement is notable. But in the granular disease the arterial stress, rising early and enormously, tells primarily and in the main on the left ventricle, though not so exclusively as often alleged. Unless perhaps in an early stage, the right ventricle partakes in some measure in the increase; though considerable enlargement of the right side is a secondary event, and a signal of systemic failure. Roy's experiment will not be forgotten, in which acute compression of the abdominal aorta raised pressure in both left and right ventricles. Dropsy in Granular kidney is rather cardiac in nature than renal. In respect of arterial pressures we have in these diseases to do with variables as yet unmeasured.

Once more: as we may have chronic parenchymatous nephritis with high arterial pressure, or with comparatively little excess, in mild early cases even with none, so conversely we may have high arterial pressures without any manifestation of renal disease. But in this important part of my argument I must tread warily, as I am entering into contested positions. Still, keeping as close as possible to accepted hypotheses, it is now generally admitted, I think, that Mahomed, von Basch, Potain, Huchard, and myself were justified in declaring that high arterial pressures may and often do arise without renal disease in any accepted clinical sense-that is, without Bright's disease. We have seen that Huchard, regarding arteriosclerosis not as an anatomical result of several diseases but as itself constituting a disease (p. 14), interpreted high arterial pressure as the first stage of it, and accordingly named this stage Presclerosis; as Mahomed, interpreting it as an introductory stage of Bright's disease, named it Prealbuminuria. My position I have explained (pp. 10-15): that in the first place arteriosclerosis is no unity, is not a "disease" in the clinical sense; and in the second place that, although it is often associated with high pressures, these are no necessary condition of it-a doctrine in which for some ten years I stood unsupported (p. 183). Then Groedel verified arteriosclerosis without high pressures, an 
independence which now seems to be generally, if rather tacitly, admitted. Had Dieulafoy discerned the different relations of arteriosclerosis in Granular kidney, in hyperpiesis, and in vascular involution without high pressures, he would have been less puzzled by the inconstancy of parallel between the arterial and the renal changes. For, even under the exorbitant pressures of Granular kidney, whatever minor spots and streaks may be visible in vessels of the larger magnitudes, ${ }^{1}$ deterioration of the arteries, outside specific areas, may be long resisted; a degree of immunity which in the muscular vessels I have attributed in part to the protection afforded by peripheral spasm. Even the thoracic aorta seems for a while to put on some measure of protective muscular hypertrophy. Dr. J. T. Macnab, in his thesis for M.B. Camb. in 1907, described a case of Granular kidney in a boy aged 11. The blood pressures were high. In August 1902 his accessible arteries were not altered ; in August 1904 he reappeared, when his arteries were definitely thickened. For some time, if there may be some general fibrous increase of the intima, there is not yet splitting of the elastica, no subintimal hyperplasia. Indeed, as the cerebral arteries are among the first to suffer (p. 333), the victim of Granular kidney may die of an apoplexy while his large arterial trunks, whatever their loss of elasticity, are still in fair preservation.

Let me repeat myself again, for a moment. In my Hunterian paper of 1894, and again in the Lane Lectures of 1896, I stated that a certain disease-that is to say, a fairly uniform recurrent series and correlation of symptoms positive and negative-is to be recognised and delineated, which, although marked from the outset by a rise of arterial pressures, does not follow the clinical course of Bright's disease in any of its modes, but manifests itself by symptoms which I have described elsewhere in this book. Nor does it, as Senator ${ }^{2}$ supposed, "if neglected" go on to "Granular" kidney; usually it is " neglected." Furthermore, that it is not an expression of arteriosclerosis, but generally speaking, if recognised in time, is curable ; and if recurrent, as frequently it is, yet even after two

1 Recent transplantation experiments in animals to prove that high blood pressures do not injure the vessels seem to me to be very fallacious.

2 Senator, Erkrankungen d. Nieren, 2nd ed., p. 306, 1902. 
or three recurrences, by diet and other vigilant precautions, it may usually be banished for good. If however, as too often is the case, it defies treatment, or is neglected, the arteries undergo such strain as to issue in arteriosclerosis. Thus arteriosclerosis is not a disease, but an event or result of several diseases ; is not a cause of excessive arterial pressures, but on the contrary, from beginning to end, is quite consistent with ordinary or even low pressures. To this disease it was, in origin independent of arteriosclerosis, that I gave the name of Senile Plethora, or Hyperpiesia, meaning by Hyperpiesia not simply a character of pressure, but a certain morbid series taken as a whole-a series of which this rise is but one of the principal characters. The question we have here to consider is whether this disease be identical in nature with " Granular Kidney," or with any chronic form of Bright's disease. This identity, or close kinship, I deny, for reasons, chiefly clinical, already given. If it be urged that the renal function is nevertheless fundamentally concerned in the disorder, this guess I neither gainsay nor confirm. It may be so, or it may not. We have as yet no evidence on which to come to a conclusion either way.

Dr. Janeway, ${ }^{1}$ after setting aside all doubtful cases, found that, of 130 patients with blood pressures about $200 \mathrm{~mm}$., in 17 no clinical evidence of renal disorder could be detected. In a later paper he says, on necropsy of the pure hyperpietics, "the renal defects, if any, may be of the most insignificant kind." I may add that the hyperpietics, in some contrast with nephritics, bear venesection well, and are much benefited by it. Josué and Block, ${ }^{2}$ of a certain high-pressure case, write that "the patient presented an instance of one of the most definite (' des plus nets') of clinical types, namely, d'hypertension pur ; without presenting any disorder directly imputable to a renal lesion," yet maintaining a very high tension. (See Preface, P.S. p. x.)

Of Hyperpiesia I have never offered an explanation, or nothing more than conjecture ; I have been content to distinguish it, as a clinical series, from the recognised forms of Bright's disease. I am far from denying that in this disease of hyperpiesia the kidneys may have some concern (p. 322). Passing over the "renal

1 Janeway, Amer. Journ. Med. Sci., May 1906.

2 Josué et Block, Arch. des mal. du cœur, mars 1908. 
inadequacy" of Clark, adopted by Huchard, as either renal disease or a vague speculation, I may mention here the argument of $\mathrm{Dr}$. Batty Shaw, in his Goulstonian Lectures of 1906, an argument which I will discuss more fully later (p. 357). He argues-putting aside problematical instances in which changes usually tending to high arterial pressures are subdued by intercurrent conditions, such as cardiac insufficiency or competing toxins-it is agreed that the cases which Huchard and Allbutt, and before them Mahomed more or less, have separated from frank Bright's disease under such names as Prealbuminuria, Presclerosis, or Hyperpiesis, do not follow the common course of disease of the kidney; yet dependent upon renal disorder of an insidious kind they are nevertheless; furthermore, on a fatal issue, some defect in the kidney will be found. The pressor element in particular is of renal origin, and is due to an escape of renal substance, or products of renal dissolution-of nephrolysis, to use a current wordinto the blood. Now Dr. Shaw's explanation may or may not be true ; my concern is to point out again that the distinction lies between Bright's disease and another malady which, if caught in time, is, if not always, yet usually curable, and when incurable abuts upon cardiac defeat or apoplexy, and does not at any stage, even of a fatal career, present uræmic symptoms; not, that is to say, anæmia, headache, nausea, lethargy, retinitis, convulsion, or coma. When Huchard states that cerebral hæmorrhage occurs only in arteriosclerosis combined with interstitial nephritis, he fails in this distinction, as he fails to distinguish between hyperpiesia and involutionary atherosclerosis not due to high pressures. Catalogues, like those of Fischer ${ }^{1}$ of Nauheim, which give us large percentages of high pressure cases in which the kidneys proved to be more or less affected, are useless unless accompanied by controls; i.e. by an equally long series and equally careful scrutiny of kidneys, during life and after death, in persons of and beyond middle life in whom pressures were not raised. Again, what does Fischer mean by "nephritis"? In many of his high pressure cases the renal defects were very slight; " small spots of fatty degeneration," "punctate areas of degeneration," and so on. All "cardiac kidneys" should be excluded, as final stages. And distinction must be made

${ }^{1}$ Fischer, Deutsche Arch. f. klin. Med. vol. cix., 1913. 
between the primary, the secondary, and the arteriosclerotic shrunken kidney. Fischer, and Münzer (loc. cit. p. 497), who agrees with him, both admit that a large percentage of the high pressure cases progress to heart defeat or apoplexy with never a clinical sign of renal disease. And neither of these authors alludes to any discrimination of decrescent from hyperpietic sclerosis, although this distinction, as Sir W. Osler says, eats into their whole argument. Osler ${ }^{1}$ refers to the " bogie of traces of albumin in elderly persons," and asks how many men over æt. 50 have kidneys histologically quite at their best ? Vaquez, ${ }^{2}$ with whom I have since had some personal conference, decisively disagrees with Strauss, who sees renal disease in all cases of arteriosclerosis. Vaquez has often found pressures even of 230-260 (Riva-Rocci) with no sign of renal disease from first to last; and Dr. Tyson gives the same witness, up to pressures of 240 (private letter to myself). Casper, ${ }^{3}$ in discussing the surgery of the kidneys, taking shrunken kidneys in the lump, wonders that some of them should be so evil, and the ill-effects of others so slow ; and wisely remarks that herein lie differences of initial diagnosis and pathology, for "some patients, in spite of this disease (" Erkrankung ") enjoy years, and decades of years, of undisturbed life." Industrious papers such as Fischer's, and Lee's 4 also, based upon large accumulations of disparate or half-analysed items, seem to me to lead nowhere. For instance, what can Dr. Lee mean by "arteriosclerotic nephritis" ? Surely no such a kidney is inflammatory. And the term "atrophic kidney" is not defined. Hensen, late of Quincke's clinic, said: "I have convinced myself of this, that a blood pressure over $200 \mathrm{~mm}$. may occur in cases of simple (" einfacher") arteriosclerosis in which clinical observation gives no support whatsoever to a diagnosis of renal disease." 5

1 Osler, W., in his well-balanced article in the Brit. Med.Journ., Nov. 2, 1912.

2 Vaquez, Arch. des mal. du cour, avr. 1913. Paper read at the Physiotherapeutic Congress at Berlin in 1913.

3 Casper, Deutsche med. Wochenschr., Nov. 17, 1910.

4 Lee, R. I., of Massachusetts Gen. Hosp., Journ. Am. Med. Ass., Oct. 7, 1911.

5 The passage runs thus (p. 506): "Dass ein Blutdruck über $200 \mathrm{~mm}$. bei einfacher Arteriosklerose vorkommt, wo die klinische Beobachtung keinerlei Anhalt für Nephritis gibt, habe ich mich überzeugt." I must add that if systolic pressures at rest run far and continually over 200 , a strong suspicion of renal disease does arise; though, with Tyson, I have notes of not a few in which on such ranges no renal symptoms appeared. 
But Hensen had still to learn that in such cases even arteriosclerosis is not a necessary factor. Whether then in essence of renal origin or not, the respective diseases-the fairly uniform series of symptoms, negative and positive-are widely different in course. And I shall argue again that in necropsy care is not taken to distinguish between the "cardiac," the atrophic or arteriosclerotic, and the "Granular" kidney technically so called, kinds distinguished by Professor Lorrain Smith, Professor Aschoff (in 1903), Dr. John Gaskell, and some other authors besides myself who believe the arteriosclerotic kidney to be within limits still fairly capable, but regard the Granular kidney as corrupt, if not actively vicious, from the beginning.

The slight or variable degrees, or the absence, of high pressures and cardiac enlargement in many cases of disease of the kidneys, when their structure may have been impaired even to extremes, have for a long time been a puzzle to clinical observers. If the heart be enlarged to any great extent, certain physicians assume that, besides the renal disease, some correlated disorder of a systemic kind, vaguely indicated as a "cachexia" (Pässler, ${ }^{1}$ Senator), must be associated with the renal disease, a condition, by the way, scarcely favourable to myocardial growth. Others, with doubtful success, look for the determining factor of the high arterial pressures in the differences of the renal elements concerned-as of the glomeruli for example, which I will consider presently; or in epithelial decay; or in the quantity of renal substance abolished; or, again, in absorption of disintegrating renal substance (Dr. B. Shaw). On one clinical distinction at any rate we are all agreed: namely, that the form of renal disease in which we find the arterial pressures constantly and regularly exorbitant, and the heart from the outset notably hypertrophied, is the "Granular," which form is in this respect also to be distinguished from other diseases ending in shrunken kidneys, such as the "small white," 2 terminal or original. In these other processes, it is true, some arterial tension and cardiac

1 Pässler u. Heineke, Verhandl. d. path. Gesellsch. zu Méran, 1905, ix. 99 (and reported in German journals of that year).

2 Professor Rose Bradford accepts the primarily contracting white kidney, indicating its often latent course in young adults. Some of Jores, Prym, and Roth's cases, under the bundle name of Schrumpfniere, seem to have been of this kind. 
enlargement are usually found, but not so early, so constantly, nor so enormously as in the "red granular" form.

As regards the amount of renal structure ; that the cardioarterial stress is not, directly at any rate, as the sum of its detrition is apparent on comparison of "Granular" cases with cases of shrunken or damaged kidneys of other kinds in which the obsolescence of renal substance may in the sum be no less. In certain kinds of renal disease, such as the amyloid, in which the proper renal elements must be universally impaired, and some obliterated, the heart may not be-in the amyloid is not-enlarged at all, nor the arterial pressures raised. And, when we look more closely at the Granular kidney proper, we find no definite relation between the demolition of the kidney and the rise of arterial pressures; the heart in these cases often grows large, and the blood pressures run high, in incipient stages before the local disease has become manifest, or importunate, or the urine much attenuated.

"Renal angio-spasm" is a hypothesis ex machina.

We have seen that Hasenfeld's hypothesis, which attributed the high pressures and enlarged heart to the geographical distribution of the arteriosclerosis, namely, in the mesenteric area, had it been valid even for ordinary cardio-arterial hypertrophies and degenerations, is quite inadequate to explain the enormous cardioarterial changes of Granular kidney; and it has been disproved by many observers, as for instance, by the well-known researches of Marchand. I have said also that Jores, in many cases of Granular kidney with large heart and high arterial pressures, had demonstrated the slightness or virtual absence of arteriosclerosis in the mesenteric area; and indeed that, speaking generally, the pancreatic, splenic, and cerebral arteries are, as a rule, more diseased than those of the bowels.

In thus turning our little lantern upon the correlations between high pressures, arteriosclerosis, and renal affections, we see a crucial point, although no instantia crucis; we meet many another baffled inquirer, each perhaps with his own guess, or scrap of information, but no signpost. What are the scraps? Well, as we have seen, that in some cases of chronic renal disease, and in many of tuberculous, or calculous, or surgical kidney, pressures do not rise at all ; that in others the rise is not 
predominant, and does not affect mainly the left ventricle but both ventricles, after the way of, and often because of, failing heart-inconsistencies in strong contrast with the definite class, and especially with the disease to which the name of Granular Kidney is given, in which from the first high pressures predominate, and consequently the left ventricle, in the manner of the so-called "concentric hypertrophy," is affected chiefly, enormously, and often until the stage of cardiac fatigue, alone. ${ }^{1}$

Still, if not eminently characteristic of them, yet it is true that in other chronic renal diseases, even in "surgical kidney," there is an inclination to a rise of arterial pressures; so we presume that in renal disease there is a something which provokes this excess; something which in the Granular kidney is a constant, in the others a variable. Is this something engendered in, or of, the kidney itself? In the view of Aufrecht, which a while ago found no inconsiderable favour, the conspicuous example of this pressure-provoking means-the Granular kidney-is but a local expression of arteriosclerosis, the renal implications of which colour the whole malady. But, like Huchard, Aufrecht also regarded arteriosclerosis as necessarily associated with high pressures, although in 1894-96 I had proved that very frequently it is not; and, in particular, that even an arteriosclerotic kidney may be, and often is, associated, from life to death, with arterial pressures normal for the age of the patient. In 222 cases of arteriosclerosis Dr. Mott found the heart and kidneys somehow affected in 141 (82 male and 59 female), but in 30 male cases and 55 female neither heart nor kidney was altered. A short time ago he kindly discussed with me 300 necropsies in his Department, in which he had noted that if, as it commonly happened in cases of atheroma, including the cerebral arteries themselves, the renal fibrosis was not that of Granular kidney, cerebral hæmorrhage was relatively infrequent. This result of a large comparison agrees with the impressions which on this subject I received when I was a Commissioner in Lunacy. Now,

1 I take this as accepted; I may add that Jores in his last (Deutsche Arch., 1909) paper carefully recorded the cardiac condition, on Müller's method, in unsorted cases of shrunken kidney, and found definite hypertrophy by no means constant. But Jores, as I have said, mixed in an uncertain number of what Lorrain Smith and I, and Aschoff and Gaskell, have separated as " arteriosclerotic" -my decrescent, or " involutionary "-kidney. 
as Dr. Mott himself observed, in pauper asylums there is some selection of cases; among the insane a very large proportion of the cases of arterial disease are not those associated with encroaching arterial pressures and large cardiac hypertrophy, whether of renal or non-renal origin; they are of a degenerative nature, with cerebral softening in the atheromatous areas. On the other hand, it is now ascertained that in the specific disease named well or ill, Granular Kidney, the arterial lesion begins in the kidney, or in the kidney with certain associated distal areas (spleen, pancreas, etc.), and that a more central arteriosclerosis is not compassed until in other districts the strain of a systemic high blood pressure has begun to tell. Yet it seems improbable that, as under the renal disorder pressures begin to increase, the strain would tell first on the remoter visceral twigs. In the renal area indeed, where the vasa afferentia branch off from the interlobular, the pressure gradient is falling, and in the tufts, which are affected early and severely, must both by friction and by extension of bed fall very quickly. High tension would, and does, make itself manifest sooner or later in the aorta and great vessels; but if the finer visceral twigs are among the first to be damaged, to initiate it must there not be some insidious humour playing on the periphery ? ${ }^{1}$ In one of Roth's cases, one of true "Granular kidney" in a man aged 30 , with blood pressures of 185-215 (death comparatively early, presumably from intercurrent causes), although the kidneys were typical, but little alteration of the larger vessels had appeared; and I have mentioned such instances. Traube's notion that constriction in the renal area would suffice to enhance general pressures was erroneous; even sudden ligation of both renal arteries does not do it. If a peripheral arteriosclerosis quickly became almost universal, it is possible that pressures might rise; but in Granular kidney such extensions come about later, although, at the outset of it, even in thirty to forty days, arterial stresses may have reached very high degrees. Certainly the high pressure is not engendered, as some have supposed, of mere chronicity.

The Glomeruli.-Weigert ${ }^{2}$ adopts the opinion, and it is one

1 See Steinbiss, p. 263.

2 Weigert, Die brightsche Nierenkrankheit, Berlin, 1906, and Volkmann's Sammlung, Nos. 162, 163. 
to which my own had independently inclined, that the secret of pressure increase lies in destruction, or suppression, of the renal parenchyma; Pässler and Heineke's conjectures also, to which I have alluded, may not be widely different. Weigert's opinion is strongly supported by pathological evidence. Aubertin, ${ }^{1}$ in a case of abuse of absinthe during the ten months before death, found a "nephrite purement epithéliale"; but the heart nevertheless was largely hypertrophied. There was no aortic nor other arterial lesion anywhere in the body. In the discussion E. Gley said that certain snake serums do a like damage to the kidney epithelium with consequent cardiac hypertrophy. Aubertin followed up the enquiry, and in the following November produced a large array of experimental facts, experiments by slow poisoning with chemical and bacterial agents. When the effects were purely epithelial (cloudy tumefaction and necrosis) enlargement of the heart ensued, even from the very outset when the epithelial changes were barely perceptible to the microscope. Pässler and Heineke's view is (loc. cit.) that the correspondence with the cardiac enlargement lies not merely in the glomerular but in the total renal defect; and Sir John Rose Bradford seems to make a similar inference from ablation experiments, in which at certain degrees pressures begin to rise ; as it would seem, from lessening the eliminating surface. Hensen quoted a case of transient acute nephritis in a girl aged 15 , in which the blood pressure rose to 180 , and two weeks later, as recovery set in, fell to 120 . I remember a case of this kind in a young woman, aged 28 or 30 . She presented acute dropsical nephritis, urine thick with albumin, epithelium, leucocytes, and casts. In a few days her heart became enlarged and the aortic second sound painfully loud. She did well, the physical signs receded, and in three months she went out to service and was reported well for a year or so, when I lost sight of her. However, Pässler and Heineke's conclusion that high pressures and definite cardiac hypertrophy arise on removal of renal tissue to a certain correlated amount is relied upon, I think, a little more confidently than as yet is quite justifiable. More verification is wanted; indeed some experimenters doubt it, or regard the variations as slight and inconstant.

\footnotetext{
${ }^{1}$ Aubertin, Soc. de Biol., juli 27, 1907.
} 
Jores, for his part, was unable to formulate any direct proportion between high pressures with cardiac increase, which often is great in comparatively early cases, and microscopical evidence of parenchymatous destruction of the kidney. That the blood pressure does not rise on extirpation of both kidneys, or on the occlusion of the renal arterial tree by the injection of paraffin into both renal arteries, ${ }^{1}$ may be attributable to the severity of the operation; and in calculous anuria the same lack of reaction time and capacity may be a valid explanation. In Aubertin's cases it was stated that the adrenals were enlarged, and that the cardiac increases may have been due not to the renal lesion, but to profusion of adrenin. Certain recent observations however have shown that, although by adrenalin great enlargement of the heart can be brought about, yet this enlargement is not good muscle, but something of a degenerative accumulation (vide p. 225). In Aubertin's cases the blood pressures during life were not recorded. In Gley's snake cases the pressures were not raised, and the adrenal bodies were not mentioned.

The contrary opinion prevails, that in glomerular lesion lies the secret of high pressure in renal disease, as was apparent at the Méran Meeting of the Deutsch. Path. Gesellsch. in $1905 .^{2}$ Ziegler $^{3}$ regarded glomerular lesion as the essential factor of high pressure, and thought that, if the vessels were clear, the impaired epithelium should recover itself. Ziegler's views command attention, but to me are inconclusive because he did not always discriminate between the granular, the arteriosclerotic, and-what we shall see is not quite the same thingthe mere senile atrophic kidney, two forms in which the glomeruli seem to suffer passively; a curious inconsistency, as he was, so far as I know, the first pathologist to recognise the "arteriosclerotic shrivelled kidney" (p. 337): a "renal arteriosclerotic atrophy" . . . "a special form of contraction of the organ" due primarily to sclerosis of the renal arteries with, usually but not necessarily, participation of areas of the intimate renal ramifications also. In Granular kidney, although it is true that lesion of the finer arteries of the organs seems to be an obligate condition, these

1 Senator, H., Zeitschr. f. Klin. Med. Bd. Ixxii., 1912.

${ }^{2}$ Verhdl. loc. cit. p. 112; F. Müller and Volhard, Mönckeberg and others.

3 Ziegler, Deutsche Arch. f. klin. Med. Bd. xxv.

VOL. I 
vessels, with others of similar magnitudes in several other regions of the body, may rather be the gate of entry of some histolytic substance, of some pernicious agent corroding unit by unit the whole gland.

We shall not be surprised to find that lesion of the glomeruli has loomed large in this enquiry. As centres of arterial disease with quasi-infarctive effects, and also of fibrous hyperplasia, these structures have caught the eye of observers; the processes howeqver do not, as Jores admits, by any means always run parallel: the hyperplasia may be far more than the arterial disease, and conversely. The double capillary system would seem to be rather an obstacle than an advantage to an organ which passes through its system ten to twenty times more blood than almost any other. Professor Starling says the glomerular function needs a pressure of some $30 \mathrm{~mm}$. Hg., a pressure which must fall below its needs when the vessels are narrowed, whereupon the glomerulus must shrink and ultimately degenerate. The longer afferents suffer first, while the shorter are still normal (J. F. Gaskell). Here we are met by the interesting argument of Professor Brodie, and of Jacobi of Tübingen, ${ }^{1}$ in which they suggest that the glomerulus, within the firm and inextensible capsule of the kidney, so far from being an obstacle, is a small hydraulic ram-." propulsor" is Brodie's term-reinforcing the heart's impulse; ${ }^{2}$ moreover that the glomerulus is not a water-spring for the tubules, nor a filter, that the water reaches the tubules by their own epithelium (Woods Smyth). Tigerstedt attributed some such aspirating capacity to the organ as a whole (Virchow's Schwamm?), so that, as he said, in spite of the narrow and contorted vascular channels, without increase of diameter or rise of pressure, the stream was quickened. That the aqueous discharge is not mere filtration is now generally accepted. ${ }^{3}$ The aqueous movements, concentrative and eliminative, depend upon the tissue colloids, variables however which usually, probably under nervous influence, are

1 Jacobi, Münch. med. Wochenschr., 1911, No. 36.

2 Dr. W. Woods Smyth of Maidstone wrote to the Brit. Med. Journal on Scpt. 23, 1911, to claim, as against Brodie (Croonian Lect., Roy. Soc., June 15, 1911) the priority of this suggestion for his brother A. W. Smyth; he referred to the American Encycl. of Biography. The hypothesis is far from verified.

3 See Höber, Physik. Chem. v. Med. Bd. i., 1907. 
rhythmical. Salts and purins make for retention (anabolic), urea for release ; and presumably space and time relations and specific molecular attachments within the cell have their values. In morbid tissues colloid accumulates, a phase which calomel seems to alter, increasing the nitrogen and phosphorus concentration of the urine, but affecting the chlorides very slightly (see Treatment of Hyperpiesis, p. 355). Sodium chloride is said to filter through the glomeruli and, by resorption of water, to concentrate in the tubular epithelium; a little salt may be reabsorbed. In acute glomerular affections chloride is retained, but, as the acuter phase passes off, it gradually comes away again. ${ }^{1}$

In the arteriosclerotic kidney also, by which I mean the state in which this organ, in association with many or most of the other parts of the body, shrinks and starves under a general vascular sclerosis, the glomeruli seem to fail in no inconsiderable numbers, so that it may not be easy to say that the sum of their defect is always behind that in Granular kidney, in which their suppression is far from being universal; yet in the one arterial pressures are raised very high, in the other little if at all. Thickening of the capsule alone may not be of grave importance, the organ may still be fairly permeable (Roth); but in Granular kidney injection of them is difficult and partial ; in the senile kidney the sclerosis does not close the tufts as does a glomerulo-nephritis.

Bähr, ${ }^{2}$ and Dickson, ${ }^{3}$ as it seems independently, experimented on the animal kidney with uranium nitrate and other poisons or toxins. Dickson, in guinea-pigs, rabbits and dogs, with uranium nitrate produced a chronic diffuse nephritis resembling that of man but not identical with it. The heart increased more or less but the arteries did not present demonstrable lesions; though some failure of their function was suspected. By differential doses of the salt, Bähr was able to throw the morbid effects severally upon the epithelium or upon the glomeruli. The vasa afferentia in his cases showed well-marked disease, but he did not discuss the coefficients

1 See Borchardt, Deutsche med. Wochenschr., Sept. 12, 1912.

2 Bähr, "Über exp. Glomerulonephritis," Ziegler's Beiträge, Bd. lv. H. 3, 1913.

3 Dickson (Toronto), Stud. Rockefeller Inst. vol. xvi., 1913. See also Janeway, "Nephr. Hypertension," Amer. Journ. Med. Sci., May 1913. 
of the heart and arterial pressures. However I quote Bähr because he and others find that various toxins have various vascular selection-vaso-tropism, as Ehrlich might put it. The injury to the glomeruli and afferent vessels seems to be due to the filtering through them of the morbific substance, and in degree, even in distribution, depends on the dose.

In estimating glomerular reduction, the personal equation of reckoning and the selection of striking instances account for some inconsistency of results. Differential counts are so tedious that the personal equation becomes dominant; moreover glomerulus disintegration differs much in amount in the several parts of the same kidney; but most recent observers - Jores, for example-find no parallel between heart enlargement and suppression of glomeruli. As however Loeb, 1 and Pässler, and Krehl ${ }^{2}$ also, still assume the rise of pressures and the growth of the left ventricle to be in direct proportion to the destruction of the glomeruli, to test this statement Jores injected a number of shrunken kidneys; in many of those unattended with cardiac hypertrophy he found the glomerular destruction to be no less ; moreover, many damaged ("verödeten ") glomeruli, whatever their remnant of functional value, were at least still permeable ; much-thickened arterioles are often still pervious. Löhlein and J. F. Gaskell also, in the present respect, have attached little importance to glomerular disease, and on experimental and clinical data look to tubular and diffuse nephritis, or nephrolysis, as the disturber of pressures. It is a point in Rautenberg's experiments, to be mentioned presently, that although rise of pressure and cardiac hypertrophy set in, the glomeruli were intact. On the other hand, in the amyloid kidney without rise of pressures, and no, or but little, cardiac increase, glomerular obliteration seems very great. In ureteral stoppages again the glomeruli retain their form for a remarkably long time; and I repeat that in the senile kidney, in which many glomeruli are obsolete, pressures rise but little. Loeb is of course well aware that in early cases of high pressure the mere obliterations in the renal area are far too limited to influence general blood pressure, while in other cases of cardio-arterial pressure and strain no evidence of renal disease can be demonstrated on necropsy;

1 Quoted by Dr. Janeway.

${ }^{2}$ Krehl, Path. Phys. p. 3. 
whereupon he postulated some wider reflexes, operating especially through the brain-an argument in which he became speculative, if not vague. However, here again let me claim that this distinguished pathologist also admits the not infrequent occurrence of high pressure cases without renal disease.

Charcot argued that glomerular defect entails defect of the whole secretory (glomerulo-tubular) unit, but this apparently reasonable guess is very uncertain. Bainbridge and Beddard ${ }^{1}$ showed that after complete occlusion of the glomeruli the tubules, if adequately supplied with oxygen, maintain their normal histological appearance, and may secrete urine. From Rautenberg's experiments, as in some cases of " surgical kidney," it would seem that without active glomerular disease the mere annulling of renal substance, if large enough, provokes a rise of arterial pressures (p. 352). Histologically, were the glomeruli in Bright's disease, say in "Granular kidney," the seat not of arteriosclerosis but of arteritis obliterans, we might have a criterion of an irritative against an atrophic lesion of these elements; but we must take it for the present from Jores and Aschoff and Gaskell that the primary arterial change is arteriosclerotic, as recognised in subintimal hyperplasia with split elastica, and in its disposition to fatty degeneration, a kind of decay to which endarteritis obliterans is less prone.

Kidney Stuff as Pressor.-I have alluded to Dr. Batty Shaw's ${ }^{2}$ assumption of a positive effect, as a hypertensive poison, of absorption of the gland into the system; a curious construing of Johnson's presumption that the "stopcock action" of the vessels served to protect the organ from a previously tainted blood. Dr. Shaw's conjecture is that kidney stuff of its own nature acts hypertensively; and that not in Bright's disease only, but also in the hyperpietic malady of Huchard and myself, the kidneys are undergoing some kind of solution and diffusion into the blood current, with the effect of general arterial constriction, and consecutively of arteriosclerosis. But surely some histological evidence of this should be produced. Prima facie the order of events is contrariwise. Dr. Shaw supposes the

1 Bainbridge and Beddard, Biochem. Journ., 1906, vol. i. p. 255. See also Bainbridge and others, Proc. R. Soc., 1913. Paper read April 24.

2 Shaw, B., Goulstonian Lectures, 1906. 
only differences of Hyperpiesia to be that this solution and diffusion are not so concentrated, or so virulent, or perhaps not so specific, as to issue in what we recognise clinically as the more familiar train of symptoms of Bright's disease. Ortner, as we have seen, declares ${ }^{1}$ that rise of pressure with arteriosclerosis is always renal disease, whether with uræmia or not. Dr. Shaw lays great stress on the experiments of Tigerstedt and Bergman to the effect that injection of extract of renal cortex, prepared by their method, drives up blood pressures to degrees unapproached by any other such organic extracts, except the pituitary. Maragliano also injected kidney pulp of dogs into rabbits, or the blood serum after tying the ureters, and inferred the presence of a nephrolysin causing nephritic symptoms. $\mathrm{He}^{2}$ made two series of experiments in this way; he injected kidney pulp of dogs into rabbits, producing-so I gather from the records-little rise of pressures, but a general cytolysis, in which the kidney was implicated. But do not these injections from one species into another seem open to the objection of violation of the idiosyncrasies of animal species Nuttall (and others)? Parisot's researches, in a book published (in 1908 after Dr. Shaw's), on Pression artérielle et sécrétion interne, so far as they are to the same effect, contain no. arguments not dealt with by Dr. Shaw; though in some facts they differ. Parisot leans to the current opinion, that the pressor effect is due not so much to an inward renal secretion or diffusion as to imperfect elimination of pressor substances in the blood, and helps out his case by looking to adrenal hyperplasia (p. 225). He notes the hypotensive effect of blocked ureters, and of very sudden and acute nephritis. By the way, he, as others have done, experimented with the liver also, and found that, in this respect, the substance had little or no effect.

However, later experiments fail to support Tigerstedt's conclusion; they are contradictory or inconclusive. Bingel and Claus $^{3}{ }^{3}$ on injection into animals of expressed juices from

1 Ortner, Wien. klin. Wochenschr., 1902.

2 Two references : (1) Virchow's Jahrb., 1903, vol. ii. p. 244 ; and (2) Gaz. degli osped., October 7, 1909 (Epitome, British Medical Journal, 1909); I have not seen the original papers. See also Bender in The Bender Lab. Rpts. vol. v., 1908.

3 Bingel und Claus, Deutsche Arch. f. klin. Med. Bd. 100. He. 3 u. 4. 
healthy or diseased kidneys, found the effects to be scarcely, or transiently, pressor. Pearce ${ }^{1}$ again, on Bingel's method, injected extract of kidney-both from healthy and diseased organsinto animals, with indecisive results; on the whole, so far as any effect on arterial pressures was perceptible, it was slight and transient, and followed by a fall. Also he injected serum from some dogs in which large portions of the kidneys had been excised, and from others in which an artificial nephritis had been induced, and at periods when the urine was free from depressor substance; but the evidence of a pressor substance, if any, was very slight. He concluded, likewise, that the kidney did not contain a pressor substance masked by a more powerful depressor constituent. We have seen that Josué and Block (loc. cit.) found depressor rather than pressor effects on injection of the serum of high-pressure subjects into rabbits. The experiments were not many, it is true, but they were definite enough. In our laboratories the researches of Professor Dixon and his colleagues and pupils have led to similar doubts and objections. We have seen however (p. 268) that Eichler did find the serum of not only nephritic but also of nephrotomised animals to contain a vaso-constrictive substance, like adrenalin ; and that Schlayer ${ }^{2}$ agreed with him. If these results be verified, they would indicate that a pressor substance may appear not from the kidney but from the absence of the kidney.

However, if with Dr. Shaw we might assume a latent renal distemper sufficient to taint the blood but not gross enough to amount to Bright's disease, we might find a solution of the problem of hyperpiesia. Dr. Shaw does not forget that renal cortex is not inexhaustible, and that, under the morbid conditions postulated, its reproduction could scarcely be very ready; but with organs thus labile, stores would presumably in no great interval of time be expended, and even before that period rather reduced than multiplied. Now the course of Hyperpiesia, as described by Huchard and myself-the disease which Dr. Shaw would draw into the net of primary renal causation-may, and frequently does, continue for years upon years-from ten to fifteen yearsbefore its final events, not of uræmia, but of cardiac defeat or

1 Pearce, Journ. of Exper. Med., 1909.

2 Schlayer, Deutsche med. Wochenschr., Nov. 14, 1907 
of cerebral hæmorrhage, appear. And I think Dr. Shaw does not give weight enough to the experience that our cases of hyperpiesia, if detected in good time, are curable, and curable repeatedly; unless perchance he supposes that our remedies act favourably by virtue of some influence on the kidneys not directly intended or recognised by us. Again, should we not find that other kinds of disturbance of a kidney,-as by a calculus or by a mass of tubercle, for instance,-by vexing the renal tissue and by promoting the assumed disintegration of the cortex and diffusing it into the blood, would run up arterial pressures? Yet, as a rule, such incidental lesions, however solvent they may be of renal tissue around them, do not raise pressure, nor deform the arteries or heart. And after all, were Dr. Batty Shaw's hypothesis to prove true, it would not set aside the practical distinctions between the series of clinical events long familiar to physicians under the name of "Granular Kidney" and the different series distinguished by Huchard and by myself as Presclerosis or Hyperpiesia.

Let me now describe Rautenberg's experiments definitely. ${ }^{1}$ Rautenberg works in Lichtheim's laboratory at Königsberg, and his report of two long series of experiments seems to prove that he is an expert investigator. At first he lost many animals (rabbits), but as he became more dexterous and more successful, about half of them survived. His improved operation consisted in tying the left ureter for three weeks, a period determined by experience. Then connection was made anew with the bladder, and the right kidney was extirpated. Some animals lived for nine months, two for a year and a quarter, one for a year and a half, one for a year and three-quarters, one for two years and a half. The blood pressures rose distinctly in about nine months, and were then systematically measured in them and in the controls. The rise proved to be proportionate to the duration; in the longer survivors it rose (from 110-122) to 160-170. On necropsy, in all these cases arterial disease was found. The heart was in like degrees enlarged up to 2 or 3 grams; but as in these animals the organ is inconstant in size, well-marked enlargements only were accepted. Alvens, ${ }^{2}$ for the same purpose,

1 Rautenberg, Deutsche med. Wochenschr., March 24, 1910.

2 Alvens, Deutsche Arch. f. klin. Med., Dez. 3, 1909. 
used gradual compression to the kidneys of animals, and found blood pressures rose parallel with the degrees of compression. Similar compression of the spleen, or other viscera, did not produce this effect. In some control experiments the nervous connections were cut, so as to eliminate afferent stimulation, but the rise took place just the same. Furthermore, contrariwise, as the compressions were relaxed the blood pressures pari passu fell ; and this in Alvens's opinion proved that the rises were not due to squeezing of juices from the kidney into the blood. As in Dr. Harvey's experiments in our own laboratories, ${ }^{1}$ the arterial disease of these experiments could not be called atherosclerosis, but a calcifying necrosis rather (p. 223). That the rise of pressures could not have been due to the arterial disease was evident in those animals which died in the earlier stages of high pressure, when the lesions of the vessels had not advanced beyond pinpoint and pinhead spots; a stage at which they could not have had any such effect. Rautenberg admits that if directly the arterial damage be a consequence of rising pressures, yet some forerunning toxic condition may make the arteries more susceptible to the abnormal stress. ${ }^{2}$ Harvey's researches however, by experiments in which compression only was used, demonstrated that mechanical stress was amply sufficient to establish this arterial lesion; and Rautenberg thinks that, in the animals under his operation, mechanical stress was, at any rate, the chief cause. But in Granular kidney, so far as the peripheral visceral arterioles are concerned, we have seen that excessive stresses cannot account for their primary atherosclerosis. Another remarkable result of Rautenberg's experiments was that even before the lapse of nine months-before, that is, any static effects of high pressures could be determined-the serum of these animals, even to dilutions of 1 in 20 , dilated the pupil of the enucleated frog's eye (Ehrmann's reaction ${ }^{3}$ ), thus suggesting that this serum

1 Harvey, A., Virchow's Archiv, 1909, Bd. cxcri.

2 It is remarkable that even in this process, of experimental abolition of renal structure by a uniformly acting cause, after death a focal distribution of the lesions was noted.

3 Ehrmann, Arch.f. exp. Pathol. und Pharm., 1905, Bd. liii. S. 97 ; and see Arch. f. ges. Physiol. Bd. cxxix., 1909. It is said however that normal blood gives more or less of Ehrmann's reaction. Schur and Wiesel (Wien. klin. Wochenschr., 1907 and 1908) attribute it to chromaffin tissue, and argue that muscular work calls forth a secretion of adrenin into the serum. 
may contain a pressor substance. ${ }^{1}$ Thevenot, ${ }^{2}$ after referring to Castaigne and Rathery's researches, injected rabbits intravenously (8-12 c.cm.), with the blood serum of nephritic patients (cases described), four times a day. He came to the following conclusions: (1) That the nephrotoxins of the blood of those suffering from nephritis are in accord with the gravity of the clinical phenomena; (2) that they are in inverse ratio to the state of permeability of the kidney-estimated according to the method of Claude and Balthazard; (3) that they are in direct relationship with the albumins in the serum ; $(4)$ that they appear to present no parallel with the amount of urea in the serum. It seems probable therefore that the nephrotoxins developed in man in the course of chronic nephritis belong, at least in part, to the albuminoids. It is possible that by Rautenberg's method the kidneys were not merely suppressed but were irritated, and so made to discharge a hypothetical " renin " into the blood; still, on the whole, they point to renal negation, rather than to renal poison, as the cause of accumulation of pressor influences in the blood. It is to be hoped that steps will be taken to verify Rautenberg's experiments.

Now, if there be but one pressor substance concerned, either this must accumulate always by fault of the kidney or there must be alternative conditions to give rise to its formation, retention, or excess. The kidney is understood to be not an elaborating, but an eliminating organ. Unless it be, as Dr. Shaw supposes, by sacrifice of its own body, it may fail to remove, but cannot manufacture, a pressor or any other poison. A failure to eliminate may, it is true, depend sometimes on transitory disorder, sometimes on incurable disease; or possibly the strain of excreting such a poison may drive the kidney into disease, though, at any rate at first, or in milder cases, one would look rather for a hypertrophy; a state however of which, in these circumstances, we have no experience. In the light of recent researches-on gout for instance-the fault seems to lie not in a failure of the kidney to excrete normal waste, but of the antecedent reductive

1 In this connection I must mention again the various interesting researches on "Sympathomimetic Amines"-for example, of Barger and Dale, Wellcome Physiological Research Laboratories [recent, but no date on my reprint (1910?)].

${ }_{2}$ Thevenot, Prov. Med., May 7, 1913, quoted Epit. Brit. Med. Journal. 
processes to render the waste into substances attractive to it. For while refusing the gouty ingredient, the kidney is often doing active work with other materials. I do not think that Widal's division of renal diseases into nitrogenous and chloride retentions respectively, if incidentally convenient, is tenable.

The opinion of observers who have worked at this subject is, on the whole, strongly in favour of some poison, whether a crude katabolic or an alien ingredient, at the back of both Granular kidney and of the associated peripheral atherosclerosis; these being concurrent effects of the hypothetical agent, as in the case of the similar, if not identical, plumbous kidney. But the more central atherosclerosis, after a while established in the large vessels, and progressing outwards, is probably less the result of poison than of consequential strain.

As I have argued in respect of the kidney, so the arteries likewise, by their peculiar limitations, are disabled from expressing as variously the various influences falling upon them; sclerosis may be their monotonous response to sundry kinds of bane. I have mentioned Abelous' ${ }^{1}$ report of a pressor substance, in the urine of normal adults-a hypothetical "hypertensine" - which, when retained in the system, as alleged by Dr. Bain, incites to rises of pressure, and may damage the kidneys. If this conclusion of Bain be verified-that in health this pressor body is eliminated, and that in high pressure cases-nephritic or hyperpietic-it is not excreted, we should be possessed of a very valuable principle; but there is much work to be done before it can take its place even as a working hypothesis. Abelous says that urohypertensine is absent from the urine in arteriosclerosis; but we have seen that " arteriosclerosis" is no clinical diagnosis. This toxic agent, Dale and Barger, and Dixon and Harvey, are disposed to identify with a certain amine of putrid meat, allied to, or identical with, ergot; this putrid matter, formed in certain circumstances in the intestines, may be absorbed into the system raising pressures by vaso-constriction, and also injuring the kidney directly. Abelous also has found such bodies in putrefying flesh. Bain described two of these pressor bodies, that of Abelous and another, both of the stem of leucine and tyrosine, as constituents of normal urine, stating that in chronic

1 Abelous and others, Comptes rendus de la Soc. de Biol., Paris, tome i., 1906. 
gout or hyperpiesis they fail to be excreted; but not by default of the kidney, as the harder work of excreting sodium chloride proceeds undisturbed. But surely the release or retention of sodium chloride is not a mere renal function; with kidneys presumably normal the salt is retained in many diseases-in pneumonia, for instance, and in certain dropsies. Salt retention is, in large part at least, a matter of the heart and circulation (p. 231), and the isotonicity of circulating fluids. Abelous' body was probably Barger's para-hydroxyl-phenylethylamine-a pressor substance. Harvey ${ }^{1}$ has experimented on rabbits with it; on some by injection, on some by the mouth. In 20 rabbits the kidneys became diseased, in degrees from arteriolar thickening only to extensive and universal change (Aschoff, Gaskell, Lorrain Smith, and others); in 13 the results were negative. In 25 the aorta had degenerated, in 10 the heart was enlarged. The duration of life in the renal positives was from 80 days to over a year, in the negatives 30-70 days; but one of these lived for 141 days. The urine produced vascular disease, first in the aorta, secondly in the renal arteries; at first without alteration of the parenchyma. Then the epithelium became impaired, the glomeruli dilated, their capsules thickened, and here and there were some round cell infiltrations. The quality of the urine and the degrees of renal lesion did not run parallel, still these results seem to be of considerable importance. Crofton ${ }^{2}$ found that certain xanthine bodies, if pressed in full doses, caused at first granular and fatty deterioration of the renal epithelium; in animals which survived six months the blood pressures rose and intertubular proliferation of the connective elements ensued. Professor Walker Hall has made experiments of a similar kind and with similar results (see his Purin Bodies, 1903). But these problems are as yet very obscure and difficult. For instance, in our laboratories Dixon and Harvey, to obtain their putrescent toxin in appreciable quantity, had to evaporate a litre of urine. Dixon traced the pressor substances to putrescence in the alimentary canal ; thence to find their way into the urine, whence they were separated by appropriate solvents. He regards them not as toxins but as crystallisable

1 Harvey, Journ. Path. and Bact. vol. xvi. No. 1, July 1911.

${ }^{2}$ Crofton, Amer. Journ. Med. Sci. vol. cxx. p. 592, 1900. 
chemical substances, such as isoamylamine. Meanwhile we shall not forget Professor Schäfer's caution that in any animal dying slowly the blood may become toxic.

Renal Infections.-We may now turn to note the effects of toxins of other and more familiar kinds ; in particular, those of the infective diseases-of scarlet fever, of diphtheria, and so forth. The focal distribution of lesion might lead us to postulate that these distempers affected the renal meshes rather by bacteria than by toxins, were it not that we have seen how the simplest lesions in the tissues may be thus scattered. However this focal discontinuity is less apparent in acute nephritis, which is probably always of toxic origin. As Ribbert observes, the trend of evidence is that in the various renal diseases toxins seem to play a much larger part than the bacteria. However this may be, the lesions of the infective diseases are disposed to attack the renal epithelium, as if directly by an acute cytolytic process ; but -as in the puerperal kidney - this corroding necrobiosis may, if time be given, associate itself with irritative overgrowth of the connective elements, so that an interstitial nephritis, not distinctively glomerular in distribution, appears. Grancher and Sargent, ${ }^{1}$ in toxic nephritis, noted the epithelial (and glomerular) seat of the disease, and the secondary nature of the interstitial changes. The primary lesion they observed in the secreting epithelium, the cells of which, if not destroyed, were swollen and fattily degenerated. Many tubules had vanished; others were shrunken or moniliform; others again thickened and abraded. Such lesions Welsh and Flexner demonstrated in diphtheria also, and opined that they might be due to bacilli plus their soluble products, or to the toxic principle alone ; or, again, that a mixed infection might occur, the diphtheria bacillus or toxin combining, for example, with a streptococcus, for multiple mischief. From such merely tubular attacks recovery may take place, indeed it is not infrequent; but, as in scarlet fever, there is a fear of a second stage, in which a perivascular cell infiltration sets in, and a diffuse nephritis, large white or secondary shrunken, marked by implication of all the renal constituents, glomerular, cellular, and fibrous. To add to this perplexity, the putrescent toxin of Dixon and Harvey,

1 Grancher and Sargent, Rev. de méd., 1901. 
although a hypertensive poison, is correlated, not with Granular kidney, but primarily with a tubular nephritis and a dropsy of the tissues, strikingly manifested in dropsical distension of the glomerular cavities. Toxins apart, if the renal artery be tied (in animals), the epithelium begins at once to degeneratea degradation due, it would seem, merely to deprivation of oxygen; if the animal survives for at least six months, an atrophic fibrous, but not a "Granular kidney" proper, is established. In practice we are well aware that we cannot keep too vigilant a guard over the integrity of the renal epithelium, as evidenced by integrity of its own and the cardio-arterial functions. For many months after apparent convalescence this vigilance, if less incessant, should at due intervals be no less searching. Happily, as ablation experiments suggest, so long as a substantial fraction of renal structure remains intact, much elimination can go on meanwhile. The patches of nephritis in the kidneys of children, consequent upon bacterial infections of the urinary tract (e.g. B. coli), are not attended with high arterial pressures, nor is the heart enlarged; there are no uræmic symptoms, and hemorrhages, if any, are not especially in the brain, but also submucous and the like.

Now in these infective renal diseases, as may be well discerned in young subjects, the arteries are not necessarily affected (see Jores's first paper, Cases XV., XVI., XVII., XVIII., both chronic and acute); not even in the scarlet fever kidney, as demonstrated in Jores's case of the child aged 5, in which nevertheless the heart was somewhat hypertrophied and dilated. Riegel, ${ }^{1}$ in the scarlatinal kidney, has demonstrated a rise of arterial pressure without any renal arteriosclerosis, and $\mathrm{Kalb}^{2}$ likewise. This is very puzzling. The explanation probably is that the scarlatinal kidney, in whatsoever stage of shrinkage, is not, and never becomes, the true primary or Granular contracted kidney; but, at worst, is a "secondary contracted" kidney, the result of a glomerulotubular nephritis. But clinically such a kidney is followed at length by the systemic arteriosclerosis of high blood pressures. Dr. J. D. Rolleston ${ }^{3}$ reports that in the majority of cases of

${ }^{1}$ Riegel, Zeitschr.f. klin. Med. Bd. vii. $\quad{ }^{2}$ Kalb, Münch. med.Wochenschr., 1903. 3 Rolleston, J. D., Journal of Childr. Dis. vol. ix., 1912. 
scarlatinal nephritis the pressures are not in excess, often indeed are under the norm. Of 33 such cases the pressures were excessive in 12 only, and excess persisted only in 3 . The renal arteries in scarlatinal kidney if often, are not always affected; in one of Jores's cases they were not. Such cases in children are largely tubular (epithelial). The toxic inflammation resembles that of cantharides, absinthe (see p. 352), mercury, and other renal poisons, which may issue in interstitial overgrowth, but only at length, in chronic cases. Such cases as these mark off the toxin of infective nephritis, without intimate renal arteriosclerosis, although making in some measure for cardio-arterial strain, from the poison-if such there be-lying behind granular kidney, in which the intimate renal arteriosclerosis and the enormous cardio-arterial strain are essential, and in some way correlated, events. In such a case as that of acute dropsical nephritis in a man, aged 29, published by Heineke, without visceral arteriosclerosis-for example, normal spleen, pancreas, etc., as determined after death-in which, on the lapse of ten or twelve months, arteriosclerosis appeared in the large and small arteries of the extremities, one is tempted to guess that the dropsical fluid itself might have contained an arteriolytic poison?

Sir John Bradford (Croonian Lecture, 1904) tells us that syphilitic kidney is commoner than we suppose. Stadler, von Romberg, and others doubt this, and suspect that some " cardiac kidneys" in syphilitic heart disease have been included. But in one clinic-I think in Leipzig ?- five "Schrumpfniere" were found in fifty-two autopsies with syphilitic aorta. In syphilitic arteritis affecting the district of the pancreas a periarteritis is seen to extend and penetrate into the substance of the organ, the process being marked by a profusion of lymphoid cells. The following case, homologous with the syphilitic pancreas, was published by Dr. Loomis. ${ }^{1}$ The father contracted syphilis twelve months before the birth of the patient, and died of it when the patient was six years old. The only other infections which occurred to the child were whooping-cough and chicken-pox. Twelve months before its death (by lobular pneumonia) albumin and casts were found in its urine, and the left ventricle of the heart was hypertrophied. The

1 Loomis, New York Path Soc. Trans., 1889. 
kidneys were about one-third of the normal size, the surface rough, the capsule thick and adherent. There was a chronic arteritis, of almost all the smaller arteries, and much intertubular connective tissue. The fibrosis was very unevenly distributed, and the cortical tissue was not diminished regularly, in some places it was hardly narrowed at all. Apparently the secreting cells were not primarily nor universally diseased, and we may doubt if such a kind of renal disease be more mischievous than the senile arteriosclerotic kidney.

Nervous Factors.-Hitherto I have said little of any nervous factor in these problems; they are baffling enough as it is, and one is unwilling to seek the obscure by way of the obscurer. Briefly, Sir John Bradford ${ }^{1}$ has pointed out how stimulation of one of many afferent nerves produces general constriction of visceral blood-vessels, including those of the kidney, with consequent rise of arterial pressure; and that other great fluctuations in the renal circulation are produced by excitation of the central ends of the depressors, or, again, of the central ends of the posterior roots of certain lower dorsal and upper lumbar segments, whereby the often assumed association of renal engorgement with general vaso-constriction, as may be the case in puerperal eclampsia, is manifestly obtained. But as yet from this side of the problem we have received little light, although we may speculate whether our hypothetical toxin or toxins raise arterial pressures directly by acting on the peripheral neuro-muscular machinery, or indirectly by the middle term of some renal product having such an operation? Of this at least we may be sure, that the intimate renal arteriosclerosis of Granular kidney is no incidental effect of vasomotor tides; that this primary and essential effect is brought about by some virus, whether, renally speaking, autogenous or exotic. Extensive vaso-constriction, with great rise of pressure in the main arterial trunks, may of course mean very low arterial pressure in the renal and other peripheral areas. Thus, contrary to current opinion, very high pressures may concur with very deficient flow of urine.

The Adrenals.-I must not omit to allude to the hypothesis

1 Bradford, Sir J. R., Goulstonian Lecture, 1898, and later papers. 
best known as that of Wiesel and Schur, favoured by Josué, that the rise of pressures in renal diseases is not attributable to the kidney at all, but is an accidental event due to a variable implication of the contiguous adrenals; or otherwise of the chromaffin system ("erhöhte Funktion der chromaffinen Gewebe "). Before Wiesel and Schur, or independently of them, this reasonable guess had been made. The constancy of pressure excess in Granular kidney might be explained by a special proclivity of this mode of nephritis to propagate itself in their direction ; or again by some community of miscarriage in the organs as pairs. The hypothesis has called forth a multitude of papers of which only a few can be noticed here. ${ }^{1}$ Eichler ${ }^{2}$ agrees with Wiesel and Schur, and so in great measure do Tacher, ${ }^{3}$ and Falter and Priestley of von Noorden's clinic. ${ }^{4}$ Pearce says after the age of 50 increase of the adrenals is found with renal disease, " almost constantly," or with arteriosclerosis, or with both. $\mathrm{He}$ regards it as compensatory, as does Pribram ${ }^{5}$ in another way. Schlayer ${ }^{6}$ on the contrary, with Meyer's delicate method of testing by arterial contractility, and Bittorf got negative results, and Meyer's method is said to be more trustworthy. Many observers question Wiesel and Schur's tests. Frank of Wiesbaden remarks upon the absence of hyperglycæmia in chronic renal disease.?

The adrenal hypothesis is contested, and I think conclusively, on the grounds both of morbid anatomy and of biochemistry. Lubarsch failed to verify a true hyperplasia, and stated that the cell conditions described by Wiesel and Schur are found in normal adrenals. We read much too vaguely of "hyperplasia," "hypertrophy," and the like. Landau, ${ }^{8}$ in 15 cases of "arteriosclerosis," found no changes in the adrenals save the sclerosis of their own vessels. Pearce, by estimation of the

1 See e.g. Vaquez and Aubertin, "Hyperplasie surrénale des néphrites hypertensives," Bull. Soc. Méd. des Hôp. de Paris, 1905; and Aubertin and Ambard in the previous year.

2 Eichler, Berl. klin. Wochschr., Nov. 11, 1907.

3 Tacher, Deutsche Arch. f. klin. Med. Bd. xcvii., 1909.

4 Falter and Priestley, Berl. klin. Wochschr., 1911, No. 47 ; see also Pearce, Stud. Bender Lab. vol. v., 1908.

5 Pribram, Münch. med. Wochenschr., Juli 25, 1911, No. 30.

6 Schlayer, Deutsche med. Wochschr., Nov. 14, 1907. See also Nos. 45 and 51.

7 Frank, E., Berl. klin. Wochschr., No. 14, 1912.

${ }^{3}$ Landau, Zeitschr. f. klin. Med., 1907.

VOL. I 
adrenals in 12 healthy persons over 50 years of age who had died by accident, tried to establish a standard, and with this standard to compare 163 adrenals in arteriosclerosis ; but unfortunately without notes of blood pressures. Stewart ${ }^{1}$ took up the tests for adrenin and submitted them to rigorous criticism and experiment. He had found that injection of serum from a case of nephritis with very high blood pressure into dogs caused in one experiment a slight fall of pressure ; in the second a rise of pressure, but so did a control injection of normal saline. Serum from a case of non-renal high pressure (Hyperpiesia) caused in a third a transitory fall of pressure ; and after cutting the vagi, no effect at all. He found the test by the enucleated frog's eyeball not discriminating enough; the urine is far from isotonic, and a solution of sodium chloride produces the same mydriatic effect. Stewart preferred the inhibiting test on gut, and the stimulant test on the uterus, but, even in normal blood, he got no evidence of adrenin. Erwin Thomas, of Marchand's laboratory, ${ }^{2}$ after a close survey of a large number of specimens, concluded that in renal disease the adrenal cortex is much oftener affected than the medulla; for instance, in 40 cases of shrunken kidney he found an enlarged cortex. But he often found the same enlargement where the kidneys were intact; or in cases of simple non-renal atherosclerosis. The medulla he found no more affected in chronic renal diseases than in relatively normal controls. In a word, he was unable to discover that either cortex or medulla behaved in ways consistent with any of these morbid series; renal, cardio-arterial, or other. (See also Elliott, p. 255 n.)

In conclusion, what issues out of this discussion to elucidate the relations between arteriosclerosis and the kidney?

May we not go this far?-That arteriosclerosis being almost the only terms in which an artery, apart from implication in surrounding tissues, can respond to irritation, we should expect it to give this peculiar response to various untoward influences. In Granular kidney it responds firstly by atherosclerosis of its intimate intravisceral twigs ; secondly, at a later period, when not these or such vessels but the systemic trunks begin to suffer under

1 Stewart, G. N., Experim. Med. vol. xiv., 1911.

2 Thomas Erwin, Ziegler's Beitr. z. Anat. vol. xlix., December 1910. 
another influence-under the stress of excessive pressures, the vessels again make the only response of which they are capable ; they also to a different injury express their reaction in similar terms of arteriosclerosis. Leaving now kidney disease and turning to the series which Huchard called "Presclerosis"-my Hyperpiesia - a series in which as yet the kidneys have not been convicted of complicity, we may find again arteriosclerosis ; but only after the mode which in Granular kidney affects the arteries of large and middle magnitudes under tensile strain, a secondary, and often preventable result. To the arteriosclerosis of hyperpiesis the kidneys, so far as yet known, stand in no correlative relation; except, as Dr. J. F. Gaskell says, that, generally speaking, the kidney is one of the organs whose arteries are affected early.

Secondly, there is yet another arteriosclerosis, or an arteriosclerosis found in quite another category-namely, the arteriosclerosis which, in 1894, I separated as the senile or decrescent form. Whether or no it be histologically identical with the kind of which we have just spoken is as yet an open question; but clinically it is distinguishable, as it arises independently of high arterial pressures, or, at any rate, under pressures not excessive for the time of life. Now in this mode of arteriosclerosis the kidney is often altered, always perhaps, more or less. This alteration, which again often puts on the aspect of a shrunken or deformed kidney, has little pathological affinity with the Granular kidney which it may simulate. Thus, besides the Granular kidney, which goes wrong with an intimate, probably toxic, arteriosclerosis of its own, there is a kidney deformed in the course of a general arteriosclerosis not its own; and, if more or less perishing by decay of its nutrient arteries, yet not primarily much amiss : a starved but not a corrupt kidney. It is but a sample of an almost universal degradation; the spleen is small and hard, the muscles and skin are wasted, the lungs are atrophied, and so on. This form some of us have long tried to differentiate, being disposed to see in it rather a negative state, a state more or less inadequate in regard of margin of safety, but sufficient for the smaller life of an elderly man. Dr. Sainsbury has emphasised "the marvellous residual vitalities of damaged organs." This kidney contains many islands of fairly 
normal renal tissue sufficient for a narrower life, a feature by which I was disposed to contrast it with the true "Granular kidney," which I had regarded as necrobiotic. But so sharp a contrast cannot quite be maintained; in the Granular kidney also there are islands of apparently normal tissue, and the essential difference seems as yet to lie, not within the compass of the kidney, but-as we have surmised-in some poison at the back of the vascular disease in the kidney and elsewhere. However this may be, the two kinds of kidney, often not unlike in aspect, at any rate superficially, are in nature radically different -a difference which, if less obvious in an effete organ, yet by the clinical series to which it belongs is, in uncomplicated cases, decisive. And in this distinction, as my citations have proved, I am far from standing alone. For instance, Dr. Andrewes ${ }^{1}$ says, "This form (the "Granular kidney") is associated with great left ventricular hypertrophy, whereas the senile type of granular kidney scarcely raises the heart weight." Then should we not discard the title "Granular" altogether?

Transitional forms, if they exist, are so infrequent as to be negligible. ${ }^{2}$

Accordingly, Professor Lorrain Smith's conclusion, in our joint paper, was that the arteriosclerotic kidney is a local phase of general arterial disease, producing in the kidney just the alterations which it produces in other organs of the body-alterations which need not amount to anything very grievous, as they are compatible with many, if sere and yellow, years of life; but that Granular kidney, on the other hand, is some form of progressive lesion of the gland as such, the structures essential to its function being attacked. In this mortal disease arteriosclerosis appears in two ways-primarily and essentially in the fine arterioles of the organ itself; secondarily and later in the systemic arteries, the effect of strain under the excessive blood pressures in which Granular kidney is always and regularly involved. The arteriosclerotic kidney, on the other hand, if it may arise in the later stage of strained arterial systems in hyperpiesia, which personally I am not ready to admit, is most commonly found in the decrescent form of arteriosclerosis.

1 Andrewes, F. W., St. Bartholomew Reports, vol. xlviii.

${ }^{2}$ Herein I disagree with Senator, loc. cit. p. 344. 
Were I to try to formulate these provisional conclusions they might run as follows :-

\section{Arteriosclerosis in Chronic Bright's Disease. ${ }^{1}$}

Class A

A (i.) Primary intimate intrarenal arteriosclerosis not due to high pressures: in Granular kidney constant; in other kinds inconstant. Found likewise in some other organs.

A (ii.) Secondary, systemic, arteriosclerosis ; consequent on high pressures: as seen also in secondary contracted kidney ("small white"). More visible in main branches.

A (iii.) A few mixed cases, not originally Bright's disease; such as renal disease supervening on a general arteriosclerosis, whether "Hyperpietic " or "Decrescent." [It is suggested by some pathologists that the primary contracted, or "Granular" kidney, properly so called, may be due not to any toxin but to long prevalence of high arterial pressures.] I am not of this opinion, unless in a very modified form.

\section{Not Bright's Disease.}

\section{Class B}

B (i.) The "arteriosclerotic kidney"; an alteration implied in general arteriosclerosis, usually of the decrescent kind without high pressures. An atrophic condition.

$B$ (ii.) Less frequently, entailed by the consecutive arteriosclerosis of excessive blood pressures (as A (ii.) ).

High pressures may be considered as (i.) obligate with primary Granular kidney; (ii.) commonly associated with other kinds of Bright's disease ; (iii.) independent of Bright's disease (e.g. "Hyperpiesia ").

B (iii.) The merely starved kidney of old persons in which fibrosis may be more evident than arteriosclerosis.

1 The term Bright's disease is used to cover the two or more primary diseases of the kidney. 


\section{CHAPTER VI}

\section{Symptoms of ARTERIOSClerosis}

IF arteriosclerosis be not a disease but a result of two or many different diseases, it is evident that, if it may have incidental consequences, consequences of a more or less mechanical order, it can scarcely have any uniform series of symptoms of its own; unless, indeed, we accept the not very probable hypothesis of a recent writer who, still influenced by the hypothesis of Virchow who saw in arteriosclerosis an inflammatory process, says that this arterial change is a substantial malady having a characteristic course attended with a subtle periodic fever. If the febrile stages of the change escape us, it is, in his opinion, because of our lack of vigilance ; for the change in the arteries advances or may advance by phases of activity and remission. That under the influences of various poisons, arteriosclerosis does often progress in this fluctuating way we have seen; and it is obvious that when later the vessels are so far damaged as to be impaired in their functions, then secondary effects, or symptoms, may arise; but various consequential perversions, the direct effects of the infection, can scarcely be regarded as symptoms of arteriosclerosis in an immediate sense. Arteriosclerosis then, a by-product of many a disease, is not, and does not itself represent, any single or self-consistent, specific symptomatic series. Whether indeed, even in its morbid anatomy, it is uniform is a question to which, as we have seen, and shall see further) we have not yet a definite answer.

Thus it is that, in beginning the chapter of Symptoms of Arteriosclerosis, I am undertaking to unravel an entangled skein, or rather to meddle with a heap of skeins, and to attempt so to separate them as to track them out singly. Fortunately 
many of these skeins, by the united labours of observers ancient and modern, have been combed already into some order; others are still confused, but with some one of them I must make a beginning.

Local Manifestations of Arteriosclerosis.-Many have been the attempts to discern the various modes of arteriosclerotic processes and their several series of consequences; some of the most ambitious of these schemes we owe to the French school. This seems to be the place to consider certain hypotheses of this kind built upon the local incidence of arterial disease. And if for illustration of these hypotheses I reflect upon the teaching of M. Teissier, ${ }^{1}$ I trust my criticisms may be regarded as a tribute to the representative views of an eminent colleague. The attraction and persuasiveness of French medical authorship depends upon a quick clinical insight; but it depends also upon a brilliant talent for system and hypothesis"l'envie de faire des sentences," - the logical ardour of which is apt to blind both author and reader to the complexity and elusiveness of biological phenomena. A logic and symmetry, which in the simpler sciences of mathematics and physics are an inspiration, may in the biological sciences be a seduction. M. Teissier has drawn up a very attractive scheme of "Clinical Forms" of arteriosclerosis, forms based not upon diversity of nature but on local distribution. Arteriosclerosis is treated throughout, after the school of Huchard, as one uniform process, of which the chief marks are "vascular hypertension, a large heart, and arteries hard and often tortuous." Such a uniformity cannot be admitted ; but let us listen now to M. Teissier. The one process, one in nature as he conceives it to be, may concentrate itself here or there, and by this diversity of distribution set up a diversity of effects. Thus the author creates a series of types, lucidly set out in elaborate tables, such as the "aphasic type," the "cardiac type," the "gastro-intestinal type," the "renal type," and so forth; the clinical events varying with the changes of the incidence of the arterial disorder; now cerebral in type, now cardiac, now abdominal, and so forth-a territorial arteriosclerosis.

\footnotetext{
1 The latest paper I have before me is "Les Formes cliniques de l'artériosclérose," Leçons prof. mai 1908, Rep. Journ. méd. Franc., 1908, pp. 629-638.
} 
When we ask what happens in each district thus affected ? what the mechanism may be? we receive a prompt answer: there is an "elevation of vascular tension in the department functionally concerned," i.e. in the temporal area, the tibial, the radial, and the rest (vide p. 30). But is this answer satisfactory? Teissier records in several cases pressures of $22 \mathrm{~cm}$. in the radial, $20 \mathrm{~cm}$. in the temporal, or the tibial, and so on ; or the pressure in the tibial was higher than in temporal or radial (see $0 . \mathrm{K}$. Williamson's observations, p. 75). Now these numbers were obtained by moving Potain's instrument from place to place, a method full of fallacy; indeed no instrument can be trusted to measure such local differences. The data are illusive. It may be true, assuredly it is true, that sclerosis is unequal in its incidence, but it is contrary to the principles of hæmodynamics to suppose that primarily local variations of wall or diameter could heap up local gradients of pressure. That alterations of the wall of a vessel modify the wall wave we know, and they may bring with them also other alterations of resistance; but blood pressures cannot thus vary from place to place. Yet it is continually reasserted that each part of the body may have its own gradient. When we ask about the mechanism are we not put off with words? Let us enquire a little further. We are told that in such a district the local blood pressure is raised; for instance, in the "type aphasique" the blood pressure in the temporal artery, and inferentially in branches within, is said to rise, say to 250 ; in the radial and tibial to 190 . If we demur that unless this rise be confined to the particular district, if the temporal district does but share in a general rise of pressure, wherein lies the peculiarity of the "type"? The rejoinder is that the rise of pressure is local, not general. But we have seen that such a local rise of pressure, measurable in inches $\mathrm{Hg}$ (" $12 \mathrm{~cm}$."), is, save as momentary fluctuation, inconceivable; it would be neutralised instantly by dilatation or derivation elsewhere. An excessive afflux of blood might of course be persistent, but this would scarcely signify a rise of lateral pressure much above the surrounding parts, and would be no less consistent with a fall of peripheral resistance. (See remarks on "Stromintensität," p. 28.) But we need not dwell on this point because the author-whom I 
am taking as representative of current opinions-says plainly that the state is not one of intense afflux of blood but of the contrary: he describes the state as one of "localised spasm," and the question is asked whether-as caused by toxin, emotion, overwork, etc.- "the hypertension provokes or betrays the localised spasm, the source of partial ischæmia leading to suppression of function"? We are not invited then to observe a large output and dilated vessels but an anæmiated area. But, if so, where, within the area, is the "hypertension"? What is stretched? A distinguished physician writes: "The pulse was very small, hard and thready, and of extremely high tension. The brachial artery could be rolled under the finger as a small hard cord." A vessel, or group of vessels, while under constriction cannot be "tense," cannot be stretched, or suffer injury by stretching. Nor can the blood pressure within be raised; the area must be one of low pressure, except so far as it may still admit a vein of a more or less high systemic pressure, which however is explicitly excluded. Dr. L. Hill corroborates the general principle when he says that to bandage the hand tightly makes no difference to the pressure readings of brachial or radial artery. Even just anterior to the constricted area the vessels cannot be tense. It may be true that if on contraction of vessels of supply to a large area the pressure head is reinforced, by plus velocity the blood supply may be maintained; but we have no knowledge of any local reflex or other mechanism to compass such a result in comparatively narrow fields. Dr. Bayliss and Professor MacWilliam have shown that compressions of an artery may give rise to vascular dilatation in the part supplied, but compression is not constriction; compression may affect the (supposed) nerve-end organs of the arterial investment, as an embolus seems to do. Indeed to raise universal pressure to get over a small field of constriction would be as uneconomical as it would be ineffectual. The only adequate reflex we can think of would be a tissue call for vascular dilatation in its field.

What would happen to arteries in areas where vaso-constriction had long been approximately complete, as in so-called "local syncopes," is a more complex problem ; and it is a problem not of physics but of biology. From what we know of the effects of 
ligature and, however imperfectly, of Raynaud's Disease and ergotism, constriction to the degree of closure results in, or more probably is correlated with, a kind of arterial lesion (Endarteritis obliterans) to which I shall make a passing reference under the head of morbid anatomy. In these diseases there are factors at work of more virulence, and a local disorder more profound, than mere sclerotic constriction. Some of the cases may be syphilitic.

I am afraid, therefore, that the French scheme of diseases by territorial arteriosclerosis will not bear close examination.

It is surprising how many authors, such even as Pal and Gouget, if not indeed authors in general, attribute arteriosclerosis to constriction, which, I repeat, must surely be a protection -at any rate against strain of the wall. An experiment of Hirsch and Thorspecken neatly proved the converse; namely, that if equal doses of adrenalin be injected into two equal rabbits of which in one the depressor has been cut and in the other not, the aorta with the intact depressor suffers far less injury. ${ }^{1}$ I suppose the notion of damage under constriction must be a griping of the tissues? But it is not easy to understand the notion, for if the damage be by squeezing, then why "tension"? Yet Pal definitely says: "When the tension is produced by contraction in a definite vascular area(!) then follow peculiar pain," etc. etc. ${ }^{2}$ My mind's eye loses sight of him.

In this, as in previous chapters, I shall observe first the two main distinctions of arteriosclerosis : the kind consecutive to hyperpiesis, and the primary or decrescent kind.

I propose then, omitting here renal diseases recognisable as such, to begin summarily, though I fear not without some repetitions, with the symptoms and signs of high pressures as correlated with arteriosclerosis-Hyperpiesis (pp. 346-7)-so far as they have been made out.

Hyperpiesis.-We have seen that Von Basch assumed the high pressures to be the consequence of the vascular lesions, but Huchard and I pointed out independently, in 1893-94, that the converse was nearer the truth; that, when arteriosclerosis and high pressures were associated, the lesion was usually the result of

1 Hirsch and Thorspecken of Göttingen, Deutsche Arch. f. kl. Med., Bd. cvii., He. 5 und 6 .

${ }^{2}$ Quotation from Osler, Lancet, Mareh 26, 1901. 
the strain. For Huchard this association was invariable; high tension first, arteriosclerosis second. My opinion was that in many cases of arteriosclerosis, perhaps in half of them, the arterial pressures were not inordinate, and probably never had been. ${ }^{1}$ When therefore Dr. Stengel and other physicians urge upon us, and most truly, that early diagnosis of arteriosclerosis is imperative, I ask: Of what arteriosclerosis ? for it is no unity, and the beginnings of its kinds are widely different and apart. If then in a substantial proportion of cases of arteriosclerosis, "high tension," even as an intermediary, is perhaps as often absent as present, in these we have surely to look for causes other than high tension. Concentrating our attention then first upon the "high tension cases," the causes-the differential causesof which I have discussed in preceding pages, can we begin to frame comparative clinical descriptions of these several classes? If we can distinguish the hyperpietics first-taking, that is, as our German brethren now concede to us, "die essentielle," as contrasted with "die nephritische Hypertonien "-we shall clear the way for differential diagnoses of the somewhat heterogeneous remainder of toxic and decrescent kinds, without excessive blood pressures.

First, let me recall that, in his classical treatise, von Basch attributed the high blood pressures solely and directly to the arteriosclerosis; and in cases of high pressure in which the accessible arteries did not exhibit sclerosis sufficient for this explanation of the pressure, he assumed a latent sclerosis of large arterial areas within, especially in the splanchnic area. And as the well-known assertions of Hasenfeld and Hirsch (vide p. 188) seemed for a while to support von Basch's conception, thus the opinion that high pressures are due to arteriosclerosis, latent or overt, gained a vogue almost universal. ${ }^{2}$ Huchard never shook himself free from this notion; and maintained the name of "Presclerosis" for the phase of high pressure which often does long precede an arteriosclerosis; thus essentially he blended

1 See v. Romberg, "Arteriosclerosis," Kongr. inn. Med., 1904 ; and Herzkrankheiten, 1906, who now admits the distinction.

2 Hasenfeld now admits that high blood pressures are not always the result of an already established arteriosclerosis or nephritis, but are often met with "as a functional hypertension," vide Zentralblatt $f$. Herz-u. Gefässkr. März 15, 1913. 
blood pressures and sclerosis as two aspects of one series. If it be replied that now this confusion is of the past, I would urge that the confusion is perpetual; were it not wearisome to do so, I could give many more illustrative quotations. Just one more recent example I will offer: Martinet (loc. cit.), after showing very definitely that high blood pressure may exist independently of known kidney or other organic lesionhis own categories are " high pressure with and without lesion" - proceeds to argue continually, in the same article, of arteriosclerosis as high blood pressure with high viscosity, and to compare it with "nephrosclerosis" as high pressure with low viscosity. Yet from the beginning I had affirmed that the conception of high pressures must be detached from its secondary and by no means inevitable implication of arteriosclerosis ; that these pressures must be regarded as significant of a certain malady originally independent of arterial lesion, but bringing this evil in its wake; and thus that the concept of arteriosclerosis should be broken up, and the lesion distributed among several diseases.

Can we, then, under the name of Hyperpiesia, or of any other appellation which hereafter may well be preferred to it, describe a Disease ; that is to say, a fairly uniform association and succession of symptoms, positive and negative? This I will endeavour to do, but the disease is often latent and stealthy, whereby indeed it has happened that the malady has not received the attention which so grave and often so mortal a visitation requires.

Whether the high blood pressures and the thickening of the accessible arteries in children, which we have encountered in a previous chapter (p. 171), is the same malady as that which we have now to describe in the adult, is open to doubt; in nature they may be the same, or akin, but this we must not assume : at any rate let us begin with the adult. In the adult, most frequently in the middle-aged, or still riper, man or woman, though sometimes in earlier adult life, we discover a state of disorder which, when it falls under our notice, has too often furtively obtained such an ascendancy in the body as to be if not beyond amelioration, yet beyond cure. One could wish its assaults were more vehement, that the symptoms were more clamant. We 
often read of " early symptoms such as a slight dyspnea or stenocardia on exertion" (I quote from a well-known recent book). Early symptoms! these are late, very late, symptoms : we are in the fifth act. Too often, it is true, the patient is not driven to the physician, or the physician is not awakened to the true state of things, until the occurrence of dyspnea, but then he awakes too late; the disease has reached its last stage secretly, a stage which thenceforth cannot be a long one. However, certain discomforts may instigate the patient to seek more timely advice, discomforts which I must try to indicate; or by some chance, such as an application for life assurance, the erudite sense of the physician may detect hyperpiesis in an early phase, in a period in which it may be completely curable, yet when, as I have said before, the patient may suppose himself to be in good health. With Dr. Christian Simpson, I saw Mr. R., æt. 56, in whom Dr. Simpson had perchance discovered that the systolic blood pressures ranged about 200 . I verified this by two instruments of my own; the diastolic seemed to be about 170 . The heart's apex was half an inch outward; the first sound much prolonged, as if seething on into a murmur (possibly there were eddies in the arch); the second very loud and clanging even at the apex. The arterial coats were little thickened, but it was difficult to stop the pulse of the brachial. He felt quite well and was doing plenty of work, but occasionally when in bed he had felt the heart's action to be labouring, and so sought advice.

It may be, though here I speak in much doubt, that for some persons a highish blood pressure is normal, pressures, at or under middle age, even up to 160-170. I shall be interested to hear the experience of my readers in this respect. Often I come across cases - some of them in physicians who had discovered it in themselves - in which systolic and diastolic pressures range thus high, and under a little excitement still higher for the while, yet in persons who nevertheless to all appearance and to all practical ends are in good health. One would apprehend that in advancing years, if the high pressures continue, there must be danger of an apoplexy. We are anxious to avoid alarms, and fusses, and to say nothing to turn a cheerful and vigorous man, often the more energetic for the ample tide 
of blood to his brain, into an anxious introspective invalid; but time after time, claudo pede perhaps, or more quickly, an economical prognosis and an indecisive treatment have been falsified by an apoplexy or a heart defeat. Of such at least are most of those who in the midst of energetic and rubicund health are struck down by the awful calamity of a hemiplegia.

It is convenient, if a little illogical, to describe first an advanced stage of Hyperpiesia, as being the most conspicuous, and thence to trace the spoor backwards. A patient of 55-70 years of age, or sometimes still younger, will tell us that in ascending hills he has found himself pulled up by shortness of breath ; perhaps also by a substernal constriction. His countenance may be healthy, or may be too florid, or may show a sallowish ground tint with a splash of ruddiness upon the malar eminences, tints apt to turn bluish on exertion. The general nutrition is usually good, often indeed with a disposition to fatness; though some of these sufferers are meagre, and their cheeks more sallow than ruddy. Or the symptom to drive the belated patient to the physician may have been a sudden hitch in his talk, or a vertigo, or a passing paresis of a hand or arm. Probably we shall be told that, if naturally of energetic temperament, he had of late lost his elasticity, had become more sluggish, fretful, and despondent, especially early in the day; he would "pout upon the morning," cheering up a little towards evening. Or the mental perversion may have been more distressing, and, even in earlier and curable stages, may have amounted to grave obsession. If he had kept a liberal table, he may not have lost appetite, but will have become more and more tempted to rouse himself with wine, to desire a glass of sherry in the forenoon; and to this excess an anxious wife, by drams, meat extracts, whipped eggs, and so forth, may have ministered only too assiduously. When pressed to describe the recent course of his health, the story is often vague and hesitating; he has been conscious of ailing, but scarcely knew how. He had not slept so well, andas I find in many of my records-he had been sensible during the night of his heart's action, especially when lying on the left side; he may complain also of a fulness of the head or dull headache, of an uneasy aching about the heart; perhaps of vertigo and some tinnitus; or again of some pursiness, or even 
panting on exertion, or after meals ; or he may speak-and this is a significant symptom - of unaccountable "bronchial colds"; and with this he may have had "biliousness," with lassitude, peevishness, and drowsiness, and perhaps one or more unaccountable attacks of nose bleeding. In women at the climacteric it is very difficult to discriminate between perturbations due to the period of life and those perhaps due to hyperpiesia. The urine may be lateritious, or alternatively profuse ; the bowels may be uncertain, sometimes costive, sometimes loose; piles may have protruded. Or a man thinks he is gouty, and talks of wandering pains about the loins or joints, and of more fixed pains in the side or back. These and such symptoms may have been attributed to "overwork" or "brainfag," and in some cases not without reason. Richard Cœur de Lion who, when not fighting, was, I fear, a glutton and a drunkard, and a bad subject for the septic wound of which he died, ${ }^{1}$ was probably a subject of hyperpiesia. After death, his heart, when placed in the gold casket, was described as "grossitudine praestans" (Gervase), and reverently admired as the fountain of his strength.

Now all these complaints are, as symptoms, too vague to dictate a diagnosis; they are consistent with many a disorder of a temporary nature; thus far, Israel-a pupil of Krehl-may plausibly demur that the symptom group is not characteristic; it may signify no more than what for many a generation has been spoken of summarily as "liver." And it may well be that this vast, manifold, and secret gland has no little to do with these cloyed passages and corrupted humours, some of them but temporary disorders, others however heading away into a permanent derangement. Or it may be, had we but looked for this feature, that in some of the states formerly confused under the name "biliousness," we should have found the blood pressures temporarily augmented; but in transitory indispositions such research is not thought necessary. A few doses of calomel clears the system; and, as we shall see, in hyperpiesia no medicine is so effectual as calomel or blue pill. If the "biliousness" becomes troublesome and stubborn, and the wary physician looks a little deeper, he may find in the pulse the crucial sign which he is seeking; it may be large, thrusting, and sustained;

${ }^{1}$ See Ramsay, Angevin Kings, p. 365. 
or small, tight, and wiry, so small perhaps as scarcely to give to the less wary observer a due sense of the often enormous pressure behind it, and the more equivocal as the narrow artery may still present to palpation a wall fairly or even quite free from sclerosis. Every now and then, or more frequently, as Dr. Mackenzie says (vide p. 390), an extrasystole may intervene, an interruption more apt to occur in slow pulses. Caeteris paribus, these pulses tend to retardation; other things however are often unequal.

The Circulatory System.-A fuller light will be thrown upon the case by the state of the heart. The heart usually presents quite decisively the features of hypertrophy, or at any rate of enlargement, especially of the left ventricle, with resonant aortic second sound; features too well known to need recount in this place. At a still later stage-for it is surprising how far this malady may surreptitiously advance, even to an apoplexy, without compelling attention, or at any rate without arousing adequate attention - the hypertrophy will have given way to a large dilatation; a mitral murmur may be coming and going, and the accessible arteries, if not protected byconstriction (p.377), will have become thickened and tortuous, at each pulsation lifting visibly from their beds. If thus the heart be yielding, such pulses to the finger will feel less "tense," the "virtual tension" of Broadbent; though, unless the patient be at the last stage of his malady, the pressure gauge will still indicate a higher number than by the finger we had supposed. The larger subcutaneous veins, as on the backs of the hands, are now often swollen, with a tumidity which is apt to fluctuate with the periods of the blood pressures. And a close inspection may detect a slight œdema on the shins; perhaps also a trace of albumin in a urine of normal specific gravity, and free, or practically free, from telltale deposits. The respiration at the pulmonary bases, if not crepitating, may be harsh and prolonged; and the percussion sounds there a little muffled. ${ }^{1}$ For this state of things, the heart, which has gallantly fought a long and losing fight, has to carry the blame of "failure." He is suffering, says the physician, from "heart failure" ; of course he is, but why? Whose fault

1 For a study of arteriosclerosis and pulmonary œdema, see Riesman, Amer. Journ. Med. Sci., January 1907. 
is that? Certainly the heart was not the primary offender. Whether it be the fault of the kidneys I have considered in the previous chapter.

Krehl says, apparently in the sense of objection, that the cardiac hypertrophy is not to be regarded as disease because it is compensatory, a strange demur; surely a disease consists in its symptoms, one of which is hypertrophy, not in our explanations of them.

The following case, which I saw over a considerable period of time with Mr. Day of Baldock, is a typical one :

Mrs. X., a lady of about 60 years of age, but looking rather younger, a moderate eater and total abstainer, of active habits, called on Mr. Day on November 18, 1894, for some temporary disorder. Mr. Day noticed that her pulse was too hard, and sent her to me. Her complexion was flushed and dusky. Her brother had died of apoplexy. Radial pulse visible, but vessel not hard nor tortuous, and to the finger, if small, was not very obviously tight; but as the rate was 124, the pressure was not very easily guessed at. By the sphygmometer, however, the full systolic pressure proved to be $240 \mathrm{~mm}$. (H and B). Heart evident over a large area, and action excessive, but apex not definitely outward. As hercomplaint was chiefly of attacks, on the least excitement, of painful flushing of the face and coldness of the feet (which warmed again as the attack passed off), I hoped that the high pressure register might be largely " nervous," vasomotor and temporary. The urine was free from albumin and casts, and, unless in phases of polyuria, of good specific gravity. The second aortic sound was slapping, but not definitely altered in quality. However, Mrs. X. did not improve; though she was sometimes free for a few days, the " attacks" would return in all weathers, even on so little an excitement as a game of cards. Passing over notes of continuously high pressures on later visits, two years afterwards my note runs as follows: Systolic pressures, taken quietly, with hands and feet warm, persisted at about $200 \mathrm{~mm}$. The artery was constricted, rolling under the finger, and the pulse sustained. The apex of the heart was now well outside the nipple line, and the action thrusting; the rate still ruled above the normal. To an eliminant and strict dietetic treatment we now added nitrites, and with some good effect. But on January 21, 1907, fulness of the head, depression, and inability to exert herself returned so severely that, in accordance with previous agreement, Mr. Day bled her to $16 \mathrm{oz}$. with remarkably good results. She spontaneously wrote me a letter of gratitude, saying she had not felt so "clear in her brain" for years, and was now active again and in good spirits.

$$
\text { voL. I }
$$


She went on well, not seeking advice for many weeks; when, unfortunately, illness in the house made a heavy call upon her, both with anxiety and work; still Mr. Day reported that the pulse was keeping softer and less sustained. But in April 1907 she was slipping back a little. I found the systolic pressure now at 200 ; but as she was nervously anxious for a good record, I thought, on this as on former visits, something should be deducted from the systolic record. The systolic pressures taken in my room were usually about $20 \mathrm{~mm}$. higher than when taken at her own home by Mr. Day. Notwithstanding, Mr. Day decided to bleed her again, and on the 29th she called with a very good report (by the way, the "vasomotor attacks" had not returned since the first bleeding). My instrument registered 180 (systolic), and no doubt, still with some nervous enhancement. But now the vessel walls were manifestly thickened, and slightly tortuous; a change possibly due to relaxation by bleeding and nitrites. On November 25 she called to know if she might be bled both spring and autumn. If not at the moment quite at her recent best, she had felt better ever since the first bleeding; her complexion also had been fresher and clearer. But the systolic and diastolic pressure were still too high; the radial wall was very thick and tortuous, and the pulse had become large and sustained. The urine was still free from albumin and casts; and no uræmic symptom, such as headache, nausea, or neuro-retinitis had appeared.

The following case, which I saw for many years with Dr. Gibbon and (afterwards) with Dr. Stiff of Bury St. Edmunds, proved also intractable:

A lady passing middle life. Pulse thrusting and sustained. Radial arteries thick and leathery, but not contracted, a condition to which the vessels attained during the years from 1889 to 1905. Complained of restlessness, shorter sleeps, apprehensions and false worries. Pain, fulness, numbness and oppressions in the head, depressions, and at times some panting. Accuses herself of "fancies," and self-regarding preoccupations. These attacks come on with phases of higher arterial pressures, and for some years were fairly readily dispelled by mercurials-with of course other means, such as diet, etc. There was never any sign of renal disease, but the heart increased. Between the attacks the pulse would soften; sometimes for a few weeks it would become almost normal as regards pressure, thick and tortuous as the vessels were. The heart also would for a while recede within narrower limits, and the action calm down. As she got older the attacks became more severe and frequent. Finally came on slight transient 
attacks of pulmonary œdema, and she died of heart defeat. This lady had been a subject of severe migraine up to middle life. I have seen many cases of this sequence, but migraine is a frequent ailment, and the negative instances-of no such sequence-are many.

As another illustration, in the third stage of the malady:

Mr. F., æt. 69 Ailing five years, previous health good. Arterial pressures had risen, with intermittent pulse. At the same time headaches and flatulency, and uneasy sleep. Urine wholly negative, but lateritious. Radials thick and tortuous, pulse sustained. Heart largely extended to left, and aortic second sound loud. Systolic murmurs, apex and base. Legs puffy, and some pulmonary œedema. Death by heart defeat. This patient had been a life abstainer, had never smoked, and was temperate in all things.

The next case is also of the third stage of hyperpiesia in a much older patient than usual. Length of history unknown.

Male, æt. 79. Seen, October 19, 1908, with Dr. Schelling, then at Sandy. Two alarming attacks of heart failure. Accessible arteries very thick and tortuous, and pulse sustained. Heart very largethree fingers outside the nipple. Beat now diffused, and a mitral murmur. Some œdema of lungs, and cardiac dyspnea. There was a long story of high pressures; but now the pulse showed Broadbent's "virtual tension." No uræmic symptoms ; urine scanty and lateritious. Death by cardiac defeat was imminent.

The following case of Hyperpiesia, also in its last stage, is taken from an article in the Lancet of September 1907 :

Male, æt. 50. Admitted to hospital, water-logged from heart failure. Heart found enlarged outwards, but no murmur. Pulse "tense"; no albumin. Necropsy; heart greatly enlarged, weighed $30 \mathrm{oz}$.; kidneys "quite competent organs." Advanced atheroma of the aorta, coronary and splenic arteries. No note of other arteries. The reporter presumed that the cardiac hypertrophy was " due to the vascular disease." $\mathrm{He}$ " could not detect with the naked eye any disease in the cardiac muscle." (No measurements of blood pressure had been taken.)

I avoided a note of the name of the reporter, because I wish here to demur (on what I think is now tardily admitted) that atheroma of the arteries does not produce cardiac hypertrophy, and that in this case the diagnosis of long-standing hyperpiesia 
had been missed, and the heart, after a long and brave fight, defeated.

These few cases, from a large collection of the kind, I offer as typical examples of pure Hyperpiesia, proving intractable. In spite of remedies, in the first case, the pressures seem likely to continue, and it is to be feared she may suffer the fate of her brother. The neurotic element in the case, and the well-marked vasomotor attacks would, without the sphygmometer, have been very misleading; I admit that at first I hoped the case might be one of mere vasomotor instability, and too readily I promised a cure. Other points of interest were the frequency of the pulse -which however is not very uncommon in these cases, and is often recorded in experimental cases of high pressure-and the advent under observation of the hypertrophy of the heart and the thickening of the radial artery. Cases caught early are usually curable, but a persistent constriction extending so far inward as the radial arteries is an unfavourable sign.

To follow up the symptoms of the circulation: the aorta and subclavians may or may not be much dilated. If dilated, the aorta rises in the thorax towards the surface, and the second sound thus becomes louder, for the stress on the end of a cylinder increases with its sectional area, and the vessel is closer to the sternum; the sound also may be not only more widely audible, as noisy at the apex as at the base, or noisier, but also altered in clang, which may depend on the degree of sclerosis of the valve and parts about it.

Friedmann ${ }^{1}$ carefully compared the conductions of the normal and abnormal ring of the cardiac sounds down the course of the aorta behind. After the age of 50 , he says, the audibility of the aortic second sound descends from the line drawn from the spine of the scapula to the third dorsal spine, down the left side of the vertebral column; and if there be much atheroma it becomes very audible and clear, down to a line drawn from the spine of the scapula to the seventh dorsal spine. For this extension of sound he gives five reasons - the state of the vessel, variations of the surrounding parts, the position of the heart, and so on. Friedmann does not pretend however to discriminate alterations of sound due to atheroma from those

1 Friedmann, Wien. klin. Wochenschr. No. 25, 1900. 
due to mere excess of aortic pressures, a difference which I fancy may be more or less perceptible.

Huchard says that a loud second sound is enough for an appreciation of high tension, and Edgren says much the same. I remember in a young woman, who was suddenly attacked by acute nephritis, that before any structural alteration could have taken place in valve or vessel, the stamping second sound at the base of the heart was most impressive; but can we distinguish such a heavy second sound from an alteration of timbre due not to enhanced aortic pressure but to atherosclerotic changes in and about the parts at normal pressure? Is there a distinctive sclerotic or syphilitic clang? In well-marked contrasts a difference of clang between mere high pressure and atheroma is appreciable; fortunately so far we are not faced by a dilemma, for the manometer, the state of the cardiac chambers, and so forth, will settle a part of the interpretation; and of course atheroma and high pressure may and often do coexist. The change of clang in atheroma may be either tinny, or, if the parts be cushioned by atheroma, muffled; in mere high pressure it is a thump. In syphilis it is "tabourka." But, even if they be real, it is not easy to put such distinctions into words. Mere accentuation of the second sound-a smart clack-even at the apex, is an untrustworthy sign; in women and in nervous persons with atonic vessels, and again in cases of approach of the great vessels to the chest wall, the second sound may be reinforced and even altered somewhat in clang. If however the reinforcement, or altered timbre, be so driven through the ventricular wall as to be remarkable at the apex, it may well have a morbid significance; and in cases of high pressure the second sound may be very distinct below the heart level. Much must depend on the state of the conducting structures; for instance, the pulmonary second sound, although aortic pressures are two or three times higher than the pulmonary, is nevertheless usually rather the louder, a difference which cannot be quite accounted for by the relative position of the pulmonary artery ; it must depend also upon different vibrations of its wall. By experienced physicians these discriminations are well recognised; what is not recognised is the difference of interpretation between the ultimate defeat, by high pressures, of a capable heart, and the gradual 
encroachment of a decrescent atheroma upon heart and vessels without any notable rise of pressures. (See Vol. II., pp. 5-6.)

In high pressures the audible characters of the first movement of the heart are too well known to need description here. To one point only I will refer; namely, the extraordinary sense of a prolonged systole. I have discussed this with Dr. James Mackenzie, who in a few cases had recognised a distinct lengthening of the systole, but not to any well-marked degree. The point is one which might easily be settled on sphygmographic tracings. The impression on the ear might lead one to interpret the prolongation as rather of the "prosphygmic period."-Anspannungszeit--but such a variation is not otherwise very probable.

On the extrasystoles of high pressure Dr. Mackenzie has written important notes in more than one place. ${ }^{1}$ He finds that these extrasystoles are usually of ventricular origin (when due to myocardial disease they may arise in the auricle). Now these ventricular extrasystoles may intrude at every alternate beat, in which case they are to be carefully distinguished from pulsus alternans; in the high-pressure cases-not, of course, in case of pulsus alternans proper-to reduce the arterial pressure by a nitrite "regularises" the pulse ; and as the effect of the drug passes off the extrasystole returns. Dr. Mackenzie's tracings show some regain of steadiness under the nitrite even after long years of stress and irregularity, although the contractile power may be failing; and of course, as coronary atheroma increases and the myocardium is starved, a true pulsus alternans may begin to complicate the record. ${ }^{2}$ But he points out also that under high pressures as, short of disease, fatigue of contractility sets in, true pulsus alternans may appear, and the weaker beats do not arrive through the cuff. To reduce the pressures, e.g. by amyl nitrite, may, or may not, then abolish the pulsus alternans, or after some little waiting at rest this relief may be obtained. $\mathrm{He}$ adds that, under high pressure, there may be a string of pre-

1 Among other papers on this subject I may refer to Mackenzie, J., Brit. Med. Journ. Oct. 20, 1906 ; an interesting article in Deutsche Arch. f. klin. Med. vol. xciv. (by an author whose name I have forgotten); Dr. Lewis' papers; Dr. Wardrop Griffith's Schorstein Lecture, 1912 ; Dr. Graham Steell's well-known studies; and many others, at home and abroad.

2 For this and other pulse symptoms see Dr. Graham Steell's excellent little Handbook on Diseases of the Heart, 1906 ; with good curves. 
mature rhythms of the pulse-abnormal irritations of auricle, ventricle, or knot; and on reduction of the pressures to normal the regular rhythm may return. He warns us however that a true pulsus alternans may be latent to the finger, although an effort, such as walking upstairs, may make it palpable. We may observe also, under high arterial pressures, geminal, triplet, and other curiosities of rhythm. I suggest that in highpressure cases the extrasystole is induced by the higher intracardial pressure which keeps the myocardium more tense, and under this loading-within limits-more irritable ; always at full cock, less under inhibitory control, so that a slighter stimulus fires it off. In this case the extrasystole is presumably of ventricular, though it might be of auricular, origin. Other causes however, such as some instability in the muscle itself, ventricular or auricular, may lead to similar premature detonations. If the extrasystole starts from the ventricle, or even from the auricle, there will be a lengthened pause--but not if it is from the sinus (H. E. Hering). ${ }^{1}$ If these extrasystoles be mixed with pulsus alternans the confusion may be disconcerting; still even then a careful graphic analysis will usually serve to disentangle it. Professor Mines has made a careful study of such complex rhythms. ${ }^{2}$ Huchard says that arrhythmia, especially "rhythmic arrhythmia," is due to cardiosclerosis; but he does not analyse the rhythms, he does not define "cardiosclerosis" (see Chap. VIII.), he attempts no proof. Dr. Mackenzie also avails himself of the term "cardiosclerosis," as a state signified by pulsus alternans, and likewise he fails to define it.

A gallop rhythm is often audible in high-pressure cases (Hyperpiesia,renal disease, etc.), a severance of the constituents of the systole into the so-called "protosystolic and deuterosystolic" sounds, without duplication of the arterial pulse, as explained many years ago by Potain. This splitting is due not to the absolute degree of systolic pressure but to an aortic pressure

1 Hering, H. E. (Prag) “ Herzalternans," etc., Zieitschr. f. exp. Path. u. Ther. Bd. X. Hft. i., 1912; a very interesting article, which has been discussed and in part accepted by others. Frédéricq, H. (Pflüger's Arch., cli. 1913) however denies Hering's explanation. He argues, from a conclusion of Dr. Gaskell that alternation is attributable to inequality of refractoriness in several bundles of the myocardium, that the apex and base may bc in opposition.

2 Mines, G. R., Proc. Roy. Soc., 1912 (paper read Oct. 28 1912). 
relatively high for the available myocardial energy - conductivity or contractility relatively minus-so that the systole is slightly "telescoped out"; or the second tap may be due to a supplementary systole which fails to reach the wrist. In high tension the aortic pressure may be rather too much for the heart; in myocardial infections the heart too weak for the aortic pressure. ${ }^{1}$ The auricular action is said to be in excess. Although then this gallop rhythm may be something of a curiosity, yet it often serves as corroborative evidence of pressures relatively high, for that heart at that moment. Yet this rhythm may be casual; not long ago I noticed it in a candidate for life insurance, a fine young man with irreproachable family history. His organs presented no other abnormality. The gallon was presumably due to some momentary discord between cardiac and aortic pressures, perhaps of nervous origin. On subsequent examinations it was not heard again.

When the walls of the aorta are taut the output varies more, and, such as it is, is not, as in the normal, engaged more or less with other coefficients. Under such conditions even digitalis might produce pulsus alternans; or, in cases where the vagus dominates the position, atropin might dispel it. However, in glancing over graphic records, I find uneven tops more often in those of aortic regurgitation, a pseudo-pulsus alternans-not the regularly and continuous alternating rhythm, an inequality probably not dependent on myocardial capacity. Strubell, in a careful essay resting upon experiment, ${ }^{2}$ says, somewhat incidentally, that it is false to say that pulsus alternans is necessarily equivalent to asystole. It " may be a species of trigeminy," or indeed, " usually is." The difference may be discernible by the test of bodily effort. Some authors say that pulsus alternans may be produced by certain drugs, chloral being one of them.

If in a case of cardiac strain consequent upon high blood pressures the heart becomes irregular-not merely by extrasystole-yet without œdema, we may suspect that it is suffering directly from some toxic influence. As in all complex motions,

1 See Pawinski, Zeitschr. f. klin. Med., 1907, Bd. Ixiv.

2 Strubell, published as an original article in Centralbl. f. Herz-u. Gefasskrankh., March 1912. See also Windle, D., Heart, ii. p. 95, etc. 
so in the circulation, many and variable are the factors which by summation or reciprocation work out in rhythm.

On another page (Chap. VIII.), I shall protest against the frequent misuse of the name " senile myocarditis," or " cardiosclerosis," names given sometimes to ill-nourished decaying hearts with broken rhythms and irregular rates, sometimes to hearts naturally sound but embarrassed or strained by high blood pressures, sometimes to myocardial fibrosis. Hence the puzzles in text-books about the inconstancy of hypertrophy, an inconstancy which, with due distinction, is explicable enough.

More or less fugitive mitral murmurs are of course to be expected in the later stages of hyperpiesia or decrescence. In these chief modes of arteriosclerosis mitral defects are respectively of two kinds: the one due to distension of the hypertrophied ventricle by high arterial pressures, the left first and then the right, with forcing of the valve; the other to sclerotic degeneration of the valve and its parts, especially of its aortic cusp. In the former case, under the abnormal strain, the cusps, like the lining of the auricle, may have undergone some fibrosis, or perhaps atheroma; in the latter the heart does not present hypertrophy nor dilatation; the chambers often show little or no alteration in volume. The right ventricle is affected in no constant proportion to the overwrought left; it slowly backs up the left, and is more or less affected the longer or the shorter the duration of the burden of the peripheral resistance. It is not necessary to assume the co-operation of some obscure cause acting directly on the myocardium.

As under perennial high pressures the heart yields, and mitral regurgitation perchance sets in, the blood pressures abate to some degree, say to $160-170$, but rarely to the level of the normal pulse. A fall to the normal under these artificial conditions would signify grave dilapidation. Even in primary mitral disease Dr. Hubert Starling, ${ }^{1}$ Dr. H. C. Mann, ${ }^{2}$ and others have shown that any considerable fall of pressures might forebode dissolution; the circulation, whatever the resistances, must be carried on; and as a matter of fact in cases of ordinary mitral disease, or of emphysema, pressures during the grip on life may be even

1 Starling, H., Lancet, 1906.

2 Mann, H. C., Guy's Hosp. Rep. vol. lxii. 
exalted, to fall in the one case with relief, in the other case, with despair. As the heart fails the velocity rate falls, and all pressures approach the mean ; pressure becomes more systolic, occupies a smaller part of the cardiac cycle, and is inconstant $;^{\mathbf{1}}$ the diastolic pressure being meanwhile in decline. Thus paradoxically, but quite intelligibly, if venesection or digitalis brings about an improvement, both systolic and diastolic pressures may fall. On the other hand strychnine may work up pressures in both phases.

As an example of the gradual declension of systolic arterial pressures as the disease (Hyperpiesia) made way, I may allude to a certain case for some years under the care of Dr. Lloyd Jones and myself.

The patient was an elderly man who had lived well, and in later years with a more sedentary life. As a rule, his radial pulse was of the large leathery and thrusting sort, the sort of pulse an elderly man in the midst of his complaints is often proud of. The case throughout was one of uncomplicated hyperpiesia, with never a sign or symptom such as we are wont to regard as renal. Latterly he had become subject to occasional attacks of paroxysmal dyspnea, not in its severest degree; and with these seizures for a day or two the pulse would become small and wiry. During the main course of his disease the systolic blood pressures ranged very high (220): in the penultimate stage they fell to about 180 ; the diastolic pressures were not recorded; in the ultimate stage the systolic pressures rarely reached 160 .

A case on which I lectured, in 1908, showed a contrary movement; but this was a renal case in a late stage. Anasarca three weeks. On admission the systolic pressure was 170 ; by rest and treatment the pressures rose to 190 , and therewith the patient's condition was much ameliorated. With the amendment the second sound became louder at the apex, and altered in timbre. In these phases the appreciations of the finger, as applied by many of us, were most untrustworthy, or even deceptive.

In some cases, whether of hyperpietic, or indeed of decrescent, or syphilitic, atherosclerosis, the aorta may be abnormally apparent or palpable in the episternal notch; if it be apparent without evidence of an atony of the great arteries, and if moreover there be some dulness on the manubrium, it signi-

1 Dr. Batty Shaw has demonstrated this inconstancy of systolic pressures in aortic regurgitation. See also p. 392. 
fies disease, syphilitic or atheromatous; but pulsation, atonic or cardio-excitatory, in this area is not very uncommon in relatively healthy persons. If there be any difference between the two radial pulses atherosclerosis is probably invading the subclavian, and the case may be one of decrescent arteriosclerosis without notably high pressures. A good deal has been said of late years on the signs of the subclavian arteries in arteriosclerosis (Vol. II., p. 199), and, for present convenience, I will not in this respect divide high pressure from decrescent atheroma. If into a baggy aorta the output of blood is hurled without buffer into the secondary branches, the subclavians may leap with a thrill, often both systolic and just perceptibly diastolic also. And with caution such pulses, if in the right subclavian only, may with other indications be taken to signify disease of the ascending aorta; if on both sides disease of the whole arch. If the subclavians are the seat of atheroma the right vessel is often pushed upwards and can be palpated (Vol. II., p. 130). We have seen that functional throbbing must be excluded, as usually can be done by other signs, the age and sex, and the story. In a man under forty-five syphilis would be suspected, in a woman functional disorder; though again and again $\mathrm{I}$ have been surprised by syphilitic infection in women, conveyed in marriage, or due to such misfortunes as may overtake nurses or doctresses.

The detection of early degrees of cardiac hypertrophy is notoriously difficult; in some cases of emphysema even a big heart may be concealed; but, if I need not do more than allude to so well-known a chapter of clinical medicine, I may touch upon a few points germane to my subject. If the abdomen be largely distended, or in pregnancy, the heart may be thrust upwards into a position almost horizontal, with the apex outwards, even enormously. Over a windy stomach the sounds, especially the second, may by a consonating tympany be strangely altered in timbre. In such a case final diagnosis must await a subsidence of the abdominal condition. Dr. Ewart $^{1}$ has pointed out that gastric distension may often be detected in the left thoracic groove, upwards and backwards, at the expense of the squeezable lung, and is to be looked for at

1 Ewart, Wm., Trans. Clin. Soc. Lond., vol. xxxiii. 
the back. Riesman ${ }^{1}$ says that a flatulent stomach may be an early symptom of hyperpiesia.

To a more permanent ambiguity I have alluded, to the elongation of a yielding aorta- "the swinging heart"; thus without any hypertrophy the heart may present its apex considerably beyond its normal site. To turn the patient from side to side will in this case alter the area of dulness. It seems pretty certain that before the appearance of atheroma, indeed before any alteration of the aorta visible even by the microscope, the vessel yields and twists a little, as if its fibres had lost quality; thus with age or deterioration the vessel slowly widens and lengthens. Schul, ${ }^{2}$ who has lately restudied this problem, attributes these effects, in my opinion, too exclusively to excess of blood pressure. A widely shifting heart depends upon too many conditions to have more than a corroborative value (Vol. II., p. 199). If the right subclavian be raised above the bone-a very variable phenomenon, as we have seen, elongated -vessels may so far be indicated. Again, very careful attention must be given to Professor Wenkebach's and Professor Keith's studies of the relations in space of heart and diaphragm.

The fallacies of percussion diagrams, especially as paraded by certain bath doctors, are now, thanks especially to Dr. James Mackenzie's animadversions, becoming better realised. Long ago von Basch pointed out the fallacies of percussive diagrams of cardiac dimensions; how a big heart may have a small area of dulness and a normal heart an excessive area ; and gently castigated " decorative diagrams," and "Virtuosenthum in der Percussionskunst." He watched the respiration very narrowly; though records of this function also lie open to like fallacies. In incipient cases of dyspnea we may generally note that an interesting conversation with the physician will reduce it, and a return to a discussion of the symptom will bring back its urgency. Even by X-ray methods (orthodiagraphy), as Franz Grödel of Nauheim ${ }^{3}$ has shown, estimates of the size of the heart may be fallacious; so that even by experts many precautions have to be taken. The diaphragmatic and respiratory excursions must be allowed

1 Riesman and others, Amer. Journ. Med. Sci., April 1913.

2 Schul, Virch. Arch., Jan. 1908 ; see also Fuchs, Zur Physiol. d. Blutgefässsystems, Jena, 1902.

3 Grödel, F., Arch. Rönt. Rays, Nos. 88-89. 
for, and in small chests the heart is less apparent than in a wide and broad one; it diminishes also during starvation, as by rigid diet, or in sickness. Franz Grödel thinks that in age the heart enlarges a little. For my own part I would emphasise in high-pressure cases the variability of the cardiac dimensions outwardly beyond the midclavicular line; how even in a few days or less the outline may recede under treatment. As physiologists, we know how the contraction volume of the heart may vary; how it may increase prodigiously, and yet be discharged on systole; under such circumstances the arterial systolic pressures may vary even by more than 50 per cent, variations presumably governed in part by reflex expansion of the periphery. Such fluctuations signify however good vessels, a good and fairly young heart, and good general metabolism. Still I have seen changes of this kind in temporary states of high arterial pressure, as during their episodical occurrence in elderly persons (p. 452). I have the impression, derived from athletic men in Cambridge, that under very active exertion of a temporary kind the wall of the left ventricle, like the muscles of the thigh, may enlarge by turgescence ; a turgescence which usually recedes quickly, but under circumstances of continuous stress may be perpetuated as a hypertrophy. In some of these cases the left ventricle has certainly seemed to increase, and after a short time to return to its normal dimensions. And when to these variables we add the many incidental embarrassments, such as emphysema, ossified ribs, or fat walls which muffle percussion sounds, and in women big breasts bothering our manipulation, we may well scrutinise some very confident clinical reports.

Pulse.-It has been said that high diastolic pressures seem to abbreviate the prosphygmic interval, the aortic and intraventricular pressures being more nearly equal at the beginning of systole (p. 390). I have made no time measurements, but I have alluded to a prolongation of systole, apparent or real, sometimes an extraordinary protraction, which I think is more or less prosphygmic. Anacroty, a phenomenon of like character, is generally recognised as a no infrequent feature of sphygmographic curves of high pressure. Dicroty is an insignificant symptom, standing in no direct relation to arteriosclerosis, high 
pressures, compensation, length of systole, or arteriosclerosis. That it is more frequent and distinct in relaxed vessels is true, but that dicroty is wholly inconsistent with high pressures is a maxim open to no little exception.

The thick corrugated and contorted radials, which suggest to the unwary observer a high resistance, if consistent with hyperpiesis are not characteristic of it, but suggest rather decrescent arteriosclerosis. I often test the blood pressure in such persons in order to confirm and emphasise its independence of arteriosclerosis. For instance, in one patient, a man aged 50, whose arteries were grotesquely deformed, and whose pulse suggested to the finger high blood pressure, but who complained of little ailment, and sought advice for some trivial cause, a man, I may add, in whom no yielding of the heart occurred then or since, in him I found the full systolic pressure barely above 120 ; the maximum oscillation lay about 90 . I may also reiterate here, in respect of inference from the radial artery, that notable arteriosclerosis in the radials and other accessible vessels is found only in about two-thirds of the cases in which this vascular disease proves to have been in existence somewhere in the tree.

How far inwards may peripheral constriction extend (p. 31) ? Certainly it may reach the brachial artery But these vascular proportions are difficult to appreciate. I have said that when, as often happens, there is no pressure gauge at hand I find some advantage in the manipulation of the brachial artery with one hand while keeping the other on the radial, pressing and releasing the vessel so as to obtain a notion of the effort required to arrest the beat. Thus my attention is often turned to the brachial; and if we estimate the main arteries roughly by the size of the limb we may find not a few cases in which the brachial seems decidedly to be narrowed in diameter; in these cases - often but not always chronic renal-the radials are very wiry.

In respect also of its walls the brachial artery in the bicipital sulcus is a useful point of observation; in hyperpiesis the wall may feel tough and incompressible but uniform; in decrescent cases, while the skilled finger may note no great tension, a tortuous degenerated vessel may leap to the eye. The temporal artery, 
or the dorsalis pedis, may thicken almost to obliteration, and thin twigs become hard cords; and this in cases in which the radial and brachial are no more than leathery and a little tortuous.

We have discussed (p. 24) the relations between pulse rate and arterial pressures, which, if not so constant as was once supposed, are yet not without importance. Dr. Core ${ }^{\mathbf{1}}$ urged forcibly that blood-pressure charts without curves of pulse rates are shorn of much of their usefulness. He published a long series of apt observations, but with some lack of summary. However, it is generally agreed that these relations are too complex, contain too many variables, for clinical maxims. In some high-pressure cases the pulse is considerably quickened. The augmentor nerve may increase the output even against rising pressures, and a slow rate be accelerated; an interference which has been demonstrated in animals by irritation of the cut sciatic. Again, a slower pulse diminishes friction, and an expanse of area, if it save some friction, may make on the whole for retardation. If then Marey's rule of inverse proportion between pulse rate and blood pressure has a certain fundamental validity, it; is so often overridden by other factors that it cannot be a governing principle. In practice, under high pressures an accelerated pulse is not at all infrequent. I have many a note of this contrast; and a trustworthy observer reported it for me in 40 out of 140 of such cases. In normal persons the difference in rate of pulse between standing and lying is usually about ten beats; the heart, as Dr. Hare of Philadelphia remarks, endeavours, as resistance falls, to keep up the pressure. But even in quite healthy persons this adjustment may be imperceptible; so far as watches and gauges can tell us, with a rising pulse, as for instance in nervous fluctuations, the pressures may remain unchanged; or with rising pressures the pulse rate may not change perceptibly (Josué and others). However, these are nice problems, and often involve psychical factors; for the present they are not of much practical importance: a more important side of the matter is the alleged perversion, in high arterial pressures, of the common relation between the pulse rates of sitting and standing, a perversion on which Huchard laid much emphasis.

1 Core, M.D. thesis, Manchester Univ., 1910. 
Huchard declared that the degree of perversion might even be used as a rough gauge of such high blood pressures. For instance, he stated that with rising pressures the parallelism between these and pulse rate failed; the pressures might rise while the rate was comparatively stable, or indeed in cases of high pressure the rate on recumbency might increase, and the recumbent rate even exceed that of the standing position. Some confusion hung about this position because Huchard and his pupils continually assumed the direct correlation of high blood pressures and arteriosclerosis. However, by other observers this alleged diagnostic difference is denied; Vaquez in France and Leonard Williams in England have been unable on repetition of these experiments to verify Huchard's rule, unless in measures which are insignificant, or under baffling variations. I have not myself made any systematic observations on the point, but my impressions are that a gradual waning of the postural difference, however interesting as an incidental observation, has not uniformity enough to be used as a criterion, or even as a measure of symptoms.

As regards the touch of the pulse, I need not lay stress upon the well-known characters of the pulse of high arterial pressure; I reiterate that vasoconstriction must protect rather than injure the arterial branches concerned. Continually we see persons, even in later life, the subjects of hyperpiesia or chronic renal disease, whose radial arteries, and other vessels of like magnitudes, however condensed by constriction, and whatever the impulsion of the blood stream within, still present coats supple to the finger. I am still compelled to urge that to call such a pulse one of "high tension" is to toy with words; a narrow constricted artery is not under tension, though the vessels more central to it may be (p. 377).

Another caution on the touch of the pulse is that the radial pulse may sink almost to extinction while in the brachial the pressure, if reduced, is fairly maintained. For instance, in hæmorrlage-say from the lung or stomach-the radial pulse upon its waning content may shrink until it becomes impalpable, and a wrist gauge quite useless, while in the brachial a pressure - for a while at any rate- of 100-110 may still be registered. On the other hand, in peripheral vasomotor slackening a wide 
radial artery may give to the finger, as we often find in the acute infections, quite a false impression of stability. ${ }^{1}$

Here I may repeat that Traube's "pulsus alternans" (see Angina Pectoris, Vol. II., pp. 334 and 372) may occur in the stage of Hyperpiesia when the heart is yielding; as under the same conditions we recognise it in chronic renal disease.

If calcification appears, as is sometimes the case in lax vessels after prolonged strain of hyperpiesis, the coats of accessible arteries may be uneven, gritty, or nodular to the touch, and the vessels more tortuous than is common in ordinary hyperpiesia. This mode of degeneration I have witnessed from time to time. But there is a subtler question, whether-as I have some reason to suppose-decrescent arteriosclerosis may set in and progress in some independence of hyperpiesia or renal disease, and the vessels thus suffer under a twofold degradation. The grounds for such suspicions suggest themselves more frequently in states of certain visceral arteries-e.g. cerebral or coronary-than in the touch of those which are accessible.

No general rule as to the rate of arterial decay is possible ; much depends upon causes, individual habits, and so forth. But I have had reason to suppose that a particular artery, e.g. the radial, may for an elderly person be fairly normal to the touch and two years later have become obviously sclerotic.

Respiratory System.-Dyspnea.-As I enter upon this paragraph I see again with the vividness of life the desperate conflict of a strong man with a paroxysm of suffocation which recently some of us witnessed in a hospital ward, during an examination. The patient, seized and throttled before he could cry out, sprang up livid to wrestle with death. The desperate conflict made the fell enemy almost visible to us. Now this way, now that, springing up in bed to fight from the edge of it, to sink back in utter exhaustion, but only to rise again panting, with the sweat streaming from him, desperately to renew the battle, the scene was almost as distressing to the bystanders as to the victim. Some one exclaimed "Angina Pectoris!"-thoracic agony it was indeed, but dyspnea is not a character of angina pectoris. Uræmic " asthma" was then suggested, but asthma, a totally different malady, it certainly was not, and uræmic in the ordinary

1 See also Passler and Rolly, Münch. med. Wochenschr., Oct. 1902, p. 1737.

vol. I

$2 \mathrm{D}$ 
sense it proved not to be : the patient was not "a nephritic." The attack, akin as such attacks may be to the paroxysmal dyspnea of Bright's disease, was in this case, as indeed it may be in Bright's disease, a paroxysm of high-pressure dyspnea. His heart was labouring and hypertrophied, the arteries thick and the arterial pressures very high. The agony was alleviated by nitroglycerine, and under repeated doses of the drug it subsided. As is common in this kind of dyspnea, the face was not cyanotic but pallid to lividity.

The subject of paroxysmal dyspnea has been brilliantly handled by Pal; but a far greater advance in our knowledge has lately been made by Lewis, Barcroft, Wolf, ${ }^{1}$ and others. These experimenters find that this dyspnea is not a cyanosis but an acid intoxication; and the same explanation may hold for the dyspnea of high altitudes. Chloride retention has no part in/this dyspnea. We may divide dyspnea by its mechanism - whether nervous (bulbar), pulmonary, circulatory, or coarsely mechanical as by pressure of some tumour; or again by its seat or origin-whether arising from pulmonary, cardiac, or cerebro-spinal disease; and so forth. Amblard, ${ }^{2}$ after describing some cases, formulates three phases of these seizures: (1) the preliminary phase; (2) the main attack; (3) the wane. During the preliminary phase the pressures run to at least $210 \mathrm{max}$. and $190 \mathrm{~min}$.; often to a maximum of 250 , or upwards, with proportionate rise of minimum. Secondly, during the attack both curves may fall; usually the minimum but little and the maximum heavily-that ominous sign of maximum falling towards minimum. In one case while the maximum fell from 230 to 190 , the minimum fell only from 180 to 160 . In these cases venesection, even copious, does not seem decisively to influence the blood pressure. Thirdly, unless a reaction set in, systolic pressure falls more and more; but the maximum may recover itself (though not to the height before the attack), the minimum remaining about the same. If the patient be closely watched between the grave attacks, smaller tides of the same kind will usually be detected. It is convenient in this place to divide dyspnea into the cardio-

1 Lewis, Barcroft, and others, Heart, vol. v. No. 1, Oct. 1913.

2 Amblard, Presse médicale, April 12, 1911, quoted Martinet (loc. cit.). 
vascular, the pulmonary and the cerebral categories. The pulmonary kind, if we exclude pulmonary disease, is due to sudden œdema of the lungs or lung, or to infarct, or occasionally to a true pulmonary apoplexy by rupture of a vessel.

We must keep a watch for pulmonary oedema, which may set in swiftly, or indeed overwhelm the patient suddenly. Vaquez (loc. cit.) quotes a case in which a large dose of nitrite of amyl, given in an attack of angina pectoris, was followed by an effusion into one lung so rapid and so large as to put the patient in extreme peril. Familiar as we are with pulmonary œdema as a chronic sign of pulmonary stasis, we may not always be quick to anticipate it as a fulminant event; but it is as such that in hyperpiesia or renal disease, like an apoplexy, it is especially apt to break forth. In the paroxysmal dyspnea of high pressures, perhaps on account of the oppression of the heart, pulmonary œdema is always to be feared. ${ }^{1}$ How far a sudden and considerable rise of arterial pressure is of itself sufficient to produce pulmonary œdema is not known; but that some connection exists between this œdema and high pressure was observed by Laennec, and even before him. ${ }^{2}$ The signs of œdema by the way are not always at the bases; they are often first audible at mid lung and, as some say, occasionally at the apex. It seems ascertained (Josué and others) that adrenalin if injected, to a certain excess, directly into the veins of animals may have this effect.

Pulmonary infarct, if affecting a considerable area, sets up a strife as distressing as paroxysmal dyspnea of central origin. In these cases hæmoptysis will usually reveal the cause; but in some cases, or on several occasions in the same patient, smaller infarcts -not so large as to cause hæmoptysis-may, if the plugging be sudden, set up sharp attacks without hæmoptysis. These may be recognised by circumscribed pleuritic rubs; the spleen may be enlarged, or the guiacum test to the urine may reveal a plug in the kidney. Asthma, a specific name often abused in careless habits of speech, is not attended by high arterial pressures, whatever the venous may be. Dr. James Adam of

1 See two instructive cases in Berl. kl. Woch. No. 52, 1909.

2 See interesting article by Riesman, D., Amer. Journ. Med. Sci., Jan. 1907 ; and an essay by Leonard Williams, Lancet, Dec. 7, 1907. 
Glasgow ${ }^{1}$ recorded the blood pressure in asthma for fifteen years, and found only 20 in which the arterial pressure was above normal. Nor are the arteries of the asthmatic very prone to thicken; usually he lives long (because of his abstinent habits ?). During the attack his radials may be constricted, or emptied; but with this we have no concern.

Another kind of high blood pressure dyspnea is that due directly to cardiac hamper-to intraventricular pressure, either positively or relatively excessive. This dyspnea, if more or less paroxysmal, or apt to come on during rest, is never so furious as the "central nervous" form, or as the two acute pulmonary forms - infarct and acute œdema; but it may simulate clot in the heart. Too often such a cardiac attack is called Angina Pectoris, because it is prone to attack the patient for the first time during the ascent of a hill, while hastening to a train, or walking against the wind; indeed a dull oppressive submammary ache may come with it. Von Basch well contrasted this cardiac dyspnea, often associated with falling pressures, with the "cerebral paroxysmal" form. After his scientific habit he made appropriate experiments upon animals, and concluded that the aortic pressure rises because the vasomotor centre is irritated by $\mathrm{CO}_{2}$ before pressure rises in the pulmonary artery. I may add that cyanotic blood is more viscous (p. 129). Then, as the left ventricle is opposed by vasoconstriction and pressure rises in the left auricle and so back to the pulmonary artery, the right ventricle distends, " and may be distended to two or three times the content of the left."

I have said that latent high pressure may be revealed by an attack of dyspnea during a trip to the mountains. One of my patients, a man of great energy and business capacity, on arriving at a Swiss Hotel at a height of some 5000 feet, was so gravely attacked by nocturnal dyspnea that, although on descent to a lower level the dyspnea passed off, on his return home he sought medical advice. We then found a very obstinate state of Hyperpiesia (ranging about $200 \mathrm{~mm}$. systolic) and a large heart; a state which at this period had become susceptible of relief but hardly of cure. The urine in every respect was normal, and no symptoms of a uræmic kind appeared. As in nearly all cases

1 Adam, Jas., Brit. Med. Journ., Jan. 13, 1912. 
of the kind, "heart failure" was diagnosed; and, as usual, this unlucky member, which by supreme effort had long been silently and successfully keeping things together, fell into disgrace.

In my experience the paroxysmal dyspnea of central origin, which in its more violent forms, even in chronic renal disease, is not frequent, in Hyperpiesia (without renal disease) is rare. The cardiac dyspnea, characteristic of the later phases of hyperpiesia, harassing as it may be, is a more chronic affair, and is not so apt to alternate with stupor and the Cheyne-Stokes phenomenon. Dyspnea is seen, as Dr. Bruce says, in " the stout, free-living man, just passing the meridian of life, whose heart is large and weak" ; a heart in the third stage of hyperpiesia, weakening but not naturally weak. At the end of a long and arduous campaign it is jaded and spent, though often game to the last.

Seizures of paroxysmal "central " dyspnea may spring up in a patient at one period of his malady, and at a later stage give way to ordinary cardiac dyspnea. Such a case I saw in January 1910 with Mr. Nash of Bedford. The notes are briefly as follows :

An Indian' officer, aged 60, temperate in all things buttobacco, some years before had become the subject of hyperpiesia. No symptoms of chronic renal disease had ever appeared. The heart was much hypertrophied, and the blood pressures ranged high. The first event to call attention to the high blood pressure was a severe epistaxis in August 1905; and a smaller hæmorrhage had occurred in 1906. In March 1907 he was seized by the first attack of paroxysmal dyspnea, not very severe. There was no notable recurrence till October 1907; this attack was of great severity, and thereafter for a while attacks of more or less severity were prone to recur. From strict diet, salines, and blue pill he obtained much relief, for a time; he could walk abroad and even mow his lawn. In the winter of 1908 the paroxysms returned, but the "panting attacks got fewer" and their place was taken by "ordinary shortness of breath" (his own words). His big heart had begun now to dilate under the high pressures, and on my visit was largely dilated; œdema also had appeared in the feet, and he had run off in condition and aspect. The arterial pressures however were still ranging well above normal.

The correlation of this paroxysmal dyspnea with reduction of the alkalinity of the blood by Lewis and Barcroft is most 
interesting, both in the value of the knowledge and as an example of genuine clinical research as contrasted with arbitrary postulates, and logical categories built up of phrases.

Another cause of dyspnea which may occur with or without arteriosclerosis is emphysema (not Asthma, see p. 403). Many years ago I pointed out how often emphysema accompanies arteriosclerosis, especially of the decrescent mode, when I suspect it to be due to degeneration of the bronchial arteries. ${ }^{1}$ Josué has noticed the same frequency, but being a wiser man proffers no explanation of the concomitance. For this emphysema a careful scrutiny should be made, for in elderly people, whose chests are rigid, it may not be obvious, although sufficient to conceal a cardiac dilatation; or to be the cause, or auxiliary, of a dyspnea otherwise interpreted. Such a patient however gets short-winded, and subject to a dry cough; a little crepitation is prone to stick at one or both pulmonary bases. The accessible arteries may be thick and tortuous, but the blood pressure not much in excess, if at all. Dr. Gregory, ${ }^{2}$ who collected cases of this coincidence for me, found that more than 50 per cent of decrescent arteriosclerotics were emphysematous. How long rising arterial stresses may precede the dyspnea is illustrated by two cases which von Basch observed for four years and twelve years respectively. In both cases the pressures were found to have risen $30-35 \mathrm{~mm}$. before respiratory symptoms set in. ${ }^{3}$ The author gives very careful descriptions of the morbid anatomy of the lungs in high-pressure cases.

In considering these problems it must be remembered that simple asphyxia - e.g. laryngeal - of itself raises the blood pressure, whether by medullary reflex, or increase of viscosity, or both together (see Viscosity, p. 129). On the other hand, if a higher arterial pressure carries more oxygen to the medulla this does not itself excite the respiration; the bulb, susceptible as it is to the smallest trace of $\mathrm{CO}_{2}$, seems indifferent to oxygen: as Barcroft and Dixon put it, the $\mathrm{CO}_{2}$ output lags behind the $\mathrm{O}$ intake. But of the nature of "toxic dyspneas" we know so little, and are hampered so much by guess-work, that I cannot discuss them further to any profit.

1 Report Brit. Med. Assoc. meeting, July 28, 1910, and previous allusions.

2 Gregory, M.D. thesis, Camb., 1911.

3 Von Basch, loc. cit. p. 285. 
Cheyne-Stokes respiration is well known as not infrequent in the extremer, though not necessarily the ultimate, phases of hyperpiesia. In the dyspneic phase the pressure rises, in the apneic it falls. Pressures may rise so high as to seem to be the direct cause of semicomatose phases, often with tidal respiration. Such phases appeared again and again in a lady whom, nearly twenty years ago, I often saw with Mr. Wallis of Cambridge. During the attacks the arterial pressures, systolic and diastolic, always excessive, ran up enormously, and she would fall into a stupor, sometimes with stertorous breathing. There were never any signs of renal disease. All these attacks she survived to die of a defeated heart and dropsy. In a case to which I have alluded on another page, as seen with Mr. Teale, complete recovery took place in spite of high pressures, paroxysmal dyspnea, Cheyne-Stokes breathing, stupors, and delirium. The patient was a very vigorous middleaged man, a free liver, and occupied in large financial concerns.

Bronchitis, or at any rate troublesome cough, is no infrequent companion of hyperpiesia ; often it is no more than a tiresome hawking and clearing; but also often a true winter (bronchial) cough with free expectoration. In some of these patients bronchitis is the way of death; such recently was the case with an old friend of my own in whom high arterial pressures had persisted for many years. The discovery of such pressures will indicate the line of treatment, which is often also the proof of the diagnosis; for dietetic reform, with calomel and other "so-called anti-gouty" means, may banish or alleviate the bronchial irritation without delay.

Hcomoptysis I have referred to as an occasional result of hyperpiesis; never I think-unless in mere specks-of decrescent arteriosclerosis. In a lady, whom I saw once in consultation, a hæmoptysis of 2 or 3 ounces first betrayed a hyperpiesis of, no doubt, some long standing. Epistaxis is more common.

The Nervous System.1-Cerebral, Seizures.-For years past observers of arterial disease have been occupied with the symptoms, convulsive or paretic but chiefly paretic, which occur in the later stage of arteriosclerosis. In $1871 \mathrm{I}$ was not the first to discriminate between the hæmorrhagic and the

\footnotetext{
1 See Stengel, Amer. Journ. Med. Sci., Feb. 1908.
} 
" obstructive" apoplexies; and, in respect of cerebral palsies, this distinction between the apoplexies of high arterial pressure and those of arterial obsolescence and thrombosis, or of embolism, a distinction in which the two chief kinds of Arteriosclerosis may be severally recognised, is essential.

As regards the lesser manifestations of disorder about the brain or head in hyperpietic and decrescent arteriosclerosis respectively, I may mention headache, vertigo, and tinnitus; they are set down in routine fashion as symptoms of arteriosclerosis, and I do not gainsay their importance. Notwithstanding, they are often overrated or misconstrued. In arteriosclerosis the ear is not infrequently affected, so that the symptoms of Menière may supervene; but these incidental phenomena are of importance only so far as the patient may be harassed by them. Headache again is a capricious and ill-defined symptom, not especially characteristic of either, or of any, form of arteriosclerosis. In my experience however subjects of migraine have seemed prone to hyperpiesis; in some of them the migraine passed in middle life into hyperpiesia; some others drifted into chronic renal disease ("Granular kidney"). "High tension headache" is said to be occipital, and aggravated not by optical errors, but by mental or bodily effort. I cannot say that in my experience such a headache, which is common enough in worried or overwrought patients, has been a special or characteristic feature either of the hyperpietic or the decrescent mode. Headaches arise in all persons from trivial and incidental causes. Of chronic renal disease it is a grievous symptom. In a few cases however I have been impressed by the insistence of a patient, afterwards attacked by an apoplexy, on a vexing pain on the side of the head opposite to his subsequent hemiplegia. It does not seem probable that arterial lesions in the brain, apart from intracranial pressure so high as to distend the membranes, can be in themselves, or their reactions, painproducing; a sudden embolism or thrombosis might be, but of its effects pain would be the least distressing and significant part. The syphilitic headache, usually worse of a night, is of course a symptom, not of cerebral arterial disease, but of the specific inflammation about the skull and its membranes. Ambrose Paré mentions the nocturnal headache of syphilis as 
often associated with deafness, twisting of the mouth, convulsion or palsies ("Vie à potence"). In renal disease, it is true, the headache seems to be, at any rate in part, a result of the excessive blood pressure; for to moderate it by a nitrite is usually to assuage the pain, an effect, by the way, which is one of the many confutations of the absurd maxim " not to treat symptoms." To abate renal headache by a nitrite not only relieves present suffering, but also enables the patient to rally and to take food; while to the general course of the malady it seems to do no harm whatever. Indeed it may ease the laboured heart. What in hyperpiesia patients often do complain of is oppressive fulness or confusion in the head.

Vertigo is likewise a symptom of indefinite meaning : it may mean anything from the little swim of atonic cephalic vessels to which neurasthenics are subject on stooping, or the passing giddiness of a dyspepsia, up to labyrinthine disease, or a small apoplexy. Stomach vertigo was something of a fad of Trousseau; probably many of his cases were labyrinthine. It is often a nice diagnostic dilemma to decide whether in a particular case the vertigo be central or peripheral, e.g. aural ; we know that labyrinthine vertigo, though usually, is not necessarily attended with deafness, nor even with tinnitus. In a case of acute vertigo (Mr. B.) which I saw with Mr. Nash of Bedford, we diagnosed a small apoplexy, partly because he had had a slight but unmistakable apoplectic seizure 12 months before. His diastolic pressure was 165-170. There were no indications of renal disease. In some cases of the vertigo of small hæmorrhage the pupils are unequal. One such case I well remember. It seems likely enough that in decrescent arterial disease minute thrombotic accidents in little twigs, as in high-pressure cases tiny hæmorrhages, would cause vertiginous attacks ; and, if in " silent" parts of the brain, without other phenomena. Arteriosclerotic vertigo has been attributed to sclerosis of the internal auditory artery, which is said to have no anastomoses. On this alleged condition, which is more likely to be of the decrescent mode, high pressure has been foisted, and decay of the twig has been confused with high-pressure vertigo of cerebral origin. In syphilitic cerebral arterial disease vertigo, almost to swoon, is frequent, and might almost be called characteristic. In this kind of disease, of the 
basilar artery especially, vertigo may be very severe, but it rarely lasts very long: the patient feels bewildered and swooning, but keeps his wits so far as to sit down, and usually in a short time is steady again; whatever the later consequences. Our forefathers were wont to distinguish between vertigo tenebrosa and vertigo caduca (syncope). Syphilitic disease of the cerebellum might, I suppose, cause severe vertigo. Labyrinthine vertigo has its own course and characters; most of the so-called arteriosclerotic vertigos are aural; I have said that it may appear without deafness. I saw lately a medical friend suffering from vertigo, clearly labyrinthine, after influenza; there was no deafness and no tinnitus. It was a great bother for a few weeks.

Tinnitus may vary from an occasional tinkle or chirp to maddening hisses or hums. It is often assumed that sclerosis of the carotid artery, where it passes through its bony canal, may set up tinnitus; but my experience of such cases is that the tinnitus, whether associated with vertigo and deafness or not, arises in the ear itself. It is rarely in rhythm with the pulse, and in elderly persons too often appears as part of the grievous affliction of "Menière's Disease." Sometimes, it is true, these symptoms appear, quickly to disappear again, whatever the blood pressures; still even then I suspect they are aural. When, on the other hand, in aortic regurgitation, even without obvious arteriosclerosis, slight degrees of it appear, the sound does synchronise with the arterial pulsations. What direct part arteriosclerosis may take in setting up the ear troubles of Menière's disease in elderly persons I must leave to the aurists to say; but again and again I find myself justified in putting to the account of the ear symptoms which had been attributed to cerebral arteriosclerosis.

" Senile Epilepsy," as an alleged symptom of arteriosclerosis, I may quickly dismiss; Stengel and others accept the association. I have little to say about it-no more indeed than to agree with the ordinary opinion that in old persons convulsive attacks resembling epilepsy are not infrequent. Whether they are, strictly speaking, epilepsy or not I cannot say; in cases in which I have been concerned no seizure has happened in my presence; besides, even by the convulsion alone the diagnosis may not be made. At any rate the simulation of epilepsy 
is often very close; moreover, as is frequent in epilepsy, the convulsions are apt to be associated, as we commonly see in the wards of asylums, with degrees of dementia (Mott and others). Of their pathogenetic conditions however we know as little as we know of those of epilepsy itself ; the guess that epilepsy is due to a vasoconstriction in certain cortical areas may be stretched to cover senile convulsions,-if such guesses be thought worth while. ${ }^{1}$ In epilepsy, save during the convulsive efforts, the blood pressure is not raised; the epileptoid convulsions of plumbism or syphilis are of course not epilepsy, common as the blunder is. I think it may be regarded as generally, if not universally, true that these senile convulsions, if a manifestation of arteriosclerosis at all, an opinion not yet proved, pertain to the decrescent or perhaps to a toxic series; possibly to a mild uræmia, or some intestinal poison, and not to hyperpiesia, as clinically distinguished from chronic renal disease.

But of sanguineous apoplexy the contrary is true ; this kind of stroke, although by no means pertaining to high arterial pressures only, for it may arise of course in intracranial gumma, in cerebral aneurysm, in contusion, in certain diseases of the blood, and some other conditions, as well as in Bright's disease, is notwithstanding a salient consequence of high pressures; and in renal disease the proximate cause of the rupture is the stress of inordinate blood pressures first undermining then bursting the vessels. Miliary aneurysms are characters of high pressure, not of decrescent cases (Mott and others). It is scarcely too much to say that in all cases of high pressure cerebral hæmorrhage is our worst apprehension; of heart strain we may take some measure, and we may stave it off, but an apoplexy lies always in ambush. It may strike suddenly, before the vascular strains had been appreciated; often indeed before they had attracted any attention.

Thus by one heavy stroke the body may be maimed, or its life extinguished; or again by a smaller attack some partial or delicate function of the body may be smitten, or transiently

1 See Vol. I., p. 161, on "Vasoconstriction in Arteriosclerosis." We are all aware of the well-known experiments of Kussmaul and Tenner on ligature of the carotids, repeated by Naunyn. But here the step from such experiments to clinical cause and effect is a long one. 
embarrassed, halting for a few hours, or but for a few minutes, and then returning to the normal concert. It has been my experience that in chronic renal disease the strokes generally fall heavily, maiming or killing the patient; those of Hyperpiesia, heavy as likewise they may be, more often than in renal disease are preluded by assaults of the same kind, but of a lighter or skirmishing order, foreboding rather than compassing the maiming or mortal attack which, however tardily, is pretty sure to follow. Omitting however chronic renal disease, which, if not alien from our subject is beside it, let us dwell more especially on the strokes and palsies of "Arteriosclerosis."

And here again I must continually urge my cardinal distinction, the fundamental distinction of which books and papers still take little heed, between the arteriosclerosis of high pressures, whether of renal disease or of Hyperpiesia, and that of the decrescent forms, whether due to toxins, to mere detrition, or to both factors together; I must speak severally of cases, as they belong fairly definitely to the high pressure or the decrescent class; a distribution which, in spite of a few mixed cases, is in accordance with the ordinary courses of nature. Excluding then renal disease in its ordinary clinical forms, let us begin with the cerebral symptoms of Hyperpiesia; the decrescent cases we will consider afterwards.

The hereditary tendency to cerebral hoomorrhage, which usually means a tendency to high arterial pressures, whether of renal origin or not, has long been recognised by the laity (p. 165). At my age, as I have said already, I look back upon family after family in which this fate seemed inexorable. Sir William Gowers, all neurologists indeed, are well aware of this bent; Gowers has said truly that apoplexy "depends upon causes that take their heredity far away from the brain in which the lesion occurs; yet the frequency in which heredity is active is most notable." Sir Hermann Weber ${ }^{1}$ reports thus of his own people: "My father died of cerebral apoplexy; his family was gouty, and for several generations the cause of death was apoplexy, or allied affection of the brain, between the ages of 68 and 75. They had all lived freely with regard to wine and food."

1 Weber, H., æt. 38, Brit. Med. Journ., Nov. 21, 1912. 
Catastrophes in disease are sudden to him only who is blind to their approaches; so it is with apoplexy-in the Latin tongue sideratio, planet-stricken. But the springing of a mine is no wonder to the sapper who laid it, nor to him who touched the button. For example, one whose blood pressures, known or unknown, have been waxing for years, and whose arteries, in the encephalic area and elsewhere, have been strained, but who may, notwithstanding, have had little sense of ailment, finds himself hindered in speech. The embarrassment is not a stutter, or usually it is not; it lies deeper. He does not laugh it off; he is startled, as by a sense of some profounder lapse. Indeed if there be also some numbness or slackness of a limb he is ready at once to suspect it to be a "stroke." Often however, in a few minutes or seconds, the embarrassment passes off. One patient, a clergyman, while preaching, hesitated, felt confused, looked anxiously about him, called up his will and pulled himself together, hoping the congregation might not have noticed it; if noticed it were, in a few minutes the incident was forgotten by the congregation, but not by the preacher, who wended his way to his physician with a sense of some arrest in his life. In such an one the physician finds an hypertrophied heart, a high blood pressure, and a radial artery constricted and wiry, or large and leathery, as the area of constriction may be. Another patient, in the midst of conversation, found himself at a loss for speech, and quietly left the room until the faculty returned; then, to reassure his family, he went back and resumed his talk without any one but himself being the wiser. Both these men were stricken down later by large apoplexies. Such cases may be given by the score. Now it is true that like omens may manifest themselves in other districts of the cerebral centres; the phenomenon may be, as I have said, a transitory numbness or slackness of a limb; or again merely a vertigo, or a bewildering confusion; but it is remarkable, at any rate so it has been in my experience, that in hyperpiesia this kind of omen appears most frequently in speech, a word which $I$ use in a general sense, not now distinguishing finely between motor and psychical spheres; often indeed in their fugitiveness these menaces escape a closer analysis. Now to what are these "intermittent claudications" due, what is the nature of the paresis? 
In one patient of mine the halting of speech was often repeated: he had several brushes of aphasia; and once by chance found himself "agraphic" without aphasia. Probably however all of these aphasic manifestations were not of the same nature, for (he too was a parson) often a halt without any confusion would come on towards the end of a prayer or short reading (he had been warned against longer services), when it may have been due to fatigue of a centre atrophied or damaged by a previous small hæmorrhage or thrombosis-a true " intermittent claudication." But to this patient I am now making but a passing and discriminative allusion, for his case was one not of hyperpiesia but of decrescent arteriosclerosis; a kind in which these simpler apraxies of atrophic centres are frequent.

These transient paretic attacks, in their various shapes, are too well known for me to tarry now longer in description of them. Of the knight Alan of Beccles Matthew Paris records that, when he raided the Abbey, gluttonously eating many dainties, "eating as none should eat," suddenly his speech failed him. Unluckily for Alan, this preliminary palsy had its later consequences; the judgment upon him was that a full seizure should follow: and so it followed; "his veins burst, and he was smitten with an apoplexy." Adam Fitzwilliam, according to this chronicler, seems to have had a similar transient attack. Apoplexy was recognised in the time of Hippocrates, who describes the plethoric habit and, in the attack, the stertorous and unrhythmical breathing. But in old days cerebral hæmorrhage was of course confused, as was inevitable, with alien conditions; even with syncope ("paralysis universalis"). By our later forefathers however this dramatic event became better and better understood, and many treatises-one of the best being that of Wepfer ${ }^{1}$-were devoted to the subject. As in the time of Matthew Paris, so in the 17th-18th century it was attributed to " intemperantia in victu et vita sedentaria " (" inter alia," of course). These descriptions had in view chiefly the cerebral hæmorrhages of high arterial pressure, for these are

1 A physician of Schaffhausen (1620-95), in whose book many years ago I found much interesting matter, as of a pioneer of neuropathology, an opinion in which Payne agreed with me. Wepfer, as Hoffmann of Halle (16661742) after him, demonstrated cerebral hæmorrhage as a cause of apoplexy. 
the most dramatic in their explosions; though of course, as was then inevitable, the hemiplegias and other palsies of cerebral atrophy and thrombosis in decrescent arteriosclerosis were classed by them under the same chapter, half distinguished perhaps as the cases in which again and again they had observed bleeding to be harmful.

In recent years, with our more intimate diagnosis, the larval forms of stroke have attracted more and more attention. Dr. Parkes Weber ${ }^{1}$ records, of a patient (M. æt. 51) suffering from high arterial pressures, that he had eight attacks of aphasia; seven with hemiparesis on the right side, one with the same on the left. Dr. Pruen, ${ }^{2}$ in an interesting survey of these and such cases, opines that "more than three-fourths" of the classical strokes have these lighter menaces as a prelude once or oftener repeated; he quotes from Gee's Aphorisms to the same effect. In my opinion Dr. Pruen lays too much stress upon mental conditions as immediate causes, as compared with, what I may for the moment call, the mechanical; and he does not, as I must insist on doing, divide the high-pressure cases from the atrophic (decrescent). By nature distinct, occasionally they may be symptomatically indistinguishable; but in Dr. Pruen's summary of associated symptoms I think this confusion is aggravated. In hospital practice of course the prelusive phenomena are apt to pass unrecorded.

Now, while still concentrating our attention on hyperpiesia, in what do these preludes consist pathologically? What are they due to? Omitting conjectures on local œdemas, local molecular fatigues, and the like, we may discuss the two more important explanations, that of local vasoconstriction, so forcibly advocated by $\mathrm{Pal},{ }^{3}$ and that of small local hæmorrhage; for small embolisms or thromboses, maladaptations of stiffened vessels, and local atrophic solutions of continuity belong rather to decrescent arteriosclerosis. For the contemplative man the hypothesis of vasoconstriction is very seductive ; without moving out of his armchair he can order it about and turn it to many

1 Weber, Parkes, Proc. Roy. Soc. Med., Feb. 1912.

2 Pruen, Lancet, Oct. 28, 1911.

3 Pal, Gefässkrisen, Leipzig, 1905-a very attractive treatise, although its large superstructure rests in great part upon unverified hypothesis. The same may be said of the shorter discussion of the subject in Meyer and Gottlieb. 
uses. As a cloud over the sun closes the sea anemone, so the handy formula of vasomotor eclipse explains this functional closure or that, as occasion requires ; an argumentum ex machina. The vessels of the brain are probably susceptible to constrictive vasomotor influence in some degrees, though in degrees which are discernible only to very sensitive methods; so slight are they indeed that for many years by skilful observers this susceptibility was denied to them. The endowment would seem therefore to be so slender as to take only a subordinate place in cerebral function, and in high-pressure cases to be quite incapable to resist any considerable areas of constriction elsewhere. In our laboratories, Dr. Cow, in verification of Barcroft's results, found that adrenalin, while constricting large areas, dilated the cerebral and coronary vessels, as it does the pulmonary (Brodie, Dixon, and Elliott). Gaskell and Barcroft think that the dilating effect is due to metabolites; I suggest that this effect is not direct but indirect, that these weaker vasomotor areas yield under the high systemic pressure. However certain authors of eminence, well aware no doubt of the spells of seductive hypotheses, adopt this guess on the lighter apoplexies by.vasomotor constriction in small areas of the brain. Sir William Osler, ${ }^{1}$ discussing the brief pareses, confusions, and sensory disorders of high-pressure cases, attributes them to spasms of arteriosclerotic vessels, assuming that in the sclerotic state arteries are not less, but more susceptible to vasomotor cramps ; a notion I have discussed elsewhere (p. 161). And he reminds us of such alleged cramps in branches of the retinal arteries, very rare and dubious observations to which I will return presently. Sir William refers to a certain patient, with such transient palsies, whose arterial pressures were excessive, but whose arteries (those accessible?) were not sclerosed; and to a second, likewise under high pressures, whose (accessible?) vessels were sclerosed, and of them says that palsies so transient " could not" be organic! Well, do not let us speak too confidently. Dr. Russell, ${ }^{2}$ likewise accepts the vasomotor hypothesis, and defends it with some fervour; although he adds that slips

1 Osler, Sir W., Canad. Med. Ass. Journ., Oct. 1911.

2 Russell, W., Brit. Med. Journ., June 4, 1904, and other essays concerning this inference. 
of the heart also may play some part in their causation. Yet surely in areas of constriction so confined, tides of cardiac action would be indifferent. He admits that the symptoms frequent the area of the internal capsule, and that in one of his cases a lesion the size of a pea was discovered. Dr. Russell goes on to distinguish atheromatous from sclerotic arteries in the brain, attributing the susceptibility to vasomotor influences to these, and denying it to those; a sclerotic artery, he says, can contract, an atheromatous cannot. With all respect, the distinction is not one which can be accepted, or not in these terms; in the cerebral vessels the process of atherosclerosis is not thus divisible, nor does the author give us post-mortem evidence in support of such a distinction. "Sclerotic" cerebral arteries might be hypermyotrophic, a condition which Dr. Russell accepts, and this change might conduce to spasm; in a fibrosed artery constriction is unlikely. Dr. Russell argues that there are tides in vasomotor activities-indeed we know there are; but this principle scarcely assists us. Nor does the appeal to migraine help us; the alleged vasomotor contraction, or dilatation, in this neuralgia is secondary to a peculiar afferent irritation, often in the eye (eye strain); it belongs to the hither side of middle life, to healthy arteries. In later life, when arteries deteriorate, migraine generally passes away.

Furthermore, vasodilators, which have been well tried, do not arrest, or even mitigate, cerebral pareses of the kind now before us. If in the high-pressure cases, as for the most part these are, the feebly innervated cerebral or coronary vessels could contract against a systemic pressure of $170-200$ or more, which is unlikely, a vasodilator should promptly release the cramp. ${ }^{1}$ In the pia mater the vessels form a network so fine that it is not easy to imagine in a small area an arrest of blood not supplemented by collaterals; partial eclipses in the terminals of the anterior and middle cerebral are conceivable, but cramp so strong and persistent in a circle so narrow, for the paresis is often of small compass, does not seem very probable; if probable, it would surely be not stedfast, but capricious, creeping hither and thither, and setting up

1 As generally agreed; but see Wiechowski, Arch. f. exp. Path. und Pharm. Bd. xIviii., 1902; and as regards coronaries, Loeb, ibid. Bd. li., 1903.

VOL. I 
dissolving views of functional squalls. And why do not such breezes blow about the common and normal brain, playing with us continually? Transient epileptic pareses are indeed quoted in proof of vasomotor causation ; incertum per incertius. That epilepsy consists essentially in cerebral vasoconstriction is itself a mere guess (p. 411), and certainly cannot carry any corollaries. Post-epileptic pareses are merely exhaustion phenomena. So far then this vasomotor interpretation, be it true or false, rests as yet on no data; indeed some plethysmographic and other experiments go rather to the contrary.

Dr. George Parker of Bristol has written on these paretic cases, ${ }^{1}$ giving some history of the subject, and adding five cases of his own. Dr. Parker is as sceptical as I am about the vasoconstrictory conjecture; he leans to the hypothesis of a local œdema.

If it had been found in these cases that fleeting palsies had manifested themselves in proportion to the cerebral arteriosclerosis discovered, without other lesion, in this or that area after death, we should have some reasons to suppose some functional link between this anatomical substratum and the apoplectiform phenomena; but necropsy gives little or no support to the vasomotor hypothesis. Very rarely indeed, so far as I know in one necropsy only (Peabody's case), is it true that a negative condition-negative as to focal lesion-has been proved in these cases of high pressure paresis. In a few other necropsies, at various intervals after such attacks, it has been asserted that in the corresponding areas of the brain no focal lesions were discovered; but these records are not precise enough to escape the demur that some focal lesion, minute in degree or faded in colour, might have been overlooked. And at best they give little hold for a functional interpretation, even were they not faced with the vastly greater number of like instances in which correlative lesions have been plainly demonstrated. In December 1913 the examiners in medicine for our M.B. degree set a certain case, from St. Bartholomew's Hospital, for commentary. A part of the history was a hemiplegia of two years' standing, with degeneration of the pyramidal tract. The brain was very closely scrutinised, very carefully sliced in thin layers

1 Parker, G., Bristol Med. Chir. Journ., March 1899. 
from vault to base, and pronounced normal. But the pathologist was not satisfied; he preserved the parts and submitted them to more searching methods, when a definite area of thrombotic effects was revealed. No ordinary survey can be accepted as evidence. If we look at the vessels of the medulla, pons, and midbrain with a lens we shall see how numerous and small they are, and how minutely subdivided, as if to provide against large oscillations of pressures. Moreover, they are peripheral to the chief fall of pressure gradient, and are further protected by some tortuosity of their branches of supply. It is improbable that so stable a network as this, with so feeble a vasomotor endowment, should be subject to instabilities so casual. As to the notion of small fleeting patches of œdema as causes of the seizures, it can scarcely pretend so far to the place of an hypothesis. In the brain of a corpse, congestions, œdemas, and the like are matters of faith or fancy. Or, if we are to guess about encephalic œdema, I suppose it would be on the lines of fluid surfeit, mentioned on another page; but it is even less easy to suppose differential fluid pressures in the brain than small areas of cortical or central arterial spasm. Areas of œdema are usually secondary to hæmorrhage, embolism, tumour, or other local lesion. In Peabody's case the stroke, upon which death immediately ensued, was no light or transient attack, it was a mortal apoplectiform seizure of right hemiplegia with coma; the statement that no lesion was present seems, uræmia apart, to stand alone against all pathological experience. In such a nontoxic case death in coma means death by intracranial pressure; and how could constriction of cerebral vessels cause an increase of intracerebral pressure? It is admitted that the cerebral arteries were badly diseased, and we are asked to suppose vessels, at best feebly innervated, beginning to show extraordinary constrictive abilities when under disease. In Sir William Osler's other cases there seems to have been no necropsy.

Now let us turn our attention to the multitude of positive, and contrary, instances. Many years ago, nearer forty than thirty, I had my first lesson in this kind of clinical series. For some little time an elderly gentleman had been under my observation for occasional transient pareses, giddinesses, and confusions, 
I remember that one morning, on rising from morning prayers, he reeled, and for some minutes was much confused; in a few days this attack, like others which had preceded it, passed over. At length he died of a grave and unmistakable apoplexy; and by good chance we obtained an autopsy. In the brain, besides the mortal clot, were found traces of no less than sixteen hæmorrhages: some were tiny, some almost faded away; most of them yellow or brownish, and evidently of old standing. Now for me this case was not only a record of curious facts, but also in itself an education. It was full of significance, clinical and pathological. Among other lessons, it taught me that tiny stains, little clumps of hæmatoidin crystals, or pigment, or tiny thromboses (p. 426) may easily be overlooked, even by a responsible anatomist, and the brain reported as " negative."

But, it may be urged, did this case teach too much? Was it not a crucial but an aberrant and too imposing an instance? If, by this, and some like experiences, I have been led to attribute transient pareses of the kind under discussion-i.e. for the most part those under high-blood pressures-too generally to hæmorrhage, what has been the experience of others, and what my own later experience? Well, apart from certain exceptions to be noted presently, the weight of evidence in favour of multiple focal lesion in these cases remains considerable. To marshal it in full would be too large a task; I must content myself with a few witnesses.

Senator, ${ }^{1}$ writing of cerebral hæmorrhage on the whole, said : "Also at times diffuse punctate hæmorrhages are found scattered throughout the whole brain." Marchand, in such brains examined after death, has often observed "numerous (zahlreichen) old apoplectic foci." Jores, in two of the reported cases in his 1904 paper, says of the first- " a high-pressure case with large heart and atrophic kidneys,"-that, with a general arteriosclerosis, including the vessels of the brain, a fresh hæmorrhage was found in the right temporal lobe; and there were also multiple old apoplectic foci (italics mine): in the second, a case of renal disease with hypertrophied left ventricle, a similar group of multiple hæmorrhages were discovered. Of another case in the same paper-a male, who died of cerebral hæmorrhage,

1 Senator, Erkrankungen d. Nervensyst. 2nd ed., 1902, s. 313. 
also a high-pressure case-we read: "kidneys, capsule easily detached (löslich), a fair amount of interstitial excess, but very little change in epithelium." Besides the final rupture " multiple old hæmorrhagic foci " were found. Kaufmann indeed, in his last edition, goes so far as to divide cerebral hæmorrhages into two classes-the Punctate Howmorrhages and the Larger Homorrhages. The punctate he describes as multiple and sharply defined. A careful article, touching this subject, by Pick ${ }^{1}$ has fallen under my notice. Pick, by a macerating method, separated the brain substance from the arterial tree; thus, in cases such as we are considering, he found that apoplexies which were mortal always came from ruptures larger than miliary aneurysms. The "miliary ruptures" he divided into (1) blood clots confined in circumvascular spaces of small twigs (as Kölliker first demonstrated), that is, false dissecting aneurysms, to be distinguished from Charcot's miliary aneurysms; (2) dissecting aneurysms : of these in his specimens many had ruptured, and clinically had caused transient palsies. As causes of death Pick considers that the importance of these small hæmorrhages has been overrated. They are never contained in sacs with walls. Dr. Forbes Robertson ${ }^{2}$ says, after months or years minute hæmorrhages may be represented only by a tiny sclerotic patch ; and, in the brains of the insane, islets are often to be seen which, although not quite satisfactorily explained, are probably of vascular origin. Out of many more such testimonies I will direct attention to an interesting paper by Naunyn ${ }^{3}$ in which, with no distinction made between one kind of arteriosclerosis and another, he describes (among other lesions of course) small scattered cerebral hæmorrhages. Vaquez, ${ }^{4}$ on high-pressure cases, says in these vertigo and "repeated cerebral hæmorrhages are characteristic." Dr. John Gaskell,, from another point of view, mentions the case of a man, with primary contracted kidney, a large heart, and extensive atherosclerosis, in whom, as the cause of death, a fresh hæmorrhage was

1 Pick, Berl. klin. Wochnschr., Feb. 21 and 28, 1910.

2 Robertson, F., Path. of Mental Dis. p. 335.

3 Naunyn, Volkmann's Samml. Ser. xix. Heft i., 1905.

4 Vaquez, Arch. des mal. du cour, avril 1913.

5 Gaskell, J. F., Thesis for M.D., 1912 ; see also Journ. Path. and Bact., 1911 (case No. 39). 
evident ; but " many old hæmorrhages were found in the basal ganglia and centrum ovale." And here he alludes to four other cases (of his own) of death by apoplexy: in the second were found (besides in all the cases the fresh hæmorrhage) signs of multiple old hæmorrhages in the basal ganglia; in the third such signs in the right lenticular nucleus, and an old "apoplectic" nodule in the putamen; in the fourth, old hæmorrhages in the pons, centrum ovale, and lenticular nucleus; and in the fifth, an old apoplectic nodule in the same nucleus: a striking list when we remember that these cases are cited in another argument not in any concern with the present question. . About ten years ago one of my pupils kindly looked up the subject for me in the records of the Manchester Royal Infirmary (but forgot to sign his report, now on my file); in 10 out of the 71 cases collected he found mention of multiple old hæmorrhages ; they were found in the pons in 5 cases; in the basal ganglia in 3 ; in the cerebellum in 1 , and in the occipital lobes and cerebellum in 1 case. Other observers mention a proclivity to tiny hæmorrhages in the pons, accidents which find some corroboration in our clinical histories. Now such, and much more, is the evidence of morbid anatomy. May we not then assume, at any rate provisionally, that in the vast majority of cases of this high-pressure kind, cases of hyperpiesia or chronic renal disease, transient pareses signify small hæmorrhages?

Let us turn back to clinical experience : is it not the experience of all of us that these transitory pareses, aphasic and otherwise, prove by their issues to be no mere swerves of function, no mere oscillation of molecules within limits of tissue consistency, nor even mere maladaptations of stiffened vessels, but to be premonitions of mortal apoplexy. Unless overborne at the heart, or cut off by some incidental event, do not these patients, practically all of them, die later of a larger hæmorrhage, a hæmorrhage of which the slighter attacks were forerunners? Dr. Pruen, in summing up his paper (loc. cit.), says, "slight strokes are always followed by ordinary ones"; meaning, no doubt, other accidents apart. Thus no case of the kind is clinically complete in which the later history is not given.

In comparing the hemiplegic with the unaffected side, Huchard, who in hæmodynamics was often much to seek, said that on the 
affected side the pressure falls to "hypotension"; while on the other the original high pressure continues. This condition he names "hemihypertension." Such partial states are of course impossible; no such contrasts could extend beyond a vasonotor change of diameter in the radial of either side, a change by which the systemic pressures would be quite unaffected.

I have remarked that these transitory pareses are often aphasic, or alalic ; still they appear in many other forms. In one case of well-established hyperpiesia I detected several small visible hæmorrhages under the skin-no very rare discoveryand on examining the retina I saw a minute hæmorrhage there also. There had been no complaint of vision in this case, and there was no renal complication; the single small hæmorrhage was the only visible eye lesion. Epistaxis is of course a wellknown signal of high blood pressure (p.442). In an extract from a French journal ${ }^{1}$ it is stated that in the course of repeated lumbar puncture-to relieve the headache of some cases of high blood pressure-evidences of meningeal hæmorrhage are not infrequent; the fluid is stained with blood, and hæmoglobin or red corpuscles may be found in it, the "remnants of older hæmorrhages." To discover red corpuscles, or hæmoglobin, 20-25 cc. are required; but in high-pressure cases the cerebrospinal fluid often jets forth in relatively large quantities. Dr. Riesman, ${ }^{2}$ in discussing these hæmorrhagic tendencies, thinks that mere high pressure with arterial disease is insufficient to produce them, and brings in a hypothetic hæmolytic toxin as an auxiliary. Some other observers are of the same opinion; but Riesman's attention was concentrated upon renal cases. In many cases of cerebral hæmorrhage in hyperpiesia it is true that the cerebral arteries, even near the rupture, shew no signs of necrosis (p. 429, Charcot).

One other critical point there is, a point upon which Cushing of Baltimore laid much emphasis, one that we dare not forget, although at the moment of an apoplexy the recollection of it may be somewhat embarrassing; this is, that under the compression of a hæmorrhage the general arterial pressure may rise from an average level enormously (even to 250-300), in order

1 La Tribune méd., Nov. 24, 1906 ; quoted Med. Rev., Feb. 1907.

2 Riesman, Amer. Journ. Med. Sci., Nov. 1907. 
for life's sake, to force blood into the bulb blanching under compression. The embarrassing dilemma may be, whether this inordinate rise of pressure is such a purely conservative feature of the moment, or is the obstinate continuance of a long period of high pressures. The form of the heart may help us in this dia. gnosis, and to decide whether to bleed or not? Still the problem is often more difficult. For instance, in a severe case of apoplexy in a man, aged 64, whom I saw with Mr. Grellet of Hitchen-an attack which on my visit was of eight days' duration, and 80 severe that, besides a left hemiplegia, the man was still torpidMr. Grellet said that on the first day of the seizure the pulse was " one of the hardest he had ever felt." Now this was not merely compensatory, for this state of the pulse had been recognised and observed by Mr. Grellet for some little time before. So with this knowledge venesection might well have seemed justifiable, or even called for. However this patient was not bled; and a consultation was called because, after the first or second day of the attack, Mr. Grellet found the pulse softening, and this so much as to arouse his apprehensions. We judged by the touch and by the stethoscope (we had no gauge at hand) that the blood pressure had fallen decidedly; the pulse had softened, the aortic second sound thinner, and the impulse of the heart more diffuse. The heart was dilating. Had the patient been bled, this change might have been attributed to the operation; indeed in this case venesection might have been harmful. Whether at length the heart was failing intrinsically, or under the cerebral oppression indirectly, it was not easy to say. (I have no note of the issue.) Celsus shrewdly remarked that in apoplexy to bleed cured or killed. However these reflections are somewhat digressive.

On the pathological modes of cerebral hæmorrhage, why cerebral hæmorrhage is very frequent, while coronary hæmorrhage is very rare, on the part miliary aneurysms take in the event, again on the alleged seasonal prevalence of apoplexy, and so forth, I have no more to say than is recorded in the books and essays at the disposal of all of us. But, in respect of blood pressures, I may allude again to the comparative frequency of seizures during the night, a tendency attributed, as in the case of angina pectoris, to a 
supposed nocturnal rise of pressure (see p. 21); but, so far as I know, we have no definite evidence of such a tide. Or it may be due to the recumbent position. As Pal, Janeway and others have pointed out, hyperpietics are more liable than other persons to critical excesses of pressure; an observation which accords with what we know of all unstable equilibriums. A remarkable case was published a few years ago, by Jackson, ${ }^{1}$ of a woman, æt. 60 , with arteriosclerosis and chronic interstitial nephritis. Her systolic pressures ordinarily ranged about 200. One day she complained of severe headache, when the pressure was read at 250 , even a little higher. For verification the observation was taken three times; while on the third trial the record was being read she fell on the floor unconscious : a fatal cerebral hæmorrhage.

Pneumonia, as I have said, is no uncommon intervention in hyperpiesia ; the following case is one of many instances :-

R. F. C., æt. 61. Obese. A keen business man. A good trencherman and heavy smoker. Four or five ozs. of whisky a day. Sent to me by Dr. Roberts of Harrogate. Went to Harrogate for eczema. Dr. R. found his pressures over 170. High frequency current and other spa treatment little service. During following winter muddled feeling in head, dizzy. No tinnitus, no deafness. Sense of insecurity in walking. Systolic pressure in my room 200, but with care brought down to 180. Pulse sustained. Most careful examination had been repeatedly made for renal disorder, but urine always between 1015-1030; no albumin, no sugar. No constipation. Heart rather big, but arteries not bad for age. Afterwards I heard from Dr. R. that a year later he had had a slight stroke with transient hemiplegia; from this he recovered so far as to return to a mild game of golf, but, after again a year's interval, he was attacked by acute pneumonia and died.

For convenience of contrast I will now bring forward the symptoms of the nervous system in Decrescent Arteriosclerosis. The symptoms of decrescent arteriosclerosis, taken as a whole, need no long description; fortunately, because it would carry us over the whole sphere of bodily function. It is not much needed because the condition is well recognised and

1 Jackson, J. M., quoted Lancet, March 28, 1903, from the Boston Med. and Surg. Journ., Feb. 26, 1903. 
familiar; and again because in character its symptoms are for the greater part negative, and consist in a drought of the body; in failing irrigation of organs such as the cardiac, the renal, the cerebro-spinal, the gastro-intestinal, and the rest: we note the withered skin, the shrunken limbs, the shuffling gait, and so forth. Or in the more rapid cases, often rather premature in age, it may consist in a kind of progressive cachexia, with pallor and emaciation, the arteries presenting universal rather than local degeneration.

An eminent physician writes, "Granular kidney, up to the age of 60, divides the honours (of apoplexy) pretty equally with atheroma." What atheroma? Certainly not with the decrescent mode. Let us then consider briefly the cerebral palsies of decrescent arteriosclerosis, the mode not correlated with inordinate pressures, and contrast them with those of high blood pressures. Broadly speaking, the plain distinction is that the palsies of this mode are not hæmorrhagic but atrophic. ${ }^{1}$ When, rarely, hæmorrhage does occur it is usually into a patch previously softened by arterial decay, and is not a primary cause of palsy or dementia; indeed as an accident it is not common, for usually the vascular supply to such an area had been already obstructed or cut off. The finer twigs are very readily blocked by tumefactions or shifting particles of decay. Stiff as the artery in such cases may be, the blood stream is often slow and small; often the heart does not exceed 10-12 ounces in weight. In high pressure cases, on the contrary, in the 64 cases of ordinary cerebral hæmorrhage in the Manchester Infirmary, which I have quoted as obtained for me by an old pupil (p. 422), the heart was hypertrophied, with or without dilatation, in 52. It was dilated simply in 5, and in the rest normal. Of the 64 cases, the kidneys were called "granular" in 40 ; in 24 they were "normal."

The great proportion of arterial disease among inmates of asylums is not associated with high pressures. Dr. Mott, in

1 "Prior et perpetua" (says Haller) " est excaecatio vasorum-aut certe si negas excaecari, tanta eorum vasorum arctitudo ut injectos etiam tenuissimos coloratos liquores negent admittere. . . . Angustia et duritas arteriarum ... ita ad arteriolas minimas minus humoris venit, et vi debiliori. ... myriades enim vasorum nunc caecae." Haller, loc. cit. He refers to his Art., Phil. Trans., n. 306. 
necropsies on 160 asylum cases of arteriosclerosis, says of the kidneys, that only in 65 was there " some degree of interstitial fibrosis, usually not very marked" ; and again "it (the kidney) seldom assumes the degree present in the small red kidney of Bright," but " is part of a general degenerative process."

But in old persons with decrescent arteriosclerosis under ordinary pressures periods of high pressure may occur, episodes which, under deobstruent treatment, usually disappear without ill consequences (see p. 452); now during such a period of high pressure a decayed vessel may give way and an apoplectic seizure occur. Such cases are not very frequent, but one clear instance I remember which I saw with $\mathrm{Mr}$. Wingate of Cambridge. Mr. Wingate said that in this lady, Mrs. J., an elderly woman with degenerate arteries without high pressure, and enjoying fair health, periods of high pressure with indisposition had appeared occasionally, and had been quickly dispelled; but on this last occasion an apoplexy had occurred which proved mortal. In another such case, a lady of more than 80 years of age, seen with Dr. Malden, such a transient episode ruptured an aortic cusp and established regurgitation (see p. 452). It is commonly said, and with apparent reason, that under peripheral vasoconstriction, as on a sudden change to cold weather, weakened cerebral vessels may be ruptured. But we have little accurate knowledge of encephalic pressures and their fluctuations. Professor Leonard Hill tells us that cerebral pressure is directly not as arterial but as venous pressure. We know that in normal life systolic pressures vary widely, often from beat to beat; but clinical experience does not indicate that such rhythmical or incidental waves push so far as to imperil even decayed peripheral arteries. For such temporary rises of systolic pressure there is ordinarily the concordant compensatory relaxation; but, as in the cases mentioned above, a week or ten days of high pressures in decayed arteries may suffice to do permanent mischief, especially if the rise be of mean pressure; a persistently high diastolic pressure may be the determining factor. We hear many warnings against straining at stool, and other such transient efforts, as immediate causes of apoplectic seizures, but I cannot say that such warnings reflect my own experience. Old people are subject 
to constipation, yet Dr. Mott finds in asylum practice that sanguineous apoplexy is marvellously infrequent, abundant on the other hand as are the cases of atrophic palsies due to arterial degeneration. In his 160 necropsies on atheromatous males the heart reached a moderate hypertrophy only in a few, and a marked hypertrophy in two only; and in 140 female cases the results were much the same. Although high-pressure cases are not of a class which drifts towards the pauper asylum, yet at Charing Cross Hospital, among the theatre people, the Covent Garden porters, persons engaged in the liquor traffic and so forth, where the hyperpietic form of disease is more common, Dr. Mott found apoplexy more frequent. Of 60 such cases of apoplexy examined after death, only about one-half, in his opinion, presented renal disease.

Marie, ${ }^{1}$ in an interesting study of the morbid anatomy of the kind of cases classed by me as decrescent, while agreeing that in them hæmorrhage is not the cause of hemiplegia, demonstrates its cause, or one of its causes, in a lacunar process : irregular cavities are formed by areas of atrophic softening, of sizes from a millet seed to a pea; one or two up to a dozen may often be detected in the central ganglia, always with arteriosclerosis. To these atrophies he attributes especially the smaller palsies or paræsthesias in such subjects. And is it not possible that, without solution of continuity, swoony sensations and passing "apraxias" may be due to a loss of the adaptive capacity of the cerebral arteries, a capacity in health of taking up and restoring energy in mutual balance with surrounding vascular areas. We are all of us, I think, disposed to suspect more than arterial decay in "softening of the brain"; something more universal and intimate; but decrescent, or in some cases a toxic, arteriosclerosis marks a large part of the process. In highpressure cases the arterial disease is not only less obliterative but stream velocity also is better maintained. Likewise, Dr. George Parker ${ }^{2}$ has argued, if a twig is plugged, its neighbours may for the moment be overcharged and by pressure anæmiate surrounding tissues, causing transient symptoms. No uniform tautness of the vessels of a part would have this action. H. W.

1 Marie, Rev. de méd., 1901.

2 Parker, G., Bristol Med.-Chir. Review, March 1909. 
Cook $^{1}$ says truly of the cerebral disorders of these arteriosclerotics without high pressures, "Such an arterial alteration frequently persists for years, even at the ages of 65 or 70 , with an unenlarged heart, or perhaps with some cardiac atrophy, without albuminuria, and with fair general health. Apoplexy is not frequent in these cases, which are typically represented by old women in whom tobacco, alcohol, syphilis and overwork do not often complicate the picture." He adds that though the vessels may prove brittle enough to break in the fingers, the systolic arterial pressure is usually between 135-160 ; in some cases only 120. These cases Cook contrasts "with the apoplexies or cardiac dilatations of big eaters," a contrast on which I have said my say. Indeed Cook virtually accepts my clinical and pathological distinctions of 1894. Charcot, in his chapter on miliary cerebral aneurysms, had said that these are infrequent in senile brain softening with obsolescent sclerotic arteries, in which cases sanguineous apoplexy also is rare. He added that it is in high pressure cases that miliary aneurysms may be found, and result in an apoplexy without obvious cerebral arteriosclerosis-i.e., high systemic pressures tell hardly even upon fairly good vessels so slender as the cerebral (p. 423).

When transient pareses occur in decrescent arteriosclerosis, they are usually due to more or less complete obstruction, in part by thickened intima, and not attended by pressure symptoms. They may be simulated by lack of concentrated effort, with slowness of utterance or other response. Thus in a case of this class, Dr. Russell ${ }^{2}$ attributed transient speechlessness to weak heart and feeble circulation (p. 417). Of this kind was Tyndall's aphasia at the Grimsel, when after a fatiguing and perilous climb he found himself unable to ask for his supper.

I have already referred to the kind instruction given to me by Dr. John Tait of Edinburgh (see p. 38), researches on which seem to throw more light on the silting up of the peripheral vessels in decrescent arteriosclerosis. Lister perceived that blood, on its issue from the body, clots because of some physical relation between it and the external surfaces with which it comes in contact. Freund of Vienna first showed that the blood of

1 Cook, H. W., Journ. Amer. Med. Assoc., Jan. 1905.

2 See Russell, H., Lancet, Nov. 15, 1912. 
venesection may be kept fluid by smearing the basin with vaseline, which is not wetted by the blood. Dr. Tait made many experiments on the adhesion of defibrinated blood to the lining of the blood vessels; as also between the cells of the blood and organic and inorganic surfaces and particles. Tait and Hewitt then estimated the amount of ether-soluble material in the lining membrane of the aorta of the ox, and, briefly, came to the conclusion that vascular endothelium is a "lipoid-membrane"; while Campbell and Tait found the same to be true of the lining of the serous cavities, contact with which does not bring about coagulation of the blood. (So far, at any rate, as rabbits are concerned, Tait thinks it improbable that a concave meniscus would be formed by the blood in the half-filled aorta.). The so-called "amœboid" movement of blood platelets, to which structures the initiation of clotting must be referred, is a simple contact phenomenon, being irreversible, like the spreading of a raindrop over a stone, and due to greater molecular adhesion between the platelet and, say, the surface of a glass slide than between plasma and the same glass surface ; such movement does not take place on lipoid surfaces, because there is no sufficient molecular adhesion. In similar fashion Tait partly explains phagocytosis, and even diapedesis. Now if we suppose that in degeneration of the vascular endothelium the lipoid quality is removed, impoverished, or altered, adhesion and thrombosis would follow.

Authors on diseases of the nervous system (Gowers, Déjérine, Naunyn ${ }^{1}$ have of late drawn more attention to enfeeblement of the legs in old persons. The patient complains of increasing weakness and insecurity of gait not due to neuritis (Erb's Dysbasia angiosclerotica); and often of paræsthesias. This shuffling and tottering often accompany or precede the vacuous face and other signs of senile dementia, but it may be well marked in persons who retain their mental faculties. In some such cases it may be difficult clearly to discriminate between the local effects of disease of the arteries of the legs and the effects of spinal atrophy due to a similar cause; however the distinction in doubtful cases is one of no great practical importance. Both vacuoles and tiny hæmorrhages (consequent upon arteriosclerosis)

1 See also article by myself in 1st ed. of my System of Med. 
have been found in the brain and spinal cord. The local symptoms of advanced arteriosclerosis of the vessels of the legs are well known. If a large artery is suddenly blocked pain and palsy of the limb may cause intolerable suffering; especially if branch after branch be occluded and perhaps inflamed, and an ischæmia or a neuritis associated with the mischief in the vessels, causing other pains and miseries-cramps, aching, darting, and tingling. But such symptoms and their causes are too familiar to need description here. These cases are nearly always of my decrescent class, and are not attended with high systemic blood pressure.

Such then is the contrast, as regards the effects upon the brain, between the arteriosclerosis of high pressures (hyperpiesia or chronic renal disease) and the decrescent mode of arteriosclerosis in which the general arterial pressure is not notably in excess. How far I have failed to impress this distinction upon my contemporaries is only too manifest. For instance, a physician of great eminence, one whose mastery of clinical medicine we all hold in respect, wrote not long ago, concerning a certain patient, as follows: "The patient, now 70 years old, presents the arteries of extreme old age-not only atheromatous but calcareous and nearly rigid, and his radials are sigmoid and tortuous ; . . . indeed they are fragile mineral tubes . . . a precarious condition ... one of these arteries may rupture to-morrow-causing apoplexy and death." Now from what has gone before it will be seen that, supposing the case to be-as probably it was-one of decrescent arteriosclerosis without high-blood pressures and hypertrophied heart, cerebral hæmorrhage was in him not a likely but an unlikely event. Such persons-as we have seen

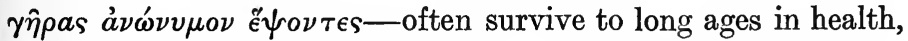
and even with fair mental energy. But what may happen to the many of this class who are less fortunate? Palsies often enough; but rarely by sanguineous apoplexy. Their vessels decay and choke; or detached bits of them are swept forwards as emboli or infarcts; and, as velocity falls more and more, and the endothelium loses its lipoid element or is eroded, many of the smaller channels are occupied by thromboses. In these degradations and atrophies lie the causes of functional failures, vague or definite, partial or general, sudden or accumulative; failures of mental and bodily energy and of disordered sensory 
and motor functions. ${ }^{1}$ And in these processes consecutive cerebral hæmorrhages, if any, are small and sporadic, and break into areas already in decay; their effects, if attended with vertigo and transient confusions, rarely issue in the deeper degrees of coma. In age collateral circulation is not ready; new vessels hardly develop as in youth; and except in a few places, such as the circle of Willis or the palmar arch, the arterial system is not rich in collaterals. The following is an example of the mode of hemiplegia in decrescent atherosclerosis :-

F. æt. 79. Seen December 17, 1907, for palsy of the right arm. This arm failed gradually, she dropped things; and speech became blurred and halting, but not so far as to prevent all conversation, as her words were correct. Never lost consciousness nor had a fit of any kind, but had paræsthetic sensations in the limb, and in other parts. Rather lachrymose at times, and attention discontinuous. Memory failing. Right arm contracted a little and reflexes exaggerated. Both arm and leg colder than left limbs; no palpable pulse in smaller vessels on either side. Radials, tibials, etc., very thick and tortuous. Brachial blood pressures 130, heart apparently normal. Urine ranged about 1020; no sugar. On a few occasions only was a trace of albumin noted; no casts could be found at any time.

That hæmorrhage is prone to occur in cerebral syphilitic disease is well known, but of the manner of its occurrence we know less. Syphilitic arteritis of the cerebral vessels tends to obstructive and obliterative effects rather than to necrosis of the media alone, of these thin vessels all three coats are involved in one diseased process. Thus it is that in syphilis hæmorrhage occurs rather in vascular gumma than as a direct outbreak from syphilitic arteries.

Ophthalmic Signs. - The state of the pupils does not help us much. Light-stop may indicate a syphilitic source; but in atherosclerosis one is not surprised to see the pupils more or less contracted; they often are so during Cheyne-Stokes breathing; or the pupillary reactions are sluggish. There seems to be some relation between blood pressure and size of pupil; rises of pressure, by straightening and lengthening the spiral vessels of the iris, may narrow the pupil ; as conversely a fall of pressure may dilate it (Waller). Even in aneurysm it is not always 1 See good study of subject by Cramer, Deutsche med. Wochenschr., Sept. 1909. 
easy to express the variable pupils in terms of mechanical pressure on the sympathetic.

I have alluded to the assertion that atherosclerotic retinal vessels have been seen to contract for a while in spasm (p. 416), and that this is the one datum for the cerebral vascular spasm hypothesis. It is therefore precious. In very rare instances the observation may have been true; but we must bear in mind that impressions from highly refractory arteries are apt to be deceptive. How is it that spasmodic caprices of vision are not more frequent? I know of only one case based upon direct evidence, and adequately described, viz. Wagenmann's case, ${ }^{1}$ relied upon by Pal to carry a very heavy burden. In a patient with arteriosclerosis (including the retinal branches) these vessels shrank away, so we are told, into fine shiny yellowish pulseless threads ; and the veins also emptied. The attacks, during one of which this was seen, produced blindness which lasted for about ten minutes, then the vessels filled as before. But the subsequent history is not given. Now in other cases, of similar history, later stages proved that the refilling of the vessel was but temporary, and a complete and soon afterwards permanent occlusion was established. ${ }^{2}$ Mr. Herbert Fisher, to corroborate certain published remarks of mine, most kindly sent me records of some cases from his own note-books, and in a subsequent correspondence wrote: “In general terms I do not think that we can distinguish between a constricted and a sclerotic artery . . . unless in blockage complete or partial." Mr. Fisher went on to say that " a history of transiently recurring attacks of amaurosis is not uncommon in cases which subsequently present themselves with all the features of an obstructed artery." ... "The sclerotic or silver wire artery . . may carry as big a stream of blood as before the sclerotic changes in its tunics, its lumen may not be constricted. I do not think that such arteries are especially liable to spasm." . . . "Thrombosed branches (showing) white lines with a threadlike red line in their centre, are quite different, and easily distinguished, from sclerotic arteries." ..." Ophthalmic surgeons would not

1 See Wagenmann's paper, "Circulationstörungen in dem Netzhautgefässe," Gräfe's Arch. Bd. xliv., 1897, p. 219.

${ }_{2}$ See Taylor James, Discussion of the Ophthalm. Soc., April 24, 1913. voL. I 
agree with the opinion that 'silver wire' retinal arteries are "pathognomonic of renal disease." Benson is said to have described arterial spasm in the retina, but $I$ have not the reference; and Elschnig 1 reports spasmodic amauroses in lead poisoning, in which disease he suggests that the muscular fibres of the vessels are irritated by the poison, and that the same events may happen in the brain. But we know that in lead poisoning the vessels suffer early, and I think it is very easy to be deceived by a gleaming light upon silvery vessels. Moreover we do not hear definitely of return of complete vision, and it is at least as likely that these amaurotic phases are results of variably deteriorating branches, with thrombotic accidents complete and incomplete. These accidents are not rare. Indeed the alleged cases of embolism of the retinal artery also, ${ }^{2}$ as distinguished from local obliterative and thrombotic states, must be accepted with caution.

At least 40 per cent, so say the ophthalmologists, of atherosclerotic subjects present sooner or later an obliterating sclerosis in the retinal branches. ${ }^{3}$ The arteries change in size, have a double outline and compress the veins at the crossings; greyishwhite opacities and streaks of exudation and possibly hæmorrhage from the vessels may be seen. ${ }^{4}$ Probably retinal signs are especially prone to occur in those cases in which the disease falls early and mainly upon the cerebral system of arteries. We have seen how partial atherosclerosis may be; that usually it is not a disease invading extensive and uniform areas of the circulation in quick succession; the arteries of the limbs may be knotted and twisted to extremes while those of the brain are intact or nearly so. For these apparent caprices of distribution we are unable to offer an explanation. In many cases of cerebral, as of coronary, atherosclerosis the parts supplied do not suffer soon and obviously if the morbid process be slow.

1 Elschnig, Wien. med. Wochenschr., 1898; quoted by Legge and Goadby, Lead Poisoning, p. 76, 1912.

2 See Hawthorne (2 cases), Practr., June 1907.

3 Vide Marple, Med. Record, March 16, 1907; and Mott, in Allbutt and Rolleston's System of Med. vol. iv. p. 621, 1910. My own vague impression is that in decrescent atherosclerosis, not of renal origin, disease of the retinal arteries may be seen sooner or later in most cases.

4 Kober, Verschluss der Netzhautzentratarterit, Leipzig, 1913. 
However, on the general value of the ophthalmoscope in clinical diagnosis, as significant now of syphilis, now of renal disease, now of simple atherosclerosis, and so on, I need not insist; these points are fully set forth in the text-books. Yet whether or no in early stages distinctions can be made between the so-called albuminuric neuroretinitis, non-renal hyperpiesia, and the retinal deterioration of simple atherosclerosis of the decrescent kind, I am not prepared to say. The "silver wire artery" is quite common in arteriosclerosis without renal disease. Mr. Parsons says the curve of intraocular pressure is longer and flatter than that of the general blood pressure; later and more prolonged.

I had some conversation on this subject at Caius with Dr. Leslie Paton, and afterwards he kindly wrote to me as follows (March 20, 1913) :

"I certainly do think that clinically the three types (i.e. of renal disease, of hyperpiesis, and of senile atherosclerosis) are distinct, but whether ophthalmoscopically one can always distinguish between them is another question. If extreme cases, at either end, be simple, there are all the intermediate grades; and, when one considers how the ophthalmoscopic changes are produced, it becomes obvious that it would be unwise to rely on the ophthalmoscope as more than an aid to diagnosis. The main changes seen are : (1) Those due directly to the presence of œdema, i.e. the loss of the finer retinal structure, the sharper striation of the nerve-fibres, the raising of the membrana limitans interna into fine folds, the disposition of these folds relative to the macula and disc, and ultimately the rupture of the fibres of Müller and the formation of the star figure.

" (2) Changes in the nerve-fibres secondary to the odema with formation of soft white areas where these have swollen up and disintegrated. (Holmes and I have dealt very fully with this in our paper in Brain, vol. xxxiii. pp. 406-412.)

“(3) Hæmorrhages, which may be either in the nerve-fibre layer or in the outer molecular layer.

"(4) The changes in the blood vessels themselves, i.e. the thickening and brightening of the arterial walls with consequent diminution of the column of blood, and the longitudinal contraction of the arteries with flattening of the curves and pulling 
away of the terminal arterioles from their tissue connections, and pressure on the veins.

"It is mainly by the variations in these four things that we can separate nephritis from arteriosclerosis with high tension, and from the senile form of arteriosclerosis. In this last form the œdema changes are but little seen, and the degeneration is probably mainly due to deficient nutrition; and degeneration is more apt to be marked at the macula than in other parts of the retina.

"To distinguish between the other two forms would require almost a treatise, but Holmes and I have said something about it in the paper I have referred to.

"Your question concerning spasm of the retinal arteries interests me greatly. I have seen temporary amaurosis due to spasm of the retinal arteries in early arteriosclerosis; but I have also seen cases where attacks of a migrainous character were associated with retinal arteriosclerosis."

Dr. Paton implies that, in his experience, spasm of the retinal arteries is associated with arteriosclerosis; I suspect, as I have said, that the ebb of the blood in such cases is due, not to spasm, but to obstructions in the vessels beginning somewhere behind the eye (see pp. 161 and 434).

Mental Symptoms.-Cerebral hæmorrhage then is the outstanding event of the atherosclerosis of hyperpiesia and of chronic renal disease; of the decrescent kind, cerebral atrophy. As regards other symptoms on the part of the brain or heart we find some further contrasts. The hyperpietic, unless after a stroke, does not become childish or vacuous as the decrescent arteriosclerotic does, or may do. The slow inexorable dissolution of mental faculties, the oil-dry lamp, characteristic of decrescent cerebral atherosclerosis, is not characteristic of the atherosclerosis of hyperpiesia or of chronic renal disease. Chronic renal disease is too well known for us to dwell upon it; in hyperpiesia, as I have said before (p. 382), dark shadows may brood over the mind disturbed by irascible or despondent moods, or by ceaseless apprehensions or glooms; clouds which gather especially of a forenoon, and lift towards evening. These disorders may engender obsessions, or even a melancholia amounting to insanity, but not in my experience the delusions 
and peculiar features of paranoia. Some years ago, Dr. Maurice Craig, beginning from the side of insanity, in his M.D. thesis at Cambridge, published a large number of very careful observations, which seemed to indicate that "melancholia"-a name which may pass for present convenience-was a disease of high arterial pressure, and in this respect differed from mania, in which pressures usually ruled about or below the normal mean. Dr. Bodington, then of the Wakefield Asylum, in his M.D. thesis in 1903, on the whole corroborated Dr. Craig's conclusions; and Dr. Longworth of the Melton Asylum, who has done much careful work on blood pressures, tells me that in the main he agrees with Craig and Bodington. ${ }^{1}$ The following case of melancholia with high pressures may serve as an instance in point :

Seen with Dr. Campbell of Cambridge. Mrs. W. (æt. 42 ?). After some temporary ailment made a slow recovery; gained flesh and looked well and rather fat. Then became sleepless and a prey to remorseful feelings; all sorts of evil suggestions spoken within herself (not voices nor fixed delusions). Much worse in a morning, better after tea. Physical examination, etc., negative, except a very hard wiry contracted radial artery; by laying the length of the finger along it, it was hardly to be arrested, it forced its way beneath. Heart two fingers outward, beating heavily; second aortic sound very loud and thumping. No albumin nor casts; urine of good specific gravity.

Other observers however have shown that we cannot accept this rule without qualification; if melancholia is often associated with high pressures the correlation is not universal. It may be that some of the cases of melancholia in which high pressures prevail are but terms in the malady of hyperpiesia; if so, they should show themselves amenable, more or less, to deobstruent and dietetic treatment, in contrast with the many other cases in which such measures prove of little avail. Only too often are we met by cases of melancholia, even acute and profound, without high blood pressures, in which alterative medication is of little service. Some of these belong to decrescent arteriosclerosis, to early stages of senile dementia, stages which often precede imbecility by some years. In these

1 Longworth, private letter, Feb. 26, 1909. 
patients however I have generally noted characters of something more than obsession, of delusion; as for instance voices, or some imaginary sin. While then I think some melancholics, and the more curable of them, are hyperpietic, a state to be discerned by clinical and therapeutical tests, yet other melancholics, and only too many, whether attended with some excess of arterial pressure or not, are of a graver and more indelible type. 'The depressed hyperpietic, though he may have well-marked obsessions, is usually more hipped than insane; and the melancholic condition rather a phase than an abiding derangement. But no doubt in all such cases we have to deal, and largely to deal, not only with the proximate causes, but also with the inherited mental bias of the individual; so that in some inmates of asylums hyperpiesis had issued in depressive insanity. Generally speaking however hyperpietic patients, if moody, are not so far deranged as to call for seclusion, though one variety may shade off insensibly into the other.

In any case, in hyperpiesia, this clouding or besetment of the mind, and loss of calmness and self-control, a condition from which the mind may emerge, and usually does emerge, uninjured, is a very different matter from the mental decay of decrescent arteriosclerosis. The arterial disease in the former condition, whether by some peculiarity of distribution, of intimacy, or of anatomical form, seems to be more conservative of endothelium and less conducive to thrombosis and obsolescence ; moreover, the high pressure itself maintains a better blood supply. Whatever the explanation, these morbid tempers apart, I repeat that in hyperpiesia insanity, if an occasional, and intermittent, term in the series, is not a very common feature. Indeed, as I have said, the higher blood pressure, alternating perhaps with periods of fretfulness or depression, yet frequently produces for a while a sense of energy, even an increased activity. In later stages, even when he is becoming puffy on exertion, the hyperpietic-here in contrast with the renal patient-is often still capable of no little endurance; he is not anæmic, and work and exercise tend to open out his peripheral circulation ; too often he keeps up his spirits, fitfully, with liberal eating and drinking. This is not at all the story of the decrescent 
form of the disease under which the man moons aimlessly about, irresolute, without mental intention enough to make up his own story. The hyperpietic patients die, of apoplexy or of heart defeat, as the case may be, with mental faculties otherwise substantially unimpaired. But for his plethora "he dieth in full strength, and his bones are moistened with marrow."

On the other hand, as many subjects of decrescent arteriosclerosis also attain a green old age without notable mental impairment, even without notable loss of memory, it would seem that in these persons we have to deal with arterial lesions partially distributed or various in degree, in the brain or in the body apart from the brain. In one patient, while the energies of the mind are unabated, thick distorted and gritty arteries are conspicuous in the limbs; in another, whose accessible arteries are in fair or good condition, the gradual waning of one mental faculty after another betrays no less conspicuously a starving of the brain, perhaps a common disintegration of cerebral vessel and cerebral substance. This is no cloud, no miasma to be dispelled, but a creeping mode of death by inches. I have spoken purposely of a common disintegration of cerebral vessel and cerebral substance, because in not a few cases one cannot but surmise that this atrophy is something of a reciprocal process, a single or united process of organic decay, so differently does it work out from the secondary atherosclerosis of hyperpiesis. In hyperpiesis the vascular injury, though grave enough to end in an apoplexy, is not penetrating enough to sap the organ of mind. In the decrescent form, on the contrary, we witness, without rises of blood pressure, without cardiac hypertrophy, not only, in respect of the brain, the lapse of memory, the torpid apprehension, the blank countenance, and the slow speech, but also the feeble gait and slurring foot, which suggest a more extensive and combined decay of vessels and nerve centres together. Finally, I include two cases of apparently healthy aged persons with extensive arteriosclerosis of the decrescent kind; in the first case perhaps of the limbs only.

Dr. Smith, late of Newport, introduced this old man to me. Att. 86. Brought up hard; fed on coarse bread, swedes, a little fat pork, and as much beer as he could get. Always a good eater. Health always good. Most of days out of doors. Eight children. 
Voice, hearing, sight all good. Good head of gray hair. "Placid temperament and fairly intellectual" (Dr. Smith). Sleep? "Yes, could sleep on a clothes-line, eight hours of a night." Pulse 68. Radials extremely atheromatous, vessels like rough tendons. Heart perhaps a trifle outward, but lungs probably atrophied. No sign of high pressure. Sounds normal. Dr. Smith had found this condition of radials in him at least twenty years before; and his brother, æt. 70, has very cordy arteries (see p. 206). Inherits longevity on maternal side. Never had chilblains, nor troubled with cold extremities.

Of the compatibility of extensive internal atherosclerosis with long life, the following case is one example :

Female, æt. 100. Recorded by Savill.1 A "healthy old woman"; died of no obvious disease, but a few spots of bronchopneumonia found post-mortem. Widespread arteriosclerosis in larger, middle, and small arteries in all areas ; in the brain even to the finest ramifications. The lumen was everywhere large, in some districts, e.g. in the common iliac, to $2 \frac{1}{2}$ times diameter. Heart rather small, not dilated, structure fairly normal. The arteries showed the usual intimal thickening, but the muscularis showed no more degradation than where in the dilated vessels it was correspondingly diminished in thickness.

Now if all this be true, and I would ask my readers to question their own clinical experience, if this contrast between the two modes of arterial disease is here again as valid as any such clinical contrasts in practical medicine can be, if this be so, are not many essays on the cerebral symptoms of arteriosclerosis obscured by a lack of this discernment? Here and there, it is true, some deeper insight is to be noted. L. W. Weber ${ }^{2}$ does contrast the cases of depression, even amounting to an abnormal sense of sin, or some ineptitude, but not to insanity, cases by no means irremediable, with those of senile decrepitude. But even he does not carry the distinction back into the different nature of the two processes; nor does he distinguish the modes of arteriosclerosis, the one marked by high blood pressures and a big heart, the other in these points negative. Dr. Alexander of Aberdeen however has made an interesting

1 Savill, Lancet, Sept. 24, 1904.

${ }^{2}$ Weber, L. W., "Arteriosklerotische Verstimmungszustände, Münch. med. Wochenschr., 1909. 
contribution to this side of the subject. ${ }^{1}$ He describes, as I did in 1894, the rises of blood pressure with the attacks of depression, together with a fall in urea excretion, and sluggish bowels. By treatment, which consists in bed, fluid diet, calomel, and salines, the pressures are reduced, and the attacks subside. Overfeeding aggravates and prolongs them. Hypnotics are mischievous. These cases he contrasts with agitated melancholia with bursts of excitement, which require a different treatment, not excluding a judicious use of opium. Yet when at Budapest International Congress, in 1909, the subject was discussed at length, the same indefiniteness of outlines prevailed. One speaker indeed did not avoid the confusion of mixing up neurasthenia (vide Diag. Vol. II., p. 61) with these arterial cases; an error avoided by Professor Cramer, whose contribution was the most important; but even he, in his argument based upon a previous article of his own, ${ }^{2}$ did not avoid the prevalent confusion of taking "arteriosclerosis" as one disease, and heaping together under this name a heterogeneous collection of cerebral effects. Between the high pressure series and the senile series he made no distinction, save to remark, without following it up, that in certain of the cases the heart is enlarged to the left, and the pulse tense; surely a remarkable difference? Throughout his description indeed one traces the separate but tangled threads of the two different processes indiscriminately mingled together. Another instance of this confusion is in respect of the high pressure and the senile paretic attacks, attacks so different in nature, but yet confused in one story. It is well perhaps here to utter the warning, which Dr. Stengel ${ }^{3}$ has emphasised, that not all disturbances occurring, perchance, in arteriosclerotic subjects are to be accepted as characters of the arterial lesions ; a caution specially necessary in arteriosclerosis, a malady of elderly persons who might, as Falstaff said, have more diseases than they knew for.

1 Alexander, H. de M., Lancet, July 5, 1902. See also Alexander and Bruce, Lancet, Aug. 24, 1901.

2 Cramer, Deutsche med. Wochenschr., Nov. 21, 1907 ; the Buda-Pest speech is published in the same journal for September 16, 1909. The earlier paper I have before me; of the Congress speech I have only the (fairly full) contemporary reports.

3 Stengel, A., Amer. Journ. Med. Sci., Feb. 1908. 
I think I need not occupy the time of the reader with a pursuit of this subject into the syphilitic kind of cerebral arterial disease. The symptoms of focal lesions of this species are already so well recognised, and so well described, as have been also the more diffuse luetic lesions of general paralysis and tabes, as to dispense with a formal recapitulation. Nor on the effects of arteriosclerosis of the limbs, as productive of paretic and paræsthetic symptoms, need I dwell : it is generally easy to discriminate these local effects from those of central origin. The alleged interdependence of arteriosclerosis and neuritis in the limbs, especially in the legs, I have discussed in another paragraph ( $p$. 304). The coincidence is an obscure one; we have but few careful observations upon it, and probably the association is one

- more of pathological curiosity than of wide significance.

Reflexes.-Dr. Moncorge of Mt. Dore ${ }^{1}$ says that the knee-jerk is, within limits, inversely as the arterial pressure, rising as pressure falls, and in high pressure cases diminishing. I have made few observations on this point, and have not read of any other series than this ; Dr. Moncorge himself confessed he had not data enough to speak confidently. If his postulate prove correct it would give us one point more in distinguishing hyperpiesia from neurasthenia with its heightened reflexes. I am under the impression that the knee-jerk may increase in the decrescent form of atherosclerosis.

Epistaxis, when it occurs early, is a very important symptom ; often the first suggestion to the physician of the hyperpiesis (renal or other) at work. It is not uncommon, but occurs in the minority of cases. It may indicate the propriety of venesection, then or at a later date. In such cases the retina should be carefully examined.

On such early signs as garrulity and tearfulness-due to loss of higher controls, as under alcohol-of repetition of old stories, due to loss of memory, and so forth, I need not dwell ; they are familiarly known, and signify the decrescent mode of arterial disorder.

Sleeplessness and torpor is well known as a common symptom of " arteriosclerosis" in all its modes. Whether by overpressure

1 Moncorge, Rev. méd. de Mt. Dore. The pages forwarded to me bore no date, but $I$ think I received them in 1902 (?) 
or underpressure, the reactions between blood and brain are in disorder. But the symptom, though frequent, is by no means general ; much must depend in each case on the manner and degree in which the local irrigation is altered. Some hyperpietics, many indeed, make no complaint about sleep; but in elderly men sleeplessness, especially in the early morning, may suggest cerebral atherosclerosis of the senile type. On the other hand, atherosclerotics of either kind may be beset by dispositions to torpor or drowsiness, apart from uræmia; too often not of a night, when drowsiness might be welcome, but in the day time, as at and after meals or during conversation. Dr. Frederick Taylor ${ }^{1}$ has drawn attention to these drowsy cases, and narrates two instances; one in a lady æt. 48, who had suffered for years from "drowsiness associated with no other morbid condition than high arterial tension," and who ultimately died of apoplexy. The other case was in a man of gross habit and high blood pressure ; he obtained some relief. Dr. Taylor discusses these cases, and, to explain them, suggests the hypothesis of some toxic influence unknown; he quotes some highly elaborate guesses of other writers. He points out correctly that the sleeplessness bears no direct relation to the high pressures, and on the whole thinks there is little support for a mere vascular hypothesis. I quite agree that in the lack of uniform sequence we perceive that other factors are at work also.

What is far worse than drowsiness, and especially characteristic of decrescent atherosclerosis, is a certain aggravation of wakefulness, a nocturnal restlessness, a fractiousness driving the sufferer from his bed to wander at large; to the sad vexation of his household. Moreover, this nocturnal excitement is not rarely associated with subdelirium or delusion, which makes matters far more trying for all concerned, and is too often a signal of dementia. This excited state, for some unknown reason, is in my experience more frequent in men than in women. In severe cases of hyperpiesia a heavy torpor or semicoma, often with delirium and Cheyne-Stokes breathing, may set in ; an alarming but not always a desperate phase. I have alluded more than once to a case of this feature of hyperpiesis without

1 Taylor, F., "Hunterian Lecture," Brit. Med. Journ., Oct. 19, 1912 ; and Practitioner, Dec. 1912. 
renal disease in which recovery was complete (p. 407); in another such case, also with periods of Cheyne-Stokes respiration, repeated attacks with these symptoms were witnessed again and again, until the patient at last died of apoplexy. In none of the preceding attacks did any paresis appear, and the indications seemed not to be of hæmorrhages but of phases of excessive blood pressure lasting a few hours at a time.

Certain paræsthetic symptoms and discomforts of arteriosclerosis, more especially of the decrescent kind, have been attributed to sclerosis of the vasa nervorum; a guess of course, but not an unlikely one.

Abdominal System.-The abdominal symptoms of hyperpiesia are few and equivocal. Flatulency is a frequent symptom in all kinds of dyspepsia, or of venous congestion of the viscera; and in cases of gluttony it is not wanting. Still it would appear to be at least as troublesome in decrescent arteriosclerosis. In gross feeders we may observe the formation of a "bow-window" which, with the heaping up of fat about the ribs and buttocks, especially observable in races originally bred in hardship, seems to be a reservoir against the empty pot; but when the pot is never empty this superfluity may become not only a nuisance, but a perilous nuisance. The fat may encroach upon the heart, or may suggest a cardio-arterial strain. The abdominal muscles slacken, aggravating the condition, and hampering the respiration. Bourguignan, of Evian, a pupil of Huchard, has observed that when under efficient treatment an arteriosclerotic patient improves in health, if no loss of weight is observed, or even desired, the girth of the abdomen is often much reduced, as may be seen by the looseness of the trouserband. But if a patient of gross habit, independently of treatment to this end, begins to emaciate, the sign is an unfavourable one, and an eye must be kept on the urine, for sugar or granular casts. I may repeat that while obese persons may be, and often are, quite free from excess of blood pressures, on the other hand, many subjects of excessive blood pressure are not obese, and may be of spare build. Thus while in the "abdominal plethora" of the older physicians, in the pursy, puffy condition of persons addicted to high living, some of them pallid, some rubicund, the bowels get sluggish, windy 
and colicky, especially about the ascending and transverse colon, with pains around the navel, and while in some of them the liver is fullish and rather tender, and the rectum hot and subject to piles, in others we see a mere insufficiency; poor nutrition, failing strength and digestion. But these patients also are subject to irregular glycosuria, and, like the "podgier" subjects may also complain of "weak heart," insomnia, weariness, tinglings in the limbs, fidgets, and so on. In some of these cases, as we shall see, hæmorrhages from the stomach or bowels may occur, without hepatic cirrhosis.

If we have heard from various writers, mostly continental, of certain clinical curiosities, phenomena so curious as to require names of much splendour, "Dyspragia intermittens angiosclerotica" for example-_" prave 'orts" indeed-we may anticipate that, in entering upon abdominal arteriosclerosis, we shall meet with the most dramatic, if not the direst, manifestations of all this sphere of disease. The victim of this magnificent malady is seized with abdominal crises comparable only with those of tabes, or of mesenteric thrombosis. He cries out of a burning pain and cramp in his belly, of distension, belchings and dyspnea, such that in more than one of these cases an exploratory operation has been performed. Usually there is obstinate constipation, but sometimes the patient is torn by a watery diarrhœea. As an example of the milder variety of the disorder, I may quote the following case recorded by one of Huchard's school as "Presclerose gastro-intestinale" :

M., æt. 35-40. Pain and tenderness in epigastrium and abdomen, worse two or three hours after meals; eructations and nausea; bad taste in mouth, and loss of appetite; vertigo and insomnia. Pulse hard, and occasionally intermittent. Accessible arteries thick, second aortic sound intensified ("hypersclerosis"). All the symptoms cleared up on theobromine, adonis vernalis, and valerian!

Barié publishes some of these equivocal cases : and it is likely enough that the atheromatous disease often discovered in them after death about the abdominal aorta and coliac axis may have contributed to the discomfort. Certainly discomfort and even distress may arise in case of extension of disease to the investments of the great vessels, with tension of these parts (see Angina Pectoris, pp. 462-3). However, although for some 
forty years I have been specially conversant with arterio-sclerotic patients, and the number of such persons seen almost day by day by English physicians is very great, yet I fail to recognise any consistent or uniform malady answering to these descriptions. Perhaps it is not the way in which the English abdomen behaves itself, but is it not as likely that many of these cases, surely multiform, have been misinterpreted? Constipation and wind are consequences of the neglect of the colon which are common enough in elderly persons, and no doubt as the activity and nutrition of the bowel become impaired these disturbances are more prone to arise. Dr. Stengel admits that one of these patients was cured by a dose of calomel. On the other hand, I have seen, we all have seen, many and many a necropsy in which extreme degeneration of the descending aorta and axis, and the gastric and mesenteric trunks had resulted in no such extravagances. My experience of advanced arteriosclerosis of the gastric vessels is that it is usually attended with the negative symptoms of loss of appetite and flesh, debility, and hypochlorhydria; symptoms which not infrequently lead to suspicion or even to diagnosis of carcinoma.

However, Ortner's papers on this subject ${ }^{1}$ have not received the attention they deserve for, if overlaboured and discursive, and, as he honestly admits, not very conclusive, he has spared no pains in the setting forth. The author is naturally influenced by the vasospasmodic hypotheses of his able colleague, Pal. Dr. Stengel, at the Toronto Meeting of the British medical Association, drew attention to Ortner's paper, and to a paper of Max Buch's, which I have not seen. ${ }^{2}$ He reported also cases of his own. Max Buch collected only 25 cases, and in these he included instances of Angina abdominalis, which Ortner rightly discarded. We are aware that in cases of atheroma of the abdominal aorta this vessel is often to be palpated, and may be tender to the touch; so much so, as to suggest tumour; this is not uncommon; but I have not observed in these cases the kind of "abdominal crises," with grave and agonising pains recorded by some authors.

1 Ortner, N., "Angiosklerose d. Darmarterien," Volkmann's Sammlung, N.F. No. 347, 1903 ; and Wien. kl. Wochenschr. vol. xv. p. 45.

${ }^{2}$ Quoted by Stengel from Progressive Medicine, Dec. 1905. 
If we assume "Claudicatio intermittens angiosclerotica abdominalis " (!), as a paretic condition, may be due to spasmodic closures of vessels, we have no evidence that a mere angiospastic anæmia, here or anywhere else, can engender violent pains. In the chapter on Angina Pectoris I shall urge that visceral pain depends on tension, on tension of the mesentery, for instance; or of an inflamed capsule, as of the liver. In an able thesis on Embolism and Thrombosis of the Abdominal vessels, maintained for our M.D. degree in 1912, Dr. Trotter adduced further evidence that such is the explanation of the agonising pains observed in certain cases, pains due to a sudden and intense distal congestion of the area (see A. Pectoris, pp. 462-3). We may assume for the present that symptoms of intestinal atony and inactivity may well be attributed to the anæmia of narrow or impervious vessels, but that severe pain, if vascular in origin, is probably due to emboli or thromboses with their congestive sequences, or enterospasm, pulling on the mesentery. Rosengart, ${ }^{1}$ in cases of colic, belching, distension, insomnia, fatigue and tingling in the limbs, symptoms not improbably attributable, at any rate in part, to sclerosis of the abdominal vessels, adds that in these he has noticed also a very fluctuating pulse, a great difference between lying and standing; and also a dilated heart, and even murmurs. These groups of symptoms remind us of those only too frequent in ordinary valvular heart disease, independent of arteriosclerosis ; it is probable that they are characteristic of any kind of circulatory disturbance affecting the function of the intestines; in cardiac disease they ensue upon venous congestion of the stomach and lower tract. Among them also are the cases we know so well, and yet so ill, which, for the present, we have to be content to call " visceral gout."

Upon local atrophies ensuing upon vascular occlusion, grave consequences may ensue, and it is rather to this mechanical decay that I would for the present attribute some of the more exorbitant symptoms to which I have alluded. We know that the arteria epiploica is liable to sclerosis and, in a certain case, which Sir Thomas Barlow will well remember, an elderly man was seized suddenly by severe pain in the left side, and died in a few hours. At the autopsy a rent was found in the stomach

1 Rosengart, Münch. med. Wochenschr., 1906, No. 20. 
near the junction of the cavity with the œsophagus; the tissue of the part had been atrophied by advanced sclerosis of the gastric system of arteries, a part of a far advanced general arteriosclerosis. Now this patient had suffered, for a year or two before, from the loss of appetite and flesh and general debility with achlorhydria suggestive of malignant disease of the stomach which I have mentioned (p. 446); but no such disease was found.

Hamorrhage from the stomach or intestines due to arterial decay in elderly persons is not a very frequent event; but cases are on record of hæmatemesis from rupture of decayed gastric coronary arteries. The region of the coronary artery is the principal source (see p. 457). Dr. Ainslie Hollis published and discussed a short series of cases of ulceration of the stomach and oesophagus in which such breaches corresponded with large "plaques" at the orifices of arterial branches going thither. Dr. Hollis however is very cautious in ascribing these ulcers, in some cases probably malignant, to the arterial disease. Still in less equivocal instances there is some evidence of such cause and effect. Bitot and Papin ${ }^{1}$ describe two cases of hæmatemesis due to gastric arteriosclerosis; in both, as the hæmorrhage was repeated, an operation was performed, but nothing else was discovered. In the second case gastroscopic examination revealed dilated and sinuous vessels, and near the altered vessels were small ulcerations which, on later examination, were found cicatrised. Bitot and Mauriac, ${ }^{2}$ referring to these two cases, publish a third, with necropsy:

Male, æt. 36. Syphilis, æt. 30. Five years later epigastric pain and hæmatemesis (ulcer suspected). P.M. General arteriosclerosis of vessels of stomach and neighbouring duodenum, which were tortuous and thrombosed. Marks of rupture were detected in places. Histologically the change was atherosclerotic (intimal). No ulcer, no miliary aneurysms.

The recorded cases of hæmatemesis in arteriosclerosis show that ulceration of the mucous membrane may accompany the vascular changes, or that blood may issue from a non-ulcerated

1 Bitot et Papin, Gaz. hebdom. des sci. méd. de Bordeaux, July 21, 1912 ; I have not looked up the original paper as it seems well reported in the Lancet, Oct. 12, 1912.

Bitot et Mauriac, Gaz. des hôp., May 13, 1913. This paper I have seen. 
surface. In this state an arteriole may present an aneurysmal dilatation; or again no such sign may be apparent. The recorded cases are for the most part in aged and obviously arteriosclerotic persons; but Hirschfeld reported a case of a man, who had suffered from hæmatemesis from the age of 18 , and died in an attack at the age of 38 , after death in whom well-marked sclerosis of the gastric arteries was found; one of them showed a small aneurysmal dilatation. Other cases on record are quoted; such as by Gallard (in 1876, but no ref.), a gastric ulcer due to rupture of a miliary aneurysm on a sclerosed coronary artery; and by Sachs ${ }^{1}$ in a man, æt. 79 , with the same condition. In none of the cases of this kind was there cirrhosis of the liver. The district of the lesser curvature seems to be most liable to this form of decay, especially, as in Barlow's case, the a. gastro-epiploica. It is remarkable that in most of the instances of the violent attacks to which I have referred, suggestions (unverified) of excessive blood pressure are made; yet surely advanced abdominal atherosclerosis would be most common in the senile (decrescent) form.

The evidence then that cramps and spasms arise in the ordinary course of senile arteriosclerosis of the bowels is slender, and by no means adequate to carry a concept so formidable, and a name so imposing. Even the alleged cases are few; and for the present it is wise to hold them in suspense, and to remember that for these few some more probable explanation may be found, such as flatulent indigestion, enterospasm, or small embolisms or thromboses such as commonly occur in the diseased arteries of the brain. Watery diarrhea is consistent with such small pluggings. Besides, enterospasm, in no connection whatever with arteriosclerosis, is no infrequent malady. Meanwhile, we shall do well not to pile speculation upon speculation by guessing at vasomotor spasms or affections of the cœliac axis of which we know nothing, and to avoid the addition of more and more enormous names to our already loaded categories.

The vessels of the pancreas are far more liable to decay than those of the intestine, and we are perhaps insufficiently alive to the consequences. A whitening of the stools, which may float in 1 Sachs, Deutsche med. Wochenschr., May 19, 1892.

voL. I 
water, or variable traces of sugar in the urine, may betray vascular decay in this area and, with it, probably an incursion of peri-arterial and other fibrous hyperplasia. ${ }^{1}$ Ottfried Müller says that in these cases the glycosuria is the result, not the cause, of the vascular failure; this is not unlikely, but the converse is often true also; in diabetics still young, even in children, without any rise of pressures, the accessible arteries not infrequently thicken (p. 279). Dr. Chalmers Watson ${ }^{2}$ states that in arteriosclerosis an examination of the urine and stools, on Cammidge's method, is often positive; this may point to sclerosis of the arteries of the pancreas. My experience in this respect agrees with that of Dr. Chalmers; I have seen these signs in one case, apparently verified by necropsy. But of course absence of sugar from the urine does not exclude "cirrhosis" of the pancreas. Dr. Bosanquet, in a paper which I have mislaid, stated, if I remember right, that he examined 100 cases of pancreatic disease, and in a considerable proportion found cirrhotic changes in the organ without a symptom of glycosuria. Of the relation of the islands of Langerhans to these conditions we have no definite knowledge.

An arteriosclerosis of the portal system, beyond the portal vein itself, is unknown to me. The vessels of the liver, for some reason, bathed in amino acids as they may be, seem little subject to sclerosis (Jores and others), and mechanical stress in them may be reduced by the interposition of the portal minor circulation. However a portal sclerosis has been described by Simmonds, ${ }^{3}$ which does not seem to be due to high arterial pressure or liver block; indeed he regards it as independent, more or less, of general arteriosclerosis. The process is intimal, with ruptures of the elastica, some secondary affection of the media, and a tendency to calcification. Simmonds attributes it to some toxic influence, from the liver (or bowel ?). It may lead to more or less extensive thrombosis, and even to varices in the stomach, and hæmatemesis. Of course this condition is not to be confused with syphilis.

In general arteriosclerosis, if a section of the liver be carefully

1 See Lepine, J., Lyon méd., Oct. 25, 1903; quoted Brit. Med. Journ., same year.

${ }^{2}$ Watson, C., Brit. Med. Journ., April 11, 1908.

3 Simmonds, Virch. Arch., March 1912. 
stained with nitrate of silver a hyperplasia of connective tissue may be perceived as a very fine reticular network; and the whole organ, though far from the density of cirrhosis proper, is harder to the touch. Furthermore, a definite cirrhosis of the liver, as a consequence of general arteriosclerosis, is described by some French authors but, so far as my information goes, without adequate proof. Some English writers have accepted this statement, but, as they explain it by an assumption of highpressure arteriosclerosis as a result of alcohol, their cases fall into the class of ordinary alcoholic cirrhosis, a disease which, kidney disease apart, we know not to be directly associated either with high pressures or with arteriosclerosis (p. 246).

I may take this occasion to refer to a malady attended with high arterial pressure from which however, in its initial stages, arteriosclerosis is probably absent. This is a kind of paroxysmal vomiting like that of migraine, but in which there is no headache, nor visual disorder. Such a case, in a lady well known to me, was most carefully and ably studied during many years by Dr. Holmes of Woburn Sands. At uncertain intervals attacks of vomiting, as severe as sea-sickness, would set in, lasting for one or two days or longer. They left after them a great prostration, so that when the interval happened to be short the patient was much reduced. They were more frequent in cold weather. Dr. Holmes always found that the blood pressures, which were permanently rather high, before a seizure became enormous; and then occasionally a little albumin would appear in the urine. These phenomena were both objectively and subjectively mitigated by sodium nitrite. This lady was subject to the attacks for many years, and in middle and later life the radial and other accessible vessels became very thick and tortuous, and the heart enlarged. Dr. Holmes was quite sure that these increases of the high pressures were due not to the irritation of the seizures but distinctly preceded them. After them they fell not indeed to normal, but to a more moderate figure. Her father was rather gouty, had migraine, and died of granular kidney. Both she herself and her father were always most abstemious persons, indeed her own asceticism seemed almost excessive; but any enrichment of the diet led to rise of pressures, and sooner or later to a vomiting attack. In 
recent years we have learned in such cases to suspect degrees of " acidosis."

The renal conditions, negative and positive, in arteriosclerosis are so important that they are discussed in a separate chapter (Chap. V. p. 309). In hyperpiesia, as contrasted with renal disease, the diurnal standard of the specific gravity of the lithatic urine, except during polyuric periods, is normal ; in later stages it may contain a little albumin, but casts are absent or rare. In the decrescent disease the urine is rather lighter.

Genitalia.-Of the uterine symptoms of arteriosclerosis I have little to say. It is stated that climacteric hæmorrhages may be due to this general change, or be associated with a normal vascular decay confined to the uterus itself. Uterine hæmorrhage due only to arterial disease in the organ has occasionally given rise to grave fears of cancer. Dr. Leonard Williams has written on this part of the subject. ${ }^{1}$ On one point only I will dwell for a moment; namely, that in certain cases of tendency to hyperpiesia, which is especially prone to occur about the menopause, a free loss of blood about these years may not be without benefit; from ancient times a similar advantage has been attributed to moderately bleeding piles. Dr. Williams says that personal observations of his own have shown a rise of blood pressure before the menstrual periods, and a fall after them; the subsidence beginning during the flow. The upper range may be about $140 \mathrm{~mm}$.

Episodic Hyperpiesia in the Elderly.-More than once I have remarked upon intercurrent transient phases of high pressures under more than one set of conditions; it is probable that we are all, or many of us, liable to transient periods of excessive pressures; but in this paragraph I wish to insist upon occasional phases of hyperpiesia in elderly persons, and to emphasise them, because in these states they may lead to an unbalanced prognosis ; moreover in them they are attended with no little immediate peril. For many years I have recognised these intercurrent phases, and learned to make allowance for them. An interesting example of this episode appeared in a very

1 Williams, L., Clinical Journal, March 3, 1909. 
comely old lady, a patient of Mr. Maund of Newmarket. Her age, if I remember right, was about 80 ; and wonderfully upright and comely she was, vigorous in mind and body and the animating head of a large family circle. Accustomed to a generous way of living, she had never found reason to deny herself what the gods gave her; if she desired a glass of champagne for her lunch it was there, and so on. Then at length she fell out of sorts; we found her with a coated tongue, depressed in spirits, unequal to her many occupations, and so on. Her pulse betrayed at once an unquestionably high blood pressure. We had no gauge at hand, but it must have approached $200 \mathrm{~mm}$. She was put under a course of calomel and salines, and a stricter dietary enjoined; with the result that in two or three weeks she was herself again. Some time later I heard that with caution in diet she had remained in good health. In this lady the radial artery was of ordinary amplitude; in another old lady, a patient of my late friend Mr. Hartley of Stortford, the same story of recent rise of pressure was given; but the radial was so constricted that I feared chronic renal disease. However, on a system of treatment adapted simply to hyperpiesia, she made a good and quick recovery; and, as a friend of her family, I knew that the improvement was abiding, and that she lived to a still riper old age. But we are not always so fortunate. In a third old lady, whom I used to see with Dr. Malden of Cambridge, such an episode of high pressure set in; this on the first occasion vanished, as we expected, under appropriate treatment. However, some time later another such attack supervened, with high arterial pressures and some slight anginiform symptoms. Again this attack also was dissipated, but, before we had time to mitigate the arterial tension, the aortic valve was forced, and a permanent aortic insufficiency established. It was interesting to see how at an age not far from fourscore the left ventricle quickly and largely hypertrophied, so that a new balance was attained. And for a few years more this lady continued in fair health and spirits, until at length she succumbed to a heavy weight of years and the aortic insufficiency. Such episodes are seen in both sexes alike.

The following notes of intercurrent high pressures was sent 
to me by a physician who had read some observations of mine on episodic high pressures in old persons :

Mrs. R., æt. over fourscore, a thin, active, vivacious, and usually a sweet old lady. But she had latterly become subject to outbreaks of peevishness, sullenness, and even bad temper, which would last for two or three days. These changes were associated with a day somnolency. Now at these times my correspondent had found that her pulse rose in pressure, and became firm and incompressible. Blue pill gave her prompt relief; alcohol made her worse. After an alterative course the arterial pressures would fall, with restoration to her normal self.

The distinguishing feature of these episodical cases in elderly persons is the absence of preceding cardiac enlargement, and not infrequently of accessible arteriosclerosis. If however decrescent arteriosclerosis be present, until the pressures are abated there may be no little anxiety lest the heart or a cerebral artery be forced. 


\section{CHAPTER VII}

\section{MORBID ANATOMY OF ARTERIOSCLEROSIS}

AT Toronto, in 1906, Arteriosclerosis was discussed in the Sections both of Medicine and of Pathology. As in the Medical Sections I had endeavoured to convince my hearers that Arteriosclerosis is not a Disease, but an issue and detriment more or less common to several diseases (pp. 13 and 374), so in the Pathological Section I expressed a suspicion that the pathological phenomena might prove to be not uniform, as the unqualified use of the name commonly implies, but twofold or manifold. That each specific cause would prove to have its own specific mode of arterial tissue reaction I scarcely supposed; it is improbable by the structure of it that arterial tissue should be capable of much variety of decay; an identical or closely similar deformation may in a particular structure be the common issue of several kinds of distemper (vide p. 324); yet from certain broad differences between the causes-between those in the main mechanical, and those which in the main are toxic, or "senile" (decrescent)—we might anticipate some various results in arterial degradation. ${ }^{1}$ For even if the decay I have called decrescent, involutionary or primary, be indeed due mainly to long accumulation of mechanical stresses, yet it might prove to have certain anatomical features, or some mode of distribution corresponding to the slow, intimate, and belated mode of its causation, to distinguish it either in character or in seat from the earlier, more incidental, and more violent strains of high tension. And again, should the decrescent kind prove to be engendered of autotoxic influences, whether chronic or more virulent and

1 See a paper by myself, Brit. Med. Journ., 1911. 
untimely, it might present certain characters different from those due simply to excessive tensile strain.

Order of Lesion.-Morbid anatomists, from the time of Rokitansky, have endeavoured to formulate some principle or rule of the order or orders in which arteriosclerosis invades the body, but as yet no principle has emerged. Its incidence may be curiously partial ; for instance, the cerebral arteries may be affected with little or no change elsewhere, and so on. The following case illustrates unequal distribution of arteriosclerosis :

I saw, with a medical friend, a middle-aged man in whom the right radial was almost obliterated by atheroma ; the left, though thickish, being fairly normal. The blood pressure was not above normal. The brachials did not show a like difference. The heart was not notably altered, if at all. The blood pressures taken in the two arms (Martin) were not unequal. He was a professional man of some kind; not a labourer nor an athlete.

The many published scales of frequency, in respect of sites, fail even in a general resemblance. The order may not be the same for hyperpiesis, for granular kidney, for the decrescent forms, and so on. High pressures seem to tell on the aorta first, and thence on the arteries of the larger magnitudes, as the stresses tell along the tree; but we do not know that this is the case; the stresses upon the peripheral vessels must depend upon vasomotor tides, general and partial. But, as I have said before, between sclerosis of main trunks and of peripheral branches there is no consistency; a remarkable conclusion pointing to large variables in causation. In renal disease, as I have already suggested, there seem to be two movements of arteriosclerosis (p. 351); the one intimate in the kidney, and pertaining to the nature of the disease, the other simply mechanical, a consequence of the high pressures. And under certain other conditions also the peripheral vessels may be widely affected while the larger are spared; often again the stem of the main vessel of a viscussuch as the coronary-may stand stiffly open while its radicals are still intact.

On the order of liability Rokitansky and Lobstein did agree, it is true ; and on this order : (1) the ascending aorta and arch; (2) the descending aorta ; (3) the splenic artery, and the crurals; (4) the coronaries; (5) the internal carotids and the vertebrals 
within the skull; (6) the uterine; (7) the brachial; (8) the spermatic; (9) the common carotids; (10) the hypogastric ; (11) the coronaries of the stomach ("very rarely"); (12) the mesenteric. The order of the mesenteric arteries, with which Dr. Mott and von Romberg agree, is notable (p. 188). On the order of the mesenteric von Romberg made a special investigation and found sclerosis in this area "sehr selten"; as also did W. C. Hodges (M.D. Thesis, Camb., 1914). (See also p. 218.) In my experience the gastric coronaries would come higher in the scale of frequency. It is important to remember that the gastric arteries may suffer fairly soon, and independently of the abdominal aorta (Potain, Teissier, Pal, Hamburger). This event, and the obscure symptoms of it, such as debility with defect of hydrochloric acid, and in one case perforation of the necrosing stomach into the pleural cavity, I have already considered (p. 448).

Dr Mott's order of decay, of all kinds of arteriosclerosis indiscriminately, is :-arteries of limbs, aorta, splenic, iliac, femoral, coronary, cerebral, uterine, brachial, gastric-mesenteric sclerosis again being rare: the limbs, that is, suffer first; of these the lower limbs before the upper, the anterior tibial being the first of all arteries to yield. His cases, collected at Claybury, chiefly consist no doubt of decrescent arteriosclerosis (p. 351). Comparative maps of the distribution of decrescent and highpressure sclerosis are much needed. The order founded upon the largest pile of unsorted material is that of Thoma, by his pupil Bergmann; it is this:-ulnaris (94 per cent), tibial. ant. (93 per cent), subclavian (88 per cent), cerebral (87 per cent), carotid int. (87 per cent), radial (86 per cent), splenic (82 per cent), popliteal (79 per cent), axillary (71 per cent), femoral (87 per cent), common carotid (68 per cent), aorta asc. (67 per cent), a. abd. (64 per cent), iliac ext. (58 per cent), brachial (55 per cent). ${ }^{1}$

As determinants of site Dr. Newton Pitt ${ }^{2}$ suggests:-difference of structure; different conditions of work; selective affinities of toxins, and finally development, on the lines of the several aortic

1 See also Harlow Brooks, New York Med. Journ., 1906, and Amer. Journ. Med. Sci., 1906. I have seen the latter paper only.

2 Pitt, N., Brit. Med. Journ., Oct. 10, 1908. 
arches. In syphilis the thoracic aorta, especially the ascending arch, suffer first; in atheroma the root of the aorta just above the sinuses and at the points of strain of the cusps: but the subdiaphragmatic part of the vessel, a part often left unopened, may suffer early. Some inferential notion of the liability of the cerebral arteries is very important for the physician, but unfortunately the incidence upon them is very irregular. Sometimes they are affected very soon, even before other vessels of other areas; sometimes, while elsewhere it is conspicuous, they show little or nothing of the disease. But as no order of liability can be accepted which is not verified by microscopical examinations, the earlier scales are far less trustworthy. A very frequent area of atheroma is the pelvic, where the sclerosis is attributed to the incessant disturbance of the vessels by the intestinal activity. If so, these arteries may be put side by side with the coronary and those of the arms and legs. Mönckeberg in 130 cases found pure medial calcification in the limb arteries (vide p. 482), with or without central atheroma; in 57 central atheroma without the peripheral form. Thus from the vessels of the arms and legs, without clinical diagnosis, we can draw no inference as to the internal vessels.

As in my paragraphs on the physics of circulatory disorder I have premised, so in respect of these on Morbid Anatomy, I do not pretend to occupy the reader with a systematic report. On the clinical side my opinions are at least first hand, the fruit of my own observation and reason; on the anatomical side I am more dependent upon the work of others. Still, before entering upon diseases of arteries, some preliminary reflections upon the normal vessel may be permitted. The structure of the normal artery is a very interesting piece of mechanical efficiency. From it the makers of other tubes, built to resist internal stresses, might have taken instruction. A big gun is built like an artery, coat upon coat, in part spiral ; and makers of all gun barrels are well aware that the metal must not be homogeneous or uniform, but twisted and fibrous. "Pure metal" is too plastic to resist extension; a compound substance, by the internal friction of its dissimilar particles, offers increase of resistance, as sandy mortar is more tenacious than so much homogeneous lime. Thus the contemplative biologist may admire this wonderful tubular 
structure which day by day, often for fourscore years or more, withstands the ninety thousand daily blows of the beating heart.

One of the qualities to which we attribute great importance in arterial function is elasticity; but we are apt to confuse elasticity with resiliency (see p. 40). The artery possesses both qualities ; no little elasticity, but far more resiliency. Elasticity is that quality by which a body resists deformation and recovers from it, and may be illustrated by the ivory balls of the billiard table ; resiliency, or lability, is the quality by which on deformation a body recovers its form, and may be illustrated by the rubber cushions. The ball is more elastic, the cushion is not brittle but springy. Like these cushions, with age, say in the aorta after æt. 60 (Herringham and Wills), the artery undergoes a slow molecular alteration. Asby frequent stretching a rubber band loses elasticity and, if stretched beyond certain limits, recovers its original form less and less entirely, so an artery, after a certain sum of tensions, recovers its proper diameters less and less entirely; especially if at times it has been extended beyond the degree known as its elastic limit, a degree which in most materials lies about halfway to breaking stress. ${ }^{1}$ How by this extensibility and resiliency (lability) energy is taken up by the vessel on its diastole, and returned to the blood on its systole, we have fully considered. Nor need I dwell again on the inverse aspect of the loss of storage as the artery loses resiliency; nor on the consequent loss of the moulding of an undulating into a continuous stream.

We have seen that to postulate a standard diameter for particular arterial sections, or for those of their several coats, has been found impracticable. The dead and the live artery, the full and the empty, differ largely and probably essentially, though on the other hand it is not easy to say when an artery is dead; as the median plain muscle will live and contract after somatic death this difficulty is increased. By contraction the intima and the elastica are thrown into folds, and the media thickens. The waviness of the elastica, therefore,

1 Continual reiteration of tensile stresses, none of which exceeds or even closely approaches strain, may nevertheless ultimately effect more or less disintegration ("fatigue "). But, important as it is for arterial pathology, this problem is not readily solved even for inorganic materials; and in life, when molecular renewals are continual, such calculations could only be made broadly and in the abstract. 
is some rough guide to the position of a vessel in tone, or in hypertrophy. But much fallacy lies in the too common use of the term "hypertrophy" in the loose sense of bigger. Professor MacWilliam found that 20 per cent plus diameter over the empty state flattens out the waviness, but beyond this degree measurement is guess-work; though an expert eye for its own guidance, not forgetting the remarkable differences due to the mode of death, between, let us say, the healthy arteries of an amputation for accident, and those from a case of death from an exhausting disease, may come to some approximate judgment. But arteries from various individuals differ a good deal. In his well-known studies of arterial qualities MacWilliam ${ }^{1}$ has demonstrated how vastly more is the play of distensibility and elongation in a relaxed than in a constricted artery, the pulsatile expansion being much larger. Thus to affect a contracted artery the blood pressure must be very high. This is indeed what we should expect, for in a constricted artery the limits of its elasticity are perhaps not nearly approached; in this position, as we have seen, equal rises of unit pressure are not followed by equal increments of cubic capacity. Indeed the differences of distension values between vessels in mean tone and in contraction are out of all reckoning. By Dr. MacWilliam's illustrations we all comprehend how in some cases, as we may observe directly in peripheral arteries which for an indefinitely long period of time have been held tightly in constriction, these vessels may present little or even no evidence of deterioration (see p. 218); although for no less a period the hydraulic pressures in the more central vessels may have had an excessive range. Such resistance to injurious stresses is often conspicuous in the radial arteries in cases of renal disease when, under these strains, the aorta, and other vessels of the larger magnitudes and less muscular structure, suffer injury, while the constricted muscular arteries of the lower magnitudes are comparatively safe. And so likewise in health, as vagus and accelerators play mutually upon the thoracic aorta and heart, tone is continuously acting as a damper to moderate the effects of incessant percussion, dilatation, and extension (Vol. II., p. 290).

1 Proc. Roy. Soc. vol. Ixx., 1902, and later papers; e.g. MacWilliam and Mackie, Brit. Med. Journ., Nov. 14, 1908, p. 1477. 
Dr. Fleming ${ }^{1}$ has suggested that the elongation of an artery may not be a physical process, or not merely such, but a vital one, a hypertrophy of length; that to meet more demand the vessel "grows" longer. Experiment showed, he said, that a certain india-rubber tube submitted to internal pressures up to $400 \mathrm{~mm}$. Hg did not elongate. Dr. Harry Campbell leans to this opinion, which at least is interesting. Yet, in so far as tortuosity may be a sign of elongation, we observe that in clinical experience it is not the arteries more active in tone or vitality which become tortuous, but those rather which are losing vitality, as in old age. If Dr. Fleming in his experiments will force the circulation through a senile rubber tube lying between relatively fixed points he will find it begin to sag. Thus under high blood pressures in the younger arteries tortuosity appears much later than in the vessels of older persons in whom the pressures may have been excessive only in proportion to the failing resiliency of the tunics. Besides, this growth explanation does not seem to be applicable to dilatation of the aorta. Tortuosity is of course a result of elongation within fixed attachments, whether physical or biological; by as much as an artery contracts when severed by so much at least can it extend itself normally when submitted to internal fluid pressure. ${ }^{2}$ Moreover, as I have said elsewhere (p. 195), if a fairly long and quite healthy artery, as of a limb, be bared, a serpentine shift of attitude can be seen by the naked eye. As a tube losing its resilience in both axes has to lie nevertheless between the same relatively fixed points, it must on elongation travel out of its bed; and if the blood pressure be very high it may tug on the fixed points to degrees approaching or surpassing the limits of normal resilience. To turn to what is perhaps a more instructive experiment, I find that metallic wires (which are easier to gauge than rubber) by flexion elongate more than by an equivalent simple traction; and this follows sooner in wires which prove microscopically to be less homogeneous. And as on arteries lateral pressures tell more than longitudinal, constriction of the spiral fibres, so long as these are healthy, by closing the spiral, shortens the tube, and prevents tortuosity (p. 196); for by so much are the

1 Fleming, Brit. Med. Journ., Feb. 17, 1906.

2 Ballance and Edmunds; see also Campbell, H., Lancet, Jan. 15, 1910. 
elastic and connective fibres slacker and safer. And of course the smaller the radius the less the bursting pressure. So it is that the vessel defends itself against these internal pressures. If tortuosity occurs in a tube of considerable diameter it will aggravate itself by the consequent increase of vortices, and so of internal friction. At any rate, as I have urged in former papers, the current opinion that, whatever the attitude of tone, in arteriosclerosis tortuosity is more or less directly an excess of systemic pressures is certainly incorrect; and this opinion Mitchell Bruce, James Barr, and Harry Campbell have accepted. Dr. Ewart 1 explicitly agrees that tortuosity is no criterion of excessive blood pressure ; he "had often seen it in hypotensive persons" (see p. 192). For a deteriorating tube normal or even slightly subnormal pressures become relatively excessive; and every day we see grotesque tortuosities in the arteries of old persons in whom the blood pressures had never exceeded at most the quasi-normal standards of later life. However these remarks are incidental and anticipatory. Meanwhile, as H. W. Cook has put it ${ }^{2}$ concerning the interaction of these conditions, a man with fair arteries under a pressure of $200 \mathrm{~mm}$. may be worse off by far than a man with well-marked sclerosed vessels under a pressure of 140 . This is a cardinal maxim on which $I$ have long insisted, and shall have more to say later.

The Arterial Coats.-Now, concerning these severally, we must not carry to any sharpness, certainly not in the aorta, the three divisions which we accept for ordinary convenience; namely, the tunica intima, the media, and the adventitia. They may be distinguishable less by the kind than by the directions of their fibres. Developmentally I understand-and from development we receive many a valuable suggestion - the artery consists of two tubes-the intima or vessel proper, and its supporting investments. If then for ordinary discussion we find it convenient to make the three divisions, we shall be led into misconceptions if we forget that these coats have very close mutual relations ; moreover, as we pass from the anatomy of the aorta to the channels of the peripheral network, they change in structure and relation. For instance, arteries exposed to flexions, or outward stresses, such as those of the limbs, or the facial, have an

1 Ewart, W., Brit. Med. Journ., Feb. 15, 1908.

2 Cook, W. H., American Medicine, 1905. 
external coat relatively thicker than, let us say, a vessel so protected as the abdominal aorta. Moreover, we have seen that the same vessel at points of stress is more padded; at bifurcations for instance, or where impact is more strenuous, as on the upper wall of the thoracic aorta, which is thicker than the floor of it. The media also is not only more definitely muscular in arteries of the third and lower magnitudes than in those of largest diameter, but in certain of the smaller vessels, such as the renal, it is more muscular and less rich in elastic fibre than in some others of comparable size, such as the radial; facts which point to differences of function. Furthermore, even in the same section, elastic and muscular fibres are more interpenetrating - per mutua nexce-than is generally supposed. The connective tissue scaffolding--from adolescence at any rate-is not subendothelial only, but all-pervading. Dr. Herringham describes the interstitial network which carries the muscular cells in a renal vessel as between the more inward strands just visible, ${ }^{1}$ and so normally fine muscular fibres may be traced. Longitudinal muscular fibres on the outward aspect Drs. Andrewes and J. F. Gaskell have demonstrated very clearly. Yet "intima" means more than many of us fully recognise. The intima is strictly that layer which lies within the inner elastic layer, as the media is that which lies between the two elastics; but as certain morbid changes in the inner elasticlayer are of cardinal importance in disease of the intima the inner elastic layers must in pathology be considered with the intima. In respect of the vasa vasorum again, as we come to minute arteries these subsidiary vessels disappear, and the twigs are, partially at any rate, nourished only from the investing lymphatic spaces and the blood stream. Thus it is that in certain forms of disease the medial fibres nearest the intima decay first; and that fibrosis occupies the same relative position. In the arterioles, in the intima of which practically little connective tissue is undeveloped into elastic fibre, we shall see clearly a conspicuous morbid change seated in the elastic layer(Jores, Marchand, Herringham and others). And in the interpretation of disease these anatomical variations are of considerable importance.

Of capillary disease, strictly speaking, it is hard to be so sure, except in respect of damaged epithelium which has another kind ${ }^{1}$ Herringham, W. P., Path. Soc., Iondon, 1901. 
of importance. That where obliterative disease attacks an arterial branch the dependent twigs must dwindle, or silt up, is of course obvious, and in this sense it was that George Balfour spoke of the obliteration of capillary areas; but of primary or peculiar capillary disease we know little. Indeed it is now asserted that in some extensive visceral areas capillaries are but perforating channels, having no proper structure. It is well known that Gull and Sutton alleged an extension of certain fibrillary or hyaline perversions and nuclear proliferations to the capillaries, and a few observers with hesitation followed them; but histological methods were not then what they are now, and for the present we must pass the matter by as undecided. In this area of the circulation the ruling influence is the cell activities.

Vasa vasorum.-In respect of the larger arteries supplied with them there ought perhaps to be a good deal to say about these vessels; that arterial health must in no inconsiderable measure be dependent upon these auxiliaries seems unquestionable. The late Hippolite Martin ${ }^{1}$ laid great, probably too great, emphasis upon their integrity as a condition of arterial efficiency, indeed he attributed atheroma to their previous disease. Rindfleisch again insisted no less on their primary importance : yet, reasonable as these opinions may seem, the evidence that they are primarily concerned in ordinary arteriosclerosis, apart from syphilis and other infectious diseases, is not convincing; nor is it growing with our familiarity with the subject. Moreover arteriosclerosis not only extends to ramifications beyond the region of the vasa vasorum, but also often, as in chronic renal disease, begins peripherally. The nutritive vasa penetrate normally about half-way into the media; but it is said that coloured granules injected into the lymph channels pass from the adventitia of a tied segment of vessel to the lumen $;^{2}$ if under normal conditions this be so, the intima depends nutritionally more or less upon the vasa vasorum, as many pathologists believe-Marchand, for instance; so that a high minimum blood pressure by squeezing them dry should starve the 1881 .

1 Martin, H., "La Pathogénie des lésions athéromateuses," Rev. méd., Paris,

${ }^{2}$ Durant, quoted Sanders, Amer. Journ. Med. Sci., Nov. 1911; see his Cases II. and IV. 
intima (p. 193). Adami and others however think this dependence, if any, to be slight, and believe the intima to be nourished from the blood stream. If so, in medial disease the intima should not pine. In my own specimens I have failed to mark any constant or even noteworthy coincidence between atheromatous patches and distribution of the vasa, odd as the incidence of the patches may be; indeed one may note that around such a patch, if in an early stage, the vessels from the adventitia seem not only patent, but on occasion even over activenot in syphilis only; or as if participating in a "subinflammatory" or subreparative process (vide p. 523). In acute arterial lesion, as in the infections for instance, the vasa vasorum are visibly distended, even to the propagation of new twigs penetrating into the swollen superepithelial layer, and in experimental infections Metchnikoff demonstrated that the vasa vasorum suffered little, if at all. ${ }^{1}$ Moreover, in decalcified atheromatous aortas in man, he found the vasa vasorum not only patent, but more extended around and even within the patches-quite good vessels, with normal epithelium and red corpuscles in their channels. (He appends two plates.) Manouélian (loc. cit. p. 305) notes with surprise the almost constant absence of morbid changes in the vasa vasorum in atheroma of the human aorta. Simnitzky, including cases of many kinds of infection other than syphilis, says the same. Again, Mönckeberg and others agree that in arteriosclerosis the vasa vasorum are generally found, unless in far advanced cases, pervious, if not intact; indeed in active cases they may be extended in ramification. And, as Brault, in his book Des Artérites, demonstrated, sclerosis occurs in vessels beyond and without vasa vasorum. When we remember, as I have said elsewhere, that the vascular network of the subadventitia is freely anastomotic, even to the formation of a cylindrical grating around the media, we shall see that disease of a vas, or even group of vasa, in the adventitia could hardly determine a patchy disposition of disease in the main vessel. But it does seem likely that in cases of high pressure, systolic and diastolic, cases in which the diastolic and systolic pulsation of the vessel itself is thus reduced to a very narrow

1 Metchnikoff, Ann. de l'Institut Pasteur, Oct. 1910.

VOL. I 
limit, and their due oscillation arrested, the free access of blood to the vasa vasorum would be hindered; if so the tunics they nourish would come short both of blood and lymph. And, though the lymph spaces, especially in the brain, do give some room for expansion, the adventitia is a tough sheath. An atrophy of a diffuse or fibrous kind might indeed thus come about, but fibrous substitution is not brought home to excess or defect in the vasa vasorum, and does not seem to be due to them. The endothelium is no doubt nourished directly by the passing blood, as are the sponge microbes in their channels. Hence probably its remarkable and most precious immunity in these diseases.

It is in the toxic cases that the part played by the vasa vasorum is most notable; by way of these the poison seems to find more or less of its distribution; although we may be surprised to find that these channels themselves convey the poison without hurt to their own integrity; they seem not to be the first seat of the mischief. In any case, to attribute ordinary arterial disease to a previous disease or thrombosis of its vasa vasorum would be but to push the question but one short step farther back-to the question why the vasa should themselves be attacked if arterioles for a while escape. So far as rises of arterial blood pressure are concerned, these subordinate vessels, subdivided and freely interlaced as they are, should suffer less from strain than the tubes they nourish. That in cases of high pressure the vasa vasorum should suffer early because they are called upon to overfeed the coats of their distressed artery - a suffering which would soon end in the defeat of their own purpose - is an opinion not indeed without supporters, but without probability. All we can say is that outward permeation of poison from the blood current is not very likely; while by way of the vasa vasorum poisoned blood would reach the media directly, and even the intima of a vessel, penetrating into their interstices; and in so far as the vasa vasorum may be affected by the causes of all arterial disease so much the worse for the parts they supply, the tissues would enjoy the less provision for repair.

Syphilis takes a peculiar, but not wholly dissimilar, course of its own. The question has been asked if the well-known trans- 
lucent or "gelatiniform" pink or red patches may be of the nature of atheroma; or if this character indicates a syphilitic infection. They may be about the size of a sixpenny-piece; isolated or grouped, often near forks. For my own part, I am not prepared as yet to accept this specific significance; the description of Dr. Mott answers to my experience, that atheroma also appears at first as a translucent or greyish layer; though it soon ripens into the opaque and cheesy stages of decay, when breaches may be made in the tenacious endothelial investment, and superficial ulcer established. From gelatiniform layer to obvious atheroma continuity seems complete; indeed both opaque and gelatiniform patches may be found in the same aorta.

However, I propose now to take the anatomy of the three coats in turn, and to dismiss first that on which I have the least to saythe adventitia; then to discuss the affections of the media; and to reserve for the last the intima, upon which a greater weight of the argument depends.

Lesions of the Adventitia then will not detain us long; it is a tough coat scarcely affected by mechanical stresses; but more attention should be given to this investment, for the blood and lymph channels which it carries; it is directly attacked, as we shall see, by certain infections, especially by syphilis and rheumatic fever. In my essay on Angina Pectoris I shall urge our need of fuller information concerning the outermost investment of the aorta, but I must anticipate these arguments a little in this place. In angina pectoris the pathologist presents us with punctiliously elaborate descriptions of the heart, pursuing his researches into its every tissue, and finest mesh of a tissue; but if we ask him about the aorta, although it is at last generally admitted that angina pectoris is peculiarly prone to appear in diseases of the aortic area, we rarely get more than off-hand naked-eye impressions of the lining of the great vessel, and not even a superficial note of its investments. While I can recall only one or two necropsies of cases of angina in which sections of the aortic area had been made, as by Dr. Poynton, only too often I read such reports in which the aorta is not even alluded to. (See Angina Pectoris, Vol. II., p. 436.)

We find ourselves therefore ill provided with pathological data concerning the outward investment of the aorta in arterial 
disease, even in syphilis. And this is the more unfortunate as the analogy of the coverings of other viscera suggests that the aortic investment may, like them, contain afferent nerve endings; indeed such structures have been found there by Thoma (see Angina Pectoris, Vol. II., p. 418). I have given definite reason to believe that the ascending arch is endowed with a peculiar sensibility to blood pressures, and plays a considerable part in their compensation (Vol. II., p. 415). Hirsch and Stadler ${ }^{1}$ have brought further evidence to prove that in this area the depressor balances the stress of every cardiac systole (every aortic diastole). On Ludwig's method, they cut the depressor in some rabbits and not in others; they then injected adrenalin into both series, and found that the aorta in those with cut depressors suffered far more strain. An interesting illustration of this comparison is given. We may presume that if in aortic regurgitation, or in syphilitic aorta, the arch is profoundly impaired, it would lose this sensitiveness and adaptability to pressure oscillations: in which case the local disease must multiply itself infinitely. From the clinical side, I had long ago formed the opinion that in many viscera, if not in all, pain has its source in the outer investment and its extensions-as in the pleura, in the meninges, in the peritoneum and mesentery, in the capsule of the liver, and in the pericardium and its extension upon the aorta. Dr. Hugh Anderson, from the side of physiology, had arrived at a similar conclusion; these investments in his view are prolongations of the skin following the in-packing of the viscera; thus visceral sensations remain as a mode of cutaneous sensation. This point of view will be more fully discussed under Angina Pectoris (Vol. II., p. 411) ; here we have to enquire how far the vascular adventitia is affected in arterial disease ; and the answer probably will be that as, syphilis apart, this lesion affects mainly the inner coats of the aorta and other vessels, if the adventitia be involved in the process, it is but occasionally; that is, when the atheroma happens to dip more deeply than is usual with it. In atherosclerosis the adventitia, if it suffer at all, seems to be the last to suffer : a little

1 Hirsch and Stadler, Deutsche Arch. f. Klin. Med. Bd. Ixxxi., 1904. On this complex problem see also Bittorf, Deutsche med. Wochenschr., No. 36, 1910, and previously Deutsche Arch. f. klin. Med., 1904, and Münch. med. Wochenschr., 1907, No. 18. Again, Bruns and Genner, Deutsche med. Wochenschr., 1910, No. 37. 
excess of connective tissue, some proliferation about the vasa vasorum, some slight cellular infiltration. In syphilis however we know that all three coats are liable to the disease (p. 301), and active proliferation, largely perivascular (vasa vasorum), is to be seen in the adventitia. H. Martin and Dieulafoy, as I have said (p. 464), believed that in atherosclerosis the intimal lesion was secondary to this affection of the vasa vasorum, at any rate in the large arteries ; but I am expressing the general opinion when I repeat that the intimal thickening comes first, and indeed that, certain infections apart, the vasa vasorum count for less than might have been expected. We know how, in chronic cases, fibrotic encroachment tends to creep from point to point, at first with a slow perivascular cell propagation, or in spots remote from vascular supply ; and in this way, in very chronic sclerosis, all three coats are liable to a slow fibrosing process, without small cell infiltration; so that in the later stages of particular cases it becomes impossible to say where the mischief originated, or what of it was primary and what secondary. The prosector may frequently note how dense and stiff the substance of the aorta becomes, cutting gristly under the knife. Still, infections apart, in ordinary atherosclerosis the lesion is in large part mechanical, and, as I have argued in more than one paragraph (e.g. p. 513), begins in the subintimal layer. However these are late phenomena and, summarily speaking, it would seem that, syphilis apart, the adventitia and its appurtenances do not play a primary, nor any very important part in atherosclerosis. I have said that Dr. Andrewes, in the iliac artery, and Dr. J. F. Gaskell, in the renal, have demonstrated how rich is the adventitia in longitudinal muscular fibres ${ }^{1}$; but of the behaviour of these fibres in disease as yet we know nothing. Longitudinal "tone" would seem to be more important in long vessels like those of the limbs. But of the media, in the next place, incomplete as our knowledge may be, there is much more to be said.

The disease named Periarteritis nodosa does not fall within my subject. Dr. Carnegie Dickson ${ }^{2}$ separates these cases into

1 Andrewes, F. W., Report on Arterial Degn., 1913, Plate II. ; and Gaskell J. F., loc. cit. Sections shown at the International Congress, 1913.

2 Dickson, C., Journ. Path. and Bact. vol. xii., 1907. 
two classes : one syphilitic, the other due to some unknown poison carried either by the vasa vasorum or by the lymph sheaths.

The Media: Hypertrophy.-Dr. Harry Campbell ${ }^{1}$ argues that more regard should be given to the relative tensions of the investing coats in the various attitudes of an artery, that their relative tensions at any given time or position are by no means one and the same. This is a true but not, $I$ think, an unobserved discrimination. No doubt as the media tightens the adventitia will be slackened, and the intima too; though this coat will be compressed between media and blood stream, a position which in former papers I have alluded to as a possible cause of injury. A thickened media is a common arterial change, but there is no general agreement in the interpretation of it: some observers, such as Johnson and Savill, have regarded this increase as a true muscular hypertrophy; others as a fibrotic hyperplasia. The chief functions of the muscular coat, first demonstrated by Henle in 1840, are distribution of irrigation in the several regions and, with the elastic fibres, resistance to lateral pressures: I have mentioned, but not assumed, a third function, that alleged by Hasebröck, who attributes to the muscular coat, at any rate in the smaller arteries, a peristaltic forwarding of the blood stream. It is however of the second of these functions we have now to speak-the resistance to lateral pressures. If these pressures persistently exceed a certain normal mean, it seems reasonable to suppose that, within limits, such stress would in the muscular arteries take shape statically, as a hypertrophy of the media. But it is a dangerous thing in biology to rest assumptions on probabilities. George Johnson's assurance of medial hypertrophy was probably more or less just (p. 312); but, among the sections which he kindly showed to me, some were ordinary arteriosclerosis, the subintimal thickening which, in certain of his drawings, we may discern even now (p. 330). Herein Gull and Sutton's researches had some advantage, though Johnson's pioneer work, ${ }^{2}$ supported by Galabin, Lancereaux, Senator, and others, tentative as it was on account of the imperfection at that time of staining and other methods of research, is to be gratefully remembered,

1 Campbell, H., Lancet, Jan. 1, 1910.

2 See Johnson, S., Med. Chir. Trans. vol. li., 1868, and many other papers. 
while we bear in mind also the still tentative quality of many of our own interpretations; for even yet observers are far from agreed as to the nature of the thickened media, how far it is muscular, how far fibrotic. Dr. Herringham, ${ }^{1}$ Dr. Cowan, and, I think, Dr. Mott, are not convinced that in any of the specimens genuine muscular hypertrophy, as distinct from fibrosis, has been demonstrated. At any rate, when at the Pathological Society, in 1904, Dr. Savill ${ }^{2}$ urged again that a true hypertrophic increase was to be seen in this coathe exhibited sections from 32 cases-he did not meet with much encouragement; his many and carefully made specimens were put in doubt, and among the experts who heard him he found few declared adherents. Since that date however more and more testimony to the frequent occurrence of a genuine hypermyotrophy in high pressure cases has been accumulating; although histologists seem still unable fully to rely upon their differential tests. Notwithstanding, the number of authoritative English and foreign pathologists who accept the correlation of a genuine hypertrophy is increasing, and in weight is already not inconsiderable.

The musculo-elastic layer also of the large arteries may grow under increase of stress. Klotz, in his last article, accepts it as definite and not infrequent, ${ }^{3}$ though after the age of 35 he doubts its quality. Jores says that in chronic renal disease the media increases with unquestionably genuine muscle; although hyperplastic connective tissue increase may come after this, and from the intimal side, forming, as it were, another layer of the intima. He insists on the careful use of differential stains, without which the precise mode of these changes escapes notice in a "homogener derber Eindruck." This form of connective overgrowth is little subject to fatty degeneration. Quincke demonstrated it in the arteries in aortic regurgitation where, at any rate in rheumatic cases, it appears to be a purely mechanical, not a toxic alteration; an observation which has been recently confirmed by others, by Merklen ${ }^{4}$ for example, who gives an excellent drawing of this alteration, extending even

1 Herringham, Pathol. Soc., London, 1901, and letters to myself.

2 Savill, Lancet, Feb. 16, 1904.

3 Klotz, O., Journ. of Path. and Bact., 1913 ; M`Cordick, Journ. Med. Res., 1914.

4 Merklen, Leçons, 1908, p. 135 
to the coronary arteries. Jores, on the other hand, in aortic regurgitation in young persons, has demonstrated a true medial arterial hypertrophy. He thinks however that it is unstable and does not long endure. ${ }^{1}$ We know that from child to adult a manifolding of the elastica is a part of genuine growth; yet under other conditions this process may be unstable, and merge into atherosclerosis. Jores agreed however that the thickening corresponded in kind to that which he had verified in early stages of high arterial pressures in disease. I may remark that Jores' opinion in this matter is of weight, seeing that he, and Thoma and others, had also observed, and by surgical and experimental ligature produced, a thickening of another seat and nature ; namely, a so-called "regenerative" increase of the fibrous constituent-" Bindegewebswucherung "-of the intima, a change which is apt to invade the media, or the room of it. I may add that in two cases Jores took portions of artery from the walls of hypertrophied hearts in young persons the subjects of valvular disease, and demonstrated, besides the multiplication of the elastic lamels, a very considerable increase also of the smooth muscular fibres associated with them. It is probable then that in high pressure cases a true if transient hypertrophy of the media does frequently occur. ${ }^{2}$ Marchand says indeed that all three coats may take on a genuine hypertrophy. For accurate appreciations, cases should be selected in which the intima is not yet much affected. Some intimal thickening, at least of a fibrous kind, is sure to be present, of senile vessels it is a universal proclivity, but the media, if a hypertrophy, should be uniformly thick, not nodular nor presenting focal lesions; and the elastica not splitting away. Indeed, integrity of the elastica may be a subordinate test of the nature of the change. ${ }^{3}$ The notion that lateral pressure, strength of pulsation, concerns the muscular coat, while the elastic and connective constituents are concerned with stretch lengthwise, cannot, I think, be confirmed.

1 Jores, Virchow's Arch. Bd. clxxviii., 1904.

${ }^{2}$ Faber, whose able book was published after this manuscript was made up for printing, says "eine Mediahypertrophie, die in der Niere in vielen Fällen so auffallend ist, dass sie als zweifellos bezeichnet werden kann," etc.

${ }^{3}$ See on this, Hallenberger, "Skler. d. Art. rad.," Arch. f. klin. Med., 1906, Bd. viii. ; and Aschoff, Beihefte z. med. Klin., 1908, Hft. i. 
A few years after Johnson's first demonstration, Ewald ${ }^{1}$ verified medial hypertrophy in renal disease, and Orth confirmed it. It was demonstrable likewise on the central side of any narrowed belt of a vessel ; so that these authors had no doubt in certain cases of a "primary and essential working hypertrophy of the media," attributable to excessive lateral pressures, not to spasm (p. 476). Johnson, who too often argued on teleological lines, attributed it to arterial spasm due to a "stopcock action" of the muscular vessels, a defence against the access of poisonous matter to the organs. Of recent English investigators, Dr. Newton Pitt, ${ }^{2}$ Professor Adami, ${ }^{3}$ Dr. Andrewes, ${ }^{4}$ Dr. Cowan, ${ }^{5}$ Dr. William Russell, ${ }^{6}$ Dr. Harry Campbell, ${ }^{7}$ and Sir John Broadbent, ${ }^{8}$ have accepted a genuine hypertrophy in cases of high pressures; in France, Létulle and Josué accept it, and in Germany, von Ziemssen, Marchand ${ }^{9}$ (in all cases of plus pressures); Friedemann ${ }^{\mathbf{1 0}}$ (in chronic renal disease), and others. ${ }^{11}$ For my own part, with due diffidence on a question of histology in which I am no expert, I believe that in high pressure cases a genuine hypertrophy of the media in the muscular type of vessel does occur, and that this new muscle is less perishable than might be supposed; but the proof is difficult, and not only because the muscularis is liable to fibrotic invasion and to degeneration. To the chief difficulties of demonstration I have alluded (p. 331) : the want of a standard diameter amid tapering vessels, the various proportions of the coats in the different vessels, especially as to the thickness of the intima, the allowances to be made for degrees of dilatation and constriction, hard to estimate, especially in specimens takenduring

1 Ewald, C. A., Virchow's Arch. Bd. lxxi., 1877.

2 Pitt, Newton, Brit. Med. Journ., Oct. 10, 1908.

3 Adami, Pathology, vol. i. p. 594.

4 Andrewes, Report to Loc. Govt. Board, 1913.

5 Cowan, J., Practitioner, March 1906; and private letter to me dated March 8, 1913, in which he says: "I am quite sure that a true medial hypertrophy may occur in high pressure cases (as in the heart)."

${ }_{6}^{6}$ Russell, W., Lancet, 1901, and Brit. Med. Journ., June 4, 1904.

7 Campbell, H., Lancet, Jan. 15, 1910, p. 195 note.

8 Broadbent, Sir John, Lancet, Feb. 20, 1904.

9 Marchand, Kongr. Inn. Med., 1904, and Med. Chir. Trans., 1907, and in his article in the 4 th edit. of Eulenburg.

10 Friedemann, Virchow's Arch. vol. clix.

11 E.g. Sanders, Amer. Journ. Med. Sci., Nov. 1911. 
long persisting rigor mortis, or bathed in water or stiffening solutions, the confusion of subintimal with medial thickening, processes which have not always been discriminated precisely, the freshness or staleness of the specimens, the various modes of preparation, and some uncertainty in our differential stains. Speaking generally, the arteries of women are smaller and thinner than those of men (Marchand). The defect in our knowledge of the normal variations of the blood vessels in different regions of the body, and at different ages, again often makes it difficult, or indeed impossible, to decide whether a given vessel is hypertrophied or not. Thus, as A. V. Meigs observes, " No textbook contains an accurate description or illustration of perfectly normal renal blood vessels, any more than of blood vessels of the other organs, and therefore any one who studies diseases of the kidney is constantly confronted with the difficulty of deciding whether a particular blood vessel is normal or diseased." This difficulty is enhanced in the case of the arterial intima, the seat par excellence of arterial disease; for not only does this coat normally present considerable differences in the arteries of different tissues, but normally it undergoes thickening with advancing years " as surely as the face becomes wrinkled." It is thick in the coronary arteries, in which atheroma appears early. Likewise it is often difficult to decide whether the adventitia be morbidly thickened or not, owing to the difficulty of determining the boundary between it and adjacent connective tissue.

Dr. Herringham relies, at any rate in part, and in early cases, upon estimation of the muscular nuclei ; these, he says, in the alleged hypertrophy are not increased in number or quality. In our laboratories Dr. Gutch had shown ${ }^{1}$ that muscular hypertrophy - at any rate in the myocardiumconsists rather in an enlargement than in a multiplication of the individual fibres; to this Dr. Herringham replied that, knowing Gutch's work, he was still of opinion that in the media of high pressure cases these fibres are neither multiplied nor enlarged. Benda also asserts that, in four cases, his stains revealed not so much muscular as fibrous increase. However, as Savill frankly said, medial hypertrophy is far from being uni-

1 Thesis for M.D. degree. Journ. of Path. and Bact., 1901. 
versal even in necropsies of granular kidney, though he did testify to its occurrence in cases of Hyperpiesia, where the kidneys had proved to be normal. When the arterial tension sustains the vessel permanently at or near its outward limit, the access of nutrition to the media must be seriously interfered with, an argument which may perhaps be extended to extreme contraction. It is because of the abnormal conditions that hypertrophy of the media, if such it be, may be stable only up to a certain period or phase-in the words of Jores, "sie bis zu einem gewissen Grade für physiologisch hält."

It is often said that this muscular hypertrophy of the vessels promotes that of the heart, or even causes it directly. The two are correlated, no doubt, but I have often found, not only a hypertrophy of the heart without that of vessels, but occasionally arterial hypertrophy without the cardiac. In this case, presumably, the arterial hypertrophy had not extended into many areas, and depended on some local cause.

Some experiments of Nothnagel, watched by Jores, are of interest in this respect. By ligature of relatively large collateral feeders Nothnagel undertook to establish a forced circulation in a definite arterial area. The arteries, thus overburdened, after no long time thickened, as compared with the corresponding vessels of the opposite side; though here again in defect of standard diameters computation of constriction cannot be free from fallacy. However, this apparent thickening both he himself and Jores pronounced to be an increase of genuine muscle-a true hypertrophy of the media. I may add that Cohnheim, in a different way, had demonstrated the same results; namely, that if a demand for compensatory activity be thrown upon small collaterals, these develop into thick-walled and strong vessels, and this by muscular growth, not by any sclerosis. Increased intermittent lateral pressure creates the demand; the afflux of blood is larger, the initial velocity is multiplied, and the vasa vasorum are replete.

It is no improbable suggestion that apparent medial thickening may in some cases be due to œdema.

Certain observers speak of a fibrotic degradation of the media, as if the muscular fibres were converted into connective fibre; this does not happen at any stage, nor under any circum- 
stances ; although, of course, a subintimal or a more general fibrosis may, and often does, encroach upon the media, penetrating the muscular layer or thrusting it back.

If a strongly contracted media be, as seems probable, that kind of arterial thickening which especially resists the cuff manometer (p. 80), the administration of a vasodilator might slacken the instrumental curve; and this possibility bears upon the question if a long-abiding constriction, without excess of lateral pressure, may bring about such hypertrophy (Russell)? That mere constriction, if persistent, would rather be unfavourable to nutrition, I have suggested; but is it clear that more tone means more effort? Dr. Hugh Anderson, in the course of some study of the iris, found that abolition of the tone of the iris for two years led to no atrophy, and he is disposed to think that excessive tone does not result in hypertrophy; he sees "no reason to suppose that one attitude of tone should lead to hypertrophy or fatigue more than another." I suggest that, although as an artery contracts there must be some transient consumption of energy, yet, the new position attained, energy is no longer consumed. Nor do the nerve centres fatigue, till near death. Traube-Hering movements persist even in profound shock. Gray and Parsons, ${ }^{1}$ it is true, in certain conditions of renal vasoconstriction, found, when vasodilatation set in, some evidence of fatigue, due, as they suspected, to a shortage of chromaffin substance; but they found none in ordinary clinical shock. That in many regions of the body certain nerves are never out of action (indefatigable) we know. Such is the case with the vagus and the depressor, and many others. The most probable opinion is that it is an increase of intermittent or oscillating lateral pressure, never sufficient to strain the muscular coat, but keeping it more active within its limits of elasticity-as, for example, by pulsating above a moderate stenosis - which would cause hypertrophy. If this be so, in case of peripheral vascular spasm so widespread as to raise arterial pressures, we should expect to find medial hypertrophy, not so much in the closely constricted portions as in portions placed more centrally where the dilating stresses come more fully into play. In the voluntary system,

1 Gray and Parsons, Arris and Gale Lect., Brit. Med. Journ., May 18, 1912. 
it is not in muscles in permanent spasm that we find hypertrophy (p. 218). ${ }^{1}$

It is not easy then to combine the hypotheses of hypermyotrophy as a result of excessive lateral pressures (Savill) and of over-constriction, independent of pressures and due to some unknown morbid stimulant(Russell), because these factors, although they may alternate, can scarcely be in action together, constriction being antagonistic to tension. We may conceive of an internal vascular reflex or reaction, whereby contractile function is called upon to antagonise extraordinary tensile stress, though such a myogenic reaction is disputable (p. 50). I have dwelt upon this point because, as we have seen in my brief remarks upon sphygmography, condensation of the arterial wall, and especially muscular constriction, may, as Dr. Russell says, interfere with the readings of the external sphygmometer (p. 80).

Although the media, independently of tone, has also its own elasticity, yet its function is largely, chiefly indeed, one lof attitudes of tone, and its deformation under pressure must be inversely as its moment of tone. Hence we perceive how erroneous is the term "arterial tension," when we mean arterial pressures.

The Media: Lesions.-After these preliminary remarks upon the limits of the normal, or quasi-normal media, we may proceed to discuss its morbid states. Of these the acuter kinds are perhaps always results of some infection. It seems that Infections, or many of them, do or may attack the media first. Wiesel, ${ }^{2}$ as we have seen, and shall see farther, made some interesting observations to this effect, upon the coronary and other arteries, in typhoid, influenza, diphtheria, scarlet fever, and septic disorders. In cases of this kind in the aorta necrosing spots are seen, the size of a pin's head or of a linseed, beginning at any rate in the media, if not confined to it. Myocarditis may become apparent a little later. In convalescence they virtually heal, passing into scar tissue, or perhaps into calcification; but Wiesel suggests that in later years such damaged spots may be

1 The few apparent exceptions to this rule have been, in my experience, cases in which muscles naturally powerful were probably no larger than before; or cases of intermittent spasm.

2 Wiesel, Wiener klin. Wochenschr., 1906, No. 24. (See also p. 287.) 
starting-points for senile arterial degeneration. Klotz's observations are in agreement with those of Wiesel, so far as diphtheria is concerned, and he noted the same change in other arteries also; but in cases of typhoid and streptococcal infections he found intimal proliferation. Other observers of arterial infection report hyaline degeneration of the media, no considerable difference. These depravations cannot well be called "mesaortitis" or mesarteritis; they are not inflammations but degradations.

The now well-known effects of adrenalin upon the media of the largest vessels in animals, by some authors regarded as toxic, are now generally interpreted, on the evidence of Harvey and others, as mechanical results of high blood pressures (p. 223). These effects of adrenalin, or mere strain, are interesting, not because they are identical with atherosclerosis in man, but because they illustrate, under the eye, the way in which such tissues break up. First, the nuclei of the muscular fibres blur, and consequently the whole fibre degenerates; so that hyaline and granular streaks and clumps, advancing, in the media, to its whole thickness and circumference, with perishing elastic fibres and empty spaces, only remain to tell of vanished cells and tissues. Such foci very soon give a lime reaction. Here and there patches of increase of connective tissue appear, possibly in part set up by the lime acting as a foreign body. If there are any intimal changes they are adjacent to the worst medial patches, and due to contiguity. The adventitia and the vasa vasorum are but little affected; and few or no leucocytes are wandering from them, though, as in human atherosclerosis, a few giant phagocytes may be seen.

But the gravest of these infectious injuries of the arteries, and especially of the media, is that of syphilis. Syphilitic arteritis is now too well known to need much description here, and I have dealt with it more fully elsewhere (p. 292). I touch upon it here only so far as concerns the media. Before my demonstration of the histology of syphilitic disease of the cerebral arteries, syphilis of arteries, as of other internal organs, had been described, macroscopically, by Wilks, in 1863 (p. 294); but his revelations were long neglected. And the enormous deformities of the aorta which we now recognise as syphilitic 
were well described by Hodgson, and later, as syphilitic, by Welch (Vol. II., p. 167). The pre-eminent part of syphilis in aneurysm, recognised plainly by Morgagni and Lancisi, has been illustrated by many writers, and re-emphasised by Dr. Drummond. By these observers especially, and also, though by no means originally, by the Kiel school, the initiation of this disease in punctate, probably "gummous," lesion of the middle coat, not always associated with evident gumma of the adventitia, has been more and more clearly demonstrated. In early cases of arterial syphilis this process, whether progressing from the adventitia inwards, or manifested by isolated granulomatous foci in the media, or again, rarely, in the intima (as in some preparations by Dr. Teacher shown at the Physicians' Association at Glasgow in 1912), is very distinct; and lately in recent cases the specific spirochæta has been detected, by Benda and after him by many other observers, in the adventitia. As elsewhere so in the vessel, we see the destructive stage succeeded, not by calcification, but by scar. In the aorta this gradual corrosion, creeping from point to point, here extinct there smouldering on, in time extends to the enormous deformity of Hodgson (Vol. II., p. 168); or, if fixed more intensely and ulceratively on one spot, so that the media gives way, a saccular aneurysm becomes the alternative, or associate, of the more diffuse blisterings and pouchings of Hodgson's disease. In these cases the test of Wassermann has now given us one more efficient means of diagnosis. Hasenfeld, at the Buda-Pest Congress in $1909,{ }^{1}$ found the Wassermann test positive in 10 per cent of all cases of "senile" aortic degeneration, and similar results are being obtained by others. But these points are discussed more fully in the section on Aortitis.

The lesions in the field about the orifice of an aneurysm are commonly more or less of the nature of atherosclerosis, and of secondary causation. And in all old syphilitic aortas specific and atheromatous lesions if not intimately blended are associated in close approximation (Vol. II., p. 206).

Although in the vast majority of cases aneurysm is syphilitic, yet in some cases it springs from other toxic causes, such as rheumatic fever (see Aortitis, Vol. II., p. 150), and now and then we come across cases of aneurysm in which a syphilitic

1 Hasenfeld, Deutsche med. Wochenschr., Oct. 8, 1908. 
origin is incredible, or indeed impossible ; of the causes of these failures of the media we know nothing, but as a healthy aorta, or one that seems after death to be healthy, may rupture, so a smaller crack might issue in an aneurysm. Aneurysm may arise also in sepsis, or that obscure disease Periarteritis nodosa; there is also a class of rare cases in which myriads of small aneurysms, in the viscera and in the limbs, arise, in consequence, as it would appear, of some infection unknown.

The resemblance of syphilitic arterial disease to ordinary atherosclerosis may be close, as Heubner and I showed in the cerebral vessels. It is in the aorta that the syphilitic features are most distinct as such. Syphilitic disease of arteries of lesser magnitudes differs more and more from that of the aorta, and not merely in those incidental modifications which depend upon size, place, and circumstance; for instance, in the cerebral it takes a form more like atherosclerosis (pp. 296-298), and without other data may be indistinguishable from it. Again, if we turn our eyes to syphilis of the common iliac we may find its state more like atheroma, though the adventitia is more thickened, and the media more fibrous. Or the adventitia may be fairly normal, and even the media, save for secondary effects, while active foci in the intima may obtrude more or less crescentically on the channel. It seems also that in syphilitic patients a more diffuse kind of medial and intimal sclerosis is prone to occur, a change more fibrous than that of atherosclerosis (vide p. 294. See also, since this was in print, Gruber, loc. cit. pp. 71-75). The following notes, kindly sent to me by Dr. Andrewes, show how difficult it may be even on necropsy to specify the difference between syphilis and atherosclerosis.

“ R. G., æt. 45, a drunkard. Wa.R. positive. P.M. Atheroma of aorta, but not far advanced. No calcification. Aorta tough but supple. Adventitia not much thickened; its vasa vasorum mostly open ; a few near junction with media definitely obliterated by arteritis, but the media for the most part actively vascularised for two-thirds of its thickness. No visible vessels in the inner third nor in the intima. No muscular atrophy nor other degeneration of the media save some thinning by encroachment of the intima near certain ' ulcerated' spots. The intima was much thickened, both generally and in patches, the outer strip (next media) being widely degenerate, with the usual phenomena. About the two or three 
' ulcers' the vascularity was strongly marked; there were abundant groups of plasma cells and large giant cells (like tubercle), with many nuclei close to the lumen. Fatty changes diffuse, not to great degree. The renal artery, taken as control, was normal; no trace of plus intima, and media normal ; elastica not split. Kidneys normal. Renal arterioles-e.g. v. afferentia, normal. My report is necessarily much abbreviated. No spirochæta found. Probably a true tertiary syphilis of the intima apart from the comparatively slight meso-aortitis." Such a case is very difficult of determination; Dr. Andrewes thought that the intimal plaque was not definitely specific, but was eaten into by a perishable kind of granulation tissue with giant cells and plasma cells; hence the " ulceration."

We have now to enter upon more disputable matter. Apart from the effects which we usually group together as infectious and septic, syphilis of course included, and independently of fibrosis, and of atherosclerosis a disease primarily of the intima although usually encroaching on the media, is there a kind of "arteriosclerosis" primarily of the media itself; a degradation originating there, having characters of its own, and taking its own way? Some pathologists deny any such separate lesion altogether; others, like Jores, Aschoff, and Marchand, while not denying a certain peculiarity in some degradations of the arteries of the limbs, seem to pay little heed to it elsewhere; in the brain, heart, and kidneys, they detect no more, or nothing but, atherosclerosis ; others, like Dr. Mott and Dr. Newton Pitt (loc. cit.), would accept and describe a particular and independent medial mode of arteriosclerosis, a mode which they compare with the arterial degeneration found in animals, especially in the herbivora, and with experimental arterial lesion. ${ }^{1}$ Again, some authors, as Dr. French, ${ }^{2}$ in distinction from medial necrosis leading to calcification, suggest that the muscle is itself liable to a non-calcifying fibrous deterioration, so that the coat is thus converted from a muscular to a connective tissue tube, a process not atherosclerosis but independent of it, and belonging primarily to the media. Dr. French's article is not olear in so far as he does not distinguish high pressure cases from the rest; he adheres to the current opinion that, when high pressures arise,

1 See Harvey, Virchow's Arch. Bd. cxcvi., 1909.

2 French, H., Lancet, July 13, 1912.

VOL. I 
they are secondary to a foregoing arterial rigidity. That the connective scaffolding of the whole vessel, of all the coats, under increasing stresses tends to trespass from the borders of physiology into pathology, so that in senile vessels connective tissue growing between the muscle fibres and from the intima may abound, and reach the adventitia, we know (p. 516); of any more peculiar process in the media, not atherosclerosis, the evidence is incomplete, and a contrast on an important scale between a fibrous arterial sclerosis and atherosclerosis is not as yet demonstrated (p. 530). Dr. French does not say how far he used the Sudan III. or other fat test. Intermediate states are infinite, and in no arteriosclerosis is connective hyperplasia absent, but normally the media contains less of this fibre than the other coats. Aschoff refuses as yet to regard any medial disease as more or other than a variety of arteriosclerosis. Jores seems indeed to have regarded the media as outside his argument. That high blood pressure is due to a fibrosis of the peripheral vessels with a consequential hypertrophy of the heart, cannot be maintained, as in many sections of this work I have shown. In the finest arterial twigs there is no fibrosis. The truth is, the old distinction between atheroma and a fibrous arteriosclerosis, or "Arterial Cirrhosis," is dead (p. 530). Connective hyperplasia in youth is formative, and with it grow the elastic and muscular fibres. After the stationary period, if it may be regenerative, e.g. after infections, it is generally substitutive, and then is associated with atherosclerosis; that is, with fatty and necrotic changes.

Mönckeberg's Sclerosis.-However, a distinction and description of a primary medial degeneration, peculiar, or almost peculiar, to the vessels of the limbs, is attributed, as by Adami, to Mönckeberg. ${ }^{1}$ Mönckeberg has the credit of the full comparison, but, as he himself points out, Orth ${ }^{2}$ first drew attention to it. As laid to the charge of the media, this alleged variety begins in a non-atherous decay of the muscular coat, with early calcification, even, it may be, with ossification, a conversion to which Adami and his pupils, especially Klotz,

1 Mönckcberg, "Über die reine Mediaverkalkung der Extremitätsarterien, und ihr Verhalten zur Arteriosklerose," Virchow's Arch. Bd. clxxi. s. 14l, 1903. See also Hübschmann, "Beitr. z. path. Anat. d. Arterienverkalkung," Ziegler's Beiträge, Bd. xxxix., 1906.

${ }_{2}^{2}$ Orth, Virchow's Arch. Bd. cxci. Hft. 2; and Lehrbuch, 1887. 
have given much attention. ${ }^{1}$ Klotz and Marchand accept "Mönckeberg's sclerosis." Klotz says that ordinarily it begins in the femoral artery; the right radial (in right-handed persons) suffers next. He had seen it in one renal artery after extirpation of the other kidney; as if it were due to "Stromintensität" acting on a relaxed vessel (p. 209). With this decay is mingled, as it were accidentally, the ordinary senile fibrosis, but no intimal change. After the infections, this is generally supposed to be the only other kind of primary medial degeneration, and its causes to lie merely in wear and tear; or in usage multiplied by obscure and indefinite influences of a toxic or chemical nature which, at some past time, had left a blight upon this tunic (vide p. 478). Such co-operative influences are suspected because visible arteriosclerosis, anything beyond universal senile fibrosis, is not quite an obligatory condition of old vessels ; and because of a suspicion, fortified by the teaching of Metchnikoff, and emphasised by Dr. Mitchell Bruce and others, that in the course of a long life the arteries cannot but be touched in one way or another by the infinite number of minor plagues and metabolic perversities which filter unseen through our frames, or arise in some no less secret obliquity of our functions (see section on Causes, p. 236). The spread of such a kind of arterial decay in comparatively young persons, a precocity which, as we have seen, is not very rare, seems by its untimeliness to point, in these instances at least, to some noxious ingredient over and above simple wear and tear. And it is probable that arteries, like lungs, hearts, and kidneys, are of very unequal values; that the common usage of life, apart from adulterations or obliquities, may find the vessels in some persons of more durable, in others of more perishable metal-the "quasi hereditaria laxitas" of Morgagni. Aschoff (loc. cit.) says, of persons normal in these respects, that arterial structure is stationary between the ages of 30 and 45 ; but that the media then becomes thinner, and presumably weaker; the elastica also undergoing some atrophy. This description seems to apply to cases which I see now and then, but are undescribed in books, cases in which the radial artery (for instance) is tortuous, even very

1 See also Oliver, G., Clin. Journ., Sept. 1908, and Marchand, Kongr. inn. Med., 1904. (I think Virchow, Cell.-Path., 1871, scarcely relevant.) 
tortuous, and yet but little thickened. In waning vasomotor tone, if pressures are moderate, or even low, the vessels get thin and slack. What we know of the involution of the uterus seems to indicate that primary decay consists in a melting down of muscularis and elastica. In the uterine process little change may be observed in the intima, any such patches being incidental, and this only in the larger branches: but the media rapidly lapses into hyaline degeneration $;^{1}$ the fibre undergoes a gelatiniform conversion, and takes in lime salts. Thoma and Westphalen tried to obtain some light from this involution, but with no very clear result ${ }^{2}$; the process is too little like arteriosclerosis to give us much help in our present problems. The notion current some twenty-five years ago of a "mesarteritis," described as a morbid vascular and cell activity with infiltrating leucocytes, and so forth, was closed by Marchand's demonstration of the different process of necrosis, dissolution, calcification, and scar. ${ }^{3}$ The part which may be taken in this issue by obliteration of the vasa vasorum of larger vessels may be auxiliary and akin, but is certainly secondary and inconstant. The specimens thus interpreted as of an arteritis may have been derived from an arterial infection; but generally in active cases the adventitia is somewhat concerned, 4 and a cellular exudate may be seen in the perivascular lymphatic spaces, a process which, by the penetration known in French as "pacifique," may invade the media almost up to the intima.

Mönckeberg, in 130 cases, found calcification of the media of limb arteries in 55 instances; of these in 43 the changes seemed strictly medial, but in 12 they were associated with intimal change also (atherosclerosis). Dr. Mott likewise speaks of "the medial type of human arteriosclerosis," " mainly" in the limbs. I have little objection to Dr. Mott's terms, but I do not go quite so far as Mönckeberg; nor does Faber.

I think this kind of arteriosclerosis, or arterionecrosis, if a distinct kind it be, pertains to the arteries of the muscular rather than

1 See Barbour, Journ. of Obstet. vol. vii. ; Findlay, Trans. Amer. Assoc. Obst. vol. xviii.; Pearce, Brit. Med. Journ., Dec. 5, 1908.

2 In pregnancies a curious and perhaps unique process has been observed; namely, a formation of new vessels within the old ones.

3 In his article in Eulenburg's Encyclopcedia.

"See however "Infections," p. 282. 
of the elastic type, and of these to the arteries of the arms and legs only; but, as we cannot as yet be sure that there is no fallacy in our observations, so "Mönckeberg's arteriosclerosis" may be but a guise assumed by atherosclerosis when arising in long vessels of this type; in such a site under disease a distinction of intimal and medial limits may be obscure or illusory, especially when connective fibre and calcification crowd in. Thus von Romberg, at the Berlin Discussion in September 1909, affirmed that atheroma, atherosclerosis, and arteriosclerosis, in whatsoever vessels, were one and the same; and that the seat of it is in the arteries most used by the individual ; rather a premature maxim. Dr. Andrewes regards Mönckeberg's decay "as the natural end of a worn-out vessel." In atherosclerosis, although in the earlier stages this may be a disease of the sub-intima only, we shall see that at the outset, or very little later, the elastica intima splits up, and disintegrates; and the depravation thence invades and destroys the media by atrophic pressure, connective proliferation, or pathological contagion (vide p. 524); a process which seems rather to be a propagation by contiguity, and a loss of mechanical advantage, than a primary vice of the media itself. Sanders (loc. cit.) made an important research into these conditions upon 300 cases (in Berlin and Munich). None of these indicated a gross or visible affection of the media at the beginning of atherosclerosis (a name which the author accepts); but after the intimal disease is established, he says, "a hypertrophy of the media may occur," and later and secondarily medial decay. Sanders indeed describes the disease of the intima almost in complete agreement with Jores, though he does not rely so much on the fat test, a test on which he is, I think, unduly sceptical. Notwithstanding, if early cases of decay in arteries of the limbs be scrutinised, focal necrotic lesions, originating in the muscularis, seem to me to have been clearly and severally made out; the muscle cell swells, undergoes fragmentation, loses its quality, and turns fatty, cloudy, hyaline, or chalky; and this while the neighbouring intima really has little, if perhaps always something, wrong with it. Hyaline change is prone to calcification. Thus Mönckeberg's sclerosis rather resembles the experimental lesions of the rabbits' aorta. In other chronic cases, of a kind 
which scarcely amounts to disease, the muscular fibre is slowly supplanted by common connective fibre. In these cases I presume the media had lost some of its tone, as in case of the appearance of this fibre in a slowly dilating myocardium (Vol. II. p. 9).

It is part of the argument then that this degenerative process in the media is essentially independent of the intima ; though, if subintimal atherosclerotic changes set in, it is obvious that stability. of the media must be menaced on its inner side also; yet hither, as even into the subintimal layer itself, the vessels of the adventitia may creep, as part of a feeble quasi-inflammatory fibrotic (reparative) reaction.

There remains however one other point of view to be considered, one advocated by Thoma, namely, that, although this particular medial disease may not lie also in the intima, yet, on the yielding of the media, the intima, to fortify the breach or to fill it up, throws out the stuff which is called atherosclerosis, and that this so blends with medial lesion as to make up arteriosclerosis; the media losing its definition, both a such and in respect of its several features. Muscle, as such, is no longer recognisable; its very nuclei may disappear, and the tunic itself may be bulged and attenuated to abolition. This is the hypothesis which, as we shall see, Thoma advocated with great ability and assiduity, and from time to time reinforced. ${ }^{1}$ We shall see that it carries us far beyond the vessels of the extremities where such considerations especially apply.

Thoma's chief point of view is that arteriosclerosis has its origin not in the intima but always in the media, the intimal changes which ensue being, in his opinion, set up to compensate the medial defect. He does not hesitate to speak of compensation in terms of an interference, as it were of some providence at watch to put in a stitch or a dab where needed; indeed this teleological notion colours all his arguments. Most of us hold not that structures in their changes are beckoned for their good hither and thither by an ally in advance, or even by a stream of intention, but depend for their survival upon such power of reserve and readaptation as they may have inherited or acquired. Thoma

1 Thoma's papers are long and rather tedious; his first papers (so far as I know) are in the 104th and 112th vols. of Virchow's Arch.; he published a long reply to his critics in the same journal, vol. 204, April 5, 1911. 
supposed that, as on yielding of media and dilatation the stream slackened, the stream would tug on the intima; such a "tug," in any case hard to suppose, is, if Dr. Tait be right (pp. 37-38 and 109), does not occur. This part of his argument, so far as comprehensible, seems to be vague and guessy. If however a readaptation comes about, it must arise out of the algebraic sum of the forces innate and contingent, some auxiliary, some inimical, according to which, and in which, the structure lives and moves. We must distinguish between fortuitous conditions, advantageous or not, and innate capacities. The term "compensation" ought not to be used save of tissues histologically normal, whatever their quantity or capacity. It is doubtful if we ought to use this word of the multiplication of the elastica after maturity, as we do not know whether it be a growth or, as Pick and Abramoff believe, a mechanical splitting; if in this latter case it be temporarily helpful it is so but by chance. It has been well said that changes of structure represent moving diagrams of the incident and innate forces at work in the structure and its milieu. Now if these forces acting on the artery-some injurious, some reparative, some impairing the media, some making up for it by, let us say, alterations in the intima-if they are, on the whole and in the balance of them, against life, the part will die; if favourable to life, it will survive. But the intimal changes must arise out of the processes at work; each step for good or bad must entail the next, which cannot be called up, as it were, by a provision. If we are to call the reaction which produces fibrous hyperplasia in an artery a "compensation," what are we to call the same reaction, due to similar causes, when it scars and maims a valve of the heart? If so be then that a thickening of the intima happens to buttress a media in defect, so much the better, as a man's life may be saved by the fall of a beam ; but when we speak of "compensation," we are not by it to understand that this end was brought about by a numen, or an intention to the end, but that it was already implied in the qualities of the moving equilibrium and the conditions under which it was working. That is, we must distinguish chance advantages from innate capacities. The action and reaction, which are obvious enough between two relatively large bodies in collision, are, if less obvious, no less true for a system of 
molecules; now, if in a system of bodies in equilibrium one of the factors varies, this variation for the moment has the system against it; but on readjustment the action and reaction constitute a "compensation," or so much of it as may be potential in the system. Thus Adami, who has some leaning towards Thoma's views, ingeniously explains that the intima thickens because of, and proportionately to, the pull on it of the yielding media. This might be conceived of a hypertrophy of connective tissue, but atherosclerosis is far more a degeneration than a hypertrophy, or repair. As physicians we are too ready to assume all readaptation to be beneficial, or if beneficial to be schematic; we cannot rid ourselves of Paleyism; yet Sir Berkeley Moynihan recently proclaimed Dame Nature to be, at any rate, a sad muff in surgery. In so far then as a moving equilibrium is such it may under incident forces rearrange itself ; but this possibility has its limits, and incident forces often set up rearrangements which are not advantageous. In our interpretations then we shall be careful not to assume provisions for future advantage, nor to impute motives to nature; we shall rather bear in mind Kant's aphorism, "In an organised product of nature all is end and all is means." The paths of science are paved with the broken stones of finalist hypotheses.

Now coming to the facts, with minds free from expectations of the useful and prepared only to discern the useful from the not useful, let us see what Thoma has to report; for in this subject he has done good service in tracing the connective and elastic tissues and their respective proportions and distributions through the vascular system. To return then to his argument; this is, that the first alteration in the artery is a yielding of the media, caused either by excessive blood pressures, by an imperfection in its own consistency, or by a fall of stream velocity; though the author's mind dwells mainly on the vascular frailty, so that he adopts the name-for this incipient phase-of Angiomalakia. In the next place he postulates a lowered nutrition of the wall, a condition difficult, even in the abstract, to detach from the first. Thirdly, the wall gives way substantially, whereby no doubt we should expect to find some further interference with the normal sources of its nutrition. Fourthly, the lumen is widened, not uniformly, but, as we have all observed, in lengths, in spindles as it were, 
and often eccentrically; whereupon ensues retardation of the blood stream, when, Thoma says, to restore the rate the vessel must contract, or be narrowed somehow. If the muscle cannot contract, or does so insufficiently, then the intima must thicken. To lateral pressures he pays little attention.

At this point of the argument Thoma leaves the sphere of disease to examine certain structural alterations of particular parts, processes which are pathological or physiological according to the circumstances of their development; the most useful for this purpose of these alterations is the physiological increase which follows the closure of the ductus botalli, a closure upon which ensue certain changes of the intima of the intervening portion of the aorta, changes correlative with the direction of the arterial stream into the new channel. Upon the nature of these changes pathologists are agreed; Thoma and Jores alike admit that they consist mainly in a thickening of the intima, but of the layer nearer the lumen; and this by an increase of the connective tissue-virtually " regenerative arteriosclerosis." But it is here also, in the deeper layers of the intima, that atherosclerosis proper begins.

Within limits then, limits probably of stress and comparative youth and health of the organism, an increase of work promotes in the artery, as from its growth up to adult life we should anticipate, a hyperplasia of connective tissue in its intima. It seems generally agreed that connective rudiments may appear in endothelial proliferation, and, unless in extreme youth, a more or less fine stratum of connective tissue is always to be seen in the intima. ${ }^{1}$ My own impressions are that, in slight and early cases, it is quite common to see a connective overgrowth internal to the elastica, and some proliferation of the intima, not in dabs where needed but often in annulose form around a vessel, seeming concentrically to narrow it a little, while the muscular coat is quite unaffected. Klotz ${ }^{2}$ decides that these changes in the intima are largely independent of change in the media; and Ophüls is of the same opinion. And so, fortuitously, in one section we may see a degenerated media alone, in another an intimal boss, or a gradually raised patch, without correspond-

1 See Adami, Pathol. 2nd ed. Also Wingate Todd (loc. cit., p. 304).

2 Klotz on hyperphasia of the intima, in Journ. Exp. Med., 1910, vol. xii. 
ing medial change; various allocations of decay and repair in the same individual. In experiments on animals wherein, even if the caged animals be in full health, the stresses are acute and excessive, this connective hyperplasia is usually overborne, prevented by degenerative or even inflammatory products; the same is seen more acutely in the infections, and more gradually in man when, by years or ill-health, redintegration of the stressed parts is less efficient: then we see not development, nor even repair, but mere degradation. Notwithstanding, the effect upon the intima is that it is thickened and, whether the thickening be of one kind or another, dilatation is reduced; whereupon, if the thickening be in due place and proportion-a condition by no means guaranteed, the normal rate of the blood stream may perchance be so far re-established. But how are we even to guess at blood rates in short lengths of a vessel subject to variable distal resistances, vasomotor influences, and systemic pressures? At this sixth phase of Thoma then we must stop for some reflection. In the necrotic long arteries Mönckeberg and his school declare that of intimal change there is none. At most we may admit, not that the intimal changes, if any, are established in order to restore the due rate of the stream in dilated parts, for Nature does not put herself out much to keep old men alive, but that, if they happen to fit, so much the better for the stream. To explain the ignoring of the media in current arterial pathology, Thoma suggests that in its attenuation it is thrust by the bloated intima so far aside as to escape due notice; in many specimens, though by no means in all, this is true.

To harmonise the apparently irregular formation, now of connective tissue, now of elastic tissue, now of necrotic debris and lime, now even of bone, Thoma offers the ingenious explanation that for each tissue there is its own critical pressure. For bone he estimates the formative pressure at 6.5 per sq. $\mathrm{mm}$. cross section; so that if pressures fell it might be reabsorbed (?) For elastic and for connective tissue-in this order-the formative pressures are somewhat less and less; and presumably under lower stresses these too would atrophy. For the unstriped muscle of the arteries, if a mean be taken between contraction and relaxation, the critical pressure would lie above elastic fibre but considerably below cartilage. All these calculations and many others, in the 
paper of 1911, are elaborately worked out in a mathematical argument of which I am no judge; but one may perceive no little interest in this way of looking at the problems. Elastic tissue, being as it is almost a mere physical substance, may lend itself to such calculations, but, speaking generally, biological problems are too elusive, too dependent upon complex contingency, for mathematical appreciations (see Preface). However, it may well be as true as it is interesting to regard the three tissues of the arteries as physiologically determined by their respective tensions, so that if one-say the media-yield, the falling tensions may become too low for its own survival, though sufficient to establish a complementary if inferior connective tissue. The tensions or pressures of the other two coats would probably be altered also, but this we must pass over. In considering these tensions Thoma includes, not only the stresses of the circulation and nutrition, but also those of the voluntary and other massive movements of the body. Hyaline degeneration of connective tissue, as described for instance by Gull and Sutton, he attributes to direct starvation, as under stretching; it is not proportional to the other changes. We have seen that this condition is very prone to calcification. Speaking generally, the more the strain, the more the coats are stretched, the more these descents of tissues, and the more the accumulations of retrograde stuff; the differences in the stuff depending on degrees of tension in parts nearer or farther from the lumen, on the tensile variations of pulsatile phases, and on the lack of nutrition.

Thoma considered also the degrees in which metabolic perversions may promote these depravities, degrees not yet ascertainable; for instance in ligature experiments (e.g. those of Pekelharing and Beneke) he thinks, probably with reason, that the degradation of the intima is due simply to insufficient blood exchange. The increase of connective tissue he regards, even in the thicker vessels of the lower extremity, as the effect of a retardin gstream; or again, as illustrated by the arteries of an amputation stump, in which case an arrested stream may by lateral pressure lead to such thickening as to close a vessel; not quite an exact analogy, by the way.

So in like manner, he argues, bulging of the media is not a consequence of a thickening intima but the condition precedent; 
when it yields the blood race slackens, and to " compensate" this the intima waxes, the waxing being not a hypertrophy directly against high pressure. Thus he interprets the primary arteriosclerosis, whether focal or diffuse ; for these are not varieties of kind, the distinction is recognised, as now generally agreed, only for broad comparison. Thoma said that he had never seen pathological thickening of the intima due to high pressures, or to lateral pressure; but here he seems not to make a clear distinction between connective tissue growth, regenerative or senile, and atherosclerosis tending to calcification; in his last paper (that of 1911) he admits that the degeneration is not wholly one of smooth muscle, but that the elastic fibres are concerned in the failure.

We do not find then, unless rarely in slow changes in young vessels, an intimal fibrosis of this hyperplastic derivation, nor areas of it with the alleged medial correspondences (Councilman). On the contrary, our specimens show that where, as in the arteries of the limbs, the yielding of the media may be more conspicuous, it is in the more distinct and early of these medial bucklings that the intimal proliferation, if perhaps never wholly absent, may be slight. I have already mentioned a section, given to me by Savill, of a cerebral arteriole, from a case of high pressure in a man of 56 , apparently somewhat thickened in its muscular coat, but otherwise normal, save at one point. Here, although the media was on the point of rupture the intima was normal ; hæmorrhage had been stayed for the moment by the adventitia, with probably a remnant of intima. Such bad spots, not buttressed by intimal hyperplasia, do occur here and there in the media, and not in arteries of the limbs only. In animal experiments, where the media is forced first, while the foci of lesion in it are tiny there is no thickening of the intima; and all experimenters (Erb, Külbs, Klotz, ${ }^{1}$ Harvey, Pearce and Stanton) seem agreed that, as the mischief proceeds and the intima is involved, there is no close correspondence in its thickened patches with the degenerated parcels of the media. Klotz says (loc. cit. p. 43): "In my own opinion the primary development of

1 Klotz, O., Journ. of Exp. Med. vol. xii., 1910; Publ. Univ. Pittsburgh Sch. of Med., 1911. 
an intimal sclerosis with plaque-like thickening followed by an atrophy of the media is far more common than a weakening of the media preceding an intimal sclerosis." "In early stages (exclusive of syphilis) there is no evidence of affections of the media but what follows on advancing disease of the intima." And at the same time it is true that experimental arterial lesions in animals cannot be compared with arteriosclerosis as known in man. As to the velocity itself, Thoma regarded it as determined by the activity of the capillary area, ${ }^{1}$ wherein substantially he agreed with the views of Broadbent (p. 200).

Nevertheless Thoma declared that if warm paraffin be poured at blood pressure into arteries affected by sclerosis it becomes evident on the casts that bucklings of the media are levelled up by the counter-swellings of the intima. The experiment is a difficult one, and the changes are such as easily to lend themselves to a preconception; suffice it to say that the subsequent observers, Klotz and Strasburger ${ }^{2}$ for example, who have repeated these experiments, have not arrived at the same conclusions, they assert rather that the patches of arteriosclerosis obev no rule of support to the media, but are distributed so far at haphazard ${ }^{3}$ : by haphazard I mean by local determinations common to many lesions, especially in the infections, which as yet are unexplained. Strasburger found vessels in early sclerosis to be less dilatable, if tested with as little disturbance as possible; and considers Thoma's experiment fallacious. Notwithstanding, we have to thank Thoma for bringing the media into the light, and in the limbs we see that its part in arterial decay is considerable.

In this not very brief but still incomplete commentary on Thoma's hypothesis I have considered his main argument, by which he would make medial deterioration depend upon stream velocity. If, he says, the velocities are continually exceeding the critical or mean velocity, the diameter of the vessel should increase; if they abide below it, the diameter

1 Thoma, Virchow's Arch. vol. 204, p. 72.

2 Strasburger, Münch. med. Wochenschr., April 9, 1907, No. 15

3 See Klotz, Trans. Assn. Amer. Physns., 1907 ; and Ophüls' " Experiments," Amer. Journ. Med. Sci., June 1906. Apt as that intimal disease is to invade the media or to deform it, Ophüls failed to find a rule that under a diseased intima the media was always or regularly weakened. I cannot, for reasons here given, quite accept Professor Adami's view in support of Thoma in the New York Med. Journ., May 15, 1909. 
should diminish, either by constriction or by the alleged morbid thickening of the wall (intima). In his view there is no "slip" between blood and wall, but adhesion and pull. But this argument is not consistent with the physics of the circulation; a retarding flow cannot exercise any traction upon the intima. Stream velocities vary largely and continually, both in the times and in the spaces of the body; there may be no adhesion between the blood and the wall (see pp. 37-38 and 109); in any case the non-corpuscular plasma skirting the wall is less viscous than the more axial and more corpuscular blood.

It seems to me that the structural variations associated with stress and overstress-or strain, phenomena which find their analogies in the more conspicuous structures of the heart, suffice, at any rate for the present, to carry forward our interpretations of these arterial diseases, without bringing in the velocity rates and other ingenuities which rest upon little evidence and moreover appear to sin against the economy of causes. If we look at the aorta we shall see "atheroma" in it more distinctly than elsewhere in the arterial tree; but that is a hardy judgment which in this large vessel sees any "compensatory" value in the diseased patches which disfigure its inner coat, patches which cannot have any effect upon the blood race, save possibly to increase friction by the formation of vortices. I have argued that stresses within normal limits, acting upon healthy tissues such as those which we are considering, thrust or pull over their molecules just so far only that these can return at once to their position of equilibrium (vide p. 194); if however this stress be largely increased, either by a single excessive pull or by a high and "fatiguing" mean of continual pulls, the molecules are thrust over to such angles that they do not quite return to their positions of equilibrium; and in this case surely we are passing out of the field of compensation altogether, and entering into that of lesion. For, let me repeat, if compensation be more than an accident it must come not of lesion, not of impaired fabric and nutrition, but of nutritive abundance or reserve; in short, it must come of physiological not of pathological means. Thus compensation in certain cases is found in a hypertrophy of the media, and in a primary yielding of the media a counter 
proliferation might be anticipated in the adventitia; ${ }^{1}$ but in the intimal changes which we have under consideration all are now agreed that little sign of proliferative reaction is to be detected, scarcely a note of an inflammatory kind; almost entirely the change is one of degradation, out of the womb of which no resources can be bred. Indeed it is in the cases in which, under higher pressures, the media is normal, or genuinely hypertrophied, that we find anything like such a fibrotic reinforcement of the intima as the hypothesis requires.

Now if my clinical view of the subject, the view described in 1894, emphasised in 1896, and often since reiterated, had received more attention, there would have been less confusion about the pathology of this subject. For Thoma also lumps all arteriosclerosis together, whether with inordinate pressures or without them, as one series. Apart from definite infections, and from inflammation of an artery, which is a different affair, I held, and with more conviction still hold, that arteriosclerosis must be separated into two main kinds at least, or at any rate regarded on two clinical aspects-the hyperpietic and the involutionary or decrescent. Putting aside for the moment borderland cases, cases in which long-continued excess of pressures has ended in decay, and a few of mixed causation, and giving our attention to less equivocal examples, we may compare the effects of high pressures in a comparatively young subject-as, for instance, in aortic regurgitation, in granular kidney, or in the malady which I have named hyperpiesia-with those of arterial decay in a person, apparently healthy, but advancing in years. We have seen (p. 334) how long in high pressure cases in a fairly young person a hypertrophied heart may drive the arteries at high pressures without producing obvious changes in their coats. And as these changes do arise, as in their due time they will, they will consist at the outset, if the case be very slow, not so much in degenerating intima, as in the physiological process of fibrous hyperplasia. And two or three years, possibly more in young subjects, may elapse before this change degenerates into atherosclerosis,

1 Round cell growth, or other signs of irritation, seen in the adventitia in arteriosclerosis are generally correlated with absorption of depraved matter from the diseased intima, not to growth. 
unless perhaps in the aorta itself ; before, that is, the subintima of arteries of smaller magnitudes will begin to show the fatty reaction, and before the sections exhibit the accumulated debris. Now in these cases there is no room for Thoma's hypothesis; the media, far from dwindling or bulging, abides, as the finger during life and the scalpel after death tell us ; strengthening itself, if time permit, either with true muscular fibre, as on physiological principles we should expect, or at any rate with the connective fibre, which, though stiffer, is less resilient. Even in middle life during a long continuance of such a high pressure malady, periods during which the finger tells us that the accessible arteries are thickened, the advent of secondary subintimal decay may be very slow, so slow that its incidence may not fall within the shortened span of the patient's life ; but the aorta and larger vessels may suffer, or the heart may succumb before the arteries generally are injured. But with senile or dystrophic arteriosclerosis the facts are otherwise; in these cases, if the first change may be attended with some addition to the connective layers, from the mingling, as we may suppose, in many pathological specimens of areas of connective tissue with areas of atheroma, it is but a transient and not a characteristic element in the affection which, even from the beginning, takes the form of the well-known senile decay, with its widespread but patchy subintimal accumulations.

In the next place, I am unable in respect of the arterial thickening to separate the effect of the dilatation of a vessel from that of the blood retardation (see p. 488) ; or were I to lay emphasis on one of these coefficients it would be rather on the dilatations. For what do we see elsewhere in hypertrophy, whether in the growth of an animal tissue or in the stem of a tree? We see that hypertrophy comes of intermittent stresses. By such alternations the nutritive juices of the part so stressed are squeezed out into its meshes, and there, according to the degree of favouring circumstances, dispose themselves to its nutrition. A tree grows by virtue of its flexions this way and that, the larger stresses, as on the lee-side of it, promoting larger outgrowths: a muscle thus grows by its alternate flexions and extensions ; and if, as in the heart, these dilate and compress the strands, more juices exude, and the part hypertrophies. Is not an artery in like case? In it also do not alternating lateral 
expansions and retractions contribute to the exudations into its interstices which, within limits, make for growth, and, out of limits, for harm, or waste? It is to be expected then that new exudation should take place on the inner curve of a bent portion of an artery, buttressing it, as in the tree more woody fibre is thrown out against the flexion; this increase being due to the bulging - the lateral pressure-not to the rate of the blood, if not without some correlations therewith. It is true that on this inner aspect the swollen patches are not of material so stable as in a branch of a tree, but the persons of whom we are now speaking are elderly, or enfeebled. The intermittency of the dilating stresses then is essential to health; the tissues must have time to rest and feed on the extruded juices; when lateral pressures-for the present we confine our view to thesebecome more and more persistent, and tensions become extreme, then the tissues and their juices, no longer enjoying full diastolic phases, degenerate. It is possible that persistent high diastolic tension with small pulse amplitudes may lead from the first to attenuation of arteries; and this I think I have seen in the aortas and radials of ill-nourished subjects, and in some rapid cases of granular kidney (pp. 483-484).

In one sense then the argument of compensation may be used; that a uniform increase of oscillating stress will, within limits, increase physiological growth so long as pulse amplitude is fairly wide, and tension on the hither side of strain; this growth is almost entirely in the form of connective fibre, possibly with some elastic and muscular constituents: the kind of tissue may perhaps, as Thoma says, be determined by the quantities of the critical pressures.

But here probably we have to bring in the shearing stresses of which I speak elsewhere ( $p$. 513), a shear between the true artery-the intima, and its accessory investments; the tubing within a coil, the structure which I have compared with that of a big gun (p. 458). To this shear it is that I would attribute the more degenerate kind of lesion between the intima and media, an exudation (atherosclerosis) hardly raised to the level of repair. We have seen that this stuff is not proved to be laid down in quantity and place corresponding to partial bucklings of the media; and, if my interpretation of its deposit

vOL. I 
be correct, it bears no relation whatever to the available lumen, and knows nothing of it. This is evident in the many surfaces of excess deposit, lumps which directly, or indirectly by promoting thrombosis, instead of improving the channel, narrow or occlude it. Indeed this capricious, and for the most part injurious silting up is especially notable in the "senile" form of arteriosclerosis, that form which most definitely suggests a primary or correlated yielding of the media.

Again often, when evidence of a yielding media is plain, we find little of the subintimal disease; there is no correspondence between these two changes: they do not correspond spot for spot. Many an arterial branch becomes not tortuous only but longer and wider. In the cerebral arteries this independence is well manifested; in them we see plainly that the subintimal and the medial degenerations are not parallel. The poverty of these arteries in muscle, the difference in structure, alters the morbid process in appearance; the intima thrusts conspicuously into the lumen. Between this simple form and atheroma of the aorta we find every gradation. It is in the hyperpietic form, in persons whose tissue reactions are still sound, that the vessels for no inconsiderable time betray little alteration; as the stress continues, a true static buttressing becomes visible in the form of increasing fibroid hyperplasia of the intima, and of increase of the media, probably at first, and in certain vessels, a muscular hypertrophy. Thus it is that for five or six years, in hyperpiesia, or in granular kidney of not very virulent degree, no great measure of atherotic degeneration may ensue, and the accessible vessels though thickish to the finger may still be smooth and fairly soft. As time goes on however atheroma adds itself to the hyperplasia; but it is only in protracted cases of such high pressures, and very late in these, that beads of calcification may appear, if they appear at all; generally in a damaged media, or intima.

If then much in these histological comparisons remains still indecisive, yet much has been done; and, on the whole evidence, it appears to me that while some loss of tone in the media, whether histologically marked or not, may precede the hurt to the intima and its degenerative hyperplasia, yet that there is only one arteriosclerosis, or atherosclerosis; that in nature we cannot divide the limb sclerosis from the central form. 
Although liable to much variety within itself, the process may be one in many gradations. We have seen that the degrees of histological changes in the media and intima do not run parallel, far from it ; and the proclivity to this mode of decay or that, as for instance to calcification, varies; but these divergences may depend on accidental conditions, such as position in the arterial tree, different build of vessel, stresses of occupations, age, and so forth. Sometimes the media alters more, as if this coat had been more tried; sometimes the intima rather, as if in this case the media had suffered less strain, primary or consecutive. In the one variety the media, when carefully stained, is perhaps never without some feature of decay, fatty or calcareous, in the other the intima does not fail to present some degree of disease. Dr. Andrewes has found in senile cases, even in the aorta, proportions of intimal and medial disease-a blendwhich is comparable or perhaps identical with the limb sclerosis of Mönckeberg. Probably in all cases the musculo-elastic layer suffers early, and proceeds to fragmentation and calcification; ${ }^{1}$ it is true, nevertheless, that in ordinary sclerosis, as the peripheral vessels are approached, the fat (Sudan) stain, so vivid in the intima of the largest vessels, becomes less conspicuous.

Calcification.-The mode of arteriosclerosis with calcification, whether due to primary failure of the media or not, the changes which, whatever their origin, make the hard, gritty, annulated, tortuous, sometimes grotesque superficial artery of elderly people, and are widespread not in the limbs only but also in the visceral arteries and aorta - "albidis maculis inchoamentis videlicet futurae ossificationis " 2-is, as I maintained in 1894, in 1896, and later dates, a state commonly associated with moderate, or relatively moderate, blood pressures; being indeed more frequent in this condition than under hyperpiesis. As regards longevity, it may be no very menacing change ; for many years it may lie chiefly in the limbs, and be consistent with energy

1 When this manuscript was in the press Faber's book came into my hands; I find he takes substantially the view I have taken, and he demonstrates that, with finer tests for fat and lime, signs of decay are to be found in both tunics in all cases. He has shown in a remarkable way that by the silver test calcification is to be detected in minute quantities at stages far earlier than we have been wont to suppose.

2 Morgagni, De Sed. Ep. xviii. 
of both mind and body. Too often however, as we have seen in the chapter on Symptoms, by silting up of vascular areas the nutrition of this organ and of that is curtailed; then, if under it existence may be indefinitely prolonged, that which makes life worth having may be starved, even to an ineffectual remnant. Notwithstanding, in other persons, degrees of this kind of arteriosclerosis which, so far as accessible vessels go, are extreme, prove to be consistent with many happy and useful years of survival.

But to proceed with calcification; in the muscle of an artery of leg or arm, even while still normal to the naked eye, the microscope may perchance reveal here and there that first stage of dissolution, the blur, which I have indicated. In the bodies of persons over the age of 50 , and in both sexes, it is often to be detected in this vascular area or in that; and over the age of 65 it has generally become obvious, more or less. Hyaline degeneration has little specific significance; this kind of decay occurs in various tissues, especially in the elastic and connective, and under various conditions; besides, it is often an artefact. It is to be distinguished from the "amyloid" by the well-known colour test. By the microscope, in sections treated by von Kossa's silver process, or by chemical analysis, calcification, in minute dots, specks or clouds, corresponding to tiny points of necrosis or to a more diffused lime affinity for failing tissue, may be found at an earlier age than we are wont to suppose, especially in the aorta; not in its medial portion only but also in the elastic and subintimal layers. The microscope detects the lime long before the eye, yet chemical analysis tells the tale sooner still; as the nitrogenous content falls the ash rises. The ash of dried atheromatous aortic substance may rise six-fold; and of the constituents of the ash the lime may rise twenty-fold. Still, in many cases, as Klotz affirms, it is true that in the muscular, as contrasted with the elastic vessels, calcification, a sign of previous muscular necrosis, may be manifest in the media when in the intima but little morbid change is perceptible, and the media is, as he says, the mainstay of the vessel; this is the variety which, as we have seen, occurs particularly in the vessels of the limbs. But in the elastic arteries, as in the aorta, this fibre is prone to suffer 
from the first, indeed is often the first to suffer. ${ }^{1}$ The fibre is not converted directly into lime but breaks down into granules, and these, and the cement substance and hyaline remnants, the salt occupies. The fat, not lipoid, varies irregularly, increasing a good deal in some medium cases.

$\mathrm{Klotz}^{2}$ is of opinion that to speak of the lime as precipitated in the tissues is too simple; that calcification arises as follows:- optically, in the atheromatous patches, cholesterin and fatty acid compounds are detected by their double refraction under polarised light ; cholesterin may be dissolved in the fatty acids, and then crystallise out, releasing these to combine with potash, soda, or lime. The soluble $\mathrm{K}$ and $\mathrm{Na}$ soaps disappear, or are re-engaged in the cycle of changes, but the $\mathrm{Ca}$ soaps, being insoluble, are deposited; then, by interaction, the phosphate of soda, with a little carbonate, and traces of phosphate and carbonate of magnesia are laid down in the tissue. The platter form is due in part to the structure of the cholesterin layers. The deposit however, whether in plates, in nodules, or more diffused, is of the same kind. In curved internal arteries with many offsets the patches are more circumscribed. But important and explanatory as these researches are, they may not include quite the whole process. If they include ionisation, segregation is hardly considered, a segregation of calcium compounds with oleic and lecythic bodies, ${ }^{3}$ without the necessary intermediation of soaps; or perhaps, more biological processes, as of ossification. Certain bacteria can decompose lime salts --even the sulphate; it is possible that in some parts such an agency may be at work, or that without them tissue dissolution may follow a similar path. Dr. Andrewes quotes Wells and Baldaux, who failed, as others have failed, in many

1 From Faber's book (p. 499 n.) I am glad to see I have not said too much concerning the calcification of the elastic fibres. Faber has found these fibres and the cement substance around them calcified even down to the finest arterioles-down to $10 \mu$, where the intima is but an epithelium lying upon an elastic lamel.

2 Klotz, Journ. Exp. Med., Nov. 1905; and Repts. Rockefeller Institute, vol. v., 1906; Dockray, Thesis for M.D., Vict. Univ., 1898. For calcification without sclerosis see Magnus-Levy, Deutsche med. Wochenschr., Nos. 26 and 27, 1914.

3 See Wells, "Pathology of Calcification," Journal Med. Research, 1906, vol. xiv. p. 491 ; Baldauf, "Chemistry of Atheroma and Calcification of Aorta," ibid. xv. 355; Stud. Benda's Hygienic Laby, 1907, p. 68; and Voorhoeve, Berl. klin. Wochenschr., 1912, No. 36. 
cases at any rate, to find calcium soaps in the aorta. Baldaux found lecithin abundantly, and this substance he regarded as the source of the phosphatic element. In diabetes calcium is carried off in the urine by the fatty acids (Ransom).

Professor Benjamin Moore, ${ }^{1}$ who has recently gone over the ground again, returns to the old opinion that calcification takes place in extravascular parts and is due to the hindrance in such parts of oxygen supply and of lymph flow. This kind of degradation, as he reminds us, appears in parts-such as fingers and toes, extravascular cartilage, circumscribed tubercle, old pus, obsolete parasitic tissues, extra-uterine fœtus, etc.-which are remote from the blood stream; and such areas he assumes in arteries of which the vasa vasorum may be narrowed or closed. There heterogeneous lees of imperfectly oxydised products are precipitated, as of oxalates and urates, as well as of stearates of calcium; though the stearates may appear first. Professor Moore thinks that in elderly persons this process may be facilitated by a higher alkalinity of the blood. It is true no doubt that connective tissue in some modes is more stable, less disposed to calcification, than in others; that of vessels thickened under high pressure is more stable in this respect than that of more degenerative modes. The determining factor may be access of nutrition, e.g. by vasa vasorum; but there seems to be some difference in the modes of connective reaction, as if between a substitutive and a hyperplastic mode.

My own observation of Dr. Harvey's ${ }^{2}$ experiments in our laboratories on arterial lesions prevents my accepting Professor Moore's conclusions without qualification. In Harvey's experiments (p. 223) strain of the thoracic aorta (in rabbits), by repeated brief compressions of the abdominal portion, led, on the central limb of it, to very early calcification, even to the curious phenomenon of true ossification, a lesion more prompt and complete than deprivation of oxygen could well account for ; and similar results are recorded in the thousand and one such experiments by means of adrenalin. Voorhoeve (loc. cit.) says that by sufficient doses in the food the lime charge of the blood

1 Moore, B., Brit. Med. Journ., April 1, 1911.

2 Harvey, H., Virch. Arch. (loc. cit.). 
can be increased, but Professor Dixon tells me that the results of our own laboratories, many times repeated, are in absolute disagreement with those of Voorhoeve, and also of Wright. On healthy people the administration of lime, as lactate or milk diet, produces no change in the blood content, and no increased coagulation of the blood. ${ }^{1}$ It is said that a plus calcium content of the blood inhibits the effect of adrenalin, but the blood of rabbits is rich in lime. And not under experiment only, but also, and not rarely, in senile decay, and even in the precocious arterial decay of young children (p. 174), due possibly to syphilis or other toxic influence, this curious development of bone may appear. ${ }^{2}$ Ziegler found cartilage in ossifying arteries. In a case reported by Bunting, ${ }^{3}$ even genuine cellular marrow was discovered, though its original seat could not be exactly demonstrated. Happily it does not fall to me now to try to explain this strange development, for a development at some growing edge it seems to be ; I can only note its occurrence as an occasional term of the series of changes in arteriosclerosis.

But as fatty atheroma is quite independent of lime, so we must not suppose that fatty accumulation is essential for calcification. We find lime deposited in the purest connective tissue, where there is no response to fatty stains; and even within stellate cells. We find it often and readily in the physical alteration called $\mathrm{k}_{\mathrm{j}}$ aline. Any one of the histological constituents of an artery, be it fibre or membrane, is susceptible of calcification. It seems probable that in many cases an albuminate of calcium is formed during necrosis of protein tissues. Calcification again may be found in particular tracts, as in the arteries of the limbs, and not in the visceral. To avoid repetition hereafter, I may say here that although I have described calcification conveniently under the head of the tunica media, yet this change is found also in the atherosclerotic degeneration of the intima.

Whatever the chain of processes in calcification, it is not an active attack upon the artery by the lime salts; it is

1 See also Dale and Laidlaw, Journ. of Path. and Bact. vol. xvi., 1912.

2 Morgagni's caution and precision are exemplified in his words on this point : "(the vessels) ostendebant aliquid aut ossei aut ad osseam naturam vergentes." But all his contemporaries in this sense spoke of "ossification."

${ }^{3}$ Bunting, Journal of Exper. Medicine, May 1906. An interesting discussion of a case in a man, æt. 72 , with a review of the literature of the subject. 
passive, and seems, as Professor Moore says, to arise wherever there is necrosis, signifying, as in tubercle or in bone, an extravascular position. Klotz says that near a vas vasis calcification does not occur. The great authorities of Virchow and Metchnikoff can hardly persuade us that it is due to liberation and transference of lime in old persons from an attenuating skeleton; nor can we attribute it to an overcharge of lime in food and blood. Gouget asserts (loc. cit.) that in the blood of arteriosclerotics no qualitative change of constitution can be discovered, whether in respect of lime, 'cholesterin, or other constituent. (But see Cholesteræmia, p. 252.) We have seen that calcification can be induced readily and acutely in the aorta of young rabbits; as however it occurs spontaneously in about 3 per cent of young captive rabbits, in using these animals for experiment this percentage has to be controlled. We cannot then attribute arterial calcification to excess of lime in the food seeking a resting-place; nor attempt to prevent it by withdrawing lime from the food, a measure which I have compared to a withdrawal of ready-money from a man whom we have left in possession of his cheque-book. ${ }^{1}$ We no longer attribute rickets to a deficiency of lime in the body; nor are the arteries affected in osteomalakia. ${ }^{2}$ Not from the attenuating bones only, there is plenty of lime available if the parts affected be in a state to attract it. Decay comes first, then petrifaction of the ruins. Animals have been fed again and again with excess of lime without any effect upon the arteries. If calcification of the aorta be more frequent in herbivora than in carnivora, in many of these at any rate, such as dogs, it is not for lack of lime, for this mineral they are continually gnawing from bones. One would suppose with Klotz that, in matter practically dead, calcification must be a mere chemical process, in no way dependent upon tissue selection. Still it is remarkable that carbonate and phosphate in the affected parts are in the same proportions as in bone and in the serum balance; as if the correlations were biological rather than merely physico-

1 Aschoff is, I find since, of the same opinion.

2 See Etienne and Robert, Arch. méd. expérim., Nov. 1911. I have mislaid, and cannot now scek, a corroborating reference to Selig and Ameseder, but I believe their paper was in the Zeitschr. f. physiol. Chemie, about 1910. 
chemical. However we do not yet know how far the physicochemical may carry us. ${ }^{1}$

It is by no means obvious, perhaps not probable, that this deposit in foci of degeneration is mischievous; as some experiments seem to prove, it may serve even to fortify a weak spot, to stop a gap; if so, to withdraw lime from the food would be an error, indeed the finer calcifying vessels do not so much rupture as silt up. Ludwig Weil ${ }^{2}$ filled calcified and normal arteries of various calibre and origin with columns of mercury of regulated heights, and found that by calcification the resistance of the arterial wall, whether to pressure or traction, was at any rate not diminished. In his experiments the vessels never broke at a calcareous patch. Aschoff also agrees that the calcification is incidentally rather protective in its effects, and that therapeutical attempts to dissipate the lime are useless if not injurious. But to argue, as some do, that the calcification is compensatory, is a contrary error. If compensatory, or reparative in purpose, it should build up the arteries of the cerebral subcentres instead of those at the base, where it is less wanted. In the brain it is a nuisance; it slackens the blood stream, favours thrombosis, and promotes occlusion of the finer twigs. It encroaches upon the endothelium, roughening it, so that thrombic layers cohere with it. As these organise or calcify, the branch is obliterated.

However these experiments and speculations, if we are to be convinced by a remarkable article by MacCordick, communicated to the British Medical Journal of October 18, 1913 by Professor Adami, are to be considerably modified. MacCordick says " as a general rule the arteries are not of a stony rigidity during life." If a tortuous, thick artery, "such as the radial" for example, " be dissected out within an hour of the patient's death, it is no doubt more resistant than the normal, but it can be bent, squeezed and curved like a moderately stiff rubber tube." . . "About ten minutes later these lengths of the vessel become absolutely rigid, possessing pipe-stem characters. They

1 But for the need of verification of the facts of calcification see Stewart, H. A., Journ. Path. and Bact. vol. xvii., July 1912.

2 Weil, L., quoted Virchow's Jahresb., 1906. See also a paper in Berl. klin. Wochenschr., 1868, by an experimenter whose name was not entered on my notes. 
crackle on compression and break on being bent." In a certain case of senile gangrene of the foot the popliteal artery was much sclerosed, but on severance bled freely, and was ligatured without difficulty. Lower down the anterior and posterior arteries were filled with recent clot, and below this they were hard and brittle; the knife cut against hard calcareous deposit similar to that in the artery exposed to the air: evidently this dense calcification had taken place before amputation. MacCordick quotes also Gruner of Montreal who, performing an autopsy immediately after death, found the aorta fairly soft and pliable; within a few hours however the specimen presented hard calcareous plates with peripheral ulceration in areas of atheromatous degeneration. The author says that the induration is identical with the setting of mortar, by the absorption of $\mathrm{CO}_{2}$ from the air. Moreover after death the reaction of the bodily tissues and fluids becomes acid. It seems then that during life the calcareous deposits are "unset"; and many puzzles are thus explained, or an answer suggested, such as the infrequency of traumatic rupture in large and tortuous vessels ; during life they are not rigid. Again, "ulceration" of the aorta without thrombosis may be thus understood, if the ulcers are post-mortem productions; the calcified areas during life are soft, but they set hard before the post-mortem contraction of the vessel takes place, when the tension of the contracting wall breaks the epithelium away from the patches.

Cholesterin and Atherosclerosis: see p. 252.

So far then there seem to be three ways, with syphilis four, in which the media becomes diseased. In the later stages of subintimal lesions it is commonly involved; or, for some obscure reasons, especially in the limbs, under causes of wear and tear, or, because of an "originally inferior quality of tubing," it may undergo the fragmentation, necrosis, cloudy swelling, and calcification described in the second place; or thirdly its muscle may be supplanted by a fibrosis penetrating between its muscular strands, and invading it from within and without; there being apparently some inverseness in the relations of medial calcification and medial fibrosis. Clinically, the kind of arteriosclerosis attributed to medial degeneration with calcification is more characteristic of 
patients suffering from what I have called " Decrescent arteriosclerosis," a disease not ordinarily attended with excessive blood pressures; the fibrotic form may be more characteristic of the arteriosclerosis of Hyperpiesia, at any rate of the earlier stages of it, and in persons not yet "senile." I do not go further than to say " more characteristic" of each respectively, because occasionally, as I have said, in late stages of protracted cases of (non-senile) Hyperpiesia we may detect scattered beads of calcification in the leathery vessels; and in the Decrescent disease excessive fibrosis may be found in the coats of not a few arteries of the muscular type, especially in the limbs; it is to be found indeed in all parts of old bodies, encroaching upon more specific tissues, even about the joints. But these two different clinical series, the hyperpietic and the decrescent, are not represented by intimal and medial lesions respectively. As we shall see, the lime may be freely deposited also in the elastic fibres and in atherosclerotic intima. In the section dealing with Disease of the Kidney I suggested that a complex organ may have but a small range, or even but one form, of response to lesions of many origins ; so that series clinically so different as the hyperpietic and the decrescent may call forth in the affected artery similar reactions. And if it be true that in the radial and tibial arteries medial degeneration may be pre-eminent, I must add that in many and many a specimen of alleged medial arteriosclerosis histological signs of a morbid intima also are evident enough ; often indeed the lesion of the media is clearly secondary to this atherosclerosis. Moreover, if it be true that pathologists have studied the aorta too exclusively, we are now in danger of making too definite a distinction between the elastic and the muscular vessels. That the aorta has muscle in abundance we all know; we are apt to forget that the muscular artery is far from poor in elastic fibre, circling and even radiating outside the elastica proper, and in a connective tissue framework woven, visibly and invisibly, throughout the whole of the wall. And if it be true that in the arteries of the limbs a sclerosis is commonly seen, of which the main feature is medial decay and calcification, so in the aorta extreme cases of this kind may from time to time be discovered which likewise present the same preeminence of this medial affection, with but slight atheroma. 
Thus, as I have said (p. 485), in the contrasted parts I submit that the difference may be one only of degree, determined by prevalence of one set of causes or another.

Finally, it is stated that traces of iron are to be found in calcified vessels; I presume the accretion to be but a pathological curiosity.

Disease of the Intima.-Arteriosclerosis, Atheroma, Atherosclerosis.

We enter now upon the main part of our pathology, and here more especially it is that we have to satisfy ourselves concerning the aspect and nature of the histological changes. At the head of this section I have taken three names as equivalent, two of which-namely, atheroma and arteriosclerosis-by some eminent authors are still regarded as signifying distinct processes, while the third-atherosclerosis-they would confine to the field of the large vessels, or repudiate altogether. These opinions must, therefore, be sifted. The considerable authority of $\mathrm{Klotz}^{1}$ is against any limitation of the name arteriosclerosis to disease of the intima; he would extend it to include at least diseases of the media (vide p. 483). We may acquiesce with some reserve, if Klotz will grant the name atherosclerosis especially for the disease of the intima, as more fully described by Langhaas, Jores, Mott, Aschoff, Marchand, Andrewes, and others, with of course its consequential effects upon the neighbouring media; though, as I have said (p. 485), the difference may be only one of relative degrees of the constituent elements. The larger the artery the more elaborate the intima. In the early stage the decay is confined to the intima; later the media and even the adventitia partake in the disease, and even slight reparative reaction, with a few new vessels, may here and there be perceptible. But in the main we see the media soon pushed back, thinning, and its elements becoming confused, first on the side nearer the intima; its fibres are less definite, and are mixed with ragged and ruptured elastic fibrils and their granular debris. But when Klotz would divide these arterial diseases into the degenerative and the productive, there, syphilis apart, I cannot follow him; for my part, as I shall presently say more fully, productive reaction, if perceptible at all, is slight and evanescent. Were it

1 Klotz, O., “Arteriosclerosis," Pittsburgh Med. Sch. Publ., N.S., 1911. 
inflammatory it should propagate itself to neighbouring parts. All arteriosclerosis, whether intimal or medial, is mainly degenerative. But Klotz, I think, does not quarrel so much with Jores' description of the intimal disease as with his comparative ignoring of the media. I have expressed my agreement in great measure with Klotz and Adami so far as the arteries of the limbs are concerned; though not as regards the arteries of other parts. I think that von Romberg also, if too categorical on this point, ${ }^{1}$ is not without justification when he roundly asserts that he knows no distinction, clinical or anatomical, between arteriosclerosis of one seat or another; that it is one disease in various proportions. But we must admit that the arterial disease of the limbs which comes on somewhat early in labouring men (p. 205), if not different in kind from atherosclerosis, is more or less different in mode.

We meet indeed not infrequently with the demur that Lobstein's name of arteriosclerosis is itself but a general term of vague meaning and loose application; but this is now, I think, an offhand criticism, one in the present state of our knowledge no longer justifiable. Although many points of detail remain for decision, the recent work of Adami and Klotz, Mott, Hollis, Cowan, Pitt, Andrewes, and others in Great Britain; of Lancereaux, Létulle, Cornil and Ranvier, Martin, Josué in France; in Germany of Langhaas, Thoma, Mönckeberg, Marchand, Aschoff, Jores-especially Jores whose test for the fatty element of the disease is crucial; of Janeway, Cook, Councilman, Thayer, Stengel in America, to name casually in each country but a few, has separated and defined "arteriosclerosis" as precisely as biological categories can be defined. On lesions of the media we have seen that this coat may perhaps have more than one mode of decay; and that an inefficiency in the media by the loss of protection, may conduce to the decay of the intima; again that the arterial diseases due to infections, and to syphilis especially, have some features more or less peculiar; and presently we shall see that the intima is capable of hypertrophy as well as of disease: yet all these processes may conveniently be included under the general name of Arteriosclerosis. This

1 Von Romberg, Deutsche med. Wochenschr., Oct. 28, 1909. 
being the case, the name Atherosclerosis, introduced by Marchand, and accepted by others well versed in the subject (Jores, Bäumler, von Romberg, Aschoff, Benda), has been adopted to signify the chief of these processes; namely, ordinary nonsyphilitic decadent lesion of the intima, whether seated in the larger vessels, and there known as atheroma, or in the smaller, where it has been called arteriosclerosis. Virchow regarded atheroma as but a farther stage of arteriosclerosis; and in recent papers $I$ have accepted the name Atherosclerosis in the specific sense I have indicated.

But it must be admitted that in this use we have not with us all pathologists expert in this matter. Huchard to the last resented the comprehension of atheroma and arteriosclerosis under one name ${ }^{1}$ he urged that atheroma is one disease-of large arteries, "arteriosclerosis" another-of the small arteries and viscera; insisting that the symptoms of the two affections were different; so that, in view of any change. in nomenclature, he would have recommended the names Atheroma and Arterio-visceral-sclerosis for the respective processes, which he regarded as distinct. $\mathrm{Klotz}^{2}$ contrasts a sclerosed radial artery (after Mönckeberg's form) with an atheromatous aorta, as examples of different processes ; a difference we have already fully discussed. Josué ${ }^{3}$ likewise denies any affinity between atheroma and arteriosclerosis, and regards them as different "entities," whatever this word may mean. But, not to pursue this point further, from many other witnesses I will cite farther only the authority of Dr. Newton Pitt, ${ }^{4}$ who looks upon "atheroma and arteriosclerosis as quite distinct diseases." Indeed the balance of contemporary opinion, at any rate in England and France, is perhaps still in agreement with these eminent dissenters, though Dr. Mott seems to decline the distinction. ${ }^{5}$ I have admitted that atheroma in the aorta, subclavian, carotid, or iliac artery for instance, and arteriosclerosis in a limb artery, such as the radial

1 Huchard, at the French Congress for Inner Medicine, Geneva, Sept. 3-6, 1908.

${ }^{2}$ Klotz, Report of Toronto Meeting, Brit. Med. Assoc., 1906.

3 Josué, Journ. Amer. Med. Assn. vol. xlii. No. 25.

${ }^{4}$ Pitt, N., Lancet, August 8, 1908; and Brit. Med. Journ., Oct. 10, 1908.

5 See also Ophüls, Amer. J. Med. Sci., June 1906; and the Broadbents, Lancet, Feb. 20, 1904. 
or anterior tibial or dorsalis pedis-diffuse and focal sclerosis, as they have been called respectively - may bear but little superficial resemblance to each other; but whether notwithstanding there be, or be not, within them a profounder likeness of kind, is a question for some further consideration. Meanwhile, it will be granted on both sides that a process, if identical in kind, but displayed, in the one case in a vessel of the elastic type, in the other upon a vessel of the muscular type, would be modified by the difference of web, and also by the different conditions of space and stress in the respective sites. Still, these are differences not of nature, but of position and pattern; the comparison of natures depends upon more intimate qualities, and upon more penetrating methods of investigation.

Other critics complain that the word atherosclerosis contains no signification of blood vessels, but custom soon settles meanings. In discussing the minute anatomy of arteriosclerosis however I will not beg the question by making use of the name atherosclerosis at this stage of the enquiry.

We are so much indebted to Jores for his work on this subject, ${ }^{1}$ that in any careful descriptions of it his papers, and those of his pupils, such as Roth and Prym, must be studied; but the reader who desires to find in small compass these results, revised and developed by another investigator distinguished in the same line of research, will find what he requires in the essay by Aschoff indicated in the note. ${ }^{2}$

At this point in the discussion however I think it fair to recall attention to the paper by Langhaas of Würzburg ${ }^{3}$ in the thirty-sixth volume of Virchow's Archives, which did not receive at the time of its publication the notice it merited. Langhaas, in a long and careful personal research, anticipated much of the later descriptions of arteriosclerosis. He demonstrated the various thickness of the intima at various ages,

1 As set forth in his book of 1903, and in his later essays, as indicated also in the chapter on "Arteriosclerosis and Disease of the Kidney" (p. 309). See also Münch. med. Wochenschr., Aug. 31, 1909.

2 Aschoff, "Atherosklerose und andere Sklerosen des Gefässsystems," Beihefte zur mediz. Klinik, Urban und Co., Wien und Berlin, 1908.

3 Beitr. z. norm. u. path. Anat. d. Arterien. As this paper is attributed by recent writers to Langhans, I have taken pains again to verify my reference. The name of the author of the article in the volume cited is plainly, whether correctly or not, Langhaas. 
comparing young and old structures, and demonstrating the excessive connective fibre in aortas over 50 years of age. $\mathrm{He}$ insisted as strongly as Jores on the subintimal seat of the lesion, and that the media was affected secondarily by squeeze, thrust, and "pressure-atrophy (durch verdrangend Druck)," with very little fatty change; the thickening being really intimal, even so that a film of intima might survive between the diseased intima and the media. He described also the increase, multiplication, and splitting of the elastica ("Zerfaserung"; see also loc. cit. p. 219) and of the several lamels, not a cellular proliferation. In view of Virchow's allegation of an arteritis he compared the signs of such irritation. He thought also, but probably in error, that the muscular fibre might degenerate into connective, or that this lower fibre might originate from muscle nuclei.

As we have presumed then that the pattern of the disease would be determined, more or less, by the pattern of the normal web, let us now briefly recall to mind the varieties of the normal structure. Broadly, we divide the arterial tree into, first, the large arteries, such as the aorta, innominate, carotid or pulmonary; vessels in which elastic tissue predominates, but is woven into a kind of felt with some connective and muscular fibres, a web connected with the lamina elastica interna; secondly, arteries of middle magnitudes, such as the radial, in which the muscular tissue begins to predominate, and, as the longitudinal fibres taper out, takes a transverse or spiral order; and, thirdly, the finest arterioles, which possess only the endothelium with its lamina elastica. On these variations of structure depend pathological variations, sometimes a little confusing. The atherosclerosis which in the smaller arteries begins in the musculoelastic layer of the intima, is seated in the aorta in the media also. Again, the process may begin in the largest arteries, or in the smallest; the larger, as in primary contracted kidney, suffering later. These oppositions of seat may correspond, as we have seen, to profound clinical differences. I have already suggested that what we may call the cell wall of the circulating blood is the intima; the media and adventitia being structures of support. The endothelium all through the tree is the same; but in the vessels of the first class the intima consists also of a 
fibro-elastic layer which, with fine muscular fibres interwoven, itself again consists of two layers, the longitudinal and the transverse, of which the longitudinal thins out. It is in this layer that atherosclerotic disease begins.

I am still of the opinion that the disease which begins here is due to tensile and shearing stresses, which arise at the plane of apposition of the intima upon its supporting and distributing coat, the media (pp. 11, 195, and 497). Thus in previous papers I have spoken of the media as a coiled spring, and suggested that the media may yield rather to longitudinal, and the intima to lateral stresses. However, writers on this subject lay too much emphasis on lateral pressures, as in distended inorganic tubes ; in life the stresses are tangential, shearing rather than bursting. Disease begins here because lesion begins here. Here the intima is sometimes folded, sometimes stretched out; sometimes lax, sometimes squeezed between a tightened media and a high blood pressure, or shifted upon its base. We have seen, in the section on Causation, by such instances as the atherosclerosis of the pulmonary artery in mitral stenosis, as the disease on the central side of a constriction in a large vessel, and again as at the orifices of forks, how such lesions, and consequent disease, arise under causes merely mechanical. The postulate that the intima suffers first because it lies beyond the vasa vasorum, which it does, will not bear examination; for it is by the lymph that tissues are nourished, and the intima, if not fed by the blood stream, is not beyond lymph irrigation.

To return to the normal anatomy; with similar convenience we may again, with Kölliker, regard the intima as consisting of two layers, the endothelial or vitally active member corresponding to the same part of the lymphatics of the serous cavities. As we pass along the length of the endothelial coat, we find, as in the other coats so also in the intima, a certain variation of its constituent layers according to the variations of its function in different areas; as, for instance, of the blood distributions, or the bearings of mechanical stress, as at the bifurcations. Logically perhaps the elastic layer of the intima ought to be regarded with the outer layers as an investment of mechanical support. 1 Elastic fibre abounds where the expansions and con-

1 That elastic and connective fibre have not a several and independent voL. I 
tractions are rhythmic, and tapers off as the flow within becomes more continuous. Moreover, stresses on the wall decrease with every decrease of diameter. Although the cerebral arteries, which are equally supported by the uniform intracephalic pressure, are thin, yet they are rather rich in elastic fibre, because in them the pulses are direct and strong. In arteries, such as those in the splanchnic area of erect animals, where the fluctuations of content and of distribution are tidal, and very great, we see the muscular fibre more predominant. Elastic fibre then, even in the smaller vessels, is concerned with pulsation; muscular fibre with machinery for blood distribution.

At this point of the argument, when we come to explain the effect of stresses, and to compare or contrast the connective and the elastic constituents of the tube, it is convenient to compare the several properties of these two tissues. The elastic tissue might more descriptively be called the resilient or labile tissue, for it has little of the strictly elastic power of resisting deformation, although it has in large measure the capacity of recovering from deformation. Its elastic limits are then very wide; and in all materials the elastic limit, however wide, is usually well under breaking point. Moreover, when inner pressure, reaching a certain degree, overcomes the elastic resistance of the wall of the vessel, the stiffer adventitia, which may be compared to the stiff cover of the armlet of a manometer, soon controls its excursion. Again, the coefficient of elasticity is not a constant, even for the same section of an artery, but varies as the state of contraction of its circular muscular fibres; in a constricted vessel the elastica is thrown into folds. The connective tissue on the other hand differs from the elastic in possessing indeed more of the elastic resistance to deformation, but much less of the rebounding capacity, the resiliency. Its elastic limits are much narrower, so that when once overstretched it may never recover, but take a permanent set at a greater length. For instance, in so far as the muscular fibre of the heart may be penetrated by connective fibre, or be supplanted by it (p. 544), so far the cavity, under excessive tensions, is

nuclear origin, but that connective fibre is converted into elastic by the addition of elastin, is a postulate beyond my competence to discuss. Vide Fuss, Virch. Arch., 1906. 
less ready to dilate, but is more liable if overstretched to take a permanent set at a longer diameter. If then an encroachment of connective tissue upon the sphere of the elastic, in offering more resistance to internal stresses, may have some conservative effect, it is at the jeopardy of irremediable overdistension. Carrel ${ }^{1}$ has dexterously transplanted short lengths of vessel-for instance of the aorta-from one animal to another, and in a certain number of them secured recovery. In these animals, when killed at much later dates, the transplanted portion was found normal in position and calibre-not dilatedbut consisting entirely of connective tissue; muscle and elastic fibre were absent. To this state more or less in later life the arteries tend, whether atherous or not. The development of connective fibre may, as we have seen, be governed by certain degrees of stress, and where such stresses most tell there the connective fibre grows, as, to use my former analogy, woody fibre grows on the trunk of a tree most on the leeward side. Thus it is that in the intima of the fœtus there is no connective tissue; and that, with the stresses of life, it grows more and more up to old age.

After these preliminaries, we can more clearly perceive the values of the quantitative distributions of the connective and the elastic constituents of the arterial tube respectively. As regards the mechanical stability of these vessels, in German opinion the elastic and connective tissues, and above all the elastic fibres, take the chief place, the muscular or musculo-fibrous tissue being less tried or less vulnerable. Although in England we do not forget the protective effect of tone upon the tube, yet it is probably upon the elastic fibres of the intima that lateral stresses begin first to tell injuriously ; and here splitting, and then fatty degeneration of these fibres, or of their cement substance, is first betrayed. In our laboratories Dr. Ricketts, ${ }^{2}$ under Dr. Dixon's superintendence, by experiments with hypertonising drugs-tobacco, squill, barium chloride-brought forward a good deal of evidence to prove that rupture of the elastic fibres is the beginning of atherosclerosis. It is at this stage that the connective elements begin to appear in excess. Elastic fibre

1 Carrel, A., Rockefeller Studies, 1912.

2 Ricketts, G. R., Journ. Path. and Bact. vol. xii., 1907. 
in its impairments seems to react rather as a physical than a vital substance, as if prone to recede within the sphere of inorganic textures; not so much to degenerate as to show effects of stress beyond its physical limits. Dr. Harvey, ${ }^{1}$ testing the physical qualities of elastic fibres, found them almost of inorganic stability (p. 521). He stretched them, buried them for a time, and so on, and found them remarkably indestructible; but the nearer a tissue approaches to the physical the less its capacity of spontaneous repair. When under excessive increments of tension the fibres deteriorated they split up into fibrils, fragments and granules, ceased to respond to their normal stains, and calcified. Notwithstanding, there is little doubt but that elastic tissue, and its cement substance, do undergo fatty degeneration (Jores, Andrews, Torhorst), especially after mechanical strain. (Harvey); and no tissue is more ready for calcification, it may be by some vital chemistry.

The connective tissue is then a useful constituent up to a certain quantity; but to increase beyond this quantity-a quantity varying with the parts of the branches-signifies strain and tissue compromise, or senility. We have much yet to learn concerning the behaviour, and perhaps to discriminate concerning the kinds, of connective tissue; when for instance, as amid these new products, it is subject to fatty degeneration, and when not so subject; when it appears as a quiet and useful surrogate of waning specific elements, and when it invades, penetrates, and strangles them; also, how far Dr. Bashford's glimpses into the functional independence of the several kinds of tissue, and a sympathy throughout the system of each with its own kind, and a participation in its fortunes, may be verified; if again such movements starting in particular spots can extend widely by sympathetic excitement; if they are cyclical, and how far subject to advances and retrogressions under harmonic, incidental, or experimental conditions?

It is of much importance therefore in this enquiry, if by these characters we are to read some part of its history, to ascertain where and in what proportions connective tissue fibre is normal to the vessel (see p. 482). In this research we find much help in that work of Thoma by which he estimated the proportions in which

1 Harvey, Henwood, Journ. Exp. Med. vol. viii., 1906. 
growth of the connective tissue entered into the normal development of the arteries. If it is in the middle coat that we see the results of infection, it is in alterations of the connective and elastic tissues of the intima that we note the first signs of strain.

Let us then, taking our departure from Langhaas, Thoma, Jores, and Adami, give a little more consideration to these conditions of the connective and elastic tissues. The connective tissue, supplementing, as we have observed, the qualities of the elastic fibre, in so far as it has with less resilience more elastic resistance, increases as with bodily growth a stouter vessel is required to withstand the increasing blood pressures. In our comparison of the virtues of parts which, for the special needs of the circulation, are all essential, resilience, which equalises the variations of pressure, is for growth at least as important as resistance; we find accordingly that arterial development in the young is more conspicuous in the elastic than in the connective layers. Some sedulous and well-directed research was required to discover the degrees in which increase of this tissue may be of the nature of genuine growth and development; as contrasted with that increase of it which becomes far more evident as years go on, usually after the third decade of life, and is not of this nature, but rather substitutive or retrogressive. In an old artery it spreads amid the muscle cells and invests even the elastic fibres. But at present it is the intima that we have more particularly under consideration. Thoma, anticipating that the increase of connective tissue would be more simple and more unequivocal in young and lively vessels subjected to definite and rather rapid increases of blood pressure and volume, directed his attention, as I have said, to the growth of the central portion of the aorta after the closure of the ductus botalli. In this, as under gravitation in the arteries of the leg, although, as we have foreseen, the elastic layers are increased, yet, with the diversion of the blood stream on the closure of the duct, a definite and normal growth of the connective tissue is also manifest. To describe it as "hypertrophic thickening," if not inaccurate is scarcely apt; for this term suggests the abnormal or incidental, whereas the process consists neither in a reaction, an adaptation, nor a degradation. By imperceptible steps during youth and adolescence this growth proceeds, and 
probably never altogether ceases. In the artery of youth elastic resistance is low, but elastic limits very wide; as growth is fulfilled the arteries are strengthened by the tissue-the connective tissue-which, conversely, has a higher resistance but narrower elastic limits. Aschoff (loc. cit.) describes the aorta of infancy as presenting well-defined and separate elastic laminæ and muscle fibres. Then, as we shall see, the elastic slowly increases, the lamels thicken, more filaments branch off, and the muscular and elastic elements become more implicated. If in the third and fourth decade of a healthy life the connective tissue be almost stationary, on the turn of middle life and during old age it begins again to increase, a feature of declining years not peculiar of course to the arterial tree; but in this declension, whether readaptive or decadent, we can no longer regard the hyperplasia as a feature of growth but one at least correlative with deterioration. In a paper by Thayer and Fabian $^{1}$ it is shown, especially on the first part of the aorta, and as concerning the radials, that in the third and fourth decades of life, not in labourers only, but also to a certain extent in all persons, connective tissue slowly advances, especially in the harder worked arterial areas. (See on the Media pp. 496-97). It insinuates itself into the elastic strands of the intima, and delicate layers and filmy fibres of it may be seen on the inside of the elastica interna, on its outer side also, and within it, splitting its layers. Again in the musculoelastic layer it penetrates between the muscular fibrils, increases in the media, and indeed penetrates the whole wall; thus for a while fortifying its structures, but at the expense of their resiliency. The stream becomes more bouncing. In the smallest twigs, in many districts at any rate, there is no connective hyperplasia, or practically none, as in these fine vessels the tissue scarcely exists ; in them degeneration begins in the elastic fibre. Hallenberger, ${ }^{2}$ a pupil of Aschoff's, in a careful research verified these conclusions, and regarded the process as the cause of the "Abspaltung" of the elastic lamels, normal and abnormal. Now this fibrosis must be clearly distinguished from inflammatory proliferation; it is a completed connective tissue

1 Thayer and Fabian, Amer. Journ. Med. Sci., Dec. 1907.

2 Hallenberger, Inaug. Diss., Marburg (date mislaid). 
mesh, looser or closer, and presents proliferative cells, some of which may have a scavenger function, but not granular nor hyaline characters. This fibrosis may be seen in parenchymatous parts around vascular branches, as if due to irritation; but again in foci away from them, as if due to innutrition. An active mode of it may be well seen in an area of hyperæmia of collaterals on a block of an arterial twig. It seems here to be molecularly less stable than under other conditions, but the degeneration around it must infect it with decay.

At what stage this change lapses from growth into repair, or even into degeneration, is an intricate question, and, as it is one more academical than practical, we may pass it by with the reflection that, as we shall see, splitting of the elastica is also a very uncertain standard of stability, and one which varies in several areas and circumstances. In some medium-sized arteries a slight development of longitudinal muscular strands within the elastica, some multiplication of the elastic lamels, and some increase of connective fibre, are not necessarily to be taken as signs of disease. Such a process may be watched in the normal subject during adolescence and early adult life. But except in the first part of the aorta, fibrosis does not appear in childhood, say under the age of 10 ; and we may suppose the age curve for this tissue to have a flat top, more or less prolonged as the individual case may be.

Jores agrees with Thoma's observations (p. 517) and, by fat and elastic stains, indicates how, without atherosclerosis, this interstitial connective tissue may precede the decay of the elastic fibres; but in conspicuous quantities he is disposed to regard connective increase as partaking of the nature of disease, or at any rate of lesion and repair. Here we may compare the fibrosis of the tricuspid valve as a sequel of mitral stenosis: in the tricuspid it is due to long continued stresses, and for a while is protective; then it merges into strain and becomes a contracting scar (p. 195). We may be sure when, as in the thicker vessels of elderly persons, the connective tissue has begun to increase out of proportion to the elastic, that physiological is passing into pathological change. On the difficulty of standards of wall, and layers of wall, I have more than once insisted, and in this context the 
difficulty is multiplied by the variations of this connective element in various topographical tracts of the vessels, and parts of vessels, the tissue being developed in longer or shorter lengths as the habitual incidences of stress require, some arteries being much thicker than others. The earlier investigators, especially of the French school, gave much weight to this supplanting by connective tissue. Débove, Létulle, Duplaix and others followed Guéneau de Mussy in supposing it to be a primary and active invasion- "la diathèse arthritique " of this observer, la diathèse fibroide of the former authors, a process distantly comparable, one may presume, to Dupuytren's contraction? If this conception partook of the French schematic tendency yet, like the similar Gull and Sutton hypothesis, it led physicians to take a larger view of the whole subject. Thoma's views of these changes are so strongly tinctured by teleological notions that our gratitude is directed to him for his facts rather than for his arguments.

As in the next place we pass from the connective to consider the elastic strands, we shall find, as we progress from the child to the man, the same ambiguous boundary between the physiological and the pathological. Summarily, as we have seen, what happens under experiment is this: the earliest changes under stretching are strain, fibrillation, splitting and granular disintegration; the associated muscular elements also degenerate; then calcification begins in and outside the elastica and encroaches on the media, the calcified fibres making little lumps. The intima may or may not be thickened already, but seems disposed to this change, especially near breaks in the calcified tissues. Finally some few elements of repair may be detected. Jores has studied these changes of the elastic strands very minutely. The single elastica from early childhood seems to grow, by splitting off new lamels ; ${ }^{\mathbf{1}}$ in the twelve months' aorta it is doubled, and both its muscular and connective fibres develop; yet the hyperplasia of arteriosclerosis, though certainly regressive, assumes to the eye a similar form; indeed the secondary layers may be more copious than the original sheet. That this splitting may be purely destructive we see in septic

1 Lamel is a convenient and already an English word. In the Oxford Dictionary the authorities for it are Grew and Henry More; and later Miss Yonge. 
conditions, when the elastic tissue, on the whole relatively resistant, is prone to split, and microbes may be seen between the layers. ${ }^{1}$ I have said that the elastic fibre is not a very living element (p. 516); it does not develop much, nor is very capable of repair. If we examine its multiplication, and here it is that the earliest pathological changes are found, we can hardly regard these multiple lamels as physiological. They are unstable, apt to blur, to lose regularity of outline, to rupture and break up into granules. And between them other structures, muscular and connective fibre, take the nuclear stain badly, and are clearly degraded; they will break indeed into vacuoles and fat. It is often difficult to say whether apparent granules are such, or cut ends of multiplied fibrils. In this kind of disintegration, according to Jores, the disease consists ; thus and otherwise, wherever it be, betraying its kinship to atheroma. In normal growth there are, as we have said, two modes of development; one of the aorta and vessels of the first magnitudes, and of the parts about certain angles of stress, the other of the vessels of middle and lesser magnitudes, such as the brachial and radial. So also the arrangement of the elastic constituent in arteries of elastic and muscular type is not identical; to resist high lateral pressures in the elastic arteries special elastic lamels are developed. Still this difference is not so great as is often asserted, and does not much affect the characters of disease. If we compare (as Jores, and Thayer and Fabian have done) aorta with radial, we find during infancy, in what we may regard as the intima of the large vessel, that a homogeneous elastic lamel soon separates from a fine network of elastic fibres surrounding muscular fibres. This is Jores' first splitting. Then elastic strips detach themselves from the homogeneous lamel. If we compare this with the radial we find a similar, if not identical mode of growth, but much slower; so that the definition attained in the aorta, say in twelve months, in the radial is not attained for ten years. But in the large artery, as we all know, delicate muscular fibres, most of them lengthwise, appear between the elastic-forming in

1 The genetic relations of connective and elastic fibre, and the manifolding of growing elastic fibre, will be found, by Schäffer, J. P., in the Journ. exp. Med., Feb. 1, 1914, vol. xix. ; and Caforio, Rif. Med., Jan. 17, 1914. 
Thoma's phrase the elastic-muscular layer of its intima. In the lesser arteries this musculo-elastic structure is seen rather at angles of stress, than in the lengths of them where, for the muscular, fine connective fibres are substituted. In the twigs the reduplication of the elastica tapers out; there we may see it degenerated simply. In youth the manifolding of the elastic elements, under the formative stimulation of stress, progresses likewise in the aorta and the small vessels respectively, but not identically nor pari passu in all the smaller vessels. Still, broadly speaking, in the smaller the elastica multiplies two to threefold; in the aorta three to fourfold, a stage which in the third decade is, in health, constant. This mode of proliferation is the hyperplastic form of Jores, though I repeat that the prefix "hyper" is rather suggestive of a phase in which this process is beginning to slip from the physiological into the pathological mode, into phases which, when more definitely morbid, constitute Jores' second form, called, not very happily, regenerative (reparative). Thus physiological growth is chiefly visible in the elastica, less so in the muscularis; and in connective fibre is distinct only in certain areas of stress; at this stage it is that elastic resistance, resilience and, with them, stability are at the best. In this efficient form the structure should be stable for some twenty years; not until about the end of the fourth decade does it begin to turn towards decline.

In disease, these features preserve no less an importance, though degradation is manifested chiefly in the behaviour of the elastic fibres, and the hitherto tenuous connective strands. The first phase is no more than a normal increase would be; in transition or suspensory phases we are guided by some intermediate and equivocal combinations. Diagnostic distinctions however can ordinarily be made plain enough ; slight degrees of splitting of the elastic lamina may be neglected, so long as no fatty reaction is given to Sudan. Dr. Cowan says that the elastica is peculiarly prone to suffer in the infections, such as tuberculosis, pneumonia, and typhoid fever, when it blurs, and loses stain characters (p. 285). Faber also lays much emphasis on this fragility of the elastica. When splitting of the elastica into layer after layer has evidently become a morbid activity, or a dilapidation (Herringham, Jores and others), we soon find 
these fibres become brittle; they break up and are separated by proliferating substance, perhaps also by some round cell infiltration, and a hyaline "between-substance" apparently allied in genesis to connective fibre, and prone to calcification. It is readily differentiated by stain from amyloid substance. These features, with some extension of vascularity from the vasa vasorum, we may regard, amid these chiefly degenerative changes, as a slight and subordinate reparative effort. As the conspicuous proliferation of the elastica bears no proportion to the degrees of need of the media, it cannot be interpreted teleologically.

Whether these "regenerative" (reparative) phenomena are or are not to be called inflammatory, depends on the sense in which this word is used. Jores does not commit himself to an opinion on this point, though he implies that the process is in part reparative. Cornil and Ranvier regarded the atheroma as the primary change, inflammatory incidents as consequent; Virchow and Ziegler thought an inflammatory preceded the atheromatous process. Köster, in 1876, appealed to increase of connective tissue and to small cell infiltration as signs of inflammation ("mesoaortitis") ; but he worked chiefly on the aorta and probably had more or less of syphilis under his eye. Besides, scavenger cells may be abundant in areas of decay. Létulle, while regarding the process as inflammatory, and also using the term " endarteritis," yet admitted that its products are of very unstable quality, if not indeed necrotic. That it is inflammatory in the full sense in which Virchow used the word "endarteritis," in the sense in which this term is properly applied to the changes in a vessel involved in an inflammatory focus - as in a tuberculous or septic process, or again as artificially produced by Dr. Mott, who painted certain vessels with irritants, or corrosives-is certainly not the case. This condition, of noncalcifying endarteritis properly so named, does not fall within my subject; nor does that terrible disease acute obliterative arteritis. A sclerosed artery is however by no means immune against an inflammatory arteritis, and the supervention of such an arteritis in an old person may suddenly determine gangrene.

The truth is that of late years our conception of inflammation has been whittled down so far that, generally speaking, it has almost lost definition. But I make now a specific allusion to 
it in view of my own opinion that this hyperplasia of the intima takes its origin in the lesion of strain, of tension, and of shear at the plane of apposition of the intima to the coats which mechanically support it in its functions (p. 513). This process may be seen not only, as we presume, in trauma, but also under other conditions of mere mechanical strain; as, for example, in the pulmonary vessels in mitral stenosis, first demonstrated, or illustrated, by Traube (p. 202). But, as a complex organ in the degree of its complexity is more and more circumscribed in its forms of response to contingencies (p. 324), reactions, the same as those of Jores' second or regenerative mode or very similiar to them, are manifested under other conditions; as in a part of an artery in thrombosis, or beyond a ligature, where internal pressure is wholly cut off; and so on. As then the morbid histological characters under many various causes may be uniform they may tell us little as to genesis, though they may indicate a greater or a lesser reparative capacity. Connective tissue increase in advancing years is a universal process, probably in obedience to several kinds either of irritation or of shortcoming, and not always of the same mode; here for instance it may signify an inflammatory or specific activity, there a substitution; or a common denominator anywhere.

But, in taking so much advantage of Jores' work, I must beware lest I commit him to opinions not his own. Jores does not identify his regenerative "proliferation" of connective tissue with arteriosclerosis; in it he had in view only a healing process, like that of a wound, and the result a scar, without atheromatous (fatty) proclivity. Perhaps we may put it this way; that Jores' regenerative connective tissue form arises in the innermost connective layer of the intima, and increases out of proportion to the elastic. The atherosclerotic process is seen at least as soon in the musculo-elastic layer between the elastic fibres and splitting them; young elements, muscular and connective, which run quickly to fatty decay. But the two processes are often simultaneous. A mere hyperplasia of the subendothelial connective tissue in which no fat response can be found is not common, but may be seen in the earlier stages of hyperpiesis, and in a true endarteritis. In thrombangitis obliterans and endarteritis proper, lesions which, except as 
syphilitic, lie outside my subject, the elastica is often intact, at any rate before stages of demolition. And this distinction seems to have some validity against Sumikawa's ${ }^{1}$ view of the process, who, after an examination of 96 cases, still urges that atherosclerosis is in nature rather a chronic inflammation than a primary degeneration. He relies on the cell proliferation and connective tissue increase which we all admit more or less (pp. 518-19). But that atherosclerosis is a result rather than a response; and, as from Kirkes and Rokitansky to Jores, pathologists are agreed that this response is best marked at first in areas of greater stress; though Sumikawa might demur that if stress be the determinant of site, it may not be the chief, or the intimate, cause of the atherosclerosis itself.

For my part, if I think infections often fall upon the media, or, as in syphilis, upon all the coats, yet I also admit the great probability that causes other than stress-poisons, toxins, or metabolic perversions-may, as it would seem with the vasa afferentia of "Granular Kidney" (p. 399), engender irritative changes in the intima in which ordinary stresses and hydraulic pressures take but a subsidiary part. As, whether by contingent causes or inherent defect, structure is enfeebled, mechanical stress, however moderate, will the sooner distort and spoil the tissues so enfeebled. An old shoe wears long on the shelf, but not long on the foot. And on the histological side my comparatively inexpert impression is that in mere repair-sound repair-the proliferative activity (e.g. small cell infiltration, etc.) is in certain cases to be seen not so saliently in and about the intima and the plane of its junction with the media as in the media itself, and still more perhaps in the adventitia with its enlivened arteriolar and lymph passages. I admit that in some few very healthy old persons we may find Jores' regenerative process in almost a pure form, especially in the arteries of the leg (e.g. posterior tibial), atherosclerosis being practically absent ; but even then we may observe that the elastic fibres have almost always, at stress points, been overstretched, are split and ruptured, and have undergone some fatty degeneration. It is said by some pathologists of consideration that a diffuse sclerosis may be detected by the microscope in cerebral arteries

1 Sumikawa, Virch. Arch., 1909, vol. cxevi. 
which to the naked eye seem normal. Some leptomeningeal fibrosis also may be discovered in such cases. These changes are evidently of very slow course, and may never give rise to symptoms.

As regards the intimal disease and the decades of life, we may turn again to Thayer and Fabian, who examined 61 morbid radial arteries at various ages, from 56 days to 83 years. But not only in the middle, in the small arteries also-such as the cerebral and coronary branches-the wall becomes thickened and unequally, patchily, discoloured. There are tracts of moderate thickening, and now and then a hump. Under the microscope "atheromatous" masses may be seen, sometimes almost closing the channel. In other tracts of these arteries, or of the renal and the like, both intima and media may have fallen into an atrophy together, or into hyaline decay. Up to the end of the fourth decade, when the second strand of the elastica, and the manifolding of it, and more or less increase of connective tissue may be observed, especially in the limbs of labourers-in one, for instance, whose age was only 22-the connective increase may have extended to the media. Still such a state, abnormal at the age of 30 , can scarcely be called abnormal at the age of 50. However, in the fifth decade more significant changes appear. The vessel may now stand open; it contains from intima to media yet more connective tissue (van Giessen's stain), which may even be supplanting the muscular fibre; the elastic strands also have multiplied and separated, and the vessel is clinically "thickened." The stress of work has begun to tell, at any rate in certain areas.

Here again, as many pathologists have stated, and as Jores by his fat stain has demonstrated, such alterations even at this comparatively early stage are not diffuse, or not only so, but are focal and patchy. Indeed calcification, on Adami's hypothesis, implies a previous fatty stage. It is a matter of common observation that in the smaller arteries cross-sections show irregular, focal protrusions, often of a crescentic section, thrusting into the lumen, even to closure of it; foci which react to the fat stain. The arterial decay of the ancient Egyptians 3000 years ago is described by Ruffer and Elliott Smith (loc. cit.; in 24 subjects) as exactly the same as the atherosclerosis 
of to-day. In these cases the earliest changes were in or close below the fenestrated membrane; they began at discrete points, and later fused into larger tracts.

If with a small scalpel we split open the finer vessels as far as we can, and examine them even with a common lens, as we pass to lesser and lesser magnitudes we shall find the thickenings by no means " uniform"; smaller as the foci become, we shall still perceive the same patchiness of disease as in the large vessels, and (on cross-section) the crescentic humps, which all respond to the fat stains. In every cabinet the specimens are many, even in very small vessels, of ruptured endothelium, with tiny craters of "ulceration." 1 Or, if we go back to the aorta and to the alleged fibrotic parcels of it - "sclerotic" in contrast with pulpy protrusions of atheroma with its "succus luteolus" 2-we may ascertain by Jores' method that there is no sclerotic thickening of the inside of a vessel in the depth of which a retrogressive fatty decay is not to be detected. It is in patches then, differing from aortic patches in size only, that the intima is thickened, that the elastic fibres have broken up and are dissolving, and that areas of coagulation necrosis, leading to calcification, have formed - probably in the deep intima encroaching on the apposite parts of the media, perhaps forming even within it; but this disputed question I will not reopen here (p. 485). My own opinion is that in atherosclerosis of the visceral type these changes displace and damage the media but do not arise in it. On the other hand the endothelium, bathed in the blood, resists these changes with rare vitality; though some hyper-activity of its cells may be perceived. In Dr. Auld's words, "the epithelial (and connective tissue) cells behave like any other tissue on which an inordinate demand is made."

Again (see p. 326) we may ask why degenerative processes, whether presenting some ineffectual reparative reactions or not, appear in patches? Difficult as the question is, we see

1 See Adami and Klotz, loc. cit., and Ribbert's Lehrbuch, p. 65. Ribbert for this affinity uses the word " analogous," but by his context he must mean homologous.

2 As described for instance by Russell, Brit. Med. Journ., Oct. 10, 1908 ; and, as reflecting recent teaching, Aitcheson, Thesis for Med. Oxon. Arch. Path. Inst., London Hospital, 1908. 
this disposition continually, and in many parts of the body. The physicist may think of segregation, or of the discrete crystallisations, or granulations of metals under long persistent and rhythmic stresses, such as beating with a mallet. Some remarks of Dr. Mott ${ }^{1}$ on foreign bodies in the blood, such as bacteria for example, seem here not irrelevant: he says that such bodies seem not to be diffused equally through the blood plasma, but to run in groups. Even in the violent and destructive but uniform strains of experimental arterial injury, some dispersion of the consequent lesions is to be observed; and here we may note Dr. Hollis's bombardments, to be discussed presently. In the larger arteries we perceive this partiality first in the streaks and tallow drops, so fully described by Ainslie Hollis, and later by Cowan, and emphasised by Jores and his pupils, Askanazy (1907), Simnitzky, and Benda (1909); spots and streaks regarded by Virchow as not atheroma, though now believed to be of this nature. These clearly represent lines or points of stress; the segregations are perhaps determined by molecular affinities or surface tensions; the connective tissue increase, if any, being subsequent. We may presume that no tissue is of perfectly uniform quality throughout. A patchy beginning of atherosclerosis is very familiar in the coronary arteries where, as elsewhere, it begins rarely, if ever, diffusely, but in parcels which seem to begin in the first part of the branch, the part most exposed to stresses from the aorta.

Dr. Hollis has closely investigated this familiar streaky distribution of early atheroma-for such it is, however superficial in appearance-in various parts of the aorta and some of the largest branches and forks; especially in the coronary and cerebral vessels, which is remarkable: in the words of Morgagni, "lineæ subobscuræ in longitudinem directæ ... et albidæ maculæ." This character is best observed in incipient cases in young subjects, when it is probably due to an infection, such as typhoid fever after which they are very frequent (p. 282). Simnitzky, in a series of necropsies on bodies of ages from 2 to 25, found them in 48 per cent. As the lesion becomes chronic, their outlines are soon blurred, so that the period of their initial significance is brief.

1 Mott, F., Journ. of Path. and Bact., 1894-95. 
This slightly raised " linear" and spotty subendothelial atheroma is to be seen in well-marked and instructive forms in the descending aorta, a part of this great vessel which, as Dr. Hollis says, and as Morgagni said before him, we are apt to leave unopened. For instance, in two of Hollis's cases (of one of which he gives an illustration) neither the state of the valves nor that of the arch itself gave any notion of the extensive atheroma which was revealed on slitting up the descending limb. Linear streaks of incipient atheroma in this limb of the great vessel are straighter than in the ostial portion, where their spiral or twisted arrangement, or when near the base transverse, signifies the eddies by which they are engendered, and of which they are the record. Posteriorly, they trend towards the floor of the arch, their long diameters being with the main stream of the blood. ${ }^{1}$ In the descending aorta of long bedridden cases, these streaks are most abundant in the region of the second intercostal artery, and are most evident on the dorsal aspect of the lining, and penetrate even to the media. One case seemed, in Dr. Hollis's opinion, to show that such atheromatous streaks may form "within a few days"; and he presumes that the "atheromatous tracery may be produced on the intima within a week." The incipient changes at the sites of branch openings begin, " not so much on the lining of the aorta, as along the sides of the branch vessel," and are probably due to vortices. These features, as we have seen in the chapter on Causes, have, in my opinion, an intimate bearing upon the mechanical factor in atheroma; but this factor in Dr. Hollis's opinion is not, or not merely, that of hydraulic stresses, but also of " bombardment by particles"- detritus or cells. Elsewhere also he speaks of bombardment "by clouds of noxious particles," and suggests that it is by the stinging of these that vaso-constriction may be stimulated and maintained, so that to the initial disease the farther evils of excessive blood pressures may gradually be added. On examining the lowest stratum of the contained blood, he finds that in supine patients, contrary to their specific lightness in comparison with the plasma, leucocytes are there accumulated, instead of floating upward to the ventral aspect. But may we not also surmise that they had gathered towards the dorsal aspect as phagocytes, in

1 See also Klotz and Manning, Journ. Path. and Bact., Oct. 1911. VOI . I 
search of toxic matter or detritus ? Of the penetration of the diseased patches themselves, and of the walls, by leucocytes, Dr. Hollis has published some interesting illustrations.

Until Jores by the Sudan III. stain demonstrated that the fat with which the tallow drop or streak is so fully charged is of universal significance in atherosclerosis, even in tracts of quite fibroid appearance where, until this test was applied, no fat was perceptible or even supposed, authors were wont to discriminate a fibrous arteriosclerosis from atheroma (p. 482). But in the fatty reaction lies a criterion of atherosclerosis in whatsoever vessel. It may be that some of the fatty specks or streaks on young aortas, if quite superficial and independent, may vanish, or leave a small scar $;^{\mathbf{1}}$ but, as they are often traced to some infection, they may also signify a deeper, but in youth remediable, disintegration. It is true notwithstanding that not only the manifolding of the elastica, but also the encroachment of the connective fibre, precede the fatty decay, and may do so by some considerable time; except in the acute infections, when fat appears quickly and abundantly, especially in the aorta. But ere long sharp outlines are blurred, the elastica, especially in its older strands, begins to accept the fat stain, and then points of fatty necrosis appear definitely. The cement substance is no doubt concerned in these degradations, but it is not easy to apportion the shares of each rudiment. The doubt if elastic fibre could break up into granular degeneration is now quite decided in the affirmative; and, as the elastica breaks up, the artery (vasomotor conditions apart), yields, lengthens, widens, and becomes tortuous. But I must reiterate that in a few sound bodies of old men the elastic fibres, although susceptible to both fatty and calcareous change, lose their quality more slowly; and for a while little or no more alteration is to be seen than an increase of connective tissue (p. 517) which, coming to the aid of a less resilient elastic and a less tonic muscular coat, may, by narrowing the excursion of the beats, withstand even better the slower current, but more bumping oscillations and somewhat higher systolic blood pressures of the elderly; although, on the other hand, it gives less help in recoil.

1 Adami, Amer. Journ. Med. Sci., Oct. 1909. 
In age therefore, as Strasburger ${ }^{1}$ says, the aorta, even when it has undergone a good deal of molecular alteration, may be smooth on its inner surface and not much widened. And although the first signs of degeneration appear in the outermost intima, contiguous to the media - on what I have called the line of shear-and so may disturb the media, yet originally and mainly the lesion pertains not, as Thoma supposed, to the media, but to the intima (vide p. 486). Sumikawa says expressly ${ }^{2}$ that arteriosclerosis (in his phrase "a chronic productive inflammation" (p. 525) passing into degeneration) begins in the layers contiguous to (angrenzenden) the media, and may extend to this coat or may not. Section after section is shown to me, by colleagues and by the readers of Dissertations, in which disease attributed to the media, which may have encroached both upon the media or its contours, and upon the lumen, really pertains to the intima. We have seen already however (p. 493) that areas of disease of the media, whether fibroid or necrotic, are by no means always contiguous to diseased patches of the intima.

In conclusion then, omitting syphilis and other infections, I repeat that arteriosclerosis takes its rise in tensile and shearing stresses which tell most at the junction of subintima and media. The mesh is stretched, its finest fibres are rent, and plasma exudes into the interspaces. If this process take place in infinitely minute measure, and in young and healthy parts, the hyperplasia may be a genuine conservative hypertrophy at the plane or angle of stress ; as also at the offsets of the intercostal arteries, or at the bifurcation of the carotid (Hollis and others). This hypertrophy resists the stress, and all goes well. But if, as in the aorta even of young persons, the stress be relatively high, and still more if the aorta be sapped by some infection, or vexed by excessive efforts in persons no longer young, or in whom the vessels were originally defective, or again, if in some disorder, effort not being excessive, the arterial pressures have ruled too high, then the consequent hyperplasia is less stable, less formative; and degeneration begins to bear a larger and larger proportion to reaction. The multiplication

1 Strasburger, "Über die Elasticität d. Aorta bei beginnender Arteriosclerosis," Mü̈nch. med. Wochenschr., 1907, No. 15; also Aschoff, loc. cit.

2 Sumikawa, Virch. Arch. Bd. 196, H. 2. 
of vasa vasorum, and the finer hyperplasia which repairs the minute disruptions of cell from cell are overborne, elasticity and tone are enfeebled, and the vessel begins to yield as a whole. Nuclear and small cell activity soon wane, and, if notable at all, are observed only on the skirts of the lesion; nuclei and fibres begin to decline their stains, and become hyaline or put on those granular characters which soon react as definite fatty specks. Thus it is that early and transient phenomena of repair merge into those of decay, hypertrophy being lost in an accumulation of waste. Thus in small arteries the lumen is often closed. And if the intima, beyond the epithelium, be nourished by imbibition, as the coat alters its nutrition is further impeded. Such fortifications, or degenerations, we observe in other parts exposed to mechanical stresses, as in the mitral valve of a hypertrophied heart; or at the attachment of the chordae tendineae and the corresponding cardiac insertions; or, again, at the insets of the aortic cusps. Of the leaf of the valve there may be a "pure sclerosis," but about its insertion atheroma; and at such spots the splitting of elastic fibre, and the rest, may be recognised as in an artery. ${ }^{1}$

While it cannot be said that toxins, autogenetic or foreign, have no part in these issues, whether arterial or elsewhere, yet in ordinary cases their part, if any, is remote and inappreciable (p. 236), while the factor of stress is salient and unmistakable. ${ }^{2}$ We can doubt no longer that the elastic fibres, naturally stiffened into strands of very low vitality, do nevertheless undergo physical and chemical degradation; and on the other hand we are told on the best authority (Aschoff and others) that, after arterial development is complete, they cannot be restored. As they grow old and lose resiliency, proliferation of the surrogate connective tissue, as normally observed in childhood, supplies their place so long as regenerative capacity holds; though, as I have said, this description must be qualified by the proviso that in the arteries of very vigorous old people the alteration may not much exceed, and may not amount to more than, connective tissue defence. In these cases

1 See e.g. Dewitzky, Virch. Arch., 1910; and the same process may be seen about a thrombus, or on the distal side of a complete obstruction.

2 See Beitzke, Virch. Arch., 1901, vol. clxiii. 
the elastica is stretched (Adami, Marchand, Jores, Herringham), and in parts may be spoilt; the vessel is thinner, and the auxiliary connective tissue increasingly manifest, but there is no fatty change, focal or diffused, in the arterial coats; the superficial fatty streaks, from which few even of young arteries are free, being of course excepted. As it is but in a very small minority, even of old men healthy up to death, that atherosclerosis is altogether absent, it would seem, when present in such subjects, to be expressive, as I have supposed in the section on Causes, of little or nothing more than prolonged mechanical wear and tear (p. 236). The amazing thing is not that the arteries suffer in process of time, but that they have developed a structure of such perennial duration.

When after these histological descriptions we return to the questions (p. 510) whether atheroma and arteriosclerosis are or are not identical in nature, and even in essential form, and if the name atherosclerosis be justified, we find that within the general category of ordinary arteriosclerosis are contained, besides atherosclerosis in its more familiar form, certain extensions of fibrosis, medial, or rather intima-medial - the more extensive connective hyperplasia being intimal in origin, arising, that is, as stellate and spindle cells between the endothelium and the elastica-and furthermore a supposed diffuse syphilitic peripheral sclerosis not as yet very distinctly demonstrated. The specific process could rarely cause any confusion; the former, whether regenerative (readaptive) or atrophic, is a subordinate feature of ordinary arteriosclerosis. We agree that the muscular fibre cannot itself lapse into connective fibre (p. 481). If subordinate and luetic forms be omitted, I think we must be convinced of the substantial identity of ordinary arteriosclerosis and atheroma. I do not forget Professor Adami's warning lest our investigations be " too aortic," and lest we come to decisions upon superficial characters. Still, if for the moment we disregard the long vessels of the limbs, recent observations agree more and more in concentrating our attention upon the intima, and in showing that sclerosis of the aorta, of its larger branches, and so on, of the coronary, cerebral, and the rest, is atheromatous; so that all such vessels may be included under the name of "Atherosclerosis." If in the long straight arteries of the limbs 
appearances at least are somewhat otherwise, if in these vessels the media prove to be the first, as it is certainly the most extensive, seat of disease, if the muscular fibre suffers and decays while connective fibre waxes (Mönckeberg, Adami, Marchand, Aschoff and others), this may be regarded as another mode of arteriosclerosis, while in certain areas transitions or blends between the kinds may be seen.

To the etymologists who will have it that the name atheroma (or atherosclerosis) shall not be extended to any process, however akin, in which pockets of porridge-like matter ( $\dot{a} \theta a \dot{\rho} \eta)$ are not to be found, we shall pay little heed; if all our words were to be cut back to their roots, language would come to an end.

Sclerosis of the veins I must pass over; on this subject indeed we have not much definite information, and, clinically speaking, it does not very nearly concern us. (On portal sclerosis, see Vol. I., p. 450.) Sclerosis of the veins and venules certainly does occur, but it is altogether minor in degree and importance to arterial sclerosis. In them it cannot, as a rule, be due to excessive internal pressures, unless from local obstruction, such as hæmorrhoids, cirrhosis of the liver, and so forth ; or again under gravitation, as commonly seen in such veins as the femoral, saphenous, or popliteal. External frictions and contusions, or compression at certain points by the contractions of the voluntary muscles may account in part for the obviously thick and dilated veins of the arms of labouring men. This thickening seems in them to consist in a subepithelial fibrosis of the intima; hyaline and fatty changes are apparently less common in the veins than in arteries. The sclerosis which arises in the pulmonary arterial system under high pressures in them and the right ventricle, as in mitral stenosis, is, as we have seen, homologous with ordinary atherosclerosis; if less constant than one might have supposed it is not infrequent; probably its minor degrees are often overlooked. 


\title{
A SYSTEM OF MEDICINE BY MANY WRITERS
}

\author{
Medium 8vo. \\ SECOND EDITION
}

EDITED BY

Sir CLIFFORD ALLBUTT, K.C.B., M.D., F.R.S.

AND

HUMPHRY DAVY ROLLESTON, M.D., F.R.C.P.

Vol. I. Prolegomena and Infectious Diseases. 25s. net.

II. Part I. Infectious Diseases (continued), Intoxications. 25s. net.

II. Part II. Tropical Diseases and Animal Parasites. 25s. net.

III. Certain General Diseases, Diseases of the Alimentary Canal and Peritoneum. 25s. net.

IV. Part I. Diseases of the Liver, Pancreas, Ductless Glands, and Kidneys. 25s. net.

IV. Part II. Diseases of the Nose, Pharynx, Larynx, Trachea, and Ear. 25s. net.

V. Diseases of the Respiratory System, Disorders of the Blood. 25s. net.

VI. Diseases of the Heart and Blood-Vessels. 25s. net.

VII. Diseases of the Muscles, The Trophoneuroses, Diseases of the Nerves, Vertebral Column, and Spinal Cord. 25s. net.

VIII. Diseases of the Brain and Mental Diseases. 25s. net.

IX. Diseases of the Skin. General Index. 25s. net.

MACMILLAN AND CO., LTD., LONDON. 


\section{A SYSTEM OF MEDICINE}

\section{SOME PRESS OPINIONS.}

$L A N C E T$.- " A work which must undoubtedly rank as a foremost treatise on the scientific medicine of to-day. It contains contributions from those whose qualifications for the task imposed on them are of the highest order, so that the reader can feel certain to find in its pages the most recent as well as the most valuable information that exists on these matters."

BRITISH MEDICAL FOURNAL.-" A work which must rank as one of the most important standard books of medical reference in the English, or indeed any language, that has appeared for many years. It may be regarded as the successor, and as occupying the same authoritative position that Reynolds' System did."

MEDICAL PRESS AND CIRCULAR.- "It is thoroughly representative of the best British medical opinions and work, and ranks amongst the foremost medical works of its time."

SCOTTISH MEDICAL AND SURGICAL FOURNAL."It is almost superfluous to commend to the medical profession a work so well known and of such established authority as Dr. Allbutt's System of Medicine. The second edition has obviously undergone the most thorough revision and amplification. . . . It is impossible in the short limits of this review to attempt to do justice to the great value of Dr. Allbutt's System. Every article which it contains is worthy of a prolonged consideration. We can only conclude by wishing the second edition the same success which has been so signally attained by the first, and by suggesting that even those who possess the first edition will do well to secure the second, which, as we have endeavoured to show, is to all intents and purposes a new book."

\section{By Sir CLIFFORD ALLBUTT, K.C.B.}

THE HISTORICAL RELATIONS OF MEDICINE AND SURGERY TO THE END OF THE SIXTEENTH CENTURY. Crown 8vo. 2s. 6d. net.

ON PROFESSIONAL EDUCATION, WITH SPECIAL REFERENCE TO MEDICINE. Crown 8vo. 2s. net. MACMILLAN AND CO., LTD., LONDON. 


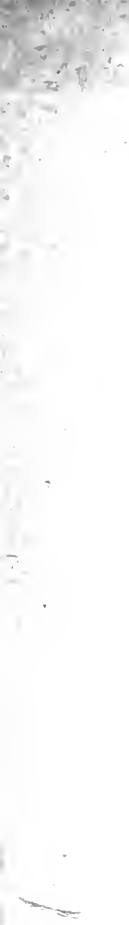

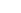




$$
\Gamma
$$




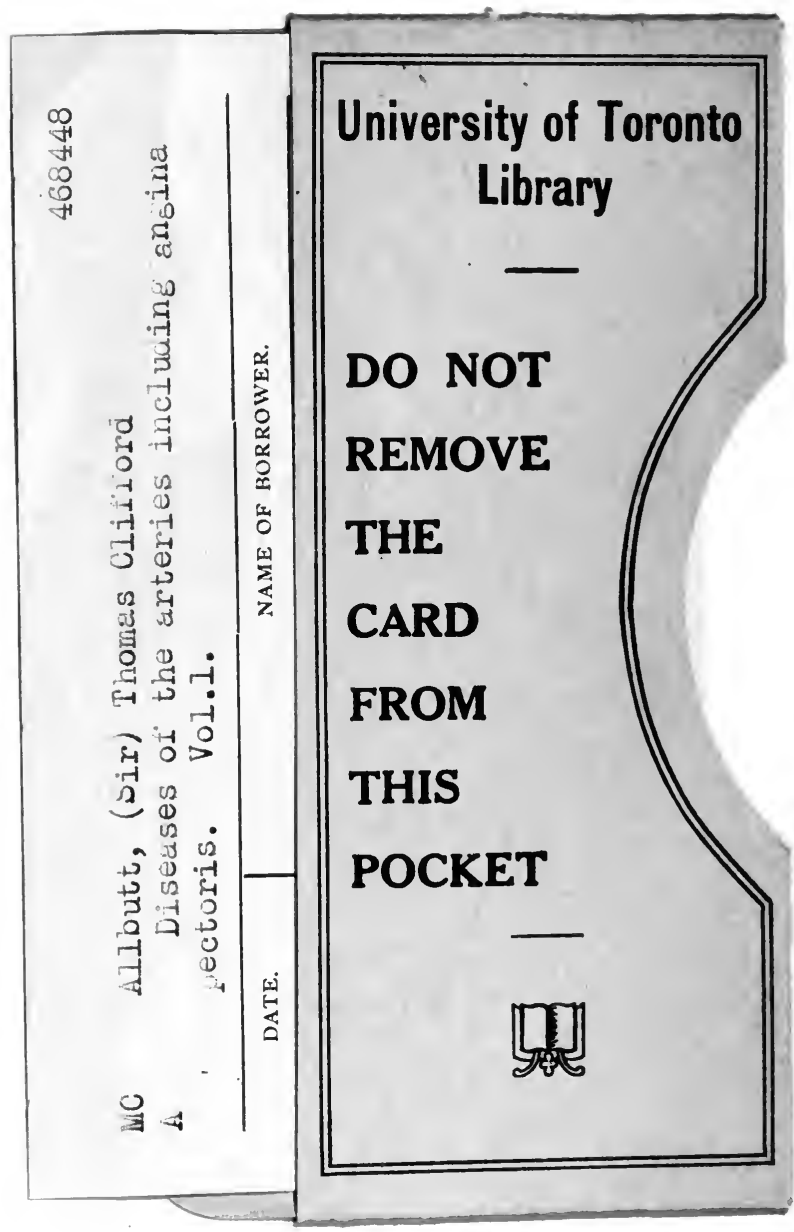


